KECLIVEO

JUR 24 10:3

\title{
Petroleum
May 1993
}

Suppisy

Monthty
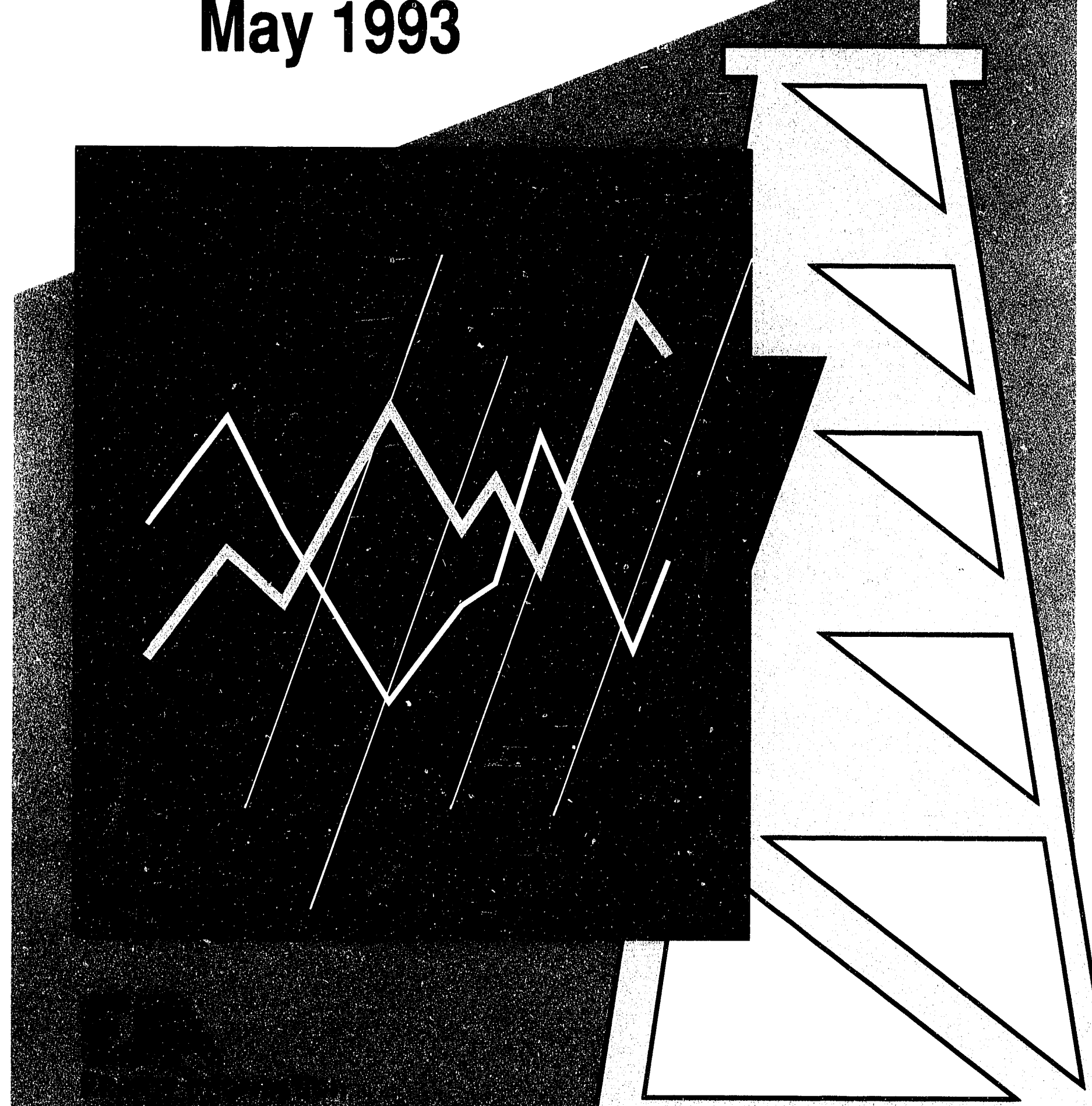
This publication and other Energy Information Administration (EIA) pliblications may be purchased from the Superintendent of Documents, U.S. Government Printing Office.

\section{All telephone orders should be directed to:}

U.S. Government Printing Office

McPherson Square Bookstore

1510 H Street N.W.

Washington, DC 20005

(202) 653-2050

FAX (202) 376-5055

9 a.m. to 4:30 p.m., eastern time, M-F
Superintendent of Documents

U.S. Government Printing Office

Washington, DC 20402

(202) $783-3238$

FAX (202) 512-2233

8 a.m. to 4:30 p.m., eastern time, M-F

All mail orders should be directed to:

U.S. Government Printing Office

P.O. Box 371954

Pittsburgh, PA 15250-7954

Complimentary subscriptions and single issues are available to certain groups of subscribers, such as public and academic libraries, Federal, State. local, and foreign governments, and the media. For further information, and for answers to questions on energy statistics, please contact EIA's National Energy Information Center. Address, telephone numbers, and hours appear below.

\author{
National Energy Information Center, EI-231 \\ Energy Information Administration \\ Forrestal Building, Room 1F-048 \\ Washington, DC 20585 \\ (202) $586-8800$
}

TTY: For people whs are deaf or hard of hearing: (202) 586-1181

9 a.m. to 5 p.m., eastern time, M-F

We thank the following for the use of their photographs and illustrations in $t^{3}$ is report.

Champlin Petroleum Co., page xi (courtesy of the American Petrolcum Institute).

Cities Service Co., page xix (courtesy of the American Petroleum Institute).

Standard Oil Co., page 1 (courtesy of the American Petroleum Institute).

Phillips 66 Co., page 33 (cnurtesy of Phillips 66 Company).

Texaco Inc., page 109 (courtesy of Texaco Inc.).

Standard Oil Co., page 113 (courtesy of the American Petroleum Institute).

Texaco Inc., page 141 (courtesy of the American Petroleum Institute).

American Petroleum Institule, page 145 (courtesy of the American Petroleum Institute).

Atlantic Richfield Co., page 151 (courtesy of the American Petroleum Institute).

Released for printing: June 4, 1993

The Petroleum Supply Monthly (ISSN 0733-0553) is published monthly by the Energy Information Administration. 1000 Independence Avenue, SW, Washington, DC 20585, and sells for $\$ 78.00$ per year (price is subject to change without advance notice). Second-class postage paid at Washington, DC 20066-9998, and at additional mailing offices. POSTMASTER: Send address changes to Petroleum Supply Monthly, Energy Information Administration, EI-231, 1000 Independence Avenuc. SW, Washington, DC 20585.

Cover: Artist's rendition of a graphic display with a profile of a drilling rig. 


\title{
Petroleum Supply Monthly
}

\author{
May 1993
}

\author{
Energy Information Administration \\ Office of Oil and Gas \\ U.S. Department of Energy \\ Washington, DC 20585
}

This report was prepared by the Energy Information Administration, the independent statistical and analytical agency within the Department of Energy. The information contained herein should not be construed as advocating or reflecting any policy position of the Department of Energy or any other organization. 


\section{Electronic Publishing System (EPUB)}

\section{User Instructions}

EPUB is an electronic publishing system maintained by the Energy Information Administration of the U.S. Department of Energy. EPUB allows the general public to electronically access selected energy data from many of EIA's statistical reports. The system is a menu-driven, bulletin board type system with extensive online help capabilities that can be accessed free of charge 24 hours a day by using a terminal or PC with an asynchronous modem. (EPUB will be taken down briefly at midnight for backup.)

\section{CONFIGURING YOUR PC SOFTWARE}

PC users must provide the following information to their communications software in order to successfully access the EPUB system. Consult your communications software documentation for information on how to correctly configure your software.

Communication Parameters:

BAUD RATE: 300 - 2400 bps

DATA BITS: 8

STOP BITS: 1

PARITY: NONE

DUPLEX: FULL

TERMINAL TYPE: examples: ANSI, ANSI-BBS, VT100

\section{ACCESS PHONE NUMBER}

Once your communications software and/or hardware has been configured, you can access EPUB by dialing (202) $586-2557$.

\section{USING EPUB}

When a connection to the system has been made, some users may find that the menu-driven instructions and the online help capabilities will provide enough information to effectively use EPUB. If needed, more extensive information may be found in the EPUB Users Guide, which is available online from the EPUB system or from:

National Energy Information Center, EI-231

Energy Information Administration

Forrestal Building, Room 1F-048

Washington, DC 20585

(202) $586-8800$

Hours: 9:00 a.m. to 5:00 p.m. Eastern Time, Monday through Friday

Telecommunications device for the hearing-impaired only:

(202) 586-1181. Hours: 9:00 a.m. to 5:00 p.m. Eastern Time, Monday through Friday

\section{EPUB ASSISTANCE}

For communications or technical assistance, call (202) 586-8959, 8:00 a.m. to 5:00 p.m. Eastern Time, Monday through Friday.

For questions about the content of EPUB reports, call (202) 586-8800, 9:00 a.m. to 5:00 p.m. Eastern Time, Monday through Friday.

\section{EPUB PROVIDES STATISTICAL INFORMATION, AS WELL AS DATA FROM THE FOLLOWING EIA PUBLICATIONS:}

Heating fuel data, updated the 2nd week of the month (April through September)

Oxygenate data, updated approximately 15 working days after the end of the month

Weekly Petroleum Status Report, updated on Wednesdays (Thursday in event of a holiday) at 5:00 p.m.

Petroleum Supply Monthly, updated on the 20th of the month

Petroleum Marketing Monthly, updated on the 20th of the month

Winter Fuels Report, updated on Thursdays (Friday in event of a holiday) at 5:00 p.r.1. (October through March)

Natural Gas Monthly, updated on the 20th of the month

Weekly Coal Production, updated on Fridays at 5:00 p.m.

Quarterly Coal Report, updated 60 days after the end of the quarter

Electric Power Monthly, updated on the 1st of the month

Monthly Energy Review, updated the last week of the month

Short Term Energy Outlook, updated 60 days after the end of the quarter ii 
The Petroleum Supply Monthly is prepared by the Petroleum Supply Division of the Office of Oil and Gas, Energy Information Administration, under the direction of Charles C. Heath (202) 586-6860.

Questions and comments concerning the contents of the Petroleum Supply Monthly may be referred to Ronald W. O'Neill (202) 586-9884, Chief of the Industry Analysis Branch, or the following specialists:

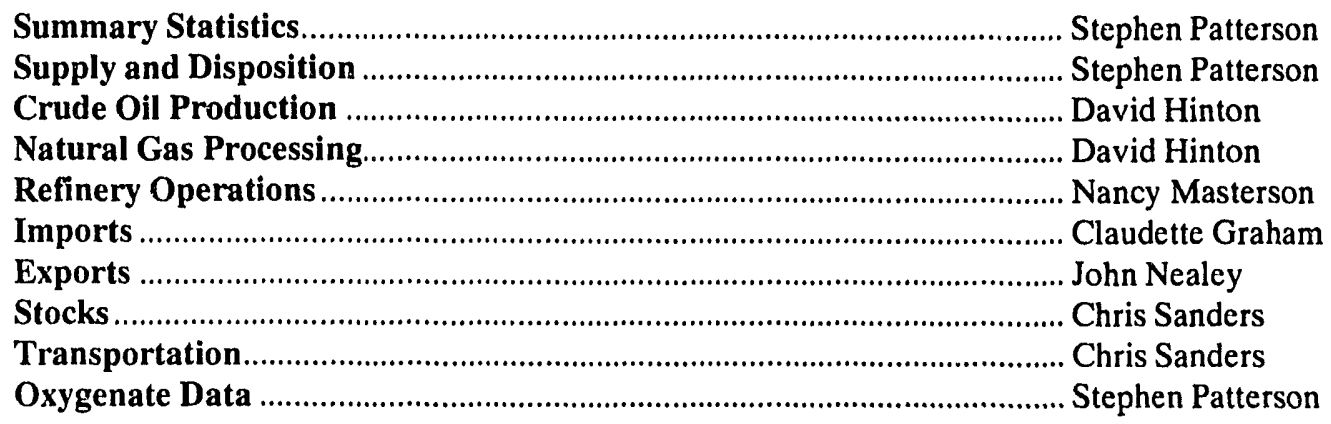

(202) 586-5994

(202) 586-5994

(202) $586-2990$

(202) $586-2990$

(202) $586-8393$

(202) 586-9649

(202) 586-9670

(202) $586-8995$

(202) 586-8995

(202) $586-5994$

Additional information on all energy statistics available from the Energy Information Administration may be obtained from the National Energy Information Center (202) 586-8800. 
Feature Article.

Highlights.

Tables

\section{Summary Statistics}

S1. Crude Oil and Petroleum Products Overview, 1981-Present

S2. Crude Oil Supply and Disposition, 1981-Present

S3. Crude Oil and Petroleum Product Imports, 1981-Present

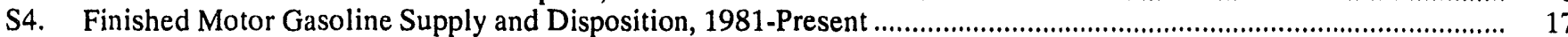

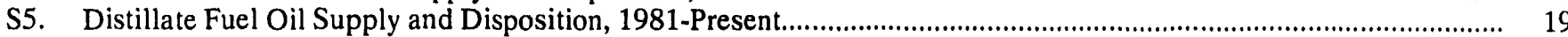

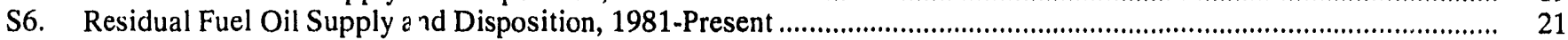

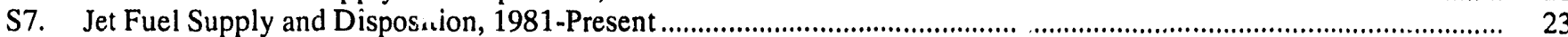

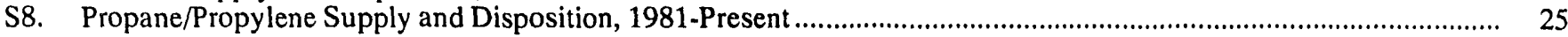

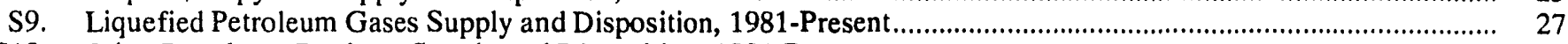

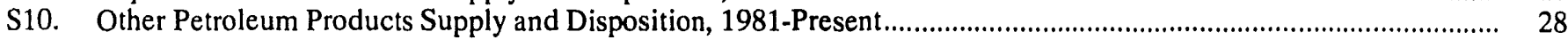

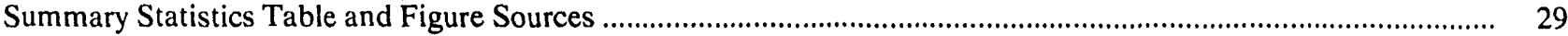

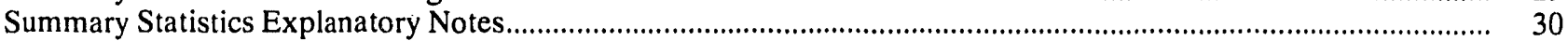

\section{Detailed Statistics}

National Statistics

1. U.S. Petroleum Balance

2. U.S. Supply, Disposition, and Ending Stocks of Crude Oil and Petroleum Products ............................................... 36

3. U.S. Year-to-Date Supply, Disposition, and Ending Stocks of Crude Oil and Petroleum Products .......................... 37

4. U.S. Daily Average Supply and Disposition of Crude Oil and Petroleum Products ............................................... 38

5 U.S. Year-to-Date Daily Average Supply and Disposition of Crude Oil and Petroleum Products.

Supply and Disposition of Crude Oil and Petroleum Products

6. PAD District I

7. Year-to-Date PAD District I

8. Daily Average PAD District I.

9. Year-to-Date Daily Average PAD District I

10. PAD District II

11. Year-to-Date PAD District II

12. Daily Average PAD District II

13. Year-to-Date Daily Average PAD District II .

14. PAD District III.

15. Year-to-Date PAD District III

16. Daily Average PAD District III

17. Year-to-Date Daily Average PAD District III

18. PAD District IV.

19. Year-to-Date PAD District IV

20. Daily Average PAD District IV.

21. Year-to-Date Daily Average PAD District IV .

22. PAD District $V$.

23. Year-to-Date PAD District V

24. Daily Average PAD District V

25. Year-to-Date Daily Average PAD District V.

Production of Crude Oil

26. Production of Crude Oil by PAD District and State

Natural Gas Processing

27. Natural Gas Plant Net Production and Stocks of Petroleum Products by PAD and Refining Districts

Refinery Operations

28. Refinery Input of Crude Oil and Petroleum Products by PAD and Refining Districts

29. Refinery Net Production of Finished Petroleum Products by PAD and Refining Districts

30. Refinery Stocks of Crude Oil and Petroleum Products by PAD and Refining Districts

31. Percent Refinery Yield of Petroleum Products by PAD and Refining Districts.... 
Imports of Crude Oil and Petroleum Products

State of Entry

32. Imports of Residual Fuel Oil by Sulfur Content

PAD District

33. Imports of Crude Oil and Petroleum Products

34. Year-to-Date Imports of Crude Oil and Petroleum Products

\section{Country of Origin}

35. United States.

36. PAD District I

37. PAD District II

38. PAD District III

39. PAD Districts IV and V

40. Year-to-Date United States

41. Year-to-Date PAD District I

42. Year-to-Date PAD District II

43. Year-to-Date PAD District III

44. Year-to-Date PAD Districts IV and V

Exports of Crude Oil and Petroleum Products

45. Exports of Crude Oil and Petroleum Products by PAD District.

46. Year-to-Date Exports of Crude Oil and Petroleum Products by PAD District .

47. Exports of Crude Oil and Petroleum Products by Destination

48. Year-to-Date Exports of Crude Oil and Petroleum Products by Destination......

Net Imports

49. Net Imports of Crude Oil and Petroleum Products into the United States by Country

50. Year-to-Date Net Imports of Crude Oil and Petroleum Products into the

United States by Country

\section{Stocks}

51. Stocks of Crude Oil and Petroleum Products by PAD District

52. Refinery, Bulk Terminal, and Natural Gas Plant Stocks of Selected Petroleum Products by PAD District and State

\section{Movements of Crude Oil and Petroleum Products}

53. Movements of Crude Oil and Petroleum Products by Pipcline, Tanker, and Barge Between PAD Districts

54. Movements of Crude Oil and Petrolcum Products by Pipeline Between PAD Districts

55. Movements of Crude Oil and Petroleum Products by Tanker and Barge Between

PAD Districts

56. Net Movements of Crude Oil and Petrolcum Products by Pipeline, Tanker, and Barge Between PAD Districts

\section{Illustrations}

S1. Petroleum Overview, March 1992-Present

S2. Petroleum Products Supplied, March 1992-Present

S3. Crude Oil Supply and Disposition, March 1992-Present

S4. Crude Oil Ending Stocks, March 1992-Present.

S5. Finished Motor Gasoline Supply and Disposition, March 1992-Present

S6. Motor Gasoline Ending Stocks, March 1992-Present

S7. Distillate Fuel Oil Supply and Disposition, March 1992-Present

S8. Distillate Fuel Oil Ending Stocks, March 1992-Present.....

S9. Residual Fuel Oil Supply and Disposition, March 1992-Present.

S10. Residual Fuel Oil Ending Stocks, March 1992-Present

S11. Jet Fuel Supply and Disposition, March 1992-Present

S12. Jet Fuel Ending Stocks, March 1992-Present .....

S13. Propane/Propylene Supply and Disposition, February 1992-Present.

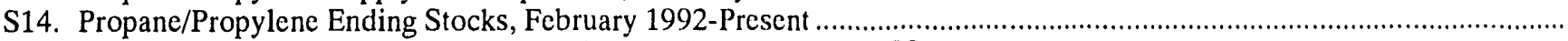

S15. Liquefied Petroleum Gases Supply and Disposition, February 1992-Present

S16 Liquefied Petroleum Gases Ending Stocks, February 1992-Present ...

\section{Appendices}

A. District Descriptions and Maps..

B. Detailed Statistics Explanatory Notes

C. Impact of Resubmissions on Major Series, 1993

D. EIA-819M, Monthly Oxygenate Telephone Report

Glossary

Definitions of Petroleum Products and Other Terms 


\section{Articles}

Feature articles on energy-related subjects are frequently included in this publication. The following articles have appeared in previous issues.

Trends in Petroleum Product Consumption

Western Countries Lead U.S. Petroleum Import Sources

U.S. Petroleum Exports Show Slight Upturn

Motor Gasoline Trends ....

Oil Imports from Saudi Arabia

Refinery Capacity Trends and Outlook

Timeliness and Accuracy of Petroleum Supply Data

Midyear Petroleum Review

Winter 1986-1987 Distillate Fuel Outlook

Recent Trends for Middle Distillates

Comparison of Independent Statistics on Petroleum Supply

U.S. Petroleum Developments: 1986

U.S. Petroleum Imports, 1986 Regional Highlights

Leading Petroleum Importers, 1986

U.S. Exports of Petroleum Products Reach Record High .

Trends in Petroleum Product Consumption

Refinery Capacity: 1987

Motor Gasoline Outlook for Summer 1987.

Motor Gasoline Trends Through 1986

Timeliness and Accuracy of Petroleum Supply Data

Midyear Petroleum Review

Petroleum Heating Fuels

Distillate Fuel Oil Outlook for Winter 1987/1988

Petroleum Exports

EIA Releases Annual Reserves Summary

Comparisons of Independent Statistics on Petroleum Supply

The Northeast-Distillate Fuel Oil Supply...

U.S. Petroleum Developments: 1987

U.S. Petroleum Import/Export Trends Through 1987

Motor Gasoline Trends Through 1987

Distillate Fuel Oil Outlook: Winter 1988/1989

Comparison of Independent Statistics on Petroleum Supply

U.S. Petroleum Developments: 1988

U.S. Petroleum Trade Trends: 1988

Timeliness and Accuracy of Petroleum Supply Data

Distillate Fuel Oil Outlook: Winter 1989/1990.

Comparisons of Independent Statistics on Petroleum Supply

U.S. Petroleum Developments: 1989.

U.S. Petroleum Trade Trends: 1989

Motor Gasoline Outlook: 1990

Timeliness and Accuracy of Petroleum Supply Data

Heating Fuel Outlook: Winter 1990-91

Comparisons of Independent Statistics on Petroleum Supply

U.S. Petroleum Developments: 1990

U.S. Petroleum Trade 1990.

Effects of the Clean Air Act's Highway Diesel Fuel Oil Provisions.

Timeliness and Accuracy of Petroleum Supply Data.

Regulation of Underground Petroleum Storage

Alternative Transportation Fuels

U.S. Petroleum Developments: 1991.

Comparisons of Independent Statistics on Petroleum Supply
January $1986^{\circ}$

January 1986

January 1986

February 1986

February 1986

March 1986

April 1986

May 1986

July 1986

July 1986

September 1986

November 1986

December 1986

December 1986

December 1986

February 1987

March 1987

March 1987

March 1987

April 1987

May 1987

July 1987

July 1987

August 1987

August 1987

September 1987

November 1987

December 1987

January 1988

February 1988

July 1988

September 1988

December 1988

January 1989

July 1989

July 1989

September 1989

December 1989

January 1990

February 1990

April 1990

July 1990

September 1990

February 1991

March 1991

June 1991

June 1991

August 1991

October 1991

February 1992

March 1992 


\section{Articles (Continued)}

U.S. Petroleum Trade, 1991

April 1992

Timeliness and Accuracy of Petroleum Supply Data

September 1992

Three Dimensional Seismology-A New Perspective

December 1992

Summer 1993 Motor Gasoline Outlook

April 1993 
Feature Article

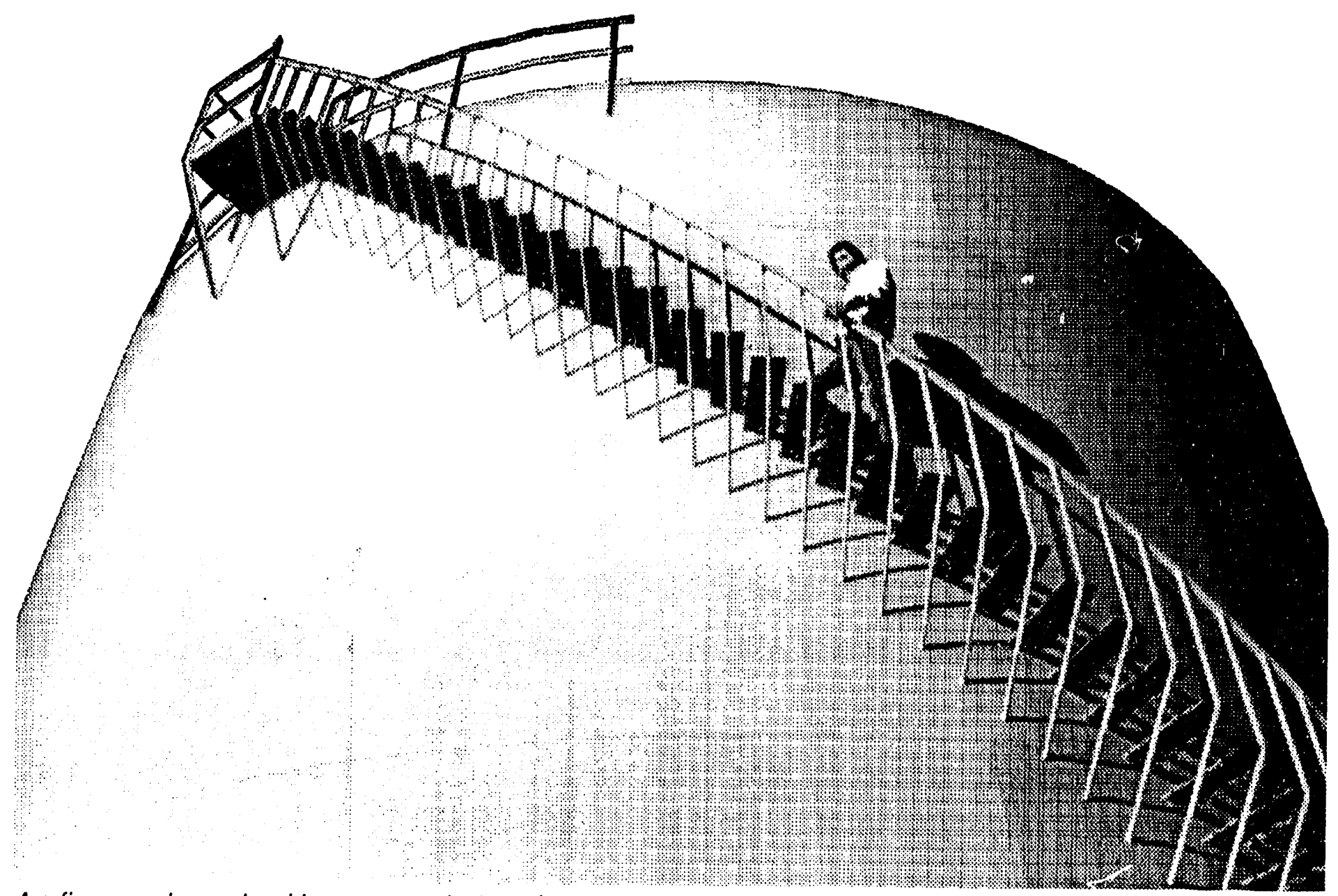




\title{
Comparisons of Independent Statistics on Petroleum Supply
}

\author{
by Robert G. Harper, III
}

\section{Introduction}

The Petroleum Supply Division (PSD) of the Energy Information Administration (EIA) collects and publishes information on petroleum supply and disposition in the United States. The information is collected through a series of surveys that make up the Petroleum Supp'y Reporting System (PSRS). The PSRS data are published in the Weekly Petroleum Status Rcport (WPSR), Petroleum Supply Monthly (PSM), and Petroleum Supply Annual (PSA).

This article compares final petroleum data published in the PSA with similar petroleum data obtained from other sources. Data comparisons are presented for 1982 through 1991, for the following series: crude oil production, crude oil imports, motor gasoline supplied, distillate fuel oil supplied, and residual fuel oil supplied.

\section{Crude Oil Production}

Crude oil production statistics (including lease condensate) from the American Petroleum Institute (API), the Oil and Gas Journal $(O G J)$ and EIA's Reserves and Natural Gas Division (RNGD) are compared with statistics from the Petroleum Supply Annual (PSA) (Table FE1). Data on crude oil production published in the PSA are based on data collected by State government agencies as well as the Minerals Management Service of the U.S. Department of the Interior, which collects data on crude oil produced on federally-owned offshore leases.

Production estimates from API are also based on data provided by State government agencies. From 1982 through 1991, API crude oil production statistics averaged 0.5 percent less than the PSA volumes. In 1991 , there was a 2.0 percent increase in API crude oil production, which caused the difference between API and PSA statistics to decrease from 1.9 percent to only 0.7 percent.

Crude oil production estimates developed by the Oil and Gas Journal (OGJ) are based on data obtained from State conservation agencies and on historical State production levels. In 1991, OGJ statistics were within 0.6 percent of PSA statistics, but for 1982 through 1991 , the average difference was only 0.2 percent less than PSA.

The Reserves and Natural Gas Division publishes the U.S. Crude Oil, Natural Gas, and Natural Gas Liquids Reserves Annual Report. Those crude oil production estimates are based on data from Form EIA-23, "Annual Survey of Domestic Oil and Gas Reserves." Survey response is mandatory. In 1991, data were received from approximately 4,150 survey respondents. The national production estimates for Form. EIA-23 were 1.6 percent lower than comparable PSA volumes. However, over the 10-year period (1982 through 1991) the RNGD and PSA statistics have

Table FE1. A Comparison of Four Crude Oll Production Data Series, 1982-1991

\begin{tabular}{|c|c|c|c|c|c|c|c|}
\hline \multirow[b]{2}{*}{ Year } & \multirow{2}{*}{ 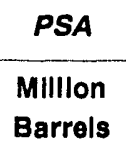 } & \multicolumn{2}{|c|}{ API } & \multicolumn{2}{|c|}{ OGJ } & \multicolumn{2}{|c|}{ RNGD } \\
\hline & & $\begin{array}{l}\text { Million } \\
\text { Barrels }\end{array}$ & $\begin{array}{c}\text { Percent } \\
\text { of PSA }\end{array}$ & $\begin{array}{l}\text { Million } \\
\text { Barrels }\end{array}$ & $\begin{array}{c}\text { Percent } \\
\text { of PSA }\end{array}$ & $\begin{array}{l}\text { Million } \\
\text { Barrels }\end{array}$ & $\begin{array}{l}\text { Percent } \\
\text { of PSA }\end{array}$ \\
\hline 1991 & 2,707 & 2,687 & 99.3 & 2,692 & 99.4 & 2,665 & 98.4 \\
\hline 1990 & 2,685 & 2,634 & 98.1 & 2,668 & 99.4 & 2,663 & 99.2 \\
\hline 1989 & 2,779 & 2,781 & 100.1 & 2,834 & 102.0 & 2,751 & 99.0 \\
\hline 1988 & 2,979 & 2,967 & 99.6 & 3,013 & 101.1 & 2,973 & 99.8 \\
\hline 1987 & 3,047 & 3,028 & 99.4 & 3,034 & 99.6 & 3,035 & 99.6 \\
\hline 1986 & 3,168 & 3,182 & 100.4 & 3,195 & 100.9 & 3,142 & 99.2 \\
\hline 1985 & 3,275 & 3,261 & 99.6 & 3,223 & 98.4 & 3,220 & 98.3 \\
\hline 1984 & 3,250 & 3,197 & 98.4 & 3,173 & 97.6 & 3,216 & 99.0 \\
\hline 1983 & 3,171 & 3,175 & 100.1 & 3,161 & 99.7 & 3,177 & 100.2 \\
\hline 1982 & 3,157 & 3,164 & 100.2 & 3,156 & 100.0 & 3,107 & 98.4 \\
\hline
\end{tabular}

Sources: PSA: Energy Information Administration, Petroleum Supply Annual, DOE/EIA-0340, 1982 through 1991, Table 2. API: American Petroleum Institute, Monthly Statistical Report, 1982 through 1991. OGJ: Oil and Gas Journal, 1982 through 1991. RNGD: U.S. Crude Oil, Natural Gas, and Natural Gas Liquids Reserves Annual Report, DOE/EIA-0216, Crude Oll, 1982 through 1991, Table 6; Lease Condensate, 1982 through 1984, Table 16; 1985, Table 14; 1986 through 1989, Table 15; 1990 through 1991, Table 16. 
remained in relatively close agreement with an average difference of only 0.9 percent. The comparison of these data series does not show any major discrepancies among the four independent sources. However, minor differences could be due to revisions and late reporting by oil field operators.

\section{Crude Oil Imports}

Data on crude oil imports are collected on survey Form EIA-814, Monthly Imports Report. Survey respondents to the form include all companies that import crude oil or petroleum products into the United States and Puerto Rico. However, for comparison purposes, statistics on imports into Puerto Rico, the Virgin Islands, and other U.S. possessions are excluded from this analysis. Approximately 860 respondents report on the Form EIA-814. The PSA statistics are compared with API statistics and the U.S. Bureau of the Census (Census) statistics for crude oil imports (Table FE2).

API data on crude oil imports are collected by a port-of-entry survey. Prior to 1985, API obtained data from reports on refinery receipts of crude oil submitted on a voluntary basis. Because the survey does not include crude oil imported by the Strategic Petroleum Reserve (SPR), data from the PSA on volumes of crude oil imported for the SPR were added to API data for comparison purposes. (See box "Information on Data Source Differences and Adjustments.") In 1991, the adjusted API data were 0.5 percent higher than PSA statistics, while the 10-year average difference was only 0.6 percent. For the third consecutive year, annual crude oil imports were above the 2 billion barrel mark.

The Bureau of the Census obtains data on crude oil imports from the U.S. Customs Service (See box "Information on Data Source
Differences and Adjustments.") In order to import crude oil or petroleum products into the United States, either U.S. Customs Form CF-7501, Entry Summary, or U.S. Customs Form CF-7505, Warehouse Withdrawal for Consumption, must be filed. Those forms are processed, tabulated, and published in report FT-246, Annual U.S. Imports for Consumption and General Imports. Data on imports into Puerto Rico and other U.S. possessions are excluded from Census Bureau data. The Census data are adjusted for comparison purposes because their geographic coverage differs from that of the PSA data. In 1991, the adjusted Census data were only 0.6 percent higher than the PSA annual volumes. The difference represents a 2.8 percent decrease from the 3.4 percent reported in 1990, although the reason for the decrease is not readily apparent. Future data comparisons will involve research into the differences in geographic coverage and their impact on the PSA/Census relationship.

\section{Product Supplied}

Product supplied, as reported in the PSA, is used to measure the volume of petroleum products available for domestic consumption. Those data are generated for each petroleum product by adding field production, refinery production, imports, and unaccounted for crude oil minus (-) stock change, crude oil losses, refinery imports and exports. Product supplied is calculated for refineries, natural gas processing plants, pipelines, and bulk terminals.

\section{Motor Gasoline Supplied}

$P S A$ statistics on motor gasoline supplied are compared with data from the EIA's Petroleum Marketing Division (PMD), the

Table FE2. A Comparison of Crude Oll Imports into United States (Excluding U.S. Possessions) Data Series, 1982-1991

\begin{tabular}{|c|c|c|c|c|c|}
\hline \multirow[b]{2}{*}{ Year } & \multirow{2}{*}{ 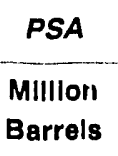 } & \multicolumn{2}{|c|}{$A P 1^{a}$} & \multicolumn{2}{|c|}{ Census $^{b}$} \\
\hline & & $\begin{array}{l}\text { Million } \\
\text { Barrels }\end{array}$ & $\begin{array}{l}\text { Percent } \\
\text { of PSA }\end{array}$ & $\begin{array}{l}\text { Million } \\
\text { Barrels }\end{array}$ & $\begin{array}{l}\text { Percent } \\
\text { of PSA }\end{array}$ \\
\hline 1991 & 2,111 & 2,122 & 100.5 & 2,124 & 100.6 \\
\hline 1990 & $2,1=!$ & 2,184 & 101.5 & 2,224 & 103.4 \\
\hline 1989 & 2,133 & 2,158 & 101.2 & 2,147 & 100.7 \\
\hline 1988 & 1,869 & 1,861 & 99.6 & 1,888 & 101.0 \\
\hline 1987 & 1,706 & 1,697 & 99.5 & 1,627 & 95.4 \\
\hline 1986 & 1,525 & 1,501 & 98.4 & 1,540 & 101.0 \\
\hline 1985 & 1,168 & 1,164 & 99.7 & 1,170 & 100.2 \\
\hline 1984 & 1,254 & 1,303 & 103.9 & 1,208 & 96.3 \\
\hline 1983 & 1,215 & 1,232 & 101.4 & 1,199 & 98.7 \\
\hline 1982 & 1,273 & 1,275 & 100.2 & 1,300 & 102.1 \\
\hline
\end{tabular}

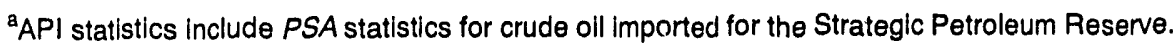

${ }^{b}$ Census statistlcs are adjusted to reflect the geographic coverage and reporting perlod of the $P S A$.

Sources: PSA: Energy Informatlon Administration, Petroleum Supply Annual, DOE/EIA-0340, 1982 through 1991, Table 2. API: American

Petroleum Institute, Monthly Statistical Report. Census: Bureau of the Census, FT-246, Annual U.S. Imports for Consumption and General Imports: TSUSA; IA-245X, Annual U.S. Imports for Consumption and General Imports: TSUSA, 1982 through 1985; IM-115 Data Tape, 1986 through 1991. 
Table FE3. A Comparison of Motor Gasoline Supplied for Domestic Use Data Series, 1982-1991

\begin{tabular}{|c|c|c|c|c|c|c|c|}
\hline \multirow[b]{2}{*}{ Year } & \multirow{2}{*}{$\begin{array}{c}\text { PSA } \\
\text { Million } \\
\text { Barrels }\end{array}$} & \multicolumn{2}{|c|}{ PMD } & \multicolumn{2}{|c|}{ API } & \multicolumn{2}{|c|}{ FHWA } \\
\hline & & $\begin{array}{l}\text { Million } \\
\text { Barrels }\end{array}$ & $\begin{array}{l}\text { Percent } \\
\text { of PSA }\end{array}$ & $\begin{array}{l}\text { Million } \\
\text { Barrels }\end{array}$ & $\begin{array}{l}\text { Percent } \\
\text { of PSA }\end{array}$ & $\begin{array}{l}\text { Million } \\
\text { Barrels }\end{array}$ & $\begin{array}{c}\text { Percent } \\
\text { of PSA }\end{array}$ \\
\hline 1991 & 2,623 & 2,870 & 109.4 & 2,621 & 99.9 & 2,665 & 101.6 \\
\hline 1990 & 2,641 & 2,919 & 110.5 & 2,650 & 100.3 & 2,739 & 103.7 \\
\hline 1989 & 2.675 & 2,873 & 107.4 & 2,683 & 100.3 & 2,722 & 101.8 \\
\hline 1988 & 2,685 & 2,874 & 107.0 & 2,678 & 99.7 & 2,702 & 100.6 \\
\hline 1987 & 2,630 & 2,750 & 104.9 & 2,611 & 99.3 & 2,677 & 101.8 \\
\hline 1986 & 2,567 & 2,733 & 106.5 & 2,580 & 100.5 & 2,630 & 102.5 \\
\hline 1985 & 2,493 & 2,691 & 107.9 & 2,476 & 99.3 & 2,553 & 102.4 \\
\hline 1924 & 2,449 & 2,552 & 104.2 & 2,459 & 100.4 & 2,498 & 102.0 \\
\hline 1983 & 2,417 & 2,495 & 103.2 & 2,420 & 100.1 & 2,434 & 100.7 \\
\hline 1982 & 2,387 & 2,451 & 102.7 & 2,376 & 99.5 & 2,413 & 101.1 \\
\hline
\end{tabular}

Sources: PSA: Energy Information Administration (EIA), Petroleum Supply Annual, DOE/EIA-0340,1982 through 1991, Table 2. PMD: Form EIA25, Prime Supplier's Monthly Report, 1982. Form EIA-782C, Monthly Report of Petroleum Products Sold into States for Consumption, Petroleum Marketing Monthly, DOE/EIA-0380, 1983 through 1985, Table 45; Petroleum Marketing Annuai, DOE/EIA-0487, 1986 through 1988, Table 45; 1989 through 1991, Table 47. API: American Petroleum Institute, Monthly Statistical Report, 1982 through 1991. FHWA: Federal Highway Administration, Highway Statistics, 1982 through 1991, Tables MF-24 and MF-21A.

American Petroleum Institute (API), and the Federal Highway Administration (FHWA) (Table FE3). PMD Form EIA-782C, Monthly Report of Petroleum Products Sold into States for Consumption, is used to monitor first sales into a State for consumption in that State. The respondent universe consists of refiners and gas plant operators, importers, and resellers or retailers. In 1991, 236 firms made up the EIA-782C survey frame. In 1991, the PMD volume of motor gasoline was approximately 9.4 percent above the $P S A$ volume. This share of $P S A$ represents a 1.1 percent decrease from 1990, but the fourth consecutive year that the difference has been 7.0 percent or greater. Downstream blending is one major reason that PMD volumes for motor gasoline are higher than PSA volumes. Blending of fuel ethanol and methyl tertiary butyl ether with unfinished gasoline often occurs downstream from refineries and may be counted in the EIA-782C data, but not in the PSA data. Another reason is double reporting on the EIA-782C survey. Some companies do not coordinate sales reporting with their customers and both may report some of the same data because they did not use the Exclusionary List to avoid double counting. The list consists of companies that are prime suppliers to other companies whose sales should be excluded if they are respondents to the survey.

API statistics on motor gasoline delivered from primary storage are published in the Monthly Statistical Report. The API statistics are similar in concept to EIA's product supplied. The data represent production plus imports for motor gasoline (adjusted for net stock change) minus exports. Those statistics are based on a historical analysis of the industry and information provided on a voluntary basis by importers of record (licensed importers) and by operators of refineries, bulk terminals, and pipelines. API and PSA staristics have consistently remained within 0.7 percent of each cther.

Data from the FHWA on total gasoline usage are based on volumes of gasoline reported to State motor fuel tax agencies by wholesale distributors. The FHWA's publication Highway' Statistics includes data on both highway and nonhighway use of gasoline, minus aviation gasoline. FHWA statistics were consistently higher than the PSA statistics ranging from a low of 0.6 percent in 1988, to a high of 3.7 percent in 1990. In 1991, the difference narrowed to 1.6 percent. For the 10-year period (1982 through 1991) the difference between PSA and FHWA data averaged 1.8 percent. Because the FHWA statistics are a wholesale measure, their data may more closely reflect the actual motor gasoline usage than does the EIA product supply series.

\section{Distillate Fuel Oil Supplied}

Statistics for distillate fuel oil (including kerosene) supplied from the Petroleum Supply Annual (PSA) are compared with EIA's PMD data on distillate fuel oil sales collected from survey Form EIA-782C and with API data on distillate fuel oil delivered from primary storage (Table FE4). Data on kerosene were discontinued as a separate category in API's Monthly Statistical Report. To adjust for this change, kerosene volumes from the PSA were added to API data for comparison purposes. API statistics on distillate fuel oil supplied generally have been comparable to PSA statistics, having stayed within 1.0 percent from 1982 through 1991.

Since 1982, the PMD statistics for first sales of distillate fuel oil and kerosene sold into States for consumption have been higher than the PSA statistics. In 1991, there was a 9.9 percent difference between PMD and PSA data, equaling that reported in 1990. For the last 10 years, the average annual difference between $P S A$ and PMD data was 6.3 percent. Double reporting on the EIA-782C survey is one reason that PMD sales are higher than PSA product supplied for distillate fuel oil. Another reason is the fungible nature of petroleum products. For example, if a product produced according to kerosene-type jet fuel specifications is sold as No. 1 
Table FE4. A Comparison of Dlstlllate Fuel Oll (Including Kerosene) Supplied Data Series, 1982-1991

\begin{tabular}{|c|c|c|c|c|c|}
\hline \multirow[b]{2}{*}{ Year } & \multirow{2}{*}{$\begin{array}{c}\text { PSA } \\
\text { Million } \\
\text { Barrels }\end{array}$} & \multicolumn{2}{|c|}{ PMD } & \multicolumn{2}{|c|}{$A P 1^{a}$} \\
\hline & & $\begin{array}{l}\text { Million } \\
\text { Barrels }\end{array}$ & $\begin{array}{l}\text { Percent } \\
\text { of PSA }\end{array}$ & $\begin{array}{l}\text { Million } \\
\text { Barrels }\end{array}$ & $\begin{array}{c}\text { Percent } \\
\text { of PSA }\end{array}$ \\
\hline 1991 & 1,083 & 1,190 & 109.9 & 1,086 & 100.3 \\
\hline 1990 & 1,118 & 1,229 & 109.9 & 1,114 & 99.6 \\
\hline 1989 & 1,183 & 1,262 & 106.7 & 1,172 & 99.1 \\
\hline 1988 & 1,178 & 1,268 & 107.6 & 1,166 & 99.0 \\
\hline 1987 & 1,121 & 1,159 & 103.4 & 1,122 & 100.1 \\
\hline 1986 & 1,100 & 1,173 & $106: 6$ & 1,111 & 101.0 \\
\hline 1985 & 9,088 & 1,160 & 106.6 & 1,090 & 100.2 \\
\hline 1984 & 1,083 & 1,154 & 106.6 & 1,093 & 100.9 \\
\hline 1983 & 1,028 & 1,045 & 101.7 & 1,027 & 99.9 \\
\hline 1982 & 1,022 & 1,054 & 103.1 & 1,031 & 100.9 \\
\hline
\end{tabular}

aAPI statistics include PSA statistics for kerosene for 1982 through 1991.

Sources: PSA: Energy Information Administration (EIA), Petroleum Supply Annual, DOE/EIA-0340, 1982 through 1991, Table 2. PMD: EIA-25, Prime Supplier's Monthly Report, 1982. Form ElA-782C, Monthly Report of Petroleum Products Sold into States for Consumption, Petroleum Marketing Monthly, 1983 through 1985, Table 46; Petroleum Marketing Annual, DOE/EIA-0487, 1986 through 1988, Table 47; 1989 through 1991 , Table 49. API: American Petroleum Institute, Monthly Statistical Report, 1982 through 1991.

distillate or kerosene, then the EIA-782C total distillate volumes would be greater than those of the PSA.

\section{Residual Fuel Oil Supplied}

Product supplied data for residual fuel oil are compared with PMD data on first sales of residual fuel oil and API data on residual fuel oil delivered (Table FE5). Until 1991, the PMD statistics for residual fuel oil were historically lower than the PSA statistics. A primary reason for the difference between PMD and PSA data may be because the PMD Form EIA-782C is a sales survey, with volumes based on transfer of ownership (equity basis), while $P S A$
Form EIA-810 is a supply survey with volumes reported based on the a amount of petroleum in custody, regardless of ownership (custody basis). Residual fuel oil imported by electric utilities for their own use may not be reported on Form EIA-782C because sellers who make such sales to electric utilities are not included in the survey frame. The difference between PSA and PMD statistics has steadily decreased from a high of 14.3 percent in 1986 , to 0.5 percent for 1991.

The API volumes of residual fuel oil supplied were close to PSA volumes over the 10-year period (1982 through 1991). Although there was a 1.9 percent increase in API volumes from that reported

Table FE5. A Comparison of Residual Fuel Oll Supplied for Domestlc Use Data Serles, 1982-1991

\begin{tabular}{|c|c|c|c|c|c|}
\hline \multirow[b]{2}{*}{ Year } & \multirow{2}{*}{$\begin{array}{c}\text { PSA } \\
\begin{array}{c}\text { Million } \\
\text { Barrels }\end{array}\end{array}$} & \multicolumn{2}{|c|}{ PMD } & \multicolumn{2}{|c|}{ API } \\
\hline & & $\begin{array}{l}\text { Million } \\
\text { Barrels }\end{array}$ & $\begin{array}{l}\text { Percent } \\
\text { of PSA }\end{array}$ & $\begin{array}{l}\text { Million } \\
\text { Barrels }\end{array}$ & $\begin{array}{c}\text { Percent } \\
\text { of PSA }\end{array}$ \\
\hline 1991 & 423 & 425 & 100.5 & 434 & 102.6 \\
\hline 1990 & 449 & 445 & 99.1 & 452 & 100.7 \\
\hline 1989 & 500 & 477 & 95.4 & 491 & 98.2 \\
\hline 1988 & 504 & 475 & 94.2 & 490 & 97.2 \\
\hline 1987 & 462 & 422 & 91.3 & 467 & 101.1 \\
\hline 1986 & 518 & 444 & 85.7 & 508 & 98.1 \\
\hline 1985 & 439 & 411 & 93.6 & 433 & 98.6 \\
\hline 1984 & 501 & 484 & 96.6 & 498 & 99.4 \\
\hline 1983 & 519 & 510 & 98.3 & 525 & 101.2 \\
\hline 1982 & 627 & 574 & 91.5 & 622 & 99.2 \\
\hline
\end{tabular}

Sources: PSA: Energy Information Administration (EIA), Petroleum Supply Annual, DOE/EIA-0340, 1982 through 1991, Table 2. PMD: Form EIA25. Prime Supplier's Monthly Report, 1982. Form ElA-782C, Monthly Report of Petroleum Products Sold into States for Consumption, Petroleum Marketing Monthly, 1983 through 1985, Table 47; Petroleum Marketing Annual, DOE/EIA-0487, 1986 through 1988, Table 46; 1989 through 1991, Table 48. API: American Petroleum Institute, Monthly Statistical Report, 1982 through 1991. 


\section{Information on Data Source Differences and Adjustments}

American Petroleum Institute: In this article, API's annual statistics are totals of initial monthly values. The initial API published monthly estimate is derived from API sources, but later API publications reflect revisions using EIA data. PSA statistics on crude oil include imports for the Strategic Petroleum Reserve (SPR) while API statistics do not. Therefore, the following figures for SPR were added to the API figures: no barrels of crude oil were reported for 1991 for the SPR, 9.8 million barrels in 1990, 20.3 million barrels in 1989, 18.8 million barrels in 1988, 26.5 million barrels in 1987, 17.6 million barrels in 1986, 43.1 million barrels in 1985, 72.0 million barrels in 1984, 85.3 million barrels in 1983, and 60.2 million barrels in 1982. The API publishes monthly estimates of motor gasoline, distillatc fuel oil, and residual fuel oil delivered from primary storage in thousand barrels per day. In 1982, the API discontinued publishing kerosene as a separate category. PSA values for kerosene supplied (17 million barrels in 1991, 16 million barrels in 1990, 31 million barrels in 1989, 35 million barrels in 1988, 35 million barrels in 1987, 36 million barrels in 1986, 42 million barrcls in 1985 and 1984, 46 million harrels in 1983, and 47 million barrels in 1982) were added to API distillate totals.

Oil and Gas Journal: The Oil and Gas Journal publishes weekly averages of crude oil production in thousand barrels per day. Those averages are used to produce monthly totals as follows: the average for each week is used as a daily production estimate for each of the days the week covers. For each month, the production estimates for days covered by the month are summed. The totals are converted from thousand to million barrels for this article.

U.S. Bureau of the Census: Data for 1982 through 1985 are from the Annual U.S. Imports for Consumption and General Imports. Since 1986, Census data have been available through the IM-115 data tape. Imports to Puerto Rico and the Virgin Islands are not included. The Census also excludes data on ${ }_{\text {. }}$ ports into the United States from Puerto Rico and the Virgin Islands.

Petroleum Marketing Division: EIA's Petroleum Marketing Division data are from the Form ElA-25, Prime Supplier's Monthly Report (computer printouts), 1982. Prime supplier usually is the supplier or producer who makes the first sale of any product into the State. In 1983, the Form EIA-782C replaced the Form EIA-25, [Monthly Report of Petroleum Products Sold into States for Consumption.]

in 1990, the average difference between PSA and API data have remained within 0.4 percent.

\section{Conclusion}

Although the 1991 PSA statistics were in relative proximity with other sources of petroleum data, there are significant differences between data reported by PSA and data reported by PMD. The make-up of the sampling frames, the inclusion or exclusion of data from related survey forms, and how survey data are compiled or aggregated are just three of the many reasons why the data submilted by a company will differ. However, for comparison purposes, it must be recognized that differenees probably will always exist given the various data collection processes employed by the respective organizations. The primary focus is to keep the data differences in perspective and within as narrow a range as possible. Future cfforts will be directed toward conducting research into relevant issues, problems or situations that will benefit the analysis of the differences and the data collection process. 


\section{Highlights}

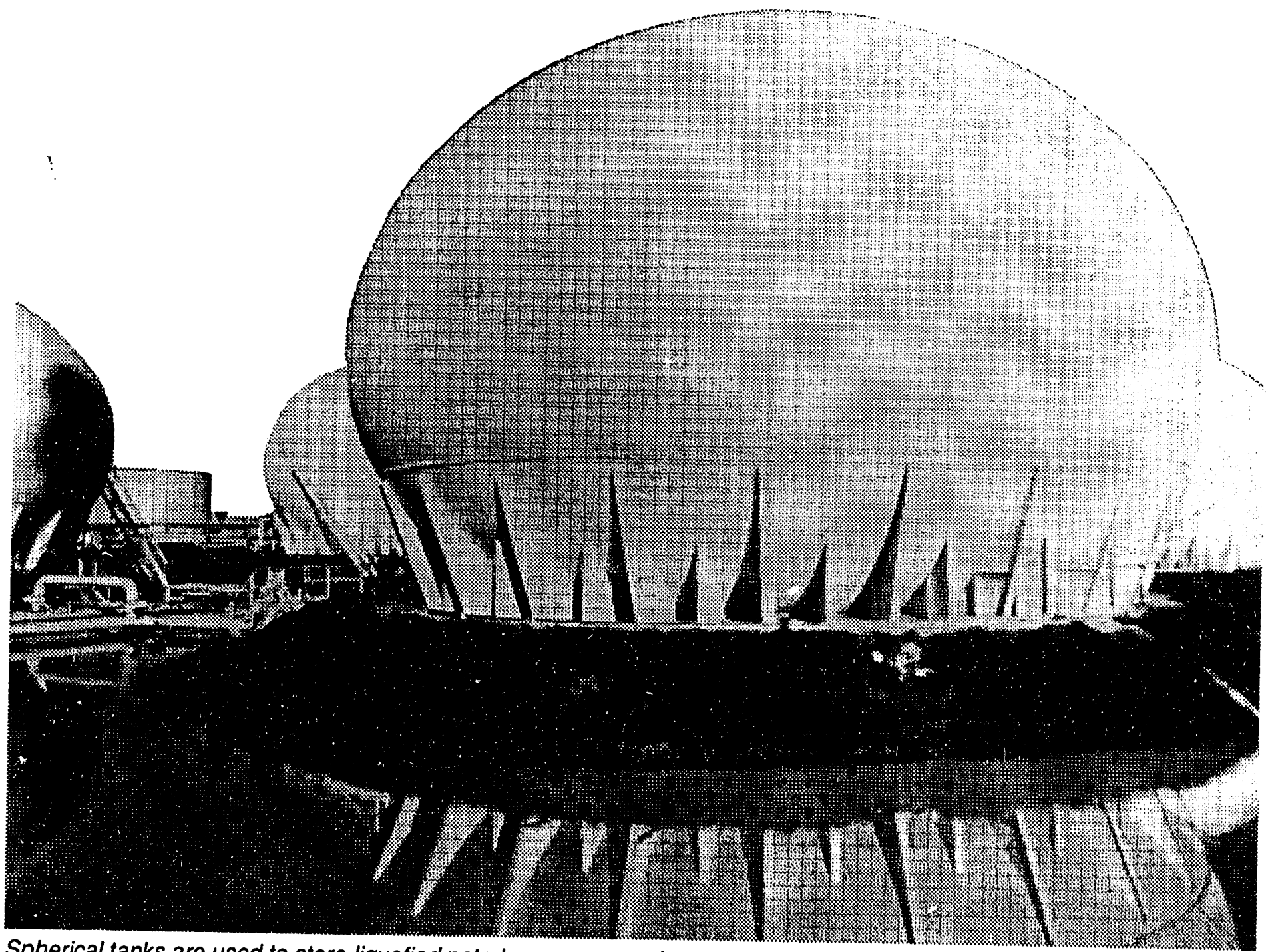

Spherical tanks are used to store liquefied petroleum gases under pressure. 


\section{Highlights}

U.S. demand for petroleum in March 1993 was 17.8 million barrels per day (measured as product supplied). This level was the highest for March since 1979. The severe snowstorm on the East Coast and colder-than-normal temperatures in many areas of the country contributed to the high demand.

Demand during the first 3 months of 1993 of 17.3 million barrels per day was 2 percent higher than for the comparable 1992 period (Table H1). The increase was due to colder winter temperatures than last year, and the recovering economy (Figure H1).

Other March and first quarter 1993 highlights include:

- Demiand for motor gasuline was the highest for March since 1989. First quarter demand was slightly higher than a year earlier. Despite stock withdrawals, inventories were higher than for the comparable period of 1991 and 1992.
- Demand for distillate fuel oil remained higher than normal in March, and first quarter demand was moderately higher than a year earlier. Stocks declined seasonally.

- High natural gas prices and the unusually cold March temperatures kept demand for residual fuel oil stable, but demand was still unseasonably low for both the month and for the first quarter of 1993.

- Total demand for liquefied petroleum gases (LPG's) decreased slightly, but propane remained strong. Stocks of propane were at a record low level, but were adequate for the end of the winter heating season.

- March and first-quarter imports of crude oil, excluding the Strategic Petroleum Reserve (SPR), were much higher than during the comparable 1992 period. Imported crude oil accounted for nearly half of domestic crude oil supplies during the first quarter of 1993.

- The refinery utilization rate increased in March, as refining margins improved.

\section{Figure H1. Quarterly Changes In Gross Domestic Product}

(Percent Change from Year Earlier)

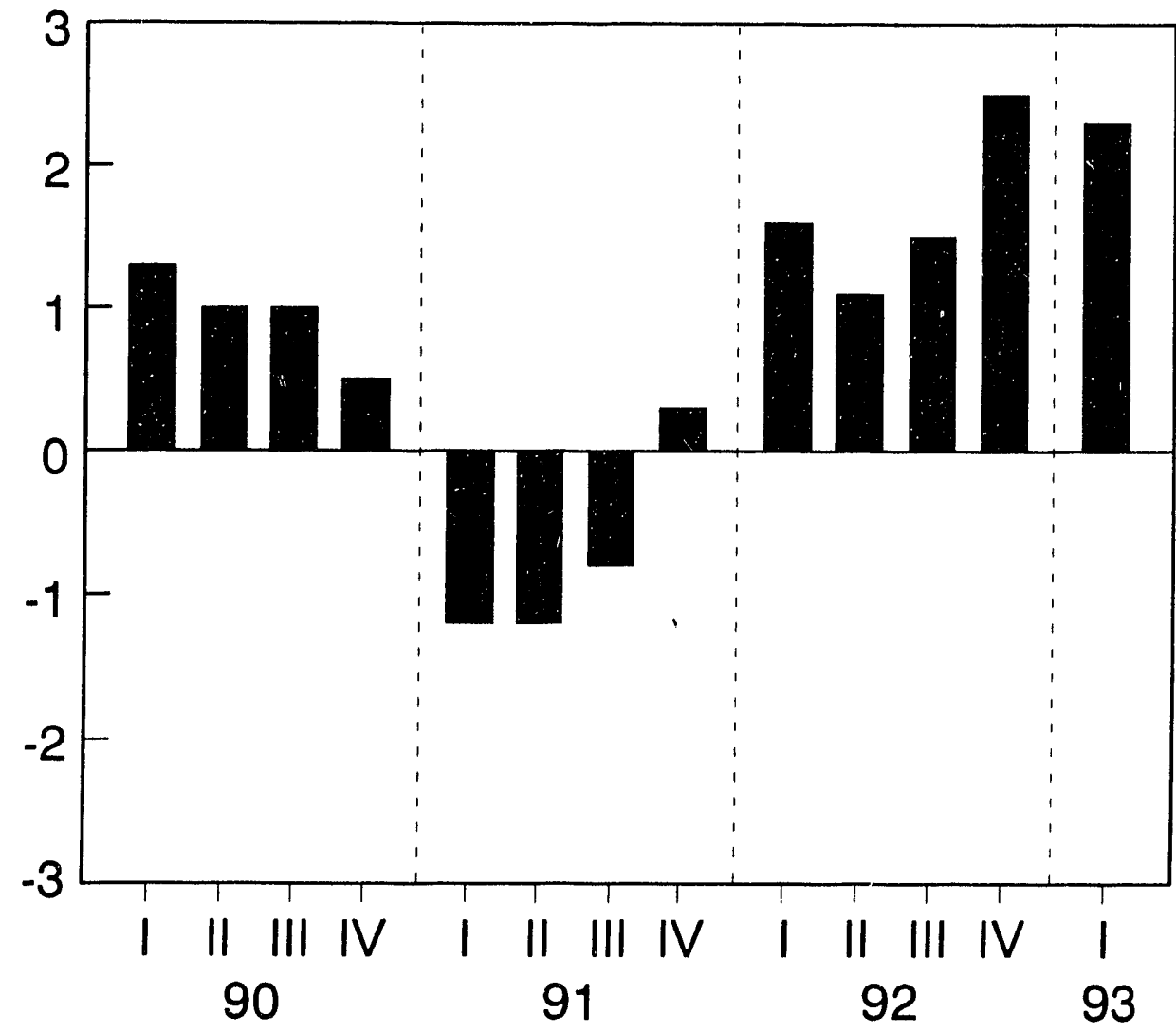

Sources: U.S. Department of Commerce, Bureau of Economic Analysis, Economic Indicators, November 1992, pp. 2 and 3, for 1990 through third quarter 1992; The Wall Street Journal, April 30, 1993, pp A2 and A6, for fourth quarter 1992 and first quarter 1993. 
Table H1. Petroleum Supply Summary

(Million Barrels per Day, Except Where Noted)

\begin{tabular}{|c|c|c|c|c|c|c|}
\hline \multirow{2}{*}{ Category } & \multicolumn{3}{|c|}{1992} & \multirow{2}{*}{1991} & \multicolumn{2}{|c|}{ January-March } \\
\hline & March & February & Difference ${ }^{a}$ & & 1992 & 1991 \\
\hline 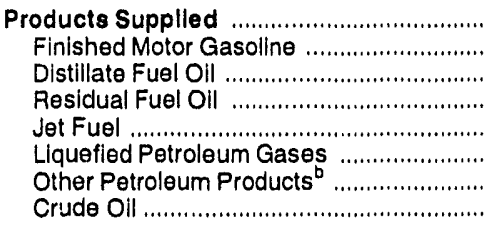 & $\begin{array}{r}17.8 \\
7.4 \\
3.5 \\
1.1 \\
1.5 \\
1.9 \\
2.4 \\
\text { (s) }\end{array}$ & $\begin{array}{r}17.6 \\
7.1 \\
3.7 \\
1.1 \\
1.5 \\
1.5 \\
2.2 \\
(\mathbf{s})\end{array}$ & $\begin{array}{r}0.2 \\
0.3 \\
-0.2 \\
-0.1 \\
(s) \\
(s) \\
0.2 \\
(s)\end{array}$ & $\begin{array}{r}16.8 \\
7.1 \\
3.2 \\
1.2 \\
1.4 \\
1.7 \\
2.2 \\
(\mathrm{~s})\end{array}$ & $\begin{array}{r}17.3 \\
7.1 \\
3.5 \\
1.1 \\
1.5 \\
1.9 \\
2.2 \\
(5)\end{array}$ & $\begin{array}{r}16.9 \\
7.0 \\
3.2 \\
1.3 \\
1.4 \\
1.9 \\
2.1 \\
\text { (s) }\end{array}$ \\
\hline 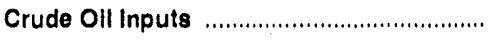 & 13.2 & 12.9 & 0.3 & 13.1 & 13.1 & 12.8 \\
\hline Operating Utllization Rate (percent) ........... & 90.8 & 88.8 & 2.0 & 89.3 & 89.6 & 87.5 \\
\hline 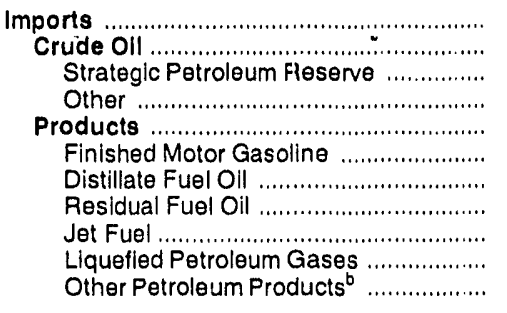 & $\begin{array}{r}8.3 \\
6.5 \\
(s) \\
6.5 \\
1.8 \\
0.2 \\
0.2 \\
0.4 \\
0.1 \\
0.1 \\
0.8\end{array}$ & $\begin{array}{r}7.9 \\
6.2 \\
0 \\
6.2 \\
1.8 \\
0.2 \\
0.2 \\
0.3 \\
0.1 \\
0.1 \\
0.8\end{array}$ & $\begin{array}{l}0.4 \\
0.4 \\
(s) \\
0.3 \\
0.1 \\
(s) \\
(s) \\
(s) \\
(s) \\
(s) \\
\text { (s) }\end{array}$ & $\begin{array}{l}7.0 \\
5.3 \\
0.0 \\
5.3 \\
1.7 \\
0.2 \\
0.2 \\
0.4 \\
0.1 \\
0.1 \\
0.7\end{array}$ & $\begin{array}{c}8.1 \\
6.3 \\
(\mathrm{~s}) \\
6.3 \\
1.8 \\
0.2 \\
0.2 \\
0.4 \\
0.1 \\
0.1 \\
0.8\end{array}$ & $\begin{array}{l}7.1 \\
5.4 \\
0.0 \\
5.4 \\
1.7 \\
0.3 \\
0.2 \\
0.4 \\
0.1 \\
0.1 \\
0.7\end{array}$ \\
\hline 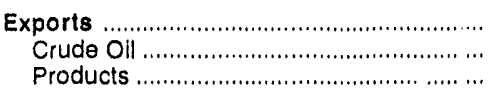 & $\begin{array}{l}0.9 \\
0.1 \\
0.8\end{array}$ & $\begin{array}{l}0.9 \\
0.2 \\
0.7\end{array}$ & $\begin{array}{l}0.1 \\
(s) \\
0.1\end{array}$ & $\begin{array}{l}0.9 \\
0.1 \\
0.8\end{array}$ & $\begin{array}{l}0.9 \\
0.1 \\
0.8\end{array}$ & $\begin{array}{l}1.0 \\
0.1 \\
0.9\end{array}$ \\
\hline 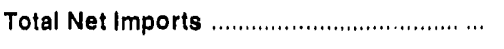 & 7.4 & 7.1 & 0.4 & 6.1 & 7.2 & 6.2 \\
\hline 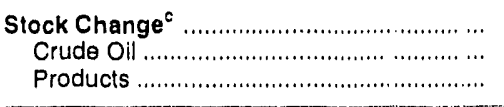 & $\begin{array}{r}-0.4 \\
0.2 \\
-0.6\end{array}$ & $\begin{array}{r}-0.6 \\
0.2 \\
-0.8\end{array}$ & $\begin{array}{r}0.2 \\
(s) \\
0.2\end{array}$ & $\begin{array}{l}-0.5 \\
-0.2 \\
-0.3\end{array}$ & $\begin{array}{r}-0.1 \\
0.2 \\
-0.3\end{array}$ & $\begin{array}{r}-0.5 \\
0.2 \\
-0.7\end{array}$ \\
\hline $\begin{array}{l}\text { Total Stocks at End of Perlod } \\
\text { (million barrels) }\end{array}$ & 1,584 & 1,595 & -12 & 1,569 & •. & 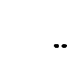 \\
\hline 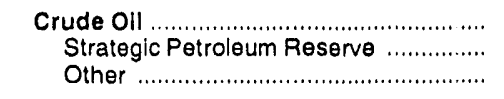 & $\begin{array}{l}915 \\
578 \\
337\end{array}$ & $\begin{array}{l}907 \\
576 \\
331\end{array}$ & $\begin{array}{l}8 \\
2 \\
6\end{array}$ & $\begin{array}{l}907 \\
569 \\
339\end{array}$ & 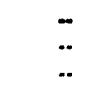 & $\ddot{*}$ \\
\hline 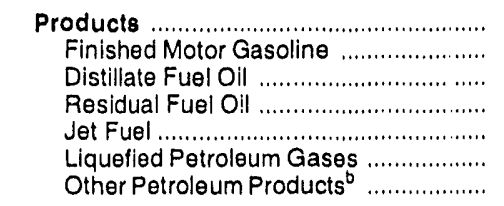 & $\begin{array}{r}669 \\
187 \\
97 \\
41 \\
41 \\
67 \\
236\end{array}$ & $\begin{array}{r}688 \\
200 \\
109 \\
42 \\
42 \\
66 \\
228\end{array}$ & $\begin{array}{r}-19 \\
-13 \\
-12 \\
-1 \\
.1 \\
(s) \\
8\end{array}$ & $\begin{array}{r}662 \\
181 \\
98 \\
40 \\
44 \\
73 \\
226\end{array}$ & $\begin{array}{l}- \\
\ddot{.} \\
\ddot{. .} \\
\ddot{. .} \\
. . \\
. .\end{array}$ & $\begin{array}{l}\ddot{-} \\
\ddot{.} \\
\ddot{.} \\
\ddot{.} \\
\ddot{.} \\
. .\end{array}$ \\
\hline
\end{tabular}

Difference is equal to volume for current month minus volume for previous month.

Includes pentanes plus, other hydiocarbons and oxygenates, unfinished olls, gasoline blending components, and all finished petroleum products except finished motor gasoline, distillate fuel oll, residual fuel oll, jet fuel, and liquefied petroleum gases.

- A negative number indicates a decrease in stocks and a positive number indicates an increase.

$(s)=$ Less than 0.05 million barrels pir day, or less than 0.05 percent, or less than 0.5 million barrels.

Note: Totals may not equal sum of components due to independent rounding.

Source: Energy Information Administration, appropriate Issues of Petroleum Supply Monthly, see Explanatory Note 2. 


\section{Motor Gasoline}

Recent economic growth served to stimulate demand for motor gasoline in March. Demand of 7.4 million barrels per day was considerably higher than in February, and was the highest level for March since 1989. Demand during the first quarter of 1993 of 7.1 million barrels per day was slightly higher than a year earlier.

Stock withdrawals to fill demand brought the level to 187 million barrels at the end of March. Stocks were moderately higher than for the comparable period of 1991 and 1992.

\section{Distillate Fuel Oil}

Demand for distillate fuel oil declined from the unusually high February level to 3.5 million barrels per day in March. Demand was higher than normal for this time of year. Solid industrial production sustained transportation use of diesel fuel at relatively high levels, even though the near-record snowfall on the East Coast disrupted highway and rail traffic for several days. Colder-than-normal temperatures also kept demand for heating oil strong. During the first quarter of 1993, winter heating needs and increases in industrial production led to an 8-percent increase in distillate fuel oil demand from the comparable period of 1992.

Stocks declined seasonally to 97 million barrels at the end of March, and were within the normal range for this time of year.

\section{Residual Fuel Oil}

Demand for residual fuel oil remained stable at 1.1 million barrels per day in March. Helping to keep demand stable were higher natural gas prices and the unusually cold March temperatures. During the first quarter of 1993, demand was unusually low, and fell 15 percent from a year earlier. An underlying weakness in demand is the decline in residual fuel oil's share of electric power generation. Over 90 percent of the petroleum consumed at electric utilities is residual fuel oil. After 1977, petroleum's share of electricity generation declined, as coal and nuclear power became more predominant (Figure H2).

\section{Liquefied Petroleum Gases}

Demand for LPG's in March was slightly lower than in February at 1.9 million barrels per day. Demand for propane declined to 1.2 million barrels per day, but was higher than normal for March because of unusually cold temperatures in the South, Midwest, and Northeast. During the first quarter of 1993, total demand for LPG's of 1.9 million barrels per day was higher than normal, primarily because of strong demand for propane. Propane demand averaged 1.2 million barrels per day, 5 percent higher than during the first quarter of 1992.

LPG stocks increased to 67 million barrels, with all components except propane increasing. Total LPG stocks remained slightly

Figure H2. Electricity Generation by Selected Fuel Source, 1977-1992

(Percent of Kilowatthours)

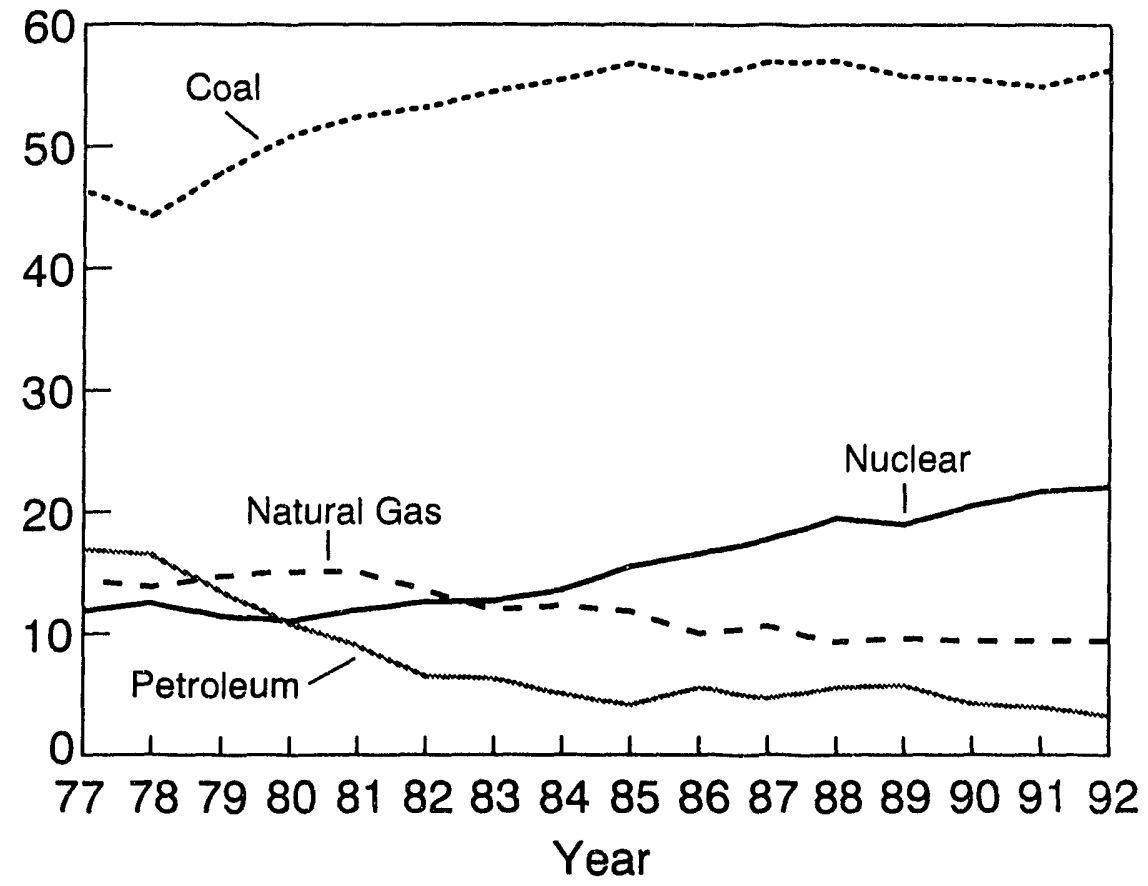

Source: Energy Information Administration, Monthly Energy Review, April 1993, Table 7.1. 
Table H2. U.S. Refinery Inputs, Capacities and Utilization Rates: 1992-1993 (Thousand Barrels per Day, Except Where Noted)

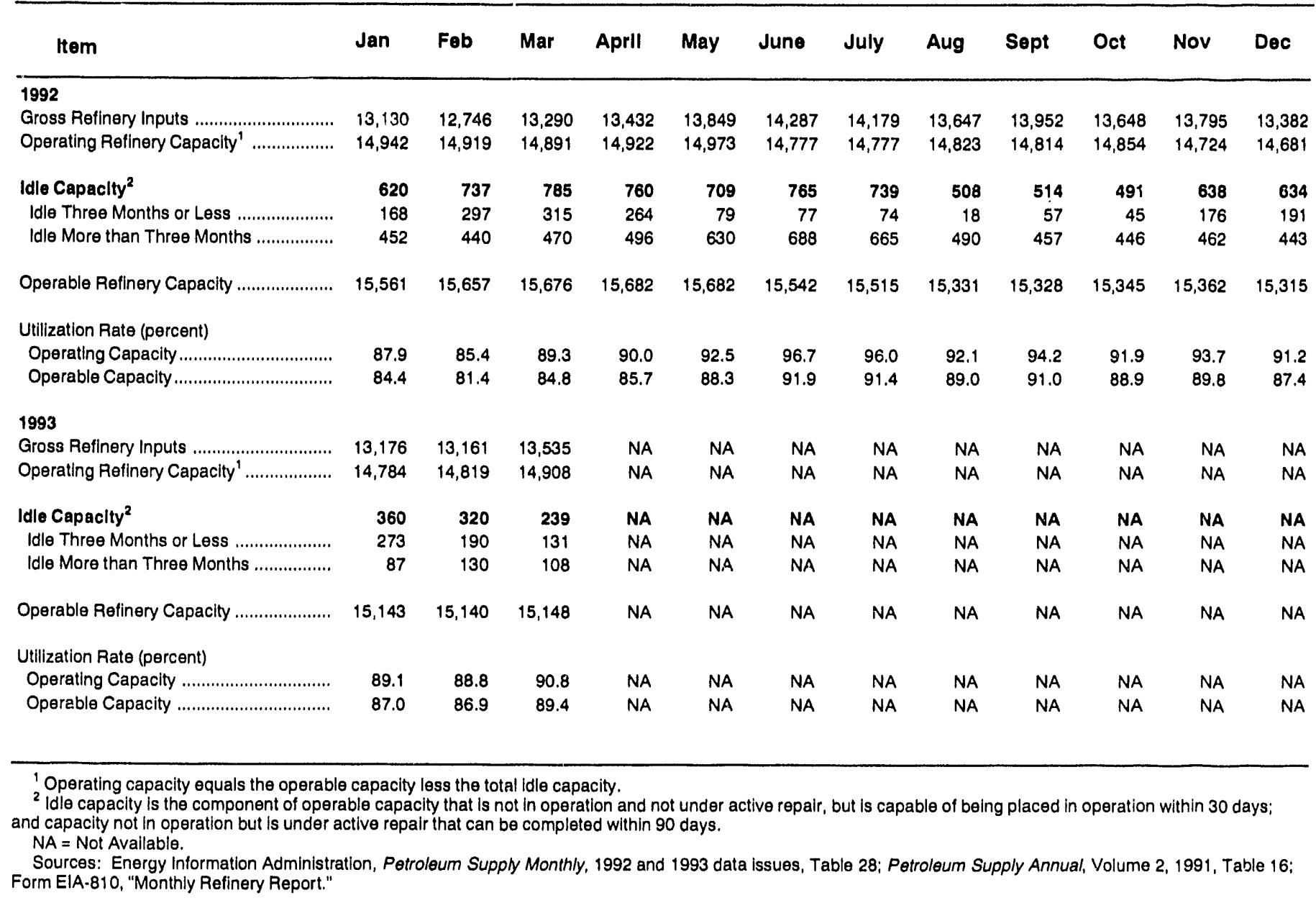

below the normal range, primarily because of record-low propane inventories. Although propane stocks at the end of March of 22 million barrels were well below the normal range for this time of year, no shortages were reported as the winter heating season drew to a close.

\section{Crude Oil}

Imports of crude oil (excluding the SPR) continued to be relatively strong, increasing to 6.5 million barrels per day in March. Imports during the first quarter of 1993 increased 16 percent from the same period in 1992. Contributing to the higher imports were stronger product demand and a 5-percent decline in domestic crude oil production during this year. Imports accounted for 47 percent of total crude oil supplies during the first quarter of 1993, compared with 42 percent a year earlier.

Stocks (excluding the SPR) increased slightly to 337 million barrels at the end of March, and were within the normal range.

\section{Refinery Utilization}

Improved refining margins contributed to an increase in the refinery utilization rate in March. Gross inputs (crude and other oils) to atmospheric crude oil distillation units increased to 13.5 million barrels per day, well above the normal seasonal level. The operating utilization rate increased to 90.8 percent. The operable utilization rate, which reflects refining capacity if all idle capacity is included, increased to 89.4 percent (Table H2). 


\section{Finished Motor Gasoline Product Supplied Adjustment}

Beginning with the reporting of January 1993 data, the Energy Information Administration (EIA) has made adjustments to the product supplied series for finished motor gasoline. It was recognized that motor gasoline statistics published by the EIA through 1992 were underreported because the reporting system was not collecting all fuel ethanol and motor gasoline blending components being blended downstream from the refinery. The EIA was able to quantify these volumes and make corrective adjustments for 1992 in 1993 . Each of these adjustments is discussed in detail following Table H3.

As a result of the changes, any comparisons of 1993 motor gasoline data to historical series must recognize the adjustments made in 1993. To assist in this comparison, the EIA has prepared a table of 1992 finished motor gasoline product supplicd on the 1993 basis (Table H3 below) showing the published numbers by month, the adjustments, and the 1993 product supplied basis number. When making a comparison of 1993 to 1992 finished motor gasoline product supplied, it is important to use the 1993 basis numbers. For example, from Table H3 the January 1993 product supplied number of 6,746 should be compared to 6,934, not the original 6,893 . The year 1992 is the only historical year for which data are available to do a comparison on the 1993 basis.

Table H3. Finished Motor Gasoline Product Supplied Adjustment, 1992 and 1993 (Thousand Barrels per Day)

\begin{tabular}{|l|c|c|c|c|c|}
\hline \multicolumn{1}{|c|}{ Months } & $\begin{array}{c}\text { Product Supplied } \\
\text { Published in PSM }\end{array}$ & $\begin{array}{c}\text { Fuel Ethanol } \\
\text { Adjustment }\end{array}$ & $\begin{array}{c}\text { Motor Gasoline Blending } \\
\text { Component Adjustment }\end{array}$ & $\begin{array}{c}\text { Product Supplied } \\
\text { 1993 Basis }\end{array}$ & Difference \\
\hline January & 6,893 & 68 & -27 & 6,934 & 41 \\
\hline February & 7,004 & 68 & -17 & 7,054 & 51 \\
\hline March & 7,145 & 62 & 72 & 7,278 & 134 \\
\hline April & 7,255 & 68 & 72 & 7,395 & 141 \\
\hline May & 7,288 & 55 & 48 & 7,391 & 104 \\
\hline June & 7,451 & 64 & 31 & 7,546 & 95 \\
\hline July & 7,607 & 52 & 93 & 7,752 & 145 \\
\hline August & 7,414 & 66 & 118 & 7,598 & 184 \\
\hline September & 7,339 & 54 & 58 & 7,451 & 112 \\
\hline October & 7,336 & 76 & 26 & 7,437 & 101 \\
\hline November & 7,119 & 91 & 81 & 7,292 & 172 \\
\hline December & 7,377 & 100 & 83 & 7,559 & 182 \\
\hline Average & 7,270 & 69 & 53 & 7,392 & 122 \\
\hline January 1993 & - & 60 & 25 & 6,746 & - \\
\hline February 1993 & - & 67 & 2 & 7,129 & - \\
\hline March 1993 & - & 70 & 42 & 7,397 & - \\
\hline
\end{tabular}

Note: Totals may not equal sum of components due to independent rounding

Source: - Fuel Ethanol Adjustment - 1992, Energy Information Administration (ElA), Petroleum Supply Monthly, Appendix D, ElA-819M, "Monthly Oxygenate Telephone Report;" 1993, EIA, Petroleum Supply Monthly. • Motor Gasoline Blending Component Adjustment - 1992, ElA, Petroleum Supply Monthly. 


\section{Fuel Ethanol Adjustment}

Prior to 1993, an estimated 60 to 70 thousand barrels per day of fuel ethanol were added to motor gasoline to produce gasohol but were not included in the Energy Information Administration's (EIA) finished motor gasoline production data. In 1992, the EIA attempted to collect these data from downstream fuel ethanol motor gasoline blenders but found that this effort was impractical and the results were inaccurate.

Beginning in January 1993, an estimate for the missing fuel ethanol blended into motor gasoline is calculated. This estimate is calculated as production (from the EIA-819M, "Monthly Oxygenate Telephone Report"), plus imports (from the EIA-814, "Monthly Imports Report"), minus inputs at refineries (from the EIA-810, "Monthly Refinery Report"), plus or minus stock change (from the EIA-819M survey). This estimate for the amount of fuel ethanol blended into motor gasoline is added to Table 1 for Natural Gas Liquids Field Production (line 14) and in the Field Production column for other motor gasoline in Tables 2 through 25 .

An adjustment is then performed to determine how much of the gasohol is used as "oxygenated" gasoline in the Environmental Protection Agency (EPA) carbon munoxide (CO) non-attainment areas specified by the Clean Air Act Amendments of 1990, and how much is used as "other" gasoline. Oxygenated gasoline is required to be used in these areas only during the winter months. Gasohol is used throughout the year and is entirely classified as "other" gasoline during the summer months. It is assumed that during the winter months (November through February), 40 percent of the gasohol will be used in the non-attainment areas and hence classified as "oxygenated" gasoline. During October, it is estimated that 20 percent of the gasohol will be produced and stored until November for use in the EPA CO non-attainment areas. These percentages are EIA estimates, which have been reviewed by industry experts.

An estimate for the total amount of gasohol produced with the ethanol is given as 10 times the estimated fuel ethanol blended (this assumes a 10 percent ethanol blend.) The amount of gasohol used as "oxygenated" gasoline is given by the total amount of gasohol times the appropriate percentage for the month as described above. This amount is added to the column labeled field production of "oxygenated gasoline" and subtracted from "other gasoline" in Tables 2 through 5.

The PAD District level detail shown in Tables 6 through 25 is obtained by allocating the national level estimates calculated above according to the percent of gasohol sales from the U.S. Department of Transportation, Federal Highway Administration, Monthly Motor Fuel Reported by States, 1991.

\section{Motor Gasoline Blending Component Adjustment}

Prior to 1993, the EIA published a "product supplied" for motor gasoline blending components. Since these components are to be blended into finished motor gasoline, there is no actual demand for this intermediate product. The EIA is correcting this series by including the quantity of "product supplied" for motor gasoline blending components with "other" finished motor gasoline. This change is accomplished in Tables 2 through 25 by adding product supplied for motor gasoline blending components to the column labeled Field Production of "other" motor gasoline, and subtracting it from the Field Production column for "motor gasoline blending components."

The blending components adjustment shown in Table H3 is product supplied for blending components published in the 1992 issues of the Petroleum Supply Monthly.

\section{Fuel Ethanol Stock Adjustment}

As discussed previously, downstream fuel ethanol motor gasoline blenders do not report on the Petroleum Supply Reporting System (PSRS). As a result, total end-of-month stocks of fuel ethanol have been underreported in the PSRS. Total stocks of fuel ethanol are assumed to be those reported by ethanol producers on the Form EIA-819M, "Monthly Oxygenate Telephone Report." The difference between the stocks reported on the EIA-819M and the stocks reported in the PSRS (from refiners, bulk terminal and pipeline operators) is added to the stocks shown for bulk terminals. 


\section{Summary Statistics}

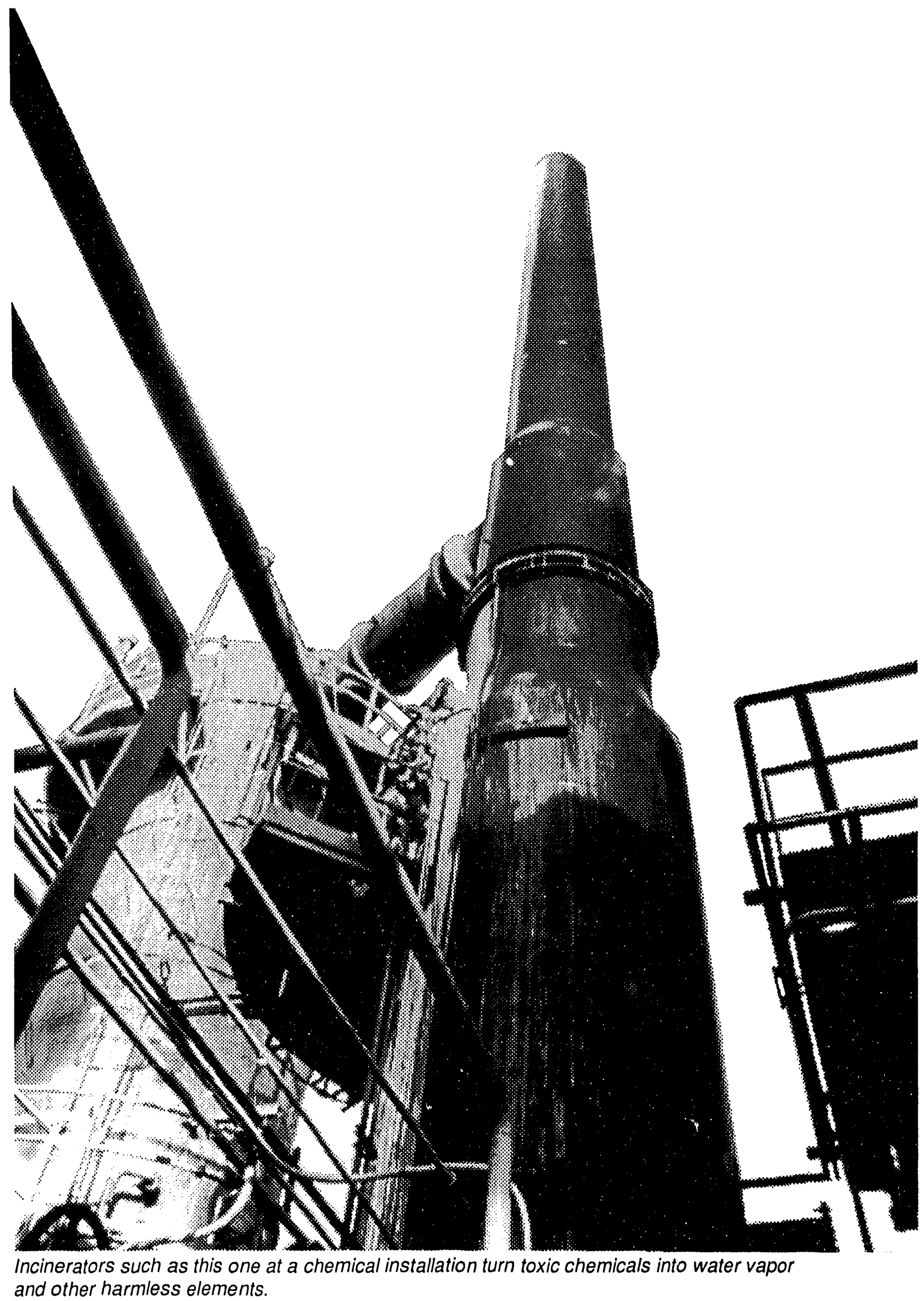


Table S1. Crude Oil and Petroleum Products Overview, 1981 - Present (Thousand Barrels per Day, Except Where Noted)

\begin{tabular}{|c|c|c|c|c|c|c|c|c|}
\hline \multirow{2}{*}{\multicolumn{2}{|c|}{ Year/Month }} & \multicolumn{3}{|c|}{ Flald Production } & \multicolumn{2}{|c|}{ Stock Change } & \multirow[b]{2}{*}{$\begin{array}{l}\text { Petroleum } \\
\text { Products } \\
\text { Supplled }\end{array}$} & \multirow{2}{*}{$\begin{array}{c}\text { Ending } \\
\text { Stocks } \\
\text { (Million Barrols) } \\
\text { Crude Oil }{ }^{d} \text { and } \\
\text { Petroloum } \\
\text { Products }\end{array}$} \\
\hline & & $\begin{array}{c}\text { Total } \\
\text { Domestic }\end{array}$ & $\begin{array}{l}\text { Crudas } \\
\text { Oll }\end{array}$ & $\begin{array}{c}\text { Natural } \\
\text { Gas Plant } \\
\text { Llquids }\end{array}$ & $\begin{array}{c}\text { Cruge } \\
\text { Oll }\end{array}$ & $\begin{array}{l}\text { Petroleum } \\
\text { Products }\end{array}$ & & \\
\hline $\begin{array}{l}1981 \\
1982 \\
1983 \\
1984 \\
1985 \\
1986 \\
1987 \\
1988 \\
1989 \\
1990\end{array}$ & $\begin{array}{l}\text { Average } \ldots \ldots \ldots \ldots \ldots . \\
\text { Average } \ldots \ldots \ldots \ldots \ldots . . \\
\text { Average } \\
\text { Average }\end{array}$ & $\begin{array}{r}10,230 \\
10,252 \\
10,299 \\
10,554 \\
10,636 \\
10,289 \\
10,008 \\
9,818 \\
9,219 \\
8,994\end{array}$ & $\begin{array}{l}8,572 \\
8,649 \\
8,688 \\
8,879 \\
8,971 \\
8,680 \\
8,349 \\
8,140 \\
7,613 \\
7,355\end{array}$ & $\begin{array}{l}1,609 \\
1,550 \\
1,559 \\
1,630 \\
1,609 \\
1,551 \\
1,595 \\
1,625 \\
1,546 \\
1,559\end{array}$ & $\begin{array}{r}9290 \\
136 \\
9214 \\
199 \\
50 \\
78 \\
128 \\
1 \\
86 \\
-35\end{array}$ & $\begin{array}{r}9.130 \\
-283 \\
9-234 \\
81 \\
-153 \\
124 \\
-87 \\
-29 \\
-129 \\
142\end{array}$ & $\begin{array}{l}16,058 \\
15,296 \\
15,231 \\
15,726 \\
15,726 \\
16,281 \\
16,685 \\
17,283 \\
17,325 \\
16,988\end{array}$ & $\begin{array}{r}1,484 \\
91,430 \\
1,454 \\
1,556 \\
1,519 \\
1,593 \\
1,607 \\
1,597 \\
1,581 \\
1,621\end{array}$ \\
\hline \multicolumn{2}{|c|}{ 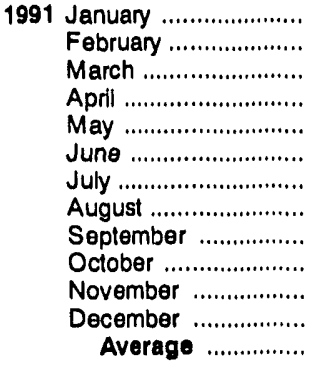 } & $\begin{array}{l}9,255 \\
9,424 \\
9,301 \\
9,262 \\
9,157 \\
9,032 \\
9,056 \\
9,027 \\
\mathbf{5}, 088 \\
9,212 \\
9,129 \\
9,089 \\
9,168\end{array}$ & $\begin{array}{l}7,500 \\
7,637 \\
7,546 \\
7,509 \\
7,409 \\
7,320 \\
7,347 \\
7,316 \\
7,368 \\
7,437 \\
7,328 \\
7,299 \\
7,417\end{array}$ & $\begin{array}{l}1,647 \\
1,695 \\
1,683 \\
1,665 \\
1,657 \\
1,627 \\
1,622 \\
1,627 \\
1,623 \\
1,686 \\
1,697 \\
1,686 \\
1,659\end{array}$ & $\begin{array}{r}-71 \\
231 \\
-239 \\
50 \\
566 \\
-299 \\
-153 \\
103 \\
.156 \\
51 \\
43 \\
.611 \\
-42\end{array}$ & $\begin{array}{r}-1,027 \\
-704 \\
-268 \\
628 \\
988 \\
546 \\
199 \\
316 \\
653 \\
-659 \\
62 \\
-365 \\
32\end{array}$ & $\begin{array}{l}16,893 \\
16,339 \\
16,212 \\
16,139 \\
16,189 \\
16,878 \\
16,971 \\
17,183 \\
16,348 \\
16,996 \\
16,730 \\
17,145 \\
16,714\end{array}$ & $\begin{array}{r}1,587 \\
1,573 \\
1,558 \\
1,578 \\
1,626 \\
1,634 \\
1,635 \\
1,648 \\
1,663 \\
1,644 \\
1,647 \\
1,617 \\
-\end{array}$ \\
\hline \multicolumn{2}{|c|}{ 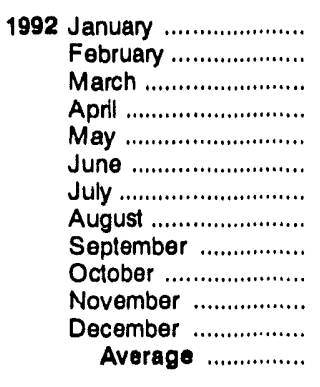 } & $\begin{array}{l}E_{9,184} \\
E_{9,170} \\
E_{9,119} \\
E_{9,086} \\
E_{8,902} \\
E_{8,926} \\
E_{8,905} \\
E_{8,677} \\
E_{8,824} \\
E_{8,971} \\
E_{8,967} \\
E_{9,034} \\
E_{8,980}\end{array}$ & $\begin{array}{l}\text { E } 7,363 \\
\text { E } 7,373 \\
E_{7,315} \\
\text { E } 7,291 \\
\text { E } 7,110 \\
\text { E } 7,138 \\
\text { E } 7,096 \\
\text { E } 6,928 \\
\text { E } 7,019 \\
\text { E } 7,065 \\
\text { E } 7,027 \\
\text { E } 7,125 \\
\text { E } 7,153\end{array}$ & $\begin{array}{l}1,686 \\
1,694 \\
1,695 \\
1,704 \\
1,701 \\
1,701 \\
1,669 \\
1,635 \\
1,660 \\
1,719 \\
1,748 \\
1,739 \\
1,696\end{array}$ & $\begin{array}{r}534 \\
176 \\
-247 \\
310 \\
.150 \\
.577 \\
249 \\
.109 \\
-180 \\
410 \\
-241 \\
-195 \\
-1\end{array}$ & $\begin{array}{r}.773 \\
.967 \\
.273 \\
75 \\
811 \\
604 \\
342 \\
131 \\
641 \\
-230 \\
67 \\
-1,209 \\
-65\end{array}$ & $\begin{array}{l}16,982 \\
16,885 \\
16,789 \\
16,772 \\
16,412 \\
16,928 \\
17,060 \\
16,937 \\
16,851 \\
17,437 \\
17,084 \\
17,913 \\
17,008\end{array}$ & $\begin{array}{r}1,608 \\
1,585 \\
1,569 \\
1,581 \\
1,601 \\
1,602 \\
1,620 \\
1,621 \\
1,635 \\
1,640 \\
1,635 \\
91,592 \\
.\end{array}$ \\
\hline \multicolumn{2}{|c|}{ 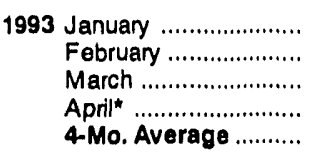 } & $\begin{array}{r}E_{9,257} \\
E_{8,948} \\
E_{9,009} \\
\text { NA } \\
\text { NA }\end{array}$ & $\begin{array}{r}E_{7,008} \\
E_{6,957} \\
\text { RE } 6,976 \\
\text { PE } 6,904 \\
\text { PE } 6,962\end{array}$ & $\begin{array}{r}1,728 \\
1,761 \\
R_{1,799} \\
E_{1,818} \\
E_{1,776}\end{array}$ & $\begin{array}{r}264 \\
219 \\
R_{246} \\
E_{337} \\
E_{267}\end{array}$ & $\begin{array}{r}9370 \\
.799 \\
-619 \\
\text { NA } \\
\text { NA }\end{array}$ & $\begin{array}{r}16,502 \\
17,577 \\
17,752 \\
\text { NA } \\
\text { NA }\end{array}$ & $\begin{array}{r}91,611 \\
1,595 \\
1,584 \\
\text { NA } \\
. .\end{array}$ \\
\hline \multicolumn{2}{|c|}{$\begin{array}{l}1992 \text { 4-Mo. Average .......... } \\
1991 \text { 4-Mo. Average .......... }\end{array}$} & $\begin{array}{r}E_{9,140} \\
\mathbf{9 , 3 0 8}\end{array}$ & $\begin{array}{r}\text { E 7,335 } \\
7,546\end{array}$ & $\begin{array}{l}1,695 \\
1,672\end{array}$ & $\begin{array}{r}193 \\
.14\end{array}$ & $\begin{array}{l}-481 \\
-342\end{array}$ & $\begin{array}{l}16,857 \\
16,399\end{array}$ & 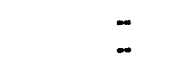 \\
\hline
\end{tabular}

a Anegative number indicates a decrease in stocks and a positive number indicates an increase.

c Includes crude oil, natural gas plant liquids, and other liquids. Beginning in 1993, fuel ethanol blended Into finlshed motor gasoline and oxygenate production from merchant MTBE plants are also included.

d Includes stocks located in the Strategic Petroleum Reserve.

- Includes crude oil for storage in the Strategic Petroleum Reserve.

1 Net Imports equal imports minus Exports.

g In January 1981 and 1983, numerous respondents were added to surveys affecting stocks reported and stock change calculations. Stock changes are calculated using new basis stock levels. Bulk terminal and plpeline stocks of oxygenales were added beginning in January 1993 . See Summary Statistics Explanatory Note 4

Footnotes continued on following page. 
Table S1. Crude Oil and Petroleum Products Overview, 1981 - Present (Continued) (Thousand Barrels per Day, Except Where Noted)

\begin{tabular}{|c|c|c|c|c|c|c|c|c|}
\hline \multirow{2}{*}{\multicolumn{2}{|c|}{ Year/Month }} & \multicolumn{3}{|c|}{ Imports } & \multicolumn{3}{|c|}{ Exports } & \multirow[b]{2}{*}{$\begin{array}{c}\text { Net } \\
\text { Imports }\end{array}$} \\
\hline & & Total & $\underset{\text { Oll }}{\text { Crude }}$ & $\begin{array}{l}\text { Potroleum } \\
\text { Products }\end{array}$ & Total & $\begin{array}{c}\text { Crude } \\
\text { Oll }\end{array}$ & $\begin{array}{l}\text { Petroleum } \\
\text { Products }\end{array}$ & \\
\hline $\begin{array}{l}1981 \\
1982 \\
1983 \\
1984 \\
1985 \\
1986 \\
1987 \\
1988 \\
1989 \\
1990\end{array}$ & 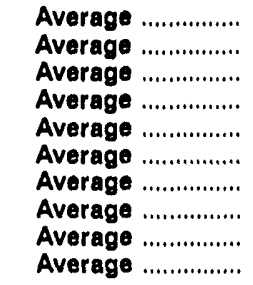 & $\begin{array}{l}5,996 \\
5,113 \\
5,051 \\
5,437 \\
5,067 \\
6,224 \\
6,678 \\
7,402 \\
8,061 \\
8,018\end{array}$ & $\begin{array}{l}4,396 \\
3,488 \\
3,329 \\
3,426 \\
3,201 \\
4,178 \\
4,674 \\
5,107 \\
5,843 \\
5,894\end{array}$ & $\begin{array}{l}1,599 \\
1,625 \\
1,722 \\
2,011 \\
1,866 \\
2,045 \\
2,004 \\
2,295 \\
2,217 \\
2,123\end{array}$ & $\begin{array}{l}595 \\
815 \\
739 \\
722 \\
781 \\
785 \\
764 \\
815 \\
859 \\
857\end{array}$ & $\begin{array}{l}228 \\
236 \\
164 \\
181 \\
204 \\
154 \\
151 \\
155 \\
142 \\
109\end{array}$ & $\begin{array}{l}367 \\
579 \\
575 \\
541 \\
577 \\
631 \\
613 \\
661 \\
717 \\
748\end{array}$ & $\begin{array}{l}5,401 \\
4,298 \\
4,312 \\
4,715 \\
4,286 \\
5,439 \\
5,914 \\
6,587 \\
7,202 \\
7,161\end{array}$ \\
\hline \multicolumn{2}{|c|}{ 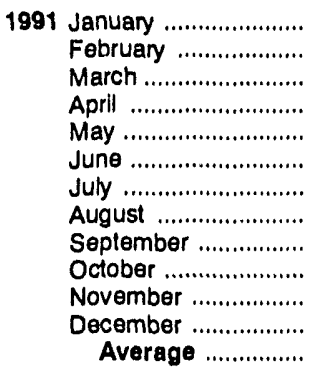 } & $\begin{array}{l}7,103 \\
6,865 \\
6,646 \\
7,418 \\
8,518 \\
8,245 \\
7,755 \\
8,670 \\
7,826 \\
7,467 \\
7,615 \\
7,337 \\
7,627\end{array}$ & $\begin{array}{l}5,296 \\
5,485 \\
5,166 \\
5,529 \\
6,363 \\
6,334 \\
5,955 \\
6,645 \\
5,812 \\
5,683 \\
5,528 \\
5,565 \\
5,782\end{array}$ & $\begin{array}{l}1,808 \\
1,380 \\
1,480 \\
1,888 \\
2,155 \\
1,911 \\
1,801 \\
2,025 \\
2,015 \\
1,784 \\
2,087 \\
1,772 \\
1,844\end{array}$ & $\begin{array}{r}1,199 \\
1,441 \\
944 \\
737 \\
1,149 \\
921 \\
963 \\
837 \\
785 \\
918 \\
926 \\
1,213 \\
1,001\end{array}$ & $\begin{array}{r}50 \\
152 \\
137 \\
162 \\
165 \\
78 \\
739 \\
139 \\
55 \\
109 \\
92 \\
126 \\
133 \\
116\end{array}$ & $\begin{array}{r}1,149 \\
1,288 \\
807 \\
575 \\
984 \\
843 \\
824 \\
783 \\
676 \\
826 \\
800 \\
1,081 \\
885\end{array}$ & $\begin{array}{l}5,904 \\
5,424 \\
5,702 \\
6,680 \\
7,369 \\
7,323 \\
6,793 \\
7,832 \\
7,042 \\
6,550 \\
6,690 \\
6,124 \\
6,626\end{array}$ \\
\hline \multicolumn{2}{|c|}{ 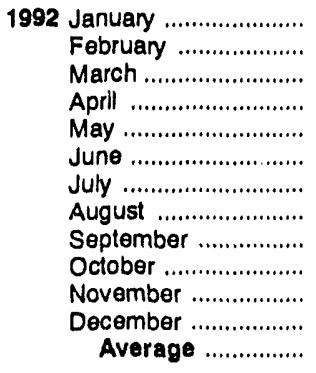 } & $\begin{array}{l}7,593 \\
6,754 \\
7,036 \\
8,067 \\
7,754 \\
7,761 \\
8,474 \\
8,256 \\
8,160 \\
8,520 \\
7,877 \\
7,828 \\
7,844\end{array}$ & $\begin{array}{l}5,885 \\
5,033 \\
5,319 \\
6,113 \\
6,025 \\
6,019 \\
6,796 \\
6,457 \\
6,206 \\
6,696 \\
6,121 \\
5,927 \\
6,054\end{array}$ & $\begin{array}{l}1,708 \\
1,721 \\
1,718 \\
1,954 \\
1,729 \\
1,742 \\
1,678 \\
1,799 \\
1,954 \\
1,824 \\
1,756 \\
1,901 \\
1,790\end{array}$ & $\begin{array}{r}1,144 \\
852 \\
912 \\
937 \\
885 \\
957 \\
929 \\
789 \\
848 \\
902 \\
995 \\
1,237 \\
950\end{array}$ & $\begin{array}{r}118 \\
22 \\
105 \\
23 \\
23 \\
106 \\
107 \\
53 \\
133 \\
68 \\
106 \\
111 \\
111 \\
89\end{array}$ & $\begin{array}{r}1,026 \\
829 \\
807 \\
914 \\
779 \\
850 \\
876 \\
657 \\
780 \\
796 \\
885 \\
1,126 \\
861\end{array}$ & $\begin{array}{l}6,449 \\
5,902 \\
6,124 \\
7,129 \\
6,869 \\
6,804 \\
7,544 \\
7,467 \\
7,312 \\
7,617 \\
6,881 \\
6,591 \\
6,895\end{array}$ \\
\hline 1993 & 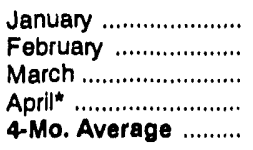 & $\begin{array}{r}7,964 \\
7,930 \\
R_{8,342} \\
E_{8,588} \\
E_{\mathbf{8 , 2 1 0}}\end{array}$ & $\begin{array}{r}6,292 \\
6,156 \\
R_{6,513} \\
E_{6,894} \\
E_{6,468}\end{array}$ & $\begin{array}{r}1,672 \\
1,775 \\
R_{1,829} \\
E_{1,694} \\
E_{1,742}\end{array}$ & $\begin{array}{r}953 \\
853 \\
905 \\
E_{875} \\
E_{898}\end{array}$ & $\begin{array}{r}129 \\
166 \\
R \quad 139 \\
E_{109} \\
E_{135}\end{array}$ & $\begin{array}{r}825 \\
687 \\
R 766 \\
E 767 \\
E_{763}\end{array}$ & $\begin{array}{r}7,011 \\
7,077 \\
R 7,437 \\
\text { E } 7,713 \\
\text { E } 7,312\end{array}$ \\
\hline \multicolumn{2}{|c|}{$\begin{array}{l}1992 \text { 4-Mo. Average .......... } \\
1991 \text { 4-Mo. Average ......... }\end{array}$} & $\begin{array}{l}7,367 \\
7,008\end{array}$ & $\begin{array}{l}5,592 \\
5,365\end{array}$ & $\begin{array}{l}1,774 \\
1,643\end{array}$ & $\begin{array}{r}963 \\
1,074\end{array}$ & $\begin{array}{r}68 \\
124\end{array}$ & $\begin{array}{l}895 \\
950\end{array}$ & $\begin{array}{l}6,403 \\
5,934\end{array}$ \\
\hline
\end{tabular}

Footnotes continued.

$R=$ Revised data. $E=$ Estimated. $P E=$ Preliminary estimate. $R E=$ Revised estimate. $N A=$ Not Available.

- See Summary Statistics Explanatory Note 1.

Notes: - Crude oil includes lease condensate. - llalics denole estimates based upon preliminary data. - Geographic coverage is the 50 States and the District of Columbia. - Totals may not equal sum of components due to independent rounding

Source: See Summary Statistics Table and Figure Sources. 
Figure S1. Petroleum Overvlew, March 1992 - Present

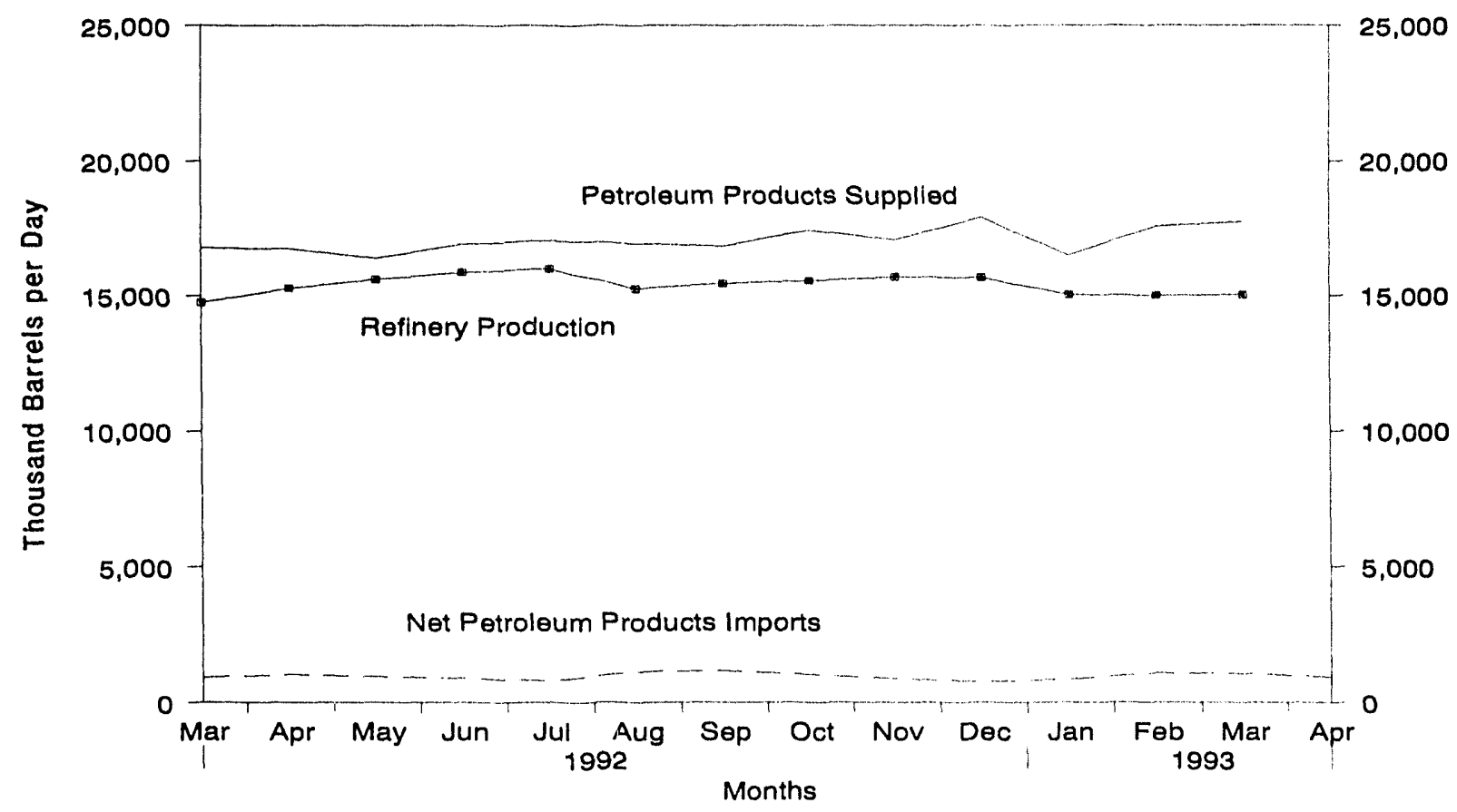

Source: Energy Information Administration, Petroleum Supply Monthly, Table S1. See Summary Statistics Table and Figure Sources.

Figure S2. Petroleum Products Supplied, March 1992 - Present

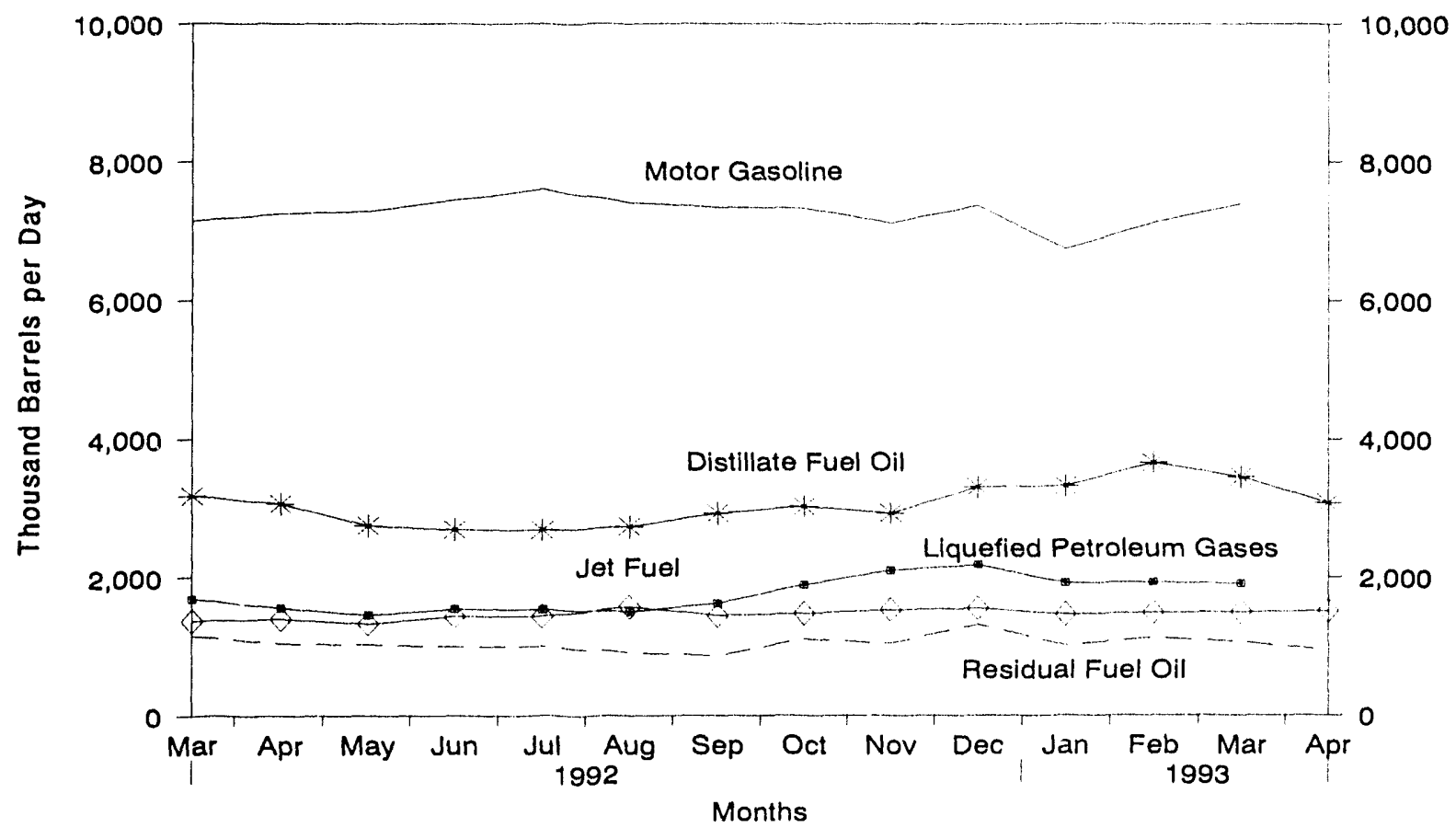

Source: Energy Information Administration, Petroleum Supply Monthly, Tables S4-S7, and S9. See Summary Statistics Table and Figure Sources. 
Figure S3. Crude Oil Supply and Disposition, March 1992 - Present

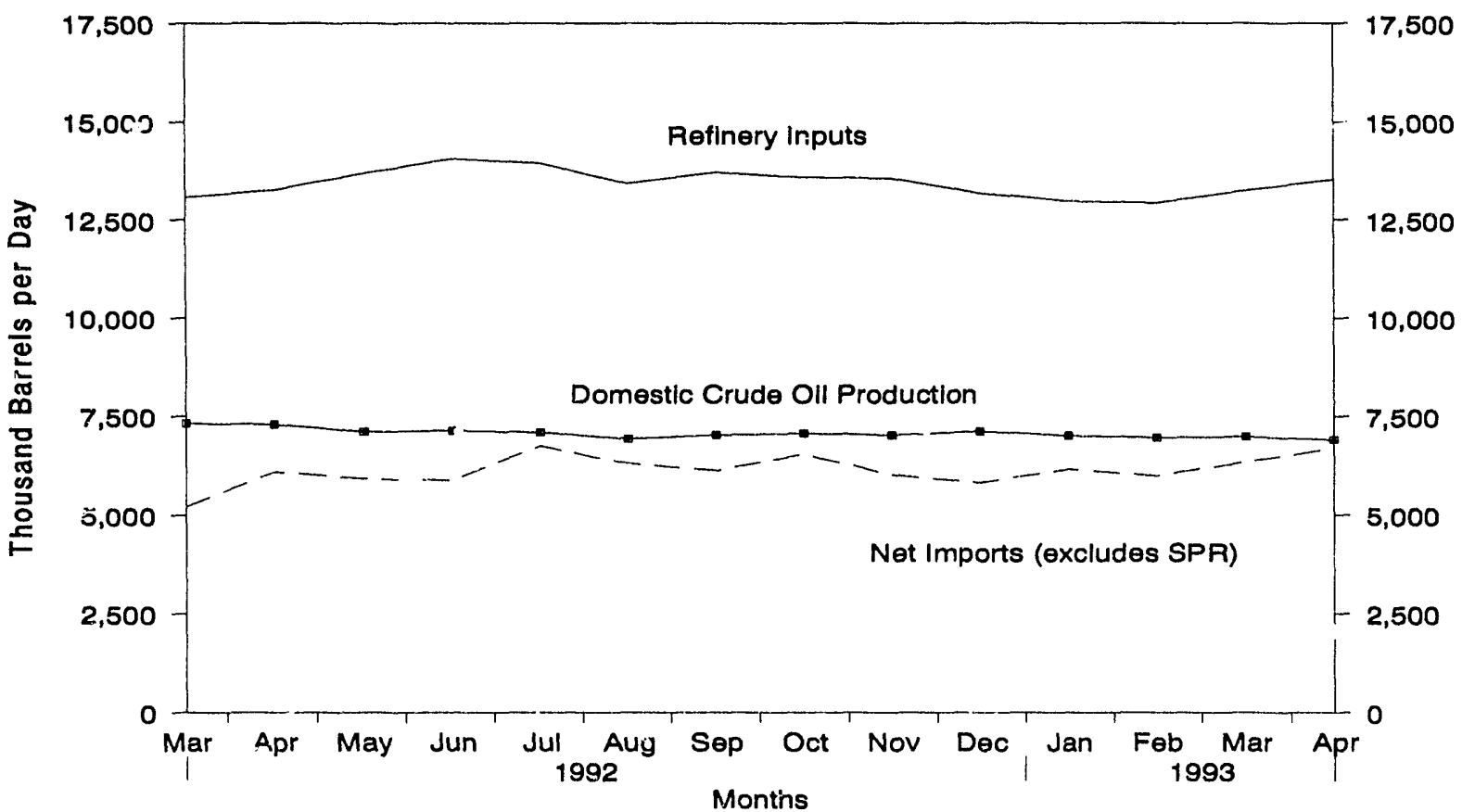

Source: Energy Information Administration. Petroleum Supply Monthly, Table S2. See Summary Statistics Table and Figure Sources.

Figure S4. Crude Oil Ending Stocks', March 1992 - Present

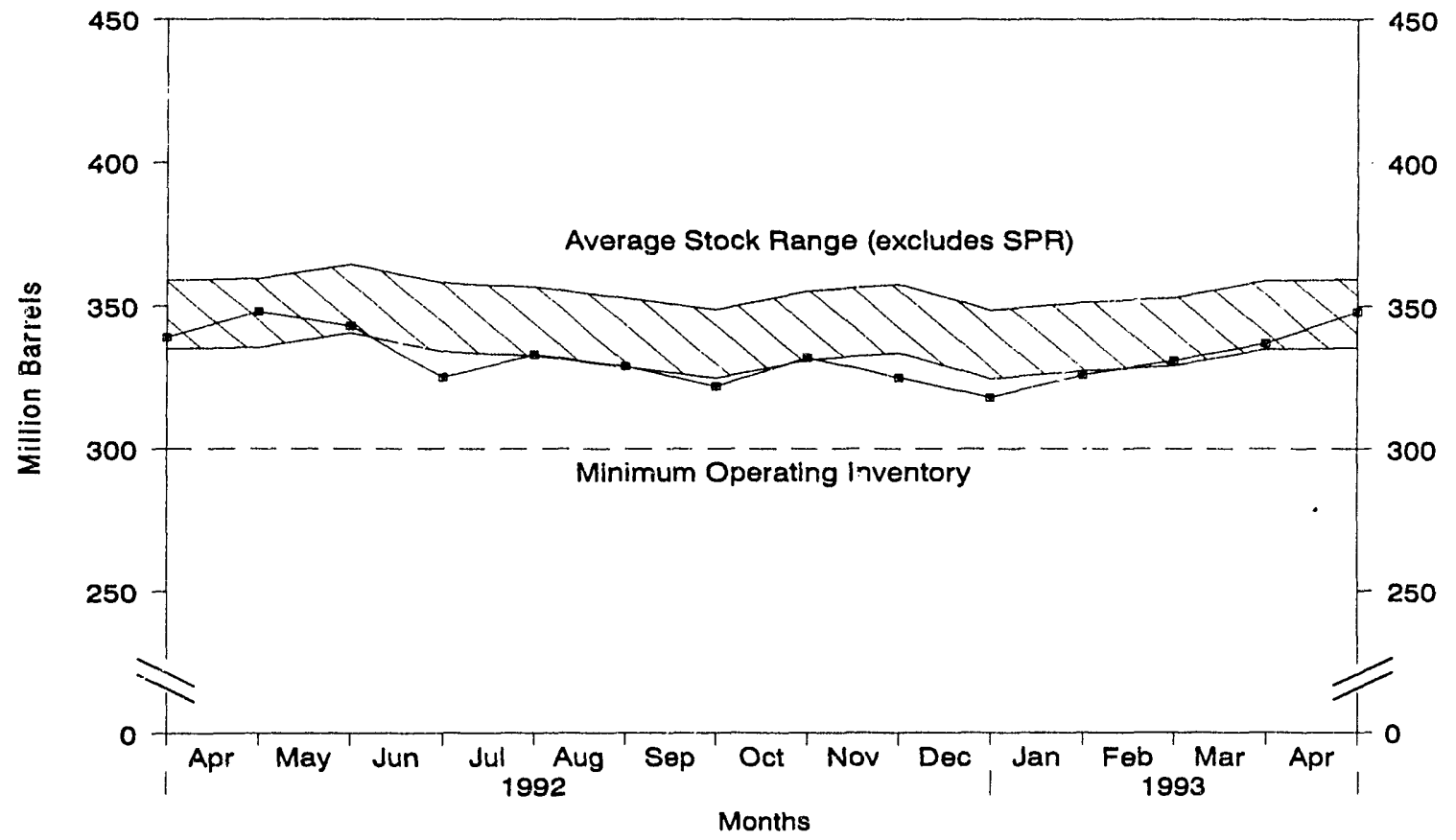

'Excludes stocks held in the Strategic Petroleum Reserve (SPR).

Note: The National Petroleum Council (NPC) defines the Minimum Operating Inventory as the inventory level below which operating problems and shortages would begin to appear in a defined distribution system. In its 1988 study, the NPC estimated this inventory level for crude oil to be 300 million barrels.

Source: Energy Information Administration, Petroleum Suppiy Monthly, Table S2. See Summary Statistics Table and Figure Sources. 
Table S2. Crude Oil Supply and Disposition, 1981 - Present (Thousand Barrels per Day, Except Where Noted)

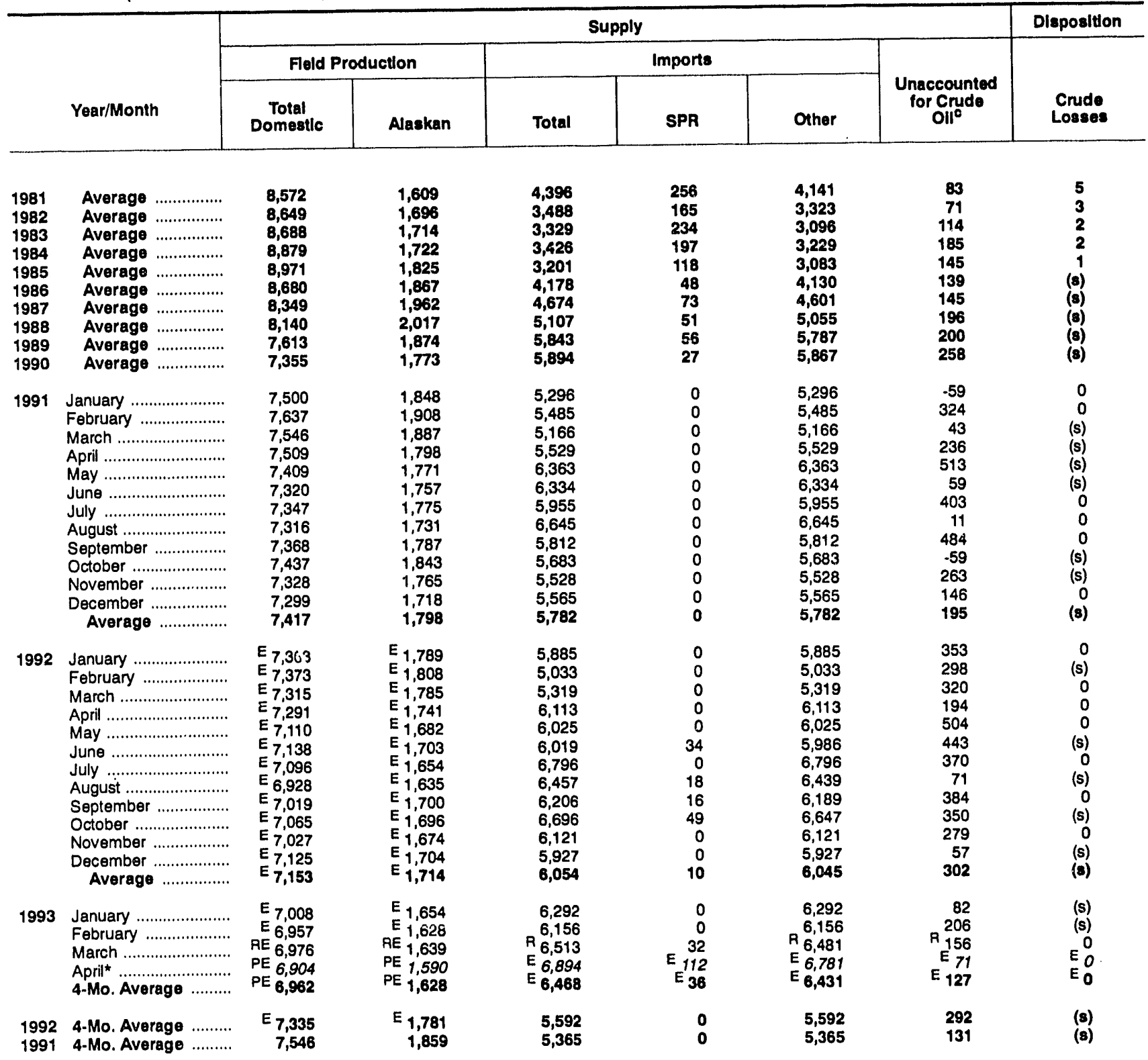

a Stocks are totals as of end of period.

c A negative number indicates a decrease in stocks and a positive number indicates an increase.

c Unacciunted for crude cil represents the difference between the supply and disposition of crude oil. Preliminary estimates of crude oil imports at the National level have historically understated final values by approxh for crude oil to overstate the final values by the same amount.

d Previously published as crude used directly.

e Stuck changes are calculated using new basis stock levels. See Summary Statistics Explanatory Note 4.

Footnotes continued on following page. 
Table S2. Crude Oil Supply and Disposition, 1981 - Present (Continued) (Thousand Barrels per Day, Except Where Noted)

\begin{tabular}{|c|c|c|c|c|c|c|c|c|c|}
\hline & \multirow[b]{3}{*}{ Year/Month } & \multicolumn{5}{|c|}{ Disposition } & \multicolumn{3}{|c|}{ Ending Stocksa (Million Barrels) } \\
\hline & & \multicolumn{2}{|c|}{ Stock Change ${ }^{b}$} & \multirow[b]{2}{*}{$\begin{array}{l}\text { Reflnery } \\
\text { Inputs }\end{array}$} & \multirow[b]{2}{*}{ Exports } & \multirow[b]{2}{*}{$\begin{array}{l}\text { Product } \\
\text { Supplied }\end{array}$} & \multirow[b]{2}{*}{ Total } & \multirow[b]{2}{*}{ SPR } & \multirow[b]{2}{*}{$\begin{array}{l}\text { Other } \\
\text { Primary }\end{array}$} \\
\hline & & SPR & Other & & & & & & \\
\hline $\begin{array}{l}1981 \\
1982 \\
1983 \\
1984 \\
1985 \\
1986 \\
1987 \\
1988 \\
1989 \\
1990\end{array}$ & 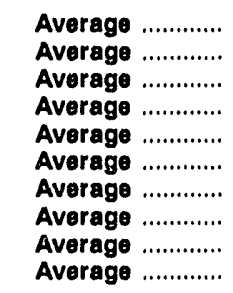 & $\begin{array}{r}336 \\
174 \\
234 \\
195 \\
117 \\
50 \\
80 \\
52 \\
56 \\
16\end{array}$ & $\begin{array}{r}e-46 \\
-38 \\
0-20 \\
4 \\
-67 \\
28 \\
49 \\
-51 \\
30 \\
-51\end{array}$ & $\begin{array}{l}12,470 \\
11,774 \\
11,685 \\
12,044 \\
12,002 \\
12,716 \\
12,854 \\
13,246 \\
13,401 \\
13,409\end{array}$ & $\begin{array}{l}228 \\
236 \\
164 \\
181 \\
204 \\
154 \\
151 \\
155 \\
142 \\
109\end{array}$ & $\begin{array}{r}d_{58} \\
d \mathbf{5 9} \\
66 \\
64 \\
60 \\
49 \\
34 \\
40 \\
28 \\
24\end{array}$ & $\begin{array}{r}594 \\
644 \\
723 \\
796 \\
814 \\
843 \\
890 \\
890 \\
921 \\
908\end{array}$ & $\begin{array}{l}230 \\
294 \\
379 \\
451 \\
493 \\
512 \\
541 \\
560 \\
580 \\
586\end{array}$ & $\begin{array}{r}363 \\
\mathbf{3 5 0} \\
344 \\
345 \\
321 \\
331 \\
349 \\
330 \\
341 \\
323\end{array}$ \\
\hline 1991 & 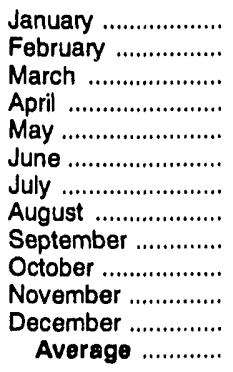 & $\begin{array}{r}0 \\
-147 \\
-422 \\
0 \\
0 \\
(s) \\
(s) \\
(s) \\
0 \\
(s) \\
(s) \\
(s) \\
47\end{array}$ & $\begin{array}{r}-71 \\
379 \\
183 \\
50 \\
566 \\
-299 \\
-153 \\
103 \\
-156 \\
51 \\
43 \\
-611 \\
5\end{array}$ & $\begin{array}{l}12,735 \\
13,046 \\
12,839 \\
13,042 \\
13,539 \\
13,918 \\
13,703 \\
13,800 \\
13,694 \\
12,896 \\
12,929 \\
13,465 \\
13,301\end{array}$ & $\begin{array}{r}50 \\
152 \\
137 \\
162 \\
165 \\
78 \\
139 \\
55 \\
109 \\
92 \\
126 \\
133 \\
116\end{array}$ & $\begin{array}{r}23 \\
17 \\
18 \\
21 \\
15 \\
16 \\
15 \\
13 \\
16 \\
22 \\
22 \\
23 \\
18\end{array}$ & $\begin{array}{r}906 \\
913 \\
905 \\
907 \\
924 \\
915 \\
911 \\
914 \\
909 \\
911 \\
912 \\
893 \\
--\end{array}$ & $\begin{array}{r}586 \\
582 \\
568 \\
568 \\
568 \\
568 \\
569 \\
569 \\
569 \\
569 \\
569 \\
569 \\
-\end{array}$ & $\begin{array}{r}320 \\
331 \\
337 \\
338 \\
356 \\
347 \\
342 \\
345 \\
341 \\
342 \\
344 \\
325 \\
-.-\end{array}$ \\
\hline 1992 & 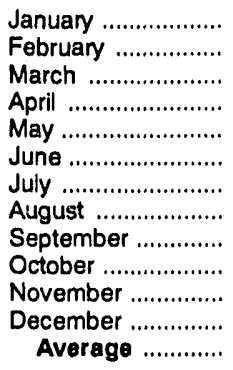 & $\begin{array}{r}\text { (s) } \\
0 \\
(s) \\
0 \\
(s) \\
34 \\
(s) \\
20 \\
43 \\
69 \\
15 \\
22 \\
17\end{array}$ & $\begin{array}{r}534 \\
176 \\
-247 \\
310 \\
-150 \\
.611 \\
249 \\
-129 \\
.224 \\
341 \\
-257 \\
.217 \\
-18\end{array}$ & $\begin{array}{l}12,923 \\
12,488 \\
13,077 \\
13,254 \\
13,673 \\
14,058 \\
13,950 \\
13,425 \\
13,710 \\
13,584 \\
13,547 \\
13,181 \\
13,408\end{array}$ & $\begin{array}{r}118 \\
22 \\
105 \\
23 \\
23 \\
106 \\
107 \\
53 \\
133 \\
68 \\
106 \\
111 \\
111 \\
89\end{array}$ & $\begin{array}{r}26 \\
17 \\
18 \\
11 \\
10 \\
12 \\
9 \\
8 \\
11 \\
10 \\
10 \\
12 \\
13\end{array}$ & $\begin{array}{r}910 \\
915 \\
907 \\
916 \\
912 \\
894 \\
902 \\
899 \\
893 \\
906 \\
899 \\
893 \\
-\end{array}$ & $\begin{array}{r}569 \\
569 \\
569 \\
569 \\
569 \\
570 \\
570 \\
570 \\
571 \\
\mathbf{5 7 4} \\
\mathbf{5 7 4} \\
\mathbf{5 7 5} \\
-\end{array}$ & $\begin{array}{r}341 \\
346 \\
339 \\
348 \\
343 \\
325 \\
333 \\
329 \\
322 \\
332 \\
325 \\
318 \\
-\end{array}$ \\
\hline 1993 & $\begin{array}{l}\text { January .................. } \\
\text { February ................ } \\
\text { March .................... } \\
\text { April ...................... } \\
\text { 4-Mo. Average ...... }\end{array}$ & $\begin{array}{r}19 \\
18 \\
R_{58} \\
E_{131}^{131} \\
E_{57}\end{array}$ & $\begin{array}{r}245 \\
202 \\
R_{188} \\
E_{205} \\
E_{210}\end{array}$ & $\begin{array}{r}12,980 \\
12,923 \\
R \quad 13,249 \\
E_{13,525} \\
E_{13,172}\end{array}$ & $\begin{array}{r}129 \\
166 \\
P_{139} \\
E_{109} 109 \\
E_{135}\end{array}$ & $\begin{array}{r}10 \\
10 \\
R_{11} \\
E_{10} \\
E_{10}\end{array}$ & $\begin{array}{r}901 \\
907 \\
R_{915} \\
E 929 \\
. .\end{array}$ & $\begin{array}{r}575 \\
576 \\
578 \\
E_{582} \\
. \cdot\end{array}$ & $\begin{array}{r}326 \\
331 \\
R 337 \\
E_{348} \\
--\end{array}$ \\
\hline $\begin{array}{l}1992 \\
1991\end{array}$ & $\begin{array}{l}\text { 4-Mo. Average ...... } \\
\text { 4-Mo. Average ..... }\end{array}$ & $\begin{array}{r}(s) \\
-143\end{array}$ & $\begin{array}{l}193 \\
130\end{array}$ & $\begin{array}{l}12,941 \\
12,911\end{array}$ & $\begin{array}{r}68 \\
124\end{array}$ & $\begin{array}{l}18 \\
20\end{array}$ & $\ddot{--}$ & $\ddot{--}$ & $\ddot{-}$ \\
\hline
\end{tabular}

Footnotes continued

$R=$ Revised data. $(s)=$ Less than 500 barrels per day. $E=$ Estimated. $P E=$ Preliminary estimate. RE = Revised estimate

SPR = Strategic Petroleum Reserve.

* See Summary Statistics Explanatory Note 1.

Notes: - Crude oil includes lease condensate. - Italics denote estimates based upon preliminary data. - Geographic coverage is the 50 States and the District of Columbia. - Totals may not equal sum of components due to independent rounding.

Source: See Summary Statistics Table and Figure Sources. 
Table S3. Crude Oil and Petroleum Product Imports, 1981 - Present (Thousand Barrels per Day)

\begin{tabular}{|c|c|c|c|c|c|c|c|c|c|}
\hline & \multirow{3}{*}{ Year/Month } & \multicolumn{8}{|c|}{ Imports from Arab-OPEC Sources } \\
\hline & & \multicolumn{2}{|c|}{ Algeria } & \multicolumn{2}{|c|}{ Iraq } & \multicolumn{2}{|c|}{ Kuwalt } & \multicolumn{2}{|c|}{ Llbya } \\
\hline & & Total & Crude Oll & Total & Crude Oll & Total & Crude Oll & Total & Crude Oll \\
\hline $\begin{array}{l}1981 \\
1982 \\
1983 \\
1984 \\
1985 \\
1986 \\
1987 \\
1988 \\
1989 \\
1990\end{array}$ & 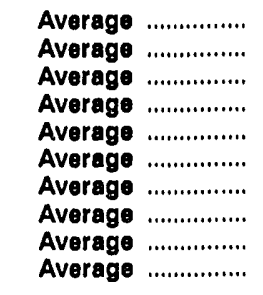 & $\begin{array}{l}311 \\
170 \\
240 \\
323 \\
187 \\
271 \\
295 \\
300 \\
269 \\
280\end{array}$ & $\begin{array}{r}261 \\
90 \\
176 \\
194 \\
84 \\
78 \\
115 \\
58 \\
60 \\
63\end{array}$ & $\begin{array}{r}(8) \\
3 \\
10 \\
12 \\
46 \\
81 \\
83 \\
345 \\
449 \\
518\end{array}$ & $\begin{array}{r}0 \\
3 \\
10 \\
12 \\
46 \\
81 \\
82 \\
343 \\
441 \\
514\end{array}$ & $\begin{array}{r}0 \\
5 \\
14 \\
36 \\
21 \\
68 \\
84 \\
92 \\
157 \\
86\end{array}$ & $\begin{array}{r}0 \\
2 \\
7 \\
24 \\
4 \\
28 \\
70 \\
80 \\
155 \\
79\end{array}$ & $\begin{array}{r}319 \\
26 \\
0 \\
1 \\
4 \\
0 \\
0 \\
0 \\
0 \\
0\end{array}$ & $\begin{array}{r}317 \\
23 \\
0 \\
0 \\
0 \\
0 \\
0 \\
0 \\
0 \\
0\end{array}$ \\
\hline 1991 & 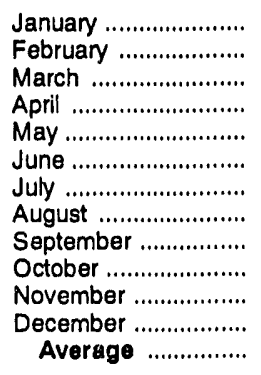 & $\begin{array}{l}327 \\
246 \\
222 \\
282 \\
308 \\
304 \\
202 \\
182 \\
205 \\
235 \\
278 \\
247 \\
253\end{array}$ & $\begin{array}{l}48 \\
20 \\
45 \\
74 \\
72 \\
37 \\
28 \\
16 \\
19 \\
53 \\
58 \\
54 \\
44\end{array}$ & $\begin{array}{l}0 \\
0 \\
0 \\
0 \\
0 \\
0 \\
0 \\
0 \\
0 \\
0 \\
0 \\
0 \\
0\end{array}$ & $\begin{array}{l}0 \\
0 \\
0 \\
0 \\
0 \\
0 \\
0 \\
0 \\
0 \\
0 \\
0 \\
0 \\
0\end{array}$ & $\begin{array}{r}0 \\
0 \\
0 \\
0 \\
0 \\
0 \\
0 \\
0 \\
34 \\
33 \\
0 \\
0 \\
6\end{array}$ & $\begin{array}{r}0 \\
0 \\
0 \\
0 \\
0 \\
0 \\
0 \\
0 \\
34 \\
33 \\
0 \\
0 \\
6\end{array}$ & $\begin{array}{l}0 \\
0 \\
0 \\
0 \\
0 \\
0 \\
0 \\
0 \\
0 \\
0 \\
0 \\
0 \\
0\end{array}$ & $\begin{array}{l}0 \\
0 \\
0 \\
0 \\
0 \\
0 \\
0 \\
0 \\
0 \\
0 \\
0 \\
0 \\
0\end{array}$ \\
\hline 1992 & $\begin{array}{l}\text { January } \\
\text { February } \\
\text { March } \ldots \ldots \ldots \ldots \ldots \ldots \\
\text { April } \ldots \ldots \ldots \ldots \ldots \ldots \ldots \ldots \\
\text { May } \ldots \ldots \ldots \ldots \ldots \ldots \ldots \ldots \\
\text { June } \\
\text { July }\end{array}$ & $\begin{array}{l}217 \\
218 \\
215 \\
182 \\
202 \\
144 \\
179 \\
261 \\
184 \\
186 \\
171 \\
203 \\
197\end{array}$ & $\begin{array}{r}37 \\
57 \\
37 \\
19 \\
7 \\
12 \\
37 \\
45 \\
19 \\
8 \\
0 \\
9 \\
24\end{array}$ & $\begin{array}{l}0 \\
0 \\
0 \\
0 \\
0 \\
0 \\
0 \\
0 \\
0 \\
0 \\
0 \\
0 \\
0\end{array}$ & $\begin{array}{l}0 \\
0 \\
0 \\
0 \\
0 \\
0 \\
0 \\
0 \\
0 \\
0 \\
0 \\
0 \\
0\end{array}$ & $\begin{array}{r}0 \\
0 \\
0 \\
0 \\
0 \\
0 \\
58 \\
66 \\
70 \\
137 \\
117 \\
165 \\
51\end{array}$ & $\begin{array}{r}0 \\
0 \\
0 \\
0 \\
0 \\
0 \\
23 \\
33 \\
33 \\
109 \\
117 \\
149 \\
39\end{array}$ & $\begin{array}{l}0 \\
0 \\
0 \\
0 \\
0 \\
0 \\
0 \\
0 \\
0 \\
0 \\
0 \\
0 \\
0\end{array}$ & $\begin{array}{l}0 \\
0 \\
0 \\
0 \\
0 \\
0 \\
0 \\
0 \\
0 \\
0 \\
0 \\
0 \\
0\end{array}$ \\
\hline 1993 & $\begin{array}{l}\text { January ................... } \\
\text { February ................... } \\
\text { March ................... } \\
\text { 3-Mo. Average .......... }\end{array}$ & $\begin{array}{l}153 \\
256 \\
185 \\
196\end{array}$ & $\begin{array}{r}28 \\
0 \\
7 \\
12\end{array}$ & $\begin{array}{l}0 \\
0 \\
0 \\
0\end{array}$ & $\begin{array}{l}0 \\
0 \\
0 \\
0\end{array}$ & $\begin{array}{l}144 \\
251 \\
316 \\
237\end{array}$ & $\begin{array}{l}129 \\
229 \\
300 \\
219\end{array}$ & $\begin{array}{l}0 \\
0 \\
0 \\
0\end{array}$ & $\begin{array}{l}0 \\
0 \\
0 \\
0\end{array}$ \\
\hline $\begin{array}{l}1992 \\
1991\end{array}$ & $\begin{array}{l}\text { 3-Mo. Average ......... } \\
\text { 3-Mo. Average ........ }\end{array}$ & $\begin{array}{l}217 \\
266\end{array}$ & $\begin{array}{l}43 \\
38\end{array}$ & $\begin{array}{l}0 \\
0\end{array}$ & $\begin{array}{l}0 \\
0\end{array}$ & $\begin{array}{l}\mathbf{0} \\
\mathbf{0}\end{array}$ & $\begin{array}{l}0 \\
0\end{array}$ & $\begin{array}{l}0 \\
0\end{array}$ & $\begin{array}{l}0 \\
0\end{array}$ \\
\hline
\end{tabular}

See footnotes at end of table. 
Table S3. Crude Oil and Petroleum Product Imports, 1981 - Present (Continued) (Thousand Barrels per Day)

\begin{tabular}{|c|c|c|c|c|c|c|c|c|c|}
\hline \multirow{3}{*}{ 更 } & \multirow{3}{*}{ Year/Month } & \multicolumn{8}{|c|}{ Imports from Arab-OPEC Sources } \\
\hline & & \multicolumn{2}{|c|}{ Oatar } & \multicolumn{2}{|c|}{$\begin{array}{l}\text { Saudl } \\
\text { Arablab }\end{array}$} & \multicolumn{2}{|c|}{$\begin{array}{c}\text { United } \\
\text { Arab } \\
\text { Emirates }\end{array}$} & \multicolumn{2}{|c|}{$\begin{array}{l}\text { Total } \\
\text { Arab } \\
\text { OPEC }\end{array}$} \\
\hline & & Total & Crude Oll & Total & Crude Oll & Total & Crude Oll & Total & Crude OII \\
\hline $\begin{array}{l}1981 \\
1982 \\
1883 \\
1984 \\
1985 \\
1986 \\
1987 \\
1988 \\
1989 \\
1990\end{array}$ & 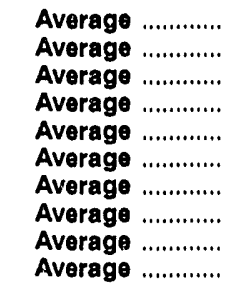 & $\begin{array}{r}7 \\
7 \\
(8) \\
5 \\
(8) \\
13 \\
0 \\
0 \\
2 \\
4\end{array}$ & $\begin{array}{r}7 \\
7 \\
0 \\
4 \\
0 \\
12 \\
0 \\
0 \\
2 \\
4\end{array}$ & $\begin{array}{r}1,129 \\
552 \\
337 \\
325 \\
168 \\
685 \\
751 \\
1,073 \\
1,224 \\
1,339\end{array}$ & $\begin{array}{r}1,112 \\
530 \\
321 \\
309 \\
132 \\
618 \\
642 \\
911 \\
1,116 \\
1,195\end{array}$ & $\begin{array}{r}81 \\
92 \\
30 \\
117 \\
45 \\
44 \\
61 \\
29 \\
28 \\
17\end{array}$ & $\begin{array}{r}77 \\
81 \\
18 \\
90 \\
35 \\
38 \\
56 \\
23 \\
21 \\
9\end{array}$ & $\begin{array}{r}1,848 \\
854 \\
632 \\
819 \\
472 \\
1,162 \\
1,274 \\
1,839 \\
2,130 \\
2,244\end{array}$ & $\begin{array}{r}1,774 \\
736 \\
533 \\
634 \\
300 \\
854 \\
965 \\
1,415 \\
1,794 \\
1,864\end{array}$ \\
\hline 1991 & 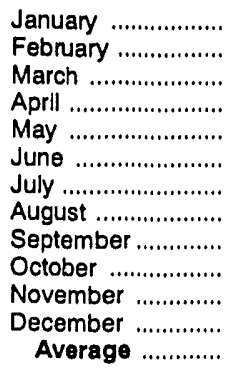 & $\begin{array}{l}0 \\
0 \\
0 \\
0 \\
0 \\
0 \\
0 \\
0 \\
0 \\
0 \\
0 \\
0 \\
0\end{array}$ & $\begin{array}{l}0 \\
0 \\
0 \\
0 \\
0 \\
0 \\
0 \\
0 \\
0 \\
0 \\
0 \\
0 \\
0\end{array}$ & $\begin{array}{l}1,934 \\
1,566 \\
1,683 \\
1,764 \\
2,258 \\
1,841 \\
1,725 \\
2,019 \\
1,708 \\
1,671 \\
1,778 \\
1,645 \\
1,802\end{array}$ & $\begin{array}{l}1,782 \\
1,538 \\
1,646 \\
1,702 \\
2,053 \\
1,795 \\
1,641 \\
1,964 \\
1,562 \\
1,545 \\
1,626 \\
1,566 \\
1,703\end{array}$ & $\begin{array}{r}0 \\
0 \\
0 \\
0 \\
0 \\
0 \\
0 \\
7 \\
0 \\
18 \\
16 \\
0 \\
3\end{array}$ & $\begin{array}{r}0 \\
0 \\
0 \\
0 \\
0 \\
0 \\
0 \\
0 \\
0 \\
18 \\
0 \\
0 \\
2\end{array}$ & $\begin{array}{l}2,261 \\
1,812 \\
1,905 \\
2,046 \\
2,566 \\
2,145 \\
1,928 \\
2,208 \\
1,947 \\
1,956 \\
2,072 \\
1,892 \\
2,064\end{array}$ & $\begin{array}{l}1,630 \\
1,559 \\
1,691 \\
1,776 \\
2,124 \\
1,832 \\
1,670 \\
1,980 \\
1,615 \\
1,649 \\
1,684 \\
1,620 \\
1,754\end{array}$ \\
\hline 1992 & 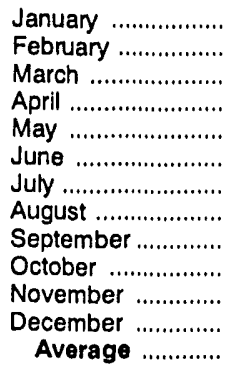 & $\begin{array}{l}0 \\
0 \\
0 \\
0 \\
0 \\
0 \\
8 \\
0 \\
0 \\
0 \\
0 \\
0 \\
1\end{array}$ & $\begin{array}{l}0 \\
0 \\
0 \\
0 \\
0 \\
0 \\
0 \\
0 \\
0 \\
0 \\
0 \\
0 \\
0\end{array}$ & $\begin{array}{l}1,971 \\
1,776 \\
1,707 \\
1,734 \\
1,764 \\
1,744 \\
1,713 \\
1,594 \\
1,593 \\
1,593 \\
1,608 \\
1,793 \\
1,716\end{array}$ & $\begin{array}{l}1,865 \\
1,687 \\
1,568 \\
1,524 \\
1,584 \\
1,610 \\
1,599 \\
1,473 \\
1,477 \\
1,482 \\
1,540 \\
1,725 \\
1,595\end{array}$ & $\begin{array}{r}18 \\
0 \\
0 \\
0 \\
0 \\
0 \\
0 \\
7 \\
0 \\
4 \\
17 \\
28 \\
6\end{array}$ & $\begin{array}{l}0 \\
0 \\
0 \\
0 \\
0 \\
0 \\
0 \\
0 \\
0 \\
0 \\
0 \\
0 \\
0\end{array}$ & $\begin{array}{l}2,206 \\
1,995 \\
1,922 \\
1,916 \\
1,966 \\
1,888 \\
1,958 \\
1,929 \\
1,847 \\
1,920 \\
1,913 \\
2,188 \\
1,971\end{array}$ & $\begin{array}{l}1,902 \\
1,745 \\
1,605 \\
1,543 \\
1,591 \\
1,621 \\
1,659 \\
1,551 \\
1,529 \\
1,599 \\
1,657 \\
1,882 \\
1,657\end{array}$ \\
\hline 1993 & $\begin{array}{l}\text { January ................ } \\
\text { February .................. } \\
\text { March .................. } \\
\text { 3-Mo. Average ....... }\end{array}$ & $\begin{array}{l}0 \\
0 \\
6 \\
2\end{array}$ & $\begin{array}{l}0 \\
0 \\
0 \\
0\end{array}$ & $\begin{array}{l}1,687 \\
1,626 \\
1,479 \\
1,597\end{array}$ & $\begin{array}{l}1,571 \\
1,480 \\
1,349 \\
1,466\end{array}$ & $\begin{array}{l}0 \\
0 \\
0 \\
0\end{array}$ & $\begin{array}{l}0 \\
0 \\
0 \\
0\end{array}$ & $\begin{array}{l}1,984 \\
2,133 \\
1,987 \\
2,032\end{array}$ & $\begin{array}{l}1,728 \\
1,709 \\
1,655 \\
1,697\end{array}$ \\
\hline $\begin{array}{l}1992 \\
1991\end{array}$ & $\begin{array}{l}\text { 3-Mo. Average ...... } \\
\text { 3-Mo. Average ...... }\end{array}$ & $\begin{array}{l}0 \\
0\end{array}$ & $\begin{array}{l}0 \\
0\end{array}$ & $\begin{array}{l}1,819 \\
1,733\end{array}$ & $\begin{array}{l}1,707 \\
1,660\end{array}$ & $\begin{array}{l}6 \\
0\end{array}$ & $\begin{array}{l}0 \\
0\end{array}$ & $\begin{array}{l}2,042 \\
1,999\end{array}$ & $\begin{array}{l}1,751 \\
1,698\end{array}$ \\
\hline
\end{tabular}

See footnotes at end of table. 
Table S3. Crude Oil and Petroleum Product Imports, 1981 - Present (Continued) (Thousand Barrels per Day)

\begin{tabular}{|c|c|c|c|c|c|c|c|c|c|}
\hline & \multirow{3}{*}{ Year/Month } & \multicolumn{8}{|c|}{ Imports from Other-OPEC Sources } \\
\hline & & \multicolumn{2}{|c|}{ Ecuador } & \multicolumn{2}{|c|}{ Gabon } & \multicolumn{2}{|c|}{ Indonesia } & \multicolumn{2}{|c|}{ Iran } \\
\hline & & Total & Crude Oll & Total & Crude OII & Total & Crude OII & Total & Crude Oll \\
\hline $\begin{array}{l}1981 \\
1982 \\
1983 \\
1984 \\
1985 \\
1986 \\
1987 \\
1988 \\
1989 \\
1990\end{array}$ & $\begin{array}{l}\text { Average } \\
\text { Average }\end{array}$..................... & $\begin{array}{l}48 \\
42 \\
61 \\
55 \\
67 \\
77 \\
29 \\
47 \\
89 \\
49\end{array}$ & $\begin{array}{l}38 \\
32 \\
56 \\
47 \\
56 \\
64 \\
23 \\
33 \\
80 \\
38\end{array}$ & $\begin{array}{l}35 \\
40 \\
59 \\
58 \\
52 \\
26 \\
35 \\
16 \\
50 \\
64\end{array}$ & $\begin{array}{l}35 \\
40 \\
59 \\
57 \\
51 \\
25 \\
35 \\
15 \\
49 \\
64\end{array}$ & $\begin{array}{l}366 \\
248 \\
338 \\
343 \\
314 \\
318 \\
285 \\
205 \\
183 \\
114\end{array}$ & $\begin{array}{r}318 \\
226 \\
315 \\
304 \\
292 \\
297 \\
262 \\
186 \\
158 \\
98\end{array}$ & $\begin{array}{r}0 \\
35 \\
48 \\
10 \\
27 \\
19 \\
98 \\
d(8) \\
0 \\
0\end{array}$ & $\begin{array}{r}0 \\
35 \\
48 \\
10 \\
27 \\
19 \\
98 \\
0(s) \\
0 \\
0\end{array}$ \\
\hline 1991 & 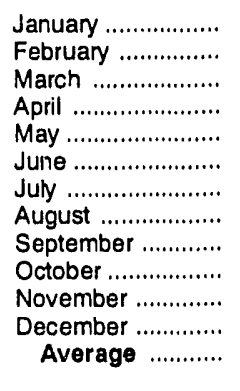 & $\begin{array}{r}18 \\
66 \\
67 \\
35 \\
109 \\
129 \\
62 \\
112 \\
31 \\
30 \\
55 \\
41 \\
63\end{array}$ & $\begin{array}{r}6 \\
55 \\
58 \\
24 \\
103 \\
126 \\
47 \\
93 \\
25 \\
24 \\
48 \\
23 \\
53\end{array}$ & $\begin{array}{r}41 \\
95 \\
29 \\
72 \\
96 \\
70 \\
137 \\
56 \\
91 \\
137 \\
91 \\
91 \\
84\end{array}$ & $\begin{array}{r}41 \\
95 \\
29 \\
72 \\
96 \\
70 \\
137 \\
56 \\
91 \\
137 \\
91 \\
91 \\
84\end{array}$ & $\begin{array}{r}70 \\
162 \\
93 \\
69 \\
97 \\
187 \\
88 \\
93 \\
83 \\
118 \\
120 \\
163 \\
111\end{array}$ & $\begin{array}{r}70 \\
153 \\
93 \\
69 \\
97 \\
187 \\
88 \\
87 \\
64 \\
91 \\
96 \\
134 \\
102\end{array}$ & $\begin{array}{r}0 \\
0 \\
0 \\
0 \\
0 \\
0 \\
81 \\
48 \\
152 \\
43 \\
64 \\
0 \\
32\end{array}$ & $\begin{array}{r}0 \\
0 \\
0 \\
0 \\
0 \\
0 \\
81 \\
48 \\
152 \\
43 \\
64 \\
0 \\
32\end{array}$ \\
\hline 1992 & 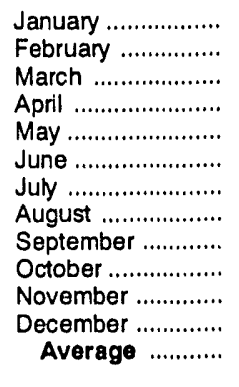 & $\begin{array}{r}23 \\
37 \\
26 \\
53 \\
51 \\
105 \\
111 \\
99 \\
97 \\
42 \\
53 \\
24 \\
60\end{array}$ & $\begin{array}{r}23 \\
24 \\
26 \\
46 \\
51 \\
101 \\
111 \\
93 \\
97 \\
36 \\
53 \\
24 \\
57\end{array}$ & $\begin{array}{r}91 \\
105 \\
25 \\
186 \\
135 \\
129 \\
143 \\
108 \\
165 \\
167 \\
114 \\
120 \\
124\end{array}$ & $\begin{array}{r}91 \\
105 \\
25 \\
186 \\
135 \\
129 \\
143 \\
108 \\
158 \\
167 \\
114 \\
120 \\
123\end{array}$ & $\begin{array}{r}125 \\
39 \\
85 \\
54 \\
155 \\
109 \\
65 \\
91 \\
57 \\
54 \\
36 \\
60 \\
78\end{array}$ & $\begin{array}{r}117 \\
39 \\
83 \\
49 \\
133 \\
102 \\
65 \\
85 \\
38 \\
43 \\
23 \\
60 \\
70\end{array}$ & $\begin{array}{l}0 \\
0 \\
0 \\
0 \\
0 \\
0 \\
0 \\
0 \\
0 \\
0 \\
0 \\
0 \\
0\end{array}$ & $\begin{array}{l}0 \\
0 \\
0 \\
0 \\
0 \\
0 \\
0 \\
0 \\
0 \\
0 \\
0 \\
0 \\
0\end{array}$ \\
\hline 1993 & $\begin{array}{l}\text { January ................. } \\
\text { February ................ } \\
\text { March .................. } \\
\text { 3-Mo. Average ..... }\end{array}$ & $\begin{array}{l}76 \\
14 \\
59 \\
51\end{array}$ & $\begin{array}{l}70 \\
14 \\
59 \\
49\end{array}$ & $\begin{array}{r}90 \\
88 \\
126 \\
102\end{array}$ & $\begin{array}{r}89 \\
88 \\
123 \\
100\end{array}$ & $\begin{array}{l}37 \\
52 \\
67 \\
52\end{array}$ & $\begin{array}{l}37 \\
51 \\
64 \\
51\end{array}$ & $\begin{array}{l}0 \\
0 \\
0 \\
0\end{array}$ & $\begin{array}{l}0 \\
0 \\
0 \\
0\end{array}$ \\
\hline $\begin{array}{r}1992 \\
1991\end{array}$ & $\begin{array}{l}\text { 3-Mo. Average ..... } \\
\text { 3-Mo. Average ..... }\end{array}$ & $\begin{array}{l}28 \\
50\end{array}$ & $\begin{array}{l}24 \\
39\end{array}$ & $\begin{array}{l}73 \\
54\end{array}$ & $\begin{array}{l}73 \\
54\end{array}$ & $\begin{array}{r}84 \\
107\end{array}$ & $\begin{array}{r}81 \\
104\end{array}$ & $\begin{array}{l}0 \\
0\end{array}$ & $\begin{array}{l}0 \\
0\end{array}$ \\
\hline
\end{tabular}

See footnotes at end of tatle. 
Table S3. Crude Oil and Petroleum Product Imports, 1981 - Present (Continued) (Thousand Barrels per Day)

\begin{tabular}{|c|c|c|c|c|c|c|c|c|c|}
\hline & \multirow{3}{*}{ Year/Month } & \multicolumn{6}{|c|}{ Imports from Other-OPEC Sources } & \multirow{2}{*}{\multicolumn{2}{|c|}{$\begin{array}{c}\text { Total } \\
\text { OPEC }\end{array}$}} \\
\hline & & \multicolumn{2}{|c|}{ Nlgerla } & \multicolumn{2}{|c|}{ Venezuala } & \multicolumn{2}{|c|}{$\begin{array}{l}\text { Total } \\
\text { Other } \\
\text { OPEC }\end{array}$} & & \\
\hline & & Total & Crude Oll & Total & Crude Oll & Total & Crude Oll & Total & Crude Oil \\
\hline $\begin{array}{l}1981 \\
1982 \\
1983 \\
1984 \\
1985 \\
1986 \\
1987 \\
1988 \\
1989 \\
1990\end{array}$ & 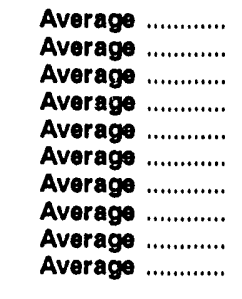 & $\begin{array}{l}620 \\
514 \\
302 \\
216 \\
293 \\
440 \\
535 \\
618 \\
815 \\
800\end{array}$ & $\begin{array}{l}611 \\
510 \\
301 \\
207 \\
280 \\
437 \\
529 \\
607 \\
800 \\
784\end{array}$ & $\begin{array}{r}406 \\
412 \\
422 \\
548 \\
605 \\
793 \\
804 \\
794 \\
873 \\
1,025\end{array}$ & $\begin{array}{l}147 \\
155 \\
164 \\
253 \\
306 \\
416 \\
488 \\
439 \\
495 \\
666\end{array}$ & $\begin{array}{l}1,476 \\
1,291 \\
1,231 \\
1,230 \\
1,358 \\
1,674 \\
1,787 \\
1,681 \\
2,010 \\
2,052\end{array}$ & $\begin{array}{r}1,149 \\
998 \\
944 \\
878 \\
1,012 \\
1,259 \\
1,435 \\
1,281 \\
1,582 \\
1,650\end{array}$ & $\begin{array}{l}3,323 \\
2,146 \\
1,862 \\
2,049 \\
1,830 \\
2,837 \\
3,060 \\
3,520 \\
4,140 \\
4,296\end{array}$ & $\begin{array}{l}2,922 \\
1,734 \\
1,477 \\
1,512 \\
1,312 \\
2,113 \\
2,400 \\
2,696 \\
3,376 \\
3,514\end{array}$ \\
\hline 1991 & 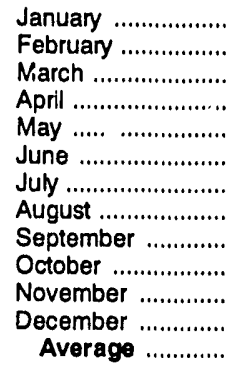 & $\begin{array}{r}504 \\
721 \\
531 \\
677 \\
860 \\
832 \\
833 \\
1,016 \\
489 \\
651 \\
704 \\
617 \\
703\end{array}$ & $\begin{array}{l}481 \\
717 \\
531 \\
649 \\
838 \\
827 \\
817 \\
983 \\
467 \\
623 \\
674 \\
593 \\
683\end{array}$ & $\begin{array}{r}1,005 \\
959 \\
998 \\
845 \\
997 \\
1,135 \\
1,102 \\
1,070 \\
1,163 \\
1,087 \\
1,065 \\
987 \\
1,035\end{array}$ & $\begin{array}{l}673 \\
686 \\
631 \\
470 \\
581 \\
705 \\
683 \\
701 \\
790 \\
777 \\
671 \\
655 \\
668\end{array}$ & $\begin{array}{l}1,637 \\
2,003 \\
1,718 \\
1,698 \\
2,158 \\
2,354 \\
2,304 \\
2,394 \\
2,009 \\
2,067 \\
2,099 \\
1,899 \\
2,028\end{array}$ & $\begin{array}{l}1,271 \\
1,705 \\
1,342 \\
1,283 \\
1,715 \\
1,915 \\
1,855 \\
1,966 \\
1,589 \\
1,694 \\
1,644 \\
1,496 \\
1,622\end{array}$ & $\begin{array}{l}3,898 \\
3,815 \\
3,623 \\
3,744 \\
4,724 \\
4,498 \\
4,232 \\
4,602 \\
3,956 \\
4,023 \\
4,171 \\
3,791 \\
4,092\end{array}$ & $\begin{array}{l}3,101 \\
3,264 \\
3,033 \\
3,059 \\
3,839 \\
3,747 \\
3,525 \\
3,946 \\
3,204 \\
3,343 \\
3,328 \\
3,116 \\
3,377\end{array}$ \\
\hline 1992 & 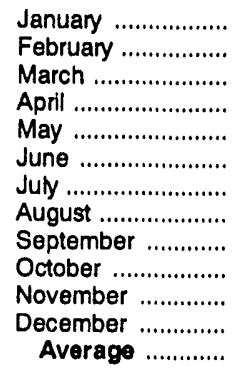 & $\begin{array}{l}593 \\
322 \\
441 \\
798 \\
773 \\
740 \\
900 \\
815 \\
774 \\
827 \\
626 \\
549 \\
681\end{array}$ & $\begin{array}{l}566 \\
303 \\
409 \\
788 \\
773 \\
740 \\
883 \\
795 \\
754 \\
813 \\
608 \\
532 \\
665\end{array}$ & $\begin{array}{l}1,105 \\
1,008 \\
1,098 \\
1,058 \\
1,031 \\
1,007 \\
1,163 \\
1,102 \\
1,341 \\
1,513 \\
1,344 \\
1,164 \\
1,162\end{array}$ & $\begin{array}{r}787 \\
655 \\
793 \\
710 \\
745 \\
694 \\
912 \\
841 \\
953 \\
1,073 \\
921 \\
763 \\
822\end{array}$ & $\begin{array}{l}1,935 \\
1,511 \\
1,676 \\
2,148 \\
2,145 \\
2,089 \\
2,381 \\
2,214 \\
2,434 \\
2,602 \\
2,174 \\
1,917 \\
2,104\end{array}$ & $\begin{array}{l}1,583 \\
1,126 \\
1,336 \\
1,779 \\
1,837 \\
1,765 \\
2,114 \\
1,922 \\
2,001 \\
2,133 \\
1,719 \\
1,499 \\
1,737\end{array}$ & $\begin{array}{l}4,141 \\
3,506 \\
3,598 \\
4,064 \\
4,111 \\
3,978 \\
4,339 \\
4,143 \\
4,281 \\
4,522 \\
4,087 \\
4,105 \\
4,076\end{array}$ & $\begin{array}{l}3,485 \\
2,871 \\
2,941 \\
3,322 \\
3,428 \\
3,387 \\
3,772 \\
3,473 \\
3,531 \\
3,732 \\
3,376 \\
3,381 \\
3,394\end{array}$ \\
\hline 1993 & $\begin{array}{l}\text { January ................ } \\
\text { February .................. } \\
\text { March .................. } \\
\text { 3-Mo. Average ...... }\end{array}$ & $\begin{array}{l}729 \\
927 \\
928 \\
859\end{array}$ & $\begin{array}{l}729 \\
913 \\
892 \\
842\end{array}$ & $\begin{array}{l}1,385 \\
1,290 \\
1,208 \\
1,294\end{array}$ & $\begin{array}{r}1,038 \\
925 \\
817 \\
927\end{array}$ & $\begin{array}{l}2,317 \\
2,372 \\
2,388 \\
2,359\end{array}$ & $\begin{array}{l}1,962 \\
1,990 \\
1,955 \\
1,968\end{array}$ & $\begin{array}{l}4,301 \\
4,505 \\
4,375 \\
4,390\end{array}$ & $\begin{array}{l}3,690 \\
3,699 \\
3,611 \\
3,666\end{array}$ \\
\hline $\begin{array}{l}1992 \\
1991\end{array}$ & $\begin{array}{l}\text { 3-Mo. Average ...... } \\
\text { 3-Mo. Average ...... }\end{array}$ & $\begin{array}{l}455 \\
581\end{array}$ & $\begin{array}{l}429 \\
571\end{array}$ & $\begin{array}{r}1,072 \\
988\end{array}$ & $\begin{array}{l}747 \\
662\end{array}$ & $\begin{array}{l}1,712 \\
1,779\end{array}$ & $\begin{array}{l}1,353 \\
1,431\end{array}$ & $\begin{array}{l}3,754 \\
3,778\end{array}$ & $\begin{array}{l}3,104 \\
3,129\end{array}$ \\
\hline
\end{tabular}

See footnotes at end of table. 
Table S3. Crude OIl and Petroleum Product Imports, 1981 - Present (Continued) (Thousand Barrels per Day)

\begin{tabular}{|c|c|c|c|c|c|c|c|c|c|c|c|c|c|}
\hline & \multirow{3}{*}{ Year/Month } & \multicolumn{12}{|c|}{ Imports from Non-OPEC Sources" } \\
\hline & & \multicolumn{2}{|c|}{ Angola } & \multicolumn{2}{|c|}{ Augtralla. } & \multicolumn{2}{|c|}{$\begin{array}{l}\text { Bahama } \\
\text { Islande }\end{array}$} & \multicolumn{2}{|c|}{ Arazll } & \multicolumn{2}{|c|}{ Canada } & \multicolumn{2}{|c|}{$\begin{array}{l}\text { China } \\
\text { People's } \\
\text { Republle }\end{array}$} \\
\hline & & Total & Crude Oll & Total & Crude Oll & Total & Crude OlI & Total & Crude Oll & Total & Crude OII & Total & Crude Oll \\
\hline $\begin{array}{l}1981 \\
1982 \\
1983 \\
1984 \\
1985 \\
1986 \\
1987 \\
1988 \\
1989 \\
1990\end{array}$ & 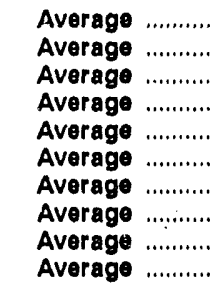 & $\begin{array}{r}49 \\
44 \\
78 \\
90 \\
110 \\
112 \\
192 \\
212 \\
284 \\
237\end{array}$ & $\begin{array}{r}45 \\
42 \\
71 \\
85 \\
104 \\
102 \\
180 \\
203 \\
279 \\
236\end{array}$ & $\begin{array}{r}5 \\
5 \\
4 \\
38 \\
37 \\
41 \\
58 \\
64 \\
36 \\
53\end{array}$ & $\begin{array}{r}0 \\
(8) \\
0 \\
25 \\
21 \\
30 \\
49 \\
59 \\
31 \\
47\end{array}$ & $\begin{array}{r}74 \\
65 \\
125 \\
88 \\
40 \\
37 \\
37 \\
32 \\
34 \\
37\end{array}$ & $\begin{array}{l}0 \\
0 \\
0 \\
0 \\
0 \\
0 \\
0 \\
0 \\
0 \\
0\end{array}$ & $\begin{array}{l}23 \\
47 \\
41 \\
60 \\
61 \\
50 \\
84 \\
98 \\
82 \\
49\end{array}$ & $\begin{array}{r}14 \\
19 \\
2 \\
(8) \\
0 \\
0 \\
0 \\
0 \\
0 \\
0\end{array}$ & $\begin{array}{l}447 \\
482 \\
547 \\
630 \\
770 \\
807 \\
848 \\
989 \\
931 \\
934\end{array}$ & $\begin{array}{l}164 \\
214 \\
274 \\
341 \\
468 \\
570 \\
608 \\
681 \\
630 \\
643\end{array}$ & $\begin{array}{l}18 \\
40 \\
34 \\
46 \\
59 \\
90 \\
82 \\
88 \\
80 \\
80\end{array}$ & $\begin{array}{r}0 \\
8 \\
6 \\
15 \\
36 \\
68 \\
63 \\
82 \\
76 \\
77\end{array}$ \\
\hline 1991 & 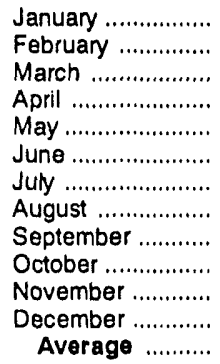 & $\begin{array}{l}232 \\
202 \\
186 \\
337 \\
220 \\
205 \\
264 \\
298 \\
230 \\
300 \\
213 \\
359 \\
254\end{array}$ & $\begin{array}{l}232 \\
202 \\
186 \\
337 \\
220 \\
205 \\
264 \\
298 \\
230 \\
300 \\
213 \\
359 \\
254\end{array}$ & $\begin{array}{r}21 \\
0 \\
0 \\
55 \\
64 \\
43 \\
20 \\
37 \\
24 \\
13 \\
25 \\
13 \\
26\end{array}$ & $\begin{array}{r}21 \\
0 \\
0 \\
55 \\
57 \\
31 \\
20 \\
22 \\
24 \\
0 \\
13 \\
13 \\
21\end{array}$ & $\begin{array}{r}25 \\
14 \\
0 \\
35 \\
42 \\
30 \\
19 \\
78 \\
29 \\
51 \\
46 \\
53 \\
35\end{array}$ & $\begin{array}{l}0 \\
0 \\
0 \\
0 \\
0 \\
0 \\
0 \\
0 \\
0 \\
0 \\
0 \\
0 \\
0\end{array}$ & $\begin{array}{r}31 \\
13 \\
0 \\
17 \\
31 \\
41 \\
21 \\
27 \\
19 \\
16 \\
45 \\
8 \\
22\end{array}$ & $\begin{array}{l}0 \\
0 \\
0 \\
0 \\
0 \\
0 \\
0 \\
0 \\
0 \\
0 \\
0 \\
0 \\
0\end{array}$ & $\begin{array}{r}978 \\
1,135 \\
1,058 \\
1,103 \\
1,027 \\
986 \\
848 \\
1,011 \\
1,137 \\
936 \\
1,107 \\
1,083 \\
1,033\end{array}$ & $\begin{array}{l}718 \\
881 \\
764 \\
768 \\
752 \\
705 \\
615 \\
694 \\
849 \\
639 \\
796 \\
759 \\
743\end{array}$ & $\begin{array}{r}68 \\
102 \\
96 \\
113 \\
119 \\
144 \\
88 \\
85 \\
91 \\
29 \\
96 \\
65 \\
91\end{array}$ & $\begin{array}{r}63 \\
96 \\
96 \\
113 \\
113 \\
139 \\
88 \\
75 \\
86 \\
24 \\
96 \\
65 \\
87\end{array}$ \\
\hline 1992 & 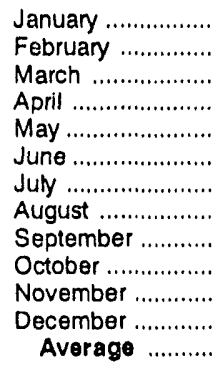 & $\begin{array}{l}360 \\
246 \\
339 \\
381 \\
264 \\
286 \\
443 \\
335 \\
248 \\
395 \\
458 \\
279 \\
336\end{array}$ & $\begin{array}{l}360 \\
246 \\
339 \\
381 \\
264 \\
286 \\
443 \\
323 \\
248 \\
395 \\
458 \\
279 \\
336\end{array}$ & $\begin{array}{r}11 \\
10 \\
0 \\
39 \\
0 \\
21 \\
20 \\
21 \\
0 \\
11 \\
53 \\
27 \\
18\end{array}$ & $\begin{array}{r}11 \\
10 \\
0 \\
22 \\
0 \\
21 \\
20 \\
21 \\
0 \\
11 \\
49 \\
27 \\
16\end{array}$ & $\begin{array}{r}63 \\
47 \\
76 \\
67 \\
46 \\
57 \\
22 \\
8 \\
8 \\
1 \\
20 \\
19 \\
36\end{array}$ & $\begin{array}{l}0 \\
0 \\
0 \\
0 \\
0 \\
0 \\
0 \\
0 \\
0 \\
0 \\
0 \\
0 \\
0\end{array}$ & $\begin{array}{r}18 \\
12 \\
0 \\
17 \\
18 \\
28 \\
25 \\
10 \\
21 \\
10 \\
32 \\
50 \\
20\end{array}$ & $\begin{array}{l}0 \\
0 \\
0 \\
0 \\
0 \\
0 \\
0 \\
0 \\
0 \\
0 \\
0 \\
0 \\
0\end{array}$ & $\begin{array}{r}1,023 \\
1,143 \\
1,094 \\
1,111 \\
972 \\
868 \\
1,036 \\
1,030 \\
1,121 \\
1,054 \\
1,032 \\
1,114 \\
1,049\end{array}$ & $\begin{array}{l}783 \\
831 \\
829 \\
833 \\
756 \\
645 \\
798 \\
762 \\
839 \\
761 \\
784 \\
816 \\
787\end{array}$ & $\begin{array}{r}144 \\
75 \\
75 \\
86 \\
124 \\
106 \\
68 \\
66 \\
80 \\
61 \\
86 \\
97 \\
89\end{array}$ & $\begin{array}{r}144 \\
69 \\
75 \\
69 \\
114 \\
95 \\
64 \\
66 \\
75 \\
61 \\
86 \\
90 \\
84\end{array}$ \\
\hline 1993 & $\begin{array}{l}\text { January ................ } \\
\text { February ................ } \\
\text { March ................ } \\
\text { 3-Mo. Average .... }\end{array}$ & $\begin{array}{l}354 \\
348 \\
408 \\
371\end{array}$ & $\begin{array}{l}354 \\
348 \\
408 \\
371\end{array}$ & $\begin{array}{l}0 \\
0 \\
0 \\
0\end{array}$ & $\begin{array}{l}0 \\
0 \\
0 \\
0\end{array}$ & $\begin{array}{l}18 \\
19 \\
30 \\
23\end{array}$ & $\begin{array}{l}0 \\
0 \\
0 \\
0\end{array}$ & $\begin{array}{r}3 \\
22 \\
27 \\
17\end{array}$ & $\begin{array}{l}0 \\
0 \\
0 \\
0\end{array}$ & $\begin{array}{l}1,034 \\
1,084 \\
1,065 \\
1,060\end{array}$ & $\begin{array}{l}778 \\
782 \\
814 \\
792\end{array}$ & $\begin{array}{l}60 \\
44 \\
79 \\
62\end{array}$ & $\begin{array}{l}60 \\
44 \\
73 \\
60\end{array}$ \\
\hline $\begin{array}{l}1992 \\
1991\end{array}$ & $\begin{array}{l}\text { 3-Mo. Average .... } \\
\text { 3-Mo. Average ... }\end{array}$ & $\begin{array}{l}316 \\
207\end{array}$ & $\begin{array}{l}316 \\
207\end{array}$ & $\begin{array}{l}7 \\
7\end{array}$ & $\begin{array}{l}7 \\
7\end{array}$ & $\begin{array}{l}63 \\
13\end{array}$ & $\begin{array}{l}0 \\
0\end{array}$ & $\begin{array}{l}10 \\
15\end{array}$ & $\begin{array}{l}0 \\
0\end{array}$ & $\begin{array}{l}1,085 \\
1,054\end{array}$ & $\begin{array}{l}814 \\
784\end{array}$ & $\begin{array}{l}98 \\
88\end{array}$ & $\begin{array}{l}97 \\
84\end{array}$ \\
\hline
\end{tabular}

See footnotes at end of table. 
Table S3. Crude OII and Petroleum Product Imports, 1981 - Present (Continued) (Thousand Barrels per Day)

\begin{tabular}{|c|c|c|c|c|c|c|c|c|c|c|c|}
\hline & \multirow{3}{*}{ Yoar/Month } & \multicolumn{10}{|c|}{ Imports from Non-OPEC Sources" } \\
\hline & & \multicolumn{2}{|c|}{ Colombla } & \multicolumn{2}{|c|}{ Italy } & \multicolumn{2}{|c|}{ Malayala } & \multicolumn{2}{|c|}{ Mexlco } & \multicolumn{2}{|c|}{ Notherlands } \\
\hline & & Total & Crude Oll & Total & Crude Oll & Total & Crude oll & Total & Crude Oll & Total & Crude Oll \\
\hline $\begin{array}{l}1981 \\
1982 \\
1983 \\
1984 \\
1985 \\
1986 \\
1987 \\
1988 \\
1989 \\
1990\end{array}$ & 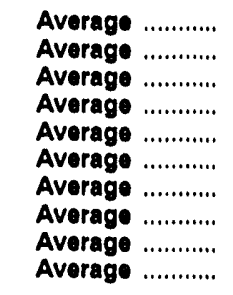 & $\begin{array}{r}1 \\
5 \\
10 \\
8 \\
23 \\
87 \\
148 \\
134 \\
172 \\
182\end{array}$ & $\begin{array}{r}0 \\
0 \\
0 \\
0 \\
0 \\
57 \\
115 \\
106 \\
136 \\
140\end{array}$ & $\begin{array}{l}11 \\
18 \\
18 \\
45 \\
60 \\
76 \\
54 \\
65 \\
34 \\
58\end{array}$ & $\begin{array}{l}0 \\
(8) \\
(8) \\
(8) \\
(8) \\
0 \\
1 \\
5 \\
3 \\
2\end{array}$ & $\begin{array}{r}36 \\
20 \\
4 \\
1 \\
3 \\
12 \\
13 \\
19 \\
39 \\
41\end{array}$ & $\begin{array}{r}33 \\
18 \\
3 \\
0 \\
1 \\
11 \\
12 \\
19 \\
39 \\
40\end{array}$ & $\begin{array}{l}522 \\
685 \\
826 \\
748 \\
816 \\
699 \\
655 \\
747 \\
767 \\
755\end{array}$ & $\begin{array}{l}469 \\
645 \\
766 \\
659 \\
715 \\
621 \\
602 \\
674 \\
716 \\
689\end{array}$ & $\begin{array}{l}30 \\
35 \\
65 \\
65 \\
58 \\
54 \\
60 \\
61 \\
49 \\
55\end{array}$ & $\begin{array}{l}\text { (8) } \\
(8) \\
3 \\
3 \\
0 \\
0 \\
0 \\
0 \\
0 \\
0\end{array}$ \\
\hline 1991 & 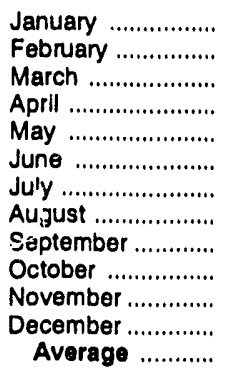 & $\begin{array}{l}194 \\
151 \\
157 \\
163 \\
163 \\
169 \\
163 \\
219 \\
168 \\
128 \\
145 \\
138 \\
163\end{array}$ & $\begin{array}{r}174 \\
98 \\
127 \\
131 \\
112 \\
124 \\
111 \\
162 \\
103 \\
80 \\
135 \\
117 \\
123\end{array}$ & $\begin{array}{l}25 \\
42 \\
29 \\
41 \\
60 \\
46 \\
54 \\
57 \\
89 \\
41 \\
15 \\
61 \\
47\end{array}$ & $\begin{array}{r}0 \\
13 \\
0 \\
12 \\
0 \\
0 \\
0 \\
11 \\
0 \\
0 \\
0 \\
0 \\
3\end{array}$ & $\begin{array}{r}0 \\
9 \\
21 \\
0 \\
66 \\
63 \\
9 \\
14 \\
10 \\
64 \\
10 \\
14 \\
24\end{array}$ & $\begin{array}{r}0 \\
9 \\
21 \\
0 \\
66 \\
63 \\
9 \\
14 \\
10 \\
64 \\
10 \\
14 \\
24\end{array}$ & $\begin{array}{l}798 \\
742 \\
795 \\
891 \\
757 \\
919 \\
835 \\
878 \\
805 \\
811 \\
716 \\
732 \\
807\end{array}$ & $\begin{array}{l}778 \\
693 \\
772 \\
819 \\
736 \\
872 \\
748 \\
797 \\
768 \\
754 \\
656 \\
708 \\
759\end{array}$ & $\begin{array}{r}6 \\
17 \\
33 \\
35 \\
45 \\
49 \\
47 \\
30 \\
44 \\
16 \\
24 \\
4 \\
29\end{array}$ & $\begin{array}{l}0 \\
0 \\
0 \\
0 \\
0 \\
0 \\
0 \\
0 \\
0 \\
0 \\
0 \\
0 \\
0\end{array}$ \\
\hline 1992 & 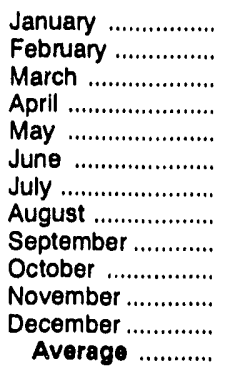 & $\begin{array}{r}158 \\
114 \\
101 \\
150 \\
57 \\
135 \\
103 \\
156 \\
177 \\
153 \\
129 \\
66 \\
125\end{array}$ & $\begin{array}{r}111 \\
92 \\
74 \\
129 \\
46 \\
114 \\
93 \\
142 \\
167 \\
132 \\
84 \\
34 \\
101\end{array}$ & $\begin{array}{l}40 \\
48 \\
44 \\
75 \\
57 \\
68 \\
36 \\
94 \\
81 \\
37 \\
33 \\
37 \\
54\end{array}$ & $\begin{array}{l}0 \\
0 \\
0 \\
0 \\
0 \\
0 \\
0 \\
0 \\
0 \\
0 \\
0 \\
0 \\
0\end{array}$ & $\begin{array}{r}0 \\
0 \\
0 \\
0 \\
5 \\
8 \\
40 \\
22 \\
17 \\
17 \\
8 \\
4 \\
10\end{array}$ & $\begin{array}{r}0 \\
0 \\
0 \\
0 \\
5 \\
8 \\
40 \\
22 \\
17 \\
17 \\
8 \\
4 \\
10\end{array}$ & $\begin{array}{l}764 \\
810 \\
846 \\
857 \\
788 \\
887 \\
830 \\
857 \\
755 \\
829 \\
762 \\
930 \\
827\end{array}$ & $\begin{array}{l}721 \\
788 \\
809 \\
795 \\
764 \\
865 \\
788 \\
790 \\
720 \\
783 \\
700 \\
888 \\
785\end{array}$ & $\begin{array}{r}31 \\
9 \\
34 \\
8 \\
27 \\
25 \\
21 \\
44 \\
38 \\
18 \\
26 \\
33 \\
26\end{array}$ & $\begin{array}{l}0 \\
0 \\
0 \\
0 \\
0 \\
0 \\
0 \\
0 \\
0 \\
0 \\
0 \\
0 \\
0\end{array}$ \\
\hline 1993 & $\begin{array}{l}\text { January ............... } \\
\text { February ................ } \\
\text { March .................. } \\
\text { 3-Mo. Average ..... }\end{array}$ & $\begin{array}{l}188 \\
148 \\
161 \\
166\end{array}$ & $\begin{array}{l}167 \\
137 \\
129 \\
145\end{array}$ & $\begin{array}{l}48 \\
34 \\
43 \\
42\end{array}$ & $\begin{array}{l}0 \\
0 \\
0 \\
0\end{array}$ & $\begin{array}{r}0 \\
0 \\
11 \\
4\end{array}$ & $\begin{array}{r}0 \\
0 \\
10 \\
4\end{array}$ & $\begin{array}{l}858 \\
807 \\
861 \\
943\end{array}$ & $\begin{array}{l}820 \\
748 \\
815 \\
796\end{array}$ & $\begin{array}{l}11 \\
18 \\
11 \\
13\end{array}$ & $\begin{array}{l}0 \\
0 \\
0 \\
0\end{array}$ \\
\hline $\begin{array}{l}1992 \\
1991\end{array}$ & $\begin{array}{l}\text { 3-Mo. Average ..... } \\
\text { 3-Mo. Average ..... }\end{array}$ & $\begin{array}{l}124 \\
168\end{array}$ & $\begin{array}{r}92 \\
134\end{array}$ & $\begin{array}{l}44 \\
31\end{array}$ & $\begin{array}{l}0 \\
4\end{array}$ & $\begin{array}{r}0 \\
10\end{array}$ & $\begin{array}{r}0 \\
10\end{array}$ & $\begin{array}{l}808 \\
780\end{array}$ & $\begin{array}{l}773 \\
749\end{array}$ & $\begin{array}{l}25 \\
19\end{array}$ & $\begin{array}{l}0 \\
0\end{array}$ \\
\hline
\end{tabular}


Table S3. Crude Oil and Petroleum Product Imports, 1981 - Present (Continued) (Thousand Barrels per Day)

\begin{tabular}{|c|c|c|c|c|c|c|c|c|c|c|c|c|c|}
\hline & \multirow{3}{*}{ Yoar/Month } & \multicolumn{12}{|c|}{ Imports from Non-OPEC Sources" } \\
\hline & & \multicolumn{2}{|c|}{$\begin{array}{c}\text { Netherlands } \\
\text { Antlles }\end{array}$} & \multicolumn{2}{|c|}{ Norway } & \multicolumn{2}{|c|}{$\begin{array}{l}\text { Puerto } \\
\text { Bice }\end{array}$} & \multicolumn{2}{|c|}{ Spaln } & \multicolumn{2}{|c|}{$\begin{array}{c}\text { Trinldad } \\
\text { and } \\
\text { Topage }\end{array}$} & \multicolumn{2}{|c|}{$\begin{array}{l}\text { United } \\
\text { Klngdom }\end{array}$} \\
\hline & & Total & Crude OII & Total & Crude OII & Total & Crude OII & Total & Crude OII & Total & Crude OII & Total & Crude Oll \\
\hline $\begin{array}{l}1981 \\
1982 \\
1983 \\
1984 \\
1985 \\
1986 \\
1987 \\
1988 \\
1989 \\
1990\end{array}$ & $\begin{array}{l}\text { Average ........... } \\
\text { Average ........... } \\
\text { Average ........... } \\
\text { Average ........... } \\
\text { Average .......... } \\
\text { Average ........... } \\
\text { Average ........... } \\
\text { Average .......... } \\
\text { Average ........... } \\
\text { Average ........... }\end{array}$ & $\begin{array}{r}197 \\
175 \\
189 \\
188 \\
40 \\
25 \\
29 \\
36 \\
42 \\
31\end{array}$ & $\begin{array}{l}0 \\
0 \\
0 \\
0 \\
0 \\
0 \\
0 \\
0 \\
0 \\
0\end{array}$ & $\begin{array}{r}119 \\
102 \\
66 \\
114 \\
32 \\
60 \\
80 \\
67 \\
138 \\
102\end{array}$ & $\begin{array}{r}114 \\
102 \\
65 \\
112 \\
31 \\
53 \\
70 \\
62 \\
127 \\
96\end{array}$ & $\begin{array}{l}62 \\
50 \\
40 \\
42 \\
28 \\
21 \\
21 \\
22 \\
32 \\
32\end{array}$ & $\begin{array}{l}0 \\
0 \\
0 \\
0 \\
0 \\
0 \\
0 \\
0 \\
0 \\
0\end{array}$ & $\begin{array}{r}1 \\
3 \\
2 \\
11 \\
29 \\
53 \\
55 \\
68 \\
67 \\
47\end{array}$ & $\begin{array}{l}(8) \\
(8) \\
(8) \\
0 \\
1 \\
0 \\
0 \\
0 \\
0 \\
0\end{array}$ & $\begin{array}{r}133 \\
112 \\
96 \\
94 \\
113 \\
125 \\
106 \\
97 \\
94 \\
96\end{array}$ & $\begin{array}{r}102 \\
92 \\
83 \\
87 \\
98 \\
93 \\
75 \\
71 \\
73 \\
76\end{array}$ & $\begin{array}{l}375 \\
456 \\
382 \\
402 \\
310 \\
350 \\
352 \\
315 \\
215 \\
189\end{array}$ & $\begin{array}{l}369 \\
441 \\
365 \\
378 \\
278 \\
317 \\
304 \\
254 \\
160 \\
155\end{array}$ \\
\hline 1991 & 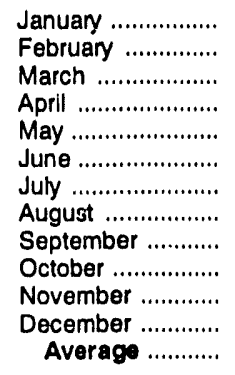 & $\begin{array}{r}103 \\
23 \\
56 \\
61 \\
113 \\
84 \\
86 \\
100 \\
67 \\
90 \\
100 \\
88 \\
81\end{array}$ & $\begin{array}{l}0 \\
0 \\
0 \\
0 \\
0 \\
0 \\
0 \\
0 \\
0 \\
0 \\
0 \\
0 \\
0\end{array}$ & $\begin{array}{r}45 \\
37 \\
25 \\
51 \\
165 \\
99 \\
69 \\
142 \\
79 \\
98 \\
73 \\
94 \\
82\end{array}$ & $\begin{array}{r}34 \\
37 \\
16 \\
35 \\
156 \\
84 \\
63 \\
136 \\
72 \\
98 \\
65 \\
88 \\
74\end{array}$ & $\begin{array}{l}22 \\
20 \\
14 \\
23 \\
42 \\
19 \\
25 \\
42 \\
34 \\
12 \\
35 \\
36 \\
27\end{array}$ & $\begin{array}{l}0 \\
0 \\
0 \\
0 \\
0 \\
0 \\
0 \\
0 \\
0 \\
0 \\
0 \\
0 \\
0\end{array}$ & $\begin{array}{l}26 \\
18 \\
13 \\
66 \\
53 \\
41 \\
22 \\
48 \\
42 \\
24 \\
19 \\
26 \\
33\end{array}$ & $\begin{array}{l}0 \\
0 \\
0 \\
0 \\
0 \\
0 \\
0 \\
0 \\
0 \\
0 \\
0 \\
0 \\
0\end{array}$ & $\begin{array}{r}75 \\
76 \\
86 \\
84 \\
61 \\
118 \\
91 \\
91 \\
119 \\
88 \\
77 \\
87 \\
88\end{array}$ & $\begin{array}{r}64 \\
76 \\
73 \\
64 \\
61 \\
104 \\
72 \\
66 \\
75 \\
76 \\
69 \\
71 \\
72\end{array}$ & $\begin{array}{r}32 \\
34 \\
48 \\
61 \\
222 \\
105 \\
228 \\
254 \\
218 \\
201 \\
84 \\
154 \\
138\end{array}$ & $\begin{array}{r}19 \\
21 \\
19 \\
37 \\
188 \\
70 \\
164 \\
217 \\
194 \\
166 \\
18 \\
151 \\
106\end{array}$ \\
\hline 1992 & 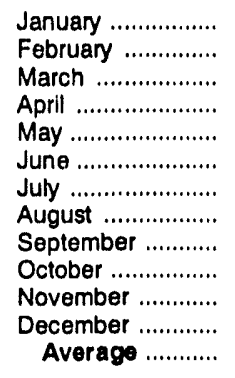 & $\begin{array}{l}40 \\
82 \\
49 \\
73 \\
59 \\
91 \\
49 \\
65 \\
60 \\
90 \\
56 \\
80 \\
66\end{array}$ & $\begin{array}{l}0 \\
0 \\
0 \\
0 \\
0 \\
0 \\
0 \\
0 \\
0 \\
0 \\
0 \\
0 \\
0\end{array}$ & $\begin{array}{r}25 \\
11 \\
11 \\
162 \\
209 \\
234 \\
194 \\
151 \\
112 \\
198 \\
120 \\
148 \\
131\end{array}$ & $\begin{array}{r}17 \\
0 \\
0 \\
147 \\
200 \\
225 \\
179 \\
134 \\
102 \\
177 \\
104 \\
133 \\
119\end{array}$ & $\begin{array}{l}32 \\
23 \\
18 \\
14 \\
22 \\
28 \\
11 \\
38 \\
37 \\
29 \\
26 \\
28 \\
26\end{array}$ & $\begin{array}{l}0 \\
0 \\
0 \\
0 \\
0 \\
0 \\
0 \\
0 \\
0 \\
0 \\
0 \\
0 \\
0\end{array}$ & $\begin{array}{l}35 \\
16 \\
37 \\
35 \\
30 \\
45 \\
18 \\
29 \\
56 \\
32 \\
36 \\
17 \\
32\end{array}$ & $\begin{array}{l}0 \\
0 \\
0 \\
0 \\
0 \\
0 \\
0 \\
0 \\
0 \\
0 \\
0 \\
0 \\
0\end{array}$ & $\begin{array}{r}108 \\
109 \\
105 \\
79 \\
69 \\
94 \\
103 \\
106 \\
84 \\
108 \\
85 \\
91 \\
85\end{array}$ & $\begin{array}{l}79 \\
76 \\
85 \\
75 \\
54 \\
74 \\
78 \\
54 \\
56 \\
71 \\
62 \\
71 \\
70\end{array}$ & $\begin{array}{r}128 \\
63 \\
79 \\
157 \\
198 \\
248 \\
353 \\
295 \\
341 \\
411 \\
336 \\
148 \\
230\end{array}$ & $\begin{array}{r}115 \\
0 \\
52 \\
128 \\
180 \\
206 \\
337 \\
282 \\
291 \\
411 \\
285 \\
110 \\
200\end{array}$ \\
\hline 1993 & $\begin{array}{l}\text { January ................. } \\
\text { February ............... } \\
\text { March ................. } \\
\text { 3-Mo. Average .... }\end{array}$ & $\begin{array}{l}73 \\
80 \\
61 \\
71\end{array}$ & $\begin{array}{l}0 \\
0 \\
0 \\
0\end{array}$ & $\begin{array}{r}70 \\
62 \\
122 \\
85\end{array}$ & $\begin{array}{r}70 \\
61 \\
115 \\
83\end{array}$ & $\begin{array}{l}37 \\
21 \\
26 \\
28\end{array}$ & $\begin{array}{l}0 \\
0 \\
0 \\
0\end{array}$ & $\begin{array}{l}44 \\
25 \\
21 \\
30\end{array}$ & $\begin{array}{l}0 \\
0 \\
0 \\
0\end{array}$ & $\begin{array}{l}59 \\
72 \\
92 \\
75\end{array}$ & $\begin{array}{l}48 \\
58 \\
71 \\
59\end{array}$ & $\begin{array}{l}228 \\
173 \\
315 \\
241\end{array}$ & $\begin{array}{l}201 \\
127 \\
281 \\
205\end{array}$ \\
\hline $\begin{array}{l}1992 \\
1991\end{array}$ & $\begin{array}{l}\text { 3-Mo. Average .... } \\
\text { 3-Mo. Average ... }\end{array}$ & $\begin{array}{l}56 \\
62\end{array}$ & $\begin{array}{l}0 \\
0\end{array}$ & $\begin{array}{l}16 \\
36\end{array}$ & $\begin{array}{r}6 \\
29\end{array}$ & $\begin{array}{l}24 \\
19\end{array}$ & $\begin{array}{l}0 \\
0\end{array}$ & $\begin{array}{l}30 \\
19\end{array}$ & $\begin{array}{l}\mathbf{0} \\
\mathbf{0}\end{array}$ & $\begin{array}{r}108 \\
79\end{array}$ & $\begin{array}{l}80 \\
71\end{array}$ & $\begin{array}{l}91 \\
38\end{array}$ & $\begin{array}{l}57 \\
20\end{array}$ \\
\hline
\end{tabular}

See footnotes at end of table. 
Table S3. Crude Oll and Petroleum Product Imports, 1981 - Present (Continued) (Thousand Barrels per Day)

\begin{tabular}{|c|c|c|c|c|c|c|c|c|c|c|c|}
\hline & \multirow{3}{*}{ Year/Month } & \multicolumn{8}{|c|}{ Imports from Non-OPEC Sources } & \multirow{2}{*}{\multicolumn{2}{|c|}{$\begin{array}{l}\text { Total } \\
\text { Imports }\end{array}$}} \\
\hline & & \multicolumn{2}{|c|}{ Augalad } & \multicolumn{2}{|c|}{$\begin{array}{l}\text { Virgin } \\
\text { lalands }\end{array}$} & \multicolumn{2}{|c|}{$\begin{array}{l}\text { Other } \\
\text { Non- } \\
\text { OPEC }\end{array}$} & \multicolumn{2}{|c|}{$\begin{array}{l}\text { Total } \\
\text { Non- } \\
\text { OPEC }\end{array}$} & & \\
\hline & & Total & Crude Oll & Total & Crude Oll & Total & Crude Oll & Total & Crude Oll & Total & Crude Oll \\
\hline $\begin{array}{l}1981 \\
1982 \\
1983 \\
1984 \\
1985 \\
1988 \\
1987 \\
1988 \\
1989 \\
1980\end{array}$ & 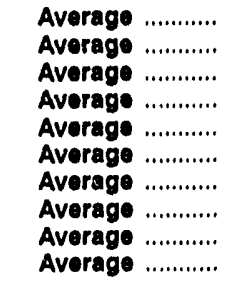 & $\begin{array}{r}5 \\
1 \\
1 \\
13 \\
8 \\
18 \\
11 \\
29 \\
48 \\
45\end{array}$ & $\begin{array}{l}(8) \\
0 \\
(8) \\
(8) \\
(8) \\
(8) \\
0 \\
0 \\
0 \\
1\end{array}$ & $\begin{array}{l}327 \\
316 \\
282 \\
294 \\
247 \\
244 \\
272 \\
242 \\
321 \\
282\end{array}$ & $\begin{array}{l}0 \\
0 \\
0 \\
0 \\
0 \\
0 \\
0 \\
0 \\
0 \\
0\end{array}$ & $\begin{array}{l}236 \\
306 \\
378 \\
411 \\
394 \\
426 \\
459 \\
487 \\
457 \\
417\end{array}$ & $\begin{array}{l}163 \\
174 \\
215 \\
210 \\
137 \\
144 \\
196 \\
186 \\
197 \\
180\end{array}$ & $\begin{array}{l}2,672 \\
2,968 \\
3,189 \\
3,388 \\
3,237 \\
3,387 \\
3,617 \\
3,882 \\
3,921 \\
3,721\end{array}$ & $\begin{array}{l}1,474 \\
1,754 \\
1,853 \\
1,914 \\
1,888 \\
2,065 \\
2,274 \\
2,411 \\
2,467 \\
2,381\end{array}$ & $\begin{array}{l}5,996 \\
5,113 \\
5,051 \\
5,437 \\
5,067 \\
6,224 \\
6,678 \\
7,402 \\
8,061 \\
8,018\end{array}$ & $\begin{array}{l}4,396 \\
3,488 \\
3,329 \\
3,426 \\
3,201 \\
4,178 \\
4,674 \\
5,107 \\
5,843 \\
5,894\end{array}$ \\
\hline 1991 & 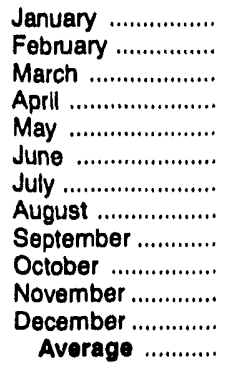 & $\begin{array}{r}28 \\
17 \\
13 \\
39 \\
42 \\
0 \\
58 \\
80 \\
23 \\
13 \\
16 \\
16 \\
29\end{array}$ & $\begin{array}{r}0 \\
0 \\
0 \\
0 \\
0 \\
0 \\
0 \\
11 \\
0 \\
0 \\
0 \\
0 \\
1\end{array}$ & $\begin{array}{l}261 \\
222 \\
214 \\
245 \\
264 \\
234 \\
191 \\
208 \\
269 \\
262 \\
264 \\
286 \\
243\end{array}$ & $\begin{array}{l}0 \\
0 \\
0 \\
0 \\
0 \\
0 \\
0 \\
0 \\
0 \\
0 \\
0 \\
0 \\
0\end{array}$ & $\begin{array}{l}235 \\
180 \\
179 \\
256 \\
239 \\
349 \\
384 \\
369 \\
374 \\
252 \\
335 \\
229 \\
282\end{array}$ & $\begin{array}{r}91 \\
96 \\
60 \\
99 \\
63 \\
189 \\
275 \\
197 \\
197 \\
139 \\
130 \\
104 \\
137\end{array}$ & $\begin{array}{l}3,205 \\
3,051 \\
3,023 \\
3,674 \\
3,794 \\
3,747 \\
3,524 \\
4,067 \\
3,871 \\
3,444 \\
3,444 \\
3,546 \\
3,535\end{array}$ & $\begin{array}{l}2,195 \\
2,221 \\
2,133 \\
2,470 \\
2,524 \\
2,587 \\
2,430 \\
2,699 \\
2,608 \\
2,340 \\
2,200 \\
2,448 \\
2,405\end{array}$ & $\begin{array}{l}7,103 \\
6,865 \\
6,646 \\
7,418 \\
8,518 \\
8,245 \\
7,755 \\
8,670 \\
7,826 \\
7,467 \\
7,615 \\
7,337 \\
7,627\end{array}$ & $\begin{array}{l}5,296 \\
5,485 \\
5,166 \\
5,529 \\
6,363 \\
6,334 \\
5,955 \\
6,645 \\
5,812 \\
5,683 \\
5,528 \\
5,565 \\
\mathbf{5 , 7 8 2}\end{array}$ \\
\hline 1992 & 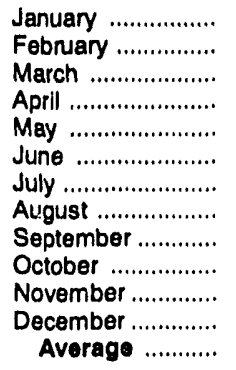 & $\begin{array}{r}17 \\
3 \\
0 \\
0 \\
0 \\
0 \\
72 \\
62 \\
53 \\
9 \\
0 \\
0 \\
18\end{array}$ & $\begin{array}{r}0 \\
0 \\
0 \\
0 \\
0 \\
0 \\
32 \\
31 \\
0 \\
0 \\
0 \\
0 \\
5\end{array}$ & $\begin{array}{l}250 \\
222 \\
202 \\
234 \\
246 \\
266 \\
278 \\
263 \\
217 \\
254 \\
274 \\
273 \\
248\end{array}$ & $\begin{array}{l}0 \\
0 \\
0 \\
0 \\
0 \\
0 \\
0 \\
0 \\
0 \\
0 \\
0 \\
0 \\
0\end{array}$ & $\begin{array}{l}206 \\
195 \\
328 \\
457 \\
452 \\
289 \\
412 \\
462 \\
372 \\
279 \\
219 \\
283 \\
330\end{array}$ & $\begin{array}{r}59 \\
50 \\
114 \\
212 \\
213 \\
95 \\
152 \\
357 \\
160 \\
144 \\
124 \\
92 \\
148\end{array}$ & $\begin{array}{l}3,452 \\
3,248 \\
3,438 \\
4,002 \\
3,643 \\
3,783 \\
4,134 \\
4,113 \\
3,879 \\
3,998 \\
3,790 \\
3,723 \\
3,769\end{array}$ & $\begin{array}{l}2,399 \\
2,162 \\
2,378 \\
2,791 \\
2,597 \\
2,633 \\
3,024 \\
2,984 \\
2,675 \\
2,964 \\
2,745 \\
2,546 \\
2,660\end{array}$ & $\begin{array}{l}7,593 \\
6,754 \\
7,036 \\
8,067 \\
7,754 \\
7,761 \\
8,474 \\
8,256 \\
8,160 \\
8,520 \\
7,877 \\
7,828 \\
7,844\end{array}$ & $\begin{array}{l}5,885 \\
5,033 \\
5,319 \\
6,113 \\
6,025 \\
6,019 \\
6,796 \\
6,457 \\
6,206 \\
6,696 \\
6,121 \\
5,927 \\
6,054\end{array}$ \\
\hline 1993 & $\begin{array}{l}\text { January .............. } \\
\text { February ................. } \\
\text { March ................. } \\
\text { 3-Mo. Average ..... }\end{array}$ & $\begin{array}{l}0 \\
0 \\
0 \\
0\end{array}$ & $\begin{array}{l}0 \\
0 \\
0 \\
0\end{array}$ & $\begin{array}{l}252 \\
244 \\
244 \\
247\end{array}$ & $\begin{array}{l}0 \\
0 \\
0 \\
0\end{array}$ & $\begin{array}{l}325 \\
223 \\
390 \\
316\end{array}$ & $\begin{array}{l}104 \\
151 \\
186 \\
146\end{array}$ & $\begin{array}{l}3,663 \\
3,425 \\
3,967 \\
\mathbf{3 , 6 9 4}\end{array}$ & $\begin{array}{l}2,602 \\
2,457 \\
2,903 \\
2,660\end{array}$ & $\begin{array}{l}7,964 \\
7,930 \\
8,342 \\
8,084\end{array}$ & $\begin{array}{l}6,292 \\
6,156 \\
6,513 \\
6,326\end{array}$ \\
\hline $\begin{array}{l}1992 \\
1991\end{array}$ & $\begin{array}{l}\text { 3-Mo. Average ..... } \\
\text { 3-Mo. Average ..... }\end{array}$ & $\begin{array}{r}7 \\
20\end{array}$ & $\begin{array}{l}0 \\
0\end{array}$ & $\begin{array}{l}225 \\
233\end{array}$ & $\begin{array}{l}0 \\
0\end{array}$ & $\begin{array}{l}244 \\
199\end{array}$ & $\begin{array}{l}75 \\
82\end{array}$ & $\begin{array}{l}3,382 \\
3,094\end{array}$ & $\begin{array}{l}2,316 \\
2,182\end{array}$ & $\begin{array}{l}7,136 \\
6,872\end{array}$ & $\begin{array}{l}5,421 \\
5,310\end{array}$ \\
\hline
\end{tabular}

Includes petroleum imported Into the United States indirectly from members of the Organization of Petroleum Exporting Countries (OPEC) primarily from Caribbean and West European areas as petroleum products that were refined from crude oil produced by OPEC.

Imports from the Neutral Zone between Kuwait and Saudl Arabla are included in imports from Saudi Arabia.

C Excludes petroleum imported into the United States indirectly from members on the Organization of Petroleum Exporting Countries (OPEC), primarily from Carbbean and West European areas, as petroleum products that were refined from crude oil produced by OPEC.

imports from other states in the former U.S.S.R. may be included in imports from Russia for the years 1981 through 1992.

- A small amount of Iranian crude oll entered the United States in January 1988 from the Virgin Islands. This oil originated in Iran and was exported to the Virgin Islands prior to the signing of Executive Order 12613 on October 29, 1987.

(s) = Less than 500 barrels per day.

Notes: - Geographic coverage is the 50 States and the District of Columbia. - Totals may not equal sum of components due to independent rounding. Source: See Summary Statistics Table and Figure Sources. 
Flgure S5. Finished Motor Gasoline Supply and Dispositton, March 1992 - Present

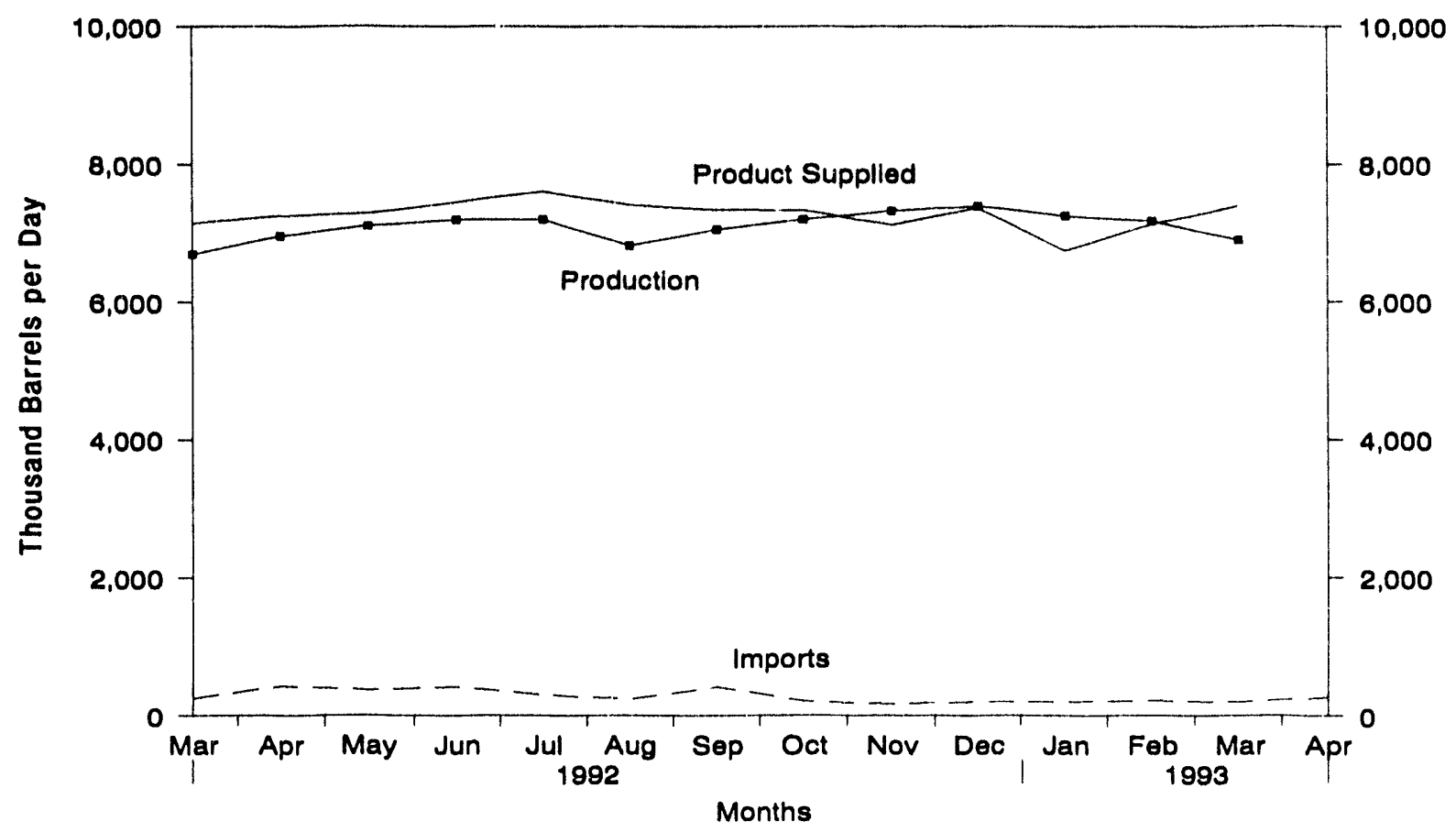

Source: Energy Information Administration, Petroleum Supply Monthly, Table S4. See Summary Statistics Table and Figure Sources.

Figure S6. Motor Gasoline Endling Stocks, March 1992 - Present

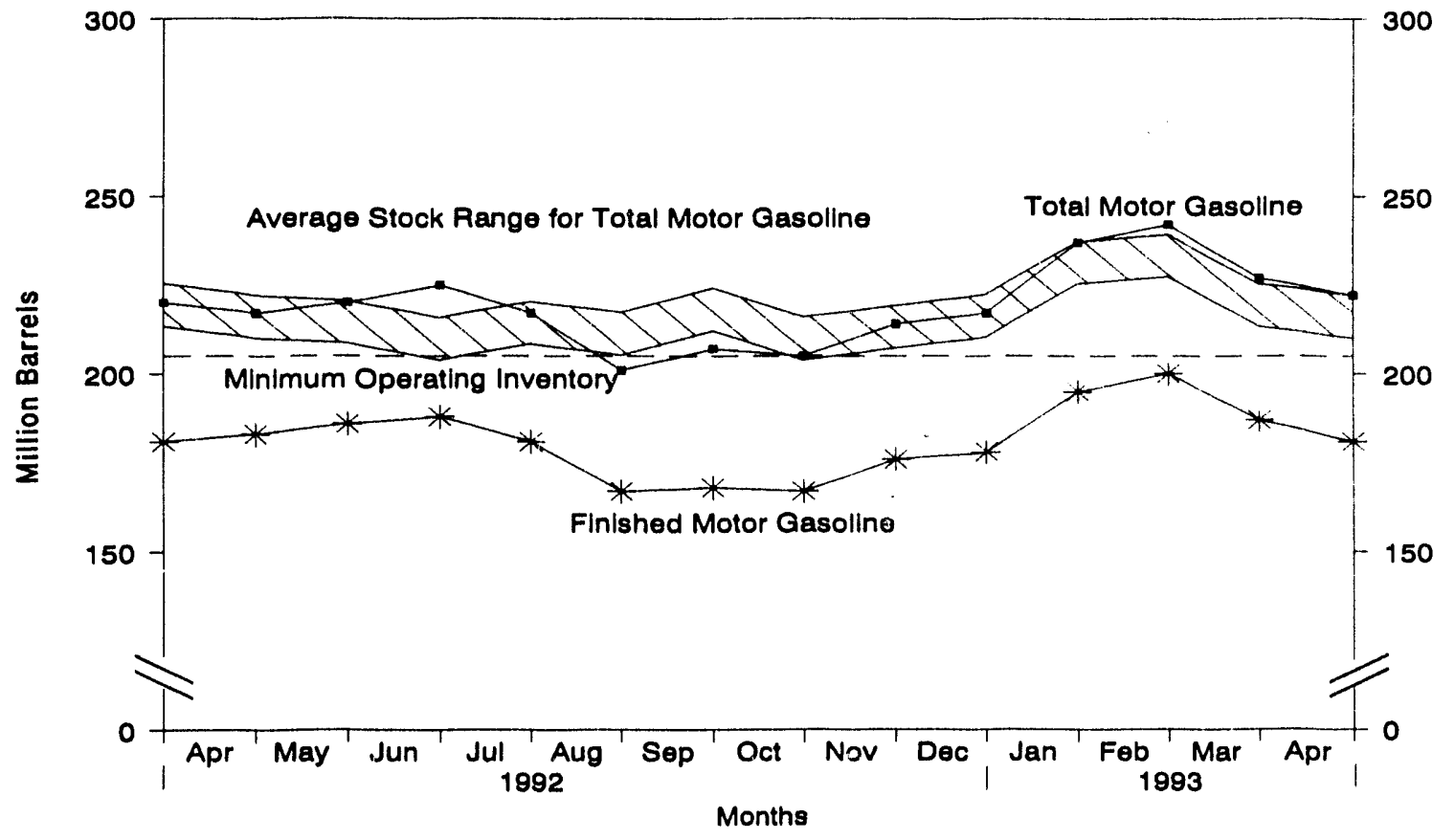

Note: - Total motor gasoline includes motor gasoline blending components and finished motor gasoline. - The National Petroleum Council (NPC) defines the Minimum Operating Inventory as the Inventory level below which operating problems and shortages would begin to appear in a defined distribution system. In its 1988 study, the NPC estimated this inventory level for total motor gasoline to be 205 million barrels.

Source: Energy Information Adminlstration, Petroleum Supply Monthly, Table S4. See Summary Statistics Table and Figure Sources. 
Table S4. Finished Motor Gasoline Supply and Disposition, 1981 - Present (Thousand Barrels per Day, Except Where Noted)

\begin{tabular}{|c|c|c|c|c|c|c|c|c|c|}
\hline & \multirow{3}{*}{ Year/Month } & \multicolumn{2}{|c|}{ Supply } & \multicolumn{3}{|c|}{ Disposition } & \multirow{2}{*}{\multicolumn{2}{|c|}{$\begin{array}{l}\begin{array}{l}\text { Ending Stocks" } \\
\text { (Milllon Barrols) }\end{array} \\
\text { Motor Gasolins }\end{array}$}} & \multirow{3}{*}{$\begin{array}{c}\begin{array}{c}\text { Ending Stocks } \\
\text { (Million Barrols) }\end{array} \\
\text { Oxygenates }\end{array}$} \\
\hline & & \multirow{2}{*}{$\begin{array}{l}\text { Total } \\
\text { Produotion }\end{array}$} & \multirow[b]{2}{*}{ Importe } & \multirow{2}{*}{$\begin{array}{c}\text { Stock } \\
\text { Change }\end{array}$} & \multirow[b]{2}{*}{ Exporte } & \multirow{2}{*}{$\begin{array}{l}\text { Product } \\
\text { Supplied }\end{array}$} & & & \\
\hline & & & & & & & Total ${ }^{\bullet}$ & Finished & \\
\hline $\begin{array}{l}1981 \\
1982 \\
1983 \\
1984 \\
1985 \\
1986 \\
1987 \\
1988 \\
1989 \\
1990\end{array}$ & 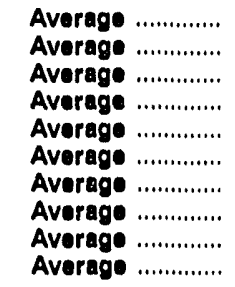 & $\begin{array}{l}6,405 \\
6,338 \\
6,340 \\
6,453 \\
6,419 \\
6,752 \\
6,841 \\
6,956 \\
6,963 \\
6,959\end{array}$ & $\begin{array}{l}157 \\
197 \\
247 \\
299 \\
381 \\
326 \\
384 \\
405 \\
369 \\
342\end{array}$ & $\begin{array}{r}1.28 \\
.25 \\
-45 \\
54 \\
-41 \\
11 \\
-15 \\
3 \\
-35 \\
10\end{array}$ & $\begin{array}{r}2 \\
20 \\
10 \\
6 \\
10 \\
33 \\
35 \\
22 \\
39 \\
55\end{array}$ & $\begin{array}{l}6,588 \\
6,539 \\
6,622 \\
6,693 \\
6,831 \\
7,034 \\
7,206 \\
7,336 \\
7,328 \\
7,235\end{array}$ & $\begin{array}{l}253 \\
235 \\
222 \\
243 \\
223 \\
233 \\
226 \\
228 \\
213 \\
220\end{array}$ & $\begin{array}{r}203 \\
1184 \\
186 \\
205 \\
190 \\
194 \\
189 \\
180 \\
177 \\
181\end{array}$ & $\begin{array}{l}\ddot{.} \\
\ddot{.} \\
\ddot{.} \\
\ddot{.} \\
\ddot{.} \\
\ddot{.}\end{array}$ \\
\hline 1991 & 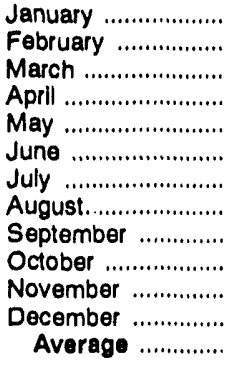 & $\begin{array}{l}6,629 \\
6,573 \\
6,643 \\
6,742 \\
7,063 \\
7,351 \\
7,274 \\
7,247 \\
7,030 \\
6,749 \\
7,018 \\
7,354 \\
6,975\end{array}$ & $\begin{array}{l}228 \\
115 \\
235 \\
381 \\
528 \\
364 \\
232 \\
385 \\
312 \\
236 \\
322 \\
216 \\
297\end{array}$ & $\begin{array}{r}162 \\
-252 \\
-236 \\
-67 \\
95 \\
160 \\
-177 \\
7 \\
195 \\
-354 \\
228 \\
267 \\
3\end{array}$ & $\begin{array}{r}50 \\
102 \\
97 \\
53 \\
59 \\
99 \\
122 \\
98 \\
63 \\
58 \\
104 \\
79 \\
82\end{array}$ & $\begin{array}{l}6,645 \\
6,838 \\
7,017 \\
7,137 \\
7,437 \\
7,456 \\
7,561 \\
7,528 \\
7,083 \\
7,281 \\
7,008 \\
7,224 \\
7,188\end{array}$ & $\begin{array}{l}225 \\
219 \\
210 \\
205 \\
209 \\
214 \\
208 \\
209 \\
216 \\
203 \\
209 \\
219 \\
--\end{array}$ & $\begin{array}{l}186 \\
179 \\
171 \\
169 \\
172 \\
177 \\
172 \\
172 \\
178 \\
167 \\
173 \\
182 \\
-\cdots\end{array}$ & $\begin{array}{l}\ddot{.} \\
\ddot{.} \\
. . \\
. . \\
. . \\
\ddot{.} \\
\ddot{.} \\
\ddot{.} \\
\ddot{.}\end{array}$ \\
\hline 1992 & 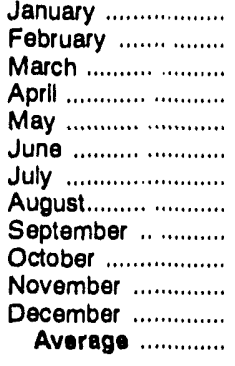 & $\begin{array}{l}7,043 \\
6,753 \\
6,694 \\
6,958 \\
7,100 \\
7,201 \\
7,197 \\
6,818 \\
7,057 \\
7,198 \\
7,323 \\
7,398 \\
7,052\end{array}$ & $\begin{array}{l}237 \\
270 \\
247 \\
428 \\
370 \\
419 \\
303 \\
240 \\
418 \\
209 \\
170 \\
202 \\
292\end{array}$ & $\begin{array}{r}300 \\
-41 \\
-275 \\
41 \\
101 \\
83 \\
-215 \\
-480 \\
51 \\
-23 \\
299 \\
38 \\
-11\end{array}$ & $\begin{array}{r}87 \\
59 \\
71 \\
90 \\
82 \\
86 \\
108 \\
123 \\
85 \\
94 \\
74 \\
184 \\
96\end{array}$ & $\begin{array}{l}6,893 \\
7,004 \\
7,145 \\
7,255 \\
7,288 \\
7,451 \\
7,607 \\
7,414 \\
7,339 \\
7,336 \\
7,119 \\
7,377 \\
7,270\end{array}$ & $\begin{array}{r}229 \\
229 \\
220 \\
217 \\
220 \\
225 \\
217 \\
201 \\
207 \\
205 \\
214 \\
217 \\
. .\end{array}$ & $\begin{array}{r}191 \\
190 \\
181 \\
183 \\
186 \\
188 \\
181 \\
167 \\
168 \\
167 \\
176 \\
178 \\
\cdots\end{array}$ & $\begin{array}{l}. . \\
. . \\
. . \\
. . \\
\ddot{.} \\
. . \\
\ddot{.} \\
\ddot{.} \\
\ddot{.} \\
.\end{array}$ \\
\hline 1993 & 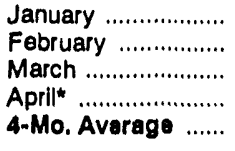 & $\begin{array}{r}7,254 \\
7,172 \\
6,897 \\
\text { NA } \\
\text { NA }\end{array}$ & $\begin{array}{r}204 \\
216 \\
R 198 \\
E_{269} \\
E_{222}\end{array}$ & $\begin{array}{r}571 \\
160 \\
R_{-411} \\
E_{-223} \\
E_{23}\end{array}$ & $\begin{array}{r}142 \\
99 \\
\mathrm{R}_{109} \\
E_{98} \\
\mathrm{E}_{112}\end{array}$ & $\begin{array}{r}6,746 \\
7,129 \\
7,397 \\
\text { NA } \\
\text { NA }\end{array}$ & $\begin{array}{r}237 \\
242 \\
\mathrm{R} 227 \\
\mathrm{E}_{222} \\
-.\end{array}$ & $\begin{array}{r}195 \\
200 \\
R \quad 187 \\
E \quad 181 \\
-\cdot\end{array}$ & $\begin{array}{r}14 \\
13 \\
14 \\
\text { NA } \\
-.\end{array}$ \\
\hline $\begin{array}{l}1992 \\
1991\end{array}$ & $\begin{array}{l}\text { 4-Mo. Average ...... } \\
\text { 4-Mo, Average ..... }\end{array}$ & $\begin{array}{l}6,863 \\
6,648\end{array}$ & $\begin{array}{l}295 \\
242\end{array}$ & $\begin{array}{r}7 \\
-95\end{array}$ & $\begin{array}{l}77 \\
75\end{array}$ & $\begin{array}{l}7,074 \\
6,809\end{array}$ & $\ddot{*}$ & $\ddot{-\bullet}$ & $\ddot{~}$ \\
\hline
\end{tabular}

\footnotetext{
Stocks are totals as of end of period.

b Beginning in 1993, motor gasoline production and product supplled Includes blending of fuel ethanol and an adjustment to correct for the Imbalance of motor gasoline blending components.

c Beginning in 1981, excludes blending components.

d A negative number indicates a decrease in stocks and a positive number indicates an increase.

- Includes motor gasoline blending components but excludes stocks of oxygenates.

In January 1981 and 1983 , numerous respondents were added to surveys aftecting stocks reported and stock change calculations. Stock changes are calculated using new basis stock levels. See Summary Statistics Explanatory Note 4.

$R=$ Revised data. $(s)=$ Less than 500 barrels per day. $E=$ Estimated. $N A=$ Not Available.

* See Summary Statlistics Explanatory Note 1.

Notes: - Italics denote estimates based upon preliminary data. - Geographic coverage is the 50 States and the District of Columbla. - Totals may not equal sum of components due to independent rounding.

Source: See Summary Statistics Table and Figure Sources.
} 
Figure S7. Distillate Fuel OII Supply and Dispositlon, March 1992 - Present

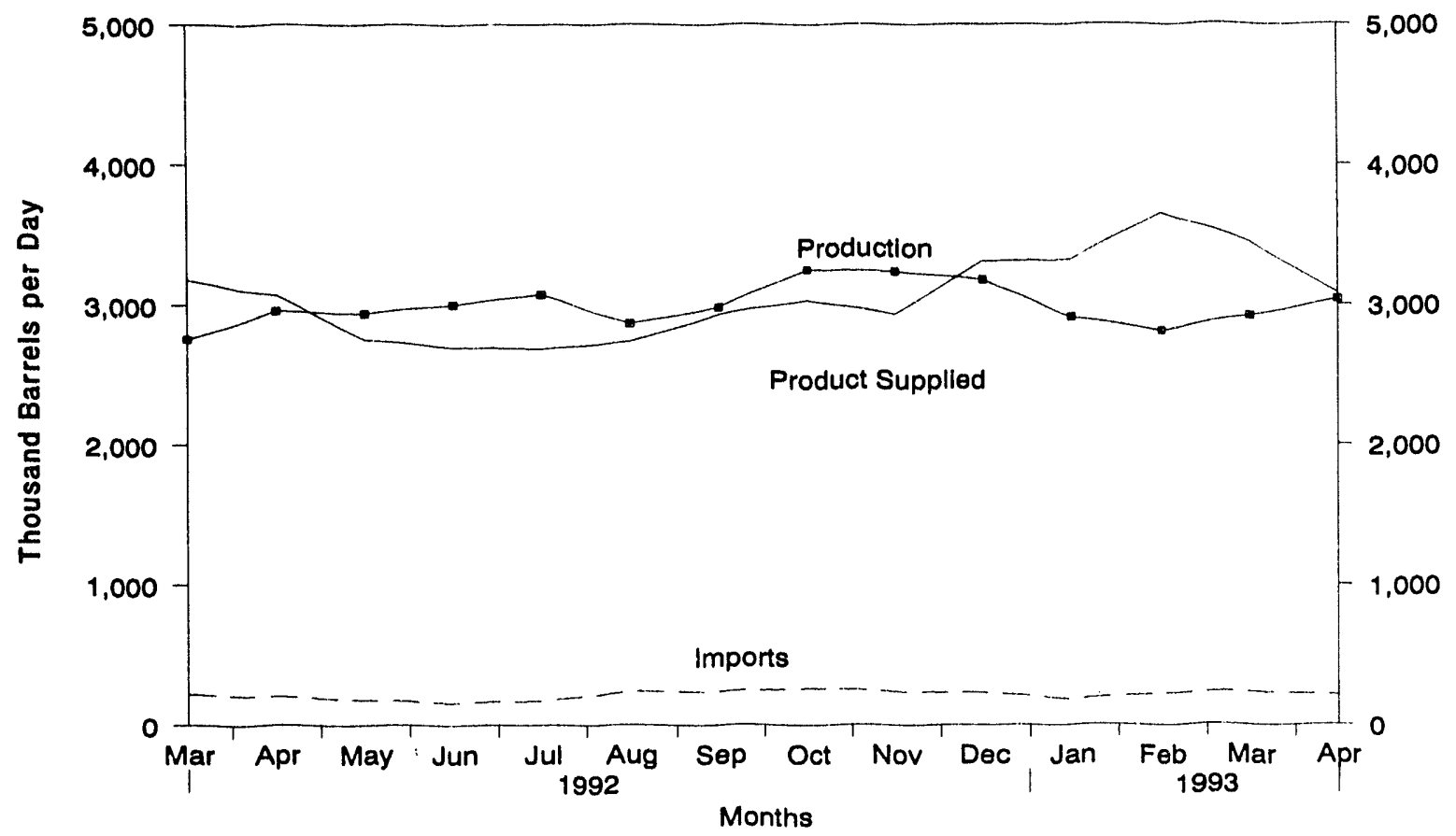

Source: Energy Information Administration, Petroleum Supply Monthly, Table S5. See Summary Statistics Table and Figure Sources.

Figure S8. Distillate Fuel Oll Ending Stocks, March 1992 - Present

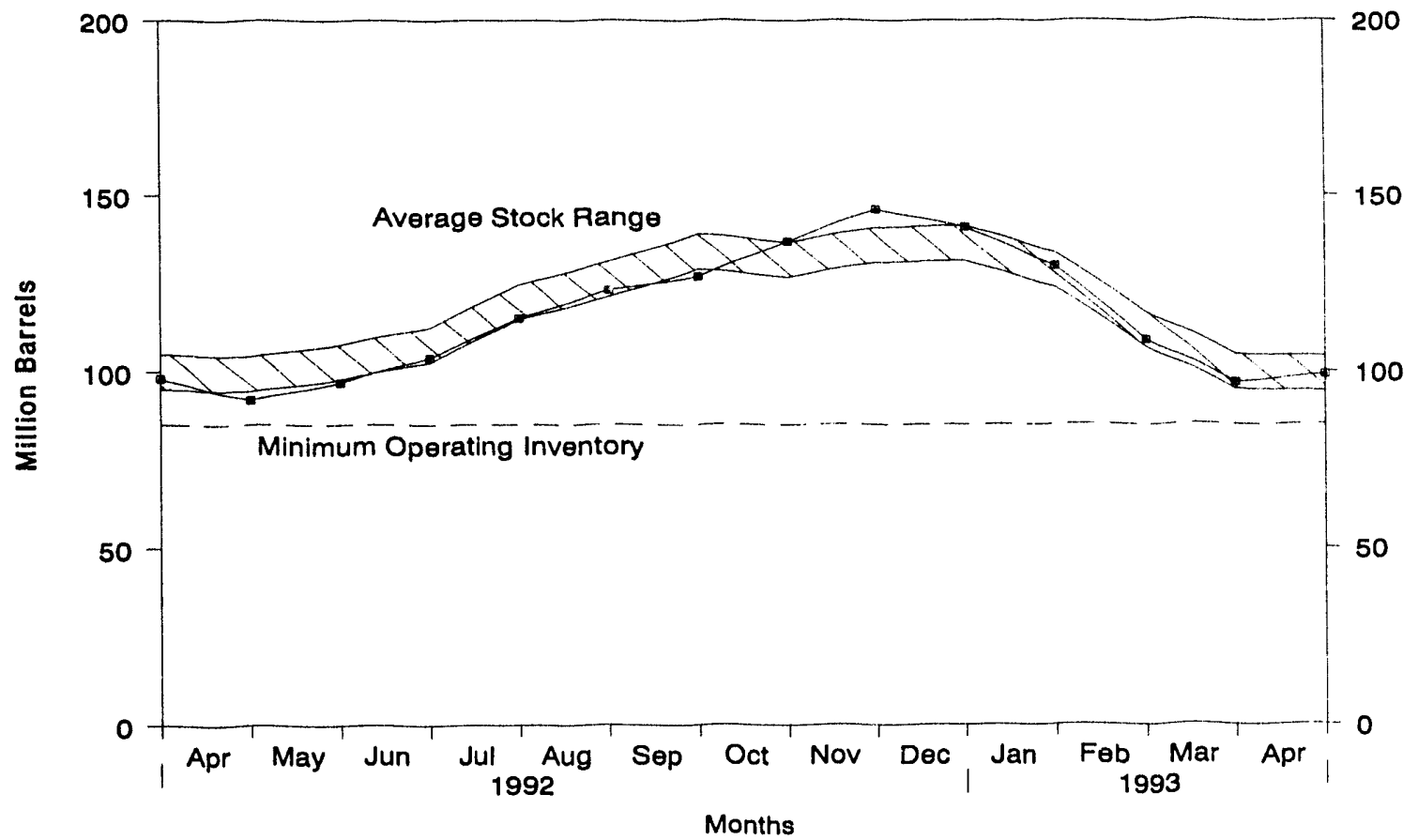

Note: The National Petroleum Council (NPC) defines the Minimum Operating Inventory as the inventory level below which operating problems and shortages would begin to appear in a defined distribution system. In its 1988 study, the NPC estimated this inventory level for distillate fuel oll to be 85 million barrels.

Source: Energy Information Administration, Petroleum Supply Monthly, Table S5. See Summary Statistics Table and Figure Sources. 
Table S5. Distillate Fuel Oll Supply andd Disposition, 1981 - Present (Thousand Barrels per Day, Excrspt Where Noted)

\begin{tabular}{|c|c|c|c|c|c|c|c|c|c|}
\hline & \multirow{3}{*}{ Yesr/Month } & \multicolumn{2}{|c|}{ Supply } & \multicolumn{3}{|c|}{ Disposition } & \multirow{2}{*}{\multicolumn{3}{|c|}{$\begin{array}{l}\text { Endling Stocksb } \\
\text { (MIllion Barrels) }\end{array}$}} \\
\hline & & \multirow[b]{2}{*}{$\begin{array}{c}\text { Total } \\
\text { Froduction }\end{array}$} & \multirow[b]{2}{*}{ Imports } & \multirow[b]{2}{*}{$\begin{array}{l}\text { Stock. } \\
\text { Change: }\end{array}$} & \multirow[b]{2}{*}{ Exports } & \multirow[b]{2}{*}{$\begin{array}{l}\text { Product } \\
\text { Supplled }\end{array}$} & & & \\
\hline & & & & & & & Total & $\begin{array}{l}0.05 \% \text { Sulfur } \\
\text { and Under }\end{array}$ & $\begin{array}{l}\text { Greater than } \\
0.05 \% \text { Sulfur }\end{array}$ \\
\hline $\begin{array}{l}1981 \\
1982 \\
1983 \\
1984 \\
1985 \\
1986 \\
1987 \\
1988 \\
1989 \\
1990\end{array}$ & 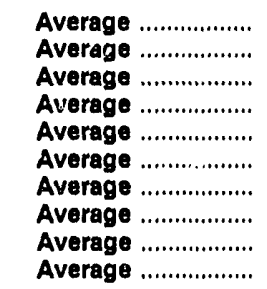 & $\begin{array}{l}2,613 \\
2,608 \\
2,456 \\
2,681 \\
2,687 \\
2,798 \\
2,731 \\
2,859 \\
2,899 \\
2,925\end{array}$ & $\begin{array}{r}173 \\
93 \\
174 \\
272 \\
200 \\
247 \\
255 \\
302 \\
306 \\
278\end{array}$ & $\begin{array}{r}d-38 \\
-35 \\
d-124 \\
57 \\
-48 \\
31 \\
-56 \\
-30 \\
-49 \\
73\end{array}$ & $\begin{array}{r}5 \\
74 \\
64 \\
51 \\
67 \\
100 \\
66 \\
69 \\
97 \\
109\end{array}$ & $\begin{array}{l}2,829 \\
2,671 \\
2,690 \\
2,845 \\
2,868 \\
2,914 \\
2,976 \\
3,122 \\
3,157 \\
3,021\end{array}$ & $\begin{array}{r}192 \\
d 179 \\
140 \\
161 \\
144 \\
155 \\
134 \\
124 \\
106 \\
132\end{array}$ & $\begin{array}{l}- \\
= \\
= \\
= \\
= \\
-\end{array}$ & $\begin{array}{l}- \\
\overline{-} \\
\overline{-} \\
\overline{-} \\
\overline{-} \\
-\end{array}$ \\
\hline 1991 & 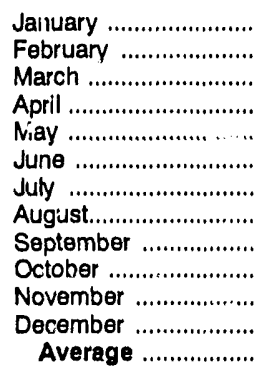 & $\begin{array}{l}2,845 \\
2,870 \\
2,86 \\
2,819 \\
2,929 \\
2,941 \\
2,998 \\
2,961 \\
3,055 \\
3,040 \\
3,103 \\
3,107 \\
2,962\end{array}$ & $\begin{array}{l}192 \\
139 \\
206 \\
258 \\
186 \\
209 \\
155 \\
168 \\
237 \\
207 \\
249 \\
252 \\
205\end{array}$ & $\begin{array}{r}-662 \\
-359 \\
-112 \\
156 \\
132 \\
225 \\
356 \\
214 \\
291 \\
-59 \\
206 \\
-30 \\
31\end{array}$ & $\begin{array}{r}332 \\
393 \\
198 \\
81 \\
218 \\
150 \\
149 \\
144 \\
136 \\
259 \\
224 \\
302 \\
215\end{array}$ & $\begin{array}{l}3,367 \\
2,976 \\
2,984 \\
2,839 \\
2,765 \\
2,775 \\
2,648 \\
2,770 \\
2,865 \\
3,047 \\
2,921 \\
3,087 \\
2,921\end{array}$ & $\begin{array}{r}112 \\
102 \\
98 \\
103 \\
107 \\
114 \\
125 \\
131 \\
140 \\
138 \\
144 \\
144 \\
-\end{array}$ & $\begin{array}{l}- \\
= \\
= \\
= \\
= \\
= \\
= \\
- \\
-\end{array}$ & $\begin{array}{l}- \\
\overline{-} \\
\overline{-} \\
- \\
- \\
- \\
- \\
-\end{array}$ \\
\hline 1992 & 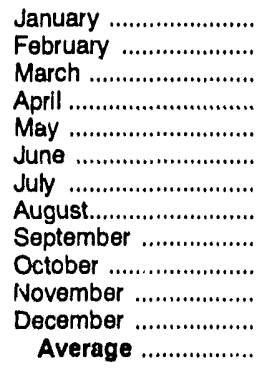 & $\begin{array}{l}2,818 \\
2,681 \\
2,753 \\
2,954 \\
2,939 \\
3,402 \\
3,073 \\
2,864 \\
2,982 \\
3,25 i \\
3,236 \\
3,179 \\
2,979\end{array}$ & $\begin{array}{l}227 \\
207 \\
218 \\
202 \\
179 \\
157 \\
172 \\
236 \\
237 \\
262 \\
236 \\
229 \\
214\end{array}$ & $\begin{array}{r}-541 \\
-629 \\
-346 \\
-190 \\
146 \\
258 \\
359 \\
237 \\
143 \\
312 \\
312 \\
-175 \\
-8\end{array}$ & $\begin{array}{l}360 \\
278 \\
138 \\
278 \\
222 \\
205 \\
201 \\
127 \\
145 \\
169 \\
230 \\
276 \\
219\end{array}$ & $\begin{array}{l}3,226 \\
3,238 \\
3,179 \\
3,068 \\
2,751 \\
2,696 \\
2,685 \\
2,736 \\
2,930 \\
3,032 \\
2,930 \\
3,308 \\
2,981\end{array}$ & $\begin{array}{r}127 \\
108 \\
98 \\
92 \\
97 \\
104 \\
115 \\
123 \\
127 \\
137 \\
146 \\
141 \\
-\end{array}$ & 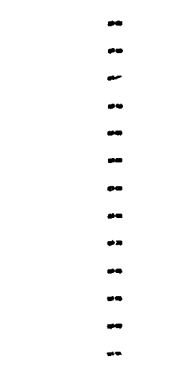 & $\begin{array}{l}- \\
- \\
= \\
= \\
- \\
- \\
- \\
- \\
-\end{array}$ \\
\hline 1993 & 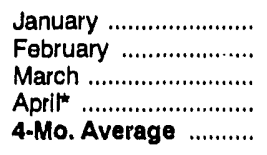 & $\begin{array}{r}2,909 \\
2,813 \\
R 2,918 \\
E_{3,034} \\
E_{2,920}\end{array}$ & $\begin{array}{r}182 \\
224 \\
R_{235} \\
E_{210} \\
E_{212}\end{array}$ & $\begin{array}{r}-336 \\
-742 \\
R-386 \\
E_{59} \\
E_{-345}\end{array}$ & $\begin{array}{r}105 \\
121 \\
R_{90} \\
E_{108} \\
E_{105}\end{array}$ & $\begin{array}{r}3,322 \\
3,658 \\
\text { R }_{3,450} \\
\text { E }_{3,077} \\
\text { S }_{3,372}\end{array}$ & $\begin{array}{r}130 \\
109 \\
97 \\
\text { E } 99 \\
-\end{array}$ & $\begin{array}{r}22 \\
16 \\
12 \\
N A \\
-\end{array}$ & $\begin{array}{r}108 \\
94 \\
85 \\
\text { NA } \\
-\end{array}$ \\
\hline $\begin{array}{l}1992 \\
1991\end{array}$ & $\begin{array}{l}\text { 4-Mo. Average .......... } \\
\text { 4-Mo. Average .......... }\end{array}$ & $\begin{array}{l}2,802 \\
2,850\end{array}$ & $\begin{array}{l}294 \\
200\end{array}$ & $\begin{array}{l}-425 \\
-244\end{array}$ & $\begin{array}{l}263 \\
249\end{array}$ & $\begin{array}{l}3,178 \\
3,045\end{array}$ & - & - & - \\
\hline
\end{tabular}

Excludes 10,000 barrels per day in 1981 and 1982 previously published as cride used directly.

Stocks are totais as of end of period.

c A negative number indicates a decrease in stocks and a nusitive number indicates an increase.

d In January 1981 and 1983, numerous reispondents weie added to surveys affecting stocks reported and stock change calculations. Stock changes are ralculaiad using new stock basis stock levels. See Surnmary Statistics Explanatory Note 4.

$R=$ Revised data. $(s)=$ Less then 500 barrels per day. $E=$ Estimated. $N A=$ Not available

* See Summary Statistics Explanatory Note 1.

Notes: - Italics denote estimates based upon preliminary data. - Geographic coverfige is the 50 States and the District of Columbla. - Totals may not equal sum of components due to independent rounding.

Source: See Summary Statistics Table and Figure Sources. 
Figure S9. Residual Fuel OII Supply and Dispositlon, March 1992 - Present

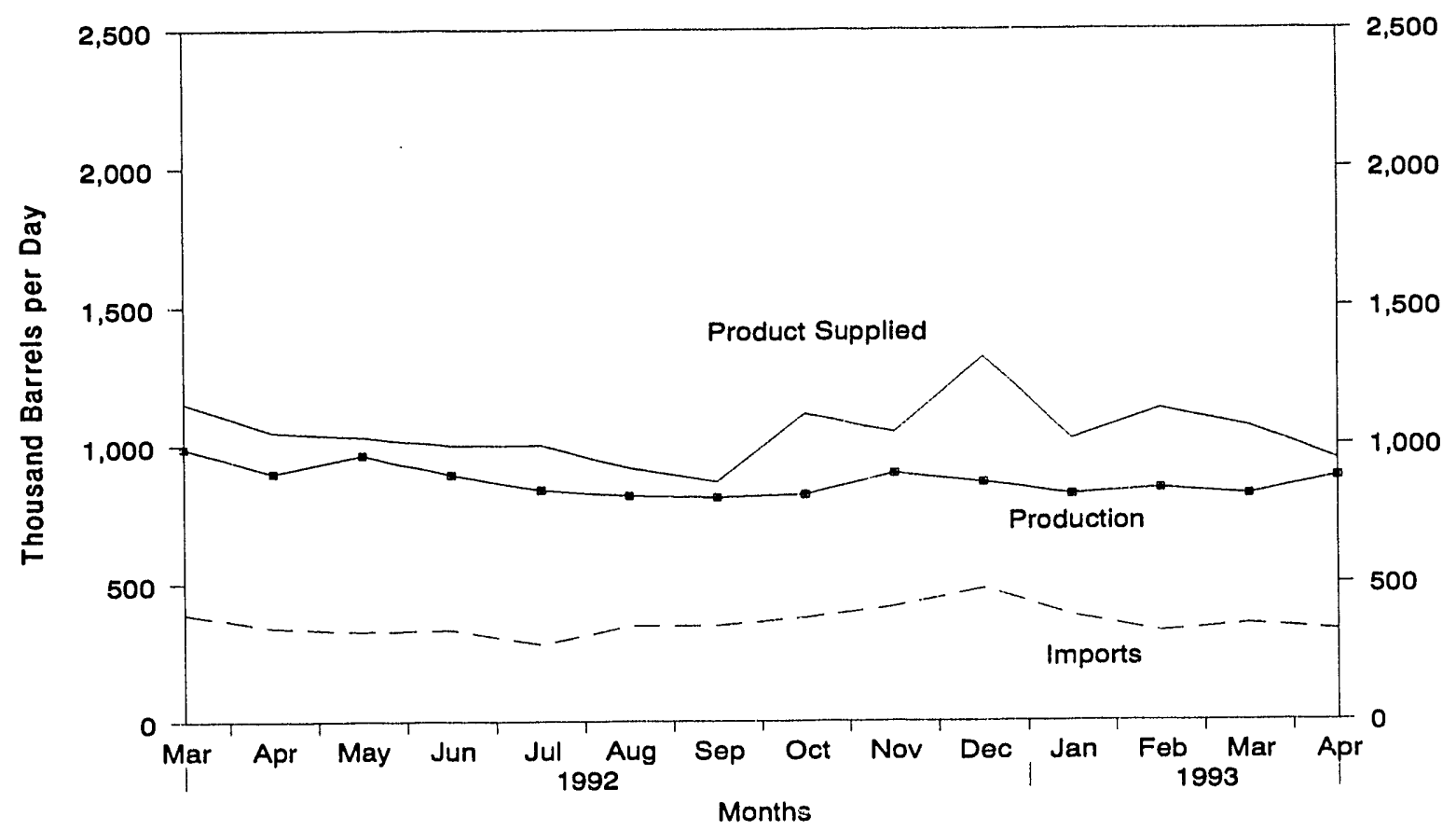

Source: Energy Information Administration, Petroleum Supply Monthly, Table S6. See Summary Statistics Table and Figure Sources.

Figure S10. Residual Fuel Oil Ending Stocks, March 1992 - Present

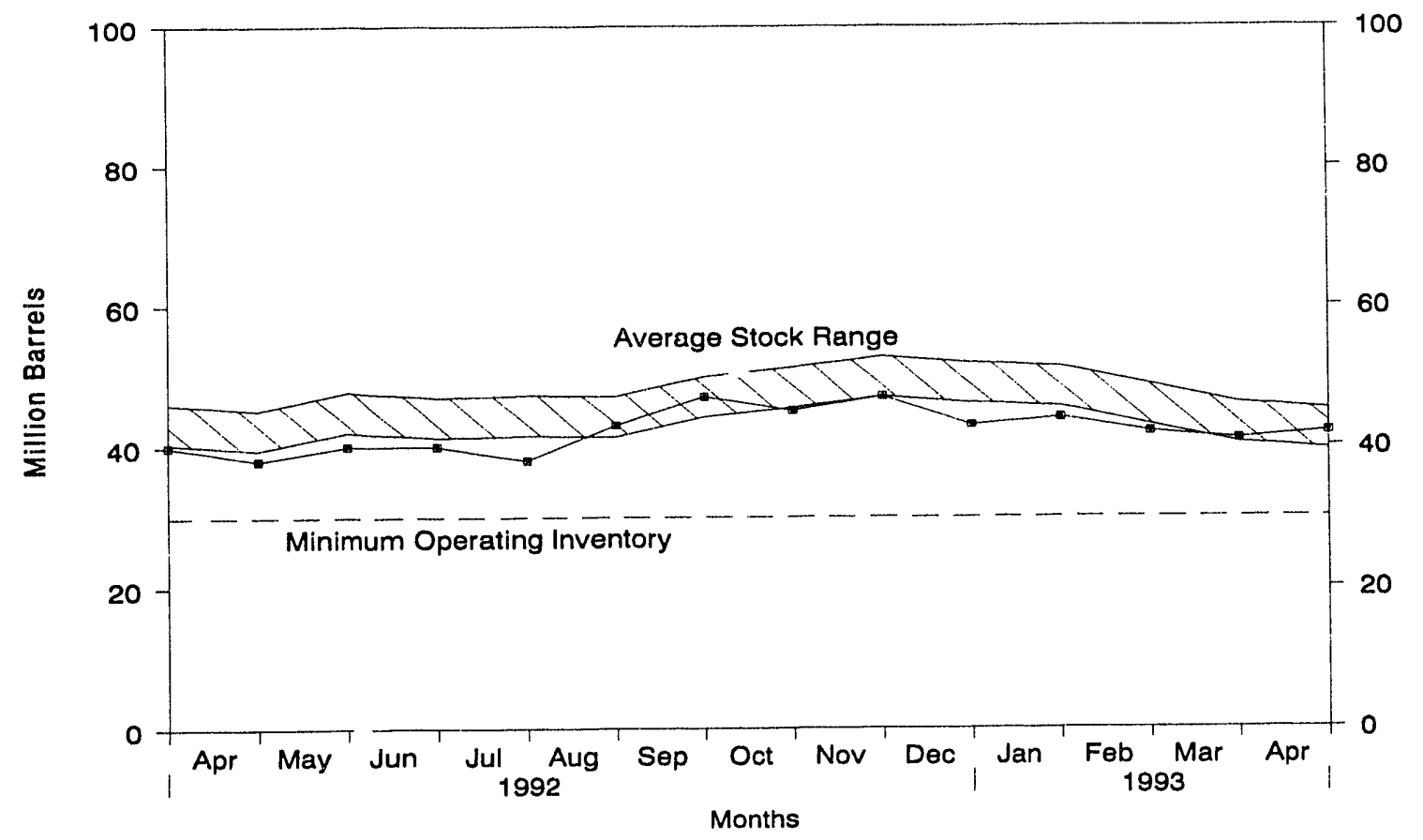

Note: The National Petroleum Council (NPC) defines the Minimum Operating Inventory is the inventory level below which operating problems and shortages would begin to appear in a defined distribution system. In its 1988 study, the NPC estimated this inventory level for residual fuel oil to be 30 million barrels.

Source: Energy Information Administration, Petroleum Supply Monthly, Table S6. See Summary Statistics Table and Figure Sources. 
Table S6. Residual Fuel OII Supply and Disposition, 1981 - Present (Thousand Barrels per Day, Except Where Noted)

\begin{tabular}{|c|c|c|c|c|c|c|c|}
\hline \multirow{2}{*}{\multicolumn{2}{|c|}{ Year/Month }} & \multicolumn{2}{|c|}{ Supply" } & \multicolumn{3}{|c|}{ Dlaposition } & \multirow[b]{2}{*}{$\begin{array}{c}\text { Endlng } \\
\text { Stockgc } \\
\text { (Million Barrels) }\end{array}$} \\
\hline & & $\begin{array}{c}\text { Total } \\
\text { Production }\end{array}$ & Importe & $\begin{array}{c}\text { Stock } \\
\text { Changeb }\end{array}$ & Exports & $\begin{array}{l}\text { Product } \\
\text { Supplled }\end{array}$ & \\
\hline $\begin{array}{l}1981 \\
1982 \\
1983 \\
1984 \\
1985 \\
1988 \\
1987 \\
1988 \\
1989 \\
1990\end{array}$ & 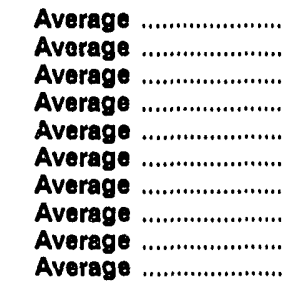 & $\begin{array}{r}1,321 \\
1,070 \\
852 \\
891 \\
882 \\
889 \\
885 \\
926 \\
954 \\
950\end{array}$ & $\begin{array}{l}800 \\
776 \\
699 \\
681 \\
510 \\
669 \\
565 \\
644 \\
629 \\
504\end{array}$ & $\begin{array}{r}d-37 \\
-32 \\
d .55 \\
12 \\
-7 \\
-8 \\
(8) \\
-8 \\
-2 \\
13\end{array}$ & $\begin{array}{l}118 \\
209 \\
185 \\
190 \\
197 \\
147 \\
186 \\
200 \\
215 \\
211\end{array}$ & $\begin{array}{l}2,088 \\
1,716 \\
1,421 \\
1,369 \\
1,202 \\
1,418 \\
1,264 \\
1,378 \\
1,370 \\
1,229\end{array}$ & $\begin{array}{r}78 \\
066 \\
49 \\
53 \\
50 \\
47 \\
47 \\
45 \\
44 \\
49\end{array}$ \\
\hline 1991 & 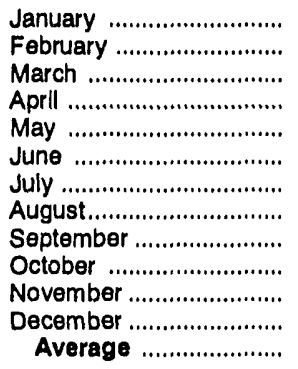 & $\begin{array}{r}1,001 \\
1,050 \\
995 \\
916 \\
929 \\
933 \\
871 \\
925 \\
838 \\
814 \\
896 \\
1,051 \\
934\end{array}$ & $\begin{array}{l}425 \\
384 \\
332 \\
416 \\
425 \\
512 \\
420 \\
599 \\
481 \\
438 \\
455 \\
547 \\
453\end{array}$ & $\begin{array}{r}-19 \\
-76 \\
-85 \\
68 \\
50 \\
-103 \\
-1 \\
68 \\
78 \\
6 \\
24 \\
28 \\
4\end{array}$ & $\begin{array}{l}320 \\
299 \\
178 \\
145 \\
300 \\
245 \\
176 \\
216 \\
168 \\
217 \\
189 \\
264 \\
226\end{array}$ & $\begin{array}{l}1,124 \\
1,211 \\
1,234 \\
1,119 \\
1,003 \\
1,303 \\
1,117 \\
1,240 \\
1,074 \\
1,029 \\
1,139 \\
1,307 \\
1,158\end{array}$ & $\begin{array}{l}48 \\
46 \\
43 \\
45 \\
47 \\
44 \\
44 \\
46 \\
48 \\
48 \\
49 \\
50 \\
-\end{array}$ \\
\hline 1992 & 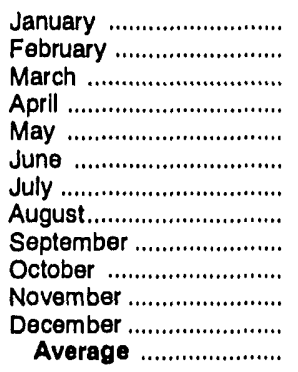 & $\begin{array}{l}964 \\
956 \\
989 \\
899 \\
964 \\
894 \\
838 \\
815 \\
809 \\
820 \\
896 \\
863 \\
892\end{array}$ & $\begin{array}{l}352 \\
487 \\
392 \\
342 \\
328 \\
334 \\
280 \\
347 \\
349 \\
376 \\
416 \\
481 \\
373\end{array}$ & $\begin{array}{r}-180 \\
-46 \\
-82 \\
-72 \\
55 \\
-2 \\
-50 \\
149 \\
145 \\
-71 \\
50 \\
-126 \\
-20\end{array}$ & $\begin{array}{r}184 \\
176 \\
310 \\
265 \\
207 \\
230 \\
169 \\
96 \\
149 \\
156 \\
216 \\
155 \\
193\end{array}$ & $\begin{array}{r}1,313 \\
1,314 \\
1,153 \\
1,048 \\
1,030 \\
1,000 \\
1,000 \\
916 \\
865 \\
1,110 \\
1,045 \\
1,316 \\
1,092\end{array}$ & $\begin{array}{r}44 \\
43 \\
40 \\
38 \\
40 \\
40 \\
38 \\
43 \\
47 \\
45 \\
47 \\
43 \\
-\end{array}$ \\
\hline 1993 & 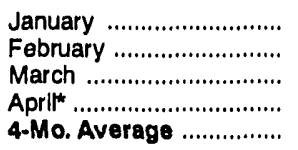 & $\begin{array}{r}820 \\
841 \\
R_{819} \\
E_{884} \\
E_{841}\end{array}$ & $\begin{array}{r}383 \\
325 \\
A_{352} \\
E_{330} \\
E_{348}\end{array}$ & $\begin{array}{r}49 \\
-75 \\
R_{-46} \\
E^{-49} \\
E_{-2}\end{array}$ & $\begin{array}{r}133 \\
113 \\
R \quad 152 \\
E 209 \\
\text { E } 152\end{array}$ & $\begin{array}{r}1,020 \\
1,128 \\
R_{1,065} \\
E_{1}^{945} \\
E_{1,038}\end{array}$ & $\begin{array}{r}44 \\
42 \\
\mathrm{P}_{41} \\
\mathrm{E}_{42} \\
-\end{array}$ \\
\hline $\begin{array}{l}1992 \\
1991\end{array}$ & 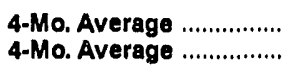 & $\begin{array}{l}953 \\
990\end{array}$ & $\begin{array}{l}392 \\
389\end{array}$ & $\begin{array}{l}-96 \\
-28\end{array}$ & $\begin{array}{l}234 \\
235\end{array}$ & $\begin{array}{l}1,207 \\
1,172\end{array}$ & $\overline{-}$ \\
\hline
\end{tabular}

Excludes 48,000 barrels per day in 1981 and 1982 previously published as crude used directly.

b A negative number indicates a decrease in stocks and a positive number indicates an increase.

c Stocks are totals as of end of period.

I In January 1981 and 1983 , numerous respondents were added to surveys affecting stocks reported and stock change calculations. Stock changes are calculated using new basis stock levels. See Summary Statistics Explanatory Note 4.

$R=$ Revised data. $(s)=$ Less than 500 barrels per day. $E=$ Estimated.

* See Summary Statistics Explanatory Note 1.

Notes: - Italics denote estimates based upon prellminary data. - Geographlc coverage is the 50 States and the District of Columbia. - Totals may not equal sum of components due to independent rounding.

Source: See Summary Statistics Table and Figure Sources. 
Figure S11. Jet Fuel Supply and Disposition, March 1992 - Present

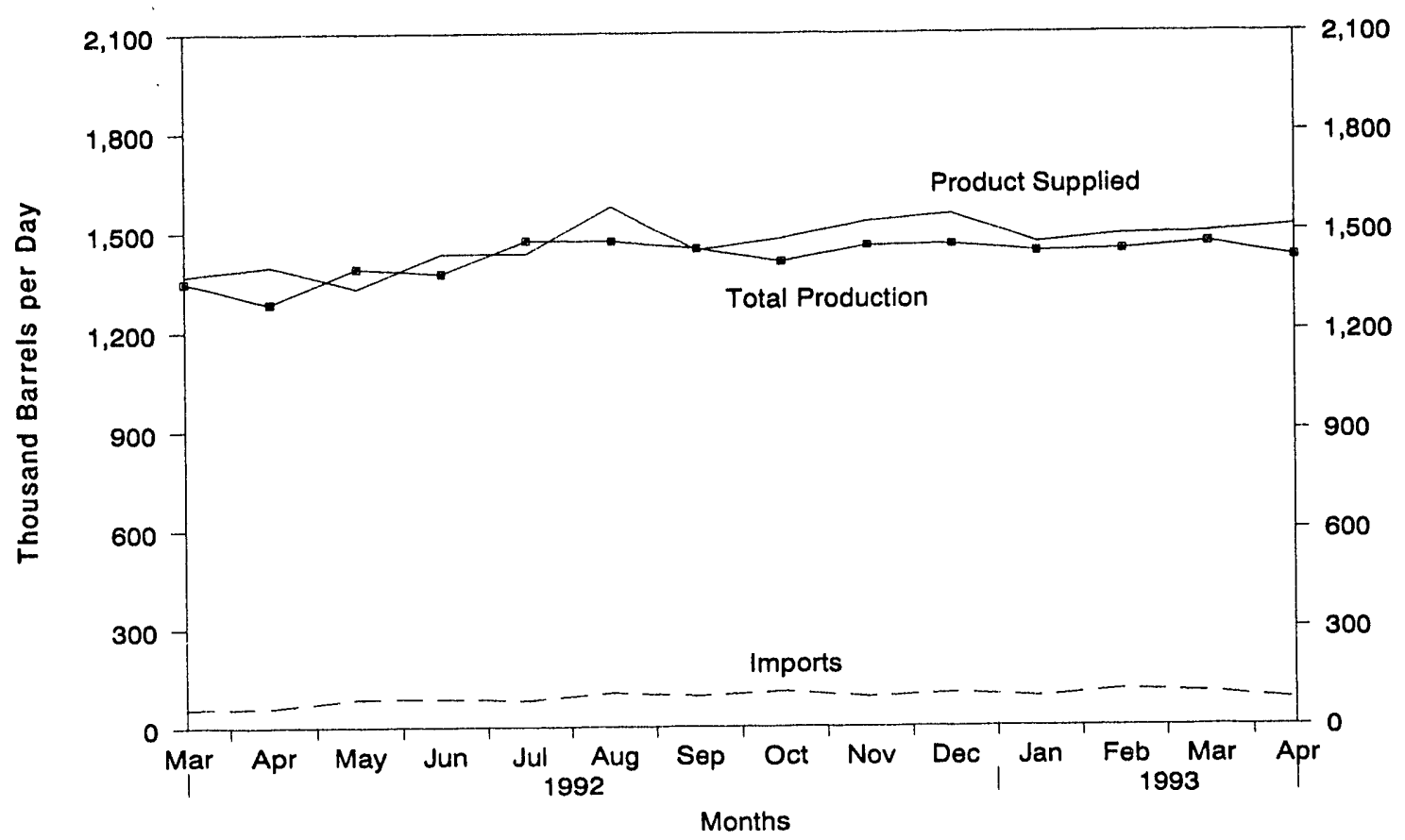

Source: Energy Information Administration, Petroleum Supply Monthly, Table S7. See Summary Statistics Table and Figure Sources.

Figure S12. Jet Fuel Ending Stocks, March 1992 - Present

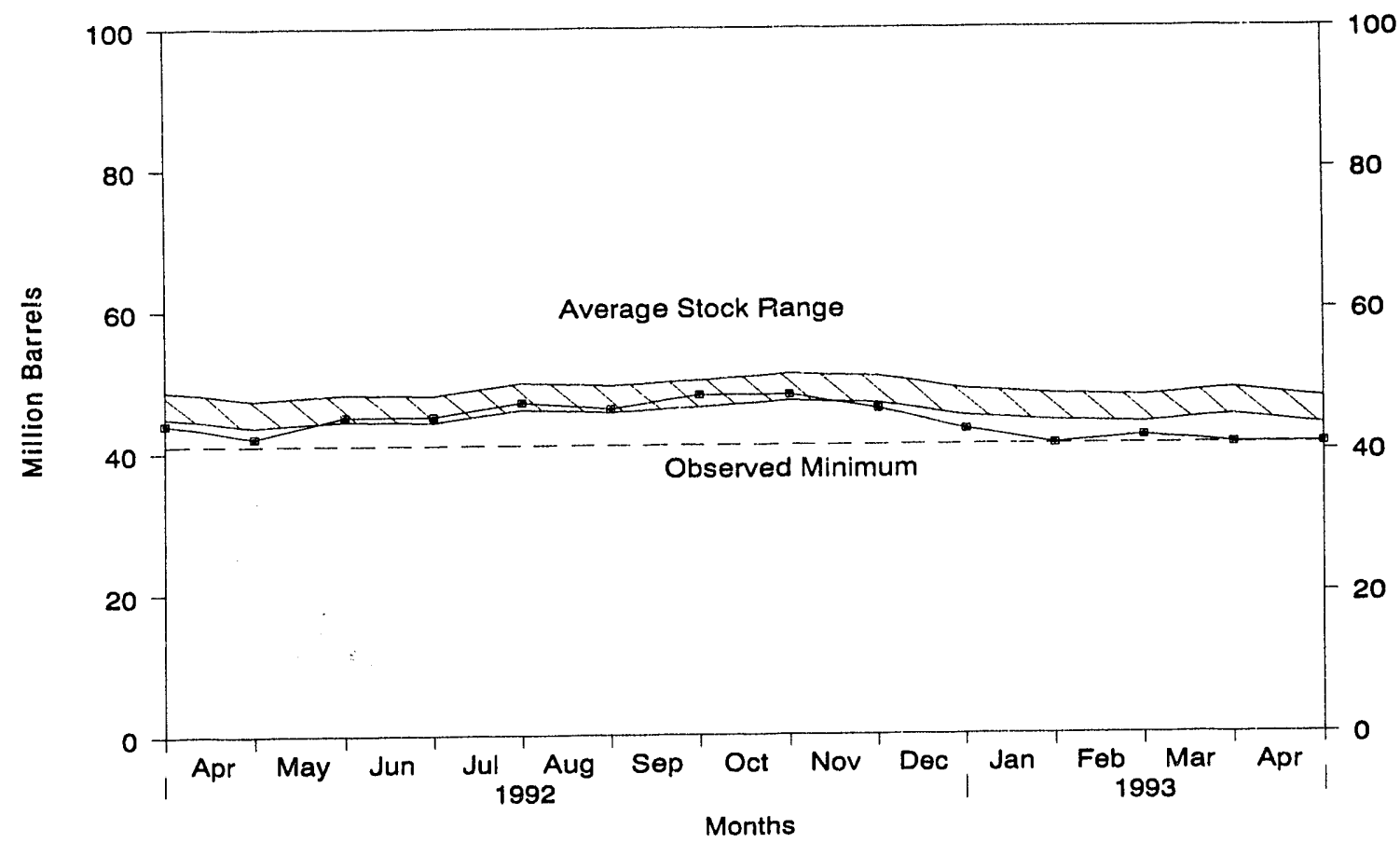

Note: The observed minimum for total stocks in the last 36-month period was 40.9 million barrels, occuring in December 1989.

Source: Energy Information Administration, Petroleum Supply Monthly, Table S7. See Summary Statistics Table and Figure Sources. 
Table S7. Jet Fuel Supply and Disposition, 1981 - Present (Thousand Barrels per Day, Except Where Noted)

\begin{tabular}{|c|c|c|c|c|c|c|c|c|c|c|}
\hline & \multirow[b]{3}{*}{ Year/Month } & \multicolumn{3}{|c|}{ Supply } & \multicolumn{4}{|c|}{ Dlsposition } & \multirow{2}{*}{\multicolumn{2}{|c|}{$\begin{array}{l}\text { Endling Stocksa } \\
\text { (Million Barrels) }\end{array}$}} \\
\hline & & \multicolumn{2}{|c|}{ Production } & \multirow[b]{2}{*}{ Imports } & \multirow[b]{2}{*}{$\begin{array}{c}\text { Stock } \\
\text { Changeb }\end{array}$} & \multirow[b]{2}{*}{ Exports } & \multicolumn{2}{|c|}{ Product Supplled } & & \\
\hline & & Total & Kerosene-Type & & & & Total & Kerosene-Type & Total & $\begin{array}{c}\text { Kerosene } \\
\text { Type }\end{array}$ \\
\hline $\begin{array}{l}1981 \\
1982 \\
1983 \\
1984 \\
1985 \\
1986 \\
1987 \\
1988 \\
1989 \\
1990\end{array}$ & 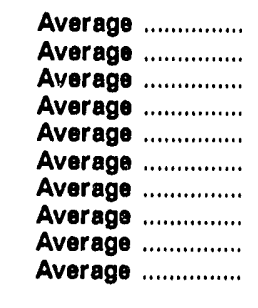 & $\begin{array}{r}968 \\
978 \\
1,022 \\
1,132 \\
1,189 \\
1,293 \\
1,343 \\
1,370 \\
1,403 \\
1,488\end{array}$ & $\begin{array}{r}775 \\
778 \\
817 \\
919 \\
983 \\
1,097 \\
1,138 \\
1,164 \\
1,197 \\
1,311\end{array}$ & $\begin{array}{r}38 \\
29 \\
29 \\
62 \\
39 \\
57 \\
67 \\
90 \\
106 \\
108\end{array}$ & $\begin{array}{r}c-4 \\
-12 \\
c(8) \\
9 \\
-4 \\
25 \\
(8) \\
-17 \\
-8 \\
31\end{array}$ & $\begin{array}{r}2 \\
6 \\
6 \\
9 \\
13 \\
18 \\
24 \\
28 \\
27 \\
43\end{array}$ & $\begin{array}{l}1,007 \\
1,013 \\
1,046 \\
1,175 \\
1,218 \\
1,307 \\
1,385 \\
1,449 \\
1,489 \\
1,522\end{array}$ & $\begin{array}{r}809 \\
804 \\
839 \\
953 \\
1,005 \\
1,105 \\
1,181 \\
1,236 \\
1,284 \\
1,340\end{array}$ & $\begin{array}{r}41 \\
c \quad 37 \\
39 \\
42 \\
40 \\
50 \\
50 \\
44 \\
41 \\
52\end{array}$ & $\begin{array}{r}34 \\
c 31 \\
32 \\
35 \\
34 \\
43 \\
42 \\
38 \\
34 \\
46\end{array}$ \\
\hline 1391 & 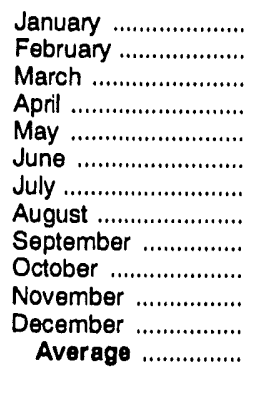 & $\begin{array}{l}1,509 \\
1,548 \\
1,299 \\
1,286 \\
1,367 \\
1,473 \\
1,426 \\
1,486 \\
1,495 \\
1,415 \\
1,433 \\
1,530 \\
1,438\end{array}$ & $\begin{array}{l}1,354 \\
1,384 \\
1,157 \\
1,135 \\
1,191 \\
1,300 \\
1,255 \\
1,316 \\
1,322 \\
1,253 \\
1,276 \\
1,357 \\
1,274\end{array}$ & $\begin{array}{l}67 \\
44 \\
65 \\
73 \\
87 \\
64 \\
67 \\
88 \\
92 \\
59 \\
56 \\
42 \\
67\end{array}$ & $\begin{array}{r}-55 \\
-108 \\
-99 \\
-8 \\
85 \\
58 \\
-47 \\
21 \\
71 \\
-66 \\
15 \\
22 \\
-9\end{array}$ & $\begin{array}{r}73 \\
159 \\
40 \\
38 \\
35 \\
13 \\
31 \\
11 \\
10 \\
50 \\
5 \\
59 \\
43\end{array}$ & $\begin{array}{l}1,559 \\
1,541 \\
1,423 \\
1,329 \\
1,334 \\
1,465 \\
1,509 \\
1,543 \\
1,506 \\
1,489 \\
1,469 \\
1,492 \\
1,471\end{array}$ & $\begin{array}{l}1,378 \\
1,360 \\
1,270 \\
1,173 \\
1,143 \\
1,280 \\
1,343 \\
1,343 \\
1,321 \\
1,319 \\
1,282 \\
1,338 \\
1,296\end{array}$ & $\begin{array}{l}50 \\
47 \\
44 \\
44 \\
47 \\
48 \\
47 \\
48 \\
50 \\
48 \\
48 \\
49 \\
-\end{array}$ & $\begin{array}{l}44 \\
41 \\
38 \\
38 \\
41 \\
43 \\
41 \\
42 \\
45 \\
43 \\
44 \\
44 \\
--\end{array}$ \\
\hline 1992 & 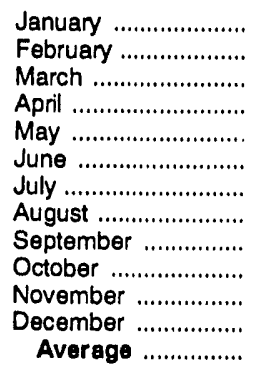 & $\begin{array}{l}1,350 \\
1,313 \\
1,347 \\
1,284 \\
1,390 \\
1,374 \\
1,473 \\
1,471 \\
1,448 \\
1,408 \\
1,457 \\
1,460 \\
1,398\end{array}$ & $\begin{array}{l}1,199 \\
1,166 \\
1,215 \\
1,131 \\
1,214 \\
1,234 \\
1,328 \\
1,339 \\
1,296 \\
1,265 \\
1,319 \\
1,333 \\
1,254\end{array}$ & $\begin{array}{r}39 \\
56 \\
56 \\
59 \\
86 \\
86 \\
81 \\
103 \\
93 \\
107 \\
90 \\
102 \\
80\end{array}$ & $\begin{array}{r}-133 \\
-63 \\
29 \\
-71 \\
120 \\
-20 \\
57 \\
-29 \\
77 \\
-9 \\
-41 \\
-101 \\
-15\end{array}$ & $\begin{array}{r}44 \\
42 \\
7 \\
18 \\
26 \\
45 \\
62 \\
28 \\
20 \\
41 \\
59 \\
112 \\
43\end{array}$ & $\begin{array}{l}1,477 \\
1,390 \\
1,367 \\
1,396 \\
1,330 \\
1,435 \\
1,435 \\
1,575 \\
1,443 \\
1,479 \\
1,529 \\
1,552 \\
1,451\end{array}$ & $\begin{array}{l}1,321 \\
1,243 \\
1,221 \\
1,247 \\
1,186 \\
1,306 \\
1,284 \\
1,423 \\
1,317 \\
1,313 \\
1,413 \\
1,407 \\
1,307\end{array}$ & $\begin{array}{r}45 \\
43 \\
44 \\
42 \\
45 \\
45 \\
47 \\
46 \\
48 \\
48 \\
46 \\
43 \\
-\end{array}$ & $\begin{array}{c}40 \\
38 \\
39 \\
37 \\
40 \\
39 \\
42 \\
41 \\
43 \\
43 \\
42 \\
39 \\
--\end{array}$ \\
\hline 1993 & 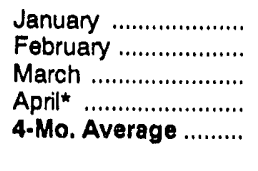 & $\begin{array}{r}1,437 \\
1,442 \\
R 1,463 \\
E_{1,420} \\
E_{1,441}\end{array}$ & $\begin{array}{r}1,306 \\
1,318 \\
R_{1,332} \\
E_{1,292} \\
1,312\end{array}$ & $\begin{array}{r}89 \\
110 \\
R \quad 102 \\
E 79 \\
E_{95}\end{array}$ & $\begin{array}{r}-73 \\
46 \\
R-29 \\
E-62 \\
E-31\end{array}$ & $\begin{array}{r}134 \\
17 \\
R_{101} \\
E_{48} \\
E_{77}\end{array}$ & $\begin{array}{r}1,464 \\
1,488 \\
R \quad 1,493 \\
E_{1,514} \\
1,490\end{array}$ & $\begin{array}{r}1,371 \\
1,346 \\
R 1,371 \\
E_{1,371} \\
E_{1,365}\end{array}$ & $\begin{array}{r}41 \\
42 \\
\mathrm{R}_{41}^{41} \\
41 \\
-\cdot\end{array}$ & $\begin{array}{r}36 \\
38 \\
\mathrm{R}_{37} \\
37 \\
. .\end{array}$ \\
\hline $\begin{array}{l}1992 \\
1991\end{array}$ & $\begin{array}{l}\text { 4-Mo. Average ......... } \\
\text { 4-Mo. Average ......... }\end{array}$ & $\begin{array}{l}1,324 \\
1,408\end{array}$ & $\begin{array}{l}1,178 \\
1,255\end{array}$ & $\begin{array}{l}52 \\
63\end{array}$ & $\begin{array}{l}-59 \\
-67\end{array}$ & $\begin{array}{l}28 \\
76\end{array}$ & $\begin{array}{l}1,408 \\
1,462\end{array}$ & $\begin{array}{l}1,258 \\
1,295\end{array}$ & $\ddot{-}$ & $\ddot{-.}$ \\
\hline
\end{tabular}

a Stocks are totals as of end of period.

- A negative number indicates a decrease in stocks and a positive number indicates an increase.

c In January 1981, 1983, and 1984, a new stock basis was established affecting stocks reported and stock change calculations. Stock changes are calculated using new basis stock levels. See Summary Statistics Explanatory Note 4.

$(s)=$ Less than 500 barrels per day. E= Estimated.

* See Summary Statistics Explanatory Note 1. Notes: - Italics denote estimates based upon preliminary data. Geographic coverage is the 50 States and the District of Columbia. - Totals may not equal
sum of components due to independent rounding.

Source: See Summary Statistics Table and Figure Sources. 
Figure S13. Propane/Propylene Supply and Disposition, February 1992 - Present

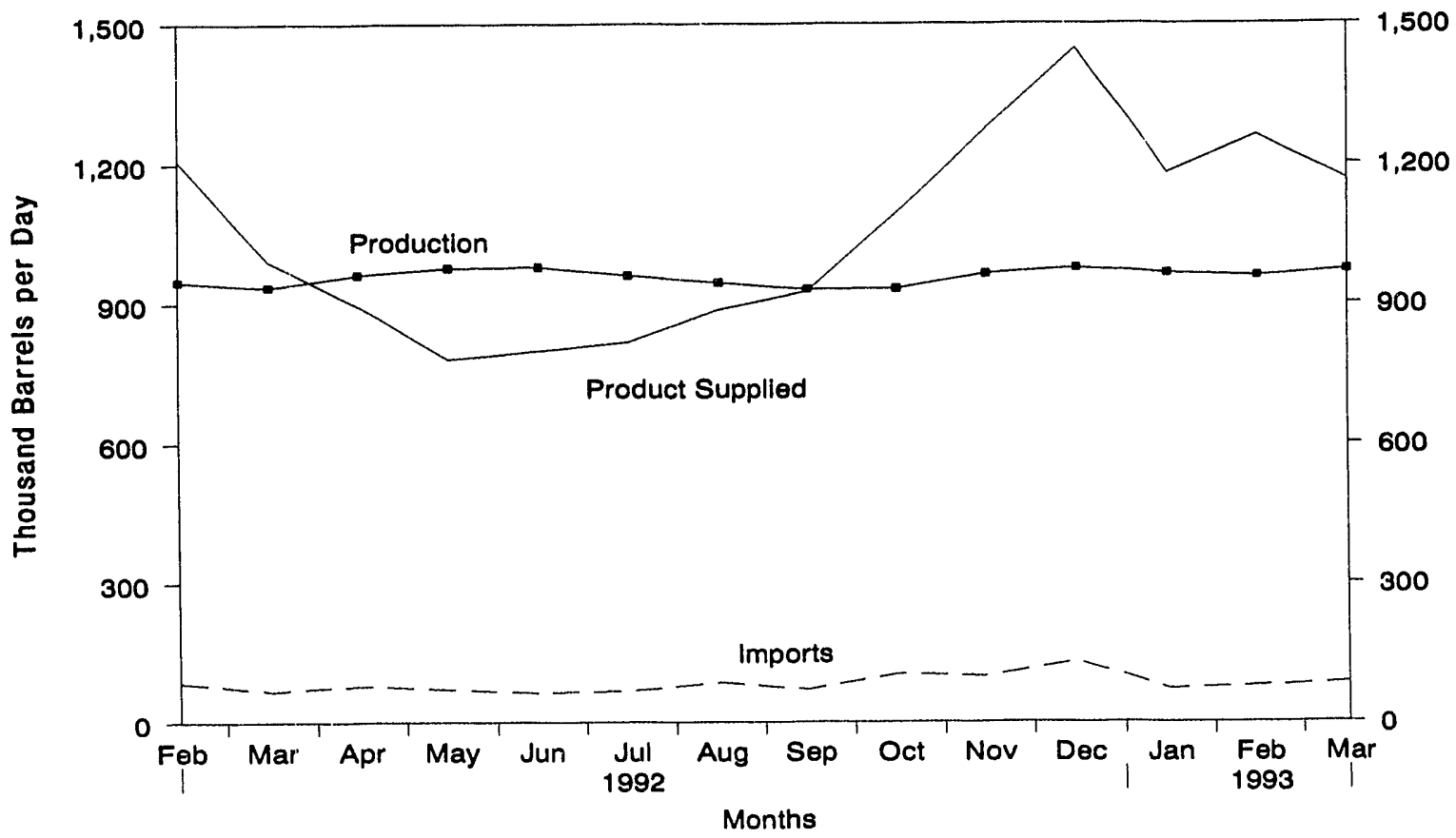

Source: Energy Information Administration, Petroleum Supply Monthly, Table S8. See Summary Statistics Table and Figure Sources.

Figure S14. Propane/Propylene Ending Stocks, February 1992 - Present

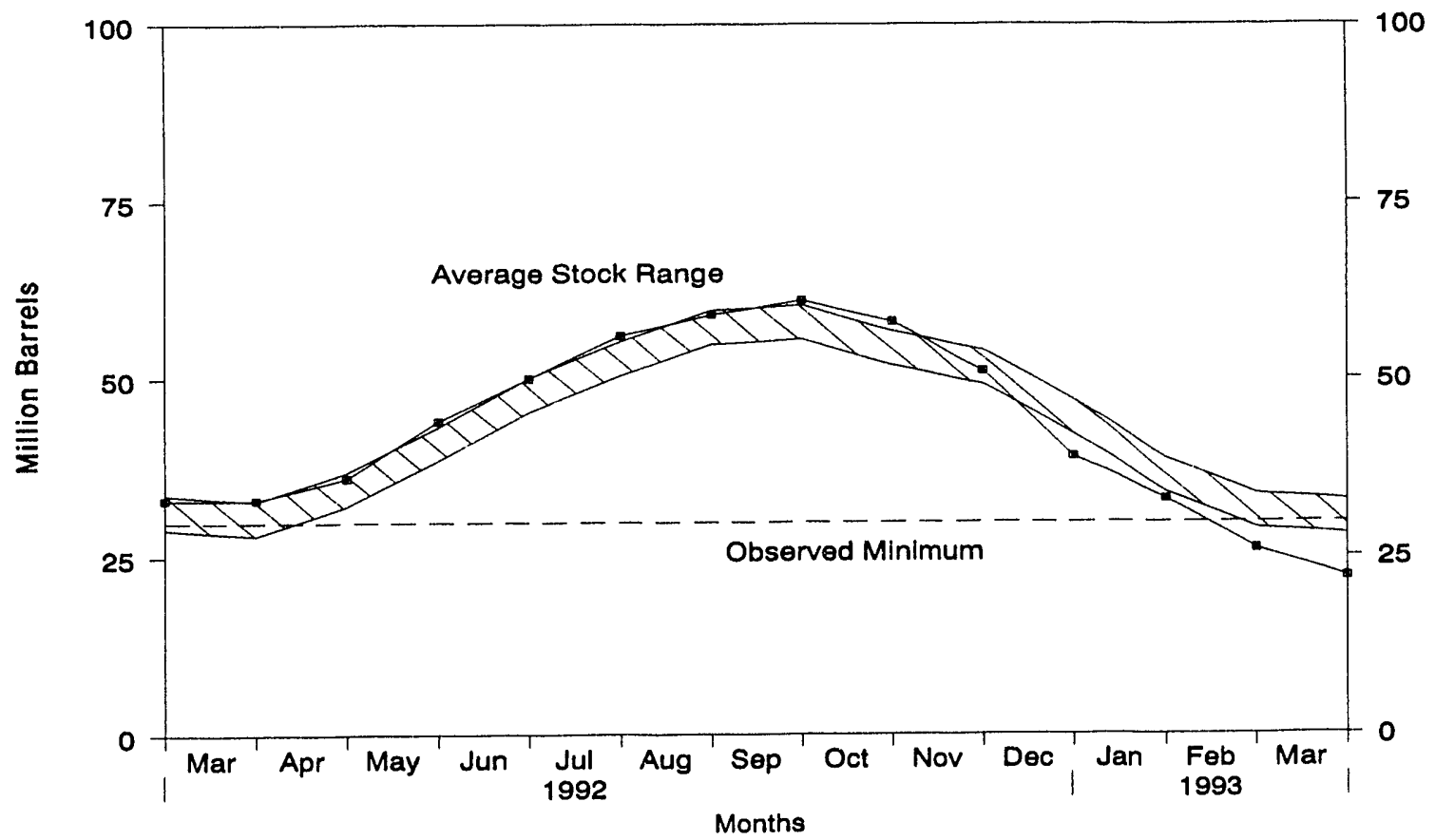

Note: The Observed Minimum for propane stocks in the last 36 month period was 29.8 million barrels, occurring in March 1991.

Source: Energy Information Administration, Petroleum Supply Monthly, Table S8. See Summary Statistics Table and Figure Sources. 
Table S8. Propane/Propylene Supply and Disposition, 1981 - Present (Thousand Barrels per Day, Except Where Noted)

\begin{tabular}{|c|c|c|c|c|c|c|c|c|}
\hline & \multirow[b]{2}{*}{ Year/Month } & \multicolumn{2}{|c|}{ Supply } & \multicolumn{4}{|c|}{ Disposition } & \multirow[b]{2}{*}{$\begin{array}{c}\text { Ending } \\
\text { Stocks } \\
\text { (Million Barrels) }\end{array}$} \\
\hline & & $\begin{array}{c}\text { Total } \\
\text { Production }\end{array}$ & Imports & $\begin{array}{l}\text { Stock } \\
\text { Change }\end{array}$ & $\begin{array}{l}\text { Reflnery } \\
\text { Inputs }\end{array}$ & Exports & $\begin{array}{l}\text { Product } \\
\text { Supplled }\end{array}$ & \\
\hline $\begin{array}{l}1981 \\
1982 \\
1983 \\
1984 \\
1985 \\
1986 \\
1987 \\
1988 \\
1989 \\
1990\end{array}$ & 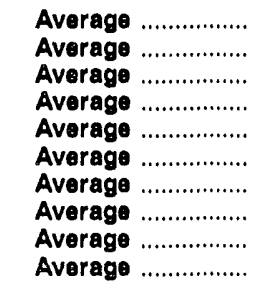 & $\begin{array}{l}745 \\
711 \\
730 \\
806 \\
816 \\
817 \\
828 \\
863 \\
862 \\
878\end{array}$ & $\begin{array}{r}70 \\
63 \\
14 \\
67 \\
67 \\
110 \\
88 \\
106 \\
111 \\
115\end{array}$ & $\begin{array}{r}c 18 \\
-59 \\
c-24 \\
c 7 \\
-50 \\
64 \\
-41 \\
7 \\
-52 \\
48\end{array}$ & $\begin{array}{r}5 \\
4 \\
4 \\
4 \\
3 \\
4 \\
8 \\
8 \\
11 \\
(8)\end{array}$ & $\begin{array}{l}18 \\
31 \\
43 \\
30 \\
48 \\
28 \\
24 \\
31 \\
24 \\
28\end{array}$ & $\begin{array}{l}773 \\
798 \\
751 \\
833 \\
883 \\
831 \\
924 \\
923 \\
990 \\
917\end{array}$ & $\begin{array}{r}76 \\
c 54 \\
c 48 \\
58 \\
39 \\
63 \\
48 \\
50 \\
32 \\
49\end{array}$ \\
\hline 1991 & 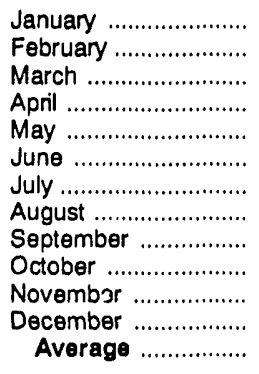 & $\begin{array}{l}920 \\
923 \\
912 \\
900 \\
922 \\
906 \\
901 \\
891 \\
905 \\
902 \\
930 \\
964 \\
915\end{array}$ & $\begin{array}{r}105 \\
90 \\
56 \\
101 \\
90 \\
81 \\
91 \\
73 \\
92 \\
146 \\
82 \\
86 \\
91\end{array}$ & $\begin{array}{r}-449 \\
-174 \\
-10 \\
179 \\
214 \\
223 \\
81 \\
40 \\
-22 \\
35 \\
-37 \\
-128 \\
3\end{array}$ & $\begin{array}{r}0 \\
0 \\
0 \\
0 \\
0 \\
0 \\
0 \\
0 \\
0 \\
0 \\
0 \\
(\mathbf{s}) \\
(8)\end{array}$ & $\begin{array}{l}51 \\
40 \\
45 \\
25 \\
31 \\
22 \\
15 \\
13 \\
14 \\
18 \\
20 \\
38 \\
28\end{array}$ & $\begin{array}{r}1,422 \\
1,147 \\
933 \\
798 \\
767 \\
741 \\
895 \\
910 \\
1,006 \\
995 \\
1,030 \\
1,139 \\
982\end{array}$ & $\begin{array}{l}35 \\
30 \\
30 \\
35 \\
42 \\
49 \\
51 \\
52 \\
52 \\
53 \\
52 \\
48 \\
--\end{array}$ \\
\hline 1992 & 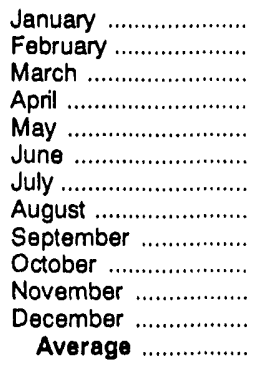 & $\begin{array}{l}946 \\
948 \\
936 \\
962 \\
977 \\
979 \\
961 \\
945 \\
931 \\
932 \\
963 \\
976 \\
954\end{array}$ & $\begin{array}{r}90 \\
86 \\
68 \\
79 \\
71 \\
64 \\
68 \\
85 \\
71 \\
104 \\
99 \\
131 \\
85\end{array}$ & $\begin{array}{r}-260 \\
-201 \\
-16 \\
120 \\
244 \\
216 \\
176 \\
118 \\
50 \\
-87 \\
-245 \\
-385 \\
-22\end{array}$ & $\begin{array}{r}(s) \\
(s) \\
2 \\
0 \\
(s) \\
(s) \\
(s) \\
(s) \\
(s) \\
(s) \\
0 \\
0 \\
(8)\end{array}$ & $\begin{array}{l}72 \\
27 \\
26 \\
24 \\
23 \\
27 \\
35 \\
25 \\
25 \\
30 \\
33 \\
45 \\
33\end{array}$ & $\begin{array}{r}1,223 \\
1,208 \\
991 \\
897 \\
781 \\
799 \\
818 \\
887 \\
926 \\
1,093 \\
1,274 \\
1,447 \\
1,028\end{array}$ & $\begin{array}{r}39 \\
33 \\
33 \\
36 \\
44 \\
50 \\
56 \\
59 \\
61 \\
58 \\
51 \\
39 \\
. .\end{array}$ \\
\hline 1993 & 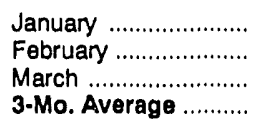 & $\begin{array}{l}965 \\
959 \\
971 \\
965\end{array}$ & $\begin{array}{l}72 \\
78 \\
85 \\
78\end{array}$ & $\begin{array}{l}-173 \\
-261 \\
-140 \\
-189\end{array}$ & $\begin{array}{l}1 \\
(s) \\
(s) \\
(8)\end{array}$ & $\begin{array}{l}31 \\
37 \\
32 \\
33\end{array}$ & $\begin{array}{l}1,179 \\
1,261 \\
1,165 \\
1,200\end{array}$ & $\begin{array}{r}33 \\
26 \\
22 \\
-\cdots\end{array}$ \\
\hline $\begin{array}{l}1992 \\
1991\end{array}$ & $\begin{array}{l}\text { 3-Mo. Average .......... } \\
\text { 3-Mo. Average ......... }\end{array}$ & $\begin{array}{l}943 \\
918\end{array}$ & $\begin{array}{l}81 \\
83\end{array}$ & $\begin{array}{l}-158 \\
-212\end{array}$ & $\begin{array}{l}1 \\
0\end{array}$ & $\begin{array}{l}42 \\
46\end{array}$ & $\begin{array}{l}1,140 \\
1,168\end{array}$ & $\ddot{~}$ \\
\hline
\end{tabular}

A negative number indicates a decrease in stocks and a positive number indicates an increase.

b Stocks are totals as of end of period.

c In January 1981, 1983, and 1984, a new stock basis was established affecting stocks reported and stock change calculations. Stock changes are calculated using new basis stock levels. See Summary Statistics Explanatory Note 4.

Notes: - Geographic coverage is the 50 States and the District of Columbia. - Totals may not equal sum of components due to independent rounding.

Source: See Summary Statistics Table and Figure Sources. 
Figure S15. Liquefled Petroleum Gases Supply and Disposition, February 1992 - Present

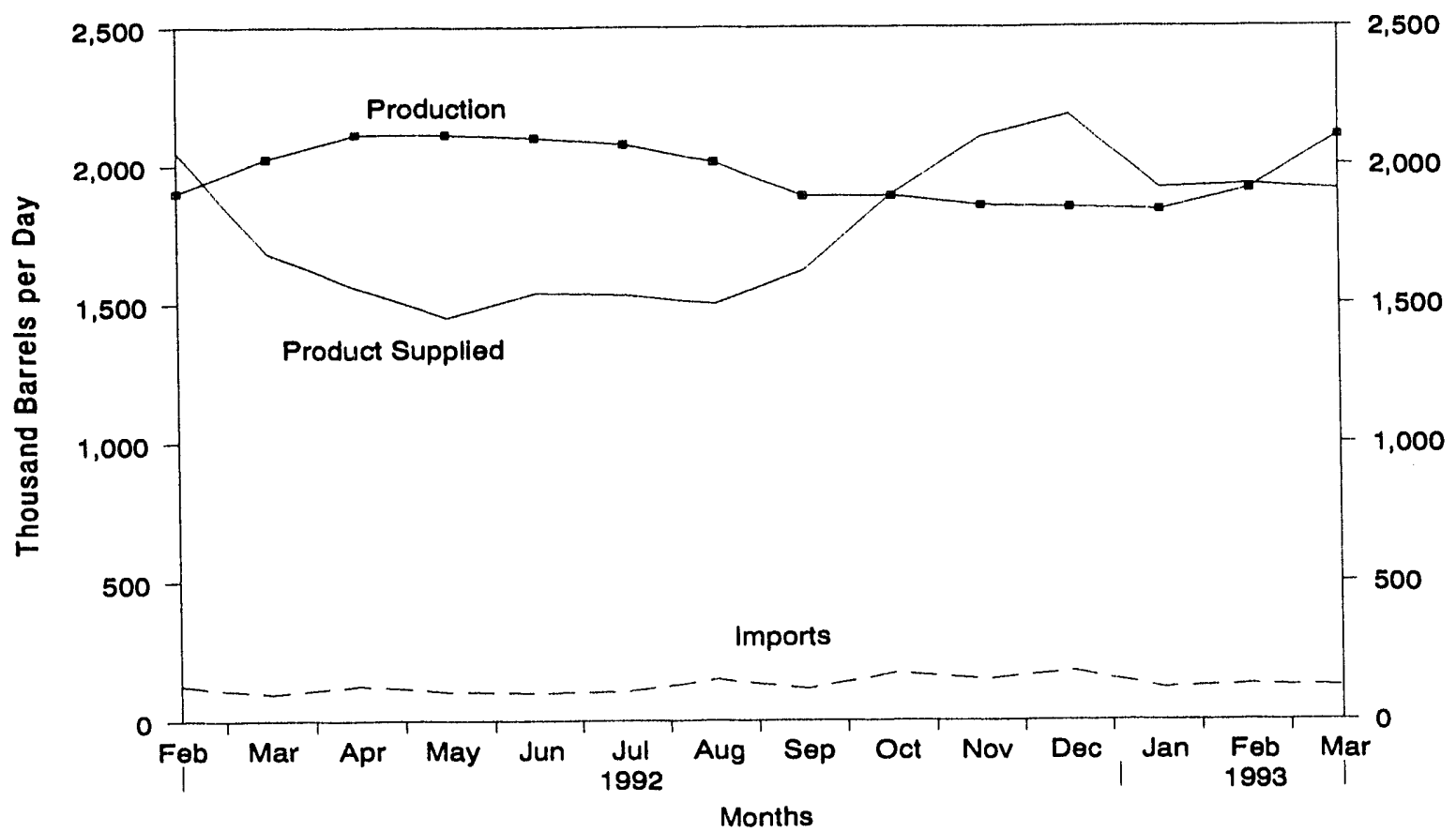

Source: Energy Information Administration, Petroleum Supply Monthly, Table S9. See Summary Statistics Table and Figure Sources.

Figure S16. Liquefied Petroleum Gases Ending Stocks, February 1992 - Present

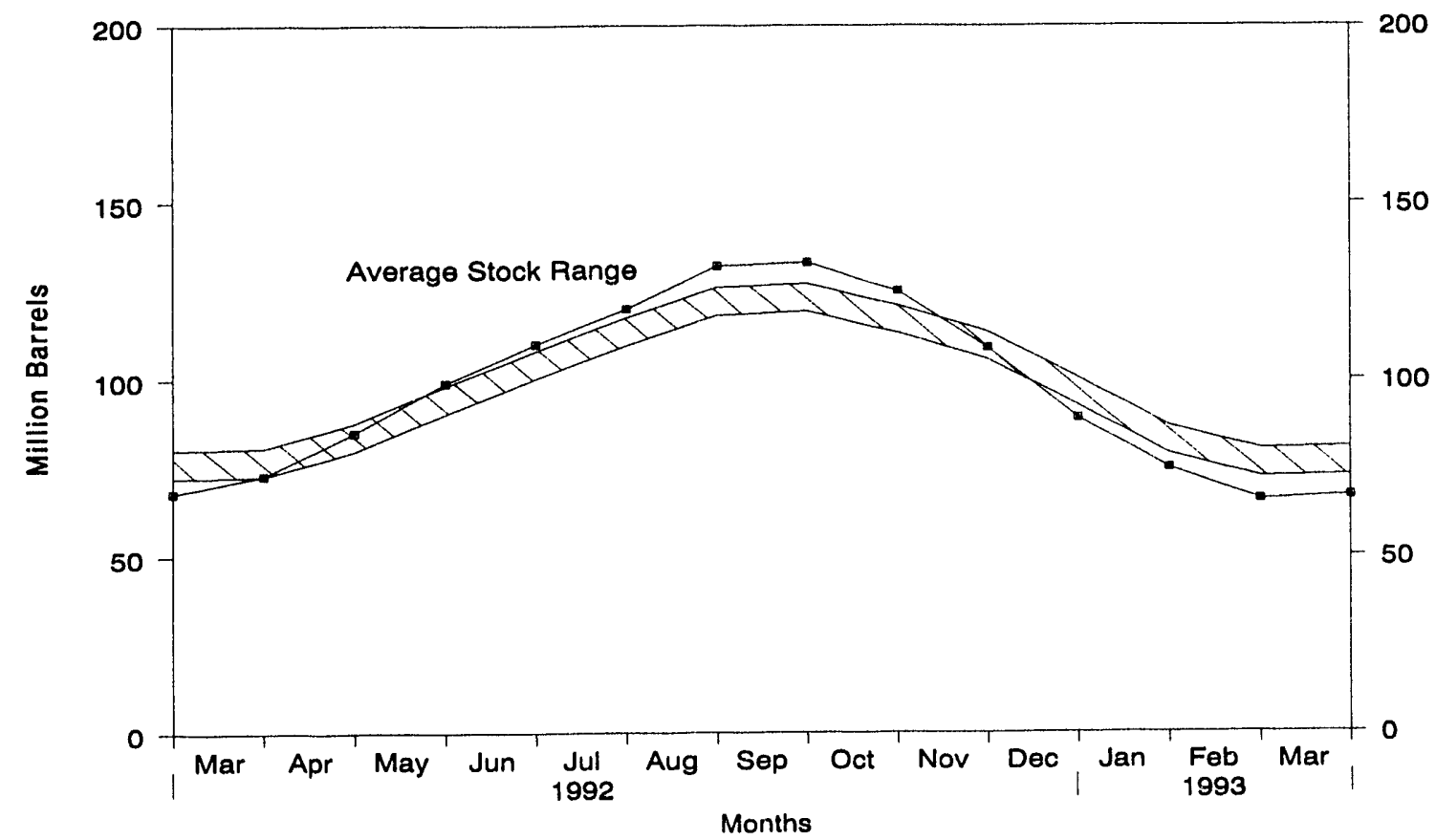

Source: Energy Information Administration, Petroleum Supply Monthly, Table S9. See Summary Statistics Table and Figure Sources. 
Table S9. Liquefied Petroleum Gases Supply and Disposition, 1981 - Present (Thousand Barrels per Day, Except Where Noted)

\begin{tabular}{|c|c|c|c|c|c|c|c|c|}
\hline & \multirow[b]{2}{*}{ Year/Month } & \multicolumn{2}{|c|}{ Supply } & \multicolumn{4}{|c|}{ Disposition } & \multirow[b]{2}{*}{$\begin{array}{c}\text { Ending } \\
\text { Stocks } \\
\text { (Million Barrels) }\end{array}$} \\
\hline & & $\begin{array}{c}\text { Total } \\
\text { Production }\end{array}$ & Imports & $\begin{array}{l}\text { Stock } \\
\text { Change }\end{array}$ & $\begin{array}{l}\text { Refinery } \\
\text { Inputs }\end{array}$ & Exports & $\begin{array}{l}\text { Product } \\
\text { Supplled }\end{array}$ & \\
\hline $\begin{array}{l}1981 \\
1982 \\
1983 \\
1984 \\
1985 \\
1986 \\
1987 \\
1988 \\
1989 \\
1990\end{array}$ & 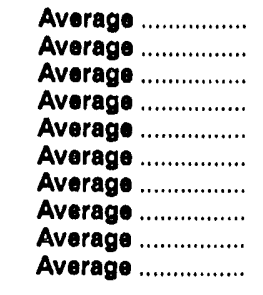 & $\begin{array}{l}1,571 \\
1,528 \\
1,642 \\
1,697 \\
1,704 \\
1,695 \\
1,748 \\
1,817 \\
1,791 \\
1,749\end{array}$ & $\begin{array}{l}244 \\
226 \\
190 \\
195 \\
187 \\
242 \\
190 \\
209 \\
181 \\
188\end{array}$ & $\begin{array}{r}c 18 \\
-111 \\
c-4 \\
c-19 \\
-75 \\
80 \\
-15 \\
1 \\
-47 \\
48\end{array}$ & $\begin{array}{l}289 \\
300 \\
253 \\
291 \\
304 \\
302 \\
304 \\
321 \\
315 \\
293\end{array}$ & $\begin{array}{l}42 \\
65 \\
73 \\
48 \\
62 \\
42 \\
38 \\
49 \\
35 \\
40\end{array}$ & $\begin{array}{l}1,468 \\
1,499 \\
1,509 \\
1,572 \\
1,599 \\
1,512 \\
1,612 \\
1,656 \\
1,668 \\
1,556\end{array}$ & $\begin{array}{r}135 \\
c 94 \\
c 101 \\
101 \\
74 \\
103 \\
97 \\
97 \\
80 \\
98\end{array}$ \\
\hline 1991 & 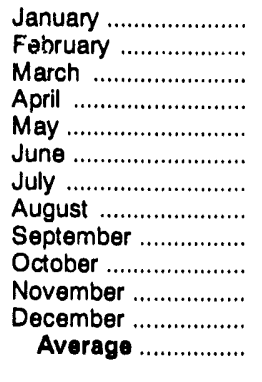 & $\begin{array}{l}1,753 \\
1,865 \\
1,942 \\
1,937 \\
1,989 \\
1,949 \\
1,913 \\
1,899 \\
1,806 \\
1,805 \\
1,789 \\
1,810 \\
1,871\end{array}$ & $\begin{array}{r}148 \\
126 \\
91 \\
154 \\
129 \\
148 \\
151 \\
143 \\
147 \\
233 \\
156 \\
139 \\
147\end{array}$ & $\begin{array}{r}-658 \\
-271 \\
113 \\
346 \\
428 \\
328 \\
211 \\
175 \\
-84 \\
33 \\
-330 \\
-488 \\
-15\end{array}$ & $\begin{array}{l}364 \\
322 \\
249 \\
237 \\
239 \\
245 \\
253 \\
255 \\
288 \\
345 \\
413 \\
437 \\
304\end{array}$ & $\begin{array}{l}56 \\
60 \\
56 \\
31 \\
45 \\
32 \\
24 \\
18 \\
31 \\
31 \\
40 \\
73 \\
41\end{array}$ & $\begin{array}{l}2,139 \\
1,880 \\
1,615 \\
1,477 \\
1,407 \\
1,492 \\
1,575 \\
1,594 \\
1,718 \\
1,629 \\
1,821 \\
1,927 \\
1,689\end{array}$ & $\begin{array}{r}78 \\
70 \\
73 \\
84 \\
97 \\
107 \\
113 \\
119 \\
116 \\
117 \\
107 \\
92 \\
\cdots\end{array}$ \\
\hline 1992 & 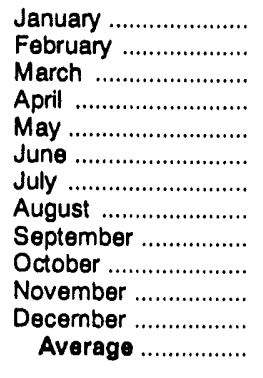 & $\begin{array}{l}1,814 \\
1,901 \\
2,025 \\
2,114 \\
2,113 \\
2,101 \\
2,077 \\
2,013 \\
1,888 \\
1,888 \\
1,853 \\
1,846 \\
1,970\end{array}$ & $\begin{array}{r}139 \\
126 \\
97 \\
126 \\
105 \\
100 \\
106 \\
148 \\
114 \\
170 \\
148 \\
176 \\
130\end{array}$ & $\begin{array}{r}-417 \\
-366 \\
158 \\
401 \\
477 \\
344 \\
343 \\
372 \\
36 \\
-239 \\
-546 \\
-659 \\
-7\end{array}$ & $\begin{array}{l}378 \\
312 \\
236 \\
235 \\
245 \\
257 \\
255 \\
233 \\
302 \\
368 \\
403 \\
451 \\
306\end{array}$ & $\begin{array}{l}80 \\
33 \\
43 \\
45 \\
44 \\
59 \\
52 \\
55 \\
45 \\
39 \\
43 \\
49 \\
49\end{array}$ & $\begin{array}{l}1,912 \\
2,048 \\
1,684 \\
1,559 \\
1,452 \\
1,541 \\
1,533 \\
1,501 \\
1,620 \\
1,892 \\
2,100 \\
2,182 \\
1,751\end{array}$ & $\begin{array}{r}78 \\
68 \\
73 \\
85 \\
99 \\
110 \\
120 \\
132 \\
133 \\
125 \\
109 \\
89 \\
\cdots\end{array}$ \\
\hline 1993 & 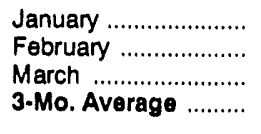 & $\begin{array}{l}1,837 \\
1,912 \\
2,106 \\
1,953\end{array}$ & $\begin{array}{l}117 \\
128 \\
123 \\
122\end{array}$ & $\begin{array}{r}-441 \\
-310 \\
9 \\
-245\end{array}$ & $\begin{array}{l}440 \\
367 \\
263 \\
356\end{array}$ & $\begin{array}{l}39 \\
55 \\
47 \\
46\end{array}$ & $\begin{array}{l}1,917 \\
1,928 \\
1,910 \\
1,918\end{array}$ & $\begin{array}{r}75 \\
66 \\
67 \\
\cdots\end{array}$ \\
\hline $\begin{array}{l}1992 \\
1991\end{array}$ & $\begin{array}{l}\text { 3-Mo. Average ......... } \\
\text { 3-Mo. Average ......... }\end{array}$ & $\begin{array}{l}1,913 \\
1,853\end{array}$ & $\begin{array}{l}121 \\
122\end{array}$ & $\begin{array}{l}-205 \\
-272\end{array}$ & $\begin{array}{l}309 \\
311\end{array}$ & $\begin{array}{l}\mathbf{5 2} \\
\mathbf{5 7}\end{array}$ & $\begin{array}{l}1,878 \\
1,878\end{array}$ & $\ddot{*}$ \\
\hline
\end{tabular}


Table S10. Other Petroleum Products Supply and Dispositlon, 1981 - Present (Thousand Barrels per Day, Except Where Noted)

\begin{tabular}{|c|c|c|c|c|c|c|c|c|}
\hline & \multirow[b]{2}{*}{ Year/Month } & \multicolumn{2}{|c|}{ Supply } & \multicolumn{4}{|c|}{ Dlapostiton } & \multirow[b]{2}{*}{$\begin{array}{c}\text { Ending } \\
\text { Stocke } \\
\text { (Mililion Barrele) }\end{array}$} \\
\hline & & $\begin{array}{l}\text { Total } \\
\text { Productlon }\end{array}$ & Imports & $\begin{array}{c}\text { Stock } \\
\text { Change }\end{array}$ & $\begin{array}{l}\text { Retinery } \\
\text { Inputs }\end{array}$ & Exports & $\begin{array}{l}\text { Products } \\
\text { Supplled }\end{array}$ & \\
\hline $\begin{array}{l}1981 \\
1982 \\
1983 \\
1984 \\
1985 \\
1986 \\
1987 \\
1988 \\
1989 \\
1990\end{array}$ & 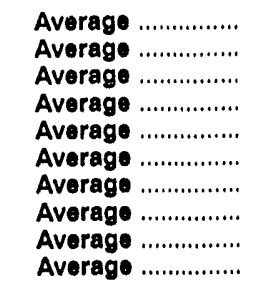 & $\begin{array}{l}2,771 \\
2,475 \\
2,437 \\
2,500 \\
2,532 \\
2,704 \\
2,737 \\
2,773 \\
2,771 \\
2,842\end{array}$ & $\begin{array}{l}188 \\
305 \\
382 \\
503 \\
550 \\
504 \\
543 \\
645 \\
627 \\
705\end{array}$ & $\begin{array}{r}c .42 \\
-68 \\
c-6 \\
c .32 \\
-62 \\
-15 \\
-1 \\
22 \\
12 \\
.32\end{array}$ & $\begin{array}{l}723 \\
787 \\
712 \\
791 \\
886 \\
888 \\
829 \\
799 \\
797 \\
887\end{array}$ & $\begin{array}{l}197 \\
205 \\
236 \\
236 \\
227 \\
291 \\
264 \\
294 \\
305 \\
289\end{array}$ & $\begin{array}{l}2,081 \\
1,856 \\
1,877 \\
2,007 \\
1,947 \\
2,045 \\
2,187 \\
2,303 \\
2,285 \\
2,402\end{array}$ & $\begin{array}{l}241 \\
c_{2} 216 \\
217 \\
198 \\
208 \\
201 \\
200 \\
208 \\
213 \\
201\end{array}$ \\
\hline 1991 & 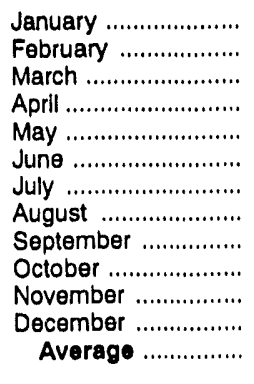 & $\begin{array}{l}2,653 \\
2,668 \\
2,576 \\
2,724 \\
2,853 \\
3,030 \\
3,029 \\
2,993 \\
3,010 \\
2,824 \\
2,750 \\
2,797 \\
2,826\end{array}$ & $\begin{array}{l}748 \\
573 \\
551 \\
607 \\
800 \\
615 \\
776 \\
642 \\
746 \\
611 \\
850 \\
577 \\
675\end{array}$ & $\begin{array}{r}204 \\
363 \\
151 \\
133 \\
198 \\
-123 \\
-143 \\
-169 \\
101 \\
-218 \\
-81 \\
-163 \\
18\end{array}$ & $\begin{array}{r}844 \\
726 \\
819 \\
753 \\
900 \\
1,092 \\
1,081 \\
1,013 \\
802 \\
944 \\
1,093 \\
1,147 \\
936\end{array}$ & $\begin{array}{l}317 \\
275 \\
239 \\
228 \\
327 \\
304 \\
321 \\
296 \\
267 \\
211 \\
238 \\
304 \\
277\end{array}$ & $\begin{array}{l}2,036 \\
1,876 \\
1,919 \\
2,217 \\
2,228 \\
2,372 \\
2,545 \\
2,496 \\
2,586 \\
2,498 \\
2,349 \\
2,085 \\
2,269\end{array}$ & $\begin{array}{r}207 \\
217 \\
222 \\
226 \\
232 \\
228 \\
224 \\
219 \\
222 \\
215 \\
213 \\
208 \\
\cdots\end{array}$ \\
\hline 1992 & 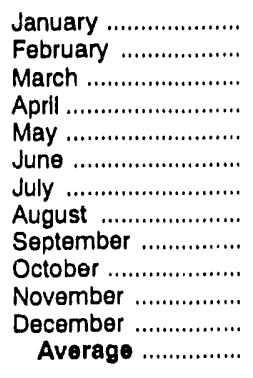 & $\begin{array}{l}2,704 \\
2,645 \\
2,735 \\
2,869 \\
2,901 \\
3,078 \\
3,162 \\
3,019 \\
3,064 \\
2,899 \\
2,875 \\
2,832 \\
2,899\end{array}$ & $\begin{array}{l}713 \\
574 \\
710 \\
797 \\
661 \\
645 \\
735 \\
726 \\
744 \\
701 \\
697 \\
711 \\
702\end{array}$ & $\begin{array}{r}197 \\
177 \\
243 \\
-34 \\
-87 \\
-60 \\
-152 \\
-118 \\
189 \\
-199 \\
-7 \\
-185 \\
-4\end{array}$ & $\begin{array}{r}815 \\
928 \\
721 \\
1,047 \\
899 \\
765 \\
973 \\
850 \\
640 \\
927 \\
964 \\
1,210 \\
895\end{array}$ & $\begin{array}{l}272 \\
240 \\
239 \\
217 \\
199 \\
225 \\
284 \\
227 \\
336 \\
295 \\
264 \\
352 \\
263\end{array}$ & $\begin{array}{l}2,135 \\
1,875 \\
2,242 \\
2,436 \\
2,551 \\
2,793 \\
2,791 \\
2,785 \\
2,642 \\
2,578 \\
2,350 \\
2,167 \\
2,447\end{array}$ & $\begin{array}{r}214 \\
219 \\
226 \\
225 \\
223 \\
221 \\
216 \\
213 \\
218 \\
212 \\
212 \\
c \\
206 \\
-.\end{array}$ \\
\hline 1993 & $\begin{array}{l}\text { January ..................... } \\
\text { February .................... } \\
\text { March ..................... } \\
\text { 3-Mo. Average ........ }\end{array}$ & $\begin{array}{l}3,026 \\
2,815 \\
2,866 \\
2,905\end{array}$ & $\begin{array}{l}698 \\
773 \\
818 \\
763\end{array}$ & $\begin{array}{l}{ }^{c} 600 \\
122 \\
243 \\
328\end{array}$ & $\begin{array}{l}829 \\
949 \\
747 \\
838\end{array}$ & $\begin{array}{l}271 \\
282 \\
269 \\
274\end{array}$ & $\begin{array}{l}2,023 \\
2,235 \\
2,425 \\
2,227\end{array}$ & $\begin{array}{r}225 \\
228 \\
236 \\
-\end{array}$ \\
\hline $\begin{array}{l}1992 \\
1991\end{array}$ & $\begin{array}{l}\text { 3-Mo. Average ........ } \\
\text { 3-Mo. Average ........ }\end{array}$ & $\begin{array}{l}2,696 \\
2,631\end{array}$ & $\begin{array}{l}668 \\
626\end{array}$ & $\begin{array}{l}206 \\
235\end{array}$ & $\begin{array}{l}819 \\
799\end{array}$ & $\begin{array}{l}250 \\
277\end{array}$ & $\begin{array}{l}2,088 \\
1,946\end{array}$ & $\ddot{-}$ \\
\hline
\end{tabular}

\footnotetext{
a A negative number indlcates a decrease in stocks and a positive number indicates an increase.

b Stocks are totals as of end of period.

c In January 1981, 1983, and 1984, new stock basis was established affecting stocks reported and stock change calculations. Stock changes are calculated using new basis stock levels. Bulk terminal and pipeline stocks of oxygenates were added beginning in January 1993. See Summary Statistics Explanatory Note 4.

Notes: - Other petroleum products includes pentanes plus, other hydrocarbons and oxygenates, unfinished olls, gasoline blending components and all finished petroleum products except finished motor gasollne, distillate fuel oll, residual fuel all, jet fuel, and liquefled petroleum gases. • Geographic coverage is the 50 States and the District of Columbla. Totals may not equal sum of components due to independent rounding.

Source: See Summary Statistics Table and Figure Sources.
} 


\section{Summary Statistics Table and Figure Sources}

Information about petroleum supply and disposition at the National level are presented in the Summary Statistics tables. Industry terminology and product definitions are listed alphabetically in the Glossary.

The data presented in these tables are from several sources and represent different levels of timeliness and data finality.

- U.S. Department of Energy, Energy Information Administration (EIA), Petroleum Supply Annual (1981 through 1991).

- EIA, Petroleum Supply Monthly (January 1992 through March 1993).
- EIA, Weekly Petroleum Supply Reporting System (except domestic crude oil production) (April 1993). A more detailed explanation is provided in Summary Statistics Explanatory Note 1.

- Domestic crude oil production estimate is based on historical statistics from State conservation agencies and the Minerals Management Service of the U.S. Department of the Interior. (January 1992 through April 1993). Refer to Summary Statistics Explanatory Note 2 for a more detailed explanation. 


\section{Summary Statistics Explanatory Notes}

The following explanatory notes are provided to assist in understanding and interpreting the data presented in the Summary Statistics section of this publication.

\section{Note 1. Preliminary Monthly Statistics Derivation}

Data collected from the Weekly Petroleum Supply Reporting System (WPSRS) are used to develop estimates of the most current monthly quantities. The forms that comprise the WPSRS are:

$\begin{array}{ll}\text { Form Number } & \text { Name } \\ \text { ELA-800 } & \text { "Weekly Refinery Report" } \\ \text { EIA-801 } & \text { "Weekly Bulk Terminal Report" } \\ \text { ELA-802 } & \text { "Weekly Product Pipeline Report" } \\ \text { EIA-803 } & \text { "Weekly Crude Oil Stocks Report" } \\ \text { ELA-804 } & \text { "Weekly Imports Report" }\end{array}$

A sample of all petroleum companies report weekly data to the Energy Information Administration (ELA) on crude oil and petroleum products stocks, refinery inputs and production, and crude oil and petroleum product imports. The sample of companies that report weekly is selected from the universe of companies that report on the comparable monthly surveys.

The sampling procedure used for the weekly system is the cut-off method. In the cut-off method, companies are ranked from largest to smallest on the basis of the quantities reported during a 12-month period. Companies are chosen for the sample beginning with the largest companies with additional companies added until the total sample coverage represents a minimum of 90 percent of each item by geographic region being measured. All monthly-from-weekly estimates are shown in italics.

In calculating monthly estimates based upon weekly submissions, an interpolation process is used to make the weekly figures comparable to the monthly. The interpolation process is designed to resolve the timing differences between the weekly and the monthly systems -- the time-of-day of reporting periods and the day-of-month of reporting periods. The end of the weekly reporting period (exactly 1 week long) is 7 a.m. Friday. The end of the monthly reporting period (one calendar month long) is 12 midnight on the last day of the month. To resolve the difference in the time-of-day of the weekly and monthly reporting periods, it is assumed that there is no activity during the period 12 midnight Thursday through 7 a.m. Friday. Thus, for the purposes of interpolation, the weekly system reporting period is assumed to end at 12 midnight on Thursday. The resolution of the day-of-month differences depends on whether the series is a cumulative one (such as production and imports) or a value at a fixed point-in-time (i.e., stocks).

For cumulative items (all items except stocks) the following method is used to calculate a monthly-from-weekly figure for a given month. First, a weight is assigned to each week in the month based on the number of days in that week that are in the month. (All intermediate weeks in a month will have a weight of seven; the beginning and ending weeks in the month may have a weight of less than seven, according to the number of days of the week that are in the month.) The weight for each week is then multiplied by the average daily volume for that week. To arrive at the monthly-from-weekly figure, a sum is taken of these weighted weekly volumes. The daily average for the monthly-from-weekly figure is calculated by dividing the total monthly-from-weekly figure by the number of days in the month.

Stock figures are not cumulative but represent inventories as of the last day of the reporting period. When the reporting week does not coincide with the end of a reporting month, an interpolation is necessary to derive a monthly-from-weekly figure for end-of-month stocks.

To derive the monthly-from-weekly stock figures, the two weekly reports that bracket the end of the month are used. Average daily stock change and the number of interpolated days are determined. The average daily stock change is defined as one-seventh of the difference between the stock level at the end of the last full week of the month and the stock level at the end of the week containing the last day of the month. The number of interpolation days is defined as the number of days between the end of the preceding weekly reporting period (midnight Thursday) and the end of the monthly reporting period. The end-of-month stock levels are then estimated as the sum of (a) the stock level reported the last full week of the month, plus (b) the number of interpolation days multiplied by the average daily stock change for the week.

The monthly-from-weekly exports data are derived from the most recent data published in the Weekly Petroleum Status Report. Beginning with statistics for the first week ending in October 1991, weekly estimates of exports are forecast using an autoregressive integrated moving-average (ARIMA) procedure. The ARIMA procedure models a value as a linear combination of its own past values and present and past values of other related time series. The most recent 5 years of 
past data are used to obtain the forecast. In addition, for the major products and crude oil, 5 years of related price data are used. The price data include some U.S. and some foreign series.

\section{Note 2. Domestic Crude Oil Production}

The Energy Information Administration (EIA) collects monthly crude oil production data on an ongoing basis. Data on crude oil production for States are reported to the EIA by State government agencies. Data on crude oil production for Federal offshore areas are reported to the EIA by the Minerals Management Service of the U.S. Department of the Interior and the Conservation Committee of California Oil Producers.

Currently, all except four crude oil producing States (Michigan, New York, Ohio, and Pennsylvania) report production on a monthly basis. These four States report crude oil production on an annual basis. Estimates of monthly crude oil production for these four States are made by the EIA using data reported on Form EIA-182, "Domestic Crude Oil First Purchase Report." After the end of each calendar year, the monthly crude oil production estimates are updated using annual reports from various State agencies, the Minerals Management Service, and the Conservation Committee of California Oil Producers. The final estimate is published in the Petroleum Supply Annual. There is a time lag of approximately 4 months between the end of the production month and the time when most monthly State crude oil production data become available.

In order to present more timely crude oil production estimates, the EIA prepares an original, forecast estimate on the first day of the production month (indicated with a "PE"). Approximately 45 days later, this original estimate of monthly crude oil production is replaced by State-level interim estimates (indicated with an "RE"). The State-level interim estimates are based on: (a) data reported by the States (e.g., production data for Alaska are typically reported to the EIA before the interim estimate is made); (b) first purchase data reported on Form EIA-182, "Domestic Crude Oil First Purchase Report;" (c) exponential or hyperbolic curve fitted projections based on recent State data; or (d) constant level projections based on the average production rate during a recent time period.

\section{Note 3. Figures}

Figures associated with the Summary Statistics tables are provided which depict the balance between supply, disposition, and ending stocks for various commodities.

The national inventory (stocks) graphs (Figures S4, S6, S8, S10, S12, S14, and S16) for crude oil, finished motor gasoline, distillate fuel oil, residual fuel oil, jet fuel, propane/propylene, and liquefied petroleum gases, in this publication include features to assist in comparing current inventory levels with past inventory levels and minimum operating levels. These features are described below.

The graphs displaying inventory levels provide the reader with actual inventory data compared to an average range from the most recent 3-year period running from January through December or from July through June. The ranges are updated every 6 months in April and October. The 3-year period is adjusted by dropping the oldest 6 months and including the most recent 6 months. The ranges also reflect seasonal variation determined from a 7-year period. The seasonal factors, which determine the shape of the upper and lower curves, are updated annually in October, using the most recent year's final monthly data.

The monthly seasonal factors are estimated by means of a seasonal adjustment technique developed at the U.S. Bureau of the Census (Census X-11). The seasonal factors are assumed to be stable (i.e., unchanging from year to year) and additive (i.e., the series is deseasonalized by subtracting the seasonal factor for the appropriate month from the reported inventory levels). The intent of deseasonalization is to remove only variation from the data. Thus, a deseasonalized series would contain the same trends, cyclical components, and irregularities as the original data.

After seasonal factors are derived, data from the most recent 3-year period (January through December or July through June) are deseasonalized. The avirage of the deseasonalized 36-month series determines the midpoint of the deseasonalized average band. The standard deviation of the deseasonalized 36 months is calculated adjusting for extreme data points. The upper curve of the average range is defined as the average plus the standard deviation. The lower curve is defined as the average plus the seasonal factors minus the standard deviation. Thus, the width of the average range is twice the standard deviation.

The lines labeled "Minimum Operating Inventory" (MOI) on the stocks graphs for crude oil, finished motor gasoline, distillate fuel oil, and residual fuel oil represent estimates of those inventory levels made by the National Petroleum Council (NPC) and published in April 1989 in a report of the NPC's Committee on Petroleum Storage \& Transportation. The NPC defines the MOI as the inventory level below which operating problems and shortages would begin to appear in a defined distribution system. The NPC report presents the findings of a study which was directed by the NPC Committee. MOI estimates presented in the report were developed by consensus through a decision-making process that relied on the judgement of Committee members based on their operating experience, on historical inventory trends, and on the results of an NPC survey of companies that provide primary inventory data to the EIA. The estimated MOI 
values are: Crude oil -- 300 million barrels; finished motor gasoline -- 205 million barrels; distillate fuel oil -- 85 million barrels; and residual fuel oil -- 30 million barrels.

The NPC did not develop a minimum operating inventory level for jet fuel stocks or propane/propylene stocks. The line labeled "observed minimum" is the lowest inventory level observed during the most recent 36 -month period as published in the Petroleum Supply Monthly.

\section{Note 4. Frames Maintenance}

In January 1981 and 1983, numerous respondents were added to bulk terminal and pipeline surveys affecting subsequent stocks reported and stock change calculations. Using the expanded coverage (new basis), the end-of-year stocks, in million barrels, would have been as listed below.

- Crude Oil: 1982-645 (Total) and 351 (Other Primary).

- Crude Oil and Petroleum Products: 1980-1,425; and 1982-1,461.

- Motor Gasoline: 1980-263 (Total) and 214 (Finished); 1982-244 (Total) and 202 (Finished).

- $\quad$ Distillate Fuel Oil: 1980-205; and 1982-186.

- $\quad$ Residual Fuel Oil: 1980-91; and 1982-69.

- Jet Fuel: 1980-42 (Total) and 36 (Kerosene-type); and 1982-39 (Total) and 32 (Kerosene-type).

- Propane/Propylene: 1980-69; and 1982-57.

- Liquefied Petroleum Gases: 1980-128; and 1982-102.

- Other Petroleum Products: 1980-207; and 1982-219.

Stock change calculations beginning in 1981 and 1983 were made using new basis stock levels.

Stocks of Alaskan crude oil in-transit were included for the first time in January 1981. The major impact of this change is on the reporting of stock change calculations. Using the expanded coverage (new basis), 1980 end-of-year crude oil stocks would have been 488 million barrels (Total) and 380 million barrels (Other Primary).

Beginning with January 1984, natural gas liquids supply and disposition data were collected on a component basis rather than a product basis. This change affected stocks reported and stock change calculations. Under the new basis, end-of-year 1983 stocks would have been:

- Propane/Propylene: 1983-55.

- Liquefied Petroleum Gases: 1983-108.

- Other Petroleum Products: 1983-210.

Beginning no later than November 1992, the Clean Air Act Amendments of 1990 require that all gasoline sold in carbon monoxide nonattainment areas have an oxygen content of 2.7 percent (by weight) during winter months. Beginning in 1995 further requirements are that only reformulated gasoline having an average oxygen content of 2.0 percent be sold in the nine worst ozone nonattainment areas.

In 1991, the Energy Information Administration (EIA) conducted a frame identifier survey of companies that produce, blend, store, or import oxygenates. The purpose of this survey was to (1) identify all U.S. producers, blenders, storers, and importers of oxygenates; and (2) collect supply and blending data for 1990 and end of 1990 inventory data on those oxygenates blended into motor gasoline. A summary of the results from the identification survey were published in the Weekly Petroleum Status Report dated February 12, 1992 and in the February 1992 issue of the Petroleum Supply Monthly.

In order to continue to provide relevant information about U.S. and regional gasoline supply, the EIA conducted a second frame identifier survey of these companies during 1992. As a result, a number of respondents were added to the monthly surveys effective in January 1993: 19 blenders, 25 stock holders, and 8 importers. This change affected stocks reported and stock change calculations. However, a new basis stock level was not calculated. 


\section{Detailed Statistics}

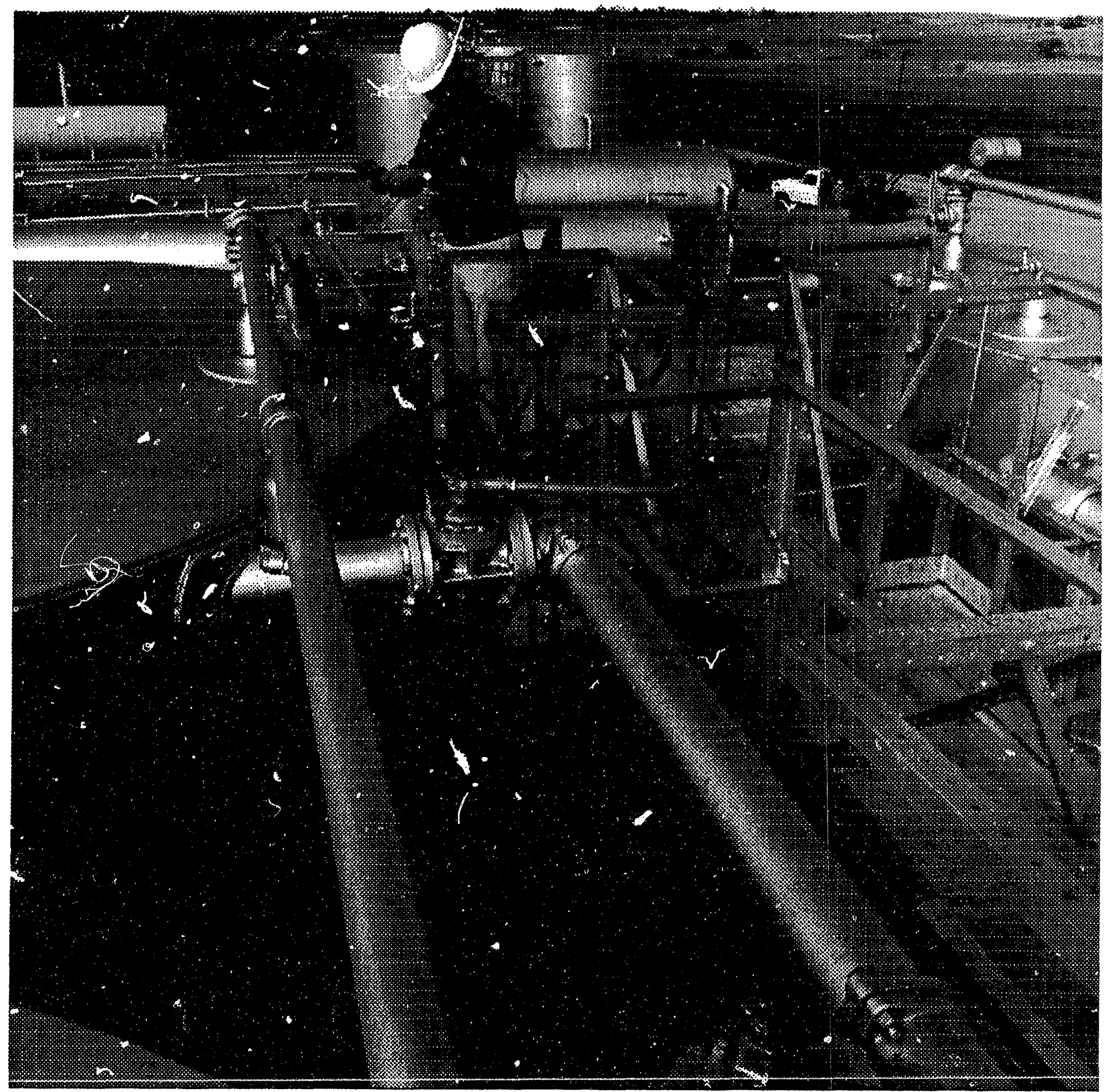

At some locations, oil skimmers and knockout tanks (in background) are used to remove waste water from the crude oil. The crude oil is then put into storage tanks and gauged. 


\begin{tabular}{|c|c|c|c|c|c|}
\hline \multirow{2}{*}{\multicolumn{2}{|c|}{ Commodity }} & \multicolumn{2}{|c|}{ Curront Month } & \multicolumn{2}{|c|}{ Yoar to Date } \\
\hline & & $\begin{array}{l}\text { Thousand } \\
\text { Barrels }\end{array}$ & $\begin{array}{c}\text { Thousand Barrole } \\
\text { per Day }\end{array}$ & $\begin{array}{l}\text { Thousand } \\
\text { Barrole }\end{array}$ & $\begin{array}{c}\text { Thousand Barrels } \\
\text { per Day }\end{array}$ \\
\hline $\begin{array}{l}(1) \\
(2) \\
(3)\end{array}$ & 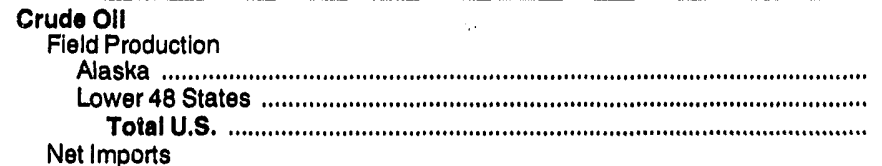 & $\begin{array}{r}E_{50,811} \\
E_{165,446} \\
E_{216,257}\end{array}$ & $\begin{array}{l}E_{1,639} \\
E_{5,337} \\
E_{6,976}\end{array}$ & $\begin{array}{r}E_{147,676} \\
E_{480,620} \\
E_{628,296}\end{array}$ & $\begin{array}{l}E_{1,641} \\
E_{5,340} \\
E_{\mathbf{6}, 981}\end{array}$ \\
\hline $\begin{array}{l}(4) \\
(5) \\
(6) \\
(7)\end{array}$ & 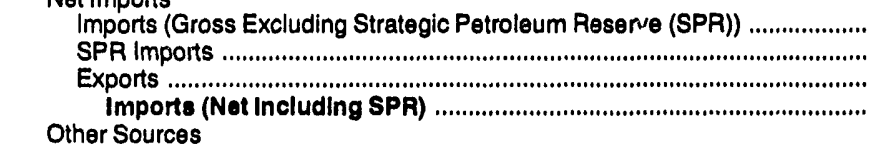 & $\begin{array}{r}200,918 \\
998 \\
4,301 \\
197,615\end{array}$ & $\begin{array}{r}5,481 \\
32 \\
139 \\
6,375\end{array}$ & $\begin{array}{r}568,335 \\
998 \\
12,932 \\
556,401\end{array}$ & $\begin{array}{r}6,315 \\
11 \\
144 \\
6,182\end{array}$ \\
\hline $\begin{array}{r}(8) \\
(9) \\
(10) \\
(11) \\
(12) \\
(13)\end{array}$ & 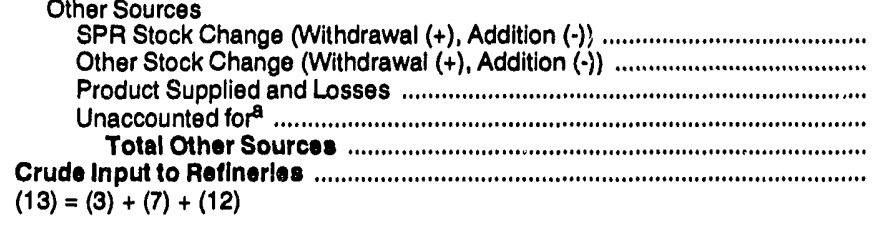 & $\begin{array}{r}-1,804 \\
-5,829 \\
-354 \\
4,837 \\
-3,150 \\
410,721\end{array}$ & $\begin{array}{r}-58 \\
-188 \\
-11 \\
156 \\
-102 \\
13,249\end{array}$ & $\begin{array}{r}-2,887 \\
-19,065 \\
-947 \\
13,148 \\
-9,751 \\
1,174,947\end{array}$ & $\begin{array}{r}-32 \\
-212 \\
-11 \\
146 \\
-108 \\
13,055\end{array}$ \\
\hline $\begin{array}{l}(14) \\
(15) \\
(16) \\
(17)\end{array}$ & 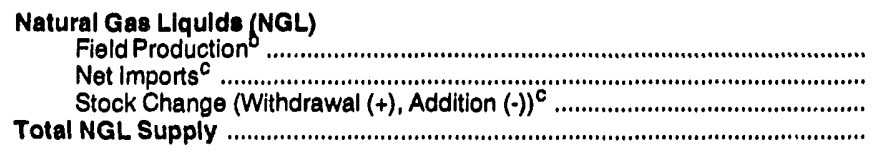 & $\begin{array}{r}59,250 \\
866 \\
-587 \\
\mathbf{3 0 , 5 2 8}\end{array}$ & $\begin{array}{r}1,911 \\
28 \\
-19 \\
1,920\end{array}$ & $\begin{array}{r}166,679 \\
2,472 \\
592 \\
169,743\end{array}$ & $\begin{array}{r}1,852 \\
27 \\
7 \\
1,886\end{array}$ \\
\hline $\begin{array}{l}(18) \\
(19) \\
(20) \\
(21) \\
(22) \\
(23)\end{array}$ & 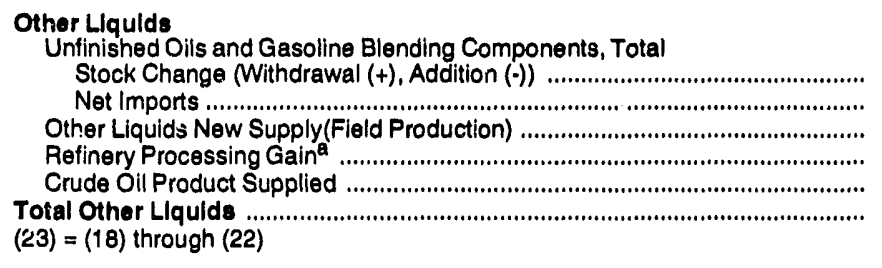 & $\begin{array}{r}-3,356 \\
16,679 \\
3,778 \\
24,084 \\
354 \\
41,539\end{array}$ & $\begin{array}{r}-108 \\
538 \\
122 \\
777 \\
11 \\
1,340\end{array}$ & $\begin{array}{r}-17,066 \\
47,847 \\
21,804 \\
69,895 \\
942 \\
123,422\end{array}$ & $\begin{array}{r}.190 \\
532 \\
242 \\
777 \\
10 \\
1,371\end{array}$ \\
\hline (24) & $\begin{array}{l}\text { Total Production of Products } \\
(24)=(13)+(17)+(23)\end{array}$ & 511,788 & 16,509 & $1,468,112$ & 16,312 \\
\hline $\begin{array}{l}(25) \\
(26) \\
(27)\end{array}$ & 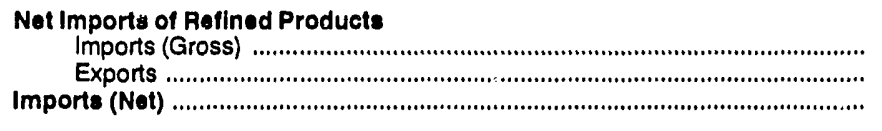 & $\begin{array}{l}38,611 \\
23,216 \\
15,395\end{array}$ & $\begin{array}{r}1,246 \\
749 \\
497\end{array}$ & $\begin{array}{r}107,000 \\
67,651 \\
39,349\end{array}$ & $\begin{array}{r}1,189 \\
752 \\
437\end{array}$ \\
\hline (28) & $\begin{array}{l}\text { Total Now Supply of Products } \\
(28)=(24)+(27)\end{array}$ & 527,184 & 17,006 & $1,507,461$ & 16,750 \\
\hline (29) & Hefined Products Stock Change (Withdrawal (+), Addition (-)) ................................ & 23,139 & 746 & 46,577 & 518 \\
\hline (30) & $\begin{array}{l}\text { Total Petroleum Products Supplled for Domestlc Use } \\
(30)=(28)+(29)\end{array}$ & 550,323 & 17,752 & $1,554,038$ & 17,267 \\
\hline $\begin{array}{l}(31) \\
(32) \\
(33) \\
(34) \\
(35) \\
(36) \\
(37) \\
(38)\end{array}$ & 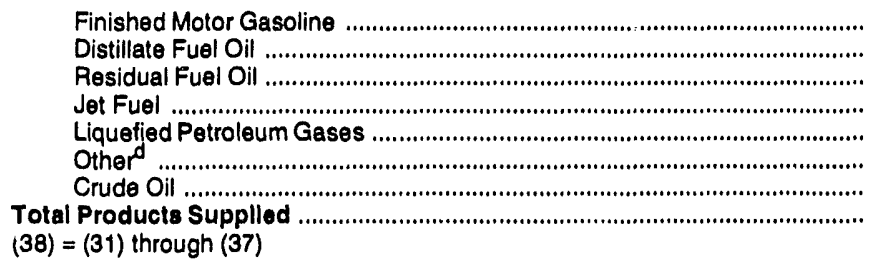 & $\begin{array}{r}229,303 \\
106,958 \\
33,020 \\
46,297 \\
59,207 \\
75,184 \\
354 \\
550,323\end{array}$ & $\begin{array}{r}7,397 \\
3,450 \\
1,065 \\
1,493 \\
1,910 \\
2,425 \\
11 \\
17,752\end{array}$ & $\begin{array}{r}638,028 \\
312,384 \\
96,238 \\
133,363 \\
172,628 \\
200,456 \\
942 \\
1,554,038\end{array}$ & $\begin{array}{r}7,089 \\
3,471 \\
1,069 \\
1,482 \\
1,918 \\
2,227 \\
10 \\
17,267\end{array}$ \\
\hline $\begin{array}{l}(39) \\
(40) \\
(41) \\
(42) \\
(43) \\
(44) \\
(45) \\
(46) \\
(47)\end{array}$ & 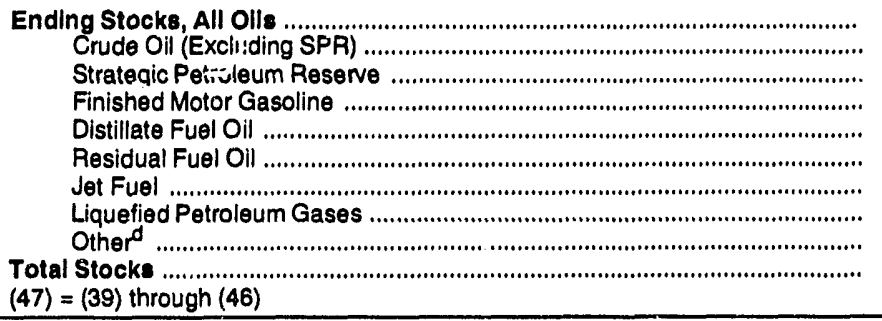 & $\begin{array}{r}337,114 \\
577,611 \\
187,039 \\
97,456 \\
40,690 \\
41,429 \\
66,610 \\
235,641 \\
1,583,590\end{array}$ & $\begin{array}{l}\ddot{.} \\
\ddot{*} \\
\ddot{.} \\
\ddot{.} \\
\ddot{*} \\
\ddot{-} \\
.\end{array}$ & $\begin{array}{r}337,114 \\
577,611 \\
187,039 \\
97,456 \\
40,690 \\
41,429 \\
66,610 \\
235,641 \\
1,583,590\end{array}$ & $\begin{array}{l}\ddot{.} \\
\ddot{. .} \\
. . \\
\ddot{.} \\
\ddot{.} \\
\ddot{.} \\
-\end{array}$ \\
\hline
\end{tabular}

a Unaccounted for crude oil represents the difference berween the supply and disposition of crude oil. Refinery processing gain represents the volumetric amount by which total output is greater than input for a given period of time. Preliminary estimates of crude oil imports at the National level have historically understated final values by approximately 50 thousand barrels per day. This causes the preliminary values of unaccounted for crude oil to overstate the final values by the same amount.

Includes fuel ethanol blended into finished motor gasoline.

c Includes products in the pentanes plus category only.

d Includes pentanes plus, other liquids, and all finished petroleum products except finished motor gasoline, distillate fuel oil, residual fuel oil, jet fuel, and liquefied petroleum gases.

(s) $=$ Less than 500 barrels per day. $E=$ Estimated.

Note: Totals may not equal sum of components due to independent rounding.

Sources: : Enorigy lníumation Adminisiration (EIA), Mionthily Petroleum Supply Reponing System. - Domestic crude oil production estimates based on historical statistics from State conservation agencies and the Minerals Management Service of the U.S. Department of the Interior. - Export data from the Bureau of the Census and Form ElA-810, "Monthly Refinery Report." 
Table 2. U.S. Supply, Disposition, and Ending Stocks of Crude Oll and Petroleum Products, March 1993

(Thousand Barrels)

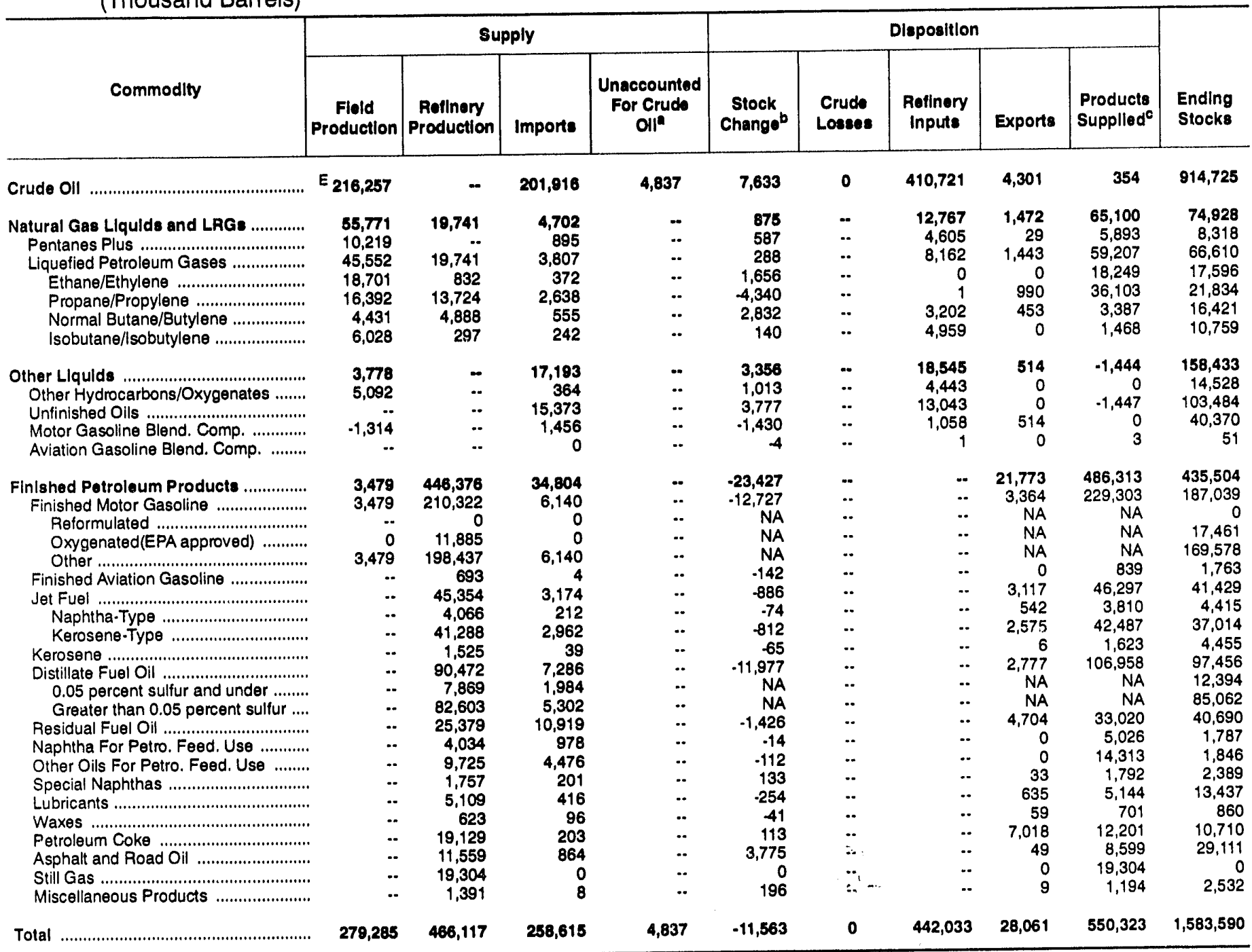

a Unaccounted for crude oil represents the difference between the supply and disposition of crude oil. Preliminary estimates of crude cii :mports at the National level have historically understated final values by approximately 50,000 barrels per day. This causes the preliminary values of unaccounted for crude oil to overstate the final values by the same amount.

A negative number indicates a decrease in stocks and a positive number indicates an increase in stocks.

c Products supplied is equal to field production, plus refinery production, plus imports, plus unaccounted for crude oil, minus stock change, minus crude losses, minus refinery inputs, minus exports.

$(s)=$ Less than 500 barrels.

$\mathrm{E}=$ Estimated.

$L R G=$ Liquefied Refinery Gas.

$N A=$ Not avallable.

Note: Totals may not equal sum of components due to independent rounding.

Sources: - Energy Information Administration (EIA) Forms EIA-810, "Monthly Refinery Report, "EIA-811, "Monthly Bulk Terminal Report," EIA-812, "Monthly Product Pipeline Report, "EIA-813, "Monthly Crude Oil Report, "EIA-814, "Monthly Imports Report," EIA-816, "Monthly Natural Gas Liquios Report," EIA-817, "Monthly Tanke and Barge Movement Report," and EIA-819M, "Monthly Oxygenate Telephone Report." - Domestic crude oil production estimates based on historical statistics from State conservation agencies and the Minerals Management Service of the U.S. Department of the Interior. - Export data from the Bureau of the Census and Form EIA.810, "Monthly Refinery Report." 
Table 3. U.S. Year-to-Date Supply, Disposition, and Ending Stocks of Crude Oll and Petroleum Products, January-March 1993 (Thousand Barrels)

\begin{tabular}{|c|c|c|c|c|c|c|c|c|c|c|}
\hline \multirow[b]{2}{*}{ Commodity } & \multicolumn{4}{|c|}{ Supply } & \multicolumn{5}{|c|}{ Dleposition } & \multirow[b]{2}{*}{$\begin{array}{l}\text { Ending } \\
\text { Stocks }\end{array}$} \\
\hline & $\begin{array}{c}\text { Fleld } \\
\text { Production }\end{array}$ & $\begin{array}{c}\text { Rellnery } \\
\text { Productlon }\end{array}$ & Imports & $\begin{array}{c}\text { Unaccounted } \\
\text { For Crude } \\
\text { Oll }^{\mathbb{a}}\end{array}$ & $\begin{array}{c}\text { Stock } \\
\text { Changeb }\end{array}$ & $\begin{array}{l}\text { Crude } \\
\text { Losses }\end{array}$ & $\begin{array}{l}\text { Retlnery } \\
\text { Inpute }\end{array}$ & Exports & $\begin{array}{l}\text { Products } \\
\text { Supplied }^{\circ}\end{array}$ & \\
\hline 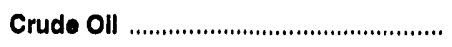 & $E_{628,296}$ & - & 569,333 & 13,148 & 21,952 & $\mathbf{5}$ & $1,174,947$ & 12,932 & 942 & 914,725 \\
\hline 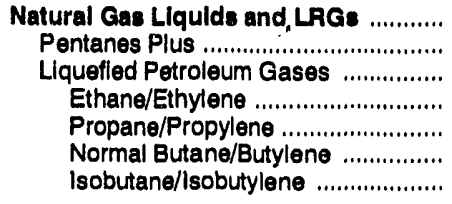 & $\begin{array}{r}158,640 \\
29,156 \\
129,484 \\
52,534 \\
46,860 \\
13,929 \\
16,161\end{array}$ & $\begin{array}{r}46,310 \\
40 \\
46,310 \\
2,266 \\
40,025 \\
3,275 \\
744\end{array}$ & $\begin{array}{r}13,578 \\
2,562 \\
11,016 \\
1,008 \\
7,054 \\
2,393 \\
561\end{array}$ & $\begin{array}{l}\ddot{.} \\
. . \\
. . \\
. . \\
. .\end{array}$ & $\begin{array}{r}-22,660 \\
-592 \\
-22,068 \\
-1,131 \\
-17,015 \\
-3,916 \\
-6\end{array}$ & $\begin{array}{l}\ddot{.} \\
. . \\
. . \\
. . \\
. . \\
. .\end{array}$ & $\begin{array}{r}46,102 \\
14,032 \\
32,070 \\
0 \\
28 \\
18,088 \\
13,954\end{array}$ & $\begin{array}{r}4,270 \\
90 \\
4,180 \\
0 \\
2,963 \\
1,217 \\
0\end{array}$ & $\begin{array}{r}190,816 \\
18,188 \\
172,628 \\
56,939 \\
107,963 \\
4,208 \\
3,518\end{array}$ & $\begin{array}{r}74,928 \\
8,318 \\
66,610 \\
17,596 \\
21,834 \\
16,421 \\
10,759\end{array}$ \\
\hline $\begin{array}{l}\text { Other Liquids .................................... } \\
\text { Other Hydrocarbons/Oxygenates ...... } \\
\text { Unfinished Olls ................................... } \\
\text { Motor Gasoline Blend. Comp. ............ } \\
\text { Avlation Gasoline Blend. Comp. ......... }\end{array}$ & $\begin{array}{r}21,804 \\
23,934 \\
\cdots \\
-2,130 \\
\cdots\end{array}$ & $\begin{array}{l}\ddot{.} \\
\ddot{.} \\
\ddot{.}\end{array}$ & $\begin{array}{r}48,664 \\
1,343 \\
44,344 \\
2,977 \\
0\end{array}$ & $\begin{array}{l}\ddot{-} \\
\ddot{.} \\
\ddot{.} \\
. .\end{array}$ & $\begin{array}{r}17,066 \\
7,646 \\
8,155 \\
1,273 \\
\cdot 8\end{array}$ & $\begin{array}{l}\cdots \\
\cdots \\
\cdots \\
\cdots\end{array}$ & $\begin{array}{r}61,386 \\
17,631 \\
44,997 \\
-1,243 \\
1\end{array}$ & $\begin{array}{r}817 \\
0 \\
0 \\
817 \\
0\end{array}$ & $\begin{array}{r}-8,801 \\
0 \\
-8,808 \\
0 \\
7\end{array}$ & $\begin{array}{r}158,433 \\
14,528 \\
103,484 \\
40,370 \\
51\end{array}$ \\
\hline 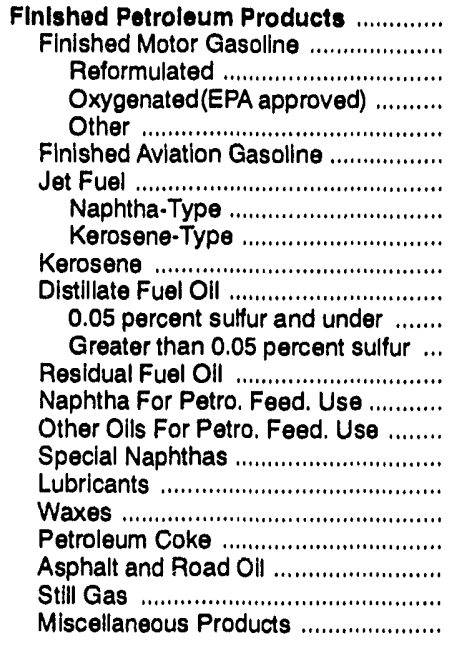 & $\begin{array}{r}8,039 \\
8,039 \\
. . \\
14,976 \\
-6,937 \\
. . \\
. . \\
. . \\
. . \\
. . \\
. . \\
. . \\
. . \\
. . \\
. . \\
. . \\
. . \\
. . \\
. . \\
. . \\
. . \\
. .\end{array}$ & $\begin{array}{r}1,306,020 \\
631,474 \\
0 \\
82,493 \\
548,981 \\
1,710 \\
130,269 \\
11,603 \\
118,666 \\
5,564 \\
259,428 \\
26,325 \\
233,103 \\
74,332 \\
11,464 \\
26,813 \\
4,532 \\
14,414 \\
1,788 \\
54,898 \\
28,967 \\
56,379 \\
3,988\end{array}$ & $\begin{array}{r}95,984 \\
18,508 \\
0 \\
0 \\
18,508 \\
7 \\
8,988 \\
669 \\
8,319 \\
133 \\
19,199 \\
4,890 \\
14,309 \\
31,888 \\
2,440 \\
10,128 \\
498 \\
1,111 \\
175 \\
238 \\
2,521 \\
0 \\
150\end{array}$ & $\begin{array}{l}. . \\
. . \\
. . \\
. . \\
. . \\
. . \\
. . \\
. . \\
. . \\
. . \\
. . \\
. . \\
. . \\
. . \\
. . \\
. . \\
. . \\
. . \\
. . \\
. .\end{array}$ & $\begin{array}{r}-24,509 \\
9,457 \\
N A \\
N A \\
N A \\
224 \\
-1,855 \\
-161 \\
-1,694 \\
-1,023 \\
-43,178 \\
N A \\
N A \\
-2,019 \\
90 \\
-47 \\
253 \\
126 \\
-49 \\
1,185 \\
11,753 \\
0 \\
574\end{array}$ & $\begin{array}{l}. . \\
. . \\
. . \\
. . \\
. . \\
. . \\
. . \\
. . \\
. . \\
. . \\
. \\
. . \\
. . \\
. . \\
. \\
. . \\
. . \\
. .\end{array}$ & $\begin{array}{l}. . \\
. . \\
. . \\
. \\
. . \\
. . \\
. . \\
. . \\
. . \\
. . \\
. . \\
. \\
. . \\
. \\
. . \\
. . \\
. . \\
. . \\
. .\end{array}$ & $\begin{array}{r}63,471 \\
10,536 \\
\mathrm{NA} \\
\mathrm{NA} \\
\mathrm{NA} \\
0 \\
7,749 \\
1,748 \\
6,002 \\
588 \\
9,421 \\
\mathrm{NA} \\
\mathrm{NA} \\
12,001 \\
0 \\
0 \\
475 \\
1,593 \\
178 \\
20,795 \\
103 \\
0 \\
32\end{array}$ & $\begin{array}{r}1,371,081 \\
638,028 \\
N A \\
N A \\
N A \\
1,493 \\
133,363 \\
10,685 \\
122,677 \\
6,132 \\
312,384 \\
N A \\
N A \\
96,238 \\
13,814 \\
36,988 \\
4,302 \\
13,806 \\
1,834 \\
33,156 \\
19,632 \\
56,379 \\
3,532\end{array}$ & $\begin{array}{r}435,504 \\
187,039 \\
0 \\
17,461 \\
169,578 \\
1,763 \\
41,429 \\
4,415 \\
37,014 \\
4,455 \\
97,456 \\
12,394 \\
85,062 \\
40,690 \\
1,787 \\
1,846 \\
2,389 \\
13,437 \\
860 \\
10,710 \\
29,111 \\
0 \\
2,532\end{array}$ \\
\hline 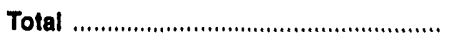 & 816,779 & $1,352,330$ & 727,558 & 13,148 & $-8,151$ & 5 & $1,282,435$ & 81,490 & $1,554,038$ & $1,583,590$ \\
\hline
\end{tabular}

a Unaccounted for crude oil represents the difference between the supply and disposition of crude oil. Preliminary estimates of crude oil imports at the National level have historically understated final values by approximately 50,000 barrels per day. This causes the preliminary values of unaccounted for crude oil to overstate the final values by the same amount.

A negative number indlcates a decrease in stocks and a positive number indicates an increase in stocks.

c Products supplied is equal to field production, plus refinery production, plus imports, plus unaccounted for crude oil, minus stock change, minus crude losses, minus refinery inputs, minus exports.

(s) $=$ Less than 500 barrels.

$E$ = Estimated

LRG = Liquefled Refinery Gas.

NA $=$ Not avallable.

Note: Totals may not equal sum of components due to independent rounding

Sources: - Energy Information Administration (EIA) Forms EIA-810, "Monthly Refinery Report," ElA-811, "Monthly Bulk Terminal Report," EIA-812, "Monthly Product Pipeline Report," EIA-813, "Monthly Crude Oil Report," EIA-814, "Monthly Imports Report," EIA-816, "Monthly Natural Gas Liquids Report," ElA-817, "Monthly Tanker arid Barge Movement Report," and ElA-819M, "Monthly Oxygenate Telephone Report." - Domestic crude oil production estimates based on historical statistics from State conservation agencies and the Minerals Management Service of the U.S. Department of the Interior. - Export data from the Bureau of the Census and Form EIA-810, "Monthly Refinery Report." 
Table 4. U.S. Daily Average Supply and Disposition of Crude Oll and Petroleum Products, March 1993

(Thousand Barrels per Day)

\begin{tabular}{|c|c|c|c|c|c|c|c|c|c|}
\hline \multirow[b]{2}{*}{ Commodity } & \multicolumn{4}{|c|}{ Supply } & \multicolumn{5}{|c|}{ Disposition } \\
\hline & $\begin{array}{c}\text { Fleld } \\
\text { Production }\end{array}$ & $\begin{array}{c}\text { Retinery } \\
\text { Production }\end{array}$ & Imports & $\begin{array}{c}\text { Unaccounted } \\
\text { For Crude } \\
\text { Olla }\end{array}$ & $\begin{array}{c}\text { Stock } \\
\text { Changeb }\end{array}$ & $\begin{array}{c}\text { Crude } \\
\text { Los8es }\end{array}$ & $\begin{array}{c}\text { Roflnery } \\
\text { Inputs }\end{array}$ & Exports & $\begin{array}{l}\text { Products } \\
\text { Suppliod' }\end{array}$ \\
\hline 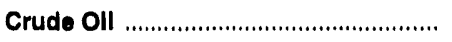 & $E_{6,976}$ & $\cdots$ & 6,513 & 156 & 246 & 0 & 13,249 & 139 & 11 \\
\hline 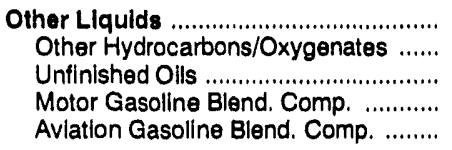 & $\begin{array}{r}122 \\
164 \\
. . \\
-42 \\
\cdots\end{array}$ & 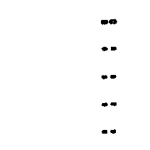 & $\begin{array}{r}555 \\
12 \\
496 \\
47 \\
0\end{array}$ & 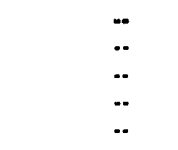 & $\begin{array}{r}108 \\
33 \\
122 \\
-46 \\
(s)\end{array}$ & $\begin{array}{l}- \\
\cdots \\
\cdots \\
\cdots \\
\cdots\end{array}$ & $\begin{array}{r}598 \\
143 \\
421 \\
34 \\
(s)\end{array}$ & $\begin{array}{r}17 \\
0 \\
0 \\
17 \\
0\end{array}$ & $\begin{array}{r}-47 \\
0 \\
-47 \\
0 \\
(\mathbf{s})\end{array}$ \\
\hline 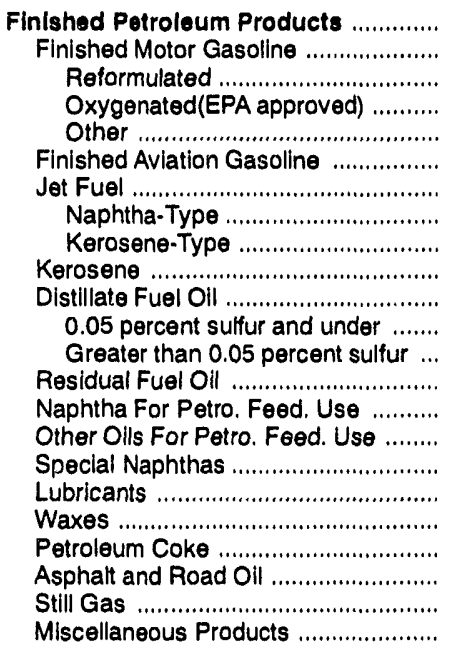 & $\begin{array}{r}112 \\
112 \\
. . \\
0 \\
112 \\
. . \\
. . \\
. . \\
. . \\
. . \\
. . \\
. . \\
. . \\
. . \\
. . \\
. . \\
. \\
. . \\
. \\
. \\
. .\end{array}$ & $\begin{array}{r}14,399 \\
6,785 \\
0 \\
383 \\
6,401 \\
22 \\
1,463 \\
131 \\
1,332 \\
49 \\
2,918 \\
254 \\
2,665 \\
819 \\
130 \\
314 \\
57 \\
165 \\
20 \\
617 \\
373 \\
623 \\
45\end{array}$ & $\begin{array}{r}1,123 \\
198 \\
0 \\
0 \\
198 \\
(s) \\
102 \\
7 \\
96 \\
1 \\
235 \\
64 \\
171 \\
352 \\
32 \\
144 \\
6 \\
13 \\
3 \\
7 \\
28 \\
0 \\
(s)\end{array}$ & $\begin{array}{l}. . \\
. . \\
. . \\
. . \\
. . \\
. . \\
. . \\
. . \\
. . \\
. . \\
. . \\
. . \\
. . \\
. . \\
. . \\
. . \\
. \\
. . \\
. . \\
. .\end{array}$ & $\begin{array}{r}-756 \\
-411 \\
\text { NA } \\
\text { NA } \\
\text { NA } \\
-5 \\
-29 \\
-2 \\
-26 \\
-2 \\
-386 \\
\text { NA } \\
\text { NA } \\
-46 \\
(s) \\
-4 \\
4 \\
-8 \\
-1 \\
4 \\
122 \\
0 \\
6\end{array}$ & $\begin{array}{l}. . \\
. . \\
. . \\
. . \\
. . \\
. . \\
. . \\
. . \\
. . \\
. . \\
. . \\
. . \\
. . \\
. . \\
. . \\
. . \\
. . \\
. . \\
. .\end{array}$ & $\begin{array}{l}. . \\
. . \\
. . \\
. . \\
. . \\
. . \\
. . \\
. . \\
. . \\
. . \\
. . \\
. . \\
. . \\
. . \\
. . \\
. . \\
. . \\
. . \\
. . \\
. .\end{array}$ & $\begin{array}{r}702 \\
109 \\
N A \\
N A \\
N A \\
0 \\
101 \\
17 \\
83 \\
(s) \\
90 \\
N A \\
N A \\
152 \\
0 \\
0 \\
1 \\
20 \\
2 \\
226 \\
2 \\
0 \\
(s)\end{array}$ & $\begin{array}{r}15,683 \\
7,397 \\
\text { NA } \\
\text { NA } \\
\text { NA } \\
27 \\
1,493 \\
123 \\
1,371 \\
52 \\
3,450 \\
\text { NA } \\
\text { NA } \\
1,065 \\
162 \\
462 \\
58 \\
166 \\
23 \\
394 \\
277 \\
623 \\
39\end{array}$ \\
\hline Total & 9,009 & 15,036 & 8,342 & 156 & -373 & 0 & 14,259 & 905 & 17,752 \\
\hline
\end{tabular}

a Unaccounted for crude oil represents the difference between the supply and disposition of crude oil. Preliminary estimates of crude oil imports at the National level have historically understated final values by approximately 50,000 barrels per day. This causes the preliminary values of unaccounted for crude oil to overstate the final values by the same amount.

- A negative number indicates a decrease in stocks and a positive number indicates an increase in stocks.

c Products supplied is equal to field production, plus refinery production, plus imports, plus unaccounted for crude oil, minus stock change, minus crude losses, minus refinery inputs, minus exports.

(s) = Less than 500 barrels per day.

$E=$ Estimated.

LRG = Liquefied Refinery Gas.

Note: Totals may not equal sum of components due to independent rounding.

Sources: - Energy Information Administration (EIA) Forms EIA-810, "Monthly Refinery Report," EIA-811, "Monthly Bulk Terminal Report," EIA-812,

"Monthly Product Pipeline Report," EIA-813, "Monthly Crude Oil Report," EIA-814, "Monthly imports Report," EIA-816, "Monthly Natural Gas

Liquids Report," EIA-817, "Monthly Tanker and Barge Movement Report," and EIA-819M, "Monthly Oxygenate Telephone Report". - Domestic

crude oil production estimates based on historical statistics from State conservation agencies and the Minerals Management Service of the U.S.

Department of the Interior. - Export data from the Bureau of the Census and Form EIA-810, "Monthly Refinery Report." 
Table 5. U.S. Year-to-Date Dally Average Supply and DIsposition of Crude OII and Petroleum Products, January-March 1993 (Thousand Barrels per Day)

\begin{tabular}{|c|c|c|c|c|c|c|c|c|c|}
\hline \multirow[b]{2}{*}{ Commodity } & \multicolumn{4}{|c|}{ Supply } & \multicolumn{5}{|c|}{ Dieposition } \\
\hline & $\begin{array}{c}\text { Fleld } \\
\text { Production }\end{array}$ & $\begin{array}{c}\text { Aofinery } \\
\text { Productlon }\end{array}$ & Importe & $\begin{array}{c}\text { Unaccounted } \\
\text { For Crude } \\
\text { Oll }\end{array}$ & $\begin{array}{c}\text { Stock } \\
\text { Changeb }\end{array}$ & $\begin{array}{l}\text { Crude } \\
\text { Loseses }\end{array}$ & $\begin{array}{l}\text { Rofinery } \\
\text { Inputs }\end{array}$ & Exports & $\begin{array}{l}\text { Products } \\
\text { Supplledc }\end{array}$ \\
\hline 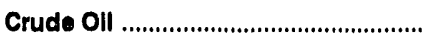 & $E_{6,981}$ & - & 6,328 & 146 & 244 & (8) & 13,055 & 144 & 10 \\
\hline 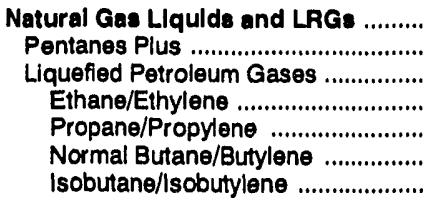 & $\begin{array}{r}1,763 \\
324 \\
1,439 \\
584 \\
521 \\
155 \\
180\end{array}$ & $\begin{array}{r}515 \\
\ddot{5} \\
515 \\
25 \\
445 \\
36 \\
8\end{array}$ & $\begin{array}{r}151 \\
28 \\
122 \\
11 \\
78 \\
27 \\
6\end{array}$ & $\begin{array}{l}\ddot{.} \\
\ddot{.} \\
\ddot{.} \\
\ddot{.} \\
\ddot{*} \\
\ddot{*}\end{array}$ & $\begin{array}{r}-252 \\
-7 \\
-245 \\
-13 \\
-189 \\
-44 \\
(8)\end{array}$ & 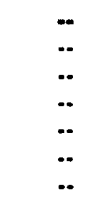 & $\begin{array}{r}512 \\
156 \\
356 \\
0 \\
(s) \\
201 \\
155\end{array}$ & $\begin{array}{r}47 \\
1 \\
46 \\
0 \\
33 \\
14 \\
0\end{array}$ & $\begin{array}{r}2,120 \\
202 \\
1,918 \\
633 \\
1,200 \\
47 \\
39\end{array}$ \\
\hline $\begin{array}{l}\text { Other Llqulde ..................................... } \\
\text { Other Hydrocarbons/Oxygenates ...... } \\
\text { Unfinished Oils ................................... } \\
\text { Motor Gasoline Blend. Comp. ........... } \\
\text { Aviation Gasoline Blend. Comp. ....... }\end{array}$ & $\begin{array}{r}242 \\
266 \\
-. \\
-24 \\
-.\end{array}$ & 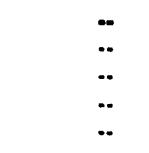 & $\begin{array}{r}541 \\
15 \\
493 \\
33 \\
0\end{array}$ & $\begin{array}{l}\ddot{ } \\
\ddot{.} \\
\ddot{.} \\
\ddot{.}\end{array}$ & $\begin{array}{r}180 \\
85 \\
91 \\
14 \\
(8)\end{array}$ & 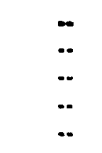 & $\begin{array}{r}682 \\
196 \\
500 \\
-14 \\
(s)\end{array}$ & $\begin{array}{l}9 \\
0 \\
0 \\
9 \\
0\end{array}$ & $\begin{array}{r}-98 \\
0 \\
-98 \\
0 \\
(s)\end{array}$ \\
\hline 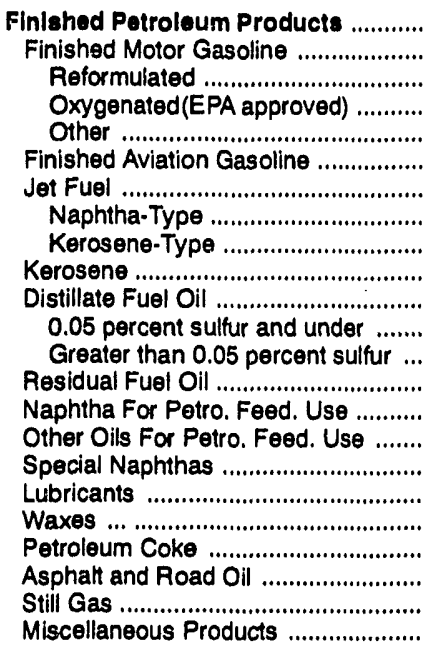 & 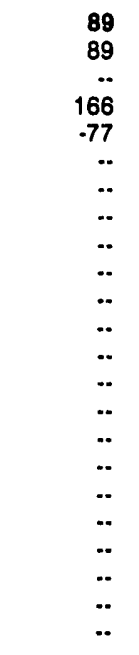 & $\begin{array}{r}14,511 \\
7,016 \\
0 \\
917 \\
6,100 \\
19 \\
1,447 \\
129 \\
1,319 \\
62 \\
2,883 \\
293 \\
2,590 \\
826 \\
127 \\
298 \\
50 \\
160 \\
20 \\
610 \\
322 \\
626 \\
44\end{array}$ & $\begin{array}{r}1,066 \\
206 \\
0 \\
0 \\
206 \\
(s) \\
100 \\
7 \\
92 \\
1 \\
213 \\
54 \\
159 \\
354 \\
27 \\
113 \\
6 \\
12 \\
2 \\
3 \\
28 \\
0 \\
2\end{array}$ & 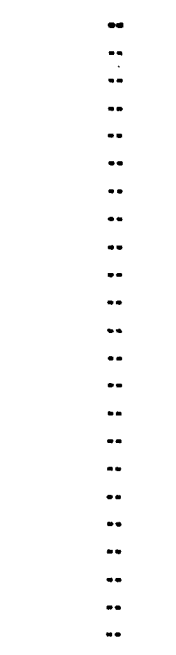 & $\begin{array}{r}-272 \\
105 \\
N A \\
N A \\
N A \\
2 \\
-21 \\
-2 \\
-19 \\
-11 \\
-480 \\
N A \\
N A \\
-22 \\
1 \\
-1 \\
3 \\
1 \\
-1 \\
13 \\
131 \\
0 \\
6\end{array}$ & 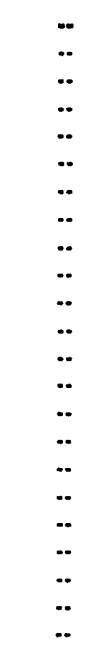 & $\begin{array}{l}. . \\
. . \\
. . \\
. . \\
. . \\
. . \\
. . \\
. . \\
. . \\
. . \\
. . \\
. . \\
. . \\
. . \\
. . \\
. . \\
. . \\
. . \\
. . \\
. . \\
. . \\
. .\end{array}$ & $\begin{array}{r}705 \\
117 \\
\text { NA } \\
\text { NA } \\
\text { NA } \\
0 \\
86 \\
19 \\
67 \\
7 \\
105 \\
\text { NA } \\
\text { NA } \\
133 \\
0 \\
0 \\
5 \\
18 \\
2 \\
231 \\
1 \\
0 \\
(5)\end{array}$ & $\begin{array}{r}15,234 \\
7,089 \\
N A \\
N A \\
N A \\
17 \\
1,482 \\
119 \\
1,363 \\
68 \\
3,471 \\
N A \\
\text { NA } \\
1,069 \\
153 \\
411 \\
48 \\
153 \\
20 \\
368 \\
218 \\
626 \\
39\end{array}$ \\
\hline 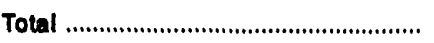 & 8,075 & 15,026 & 8,084 & 146 & -91 & (8) & 14,249 & 905 & 17,267 \\
\hline
\end{tabular}

a Unaccounted for crude oll represents the difference between the supply and disposition of crude oil. Prellminary estimates of crude oil imports at the National level have historically understated final values by approximately 50,000 barrels per day. This causes the preliminary values of unaccounted for crude oll to overstate the final values by the same amount.

b A negative number indicates a decrease in stocks and a positive number indlcates an increase in stocks.

c Products supplied is equal to field production, plus refinery production, plus imports, plus unaccounted for crude oll, minus stock change, minus crude losses, minus refinery inputs, minus exports.

(s) = Less than 500 barrels per day.

$E=$ Estlmated.

LRG = Liquefied Refinery Gas.

Note: Totals may not equal sum of components due to independent rounding.

Sources: - Energy Information Administration (EIA) Forms EIA-810, "Monthly Refinery Report," ElA-811, "Monthly Bulk Terminal Report," EIA-812, "Monthly Product Pipeline Report," EIA-813, "Monthly Crude Oll Report," EIA-814, "Monthly Imports Report," EIA-816, "Monthly Natural Gas Liquids Report," ElA-817, "Monthly Tanker and Barge Movement Report," and ElA-819M, "Monthly Oxygenate Telephone Report". • Domestic crude oll production estimates based on historical statlstics from State conservation agencies and the Minerals Management Service of the U.S. Department of the Interlor. - Export data from the Bureau of the Census and Form EIA-810, "Monthly Refinery Report." 
Table 6. PAD District I-Supply, Disposition, and Ending Stocks of Crude Oll and Petroleum Products, March 1993

(Thousand Barrels)

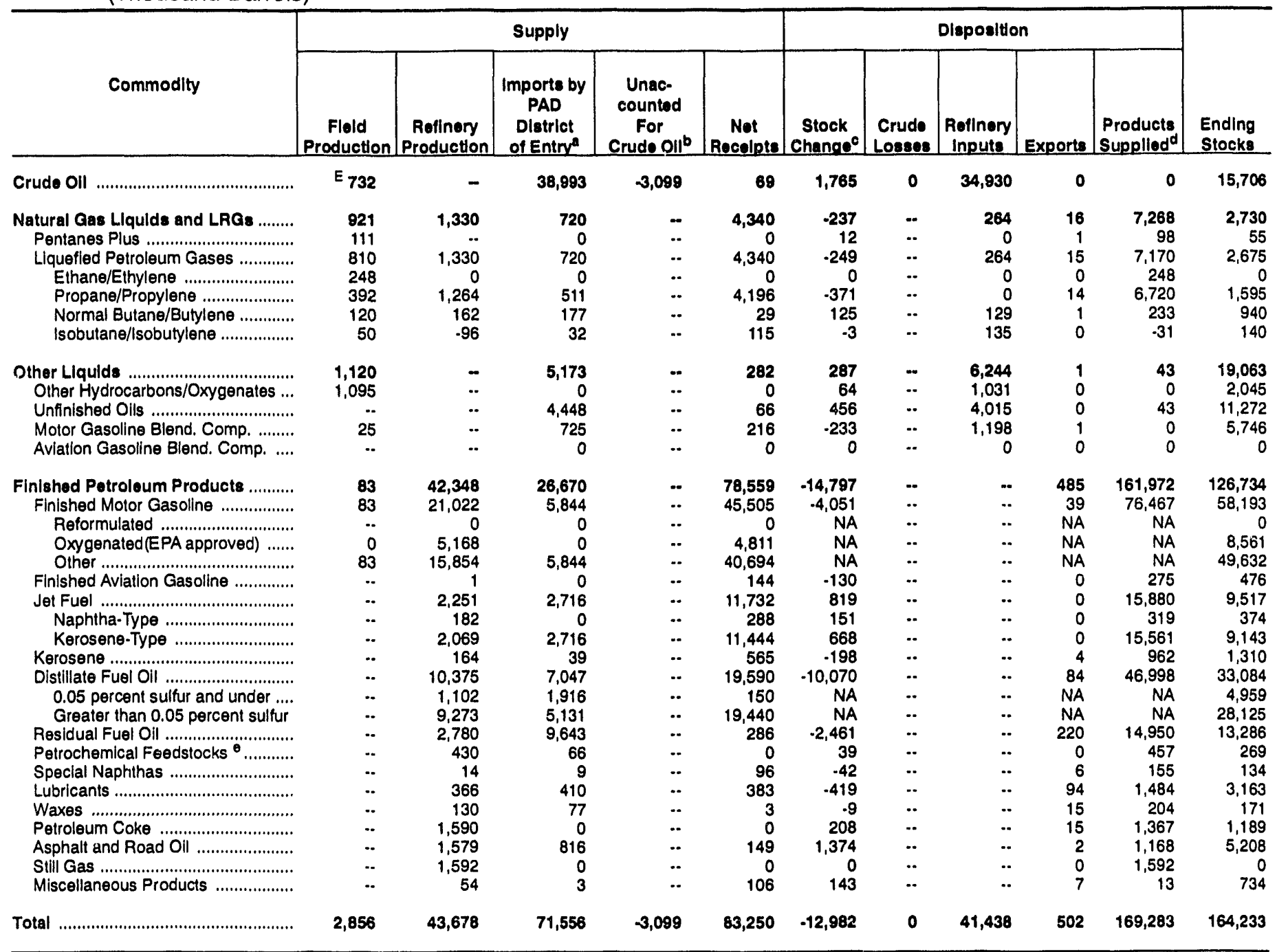

a Represents the PAD District in which the material entered the United States and not necessarlly where the crude oll or product is processed and/or consumed.

b Unaccounted for crude oil represents the difference between the supply and disposition of crude oil.

c A negative number indicates a decrease in stocks and a positive number indicates an increase in stocks.

d Products supplied is equal to field production, plus refinery production, plus imports, plus unaccounted for crude oll, plus net recelpts, minus stock change, minus crude losses, minus refinery inputs, minus exports.

Includes naphtha less than $401^{\circ} \mathrm{F}$ endpoint and other olls equal to or greater than $401^{\circ} \mathrm{F}$ endpoint.

(s) = Less than 500 barrels.

$E=$ Estimated.

LRG = Liquefied Refinery Gas.

Note: Totals may not equal sum of components due !o independent rounding.

Sources: - Energy Information Administration (EIA) Forms EIA-810, "Monthly Refinery Report," EIA-811, "Monthly Bulk Terminal Report," EIA-812, "Monthly Product Pipeline Report," ElA-813, "Monthly Crucie OIl Report," EIA-814, "Monthly Imports Report," EIA-816, "Monthly Natural Gas Liquids Report," ElA-817, "Monthly Tanker and Barge Movement Report," anc ElA.- 19M, "Monthly Oxygenate Telephone Report". - Domestlc crude oil production estimates based on historical statistics from State conservation agenclen and the Minerals Management Service of the U.S. Department of the Interlor. - Export data from the Bureau of the Census and Form EIA.810, "Monthly Refinery Feport." 
Table 7. PAD District I-Year-to-Date Supply, Disposition, and Ending Stocks of Crude OIl and Petroleum Products, January-March 1993

(Thousand Barrels)

\begin{tabular}{|c|c|c|c|c|c|c|c|c|c|c|c|}
\hline \multirow[b]{2}{*}{ Commodity } & \multicolumn{5}{|c|}{ Supply } & \multicolumn{5}{|c|}{ Disposition } & \multirow[b]{2}{*}{$\begin{array}{l}\text { Endlng } \\
\text { Stock8 }\end{array}$} \\
\hline & $\begin{array}{c}\text { Fiold } \\
\text { Production }\end{array}$ & $\begin{array}{c}\text { Roflnery } \\
\text { Production }\end{array}$ & $\begin{array}{c}\text { Imports by } \\
\text { PAD } \\
\text { District } \\
\text { of Entry }\end{array}$ & $\begin{array}{l}\text { Unac- } \\
\text { countod } \\
\text { For } \\
\text { Crude oll }\end{array}$ & $\begin{array}{c}\text { Net } \\
\text { Recelpts }\end{array}$ & $\begin{array}{c}\text { Stock } \\
\text { Changec }\end{array}$ & $\begin{array}{c}\text { Crude } \\
\text { Losses }\end{array}$ & $\begin{array}{c}\text { Refinery } \\
\text { Inputs }\end{array} \mid$ & Exports & $\begin{array}{l}\text { Products } \\
\text { Supplled }\end{array}$ & \\
\hline Crude Oll ................................................ & $E_{2,319}$ & - & 106,263 & $-2,350$ & 4 & 1,679 & 0 & 104,557 & 0 & 0 & 15,706 \\
\hline 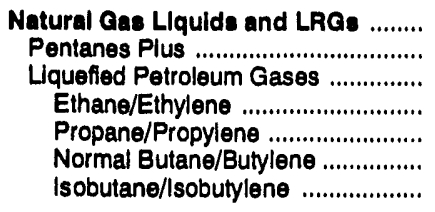 & $\begin{array}{r}2,683 \\
327 \\
2,356 \\
738 \\
1,102 \\
383 \\
133\end{array}$ & $\begin{array}{r}3,351 \\
.0 \\
3,351 \\
0 \\
3,882 \\
-396 \\
-135\end{array}$ & $\begin{array}{r}2,353 \\
0 \\
2,353 \\
0 \\
1,812 \\
508 \\
33\end{array}$ & $\begin{array}{l}\ddot{.} \\
\ddot{.} \\
\ddot{.} \\
\ddot{.} \\
\ddot{.}\end{array}$ & $\begin{array}{r}12,550 \\
0 \\
12,550 \\
0 \\
11,905 \\
530 \\
115\end{array}$ & $\begin{array}{r}-2,244 \\
6 \\
-2,250 \\
0 \\
-2,098 \\
-162 \\
10\end{array}$ & $\begin{array}{l}\ddot{.} \\
. . \\
. . \\
. . \\
. . \\
. .\end{array}$ & $\begin{array}{r}1,243 \\
0 \\
1,243 \\
0 \\
0 \\
918 \\
325\end{array}$ & $\begin{array}{r}60 \\
6 \\
55 \\
0 \\
50 \\
5 \\
0\end{array}$ & $\begin{array}{r}21,878 \\
315 \\
21,562 \\
738 \\
20,749 \\
264 \\
.189\end{array}$ & $\begin{array}{r}2,730 \\
55 \\
2,675 \\
0 \\
1,595 \\
940 \\
140\end{array}$ \\
\hline $\begin{array}{l}\text { Other Liqulds ................................... } \\
\text { Other Hydrocarbons/Oxygenates ...... } \\
\text { Unfinished Olls ................................. } \\
\text { Motor Gasollne Blend. Comp. ........... } \\
\text { Aviation Gasollne Blend. Comp. ........ }\end{array}$ & $\begin{array}{r}5,486 \\
4,646 \\
\cdots \\
850 \\
-\cdots\end{array}$ & $\begin{array}{l}- \\
\ddot{-} \\
\ddot{-} \\
\cdots\end{array}$ & $\begin{array}{r}16,585 \\
415 \\
14,126 \\
2,054 \\
0\end{array}$ & $\begin{array}{l}\cdots \\
\ddot{.} \\
\ddot{.} \\
. .\end{array}$ & $\begin{array}{r}723 \\
0 \\
434 \\
289 \\
0\end{array}$ & $\begin{array}{r}95 \\
833 \\
128 \\
-866 \\
0\end{array}$ & 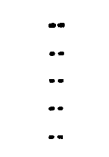 & $\begin{array}{r}22,599 \\
4,228 \\
14,315 \\
4,056 \\
0\end{array}$ & $\begin{array}{l}3 \\
0 \\
0 \\
3 \\
0\end{array}$ & $\begin{array}{r}117 \\
0 \\
117 \\
0 \\
0\end{array}$ & $\begin{array}{r}19,063 \\
2,045 \\
11,272 \\
5,746 \\
0\end{array}$ \\
\hline 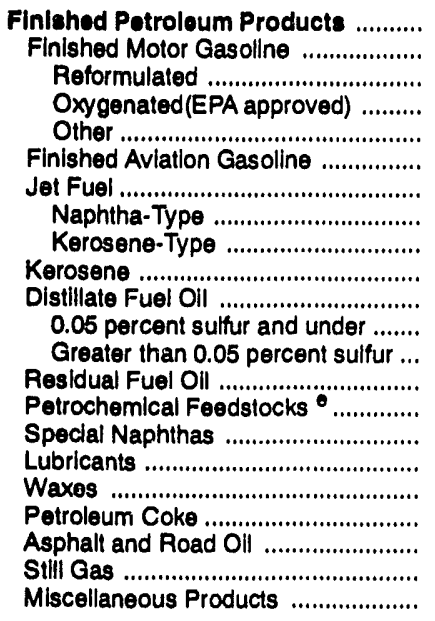 & 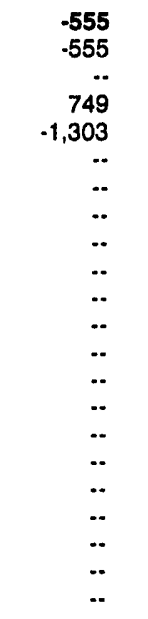 & $\begin{array}{r}131,533 \\
67,389 \\
0 \\
23,276 \\
44,113 \\
24 \\
7,367 \\
633 \\
6,734 \\
600 \\
31,235 \\
3,990 \\
27,245 \\
9,283 \\
1,388 \\
0 \\
1,427 \\
402 \\
4,457 \\
2,716 \\
5,090 \\
155\end{array}$ & $\begin{array}{r}72,679 \\
16,923 \\
0 \\
0 \\
16,923 \\
0 \\
7,831 \\
314 \\
7,517 \\
133 \\
18,051 \\
4,332 \\
13,719 \\
25,723 \\
333 \\
28 \\
1,030 \\
135 \\
0 \\
2,356 \\
0 \\
136\end{array}$ & $\begin{array}{l}. . \\
. . \\
. . \\
. . \\
. \\
. . \\
. \\
. . \\
. \\
. . \\
. . \\
. . \\
. . \\
. . \\
. \\
. . \\
. . \\
. . \\
. .\end{array}$ & $\begin{array}{r}230,341 \\
132,913 \\
0 \\
24,984 \\
107,929 \\
274 \\
32,651 \\
948 \\
31,703 \\
1,903 \\
59,242 \\
3,203 \\
56,039 \\
1,184 \\
-84 \\
284 \\
1,102 \\
12 \\
0 \\
554 \\
0 \\
306\end{array}$ & $\begin{array}{r}-30,909 \\
3,663 \\
N A \\
N A \\
N A \\
78 \\
-183 \\
87 \\
-270 \\
-916 \\
-31,920 \\
N A \\
N A \\
-3,829 \\
48 \\
-115 \\
-181 \\
1 \\
188 \\
2,023 \\
0 \\
234\end{array}$ & $\begin{array}{l}. . \\
. . \\
. . \\
. . \\
. . \\
. . \\
. . \\
. . \\
. . \\
. . \\
. . \\
. . \\
. . \\
. . \\
. . \\
. . \\
. . \\
. . \\
. .\end{array}$ & $\begin{array}{l}. . \\
. . \\
. . \\
. . \\
. . \\
. . \\
. . \\
. . \\
. . \\
. . \\
. . \\
. . \\
. . \\
. . \\
. . \\
. . \\
. . \\
. . \\
. . \\
. . \\
. .\end{array}$ & $\begin{array}{r}2,535 \\
127 \\
N A \\
N A \\
N A \\
0 \\
235 \\
1 \\
234 \\
18 \\
127 \\
N A \\
N A \\
1,242 \\
0 \\
16 \\
405 \\
36 \\
300 \\
7 \\
0 \\
22\end{array}$ & $\begin{array}{r}462,372 \\
212,880 \\
N A \\
N A \\
N A \\
220 \\
47,797 \\
1,807 \\
45,990 \\
3,534 \\
140,321 \\
N A \\
N A \\
38,777 \\
1,589 \\
411 \\
3,335 \\
512 \\
3,969 \\
3,596 \\
5,090 \\
341\end{array}$ & $\begin{array}{r}126,734 \\
58,193 \\
0 \\
8,561 \\
49,632 \\
476 \\
9,517 \\
374 \\
9,143 \\
1,310 \\
33,084 \\
4,959 \\
28,125 \\
13,286 \\
269 \\
134 \\
3,163 \\
171 \\
1,189 \\
5,208 \\
0 \\
734\end{array}$ \\
\hline 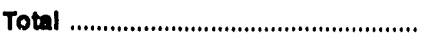 & 9,944 & 134,884 & 197,890 & $-2,350$ & 243,618 & $-31,378$ & 0 & 128,399 & 2,598 & 484,367 & 164,233 \\
\hline
\end{tabular}

Represents the PAD District in which the material entered the United States and not necessarily where the crude oil or product is processed and/or consumed.

Unaccounted for crude oil represents the difference between the supply and disposition of crude oil.

c A negative number indicates a decrease in stocks and a positive number indicates an increase in stocks.

d Products supplied is equal to field production, plus refinery production, plus imports, plus unaccounted for crude oil, plus net receipts, minus stock change, minus crude losses, minus refinery inputs, minus exports.

- Includes naphtha less than $401^{\circ} \mathrm{F}$ endpoint and other oils equal to or greater than $401^{\circ} \mathrm{F}$ endpoint.

(s) $=$ Less than 500 barrels.

$E=$ Estimated.

LRG = Liquefied Refinery Gas.

Note: Totals may not equal sum of components due to independent rounding.

Sources: - Energy Information Administration (EIA) Forms EIA-810, "Monthly Refinery Report," EIA-811, "Monthly Bulk Terminal Report," EIA-812 "Monthly Product Pipeline Report," EIA-813, "Monthly Crude Oil Report," EIA-814, "Monthly Imports Report, "EIA-816, "Monthly Natural Gas Liquids Report," EIA-817, "Monthly Tanker and Barge Movement Report," and EIA-819M, "Monthly Oxygenate Telephone Report". - Domestic crude oil production estimates based on historical statistics from State conservation agencies and the Minerals Management Service of the U.S. Department of the interior. - Export data from the Bureau of the Census and Form EIA.B10, "Monthly Refinery Report." 
Table 8. PAD District 1-Dally Average Supply and Disposition of Crude Oll and Petroleum Products, March 1993

(Thousand Barrels per Day)

\begin{tabular}{|c|c|c|c|c|c|c|c|c|c|c|}
\hline \multirow[b]{2}{*}{ Commodity } & \multicolumn{5}{|c|}{ Supply } & \multicolumn{5}{|c|}{ Disposition } \\
\hline & $\begin{array}{c}\text { Field } \\
\text { Production }\end{array}$ & $\begin{array}{c}\text { Roflnery } \\
\text { Productlon }\end{array}$ & $\begin{array}{c}\text { Imports by } \\
\text { PAD } \\
\text { Dletrlct } \\
\text { of Entry }\end{array}$ & $\begin{array}{l}\text { Unac- } \\
\text { counted } \\
\text { For } \\
\text { Crude Oll }\end{array}$ & $\begin{array}{c}\text { Not } \\
\text { Recelpto }\end{array}$ & $\begin{array}{c}\text { Stock } \\
\text { Change }\end{array}$ & $\begin{array}{l}\text { Crude } \\
\text { Losedes }\end{array}$ & $\begin{array}{c}\text { Refinery } \\
\text { Inpute }\end{array}$ & Exports & $\begin{array}{l}\text { Products } \\
\text { Supplled }\end{array}$ \\
\hline Crude Oll & $E_{24}$ & - & 1,258 & -100 & 2 & 57 & 0 & 1,127 & 0 & 0 \\
\hline 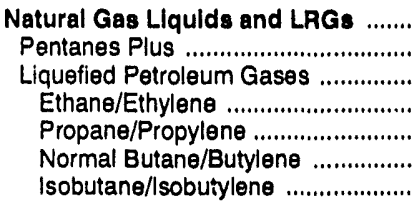 & $\begin{array}{r}30 \\
4 \\
26 \\
8 \\
13 \\
4 \\
2\end{array}$ & $\begin{array}{r}43 \\
- \\
43 \\
0 \\
41 \\
5 \\
.3\end{array}$ & $\begin{array}{r}23 \\
0 \\
23 \\
0 \\
16 \\
6 \\
1\end{array}$ & $\begin{array}{l}\ddot{.} \\
\ddot{.} \\
\ddot{.} \\
\ddot{.} \\
\ddot{-}\end{array}$ & $\begin{array}{r}140 \\
0 \\
140 \\
0 \\
135 \\
1 \\
4\end{array}$ & $\begin{array}{r}-8 \\
(s) \\
-8 \\
0 \\
-12 \\
4 \\
(s)\end{array}$ & $\begin{array}{l}\cdots \\
\ddot{.} \\
\ddot{.} \\
\ddot{.} \\
\ddot{*} \\
\ddot{*}\end{array}$ & $\begin{array}{l}9 \\
0 \\
9 \\
0 \\
0 \\
4 \\
4\end{array}$ & $\begin{array}{r}1 \\
(s) \\
(s) \\
0 \\
(s) \\
(s) \\
0\end{array}$ & $\begin{array}{r}234 \\
3 \\
231 \\
8 \\
217 \\
8 \\
-1\end{array}$ \\
\hline $\begin{array}{l}\text { Other Llqulds ................................. } \\
\text { Other Hydrocarbons/Oxygenates ..... } \\
\text { Unfinished Oils .................................. } \\
\text { Motor Gasoline Blend. Comp. ........... } \\
\text { Aviation Gasoline Blend. Comp. ........ }\end{array}$ & $\begin{array}{r}36 \\
35 \\
\ddot{1} \\
\cdots\end{array}$ & $\begin{array}{l}\ddot{-} \\
\ddot{.} \\
\ddot{.} \\
\ddot{*}\end{array}$ & $\begin{array}{r}167 \\
0 \\
143 \\
23 \\
0\end{array}$ & 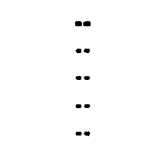 & $\begin{array}{l}9 \\
0 \\
2 \\
7 \\
0\end{array}$ & $\begin{array}{r}9 \\
2 \\
15 \\
.8 \\
0\end{array}$ & 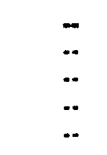 & $\begin{array}{r}201 \\
33 \\
130 \\
39 \\
0\end{array}$ & $\begin{array}{r}\text { (8) } \\
0 \\
0 \\
(s) \\
0\end{array}$ & $\begin{array}{l}1 \\
0 \\
1 \\
0 \\
0\end{array}$ \\
\hline 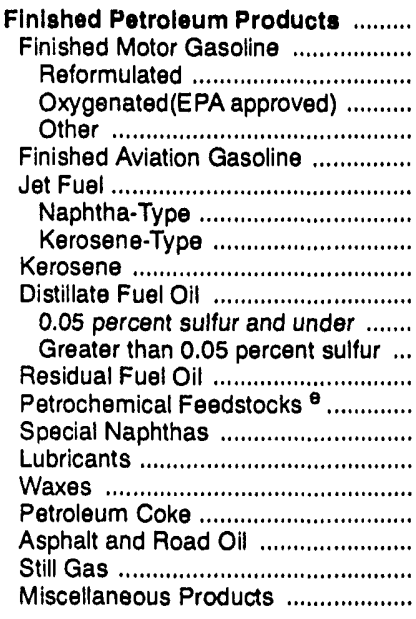 & 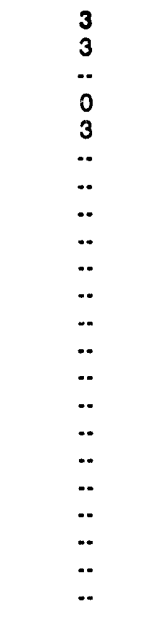 & $\begin{array}{r}1,366 \\
678 \\
0 \\
167 \\
511 \\
(s) \\
73 \\
6 \\
67 \\
5 \\
335 \\
36 \\
299 \\
90 \\
14 \\
(s) \\
12 \\
4 \\
51 \\
51 \\
51 \\
2\end{array}$ & $\begin{array}{r}860 \\
189 \\
0 \\
0 \\
189 \\
0 \\
88 \\
0 \\
88 \\
1 \\
227 \\
62 \\
166 \\
311 \\
2 \\
(s) \\
13 \\
2 \\
0 \\
26 \\
0 \\
(s)\end{array}$ & $\begin{array}{l}. . \\
. . \\
. . \\
. . \\
. . \\
. . \\
. . \\
. . \\
. . \\
. \\
. . \\
. \\
. . \\
. . \\
. . \\
. . \\
. . \\
. . \\
. . \\
. .\end{array}$ & $\begin{array}{r}2,534 \\
1,468 \\
0 \\
155 \\
1,313 \\
5 \\
378 \\
9 \\
369 \\
18 \\
632 \\
5 \\
627 \\
9 \\
0 \\
3 \\
12 \\
(5) \\
0 \\
5 \\
0 \\
3\end{array}$ & $\begin{array}{r}-477 \\
-131 \\
N A \\
N A \\
N A \\
-4 \\
26 \\
5 \\
22 \\
-6 \\
.325 \\
N A \\
\text { NA } \\
-79 \\
1 \\
-1 \\
-14 \\
(5) \\
7 \\
44 \\
0 \\
5\end{array}$ & $\begin{array}{l}. . \\
. . \\
. . \\
. . \\
. . \\
. . \\
. . \\
. . \\
. . \\
. . \\
. . \\
. . \\
. . \\
. . \\
. . \\
. . \\
. . \\
. . \\
. .\end{array}$ & $\begin{array}{l}. . \\
. . \\
. . \\
. . \\
. . \\
. . \\
. . \\
. . \\
. . \\
. . \\
. . \\
. . \\
. . \\
. . \\
. . \\
. . \\
. . \\
. . \\
. .\end{array}$ & $\begin{array}{r}16 \\
1 \\
N A \\
N A \\
N A \\
0 \\
0 \\
0 \\
0 \\
(s) \\
3 \\
N A \\
N A \\
7 \\
0 \\
(s) \\
3 \\
(s) \\
(s) \\
(s) \\
0 \\
(s)\end{array}$ & $\begin{array}{r}5,225 \\
2,467 \\
\text { NA } \\
\text { NA } \\
\text { NA } \\
9 \\
512 \\
10 \\
502 \\
31 \\
1,516 \\
\text { NA } \\
\text { NA } \\
482 \\
15 \\
5 \\
48 \\
7 \\
44 \\
38 \\
51 \\
(s)\end{array}$ \\
\hline Total & 92 & 1,409 & 2,308 & -100 & 2,685 & -419 & 0 & 1,337 & 16 & 5,461 \\
\hline
\end{tabular}

a Represents the PAD District in which the material entered the United States and not necessarily where the crude oil or product is processed and/or consumed.

b Unaccounted for crude oil represents the difference between the supply and disposition of crude oil.

c A negative number indicates a decrease in stocks and a positive number indicates an increase in stocks.

d Products supplied is equal to field production, plus refinery production, plus imports, plus unaccounted for crude oil, plus net receipts, minus stock change, minus crude losses, minus refinery inputs, minus exports.

$\theta$ Includes naphtha less than $401^{\circ} \mathrm{F}$ endpoint and other oils equal to or greater than $401^{\circ} \mathrm{F}$ endpoint.

(s) = Less than 500 barrels per day.

$E=$ Estimated.

$L R G=$ Liquefied Refinery Gas.

Note: Totals may not equal sum of components due to independent rounding.

Sources: - Energy Information Administration (EIA) Forms EIA-810, "Monthly Refinery Report," EIA-811, "Monthly Bulk Terminal Report," EIA-812, "Monthly Product Pipeline Report," EIA-813, "Monthly Crude Oil Report, "EIA-814, "Monthly Imports Report," ElA-816, "Monthly Natural Gas Liquids Report," ElA-817, "Monthly Tanker and Barge Movement Report," and EIA-819M, "Monthly Oxygenate Telephone Report". - Domestic crude oil production estimates based on historical statistics from State conservation agencies and the Minerals Management Service of the U.S. Department of the Interior. - Export data from the Bureau of the Census and Form EIA-810, "Monthly Refinery Report." 
Table 9. PAD District L-Year-to-Date Dally Average Supply and Disposition of Crude Oll and Petroleum Products, January-March 1993

(Thousand Barrels per Day)

\begin{tabular}{|c|c|c|c|c|c|c|c|c|c|c|}
\hline \multirow[b]{2}{*}{ Commodity } & \multicolumn{5}{|c|}{ Supply } & \multicolumn{5}{|c|}{ Dlsposition } \\
\hline & $\begin{array}{c}\text { Flold } \\
\text { Productlon }\end{array}$ & $\begin{array}{c}\text { Reflnery } \\
\text { Productlon }\end{array}$ & $\begin{array}{c}\text { Importe by } \\
\text { PAD } \\
\text { Distrlet } \\
\text { of Entre }\end{array}$ & $\begin{array}{l}\text { Unac- } \\
\text { counted } \\
\text { For } \\
\text { Cnude Oll } \\
\end{array}$ & $\begin{array}{c}\text { Not } \\
\text { Recelpte }\end{array}$ & $\begin{array}{c}\text { Stock } \\
\text { Change }\end{array}$ & $\begin{array}{l}\text { Crude } \\
\text { Lesens }\end{array}$ & $\begin{array}{c}\text { Reflnery } \\
\text { Inpute }\end{array}$ & Exports & $\begin{array}{l}\text { Producte } \\
\text { Suppolled }\end{array}$ \\
\hline Crude Oll & $E_{26}$ & .. & 1,181 & .26 & (e) & 19 & 0 & 1,162 & 0 & 0 \\
\hline 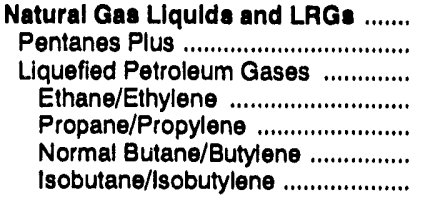 & $\begin{array}{r}30 \\
4 \\
26 \\
8 \\
12 \\
4 \\
1\end{array}$ & $\begin{array}{r}37 \\
-. \\
37 \\
0 \\
43 \\
-4 \\
-2\end{array}$ & $\begin{array}{r}26 \\
0 \\
26 \\
0 \\
20 \\
6 \\
(\mathbf{s})\end{array}$ & $\begin{array}{l}\ddot{.} \\
\ddot{.} \\
\ddot{.} \\
\ddot{.} \\
\ddot{.}\end{array}$ & $\begin{array}{r}139 \\
0 \\
139 \\
0 \\
132 \\
6 \\
1\end{array}$ & $\begin{array}{r}-25 \\
(s) \\
-25 \\
0 \\
-23 \\
-2 \\
(s)\end{array}$ & $\begin{array}{l}\ddot{.} \\
\ddot{.} \\
\ddot{.} \\
\ddot{.} \\
\ddot{.}\end{array}$ & $\begin{array}{r}14 \\
0 \\
14 \\
0 \\
0 \\
10 \\
4\end{array}$ & $\begin{array}{r}1 \\
(s) \\
1 \\
0 \\
1 \\
(s) \\
0\end{array}$ & $\begin{array}{r}243 \\
4 \\
240 \\
8 \\
231 \\
3 \\
-2\end{array}$ \\
\hline 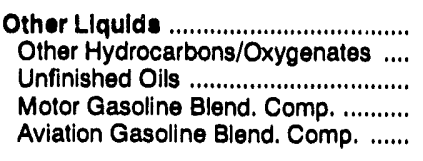 & $\begin{array}{r}61 \\
52 \\
\ddot{9} \\
-.\end{array}$ & $\begin{array}{l}\ddot{.} \\
\ddot{.} \\
\ddot{.}\end{array}$ & $\begin{array}{r}184 \\
5 \\
157 \\
23 \\
0\end{array}$ & $\begin{array}{l}\ddot{.} \\
\ddot{.} \\
\ddot{.} \\
\ddot{.}\end{array}$ & $\begin{array}{l}8 \\
0 \\
5 \\
3 \\
0\end{array}$ & $\begin{array}{r}1 \\
9 \\
1 \\
-10 \\
0\end{array}$ & 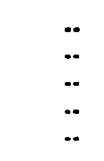 & $\begin{array}{r}251 \\
47 \\
159 \\
45 \\
0\end{array}$ & $\begin{array}{r}(8) \\
0 \\
0 \\
(\mathbf{s}) \\
0\end{array}$ & $\begin{array}{l}1 \\
0 \\
1 \\
0 \\
0\end{array}$ \\
\hline 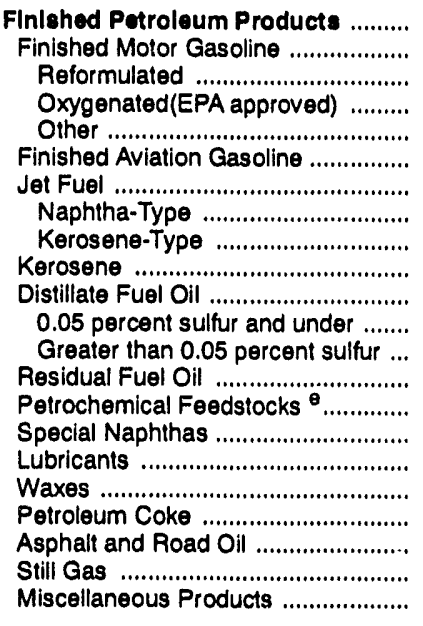 & 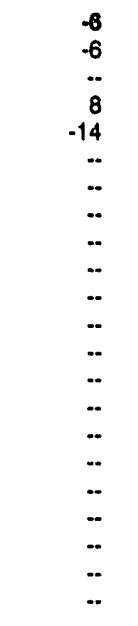 & $\begin{array}{r}1,461 \\
749 \\
0 \\
259 \\
490 \\
(\mathbf{s}) \\
82 \\
7 \\
75 \\
7 \\
347 \\
44 \\
303 \\
103 \\
15 \\
0 \\
16 \\
4 \\
50 \\
30 \\
57 \\
2\end{array}$ & $\begin{array}{r}808 \\
188 \\
0 \\
0 \\
0 \\
188 \\
0 \\
07 \\
87 \\
3 \\
84 \\
1 \\
201 \\
48 \\
152 \\
286 \\
4 \\
(8) \\
11 \\
2 \\
0 \\
26 \\
26 \\
0 \\
2\end{array}$ & 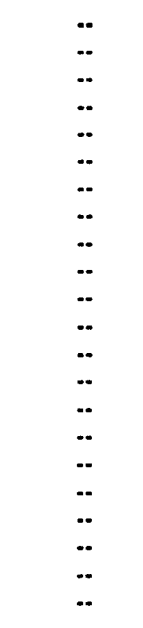 & $\begin{array}{r}2,559 \\
1,477 \\
0 \\
278 \\
1,199 \\
3 \\
363 \\
11 \\
352 \\
21 \\
658 \\
36 \\
623 \\
13 \\
-1 \\
3 \\
12 \\
(s) \\
0 \\
6 \\
0 \\
3\end{array}$ & $\begin{array}{r}-343 \\
41 \\
N A \\
N A \\
N A \\
1 \\
-2 \\
1 \\
-3 \\
-10 \\
-355 \\
N A \\
\text { NA } \\
-43 \\
1 \\
-1 \\
-2 \\
(s) \\
2 \\
22 \\
0 \\
3\end{array}$ & 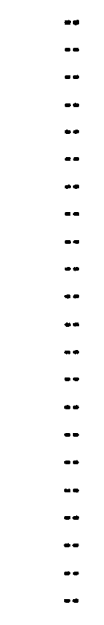 & 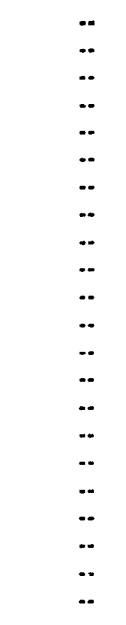 & $\begin{array}{r}28 \\
1 \\
N A \\
N A \\
N A \\
0 \\
3 \\
(s) \\
3 \\
(s) \\
1 \\
N A \\
N A \\
14 \\
0 \\
\text { (s) } \\
4 \\
\text { (s) } \\
3 \\
\text { (s) } \\
0 \\
\text { (s) }\end{array}$ & $\begin{array}{r}5,137 \\
2,365 \\
\text { NA } \\
\text { NA } \\
\text { NA } \\
2 \\
531 \\
20 \\
511 \\
39 \\
1,559 \\
\text { NA } \\
\text { NA } \\
431 \\
18 \\
5 \\
37 \\
6 \\
44 \\
40 \\
57 \\
4\end{array}$ \\
\hline Total & 110 & 1,499 & 2,199 & .26 & 2,707 & 349 & 0 & 1,427 & 29 & 5,382 \\
\hline
\end{tabular}

a Represents the PAD District in which the material entered the United States and not necessarily where the crude oil or product is processed and/or consumed.

b Unaccounted for crude oil represents the difference between the supply and disposition of crude oil.

c A negative number indicates a decrease in stocks and a positive number indicates an increase in stocks.

d Products supplied is equal to field production, plus refinery production, plus imports, plus unaccounted for crude oil, plus net receipts, minus stock change, minus crude losses, minus refinery inputs, minus exports.

$\theta$ Includes naphtha less than $401^{\circ} \mathrm{F}$ endpoint and other olls equal to or greater than $401^{\circ} \mathrm{F}$ endpoint.

(s) $=$ Less than 500 barrels per day.

$E=$ Estimated

$L R G=$ Liquefied Refinery Gas.

Note: Totals may not equal sum of components due to independent rounding.

Sources: - Energy Information Administration (EIA) Forms EIA-810, "Monthly Refinery Report," EIA-811, "Monthly Bulk Terminal Report," EIA-812, "Monthly Product Plpellne Report," EIA-813, "Monthly Crude Oil Report," EIA-814, "Monthly Imports Report," EIA-816, "Monthly Natural Gas Liquids Report," EIA-817, "Monthly Tanker and Barge Movement Report," and EIA-819M, "Monthly Oxygenate Telephone Report". - Domestic crude oil production estimates based on historical statistics from State conservation agencies and the Minerals Management Service of the U.S. Department of the Interior. • Export data from the Bureau of the Census and Form ElA-810, "Monthly Refinery Report." 
Table 10. PAD District II-Supply, Disposition, and Ending Stocks of Crude Oll and Petroleum Products, March 1993

(Thousand Barrels)

\begin{tabular}{|c|c|c|c|c|c|c|c|c|c|c|c|}
\hline \multirow[b]{2}{*}{ Commodlty } & \multicolumn{5}{|c|}{ Supply } & \multicolumn{5}{|c|}{ Dlaposition } & \multirow[b]{2}{*}{$\begin{array}{l}\text { Ending } \\
\text { Stocke }\end{array}$} \\
\hline & $\begin{array}{c}\text { Fiald } \\
\text { Production }\end{array}$ & $\begin{array}{c}\text { Rellnery } \\
\text { Productlon }\end{array}$ & $\begin{array}{c}\text { Imports by } \\
\text { PAD } \\
\text { Dlatrlet } \\
\text { of Entro }\end{array}$ & $\begin{array}{c}\text { Unac- } \\
\text { counted } \\
\text { For } \\
\text { Crude Oll }\end{array}$ & $\begin{array}{c}\text { Not } \\
\text { Recelpte }\end{array}$ & $\begin{array}{c}\text { Stock } \\
\text { Changec }\end{array}$ & $\begin{array}{l}\text { Crude } \\
\text { Logses }\end{array}$ & $\begin{array}{c}\text { Reflnery } \\
\text { Inpute }\end{array}$ & Exports & $\begin{array}{l}\text { Products } \\
\text { Supplled }\end{array}$ & \\
\hline 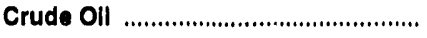 & ${ }^{E} 20,455$ & - & 19,648 & 1,044 & 56,617 & 3,115 & 0 & 94,602 & 46 & 0 & 73,747 \\
\hline $\begin{array}{l}\text { Natural Gas Liquide and LRGe .......... } \\
\text { Pentanes Plus .................................. } \\
\text { Liquefied Petroleum Gases ............. } \\
\text { Ethane/Ethylene ............................ } \\
\text { Propane/Propylene .................... } \\
\text { Normal Butane/Butylene ................ } \\
\text { Isobulane/lsobutylene .................. }\end{array}$ & $\begin{array}{r}10,439 \\
1,353 \\
9,086 \\
3,357 \\
3,807 \\
1,146 \\
776\end{array}$ & $\begin{array}{r}3,807 \\
30 \\
3,807 \\
0 \\
3,070 \\
611 \\
126\end{array}$ & $\begin{array}{r}2,081 \\
42 \\
2,039 \\
372 \\
1,447 \\
131 \\
89\end{array}$ & $\begin{array}{l}\ddot{ } \\
\ddot{.} \\
\ddot{.} \\
\ddot{.} \\
\ddot{.}\end{array}$ & $\begin{array}{r}1,509 \\
479 \\
1,030 \\
\cdot 1,166 \\
1,796 \\
.64 \\
464\end{array}$ & $\begin{array}{r}468 \\
.33 \\
499 \\
17 \\
.269 \\
433 \\
318\end{array}$ & $\begin{array}{l}\ddot{.} \\
. . \\
. . \\
\ddot{.} \\
. .\end{array}$ & $\begin{array}{r}2,749 \\
786 \\
1,963 \\
0 \\
0 \\
630 \\
1,333\end{array}$ & $\begin{array}{r}238 \\
27 \\
209 \\
0 \\
83 \\
126 \\
0\end{array}$ & $\begin{array}{r}14,385 \\
1,094 \\
13,291 \\
2,546 \\
10,306 \\
635 \\
.196\end{array}$ & $\begin{array}{r}21,614 \\
3,294 \\
18,320 \\
4,242 \\
7,384 \\
3,898 \\
2,796\end{array}$ \\
\hline $\begin{array}{l}\text { Other Llquide ................................... } \\
\text { Other Hydrocarbons/Oxygenates ..... } \\
\text { Unfinished Olls .................................... } \\
\text { Molor Gasoline Blend. Comp. .......... } \\
\text { Aviation Gasoline Blend. Comp. ........ }\end{array}$ & $\begin{array}{r}-837 \\
162 \\
. . \\
.999 \\
. .\end{array}$ & $\begin{array}{l}\ddot{.} \\
\ddot{.} \\
. .\end{array}$ & $\begin{array}{r}104 \\
0 \\
102 \\
2 \\
0\end{array}$ & $\begin{array}{l}- \\
\ddot{.} \\
. . \\
. .\end{array}$ & $\begin{array}{r}776 \\
0 \\
96 \\
680 \\
0\end{array}$ & $\begin{array}{r}2,203 \\
-142 \\
2,468 \\
-125 \\
2\end{array}$ & $\begin{array}{l}\ddot{*} \\
. . \\
. .\end{array}$ & $\begin{array}{r}-977 \\
304 \\
-1,085 \\
-192 \\
-4\end{array}$ & $\begin{array}{l}0 \\
0 \\
0 \\
0 \\
0\end{array}$ & $\begin{array}{r}-1,183 \\
0 \\
-1,185 \\
0 \\
2\end{array}$ & $\begin{array}{r}26,946 \\
1,300 \\
17,214 \\
8,426 \\
6\end{array}$ \\
\hline 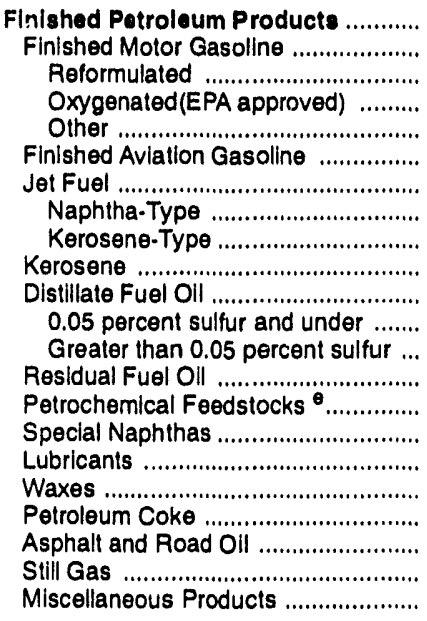 & $\begin{array}{r}2,644 \\
2,644 \\
. \\
0 \\
2,644 \\
. . \\
. . \\
. . \\
. . \\
. . \\
. . \\
. . \\
. . \\
. . \\
. . \\
. . \\
. \\
. . \\
.\end{array}$ & $\begin{array}{r}97,008 \\
48,906 \\
0 \\
2,537 \\
46,369 \\
79 \\
6,513 \\
483 \\
6,030 \\
849 \\
22,437 \\
425 \\
22,012 \\
2,396 \\
1,402 \\
468 \\
851 \\
70 \\
3,771 \\
4,882 \\
3,995 \\
389\end{array}$ & $\begin{array}{r}348 \\
59 \\
0 \\
0 \\
59 \\
1 \\
71 \\
71 \\
0 \\
0 \\
110 \\
38 \\
72 \\
2 \\
32 \\
53 \\
6 \\
7 \\
0 \\
0 \\
0 \\
5\end{array}$ & $\begin{array}{l}. . \\
. . \\
. . \\
. . \\
. . \\
. . \\
. . \\
. . \\
. . \\
. \\
. . \\
. \\
. . \\
. \\
. . \\
. \\
. . \\
. \\
. . \\
. .\end{array}$ & $\begin{array}{r}21,676 \\
14,207 \\
0 \\
0 \\
14,207 \\
91 \\
2,324 \\
43 \\
2,281 \\
110 \\
4,873 \\
.452 \\
5,325 \\
-347 \\
0 \\
25 \\
245 \\
0 \\
0 \\
83 \\
0 \\
65\end{array}$ & $\begin{array}{r}-664 \\
-2,544 \\
N A \\
N A \\
N A \\
3 \\
-367 \\
-98 \\
-269 \\
219 \\
.159 \\
N A \\
N A \\
.90 \\
90 \\
77 \\
124 \\
7 \\
.73 \\
2,016 \\
0 \\
33\end{array}$ & $\begin{array}{l}. . \\
. . \\
. . \\
. . \\
. . \\
. . \\
. . \\
. . \\
. . \\
. . \\
. . \\
.0 \\
. . \\
.0 \\
. . \\
. . \\
.0 \\
. .\end{array}$ & $\begin{array}{l}. . \\
. . \\
. . \\
. . \\
. . \\
. . \\
. . \\
. . \\
. . \\
. . \\
. . \\
. . \\
. . \\
. . \\
. . \\
. . \\
. . \\
. . \\
. .\end{array}$ & $\begin{array}{r}338 \\
16 \\
\text { NA } \\
\text { NA } \\
\text { NA } \\
0 \\
2 \\
1 \\
(s) \\
1 \\
(s) \\
\text { NA } \\
\text { NA } \\
1 \\
0 \\
8 \\
42 \\
3 \\
244 \\
21 \\
0 \\
\text { (s) }\end{array}$ & $\begin{array}{r}122,000 \\
68,344 \\
N A \\
N A \\
N A \\
168 \\
9,273 \\
694 \\
8,580 \\
739 \\
27,579 \\
N A \\
N A \\
2,140 \\
1,344 \\
461 \\
936 \\
67 \\
3,600 \\
2,928 \\
3,995 \\
426\end{array}$ & $\begin{array}{r}112,275 \\
50,624 \\
0 \\
1,856 \\
48,768 \\
483 \\
8,369 \\
899 \\
7,470 \\
1,488 \\
28,955 \\
1,605 \\
27,350 \\
2,756 \\
293 \\
485 \\
2,040 \\
111 \\
2,723 \\
13,674 \\
0 \\
274\end{array}$ \\
\hline 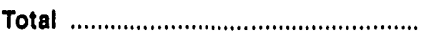 & 32,701 & 100,815 & 22,179 & 1,044 & 80,578 & 5,120 & 0 & 96,374 & 621 & 135,202 & 234,582 \\
\hline
\end{tabular}

Represents the PAD District in which the material entered the United States and not necessarily where the crude oll or product is processed and/or consumed.

b Unaccounted for crude oll represents the difference between the supply and disposition of crude oll.

c A negative number indicates a decrease in stocks and a positive number indicates an increase in stocks.

Droducts supplied is equal to fleld production, plus refinery production, plus imports, plus unaccounted for crude oll, plus net receipts, minus stock change, minus crude losses, minus refinery inputs, minus exports.

- Includes naphtha less than $401^{\circ} \mathrm{F}$ endpoint and other olls equal to or greater than $401^{\circ} \mathrm{F}$ endpoint.

(s) = Less than 500 barrels.

$E=$ Estimated

$L R G=$ Llquefied Refinery Gas.

Note: Totals may not equal sum of components due to independent rounding.

Sources: - Energy Information Administration (EIA) Forms ElA-810, "Monthly Refinery Report," ElA.811, "Monthly Bulk Terminal Report," EIA-812, "Monthly Product Pipeline Report," EIA-813, "Monthly Crude Oll Report," EIA-814, "Monthly Imports Report," EIA-816, "Monthly Natural Gas Liquids Report," EIA.817, "Monthly Tanker and Barge Movement Report," and EIA-819M, "Monthly Oxygenate Telephone Report". - Domestic crude oll production estimates based on historical statistics from State conservation agencies and the Minerals Management Service of the U.S. Department of the Interlor. - Export data from the Bureau of the Census and Form EIA.810,

"Monthly Refinery Report." 
Table 11. PAD Dlstrlct II-Year-to-Date Supply, Dlsposition, and Ending Stocks of Crude Oll and Petroleum Products, January-March 1993 (Thousand Barrels)

\begin{tabular}{|c|c|c|c|c|c|c|c|c|c|c|c|}
\hline \multirow[b]{2}{*}{ Cominodity } & \multicolumn{5}{|c|}{ Supply } & \multicolumn{5}{|c|}{ Dleposition } & \multirow[b]{2}{*}{$\begin{array}{l}\text { Ending } \\
\text { Stocks }\end{array}$} \\
\hline & $\begin{array}{c}\text { Flold } \\
\text { Production }\end{array}$ & $\begin{array}{c}\text { Rolinery } \\
\text { Produetion }\end{array}$ & $\begin{array}{c}\text { Imports by } \\
\text { PAD } \\
\text { Diatrict } \\
\text { of Entry }\end{array}$ & $\begin{array}{l}\text { Unse- } \\
\text { counted } \\
\text { For } \\
\text { Crude oll }\end{array}$ & $\begin{array}{c}\text { Net } \\
\text { Recelpte }\end{array}$ & $\begin{array}{c}\text { Stock } \\
\text { Change }\end{array}$ & $\begin{array}{l}\text { Crude } \\
\text { Losenes }\end{array}$ & $\begin{array}{c}\text { Aoflnery } \\
\text { Inpute }\end{array}$ & Exports & $\begin{array}{l}\text { Products } \\
\text { Supplied d }\end{array}$ & \\
\hline Crude Oll & $E_{88,224}$ & •. & $\mathbf{s 3}, 001$ & 2,289 & 164,631 & 4,261 & 0 & 273,612 & 272 & 0 & $\mathbf{7 3 , 7 4 7}$ \\
\hline 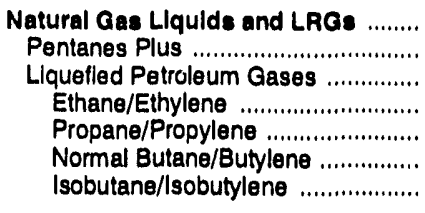 & $\begin{array}{r}29,264 \\
3,900 \\
25,364 \\
9,149 \\
10,702 \\
3,469 \\
2,044\end{array}$ & $\begin{array}{r}9,534 \\
\ddot{0} \\
9,534 \\
0 \\
9,196 \\
75 \\
263\end{array}$ & $\begin{array}{r}6,473 \\
124 \\
6,349 \\
962 \\
4,041 \\
1,159 \\
187\end{array}$ & $\begin{array}{l}. . \\
. . \\
. . \\
. . \\
. . \\
. . \\
. \cdot\end{array}$ & $\begin{array}{r}\mathbf{5 , 1 3 9} \\
2,517 \\
2,622 \\
-4,540 \\
\mathbf{5 , 4 3 3} \\
\mathbf{5 5 5} \\
1,174\end{array}$ & $\begin{array}{r}.6,446 \\
204 \\
\cdot 6,650 \\
-570 \\
-4,212 \\
-1,884 \\
16\end{array}$ & $\begin{array}{l}\ddot{.} \\
\ddot{.} \\
\ddot{.} \\
. . \\
\ddot{.}\end{array}$ & $\begin{array}{r}11,813 \\
2,556 \\
9,257 \\
0 \\
0 \\
5,420 \\
3,837\end{array}$ & $\begin{array}{r}512 \\
81 \\
430 \\
0 \\
222 \\
209 \\
0\end{array}$ & $\begin{array}{r}44,531 \\
3,700 \\
40,832 \\
6,141 \\
33,362 \\
1,514 \\
.185\end{array}$ & $\begin{array}{r}21,614 \\
3,294 \\
18,320 \\
4,242 \\
7,384 \\
3,898 \\
2,796\end{array}$ \\
\hline $\begin{array}{l}\text { Other Lquulds .................................. } \\
\text { Other Hydrocarbons/Oxygenates ..... } \\
\text { Unfinished Olls ................................ } \\
\text { Motor Gasollne Blend. Comp. .......... } \\
\text { Avlation Gasoline Blend. Comp. ........ }\end{array}$ & $\begin{array}{r}.153 \\
1,647 \\
.0 \\
.1,800 \\
\ldots\end{array}$ & $\begin{array}{l}\ddot{.} \\
\ddot{.} \\
\ddot{.} \\
\ddot{.}\end{array}$ & $\begin{array}{r}373 \\
0 \\
370 \\
3 \\
0\end{array}$ & $\begin{array}{l}\ddot{*} \\
\ddot{.} \\
\ddot{.} \\
\ddot{*}\end{array}$ & $\begin{array}{r}1,745 \\
0 \\
126 \\
1,619 \\
0\end{array}$ & $\begin{array}{r}4,609 \\
849 \\
2,908 \\
851 \\
1\end{array}$ & $\begin{array}{l}- \\
. . \\
. . \\
. .\end{array}$ & $\begin{array}{r}1,042 \\
798 \\
1,279 \\
\cdot 1,029 \\
\cdot 6\end{array}$ & $\begin{array}{r}(8) \\
0 \\
0 \\
(s) \\
0\end{array}$ & $\begin{array}{r}-3,686 \\
0 \\
-3,691 \\
0 \\
5\end{array}$ & $\begin{array}{r}26,946 \\
1,300 \\
17,214 \\
8,426 \\
6\end{array}$ \\
\hline 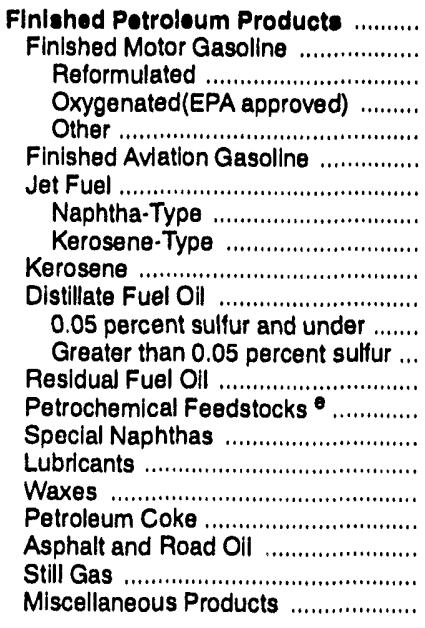 & $\begin{array}{r}6,291 \\
6,291 \\
. . \\
11,382 \\
-5,091 \\
. . \\
. . \\
. . \\
. . \\
. . \\
. . \\
. . \\
. . \\
. . \\
. . \\
. . \\
. . \\
. . \\
. . \\
. . \\
. . \\
. .\end{array}$ & $\begin{array}{r}289,991 \\
151,171 \\
0 \\
7,901 \\
143,270 \\
231 \\
18,555 \\
1,302 \\
17,253 \\
2,886 \\
65,297 \\
4,272 \\
61,025 \\
7,124 \\
4,189 \\
1,216 \\
2,133 \\
168 \\
11,067 \\
13,057 \\
11,792 \\
1,105\end{array}$ & $\begin{array}{r}1,379 \\
263 \\
0 \\
0 \\
263 \\
4 \\
214 \\
214 \\
0 \\
0 \\
574 \\
318 \\
256 \\
26 \\
47 \\
178 \\
40 \\
19 \\
0 \\
0 \\
0 \\
14\end{array}$ & $\begin{array}{l}. . \\
. . \\
. . \\
. . \\
. . \\
. . \\
. . \\
. . \\
. . \\
. . \\
. . \\
. . \\
. . \\
. . \\
. . \\
. . \\
. . \\
. . \\
. . \\
. .\end{array}$ & $\begin{array}{r}54,800 \\
38,988 \\
0 \\
-146 \\
39,134 \\
250 \\
6,376 \\
111 \\
6,265 \\
221 \\
9,817 \\
40 \\
9,777 \\
-1,705 \\
84 \\
56 \\
548 \\
0 \\
0 \\
83 \\
0 \\
82\end{array}$ & $\begin{array}{r}8,241 \\
4,351 \\
N A \\
N A \\
N A \\
103 \\
-964 \\
-116 \\
-848 \\
2 \\
-2,373 \\
N A \\
N A \\
-257 \\
92 \\
50 \\
134 \\
-14 \\
104 \\
6,925 \\
0 \\
88\end{array}$ & $\begin{array}{l}. . \\
. . \\
. . \\
. . \\
. . \\
. . \\
. . \\
. . \\
. \\
. . \\
. \\
. \\
. . \\
. \\
. . \\
. \\
. \\
. . \\
. . \\
. \\
. .\end{array}$ & $\begin{array}{l}. . \\
. . \\
. . \\
. . \\
. . \\
. . \\
. . \\
. . \\
. . \\
. . \\
. \\
. . \\
. . \\
. . \\
. . \\
. . \\
. . \\
. . \\
. . \\
. .\end{array}$ & $\begin{array}{r}913 \\
190 \\
N A \\
N A \\
N A \\
0 \\
77 \\
29 \\
47 \\
2 \\
4 \\
N A \\
N A \\
50 \\
0 \\
21 \\
102 \\
10 \\
425 \\
31 \\
0 \\
(s)\end{array}$ & $\begin{array}{r}343,307 \\
192,172 \\
N A \\
N A \\
N A \\
382 \\
26,032 \\
1,714 \\
24,319 \\
3,103 \\
78,057 \\
N A \\
N A \\
5,652 \\
4,228 \\
1,379 \\
2,485 \\
181 \\
10,538 \\
6,184 \\
11,792 \\
1,113\end{array}$ & $\begin{array}{r}112,275 \\
50,624 \\
0 \\
1,856 \\
48,768 \\
483 \\
8,369 \\
899 \\
7,470 \\
1,488 \\
28,955 \\
1,605 \\
27,350 \\
2,756 \\
293 \\
485 \\
2,040 \\
111 \\
2,723 \\
13,674 \\
0 \\
274\end{array}$ \\
\hline 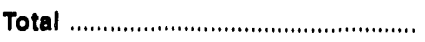 & 93,628 & 299,525 & 61,226 & 2,289 & 226,315 & 10,665 & 0 & 286,467 & 1,696 & 384,152 & 234,582 \\
\hline
\end{tabular}

Represents the PAD District in which the materlal entered the United States and not necessarily where the crude oll or product is processed and/or consumed.

b Unaccounted for crude oll represents the difference between the supply and disposition of crude oll.

c A negative number indicates a decrease in stocks and a positive number indicates an Increase in stocks.

Products supplled is equal to field production, plus refinery production, plus imports, plus unaccounted for crude oil, plus net receipts, minus stock change, minus crude losses, minus refinery inputs, minus exports.

- Includes naphtha less than $401^{\circ} \mathrm{F}$ endpoint and other olls equal to or greater than $401^{\circ} \mathrm{F}$ endpoint.

(s) $=$ Less than 500 barrels.

$E$ : Estimated

LRG = Liquefied Refinery Gas.

Note: Totals may not equal sum of components due to Independent rounding.

Sources: • Energy Information AdmInlstratlon (EIA) Forms EIA-810, "Monthly Refinery Report," ElA-814, "Monthly Bulk Terminal Report," ElA-812, "Monthly Product Pipeline Report," EIA-813, "Monthly Crude Oll Report," EIA-814, "Monthly Imports Report," EIA-816, "Monthly Natural Gas Liquids Report," EIA-817, "Monthly Tanker and Barge Movement Report," and EIA.819M, "Monthly Oxygenate Telephone Report". - Domestic crude oil production estimates based on historical statistics from State conservation agencies and the Minerals Management Service of the U.S. Department of the interlor. - Export data from the Bureau of the Census and Form EIA-810, "Monthly Refinery Report." 
Table 12. PAD District II-Daily Average Supply and Disposition of Crude Oil and Petroleum Products, March 1993 (Thousand Barrels per Day)

\begin{tabular}{|c|c|c|c|c|c|c|c|c|c|c|}
\hline \multirow[b]{2}{*}{ Commodity } & \multicolumn{5}{|c|}{ Supply } & \multicolumn{5}{|c|}{ Dleposition } \\
\hline & $\begin{array}{c}\text { Fiold } \\
\text { Productlon }\end{array}$ & $\begin{array}{c}\text { Rofinery } \\
\text { Productlon }\end{array}$ & $\begin{array}{c}\text { Imports by } \\
\text { PAD } \\
\text { Distrlct } \\
\text { of Entre }\end{array}$ & $\begin{array}{c}\text { Unac- } \\
\text { counted } \\
\text { For } \\
\text { Cnude } 0 \|^{b}\end{array}$ & $\begin{array}{c}\text { Net } \\
\text { Recelote }\end{array}$ & $\begin{array}{c}\text { Stock } \\
\text { Change }\end{array}$ & $\begin{array}{l}\text { Crude } \\
\text { Lerees }\end{array}$ & $\begin{array}{c}\text { Reflinery } \\
\text { Inpute }\end{array}$ & Expente & $\begin{array}{l}\text { Products } \\
\text { Supplled }\end{array}$ \\
\hline Crude Oll ................................................. & $E_{680}$ & $\cdots$ & 634 & 34 & 1,826 & 100 & 0 & 3,052 & 1 & 0 \\
\hline $\begin{array}{l}\text { Natural Gas Llquids and LRGs ........ } \\
\text { Pentanes Plus ................................... } \\
\text { Lquefied Petroleum Gases ............. } \\
\text { Ethane/Ethylene ............................ } \\
\text { Propane/Propylene ...................... } \\
\text { Normal Butane/Butylene ................ } \\
\text { Isobutane/lsobutylene .................. }\end{array}$ & $\begin{array}{r}337 \\
44 \\
293 \\
108 \\
123 \\
37 \\
25\end{array}$ & $\begin{array}{r}123 \\
123 \\
0 \\
99 \\
20 \\
4\end{array}$ & $\begin{array}{r}67 \\
1 \\
68 \\
12 \\
47 \\
4 \\
3\end{array}$ & 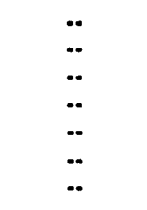 & $\begin{array}{r}49 \\
15 \\
33 \\
-38 \\
58 \\
-2 \\
15\end{array}$ & $\begin{array}{r}15 \\
-1 \\
16 \\
1 \\
-9 \\
14 \\
10\end{array}$ & $\begin{array}{l}\ddot{.} \\
\ddot{.} \\
. . \\
. . \\
\ddot{.} \\
.\end{array}$ & $\begin{array}{r}89 \\
25 \\
63 \\
0 \\
0 \\
20 \\
43\end{array}$ & $\begin{array}{l}8 \\
1 \\
7 \\
0 \\
3 \\
4 \\
0\end{array}$ & $\begin{array}{r}464 \\
35 \\
429 \\
82 \\
332 \\
20 \\
-6\end{array}$ \\
\hline $\begin{array}{l}\text { Other Llquids .................................. } \\
\text { Other Hydrocarbons/Oxygenates .... } \\
\text { Unfinished Oils .................................. } \\
\text { Motor Gasoline Blend. Comp. ........... } \\
\text { Aviation Gasoline Biend. Comp. ....... }\end{array}$ & $\begin{array}{r}-27 \\
5 \\
\ddot{32} \\
-.\end{array}$ & $\begin{array}{l}\ddot{.} \\
\ddot{-} \\
\ddot{-} \\
\ddot{-}\end{array}$ & $\begin{array}{r}3 \\
0 \\
3 \\
\text { (s) } \\
0\end{array}$ & $\begin{array}{l}\ddot{*} \\
\ddot{-} \\
\ddot{*}\end{array}$ & $\begin{array}{r}25 \\
0 \\
3 \\
22 \\
0\end{array}$ & $\begin{array}{r}71 \\
-5 \\
80 \\
-4 \\
(s)\end{array}$ & $\begin{array}{l}\ddot{.} \\
\ddot{*} \\
\ddot{.} \\
\ddot{-}\end{array}$ & $\begin{array}{r}-32 \\
10 \\
-35 \\
.6 \\
(s)\end{array}$ & $\begin{array}{l}0 \\
0 \\
0 \\
0 \\
0\end{array}$ & $\begin{array}{r}38 \\
0 \\
38 \\
0 \\
(3)\end{array}$ \\
\hline 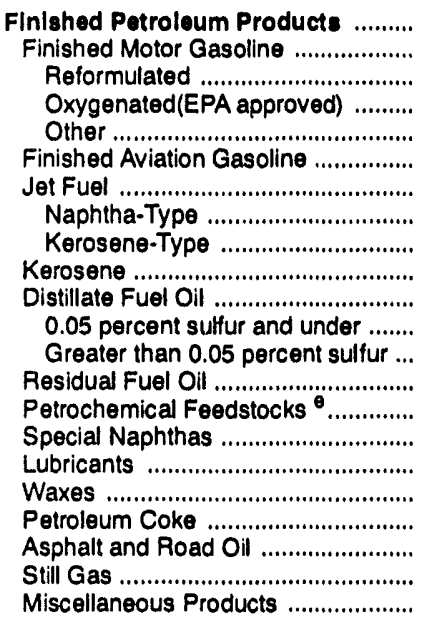 & $\begin{array}{r}85 \\
85 \\
. . \\
0 \\
85 \\
.- \\
. . \\
.- \\
. . \\
. . \\
. . \\
. . \\
. . \\
. . \\
. . \\
. . \\
. . \\
.- \\
. . \\
. . \\
. .\end{array}$ & $\begin{array}{r}3,129 \\
1,578 \\
0 \\
82 \\
1,496 \\
3 \\
210 \\
16 \\
195 \\
27 \\
724 \\
14 \\
710 \\
77 \\
45 \\
15 \\
27 \\
2 \\
122 \\
157 \\
129 \\
13\end{array}$ & $\begin{array}{r}11 \\
2 \\
0 \\
0 \\
2 \\
(s) \\
2 \\
2 \\
0 \\
0 \\
4 \\
1 \\
2 \\
(s) \\
1 \\
2 \\
(s) \\
\text { (s) } \\
0 \\
0 \\
0 \\
\text { (s) }\end{array}$ & $\begin{array}{l}. . \\
. . \\
. . \\
. . \\
. . \\
. . \\
\ddot{.} \\
. . \\
. . \\
. . \\
. . \\
. . \\
. . \\
. . \\
. . \\
. . \\
. . \\
. .\end{array}$ & $\begin{array}{r}699 \\
458 \\
0 \\
0 \\
458 \\
3 \\
75 \\
1 \\
74 \\
4 \\
157 \\
-15 \\
172 \\
-11 \\
0 \\
1 \\
8 \\
0 \\
0 \\
3 \\
0 \\
2\end{array}$ & $\begin{array}{r}-21 \\
-82 \\
N A \\
N A \\
N A \\
(s) \\
-12 \\
-3 \\
-9 \\
7 \\
-5 \\
N A \\
N A \\
-3 \\
3 \\
2 \\
4 \\
(s) \\
-2 \\
65 \\
0 \\
1\end{array}$ & $\begin{array}{l}. . \\
. . \\
. . \\
. . \\
. . \\
. . \\
. . \\
. . \\
. . \\
. . \\
. . \\
. . \\
. . \\
. . \\
. . \\
. . \\
. . \\
. .\end{array}$ & $\begin{array}{l}. . \\
. . \\
. . \\
. . \\
.- \\
.- \\
. . \\
. . \\
. . \\
. . \\
.- \\
. . \\
.- \\
. . \\
. . \\
.- \\
. . \\
.\end{array}$ & $\begin{array}{r}11 \\
1 \\
N A \\
N A \\
N A \\
0 \\
(s) \\
(s) \\
(s) \\
(s) \\
(s) \\
N A \\
N A \\
(s) \\
0 \\
(s) \\
1 \\
(s) \\
8 \\
1 \\
0 \\
(s)\end{array}$ & $\begin{array}{r}3,935 \\
2,205 \\
N A \\
N A \\
N A \\
5 \\
299 \\
22 \\
277 \\
24 \\
890 \\
N A \\
N A \\
69 \\
43 \\
15 \\
30 \\
2 \\
116 \\
94 \\
129 \\
14\end{array}$ \\
\hline Total ....................................................... & 1,055 & 3,252 & 715 & 34 & 2,599 & 165 & 0 & 3,109 & 20 & 4,361 \\
\hline
\end{tabular}

a Represents the PAD District in which the material entered the United States and not necessarily where the crude oil or product is processed and/or consumed.

b Unaccounted for crude oil represents the difference between the supply and disposition of crude oil.

c Anegative number indicates a decrease in stocks and a positive number indicates an increase in stocks.

Products supplied is equal to field production, plus refinery production, plus imports, plus unaccounted for crude oil, plus net receipts, minus stock change, minus crude losses, minus refinery inputs, minus exports.

- Includes naphtha less than $401^{\circ} \mathrm{F}$ endpoint and other oils equal to or greater than $401^{\circ} \mathrm{F}$ endpoint.

(s) = Less than 500 barrels per day.

$\mathrm{E}$ = Estimated.

$L R G=$ Liquefied Refinery Gas.

Note: Totals may not equal sum of components due to independent rounding.

Sources: - Energy Information Administration (EIA) Forms EIA-810, "Monthly Refinery Report," EIA-811, "Monthly Bulk Terminal Report," EIA-812, "Monthly Product Pipeline Report," EIA-813, "Monthly Crude Oil Report," EIA-814, "Monthly Imports Report," ElA-816, "Monthly Natural Gas Liquids Report," EIA-817, "Monthly Tanker and Barge Movement Report," and EIA-819M. "Monthly Oxygenate Telephone Report". - Domestic crude oil production estimates based on historical statistics from State conservation agencies and the Minerals Management Service of the U.S. Department of the Interior. - Export data from the Bureau of the Census and Form EIA-810, "Monthly Refinery Report." 
Table 13. PAD District II-Year-to-Date Daily Average Supply and Disposition of Crude Oll and Petroleum Products, January-March 1993 (Thousand Barrels per Day)

\begin{tabular}{|c|c|c|c|c|c|c|c|c|c|c|}
\hline \multirow[b]{2}{*}{ Commodity } & \multicolumn{5}{|c|}{ Supply } & \multicolumn{5}{|c|}{ Dleposition } \\
\hline & $\begin{array}{c}\text { Flold } \\
\text { Productlon }\end{array}$ & $\begin{array}{c}\text { Rofinery } \\
\text { Productlon }\end{array}$ & $\begin{array}{c}\text { Importe by } \\
\text { PAD } \\
\text { District } \\
\text { of Entry }\end{array}$ & $\begin{array}{l}\text { Unac. } \\
\text { counted } \\
\text { For } \\
\text { Crude } 01^{b} \\
\end{array}$ & $\begin{array}{c}\text { Net } \\
\text { Recelpte }\end{array}$ & $\begin{array}{c}\text { Stock } \\
\text { Chango }\end{array}$ & $\begin{array}{l}\text { Crude } \\
\text { Loseres }\end{array}$ & $\begin{array}{c}\text { Refinery } \\
\text { Inpute }\end{array}$ & Experte & $\begin{array}{l}\text { Producte } \\
\text { Supplled }\end{array}$ \\
\hline Crude Oll & . E 647 & .• & 589 & 26 & 1,829 & 47 & $n$ & 3,040 & 3 & 0 \\
\hline 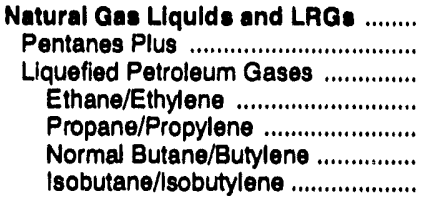 & $\begin{array}{r}325 \\
43 \\
282 \\
102 \\
119 \\
39 \\
23\end{array}$ & $\begin{array}{r}106 \\
106 \\
0 \\
0 \\
102 \\
1 \\
3\end{array}$ & $\begin{array}{r}72 \\
1 \\
71 \\
11 \\
45 \\
13 \\
2\end{array}$ & $\begin{array}{l}\ddot{.} \\
\ddot{*} \\
\ddot{.} \\
\ddot{.} \\
\ddot{*}\end{array}$ & $\begin{array}{r}57 \\
28 \\
29 \\
-50 \\
60 \\
6 \\
13\end{array}$ & $\begin{array}{r}.72 \\
2 \\
.74 \\
-6 \\
.47 \\
.21 \\
(3)\end{array}$ & $\begin{array}{l}\ddot{.} \\
\ddot{.} \\
\ddot{.} \\
\ddot{.} \\
\ddot{.}\end{array}$ & $\begin{array}{r}131 \\
28 \\
103 \\
0 \\
0 \\
60 \\
43\end{array}$ & $\begin{array}{l}6 \\
1 \\
5 \\
0 \\
2 \\
2 \\
0\end{array}$ & $\begin{array}{r}495 \\
41 \\
454 \\
68 \\
371 \\
17 \\
-2\end{array}$ \\
\hline 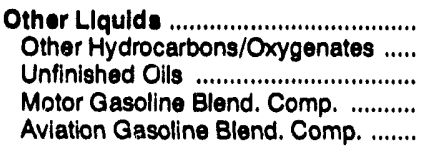 & $\begin{array}{r}.2 \\
18 \\
. . \\
.20 \\
. .\end{array}$ & $\begin{array}{l}\ddot{.} \\
\ddot{.} \\
\ddot{.}\end{array}$ & $\begin{array}{r}4 \\
0 \\
4 \\
(s) \\
0\end{array}$ & $\begin{array}{l}\ddot{.} \\
\ddot{.} \\
\ddot{.} \\
\ddot{.}\end{array}$ & $\begin{array}{r}19 \\
0 \\
1 \\
18 \\
0\end{array}$ & $\begin{array}{r}51 \\
9 \\
32 \\
9 \\
(\mathbf{s})\end{array}$ & $\begin{array}{l}\ddot{.} \\
\ddot{.} \\
\ddot{.}\end{array}$ & $\begin{array}{r}12 \\
9 \\
14 \\
-11 \\
(s)\end{array}$ & $\begin{array}{r}(s) \\
0 \\
0 \\
(s) \\
0\end{array}$ & $\begin{array}{r}-41 \\
0 \\
.41 \\
0 \\
(s)\end{array}$ \\
\hline 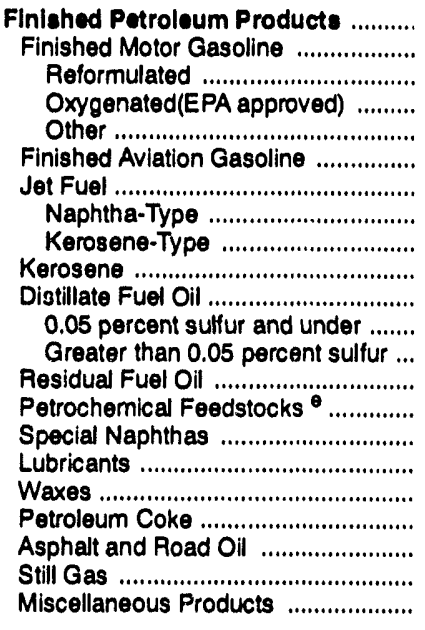 & 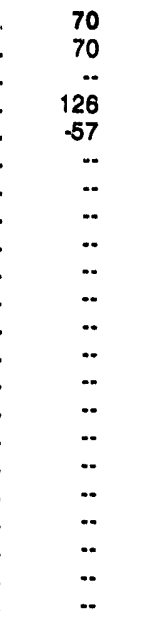 & $\begin{array}{r}3,222 \\
1,680 \\
0 \\
88 \\
1,592 \\
3 \\
3 \\
206 \\
14 \\
192 \\
32 \\
726 \\
47 \\
678 \\
79 \\
47 \\
14 \\
24 \\
2 \\
123 \\
145 \\
131 \\
12\end{array}$ & $\begin{array}{r}15 \\
3 \\
0 \\
0 \\
3 \\
(s) \\
2 \\
2 \\
0 \\
0 \\
6 \\
4 \\
3 \\
(s) \\
1 \\
2 \\
(s) \\
(s) \\
0 \\
0 \\
0 \\
\text { (s) }\end{array}$ & 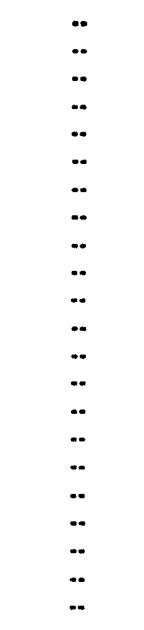 & $\begin{array}{r}609 \\
433 \\
0 \\
-2 \\
435 \\
3 \\
71 \\
1 \\
1 \\
70 \\
2 \\
109 \\
(5) \\
109 \\
-19 \\
1 \\
1 \\
6 \\
0 \\
0 \\
1 \\
0 \\
1\end{array}$ & $\begin{array}{r}92 \\
90 \\
N A \\
N A \\
N A \\
1 \\
-11 \\
-1 \\
-9 \\
(s) \\
-26 \\
\text { NA } \\
\text { NA } \\
3 \\
1 \\
1 \\
1 \\
\text { (s) } \\
1 \\
77 \\
0 \\
1\end{array}$ & $\begin{array}{l}. . \\
. . \\
. . \\
. . \\
. . \\
. . \\
. . \\
. . \\
\ddot{.} \\
. \\
\ddot{.} \\
. \\
. \\
. . \\
\ddot{.} \\
\ddot{.}\end{array}$ & 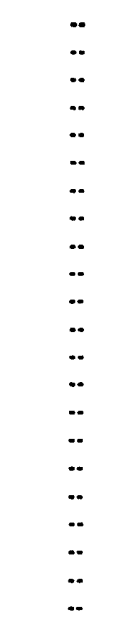 & $\begin{array}{r}10 \\
2 \\
\text { NA } \\
\text { NA } \\
\text { NA } \\
0 \\
1 \\
\text { (s) } \\
1 \\
\text { (s) } \\
\text { (s) } \\
\text { NA } \\
\text { NA } \\
1 \\
0 \\
\text { (s) } \\
1 \\
\text { (s) } \\
5 \\
\text { (s) } \\
0 \\
\text { (s) }\end{array}$ & $\begin{array}{r}3,815 \\
2,135 \\
\text { NA } \\
\text { NA } \\
\text { NA } \\
4 \\
289 \\
19 \\
270 \\
270 \\
34 \\
867 \\
\text { NA } \\
\text { NA } \\
63 \\
47 \\
15 \\
28 \\
2 \\
217 \\
117 \\
69 \\
131 \\
12\end{array}$ \\
\hline Total & 1,040 & 3,328 & 680 & 25 & 2,515 & 119 & 0 & 3,183 & 19 & 4,268 \\
\hline
\end{tabular}

Represents the PAD District in which the material entered the United States and not necessarily where the crude oil or product is processed and/or consumed.

Unaccounted for crude oil represents the difference between the supply and disposition of crude oil.

c A negative number indlcates a decrease in stocks and a postive number Indicates an increase in siocks.

Products supplied is equal to fleld production, plus refinery production, plus imports, plus unaccounted for crude oil, plus net receipts, minus stock change, minus crude losses, minus refinery Inputs, minus exports.

- Includes naphtha less than $401^{\circ} \mathrm{F}$ endpoint and other olls equal to or greater than $401^{\circ} \mathrm{F}$ endpoint.

(s) = Less than 500 barrels per day.

$E$ = Estimated.

LRG a Liquefled Refinery Gas.

Note: Totals may not equal sum of components due to Independent rounding.

Sources: - Energy Information Administration (EIA) Forms EIA-810, "Monthly Refinery Report," ElA.811, "Monthly Bulk Terminal Report," EIA.812, "Monthly Product Pipeline Report," ElA-813, "Monthly Crude Oll Report," ElA-814, "Monthly Imports Report," EIA-816, "Monthly Natural Gas Liquids Report," ElA-817, "Monthly Tanker and Barge Movement Report," and EIA-819M, "Monthly Oxygenate Telephone Report". "Dornestlc crude oll production estimates based on historical statistics from State conservation agencies and the Minerals Management Service of the U.S. Department of the Interlor. - Export data from the Bureau of the Consus and Form EIA.810, "Monthly Pefinery Peport." 
Table 14. PAD District III-Supply, Disposition, and Ending Stocks of Crude Oil and Petroleum Products, March 1993

(Thousand Barrels)

\begin{tabular}{|c|c|c|c|c|c|c|c|c|c|c|c|}
\hline \multirow{2}{*}{ Commodity } & \multicolumn{5}{|c|}{ Supply } & \multicolumn{5}{|c|}{ Disposition } & \multirow[b]{2}{*}{$\begin{array}{l}\text { Ending } \\
\text { Stocks }\end{array}$} \\
\hline & $\begin{array}{c}\text { Fleld } \\
\text { Production }\end{array}$ & $\begin{array}{c}\text { Refinery } \\
\text { Production }\end{array}$ & $\begin{array}{c}\text { Imports by } \\
\text { PAD } \\
\text { District } \\
\text { of Entry }\end{array}$ & $\begin{array}{c}\text { Unac- } \\
\text { counted } \\
\text { For } \\
\text { Crude Oll } \\
\end{array}$ & $\begin{array}{c}\text { Not } \\
\text { Recelots }\end{array}$ & $\begin{array}{c}\text { Stock } \\
\text { Change" }\end{array}$ & $\begin{array}{l}\text { Crude } \\
\text { Logseg }\end{array}$ & $\begin{array}{c}\text { Refinery } \\
\text { Inputs }\end{array}$ & Exports & {$\left[\begin{array}{l}\text { Products } \\
\text { Supplied }\end{array}\right]$} & \\
\hline Crude Oll & $E_{101,897}$ & -. & 134,487 & 2,032 & $-46,314$ & 2,822 & 0 & 189,280 & 0 & 0 & 740,304 \\
\hline 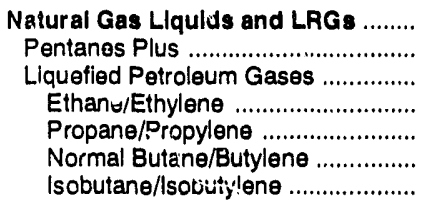 & $\begin{array}{r}36,503 \\
6,066 \\
30,437 \\
13,689 \\
10,489 \\
1,857 \\
4,402\end{array}$ & $\begin{array}{r}11,392 \\
10 \\
11,392 \\
832 \\
7,806 \\
2,550 \\
204\end{array}$ & $\begin{array}{r}1,624 \\
775 \\
849 \\
0 \\
576 \\
172 \\
101\end{array}$ & $\begin{array}{l}\ddot{.} \\
\ddot{.} \\
\ddot{.} \\
. . \\
\ddot{.} \\
.\end{array}$ & $\begin{array}{r}-3,112 \\
-179 \\
-2,933 \\
2,454 \\
-5,410 \\
379 \\
-356\end{array}$ & $\begin{array}{r}224 \\
603 \\
-379 \\
1,627 \\
-3,643 \\
1,973 \\
-336\end{array}$ & $\begin{array}{l}\cdots \\
\cdots \\
-. \\
. . \\
\cdots \\
\cdots \\
-.\end{array}$ & $\begin{array}{r}6,467 \\
2,350 \\
4,117 \\
0 \\
1 \\
1,357 \\
2,759\end{array}$ & $\begin{array}{r}747 \\
0 \\
747 \\
0 \\
661 \\
86 \\
0\end{array}$ & $\begin{array}{r}38,969 \\
3,709 \\
35,260 \\
15,348 \\
16,442 \\
1,542 \\
1,928\end{array}$ & $\begin{array}{r}47,172 \\
4,767 \\
42,405 \\
13,158 \\
12,212 \\
9,942 \\
7,093\end{array}$ \\
\hline $\begin{array}{l}\text { Other Llquids .............................. } \\
\text { Other Hydrocarbons/Oxygenates .... } \\
\text { Unfinished Oils ............................... } \\
\text { Motor Gasoline Blend. Comp. ........... } \\
\text { Aviation Gasoline Blend. Comp. ....... }\end{array}$ & $\begin{array}{r}2,451 \\
2,872 \\
\cdots \\
-421 \\
\cdots\end{array}$ & $\begin{array}{l}\cdots \\
\cdots \\
\cdots \\
-. \\
-\cdot\end{array}$ & $\begin{array}{r}10,636 \\
0 \\
9,943 \\
693 \\
0\end{array}$ & $\begin{array}{l}\ddot{-} \\
\ddot{.} \\
. . \\
. .\end{array}$ & $\begin{array}{r}-1,207 \\
0 \\
-260 \\
-947 \\
0\end{array}$ & $\begin{array}{r}-447 \\
512 \\
-138 \\
-824 \\
3\end{array}$ & $\begin{array}{l}\cdots \\
\ddot{.} \\
. . \\
\ddot{*}\end{array}$ & $\begin{array}{r}12,712 \\
2,360 \\
10,461 \\
-105 \\
-4\end{array}$ & $\begin{array}{r}254 \\
0 \\
0 \\
254 \\
0\end{array}$ & $\begin{array}{r}-639 \\
0 \\
-640 \\
0 \\
1\end{array}$ & $\begin{array}{r}72,180 \\
6,810 \\
49,842 \\
15,486 \\
42\end{array}$ \\
\hline 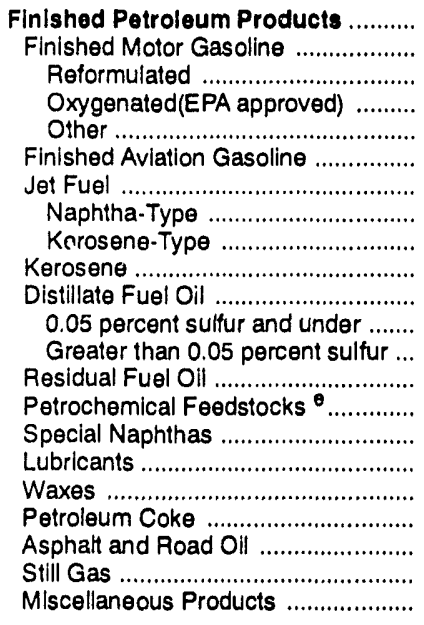 & $\begin{array}{r}594 \\
594 \\
. \\
0 \\
594 \\
. . \\
. . \\
. . \\
. . \\
. . \\
. . \\
. . \\
. . \\
. . \\
. . \\
. . \\
. . \\
. . \\
. . \\
. . \\
. . \\
. .\end{array}$ & $\begin{array}{r}209,031 \\
96,728 \\
0 \\
4,654 \\
92,074 \\
422 \\
22,424 \\
2,218 \\
20,206 \\
392 \\
40,767 \\
2,817 \\
37,950 \\
10,416 \\
11,743 \\
1,206 \\
3,144 \\
300 \\
8,742 \\
3,266 \\
8,830 \\
651\end{array}$ & $\begin{array}{r}6,701 \\
0 \\
0 \\
0 \\
0 \\
0 \\
383 \\
141 \\
242 \\
0 \\
0 \\
0 \\
0 \\
609 \\
5,356 \\
131 \\
0 \\
6 \\
181 \\
35 \\
0 \\
0\end{array}$ & $\begin{array}{l}. . \\
. . \\
. . \\
. . \\
. . \\
. . \\
. . \\
. . \\
. . \\
. . \\
. . \\
. . \\
. . \\
. . \\
. . \\
. . \\
. . \\
. \\
. . \\
.\end{array}$ & $\begin{array}{r}-103,591 \\
-61,724 \\
0 \\
-4,811 \\
-56,913 \\
-243 \\
-14,970 \\
-377 \\
-14,593 \\
-665 \\
-24,895 \\
-125 \\
-24,770 \\
61 \\
0 \\
-121 \\
-628 \\
-3 \\
0 \\
-232 \\
0 \\
-171\end{array}$ & $\begin{array}{r}-8,058 \\
-4,197 \\
N A \\
N A \\
N A \\
23 \\
-2,424 \\
-114 \\
-2,310 \\
-84 \\
-1,478 \\
N A \\
N A \\
100 \\
-188 \\
105 \\
92 \\
.56 \\
-250 \\
222 \\
0 \\
77\end{array}$ & $\begin{array}{l}. . \\
. . \\
. . \\
. . \\
. . \\
. . \\
. . \\
. . \\
. . \\
. . \\
. . \\
. . \\
. . \\
. . \\
. . \\
. . \\
. \\
. . \\
. . \\
.\end{array}$ & $\begin{array}{l}. . \\
. . \\
. . \\
. . \\
. . \\
. . \\
. . \\
. . \\
. . \\
. \\
. . \\
. \\
. . \\
. . \\
. . \\
. . \\
. . \\
. . \\
. . \\
.\end{array}$ & $\begin{array}{r}10,353 \\
2,849 \\
\mathrm{NA} \\
\mathrm{NA} \\
\mathrm{NA} \\
0 \\
1,499 \\
16 \\
1,482 \\
0 \\
846 \\
\mathrm{NA} \\
\mathrm{NA} \\
2,001 \\
0 \\
15 \\
306 \\
31 \\
2,799 \\
7 \\
0 \\
(\mathrm{~s})\end{array}$ & $\begin{array}{r}110,440 \\
36,946 \\
N A \\
N A \\
N A \\
156 \\
8,762 \\
2,080 \\
6,683 \\
-189 \\
16,504 \\
N A \\
N A \\
8,985 \\
17,287 \\
1,096 \\
2,118 \\
328 \\
6,374 \\
2,840 \\
8,830 \\
403\end{array}$ & $\begin{array}{r}124,847 \\
50,072 \\
0 \\
4,925 \\
45,147 \\
388 \\
13,664 \\
1,622 \\
12,042 \\
1,533 \\
23,137 \\
2,828 \\
20,309 \\
15,633 \\
2,895 \\
1,707 \\
6,402 \\
403 \\
2,791 \\
4,977 \\
0 \\
1,245\end{array}$ \\
\hline 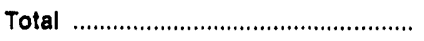 & 141,445 & 220,423 & 153,448 & 2,032 & $.154,224$ & $-5,459$ & 0 & 208,459 & 11,354 & 148,770 & 984,503 \\
\hline
\end{tabular}

a Represents the PAD District in whilch the material entered the United States and not necessarily where the crude oil or product is processed and/or consumed.

c A negative number indicates a decrease in stocks and a posittve number indicates an increase in stocks.

d Products supplied is equal to field production, plus refinery production, plus imports, plus unaccounted for crude oil, plus net receipts, minus stock change, minus crude losses, minus refinery inputs, minus exports.

Includes naphtha less than $401^{\circ} \mathrm{F}$ endpoint and other olls equal to or greater than $401^{\circ} \mathrm{F}$ ondpoint.

(s) = Less than 500 barrels.

$E=$ Estimated.

LRG = Llquefied Refinery Gas.

Note: Totals may not equal sum of components due to independent rounding.

Sources: - Energy Information Adminlstration (EIA) Forms EIA-810, "Monthly Retinery Report," EIA-811, "Monthly Bulk Terminal Report," EIA-812, "Monthly Product Pipellne Report," EIA-813, "Monthly Crude Oil Report," EIA-814, "Monthly Imports Report," EIA-816, "Monthly Natural Gas Liquids Report," ElA.817, "Monthly Tanker and Barge Movement Report," and EIA-819M, "Monthly Oxygenate Telephone Report". - Domestic crude oil production estimates based on historical statistics trom State conservation agencies and the Minerals Management Service of the U.S. Department of the Interior. - Export data from the Bureau of the Census and Form ElA.810, "Monthly Refinery Report." 
Table 15. PAD District III-Yeai-to-Date Supply, Disposition, and Ending Stocks of Crude Oll and Petroleum Products, January-March 1993 (Thousand Barrels)

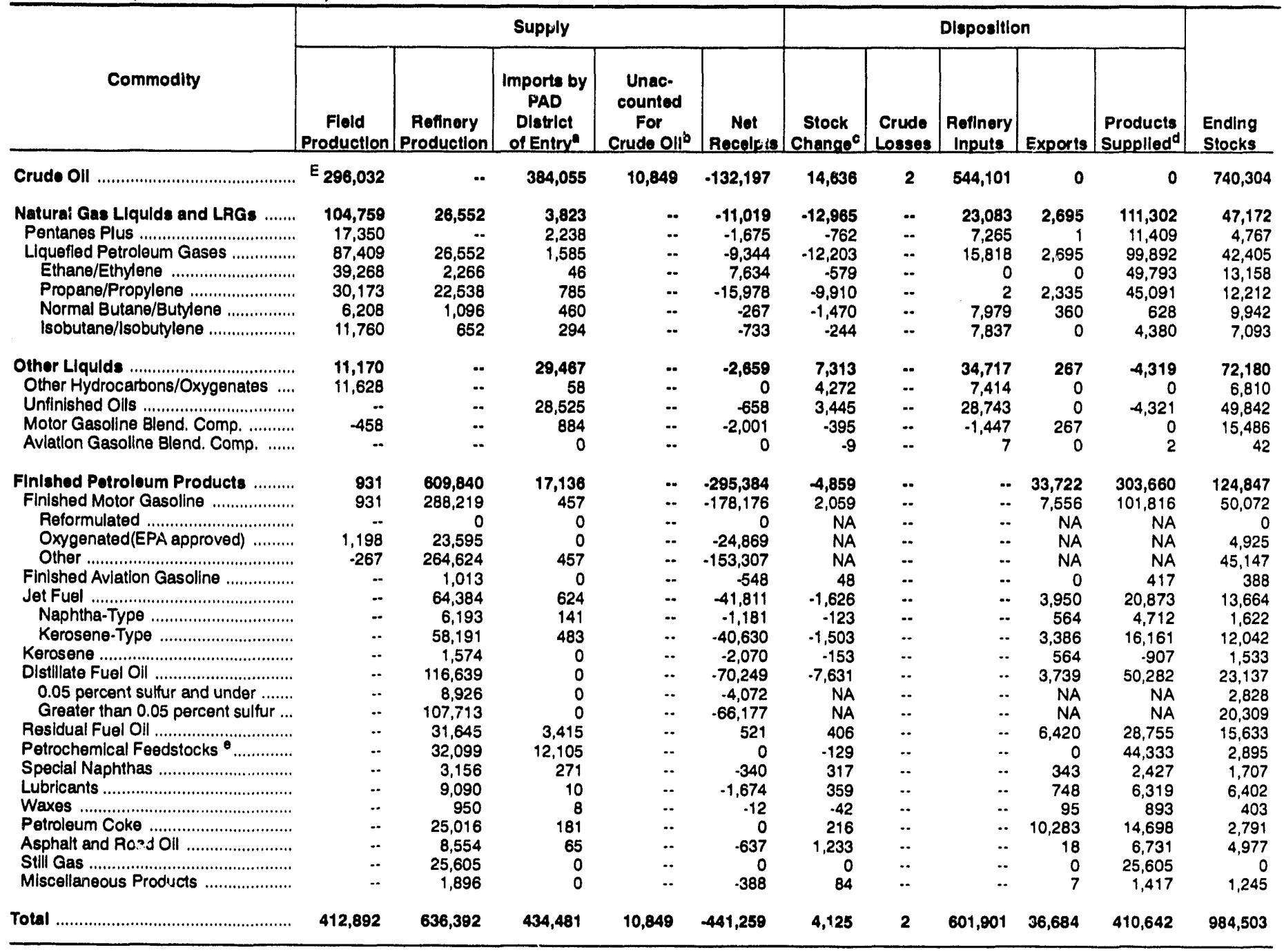

Represents the PAD District in which the material entered the United States and not necessarily where the crude oll or product is processed and/or consumed.

b Unaccounted for crude oil represents the difference between the supply and disposition of crude oil.

c A negative number indicates a decrease in stocks and a positive number indicates an Increase in stocks.

Products supplied is equal to fleld production, plus refinery production, plus imports, plus unaccounted for crude oil, plus net receipts, minus stock change, minus crude losses, minus refinery incuts, minus exports.

Includes naphtha less than $401^{\circ} \mathrm{F}$ endpoint and other oils equal to or greater than $401^{\circ} \mathrm{F}$ endpoint.

(8) = Less than 500 barrels.

$E=$ Estimated.

LRG = Liquefied Refinery Gas.

Note: Totals may not eiual sum of components due to independent rounding.

Sources: - Energy Information Administration (EIA) Forms EIA-810, "Monthly Refinery Report," EIA-811, "Monthly Bulk Terminal Report," EIA-812, "Monthly Product Plpeline Report," EIA-813, "Monthly Crude Oil Report," EIA-814, "Monthly Imports Report," ElA-816, "Monthly Natural Gas Liquids Report," EIA-817, "Monthly Tanker and Barge Movement Report," and EIA-819M, "Monthly Oxygenate Telephone Report". - Domestic crude oil production estimates based on historical statistics from State conservation agencies and the Minerals Management Service of the U.S. Department of the Interlor. - Export data from the Bureau of the Census and Form ElA.810, "Monthly Refinery Report." 
Table 16. PAD District III-Dally Average Supply and Disposition of Crude Oll and Petroleum Products, March 1993

(Thousand Barrels per Day)

\begin{tabular}{|c|c|c|c|c|c|c|c|c|c|c|}
\hline \multirow[b]{2}{*}{ Commodity } & \multicolumn{5}{|c|}{ Supply } & \multicolumn{5}{|c|}{ Disposition } \\
\hline & \begin{tabular}{|c|} 
Fleld \\
Production \\
\end{tabular} & $\begin{array}{c}\text { Relinery } \\
\text { Productlon }\end{array}$ & $\begin{array}{c}\text { Imports by } \\
\text { PAD } \\
\text { District } \\
\text { of Entry }\end{array}$ & $\begin{array}{l}\text { Unac- } \\
\text { counted } \\
\text { For } \\
\text { Crude } 0 \|^{b}\end{array}$ & $\begin{array}{c}\text { Not } \\
\text { Recelpts }\end{array}$ & $\begin{array}{c}\text { Stock } \\
\text { Changec }\end{array}$ & $\begin{array}{l}\text { Crude } \\
\text { Losses }\end{array}$ & $\begin{array}{c}\text { Reflinery } \\
\text { Inputs }\end{array}$ & Exports & $\begin{array}{l}\text { Products } \\
\text { Supplled d }\end{array}$ \\
\hline 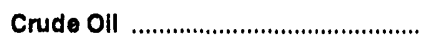 & $E_{3,287}$ & 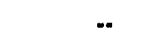 & 4,338 & 66 & $-1,494$ & 91 & 0 & 6,106 & 0 & 0 \\
\hline $\begin{array}{l}\text { Natural Gas Liquids and LRGs } \ldots . \ldots . . \\
\text { Pentanes Plus ............................... } \\
\text { Liquefied Petroleum Gases ............. } \\
\text { Ethane/Ethylene .......................... } \\
\text { Propane/Propylene ..................... } \\
\text { Normal Butane/Butylene .............. } \\
\text { Isobutane/Isobutylene }\end{array}$ & $\begin{array}{r}1,178 \\
196 \\
982 \\
442 \\
338 \\
60 \\
142\end{array}$ & $\begin{array}{r}367 \\
\ddot{3} \\
367 \\
27 \\
252 \\
82 \\
7\end{array}$ & $\begin{array}{r}52 \\
25 \\
27 \\
0 \\
19 \\
6 \\
3\end{array}$ & $\begin{array}{l}\ddot{.} \\
\ddot{. .} \\
\ddot{.} \\
\ddot{. .} \\
\ddot{.}\end{array}$ & $\begin{array}{r}-100 \\
-6 \\
-95 \\
79 \\
-175 \\
12 \\
-11\end{array}$ & $\begin{array}{r}7 \\
19 \\
-12 \\
52 \\
-118 \\
64 \\
-11\end{array}$ & $\begin{array}{l}\ddot{. .} \\
\ddot{. .} \\
\ddot{. .} \\
\ddot{. .} \\
. .\end{array}$ & $\begin{array}{r}209 \\
76 \\
133 \\
0 \\
(s) \\
44 \\
89\end{array}$ & $\begin{array}{r}24 \\
0 \\
24 \\
0 \\
21 \\
3 \\
0\end{array}$ & $\begin{array}{r}1,257 \\
120 \\
1,137 \\
495 \\
530 \\
50 \\
62\end{array}$ \\
\hline $\begin{array}{l}\text { Other Llquids .................................. } \\
\text { Other Hydrocarbons/Oxygenates .... } \\
\text { Unfinished Oils ................................. } \\
\text { Motor Gasoline Blend. Comp. .......... } \\
\text { Aviation Gasoline Blend. Comp. ...... }\end{array}$ & $\begin{array}{r}79 \\
93 \\
-. \\
-14 \\
-\cdot\end{array}$ & 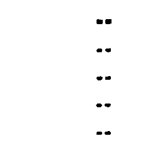 & $\begin{array}{r}343 \\
0 \\
321 \\
22 \\
0\end{array}$ & $\begin{array}{l}\ddot{. .} \\
\ddot{. .} \\
. . \\
. .\end{array}$ & $\begin{array}{r}-39 \\
0 \\
-8 \\
-31 \\
0\end{array}$ & $\begin{array}{r}-14 \\
17 \\
-4 \\
-27 \\
\text { (s) }\end{array}$ & $\begin{array}{l}\ddot{.} \\
\ddot{*} \\
\ddot{*} \\
. .\end{array}$ & $\begin{array}{r}410 \\
76 \\
337 \\
-3 \\
(s)\end{array}$ & $\begin{array}{l}8 \\
0 \\
0 \\
8 \\
0\end{array}$ & $\begin{array}{r}-21 \\
0 \\
-21 \\
0 \\
(\mathbf{s})\end{array}$ \\
\hline 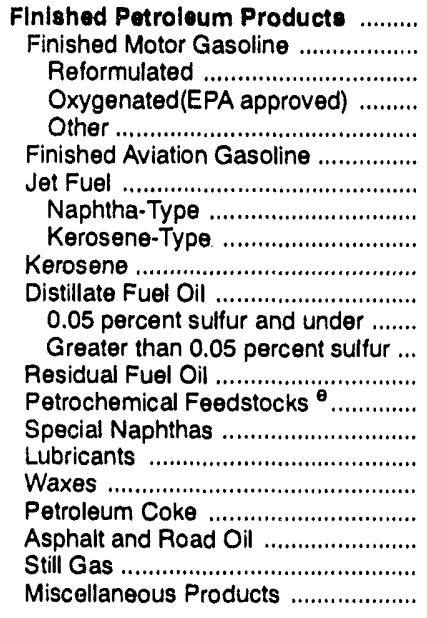 & 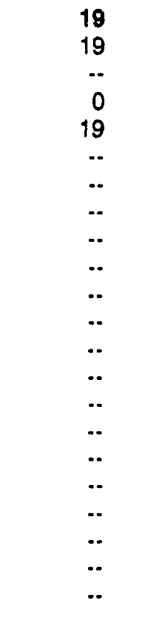 & $\begin{array}{r}6,743 \\
3,120 \\
0 \\
150 \\
2,970 \\
14 \\
723 \\
72 \\
652 \\
13 \\
1,315 \\
91 \\
1,224 \\
336 \\
379 \\
39 \\
101 \\
10 \\
282 \\
105 \\
285 \\
21\end{array}$ & $\begin{array}{r}216 \\
0 \\
0 \\
0 \\
0 \\
0 \\
12 \\
5 \\
8 \\
0 \\
0 \\
0 \\
0 \\
20 \\
173 \\
4 \\
0 \\
(\mathrm{~s}) \\
6 \\
1 \\
0 \\
0\end{array}$ & $\begin{array}{l}. . \\
. . \\
. . \\
. . \\
. . \\
. . \\
. . \\
. . \\
. . \\
. . \\
. . \\
. . \\
. . \\
. . \\
. . \\
. . \\
. . \\
. . \\
. . \\
. .\end{array}$ & $\begin{array}{r}-3,342 \\
-1,991 \\
0 \\
-155 \\
-1,836 \\
-8 \\
-483 \\
-12 \\
.471 \\
-21 \\
-803 \\
-4 \\
.799 \\
2 \\
0 \\
-4 \\
-20 \\
(\mathrm{~s}) \\
0 \\
-7 \\
0 \\
-6\end{array}$ & $\begin{array}{r}-260 \\
-135 \\
N A \\
N A \\
N A \\
1 \\
.78 \\
-4 \\
.75 \\
-3 \\
-48 \\
N A \\
N A \\
3 \\
-6 \\
3 \\
3 \\
-2 \\
-8 \\
7 \\
0 \\
2\end{array}$ & $\begin{array}{l}. . \\
. . \\
. . \\
. . \\
. . \\
. . \\
. . \\
. . \\
. . \\
. . \\
. . \\
. . \\
. . \\
. . \\
. . \\
. . \\
. . \\
. . \\
. . \\
. . \\
. .\end{array}$ & $\begin{array}{l}. . \\
. . \\
. . \\
. . \\
. . \\
. . \\
. . \\
. . \\
. . \\
. . \\
. . \\
. . \\
. . \\
. . \\
. . \\
. . \\
. . \\
. . \\
. .\end{array}$ & $\begin{array}{r}334 \\
92 \\
N A \\
N A \\
N A \\
0 \\
48 \\
1 \\
48 \\
0 \\
27 \\
\text { NA } \\
\text { NA } \\
65 \\
0 \\
(s) \\
10 \\
1 \\
90 \\
\text { (s) } \\
0 \\
\text { (s) }\end{array}$ & $\begin{array}{r}3,563 \\
1,192 \\
N A \\
N A \\
N A \\
5 \\
283 \\
67 \\
216 \\
-6 \\
532 \\
N A \\
N A \\
290 \\
558 \\
35 \\
68 \\
11 \\
206 \\
92 \\
285 \\
13\end{array}$ \\
\hline 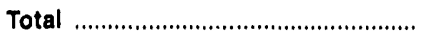 & 4,563 & 7,110 & 4,950 & 66 & $-4,975$ & .176 & 0 & 6,724 & 366 & 4,799 \\
\hline
\end{tabular}

Represents the PAD District in which the material entered the United States and not necessarily where the crude oil or product is processed and/or consumed.

- Unaccounted for crude oil represents the difference between the supply and disposition of crude oil.

c A negative number indicates a decrease in stocks and a positive number indicates an increase in stocks.

Products supplied is equal to field production, plus refinery production, plus imports, plus unaccounted for crude oil, plus net receipts, minus stock change minus crude losses, minus refinery inputs, minus exports.

$\theta$ Includes naphtha less than $401^{\circ} \mathrm{F}$ endpoint and other oils equal to or greater than $401^{\circ} \mathrm{F}$ endpoint.

$(s)=$ Less than 500 barrels per day.

$E=$ Estimated.

LRG = Liquefied Refinery Gas.

Note: Totals may not equal sum of components due to independent rounding.

Sources: - Energy Iniormation Administration (EIA) Forms EIA-810, "Monthly Refinery Report," ElA-811, "Monthly Bulk Terminal Report," EIA-812, "Monthly Product Pipeline Report," EIA-813, "Monthly Crude Oil Report," EIA-814, "Monthly Imports Report," EIA-816, "Monthly Natural Gas Liquids Report," EIA-817, "Monthly Tanker and Barge Movement Report," and E|A-819M, "Monthly Oxygenate Telephone Report". - Domestic crude oil production estimates based on historical statistics from State conservation agencies and the Minerals Management Service of the U.S. Department of the Interior. - Export data from the Bureau of the Census and Form ElA-810, "Monthly Refinery Report." 
Table 17. PAD District III-Year-to-Date Dally Average Supply and Disposition of Crude Oll and Petroleum Products, January-March 1993 (Thousand Barrels per Day)

\begin{tabular}{|c|c|c|c|c|c|c|c|c|c|c|}
\hline \multirow[b]{2}{*}{ Commodity } & \multicolumn{5}{|c|}{ Supply } & \multicolumn{5}{|c|}{ Dlsposition } \\
\hline & $\begin{array}{c}\text { Flold } \\
\text { Production }\end{array}$ & $\begin{array}{c}\text { Roflnery } \\
\text { Production }\end{array}$ & $\begin{array}{c}\text { importo by } \\
\text { PAD } \\
\text { Dlstrict } \\
\text { of Entry }\end{array}$ & $\begin{array}{l}\text { Unac- } \\
\text { counted } \\
\text { For } \\
\text { Crude Oll } \\
\end{array}$ & $\begin{array}{c}\text { Net } \\
\text { Recelpts }\end{array}$ & $\begin{array}{c}\text { Stock } \\
\text { Change }\end{array}$ & $\begin{array}{c}\text { Crude } \\
\text { Losses } \\
\end{array}$ & $\begin{array}{c}\text { Refinery } \\
\text { Inputa }\end{array}$ & Exports & $\begin{array}{l}\text { Products } \\
\text { Supplled }\end{array}$ \\
\hline 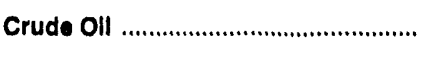 & $E_{3,289}$ & 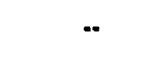 & 4,267 & 121 & $-1,469$ & 163 & (8) & 6,046 & 0 & 0 \\
\hline $\begin{array}{l}\text { Natural Gas Llquide and LRGe ......... } \\
\text { Pentanes Plus .................................. } \\
\text { Liquefied Petroleum Gases ............. } \\
\text { Ethane/Ethylene .............................. } \\
\text { Propane/Propylene ...................... } \\
\text { Normal Butane/Butylene ............... } \\
\text { Isobutane/lsobutylene .................... }\end{array}$ & $\begin{array}{r}1,164 \\
193 \\
971 \\
436 \\
335 \\
69 \\
131\end{array}$ & $\begin{array}{r}295 \\
295 \\
25 \\
250 \\
12 \\
7\end{array}$ & $\begin{array}{r}42 \\
25 \\
18 \\
1 \\
9 \\
5 \\
3\end{array}$ & $\begin{array}{l}\cdots \\
\cdots \\
. . \\
. . \\
. . \\
.- \\
.-\end{array}$ & $\begin{array}{r}-122 \\
-19 \\
-104 \\
85 \\
.178 \\
-3 \\
.8\end{array}$ & $\begin{array}{r}-144 \\
-8 \\
-136 \\
-6 \\
-110 \\
-16 \\
-3\end{array}$ & $\begin{array}{l}. . \\
\ldots \\
. . \\
. . \\
. . \\
\ldots\end{array}$ & $\begin{array}{r}256 \\
81 \\
176 \\
0 \\
(s) \\
89 \\
87\end{array}$ & $\begin{array}{r}30 \\
(s) \\
30 \\
0 \\
26 \\
4 \\
0\end{array}$ & $\begin{array}{r}1,237 \\
127 \\
1,110 \\
553 \\
501 \\
7 \\
49\end{array}$ \\
\hline $\begin{array}{l}\text { Other Llqulds ................................... } \\
\text { Other Hydrocarbons/Oxygenates ...... } \\
\text { Unfinished Oils ................................ } \\
\text { Motor Gasoline Blend. Comp. ........... } \\
\text { Aviation Gascline Blend. Comp. ....... }\end{array}$ & $\begin{array}{r}124 \\
129 \\
-. \\
-.5\end{array}$ & 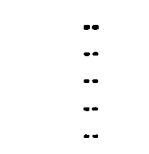 & $\begin{array}{r}327 \\
1 \\
317 \\
10 \\
0\end{array}$ & $\begin{array}{l}\ddot{.} \\
\ddot{*} \\
\ddot{*} \\
\ddot{*}\end{array}$ & $\begin{array}{r}-30 \\
0 \\
.7 \\
.22 \\
0\end{array}$ & $\begin{array}{r}81 \\
47 \\
38 \\
-4 \\
\text { (s) }\end{array}$ & 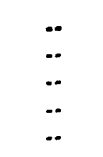 & $\begin{array}{r}386 \\
82 \\
319 \\
-16 \\
(s)\end{array}$ & $\begin{array}{l}3 \\
0 \\
0 \\
3 \\
0\end{array}$ & $\begin{array}{r}-48 \\
0 \\
-48 \\
0 \\
(s)\end{array}$ \\
\hline 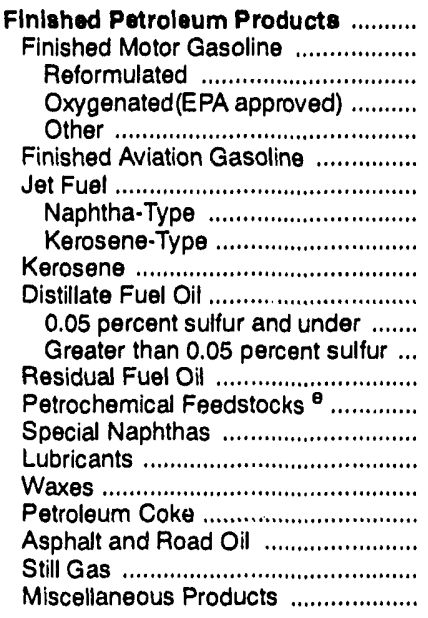 & 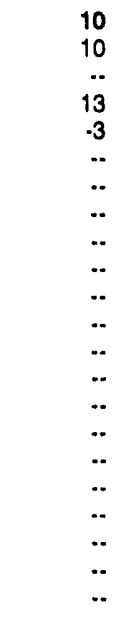 & $\begin{array}{r}6,776 \\
3,202 \\
0 \\
262 \\
2,940 \\
11 \\
715 \\
69 \\
647 \\
17 \\
1,296 \\
99 \\
1,197 \\
352 \\
357 \\
35 \\
101 \\
11 \\
278 \\
95 \\
285 \\
21\end{array}$ & $\begin{array}{r}190 \\
5 \\
0 \\
0 \\
5 \\
0 \\
7 \\
2 \\
5 \\
0 \\
0 \\
0 \\
0 \\
38 \\
135 \\
3 \\
(s) \\
(s) \\
2 \\
1 \\
0 \\
0\end{array}$ & $\begin{array}{l}. . \\
. . \\
. . \\
. . \\
. . \\
. . \\
. . \\
. . \\
. . \\
. . \\
. . \\
. . \\
. . \\
. . \\
. . \\
. . \\
. . \\
. . \\
. . \\
. .\end{array}$ & $\begin{array}{r}3,282 \\
-1,980 \\
0 \\
-276 \\
-1,703 \\
-6 \\
-465 \\
-13 \\
-451 \\
-23 \\
-781 \\
-45 \\
-735 \\
6 \\
0 \\
-4 \\
-19 \\
(\mathrm{~s}) \\
0 \\
-7 \\
0 \\
-4\end{array}$ & $\begin{array}{r}-54 \\
23 \\
N A \\
N A \\
N A \\
1 \\
-18 \\
-1 \\
-17 \\
-2 \\
-85 \\
N A \\
N A \\
5 \\
-1 \\
4 \\
4 \\
(s) \\
2 \\
14 \\
0 \\
1\end{array}$ & $\begin{array}{l}. . \\
. . \\
. . \\
. . \\
. . \\
. . \\
. . \\
. . \\
. . \\
. . \\
. \\
. . \\
. \\
. . \\
. . \\
. . \\
. . \\
. .\end{array}$ & $\begin{array}{l}. . \\
. . \\
. . \\
. . \\
. . \\
. . \\
. . \\
. . \\
. . \\
. . \\
. . \\
. \\
. . \\
. \\
. . \\
. \\
. . \\
. . \\
. . \\
. . \\
. .\end{array}$ & $\begin{array}{r}375 \\
84 \\
N A \\
N A \\
N A \\
0 \\
44 \\
6 \\
38 \\
6 \\
42 \\
N A \\
N A \\
71 \\
0 \\
4 \\
C \\
1 \\
114 \\
\text { (s) } \\
0 \\
\text { (s) }\end{array}$ & $\begin{array}{r}3,374 \\
1,131 \\
N A \\
N A \\
N A \\
5 \\
232 \\
52 \\
180 \\
-10 \\
559 \\
N A \\
N A \\
319 \\
493 \\
27 \\
70 \\
10 \\
163 \\
75 \\
285 \\
16\end{array}$ \\
\hline 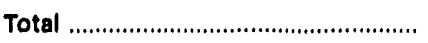 & 4,588 & 7,071 & 4,828 & 121 & $-4,903$ & 46 & (8) & 6,688 & 408 & 4,563 \\
\hline
\end{tabular}

a Represents the PAD District In which the material entered the United States and not necessarily where the crude oil or product is processed and/or consumed.

b Unaccounted for crude oil represents the diference between the supply and disposition of crude oil.

c A negative number indicates a decrease in stocks and a positive number indicates an increase in stocks.

d Products supplied is equal to field production, plus refinery production, plus imports, plus unaccounted for crude oil, plus net receipts, minus stock change, minus crude losses, minus refinery inputs, minus exports.

$\theta$ Includes naphtha less than $401^{\circ} \mathrm{F}$ endpoint and other olls equal to or greater than $401^{\circ} \mathrm{F}$ endpoint.

(s) $=$ Less than 500 barrels per day.

$E=$ Estimated.

$L R G=$ Liquefied Refinery Gas.

Note: Totals may not equal sum of components due to independent rounding.

Sources: - Energy Information Administration (EIA) Forms EIA-810, "Monthly Refinery Report," EIA-811, "Monthly Bulk Terminal Report," ElA-812, "Monthly Product Pipeline Report," EIA-813, "Monthly Crude Oll Report," EIA-814, "Monthly Imports Report," ElA-816, "Monthly Natural Gas Liquids Report," ElA-817, "Monthly Tanker and Barge Movement Report," and EIA-819M, "Monthly Oxygenate Telephone Report". - Domestic crude oil production estimates based on historical statistics from State conservation agencies and the Minerals Management Service of the U.S. Department of the Interior. - Export data from the Bureau of the Census and Form EIA-810, "Monthly Refinery Report." 
Table 18. PAD District IV-Supply, Disposition, and Ending Stocks of Crude Oil and Petroleum Products, March 1993

(Thousand Barrels)

\begin{tabular}{|c|c|c|c|c|c|c|c|c|c|c|c|}
\hline \multirow[b]{2}{*}{ Commodity } & \multicolumn{5}{|c|}{ Supply } & \multicolumn{5}{|c|}{ Disposition } & \multirow[b]{2}{*}{$\begin{array}{l}\text { Endlng } \\
\text { Stocke }\end{array}$} \\
\hline & $\begin{array}{c}\text { Fleid } \\
\text { Production }\end{array}$ & $\begin{array}{c}\text { Rofinary } \\
\text { Production }\end{array}$ & $\begin{array}{c}\text { Importe by } \\
\text { PAD } \\
\text { Dlstrict } \\
\text { of Entry }\end{array}$ & $\begin{array}{l}\text { Unac- } \\
\text { counted } \\
\text { For } \\
\text { Crude oll }\end{array}$ & $\begin{array}{c}\text { Not } \\
\text { Recelpte }\end{array}$ & $\begin{array}{c}\text { Stock } \\
\text { Changec }\end{array}$ & $\begin{array}{l}\text { Crude } \\
\text { Losses }\end{array}$ & $\begin{array}{c}\text { Reflnery } \\
\text { Inpute }\end{array} \mid$ & Exports & $\begin{array}{l}\text { Products } \\
\text { Supplled }\end{array}$ & \\
\hline 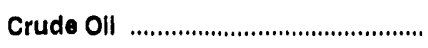 & $E_{13,200}$ & - & 2,649 & 1,898 & $-4,067$ & 61 & 0 & 13,619 & 0 & 0 & 12,358 \\
\hline 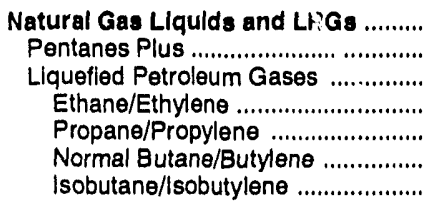 & $\begin{array}{r}4,628 \\
1,011 \\
3,617 \\
1,405 \\
1,388 \\
497 \\
327\end{array}$ & $\begin{array}{r}237 \\
-. \\
237 \\
0 \\
215 \\
3 \\
19\end{array}$ & $\begin{array}{r}261 \\
78 \\
183 \\
0 \\
100 \\
75 \\
8\end{array}$ & $\begin{array}{l}\ddot{.} \\
\ddot{.} \\
\ddot{.} \\
\ddot{.} \\
\ddot{.}\end{array}$ & $\begin{array}{r}-2,737 \\
-300 \\
-2,437 \\
-1,288 \\
-582 \\
-344 \\
-223\end{array}$ & $\begin{array}{r}80 \\
-9 \\
89 \\
12 \\
1 \\
40 \\
36\end{array}$ & 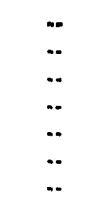 & $\begin{array}{r}508 \\
191 \\
317 \\
0 \\
0 \\
204 \\
113\end{array}$ & $\begin{array}{l}1 \\
1 \\
0 \\
0 \\
0 \\
0 \\
0\end{array}$ & $\begin{array}{r}1,800 \\
606 \\
1,194 \\
105 \\
1,120 \\
-13 \\
-18\end{array}$ & $\begin{array}{r}1,198 \\
137 \\
1,061 \\
196 \\
374 \\
300 \\
191\end{array}$ \\
\hline $\begin{array}{l}\text { Other Llquids .................................. } \\
\text { Other Hydrocarbons/Oxygenates ..... } \\
\text { Unfinished Olls ................................... } \\
\text { Motor Gasollne Blend. Comp. .......... } \\
\text { Aviation Gasoline Blend. Comp. ....... }\end{array}$ & $\begin{array}{r}17 \\
84 \\
-. \\
-67 \\
-.\end{array}$ & $\begin{array}{l}- \\
\ddot{-} \\
\ddot{.} \\
.-\end{array}$ & $\begin{array}{l}0 \\
0 \\
0 \\
0 \\
0\end{array}$ & $\begin{array}{l}\ddot{. .} \\
\ddot{.} \\
\ddot{.} \\
. .\end{array}$ & $\begin{array}{l}0 \\
0 \\
0 \\
0 \\
0\end{array}$ & $\begin{array}{r}440 \\
19 \\
378 \\
43 \\
0\end{array}$ & $\begin{array}{l}\cdots \\
\cdots \\
\ddot{*} \\
\cdots\end{array}$ & $\begin{array}{r}-461 \\
65 \\
-416 \\
-110 \\
0\end{array}$ & $\begin{array}{l}0 \\
0 \\
0 \\
0 \\
0\end{array}$ & $\begin{array}{r}38 \\
0 \\
38 \\
0 \\
0\end{array}$ & $\begin{array}{r}5,477 \\
146 \\
2,926 \\
2,405 \\
0\end{array}$ \\
\hline 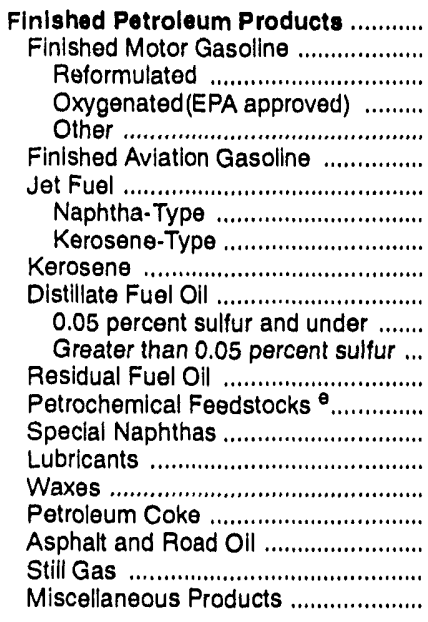 & $\begin{array}{r}110 \\
110 \\
. . \\
0 \\
110 \\
. . \\
. . \\
. . \\
. . \\
. . \\
. . \\
. . \\
. . \\
. . \\
. . \\
. . \\
. . \\
. . \\
.\end{array}$ & $\begin{array}{r}13,863 \\
6,705 \\
0 \\
111 \\
6,594 \\
18 \\
1,205 \\
378 \\
827 \\
42 \\
3,493 \\
334 \\
3,159 \\
485 \\
23 \\
0 \\
0 \\
46 \\
401 \\
763 \\
612 \\
70\end{array}$ & $\begin{array}{r}67 \\
11 \\
0 \\
0 \\
11 \\
0 \\
0 \\
0 \\
0 \\
0 \\
52 \\
30 \\
22 \\
0 \\
0 \\
0 \\
0 \\
4 \\
0 \\
0 \\
0 \\
0\end{array}$ & $\begin{array}{l}. . \\
. . \\
. . \\
. . \\
. . \\
. . \\
. . \\
. . \\
. . \\
\ddot{.} \\
\ddot{.} \\
\ddot{. .} \\
. . \\
. . \\
. . \\
. . \\
. . \\
. .\end{array}$ & $\begin{array}{r}982 \\
428 \\
0 \\
0 \\
428 \\
8 \\
547 \\
-165 \\
712 \\
-10 \\
9 \\
427 \\
-418 \\
0 \\
0 \\
0 \\
0 \\
0 \\
0 \\
0 \\
0 \\
0\end{array}$ & $\begin{array}{r}522 \\
42 \\
N A \\
\text { NA } \\
\text { NA } \\
1 \\
171 \\
53 \\
118 \\
4 \\
-26 \\
\text { NA } \\
\text { NA } \\
126 \\
0 \\
0 \\
0 \\
0 \\
6 \\
34 \\
159 \\
0 \\
5\end{array}$ & $\begin{array}{l}. . \\
. . \\
. . \\
. . \\
. . \\
. . \\
. . \\
. . \\
. . \\
\ddot{. .} \\
\ddot{. .} \\
. . \\
. . \\
. . \\
. . \\
. .\end{array}$ & 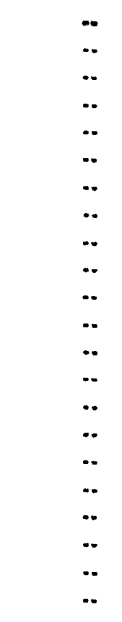 & $\begin{array}{r}11 \\
\text { (s) } \\
\text { NA } \\
\text { NA } \\
\text { NA } \\
0 \\
0 \\
0 \\
0 \\
0 \\
1 \\
N A \\
\text { NA } \\
0 \\
0 \\
(s) \\
5 \\
0 \\
4 \\
1 \\
0 \\
0\end{array}$ & $\begin{array}{r}14,490 \\
7,212 \\
\mathrm{NA} \\
\mathrm{NA} \\
\mathrm{NA} \\
25 \\
1,581 \\
160 \\
1,421 \\
28 \\
3,579 \\
\mathrm{NA} \\
\mathrm{NA} \\
359 \\
23 \\
(\mathrm{~s}) \\
-5 \\
44 \\
363 \\
603 \\
612 \\
65\end{array}$ & $\begin{array}{r}12,288 \\
4,993 \\
0 \\
300 \\
4,693 \\
45 \\
931 \\
348 \\
583 \\
78 \\
2,377 \\
458 \\
1,919 \\
405 \\
1 \\
1 \\
0 \\
31 \\
305 \\
3,113 \\
0 \\
8\end{array}$ \\
\hline Total & 17,955 & 14,100 & 2,977 & 1,898 & $-5,822$ & 1,103 & 0 & 13,666 & 11 & 16,328 & 31,321 \\
\hline
\end{tabular}

a Represents the PAD District in which the material entered the United States and not necessarily where the crude oil or product is processed and/or consumed.

b Unaccounted for crude oil represents the difference between the supply and dlsposition of crude oil.

- A negative number indicates a decrease in stocks and a positive number indicates an increase in stocks.

d Products supplied is equal to field production, plus refinery production, plus imports, plus unaccounted for crude oil, plus net receipts, minus stock change, minus crude losses, minus refinery inputs, minus exports.

Includes naphtha less than $401^{\circ} \mathrm{F}$ endpoint and other olls equal to or greater than $401^{\circ} \mathrm{F}$ endpoint.

(s) = Less than 500 barrels.

$E=$ Estimated.

$L R G=$ Llquefied Refinery Gas.

Note: Totals may not equal sum of components due to Independent rounding.

Sources: - Energy Information Administration (EIA) Forms EIA-810, "Monthly Refinery Report," EIA-811, "Monthly Bulk Terminal Report," ElA-812, "Monthly Product Pipeline Report," EIA-813, "Monthly Crude Oil Report," EIA-814, "Monthly Imports Report," EIA-816, "Monthly Natural Gas Liquids Report," ElA-817, "Monthly Tanker and Barge Movement Report," and ElA-819M, "Monthly Oxygenate Telephone Report". - Domestic crude oil production estimates based on historical statistics from State conservation agencies and the Minerals Managemeni Service of the U.S. Department of the Interior. - Export data from the Bureau of the Census and Form EIA-810, "Monthly Refinery Report." 
Table 19. PAD District IV-Year-to-Date Supply, Disposition, and Ending Stocks of Crude OIl and Petroleum Products, January-March 1993 (Thousand Barrels)

\begin{tabular}{|c|c|c|c|c|c|c|c|c|c|c|c|}
\hline \multirow[b]{2}{*}{ Commodity } & \multicolumn{5}{|c|}{ Supply } & \multicolumn{5}{|c|}{ Dlaposition } & \multirow[b]{2}{*}{$\begin{array}{l}\text { Ending } \\
\text { Stocks } \\
\end{array}$} \\
\hline & $\begin{array}{c}\text { Flold } \\
\text { Production }\end{array}$ & $\begin{array}{c}\text { Refinery } \\
\text { Production }\end{array}$ & $\begin{array}{c}\text { Imports by } \\
\text { PAD } \\
\text { Diatrict } \\
\text { of Entry }\end{array}$ & $\begin{array}{c}\text { Unac- } \\
\text { counted } \\
\text { For } \\
\text { Crude } \text { OII }^{b}\end{array}$ & $\begin{array}{c}\text { Not } \\
\text { Recelpte }\end{array}$ & $\begin{array}{c}\text { Stock } \\
\text { Changec }\end{array}$ & $\begin{array}{c}\text { Crude } \\
\text { Losses }\end{array}$ & $\begin{array}{c}\text { Reflnery } \\
\text { Inpute }\end{array}$ & Exports & $\mid \begin{array}{l}\text { Products } \\
\text { Supplled }\end{array}$ & \\
\hline 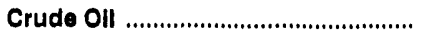 & $E_{38,958}$ & - & 7,584 & 3,615 & $-11,410$ & 851 & 0 & 37,797 & 0 & 0 & 12,358 \\
\hline $\begin{array}{l}\text { Natural Gas Llquids and LRGs ....... } \\
\text { Pentanes Plus .................................. } \\
\text { Llquefled Petroleum Gases ............ } \\
\text { Ethane/Ethylene .............................. } \\
\text { Propane/Propylene ..................... } \\
\text { Normal Butane/Butylene ............... } \\
\text { Isobutane/lsobutylene ................... }\end{array}$ & $\begin{array}{r}12,513 \\
2,745 \\
9,768 \\
3,373 \\
3,974 \\
1,504 \\
917\end{array}$ & $\begin{array}{r}402 \\
402 \\
0 \\
627 \\
-222 \\
.3\end{array}$ & $\begin{array}{r}881 \\
200 \\
681 \\
0 \\
397 \\
260 \\
24\end{array}$ & 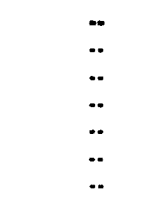 & $\begin{array}{r}6,670 \\
-842 \\
-5,828 \\
-3,094 \\
-1,360 \\
.818 \\
-556\end{array}$ & $\begin{array}{r}-80 \\
-25 \\
.65 \\
18 \\
-119 \\
15 \\
21\end{array}$ & $\begin{array}{l}\ddot{.} \\
\ddot{.} \\
\ddot{.} \\
\ddot{.} \\
. \\
. .\end{array}$ & $\begin{array}{r}1,859 \\
625 \\
1,234 \\
0 \\
0 \\
916 \\
318\end{array}$ & $\begin{array}{l}2 \\
2 \\
0 \\
0 \\
0 \\
0 \\
0\end{array}$ & $\begin{array}{r}5,355 \\
1,501 \\
3,854 \\
261 \\
3,757 \\
-207 \\
43\end{array}$ & $\begin{array}{r}1,198 \\
137 \\
1,061 \\
196 \\
374 \\
300 \\
191\end{array}$ \\
\hline $\begin{array}{l}\text { Other Llqulds ................................... } \\
\text { Other Hydrocarbons/Oxygenates .... } \\
\text { Unfinished Oils ................................... } \\
\text { Motor Gasoline Blend. Comp. ......... } \\
\text { Aviation Gasoline Blend. Comp. ....... }\end{array}$ & $\begin{array}{r}159 \\
393 \\
-234 \\
. .\end{array}$ & $\begin{array}{l}\ddot{ } \\
\ddot{.} \\
\ddot{.} \\
\ddot{.}\end{array}$ & $\begin{array}{l}0 \\
0 \\
0 \\
0 \\
0\end{array}$ & $\begin{array}{l}\ddot{-.} \\
. . \\
. . \\
. .\end{array}$ & $\begin{array}{l}0 \\
0 \\
0 \\
0 \\
0\end{array}$ & $\begin{array}{r}1,114 \\
44 \\
529 \\
541 \\
0\end{array}$ & $\begin{array}{l}\ddot{.} \\
\ddot{.} \\
\ddot{.} \\
. .\end{array}$ & $\begin{array}{r}.906 \\
349 \\
.480 \\
.775 \\
0\end{array}$ & $\begin{array}{l}0 \\
0 \\
0 \\
0 \\
0\end{array}$ & $\begin{array}{r}-49 \\
0 \\
-49 \\
0 \\
0\end{array}$ & $\begin{array}{r}5,477 \\
146 \\
2,926 \\
2,405 \\
0\end{array}$ \\
\hline 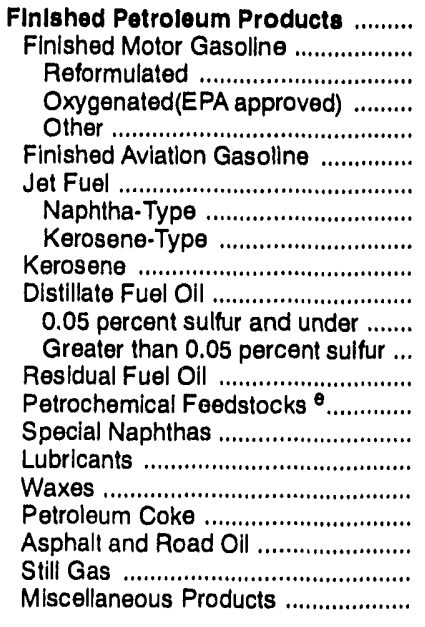 & $\begin{array}{r}352 \\
352 \\
300 \\
53 \\
. . \\
. . \\
. . \\
. . \\
. . \\
. . \\
. . \\
. . \\
. . \\
. . \\
. \\
. . \\
. \\
. . \\
.\end{array}$ & $\begin{array}{r}39,616 \\
19,577 \\
0 \\
1,798 \\
17,779 \\
36 \\
3,314 \\
938 \\
2,376 \\
240 \\
9,743 \\
869 \\
8,874 \\
1,119 \\
66 \\
0 \\
0 \\
76 \\
1,262 \\
2,219 \\
1,759 \\
205\end{array}$ & $\begin{array}{r}245 \\
28 \\
0 \\
0 \\
28 \\
0 \\
0 \\
0 \\
0 \\
0 \\
210 \\
121 \\
89 \\
0 \\
0 \\
0 \\
0 \\
7 \\
0 \\
0 \\
0 \\
0\end{array}$ & $\begin{array}{l}. . \\
. . \\
. . \\
. . \\
. . \\
. . \\
. . \\
. . \\
. . \\
. . \\
. . \\
. . \\
. . \\
. . \\
. \\
. . \\
. . \\
. .\end{array}$ & $\begin{array}{r}1,796 \\
698 \\
0 \\
31 \\
667 \\
24 \\
1,640 \\
-443 \\
2,083 \\
-54 \\
.512 \\
829 \\
-1,341 \\
0 \\
0 \\
0 \\
0 \\
0 \\
0 \\
0 \\
0 \\
0\end{array}$ & $\begin{array}{r}1,466 \\
403 \\
N A \\
N A \\
N A \\
0 \\
96 \\
51 \\
45 \\
61 \\
-338 \\
N A \\
N A \\
48 \\
-1 \\
0 \\
0 \\
-13 \\
72 \\
1,132 \\
0 \\
6\end{array}$ & $\begin{array}{l}. . \\
. . \\
. . \\
. . \\
. . \\
. . \\
. . \\
. . \\
. . \\
. \\
. . \\
. \\
. . \\
. . \\
. \\
. . \\
. \\
. . \\
. . \\
. .\end{array}$ & 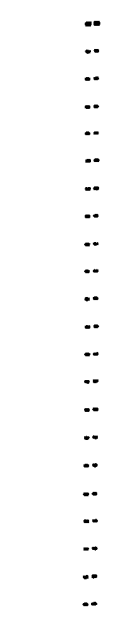 & $\begin{array}{r}22 \\
1 \\
N A \\
N A \\
N A \\
0 \\
0 \\
0 \\
0 \\
0 \\
1 \\
N A \\
N A \\
0 \\
0 \\
1 \\
11 \\
0 \\
6 \\
3 \\
0 \\
0\end{array}$ & $\begin{array}{r}40,522 \\
20,252 \\
N A \\
N A \\
N A \\
60 \\
4,858 \\
444 \\
4,414 \\
125 \\
9,778 \\
N A \\
N A \\
1,071 \\
67 \\
-1 \\
-11 \\
96 \\
1,184 \\
1,084 \\
1,759 \\
199\end{array}$ & $\begin{array}{r}12,288 \\
4,993 \\
0 \\
300 \\
4,693 \\
45 \\
931 \\
348 \\
583 \\
78 \\
2,377 \\
458 \\
1,919 \\
405 \\
1 \\
1 \\
0 \\
31 \\
305 \\
3,113 \\
0 \\
8\end{array}$ \\
\hline 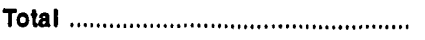 & 51,983 & 40,018 & 8,710 & 3,615 & $-16,284$ & 3,441 & 0 & 38,750 & 24 & 45,827 & 31,321 \\
\hline
\end{tabular}

a Represents the PAD District in which the material entered the United States and not necessarlly where the crude oil or product is processed and/or consumed.

b Unaccounted for crude oil represents the difference between the supply and disposition of crude oil.

c A negative number indicates a decrease in stocks and a positive number Indicates an increase in stocks.

d Products supplled is equal to field production, plus refinery production, plus imports, plus unaccounted for crude oll, plus net receipts, minus stock change, minus crude losses, minus refinery inputs, minus exports.

$\theta$ Includes naphtha less than $401^{\circ} \mathrm{F}$ endpoint and other oils equal to or greater than $401^{\circ} \mathrm{F}$ endpoint.

(s) = Less than 500 barrels.

$\mathrm{E}=$ Estimated.

LRG = Liquefied Refinery Gas.

Note: Totals may not equal sum of components due to independent rounding.

Sources: - Energy Information Administration (EIA) Forms EIA-810, "Monthly Refinery Report," EIA-811, "Monthly Bulk Terminal Report," EIA-812, "Monthly Product Pipeline Report," EIA-813, "Monthly Crude Oil Report," EIA-814, "Monthly Imports Report," ElA-816, "Monthly Natural Gas Liquids Report," ElA-817, "Monthly Tanker and Barge Movement Report," and EIA-819M, "Monthly Oxygenate Telephone Report". - Domestic crude oil production estimates based on historical statistics from State conservation agencles and the Minerals Management Service of the U.S. Department of the Interior. - Export data from the Bureau of the Census and Form EIA.810. "Monthly Refinery Report." 
Table 20. PAD District IV-Dally Average Supply and Disposition of Crude Oll and Petroleum Products, March 1993 (Thousand Barrels per Day)

\begin{tabular}{|c|c|c|c|c|c|c|c|c|c|c|}
\hline \multirow[b]{2}{*}{ Commodity } & \multicolumn{5}{|c|}{ Supply } & \multicolumn{5}{|c|}{ Disposition } \\
\hline & $\begin{array}{c}\text { Flald } \\
\text { Production }\end{array}$ & $\begin{array}{c}\text { Pofinory } \\
\text { Productlon }\end{array}$ & $\begin{array}{c}\text { Importa by } \\
\text { PAD } \\
\text { Distrlot } \\
\text { of Entry }\end{array}$ & $\begin{array}{l}\text { Unac- } \\
\text { counted } \\
\text { For } \\
\text { Crude } 011^{b}\end{array}$ & $\begin{array}{c}\text { Not } \\
\text { Aecelpte }\end{array}$ & $\begin{array}{c}\text { Stock } \\
\text { Chance }\end{array}$ & $\begin{array}{l}\text { Crude } \\
\text { Losses } \\
\end{array}$ & $\begin{array}{c}\text { Rofinery } \\
\text { Inputs }\end{array}$ & Exponts & $\begin{array}{l}\text { Products } \\
\text { Supplledd }\end{array}$ \\
\hline 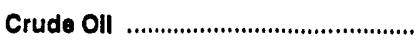 & $E_{428}$ & $\infty$ & 85 & 61 & -131 & 2 & 0 & 439 & 0 & 0 \\
\hline $\begin{array}{l}\text { Natural Gas Llquide and LRGe ......... } \\
\text { Pentanes Plus ................................... } \\
\text { Liquefied Petroleum Gases .............. } \\
\text { Ethane/Ethylene .......................... } \\
\text { Propane/Propylene ....................... } \\
\text { Normal Butane/Butylene .............. } \\
\text { Isobutane/lsobutylene .................. }\end{array}$ & $\begin{array}{r}149 \\
33 \\
117 \\
45 \\
45 \\
16 \\
11\end{array}$ & $\begin{array}{r}8 \\
\ddot{8} \\
0 \\
7 \\
(8) \\
1\end{array}$ & $\begin{array}{r}8 \\
3 \\
6 \\
0 \\
3 \\
2 \\
(8)\end{array}$ & $\begin{array}{l}- \\
\ddot{.} \\
\ddot{. .} \\
\ddot{.} \\
\ddot{.}\end{array}$ & $\begin{array}{r}-88 \\
-10 \\
-79 \\
-42 \\
-19 \\
-11 \\
-7\end{array}$ & $\begin{array}{r}3 \\
(8) \\
3 \\
(8) \\
(8) \\
1 \\
1\end{array}$ & 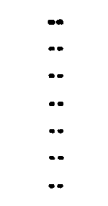 & $\begin{array}{r}16 \\
6 \\
10 \\
0 \\
0 \\
7 \\
4\end{array}$ & $\begin{array}{c}\text { (s) } \\
\text { (s) } \\
0 \\
0 \\
0 \\
0 \\
0\end{array}$ & $\begin{array}{r}58 \\
20 \\
39 \\
3 \\
36 \\
(9) \\
-1\end{array}$ \\
\hline $\begin{array}{l}\text { Other Llquids ................................... } \\
\text { Other Hydrocarbons/Oxygenates .... } \\
\text { Unfinished Oils ................................. } \\
\text { Motor Gasoline Blend. Comp. .......... } \\
\text { Avlation Gasoline Blend. Comp. ...... }\end{array}$ & $\begin{array}{l}1 \\
3 \\
\ddot{-} \\
-2\end{array}$ & $\begin{array}{l}\cdots \\
\cdots \\
\cdots \\
\cdots\end{array}$ & $\begin{array}{l}0 \\
0 \\
0 \\
0 \\
0\end{array}$ & $\begin{array}{l}- \\
\ddot{.} \\
\ddot{.} \\
\ddot{.}\end{array}$ & $\begin{array}{l}0 \\
0 \\
0 \\
0 \\
0\end{array}$ & $\begin{array}{r}14 \\
1 \\
12 \\
1 \\
0\end{array}$ & $\begin{array}{l}\ddot{.} \\
\ddot{.} \\
\ddot{.} \\
.-\end{array}$ & $\begin{array}{r}-15 \\
2 \\
-13 \\
-4 \\
0\end{array}$ & $\begin{array}{l}0 \\
0 \\
0 \\
0 \\
0\end{array}$ & $\begin{array}{l}1 \\
0 \\
1 \\
0 \\
0\end{array}$ \\
\hline 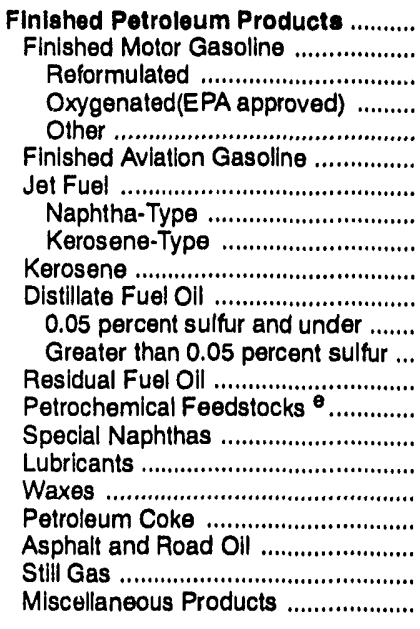 & $\begin{array}{l}4 \\
4 \\
. \\
0 \\
4 \\
. . \\
. . \\
. . \\
. . \\
. . \\
. . \\
. . \\
. . \\
. . \\
. . \\
. . \\
. .\end{array}$ & $\begin{array}{r}447 \\
216 \\
0 \\
4 \\
213 \\
1 \\
39 \\
12 \\
27 \\
1 \\
113 \\
11 \\
102 \\
16 \\
1 \\
0 \\
0 \\
1 \\
13 \\
25 \\
20 \\
2\end{array}$ & $\begin{array}{r}2 \\
(8) \\
0 \\
0 \\
(8) \\
0 \\
0 \\
0 \\
0 \\
0 \\
2 \\
1 \\
1 \\
0 \\
0 \\
0 \\
0 \\
(8) \\
0 \\
0 \\
0 \\
0\end{array}$ & $\begin{array}{l}- \\
. \\
. \\
. \\
. \\
. \\
. \\
. \\
. \\
. \\
. \\
. \\
. \\
. \\
. \\
. \\
. \\
. \\
. \\
. \\
.\end{array}$ & $\begin{array}{r}32 \\
14 \\
0 \\
0 \\
14 \\
(8) \\
18 \\
-5 \\
23 \\
(8) \\
(8) \\
14 \\
-13 \\
0 \\
0 \\
0 \\
0 \\
0 \\
0 \\
0 \\
0 \\
0\end{array}$ & $\begin{array}{r}17 \\
1 \\
N A \\
N A \\
N A \\
(s) \\
6 \\
2 \\
4 \\
(s) \\
-1 \\
N A \\
N A \\
4 \\
0 \\
0 \\
0 \\
(s) \\
1 \\
5 \\
0 \\
(s)\end{array}$ & 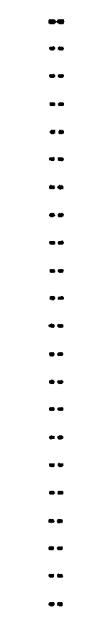 & 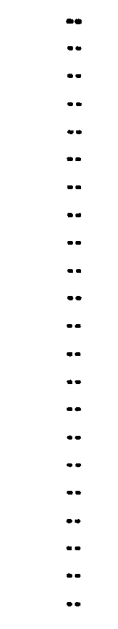 & $\begin{array}{r}(s) \\
(s) \\
N A \\
N A \\
N A \\
0 \\
0 \\
0 \\
0 \\
0 \\
(s) \\
N A \\
\text { NA } \\
0 \\
0 \\
(s) \\
(s) \\
0 \\
(s) \\
(s) \\
0 \\
0\end{array}$ & $\begin{array}{r}467 \\
233 \\
\text { NA } \\
\text { NA } \\
\text { NA } \\
1 \\
51 \\
5 \\
46 \\
1 \\
115 \\
\text { NA } \\
\text { NA } \\
12 \\
1 \\
(s) \\
(s) \\
1 \\
12 \\
19 \\
20 \\
2\end{array}$ \\
\hline 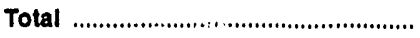 & 578 & 455 & 86 & 61 & -188 & 36 & $\mathbf{0}$ & 441 & (a) & 527 \\
\hline
\end{tabular}

Represents the PAD District in which the material entered the United States and not necessarily where the crude oll or product is processed and/or consumed.

b Unaccounted for crude oll represents the difference between the supply and disposition of crude oil.

c A negative number Indlcates a decrease in stocks and a positive number indicates an Increase in stocks.

d Products supplied is equal to field production, plus refinery production, plus imports, plus unaccounted for crude oll, plus net receipts, minus stock change, minus crude losses, minus refinery inputs, minus exports.

- Includes naphtha less than $401^{\circ} \mathrm{F}$ endpoint and other olls equal to or greater than $401^{\circ} \mathrm{F}$ endpoint.

$(s)=$ Less than 500 barrels per day.

$E$ = Estimated.

LRG = Liquefled Refinery Gas.

Note: Totals may not equal sum of components due to Independent rounding.

Sources: - Enei'gy Information Administration (EIA) Forms ElA.810, "Monthly Refinery Report," ElA-811, "Monthly Bulk Terminal Report," EIA-812, "Monthly Product Pipellne Report," EIA-813, "Monthly Crude Oll Report," EIA-814, "Monthly Imports Report," EIA-816, "Monthly Natural Gas Liquids Report," EIA-817, "Monthly Tanker and Barge Movement Report," and EIA.819M, "Monthly Oxygenate Telephone Report". - Domestic crude oil productlon estimates based on historical statistics from State conservation agencles and the Minerals Management Service of the U.S. Department of the Interlor. - Export data from the Bureau of the Census and Form EIA-810, "Monthly Refinery Report." 
Table 21. PAD District IV-Year-to-Date Dally Average Supply and Disposition of Crude Oll and Petroleum Products, January-March 1993 (Thousand Barrels per Day)

\begin{tabular}{|c|c|c|c|c|c|c|c|c|c|c|}
\hline \multirow[b]{2}{*}{ Commodlty } & \multicolumn{5}{|c|}{ Supply } & \multicolumn{5}{|c|}{ Dlapoaltion } \\
\hline & $\begin{array}{c}\text { Field } \\
\text { Production }\end{array}$ & $\begin{array}{c}\text { Aotlnory } \\
\text { Productlon }\end{array}$ & $\begin{array}{c}\text { Imports by } \\
\text { PAD } \\
\text { Diorrict } \\
\text { of Entry }\end{array}$ & $\begin{array}{l}\text { Unac- } \\
\text { counted } \\
\text { For } \\
\text { Crude oll }\end{array}$ & $\begin{array}{c}\text { Not } \\
\text { Recelpte }\end{array}$ & $\begin{array}{c}\text { Stock } \\
\text { Change }\end{array}$ & $\begin{array}{l}\text { Crude } \\
\text { Longes }\end{array}$ & $\begin{array}{c}\text { Refinery } \\
\text { Inpute }\end{array}$ & Exporte & $\begin{array}{l}\text { Products } \\
\text { Supplled }\end{array}$ \\
\hline 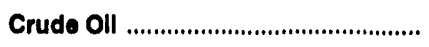 & $E_{433}$ & $\bullet$ & 84 & 40 & -127 & 11 & 0 & 420 & 0 & 0 \\
\hline $\begin{array}{l}\text { Natural Gas Llqulds and LRGs } \\
\text { Pentanes Plus ..................................... } \\
\text { Llquefled Petroleum Gases ............... } \\
\text { Ethane/Ethylene .......................... } \\
\text { Propane/Propylene ...................... } \\
\text { Normal Butane/Butylene ............... } \\
\text { Isobutane/lsobutylene .................. }\end{array}$ & $\begin{array}{r}139 \\
31 \\
109 \\
37 \\
44 \\
17 \\
10\end{array}$ & $\begin{array}{r}4 \\
\ddot{4} \\
4 \\
0 \\
7 \\
-2 \\
(8)\end{array}$ & $\begin{array}{r}10 \\
2 \\
8 \\
0 \\
4 \\
3 \\
(s)\end{array}$ & $\begin{array}{l}\ddot{ } \\
\ddot{.} \\
\ddot{.} \\
\ddot{.} \\
\ddot{.}\end{array}$ & $\begin{array}{r}-74 \\
-9 \\
-65 \\
-34 \\
.15 \\
.9 \\
-6\end{array}$ & $\begin{array}{l}-1 \\
(s) \\
-1 \\
(s) \\
-1 \\
\text { (s) } \\
\text { (s) }\end{array}$ & $\begin{array}{l}\ddot{-} \\
\ddot{.} \\
\ddot{.} \\
\ddot{.} \\
\ddot{.-}\end{array}$ & $\begin{array}{r}21 \\
7 \\
14 \\
0 \\
0 \\
10 \\
4\end{array}$ & $\begin{array}{c}\text { (8) } \\
\text { (s) } \\
0 \\
0 \\
0 \\
0 \\
0\end{array}$ & $\begin{array}{r}59 \\
17 \\
43 \\
3 \\
42 \\
-2 \\
\text { (s) }\end{array}$ \\
\hline $\begin{array}{l}\text { Other Llquids ................................... } \\
\text { Other Hydrocarbons/Oxygenates ...... } \\
\text { Unflnished Oils ................................. } \\
\text { Motor Gasoline Blend. Comp. .......... } \\
\text { Aviation Gasollne Blend. Comp. ........ }\end{array}$ & $\begin{array}{l}2 \\
4 \\
-. \\
-3 \\
-\cdots\end{array}$ & $\begin{array}{l}\cdots \\
\cdots \\
\cdots \\
\cdots\end{array}$ & $\begin{array}{l}0 \\
0 \\
0 \\
0 \\
0\end{array}$ & 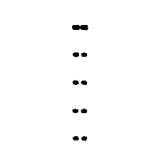 & $\begin{array}{l}0 \\
0 \\
0 \\
0 \\
0\end{array}$ & $\begin{array}{r}12 \\
(3) \\
6 \\
6 \\
0\end{array}$ & $\begin{array}{l}\ddot{ } \\
\ddot{ } \\
\ddot{ } \\
\ddot{.}\end{array}$ & $\begin{array}{r}-10 \\
4 \\
-5 \\
.9 \\
0\end{array}$ & $\begin{array}{l}0 \\
0 \\
0 \\
0 \\
0\end{array}$ & $\begin{array}{r}-1 \\
0 \\
-1 \\
0 \\
0\end{array}$ \\
\hline 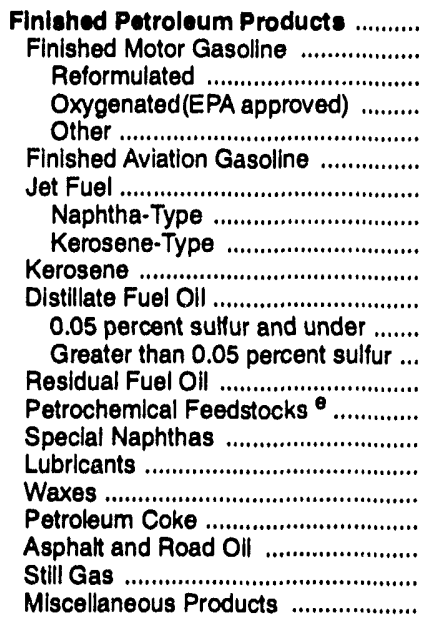 & $\begin{array}{l}4 \\
4 \\
. . \\
3 \\
1 \\
. . \\
. . \\
. . \\
. . \\
. . \\
. . \\
. . \\
. . \\
. . \\
. . \\
. . \\
. . \\
. . \\
. . \\
. .\end{array}$ & $\begin{array}{r}440 \\
218 \\
0 \\
20 \\
198 \\
(9) \\
37 \\
10 \\
26 \\
3 \\
108 \\
10 \\
99 \\
12 \\
1 \\
0 \\
0 \\
1 \\
14 \\
25 \\
20 \\
2\end{array}$ & $\begin{array}{r}3 \\
(s) \\
0 \\
0 \\
(s) \\
0 \\
0 \\
0 \\
0 \\
0 \\
2 \\
1 \\
1 \\
0 \\
0 \\
0 \\
0 \\
(s) \\
0 \\
0 \\
0 \\
0\end{array}$ & 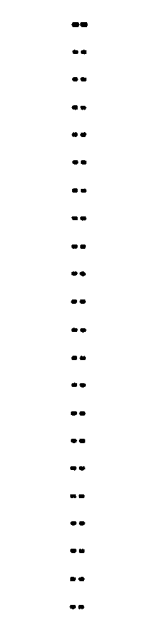 & $\begin{array}{r}20 \\
8 \\
0 \\
(8) \\
7 \\
(8) \\
18 \\
-5 \\
23 \\
-1 \\
-6 \\
9 \\
-15 \\
0 \\
0 \\
0 \\
0 \\
0 \\
0 \\
0 \\
0 \\
0\end{array}$ & $\begin{array}{r}16 \\
4 \\
N A \\
N A \\
N A \\
0 \\
1 \\
1 \\
1 \\
1 \\
-4 \\
N A \\
\text { NA } \\
1 \\
(s) \\
0 \\
0 \\
\text { (s) } \\
1 \\
13 \\
0 \\
\text { (s) }\end{array}$ & 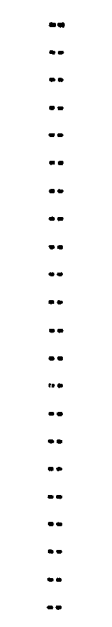 & 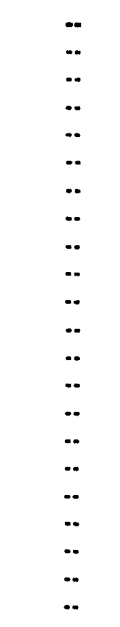 & $\begin{array}{r}(s) \\
(s) \\
N A \\
N A \\
N A \\
0 \\
0 \\
0 \\
0 \\
0 \\
(s) \\
N A \\
\text { NA } \\
0 \\
0 \\
(s) \\
(s) \\
0 \\
(s) \\
(s) \\
0 \\
0\end{array}$ & $\begin{array}{r}450 \\
225 \\
\text { NA } \\
\text { NA } \\
\text { NA } \\
1 \\
54 \\
5 \\
49 \\
1 \\
109 \\
\text { NA } \\
\text { NA } \\
12 \\
1 \\
(s) \\
(s) \\
1 \\
13 \\
12 \\
20 \\
2\end{array}$ \\
\hline 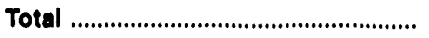 & 578 & 445 & 97 & 40 & -181 & 38 & 0 & 431 & (s) & 509 \\
\hline
\end{tabular}

a Represents the PAD District in which the material entered the United States and not necessarily where the crude oil or product is processed and/or consumed.

b Unaccounted for crude oll represents the difference between the supply and disposition of crude oil.

c. A negative number indicates a decrease in stocks and a positive number indlcates an increase in stocks.

d Products supplied is equal to field production, plus refinery production, plus imports, plus unaccounted for crude oil, plus net recelpts, minus stock change, minus crude losses, minus refinery inputs, minus exports.

- Includes naphtha less than $401^{\circ} \mathrm{F}$ endpoint and other olls equal to or greater than $401^{\circ} \mathrm{F}$ endpolnt.

(s) = Less than 500 barrels per day.

$E=$ Estimated.

$L R G=$ Liquefled Refinery Gas.

Note: Totals may not equal sum of components due to independent rounding.

Sources: - Energy Information Administration (EIA) Forms ElA-810, "Monthly Refinery Report," EIA-811, "Monthly Bulk Terminal Report," EIA-812, "Monthly Product Pipeline Peport," EIA-813, "Monthly Crude Oll Report," EIA-814, "Monthly Imports Report," ElA-816, "Monthly Natural Gas Liquids Report," ElA-817, "Monthly Tanker and Barge Movement Report," and EIA-819M, "Monthly Oxygenate Telephone Report". - Domestic crude oil production estimates based on historical statistics from State consenvation agencies and the Minerals Management Service of the U.S. Depsitment of the Interior. • Export data from the Bureau of the Census and Form EIA-810, "Monthly Refinery Report." 
Table 22. PAD District V-Supply, Disposition, and Ending Stocks of Crude Oll and Petroleum Products, March 1993

(Thousand Barrels)

\begin{tabular}{|c|c|c|c|c|c|c|c|c|c|c|c|}
\hline \multirow[b]{2}{*}{ Commodity } & \multicolumn{5}{|c|}{ Supply } & \multicolumn{5}{|c|}{ Dleposition } & \multirow[b]{2}{*}{$\begin{array}{l}\text { Ending } \\
\text { Stocks }\end{array}$} \\
\hline & $\begin{array}{c}\text { Flold! } \\
\text { Production }\end{array}$ & $\begin{array}{c}\text { Reflinery } \\
\text { Production }\end{array}$ & $\begin{array}{c}\text { Importe by } \\
\text { PAD } \\
\text { Distrlet } \\
\text { of Entrye }\end{array}$ & $\begin{array}{l}\text { Unac- } \\
\text { counted } \\
\text { For } \\
\text { Crude Oll } \\
\end{array}$ & $\begin{array}{c}\text { Not } \\
\text { Recelpts }\end{array}$ & $\begin{array}{c}\text { Stock } \\
\text { Chance }\end{array}$ & $\begin{array}{l}\text { Crude } \\
\text { Losees }\end{array}$ & $\begin{array}{c}\text { Rofinory } \\
\text { Inpute }\end{array}$ & Export: & $\begin{array}{l}\text { Products } \\
\text { Supplledd }\end{array}$ & \\
\hline 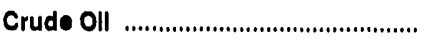 & $E_{79,974}$ & $\bullet$ & 6,139 & 2,961 & $-6,305$ & .130 & 0 & 78,290 & 4,255 & 354 & 72,610 \\
\hline 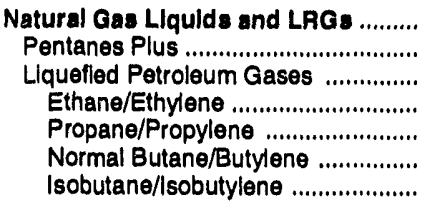 & $\begin{array}{r}3,280 \\
1,678 \\
1,602 \\
2 \\
316 \\
811 \\
473\end{array}$ & $\begin{array}{r}2,975 \\
\cdots \\
2,975 \\
0 \\
1,369 \\
1,562 \\
44\end{array}$ & $\begin{array}{r}16 \\
0 \\
16 \\
0 \\
4 \\
0 \\
12\end{array}$ & $\begin{array}{l}\ddot{.} \\
\ddot{.} \\
\ddot{.} \\
\ddot{.} \\
\ddot{.}\end{array}$ & $\begin{array}{l}0 \\
0 \\
0 \\
0 \\
0 \\
0 \\
0\end{array}$ & $\begin{array}{r}342 \\
14 \\
328 \\
0 \\
.58 \\
261 \\
125\end{array}$ & $\begin{array}{l}\ddot{.} \\
\ddot{.} \\
\ddot{.} \\
. . \\
. . \\
. .\end{array}$ & $\begin{array}{r}2,779 \\
1,278 \\
1,501 \\
0 \\
0 \\
882 \\
619\end{array}$ & $\begin{array}{r}472 \\
0 \\
472 \\
0 \\
233 \\
239 \\
0\end{array}$ & $\begin{array}{r}2,678 \\
386 \\
2,292 \\
2 \\
1,514 \\
991 \\
-215\end{array}$ & $\begin{array}{r}2,214 \\
65 \\
2,149 \\
0 \\
269 \\
1,341 \\
539\end{array}$ \\
\hline $\begin{array}{l}\text { Other Llqulds ................................... } \\
\text { Other Hydrocarbons/Oxygenates ..... } \\
\text { Unflinished Olis ................................... } \\
\text { Motor Gasoline Blend. Comp. .......... } \\
\text { Avlation Gasoline Blend. Comp. ....... }\end{array}$ & $\begin{array}{r}1,028 \\
879 \\
149 \\
\cdots\end{array}$ & 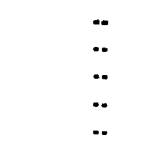 & $\begin{array}{r}1,280 \\
364 \\
880 \\
36 \\
0\end{array}$ & $\begin{array}{l}\ddot{ } \\
\ddot{.} \\
. .\end{array}$ & $\begin{array}{r}149 \\
0 \\
98 \\
51 \\
0\end{array}$ & $\begin{array}{r}873 \\
560 \\
613 \\
-291 \\
-9\end{array}$ & $\begin{array}{l}\ddot{.} \\
\ddot{.} \\
\ddot{.} \\
\ddot{*}\end{array}$ & $\begin{array}{r}1,027 \\
683 \\
68 \\
267 \\
9\end{array}$ & $\begin{array}{r}260 \\
0 \\
0 \\
260 \\
0\end{array}$ & $\begin{array}{r}297 \\
0 \\
297 \\
0 \\
0\end{array}$ & $\begin{array}{r}34,767 \\
4,227 \\
22,230 \\
8,307 \\
3\end{array}$ \\
\hline 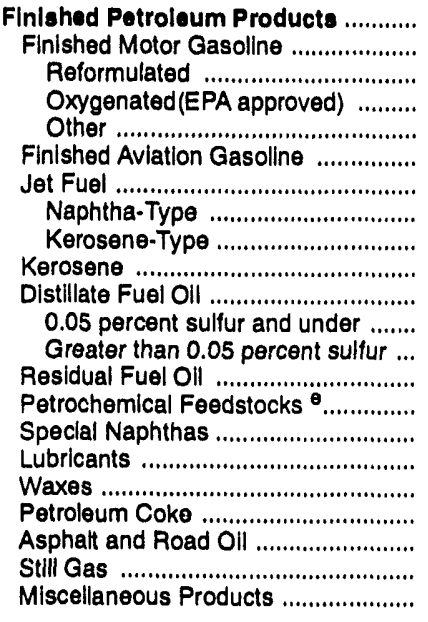 & 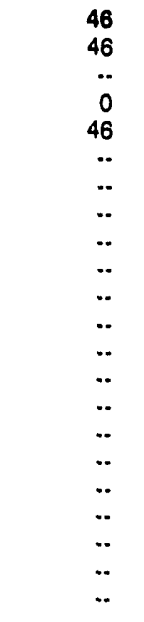 & $\begin{array}{r}84,126 \\
36,961 \\
0 \\
-585 \\
37,546 \\
173 \\
12,961 \\
805 \\
12,156 \\
78 \\
13,400 \\
3,191 \\
10,209 \\
9,302 \\
161 \\
69 \\
748 \\
77 \\
4,625 \\
1,069 \\
4,275 \\
227\end{array}$ & $\begin{array}{r}1,020 \\
226 \\
0 \\
0 \\
226 \\
3 \\
4 \\
0 \\
4 \\
0 \\
77 \\
0 \\
77 \\
665 \\
0 \\
8 \\
0 \\
2 \\
22 \\
13 \\
0 \\
0\end{array}$ & $\begin{array}{l}. . \\
. . \\
. . \\
. . \\
. . \\
. . \\
. . \\
. . \\
. . \\
. . \\
. . \\
. . \\
. . \\
. . \\
. . \\
. . \\
. . \\
. . \\
. . \\
. . \\
. .\end{array}$ & $\begin{array}{r}2,374 \\
1,584 \\
0 \\
0 \\
1,584 \\
0 \\
367 \\
211 \\
156 \\
0 \\
423 \\
0 \\
423 \\
0 \\
0 \\
0 \\
0 \\
0 \\
0 \\
0 \\
0 \\
0\end{array}$ & $\begin{array}{r}-430 \\
-1,977 \\
N A \\
N A \\
N A \\
-39 \\
915 \\
-66 \\
981 \\
-6 \\
-244 \\
N A \\
N A \\
899 \\
-67 \\
-7 \\
.51 \\
11 \\
194 \\
4 \\
0 \\
-62\end{array}$ & $\begin{array}{l}. . \\
. . \\
. . \\
. . \\
. . \\
. . \\
. . \\
. . \\
. . \\
. . \\
. . \\
. . \\
. . \\
. . \\
. . \\
. . \\
. . \\
. . \\
. . \\
. .\end{array}$ & $\begin{array}{l}. . \\
. . \\
. . \\
. . \\
. . \\
. . \\
. . \\
. . \\
. . \\
. . \\
. . \\
. . \\
. . \\
. . \\
. . \\
. .\end{array}$ & $\begin{array}{r}10,585 \\
460 \\
N A \\
N A \\
N A \\
0 \\
1,617 \\
525 \\
1,092 \\
1 \\
1,845 \\
N A \\
N A \\
2,482 \\
0 \\
4 \\
189 \\
10 \\
3,957 \\
19 \\
0 \\
1\end{array}$ & $\begin{array}{r}77,411 \\
40,334 \\
N A \\
N A \\
N A \\
215 \\
10,800 \\
557 \\
10,243 \\
83 \\
12,299 \\
N A \\
N A \\
6,586 \\
228 \\
80 \\
610 \\
58 \\
496 \\
1,059 \\
4,275 \\
288\end{array}$ & $\begin{array}{r}59,360 \\
23,157 \\
0 \\
1,819 \\
21,338 \\
371 \\
8,948 \\
1,172 \\
7,776 \\
46 \\
9,903 \\
2,544 \\
7,359 \\
8,610 \\
175 \\
62 \\
1,832 \\
144 \\
3,702 \\
2,139 \\
0 \\
271\end{array}$ \\
\hline 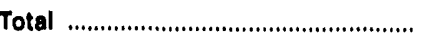 & 84,327 & 87,101 & 8,455 & 2,961 & $-3,782$ & 655 & 0 & 82,096 & 15,572 & 80,740 & 168,951 \\
\hline
\end{tabular}

a Represents the PAD District in which the material entered the United States and not necessarily where the crude oil or product is processed and/or consumed.

b Unaccounted for crude oll represents the difference between the supply and disposition of crude oil.

c A negative number indicates a decrease in stocks and a positive number indicates an increase in stocks.

d Products supplled is equal to fleld production, plus refinery production, plus imports, plus unaccounted for crude oll, plus net receipts, minus stock change, minus crude losses, minus refinery inputs, minus exports.

Includes naphtha less than $401^{\circ} \mathrm{F}$ endpoint and other oils equal to or greater than $401^{\circ} \mathrm{F}$ endpoint.

(s) = Less than 500 barrels.

$E=$ Estimated.

LRG = Llquefied Refinery Gas.

Note: Totals may not equal sum of components due to independent rounding.

Sources: - Energy Information Administration (ElA) Forms ElA-810, "Monthly Refinery Report," ElA-811, "Monthly Bulk Terminal Report," ElA-812, "Monthly Product Pipeline Report," EIA-813, "Monthly Crude Oll Report," EIA-814, "Monthly Imports Report," EIA-816, "Monthly Natural Gas Liquids Report," EIA-817, "Monthly Tanker and Barge Movement Report," and EIA-819M, "Monthly Oxygenate Telephone Report". - Domestic crude oll production estlmates based on historical statistics from State conservation agencles and the Minerals Management Service of the U.S. Department of the Interior. - Export data from the Bureau of the Census and Form ElA-810, "Monthly Refinery Report." 
Table 23. PAD District V-Year-to-Date Supply, Disposition, and Ending Stocks of Crude OII and Petroleum Products, January-March 1993

(Thousand Barrels)

\begin{tabular}{|c|c|c|c|c|c|c|c|c|c|c|c|}
\hline \multirow[b]{2}{*}{ Commodlty } & \multicolumn{5}{|c|}{ Suppiy } & \multicolumn{5}{|c|}{ Disposition } & \multirow[b]{2}{*}{$\begin{array}{l}\text { Endling } \\
\text { Stocks }\end{array}$} \\
\hline & $\begin{array}{c}\text { Flold } \\
\text { Production }\end{array}$ & $\begin{array}{c}\text { Refinery } \\
\text { Productlon }\end{array}$ & $\begin{array}{c}\text { Imports by } \\
\text { PAD } \\
\text { Dlstrict } \\
\text { of Entry }\end{array}$ & $\begin{array}{c}\text { Unac- } \\
\text { counted } \\
\text { For } \\
\text { Crude Oll }\end{array}$ & $\begin{array}{c}\text { Net } \\
\text { Recelpts }\end{array}$ & $\begin{array}{c}\text { Stock } \\
\text { Change }\end{array}$ & $\begin{array}{c}\text { Crude } \\
\text { Losses }\end{array}$ & $\begin{array}{c}\text { Peflnery } \\
\text { Inputs }\end{array}$ & Exports & $\begin{array}{l}\text { Products } \\
\text { Supplled }\end{array}$ & \\
\hline 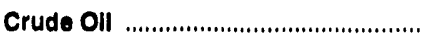 & $E_{232,763}$ & 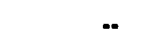 & 18,430 & $-1,256$ & $-21,028$ & 425 & 3 & 214,880 & 12,860 & 942 & 72,610 \\
\hline $\begin{array}{l}\text { Natural Cas Llquide and LRGs .......... } \\
\text { Pentanes Plus .................................. } \\
\text { Llquefled Petroleum Gases ............. } \\
\text { Ethane/Ethylene ............................ } \\
\text { Propane/Propylene ...................... } \\
\text { Normal Butane/Butylene ............... } \\
\text { Isobutane/lsobutylene .................. }\end{array}$ & $\begin{array}{r}9,421 \\
4,834 \\
4,587 \\
6 \\
909 \\
2,365 \\
1,307\end{array}$ & $\begin{array}{r}6,471 \\
0 . \\
6,471 \\
0 \\
3,782 \\
2,722 \\
33\end{array}$ & $\begin{array}{r}48 \\
0 \\
48 \\
0 \\
19 \\
6 \\
23\end{array}$ & $\begin{array}{l}\ddot{.} \\
\ddot{.} \\
\ddot{.} \\
\ddot{.} \\
. .\end{array}$ & $\begin{array}{l}0 \\
0 \\
0 \\
0 \\
0 \\
0 \\
0\end{array}$ & $\begin{array}{r}-915 \\
.15 \\
.900 \\
0 \\
-676 \\
-415 \\
191\end{array}$ & $\begin{array}{l}\ddot{.} \\
\ddot{.} \\
\ddot{.} \\
\ddot{.} \\
\ddot{.}\end{array}$ & $\begin{array}{r}8,104 \\
3,586 \\
4,518 \\
0 \\
26 \\
2,855 \\
1,637\end{array}$ & $\begin{array}{r}1,000 \\
0 \\
1,000 \\
0 \\
357 \\
643 \\
0\end{array}$ & $\begin{array}{r}7,751 \\
1,263 \\
6,488 \\
6 \\
5,003 \\
2,010 \\
-531\end{array}$ & $\begin{array}{r}2,214 \\
65 \\
2,149 \\
0 \\
269 \\
1,341 \\
539\end{array}$ \\
\hline $\begin{array}{l}\text { Other Liqulds .................................... } \\
\text { Other Hydrocarbons/Oxygenates ..... } \\
\text { Unfinished Olis .................................... } \\
\text { Motor Gasollne Blend. Comp. .......... } \\
\text { Aviation Gasoline Blend. Comp. ........ }\end{array}$ & $\begin{array}{r}5,133 \\
5,620 \\
\ldots \\
-487 \\
\ldots\end{array}$ & $\begin{array}{l}\cdots \\
\ddot{.} \\
\ddot{.} \\
\ddot{.}\end{array}$ & $\begin{array}{r}2,229 \\
870 \\
1,323 \\
36 \\
0\end{array}$ & $\begin{array}{l}\ddot{.} \\
\ddot{.} \\
\ddot{.} \\
\ddot{.}\end{array}$ & $\begin{array}{r}191 \\
0 \\
98 \\
93 \\
0\end{array}$ & $\begin{array}{r}3,935 \\
1,648 \\
1,145 \\
1,142 \\
0\end{array}$ & $\begin{array}{l}\ddot{.} \\
\ddot{.} \\
. .\end{array}$ & $\begin{array}{r}3,934 \\
4,842 \\
1,140 \\
-2,048 \\
0\end{array}$ & $\begin{array}{r}548 \\
0 \\
0 \\
548 \\
0\end{array}$ & $\begin{array}{r}-864 \\
0 \\
-864 \\
0 \\
0\end{array}$ & $\begin{array}{r}34,767 \\
4,227 \\
22,230 \\
8,307 \\
3\end{array}$ \\
\hline 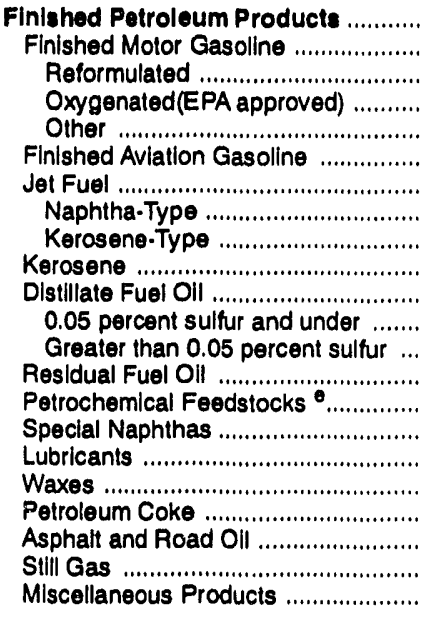 & $\begin{array}{r}1,019 \\
1,019 \\
. . \\
1,348 \\
329 \\
. . \\
. . \\
. . \\
. . \\
. . \\
. . \\
. . \\
. . \\
. . \\
. . \\
. . \\
. . \\
. . \\
. . \\
. .\end{array}$ & $\begin{array}{r}235,040 \\
105,118 \\
0 \\
25,923 \\
79,195 \\
406 \\
36,649 \\
2,537 \\
34,112 \\
264 \\
36,514 \\
8,268 \\
28,246 \\
25,161 \\
535 \\
160 \\
1,764 \\
192 \\
13,096 \\
2,421 \\
12,133 \\
627\end{array}$ & $\begin{array}{r}4,545 \\
837 \\
0 \\
0 \\
837 \\
3 \\
319 \\
0 \\
319 \\
0 \\
364 \\
119 \\
245 \\
2,724 \\
83 \\
21 \\
31 \\
6 \\
57 \\
100 \\
0 \\
0\end{array}$ & $\begin{array}{l}. . \\
. . \\
. . \\
. . \\
. . \\
. . \\
. \\
. . \\
. . \\
. . \\
. . \\
. . \\
. . \\
. . \\
. . \\
. . \\
. . \\
. .\end{array}$ & $\begin{array}{r}8,447 \\
5,577 \\
0 \\
0 \\
5,577 \\
0 \\
1,144 \\
565 \\
579 \\
0 \\
1,702 \\
0 \\
1,702 \\
0 \\
0 \\
0 \\
24 \\
0 \\
0 \\
0 \\
0 \\
0\end{array}$ & $\begin{array}{r}1,552 \\
-1,019 \\
N A \\
N A \\
N A \\
-5 \\
822 \\
-60 \\
882 \\
-17 \\
.916 \\
N A \\
N A \\
1,613 \\
33 \\
1 \\
-186 \\
19 \\
605 \\
440 \\
0 \\
162\end{array}$ & $\begin{array}{l}. . \\
. . \\
. . \\
. . \\
. \\
. . \\
. . \\
. . \\
. \\
. . \\
. \\
. \\
. \\
. . \\
. \\
. . \\
. . \\
. . \\
. .\end{array}$ & $\begin{array}{l}\ddot{.} \\
. . \\
. . \\
. . \\
. . \\
. . \\
. . \\
. . \\
. . \\
. . \\
. . \\
. . \\
. . \\
. . \\
. . \\
. . \\
. . \\
. . \\
. . \\
. \cdot\end{array}$ & $\begin{array}{r}26,279 \\
2,663 \\
N A \\
N A \\
N A \\
0 \\
3,488 \\
1,153 \\
2,335 \\
3 \\
5,550 \\
N A \\
N A \\
4,288 \\
0 \\
95 \\
327 \\
37 \\
9,781 \\
44 \\
0 \\
2\end{array}$ & $\begin{array}{r}221,220 \\
110,908 \\
\mathrm{NA} \\
\mathrm{NA} \\
\mathrm{NA} \\
414 \\
33,802 \\
2,009 \\
31,793 \\
278 \\
33,946 \\
\mathrm{NA} \\
\mathrm{NA} \\
21,984 \\
585 \\
85 \\
1,678 \\
142 \\
2,767 \\
2,037 \\
12,133 \\
463\end{array}$ & $\begin{array}{r}59,360 \\
23,157 \\
0 \\
1,819 \\
21,338 \\
371 \\
8,948 \\
1,172 \\
7,776 \\
46 \\
9,903 \\
2,544 \\
7,359 \\
8,610 \\
175 \\
62 \\
1,832 \\
144 \\
3,702 \\
2,139 \\
0 \\
271\end{array}$ \\
\hline Total & 248,336 & 241,511 & 25,252 & $-1,255$ & $-12,380$ & 4,997 & 3 & 226,818 & 40,487 & 229,049 & 168,951 \\
\hline
\end{tabular}

aepresents the PAD District in whlch the materlal entered the United States and not necessarily where the crude oil or product is processed and/or consumed.

b Unaccounted for crude oil represents the difference between the supply and disposition of crude oil.

c A negative number indicates a decrease in stocks and a positive number indicates an increase in stocks.

d Products supplied is equal to field production, plus refinery production, plus imports, plus unaccounted for crude oll, plus net recelpts, minus stock change, minus crude losses, minus refinery inputs, minus exports.

- Includes naphtha less than $401^{\circ} \mathrm{F}$ endpoint and other olls equal to or greater than $401^{\circ} \mathrm{F}$ endpoint.

(s) = Less than 500 barrels.

$E=$ Estimated.

LRG = Liquefied Refinery Gas.

Note: Totals may not equal sum of components due to independent rounding.

Sources: - Energy Informatlon Administration (EIA) Forms EIA-810, "Monthly Refinery Report," EIA-811, "Monthly Bulk Terminal Report," EIA-812, "Monthly Product Plpeline Report," EIA-813, "Monthly Crude Oll Report," ElA-814, "Monthly Imports Report," ElA-816, "Monthly Natural Gas Liquids Report " ElA-817 "Monthly Tanker and Barge Movement Report," and EIA-819M, "Monthly Oxygenale Telephone Report". - Domestlc crude oil production estimates based on historical statistlcs from State conservation agencles and the Minerals Management Service of the U.S. Department of the Interlor. - Export data from the Bureau of the Census and Form EIA.810, "Monthly Refinery Report." 
Table 24. PAD District V - Dally Average Supply and Dlsposition of Crude OIl and Petroleum Products, March 1993 (Thousand Barrels per Day)

\begin{tabular}{|c|c|c|c|c|c|c|c|c|c|c|}
\hline \multirow[b]{2}{*}{ Commodlty } & \multicolumn{5}{|c|}{ Supply } & \multicolumn{5}{|c|}{ Dleposition } \\
\hline & $\begin{array}{c}\text { Fiold } \\
\text { Productlon }\end{array}$ & $\begin{array}{c}\text { Rennery } \\
\text { Productlon }\end{array}$ & $\begin{array}{c}\text { Imports by } \\
\text { PAD } \\
\text { District } \\
\text { of Entry }\end{array}$ & $\begin{array}{l}\text { Unac- } \\
\text { counted } \\
\text { For } \\
\text { Crude } 0 \|^{b}\end{array}$ & $\begin{array}{c}\text { Not } \\
\text { Becelpis }\end{array}$ & $\begin{array}{c}\text { Stock } \\
\text { Changec }\end{array}$ & $\begin{array}{c}\text { Crude } \\
\text { Losses }\end{array}$ & $\begin{array}{c}\text { Retinery } \\
\text { Inpuls }\end{array}$ & Exports & $\begin{array}{l}\text { Products } \\
\text { Supplled d }\end{array}$ \\
\hline 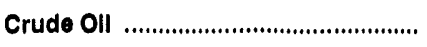 & $\mathbf{E}_{\mathbf{2 , 5 8 0}}$ & - & 198 & 96 & -203 & -4 & 0 & 2,525 & 137 & 11 \\
\hline 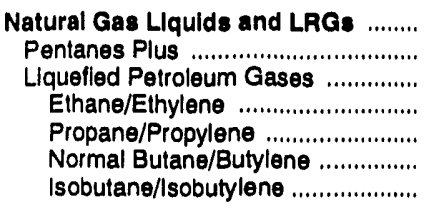 & $\begin{array}{r}106 \\
54 \\
52 \\
(s) \\
10 \\
26 \\
15\end{array}$ & $\begin{array}{r}98 \\
\ddot{96} \\
0 \\
44 \\
50 \\
1\end{array}$ & $\begin{array}{r}1 \\
0 \\
1 \\
0 \\
\text { (s) } \\
0 \\
(8)\end{array}$ & $\begin{array}{l}\ddot{-} \\
\ddot{.} \\
\ddot{.} \\
\ddot{.} \\
. \\
. .\end{array}$ & $\begin{array}{l}0 \\
0 \\
0 \\
0 \\
0 \\
0 \\
0\end{array}$ & $\begin{array}{r}11 \\
(s) \\
11 \\
0 \\
.2 \\
8 \\
4\end{array}$ & $\begin{array}{l}\ddot{.} \\
. . \\
. . \\
\ddot{.} \\
\ddot{.}\end{array}$ & $\begin{array}{r}90 \\
41 \\
48 \\
0 \\
0 \\
28 \\
20\end{array}$ & $\begin{array}{r}15 \\
0 \\
15 \\
0 \\
8 \\
8 \\
0\end{array}$ & $\begin{array}{l}86 \\
12 \\
74 \\
\text { (s) } \\
49 \\
32 \\
-7\end{array}$ \\
\hline $\begin{array}{l}\text { Other Llquids ................................. } \\
\text { Other Hydrocarbons/Oxygenates ..... } \\
\text { Unfinished Olls ................................ } \\
\text { Motor Gasoline Blend. Comp. .......... } \\
\text { Aviation Gasoline Blend. Comp. ........ }\end{array}$ & $\begin{array}{c}33 \\
28 \\
\ddot{5} \\
\cdots\end{array}$ & 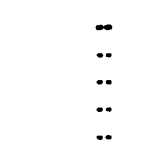 & $\begin{array}{r}41 \\
12 \\
28 \\
1 \\
0\end{array}$ & $\begin{array}{l}- \\
\ddot{.} \\
\ddot{.} \\
\ddot{.}\end{array}$ & $\begin{array}{l}\mathbf{5} \\
0 \\
3 \\
2 \\
0\end{array}$ & $\begin{array}{r}28 \\
18 \\
20 \\
.9 \\
(8)\end{array}$ & $\begin{array}{l}\ddot{.} \\
\ddot{.} \\
\ddot{.} \\
\ddot{*}\end{array}$ & $\begin{array}{r}33 \\
22 \\
2 \\
9 \\
(s)\end{array}$ & $\begin{array}{l}8 \\
0 \\
0 \\
8 \\
0\end{array}$ & $\begin{array}{r}10 \\
0 \\
10 \\
0 \\
0\end{array}$ \\
\hline 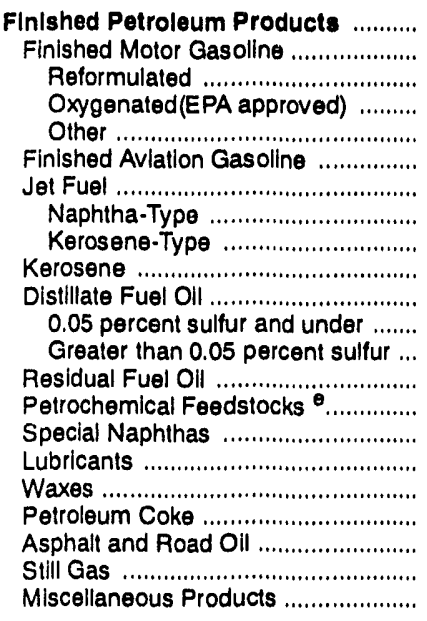 & $\begin{array}{l}1 \\
1 \\
.0 \\
0 \\
1 \\
. . \\
. . \\
. . \\
. . \\
. . \\
. . \\
. . \\
. . \\
. . \\
. . \\
. . \\
. . \\
. . \\
. .\end{array}$ & $\begin{array}{r}2,714 \\
1,192 \\
0 \\
-19 \\
1,211 \\
6 \\
418 \\
26 \\
392 \\
3 \\
432 \\
103 \\
329 \\
300 \\
5 \\
2 \\
24 \\
2 \\
149 \\
34 \\
138 \\
7\end{array}$ & $\begin{array}{r}33 \\
7 \\
0 \\
0 \\
7 \\
(s) \\
(s) \\
0 \\
(s) \\
0 \\
2 \\
0 \\
2 \\
21 \\
0 \\
(s) \\
0 \\
(s) \\
1 \\
(s) \\
0 \\
0\end{array}$ & $\begin{array}{l}. . \\
. . \\
. . \\
. . \\
. . \\
. . \\
. . \\
. . \\
. . \\
. . \\
. . \\
. . \\
. . \\
. . \\
. . \\
. . \\
. .\end{array}$ & $\begin{array}{r}77 \\
51 \\
0 \\
0 \\
51 \\
0 \\
12 \\
7 \\
5 \\
0 \\
14 \\
0 \\
14 \\
0 \\
0 \\
0 \\
0 \\
0 \\
0 \\
0 \\
0 \\
0\end{array}$ & $\begin{array}{r}-14 \\
-64 \\
\text { NA } \\
\text { NA } \\
\text { NA } \\
-1 \\
30 \\
-2 \\
32 \\
(s) \\
-8 \\
\text { NA } \\
\text { NA } \\
29 \\
-2 \\
(s) \\
-2 \\
\text { (s) } \\
6 \\
\text { (s) } \\
0 \\
-2\end{array}$ & $\begin{array}{l}. . \\
. . \\
. . \\
. . \\
. . \\
. . \\
. . \\
. . \\
. \\
. . \\
. \\
. . \\
. . \\
. . \\
. . \\
. . \\
. . \\
. . \\
. .\end{array}$ & $\begin{array}{l}. . \\
. . \\
. . \\
. . \\
. . \\
. . \\
. . \\
. . \\
. . \\
. . \\
. . \\
. . \\
. . \\
\ddot{.} \\
\ddot{.} \\
. . \\
. . \\
. . \\
. .\end{array}$ & $\begin{array}{r}341 \\
15 \\
\text { NA } \\
\text { NA } \\
\text { NA } \\
0 \\
52 \\
17 \\
35 \\
(s) \\
60 \\
\text { NA } \\
\text { NA } \\
80 \\
0 \\
(s) \\
6 \\
(s) \\
128 \\
1 \\
0 \\
(s)\end{array}$ & $\begin{array}{r}2,497 \\
1,301 \\
N A \\
N A \\
N A \\
7 \\
348 \\
18 \\
330 \\
3 \\
397 \\
N A \\
N A \\
212 \\
7 \\
3 \\
20 \\
2 \\
16 \\
34 \\
138 \\
9\end{array}$ \\
\hline 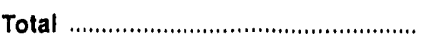 & 2,720 & 2,810 & 273 & 96 & -122 & 21 & 0 & 2,648 & 502 & 2,605 \\
\hline
\end{tabular}

a Represents the PAD District in which the materlal entered the United States and not necessarily where the crude oil or product is processed and/or consumed.

Unaccounted for crude oll represents the difference between the supply and disposition of crude oll.

c A negative number indicates a decrease in stocks and a positive number indicates an increase in stocks.

d Products supplied is equal to field production, plus refinery production, plus imports, plus unaccounted for crude oil, plus net receipts, minus stock change, minus crude losses, minus refinery inputs, minus exports.

Includes naphtha less than $401^{\circ} \mathrm{F}$ endpoint and other oils equal to or greater than $401^{\circ} \mathrm{F}$ endpoint.

(s) = Less than 500 barrels per day.

$E=$ Estimated.

LRG = Liquefied Refinery Gas.

Note: Totals may not equal sum of components due to independent rounding.

Sources: - Energy Information Administration (EIA) Forms EIA-B10, "Monthiy Refinery Report," ElA-811, "Monthly Bulk Terminal Report," ElA-812, "Monthly Product Pipeline Report," EIA-813, "Monthly Crude Oll Report," EIA-814, "Monthly Imports Report," ElA-816, "Monthly Natural Gas Liquids Report," EIA-817, "Monthly Tanker and Barge Movement Report," and EIA-819M, "Monthly Oxygenate Telephone Report". - Domestlc crude oil production estimates based on historical statistics from State conservation agencles and the Minerals Management Service of the U.S. Department of the Interior. - Export data from the Bureau of the Census and Form EIA-810, "Monthly Refinery Report." 
Table 25. PAD District V - Year-to-Date Dally Average Supply and Disposition of Crude Oll and Petroleum Products, January-March 1993

(Thousand Barrels per Day)

\begin{tabular}{|c|c|c|c|c|c|c|c|c|c|c|}
\hline \multirow[b]{2}{*}{ Commodlty } & \multicolumn{5}{|c|}{ Supply } & \multicolumn{5}{|c|}{ Dlsposition } \\
\hline & $\begin{array}{c}\text { Flold } \\
\text { Production }\end{array}$ & $\begin{array}{c}\text { Pofinery } \\
\text { Productlon }\end{array}$ & $\begin{array}{c}\text { Imports by } \\
\text { PAD } \\
\text { Dlstrlot } \\
\text { of Enter }\end{array}$ & $\begin{array}{l}\text { Unac- } \\
\text { counted } \\
\text { For } \\
\text { Crude } 0 \|^{b}\end{array}$ & $\begin{array}{c}\text { Not } \\
\text { Recelote }\end{array}$ & $\begin{array}{c}\text { Stock } \\
\text { Change }\end{array}$ & $\begin{array}{l}\text { Crude } \\
\text { Lesees } \\
\end{array}$ & $\begin{array}{c}\text { Refinery } \\
\text { Inpute }\end{array}$ & Exports & $\begin{array}{l}\text { Preducts } \\
\text { Supplled }^{d}\end{array}$ \\
\hline Crude Oll ................................................... & $E_{2,586}$ & $\cdots$ & 205 & -14 & 234 & $\mathbf{5}$ & (0) & 2,388 & 141 & 10 \\
\hline $\begin{array}{l}\text { Natural Gas Llqulde and LRGe .......... } \\
\text { Pentanes Plus ................................... } \\
\text { Liquetied Petroleum Gases ............... } \\
\text { Ethane/Ethyene ............................. } \\
\text { Propane/Propylene ...................... } \\
\text { Normal Butane/Butylene ................ } \\
\text { Isobutane//sobutylene .................. }\end{array}$ & $\begin{array}{r}105 \\
54 \\
51 \\
(8) \\
10 \\
26 \\
15\end{array}$ & $\begin{array}{r}72 \\
72 \\
72 \\
0 \\
42 \\
30 \\
(8)\end{array}$ & $\begin{array}{r}1 \\
0 \\
1 \\
0 \\
(8) \\
(s) \\
(s)\end{array}$ & 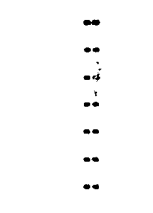 & $\begin{array}{l}0 \\
0 \\
0 \\
0 \\
0 \\
0 \\
0\end{array}$ & $\begin{array}{r}-10 \\
(3) \\
-10 \\
0 \\
-8 \\
-5 \\
2\end{array}$ & 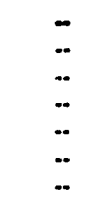 & $\begin{array}{r}90 \\
40 \\
50 \\
0 \\
(s) \\
32 \\
18\end{array}$ & $\begin{array}{r}11 \\
0 \\
11 \\
0 \\
4 \\
7 \\
0\end{array}$ & $\begin{array}{l}86 \\
14 \\
72 \\
\text { (s) } \\
56 \\
22 \\
-6\end{array}$ \\
\hline $\begin{array}{l}\text { Other Llquide .................................... } \\
\text { Other Hydrocarbons/Oxygenates ..... } \\
\text { Unfinished Oils ................................... } \\
\text { Motor Gasoline Blend. Comp. ........... } \\
\text { Aviation Gasoline Blend. Comp. ....... }\end{array}$ & $\begin{array}{r}57 \\
62 \\
-. \\
-5 \\
-.\end{array}$ & 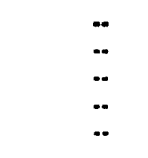 & $\begin{array}{r}25 \\
10 \\
15 \\
(9) \\
0\end{array}$ & $\begin{array}{l}\cdots \\
\cdots \\
\cdots \\
\cdots\end{array}$ & $\begin{array}{l}2 \\
0 \\
1 \\
1 \\
0\end{array}$ & $\begin{array}{r}44 \\
18 \\
13 \\
13 \\
0\end{array}$ & $\begin{array}{l}- \\
\cdots \\
\cdots \\
\cdots\end{array}$ & $\begin{array}{r}44 \\
54 \\
13 \\
-23 \\
0\end{array}$ & $\begin{array}{l}6 \\
0 \\
0 \\
6 \\
0\end{array}$ & $\begin{array}{r}-10 \\
0 \\
-10 \\
0 \\
0\end{array}$ \\
\hline 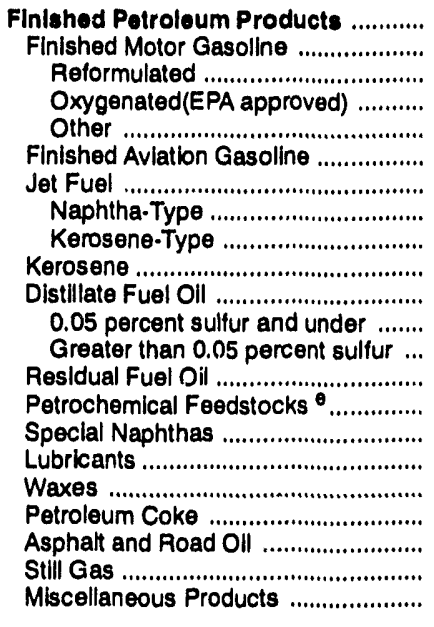 & $\begin{array}{l}11 \\
11 \\
. . \\
15 \\
.4 \\
. . \\
. . \\
. . \\
. . \\
. . \\
. . \\
. . \\
. . \\
. . \\
. \\
. . \\
. \\
. \\
. . \\
. .\end{array}$ & $\begin{array}{r}2,612 \\
1,168 \\
0 \\
288 \\
880 \\
5 \\
507 \\
28 \\
379 \\
3 \\
406 \\
92 \\
314 \\
280 \\
6 \\
2 \\
2 \\
20 \\
2 \\
146 \\
27 \\
135 \\
7\end{array}$ & $\begin{array}{r}51 \\
9 \\
0 \\
0 \\
9 \\
(8) \\
4 \\
0 \\
4 \\
0 \\
4 \\
1 \\
3 \\
30 \\
1 \\
(8) \\
(8) \\
(8) \\
1 \\
1 \\
0 \\
0\end{array}$ & $\begin{array}{l}. . \\
. . \\
. . \\
. . \\
. . \\
\ddot{. .} \\
\ddot{.} \\
\ddot{.} \\
\ddot{.} \\
\ddot{.} \\
\ddot{.} \\
. . \\
. . \\
\ddot{.} \\
. .\end{array}$ & $\begin{array}{r}94 \\
62 \\
0 \\
0 \\
62 \\
0 \\
13 \\
6 \\
6 \\
0 \\
19 \\
0 \\
19 \\
0 \\
0 \\
0 \\
(8) \\
0 \\
0 \\
0 \\
0 \\
0\end{array}$ & $\begin{array}{r}17 \\
-11 \\
\text { NA } \\
\text { NA } \\
\text { NA } \\
(s) \\
9 \\
-1 \\
10 \\
(s) \\
-10 \\
N A \\
\text { NA } \\
18 \\
(s) \\
(s) \\
-2 \\
(s) \\
7 \\
5 \\
0 \\
2\end{array}$ & $\begin{array}{l}. . \\
. . \\
. . \\
. . \\
. . \\
. . \\
. . \\
. . \\
. . \\
. . \\
. . \\
. . \\
. . \\
. . \\
. . \\
. . \\
. . \\
. . \\
. .\end{array}$ & $\begin{array}{l}. . \\
. . \\
. . \\
. . \\
. . \\
. . \\
. . \\
. . \\
. . \\
. . \\
. . \\
. . \\
. . \\
. . \\
. . \\
. . \\
. . \\
. . \\
\ddot{. .} \\
. .\end{array}$ & $\begin{array}{r}292 \\
30 \\
\text { NA } \\
\text { NA } \\
\text { NA } \\
0 \\
39 \\
13 \\
26 \\
(s) \\
62 \\
\text { NA } \\
\text { NA } \\
48 \\
0 \\
1 \\
4 \\
\text { (s) } \\
109 \\
\text { (s) } \\
0 \\
\text { (s) }\end{array}$ & $\begin{array}{r}2,458 \\
1,232 \\
N A \\
N A \\
N A \\
5 \\
376 \\
22 \\
353 \\
3 \\
377 \\
N A \\
N A \\
244 \\
7 \\
1 \\
19 \\
2 \\
31 \\
23 \\
135 \\
5\end{array}$ \\
\hline Total & 2,759 & 2,683 & 281 & -14 & -138 & 56 & (s) & 2,521 & 450 & 2,545 \\
\hline
\end{tabular}

Represents the PAD District in which the material entered the United States and not necessarily where the crude oil or product is processed and/or consumed.

b Unaccounted for crude oil represents the difference between the supply and disposition of crude oil.

c A negative number indicates a decrease in stocks and a positive number indicates an increase in stocks.

Products supplied is equal to field production, plus refinery production, plus imports, plus unaccounted for crude oil, plus net receipts, minus stock change, minus crude losses, minus refinery inputs, minus exports.

- Includes naphtha less than $401^{\circ} \mathrm{F}$ endpoint and other oils equal to or greater than $401^{\circ} \mathrm{F}$ endpoint.

$(s)=$ Less than 500 barrels per day.

$E=$ Estimated

$L R G=$ Liquefied Refinery Gas.

Note: Totals may not equal sum of components due to independent rounding.

Sources: - Energy Information Administration (E|A) Forms E|A-810, "Monthly Refinery Report," EIA-811, "Monthly Bulk Terminal Report, " E|A-812, "Monthly Product Pipeline Report," EIA-813, "Monthly Crude Oil Report," ElA-814, "Monthly Imports Report, "ElA-816, "Monthly Natural Gas Liquids Report," ElA-817, "Monthly Tanker and Barge Movement Report," and ElA-819M, "Monthly Oxygenate Telephone Report". - Domestic crude oil production estimates based on historical statistics from State conservation agencies and the Minerals Management Service of the U.S. Department of the Interior. - Export data from the Bureau of the Census and Form EIA-810, "Monthly Refinery Report." 
Table 26. Production of Crude Oll by PAD District and State (Thousand Barrels)

\begin{tabular}{|c|c|c|}
\hline \multirow[b]{2}{*}{ PAD Distrlct and State } & \multicolumn{2}{|c|}{ January 1993} \\
\hline & Total & $\begin{array}{c}\text { Dally } \\
\text { Average }\end{array}$ \\
\hline 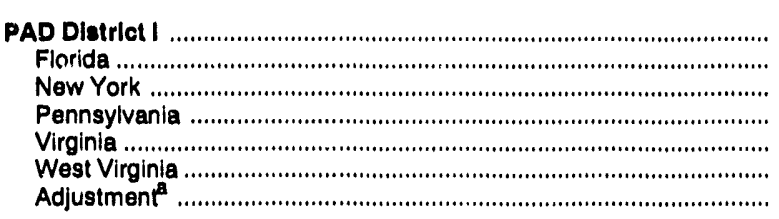 & $\begin{array}{r}E_{816} \\
485 \\
E_{29} \\
E_{164} \\
E_{1} \\
-31 \\
-31\end{array}$ & $\begin{array}{r}E_{26} \\
E_{1}^{16} \\
E_{6} \\
E_{(s)} \\
-1\end{array}$ \\
\hline $\begin{array}{l}\text { PAD Dlstrlot II } \\
\text { Illinois ... } \\
\text { Indiana } \\
\text { Kansas .... } \\
\text { Kentucky } \\
\text { Michigan } \\
\text { Missouri } \\
\text { Nebraska } \\
\text { North Dakota } \\
\text { Ohio } \\
\text { Oklahoma } \\
\text { South Dakota } \\
\text { Tennessee } \\
\text { Adjustment }\end{array}$ & $\begin{array}{r}19,914 \\
1,600 \\
247 \\
4,087 \\
350 \\
E_{1} 994 \\
11 \\
423 \\
2,663 \\
703 \\
8,170 \\
128 \\
36 \\
402\end{array}$ & $\begin{array}{r}E_{642} \\
52 \\
8 \\
132 \\
11 \\
E_{35} \\
E_{(9)} \\
14 \\
86 \\
E_{23} \\
264 \\
4 \\
1 \\
13\end{array}$ \\
\hline $\begin{array}{l}\text { PAD District III } \\
\text { Alabama } \\
\text { Arkansas } \\
\text { Louisiana }{ }^{b} \\
\text { Mississippi } \\
\text { New Mexico } \\
\text { Texas } \\
\text { Federal Offshore PAD District III } \\
\text { Adjustment }\end{array}$ & $\begin{array}{r}102,125 \\
1,622 \\
E_{916} \\
11,960 \\
1,984 \\
5,848 \\
53,378 \\
25,646 \\
771\end{array}$ & $\begin{array}{r}E_{3,294} \\
E_{30} \\
E_{386} \\
64 \\
189 \\
1,722 \\
E_{827}^{2} \\
25\end{array}$ \\
\hline 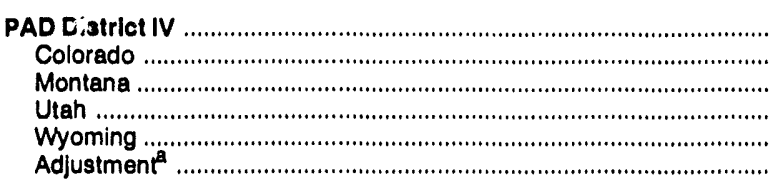 & $\begin{array}{r}E_{13,666} \\
E_{2,302} \\
1,448 \\
1,853 \\
7,553 \\
510\end{array}$ & $\begin{array}{r}E_{441} \\
E_{74} \\
47 \\
60 \\
244 \\
16\end{array}$ \\
\hline 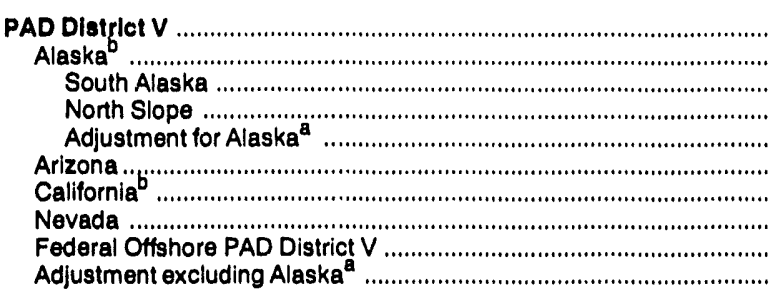 & $\begin{array}{r}E \\
E 0,714 \\
51,280 \\
1,238 \\
50,045 \\
-3 \\
6 \\
24,865 \\
252 \\
3,747 \\
561\end{array}$ & $\begin{array}{r}E_{2,604} \\
1,654 \\
40 \\
1,614 \\
(s) \\
(s) \\
802 \\
8 \\
121 \\
18\end{array}$ \\
\hline (n) & $E_{217,235}$ & $E_{7,008}$ \\
\hline
\end{tabular}

These adjustments are used to reconcile the national and PAD District level sums of the State data with the independently estimated U.S. and Alaskan figures shown in the Summary Statistics portion of this issue and with the PAD District level figures published in a previous issue. Revised data at the State, PAD District, and national levels will be published without adjustments in the Petroleum Supply Annual.

Includes the following current month offshore production (thousand barrels): Alaska: State - 4,690; California:

State - 1,739; Louisiana: State - E1,964; Texas: State -175; U.S. Total, including Federal offshore - E37,962.

(s) = Less than 500 barrels or less than 500 barrels per day.

$E$ Estimated.

Note: Totals may not equal sum of components due to independent rounding.

Sources: State government agencles, U.S. Department of the Interior, Minerals Management Service and the Conservation Committee of California Oll Producers. 
Table 27. Natural Gas Plant Net Production and Stocks of Petroleum Products by PAD and Refining Distrlcts, March 1993

(Thousand Barrels)

\begin{tabular}{|c|c|c|c|c|c|c|c|c|c|}
\hline \multirow[b]{2}{*}{ Commodity } & \multicolumn{4}{|c|}{ PAD Dlatrice I } & \multicolumn{5}{|c|}{ PAD Dlatrlet II } \\
\hline & $\begin{array}{l}\text { East } \\
\text { Coast }\end{array}$ & \multicolumn{2}{|c|}{$\begin{array}{c}\text { Appalachlan } \\
\text { No. } 1\end{array}$} & Total & Ind., III., Ky. & \multicolumn{2}{|c|}{$\begin{array}{l}\text { MInn., Wle., } \\
\text { N. Dak., S. Dak. }\end{array}$} & $\begin{array}{c}\text { Okla., Kano., } \\
\text { Mo. }\end{array}$ & Total \\
\hline \multirow{3}{*}{ 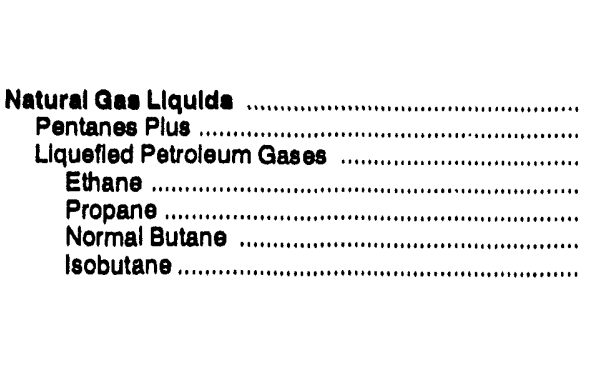 } & \multicolumn{9}{|c|}{ Not Production } \\
\hline & $\begin{array}{r}209 \\
35 \\
174 \\
53 \\
73 \\
38 \\
10\end{array}$ & & & $\begin{array}{r}921 \\
111 \\
810 \\
248 \\
392 \\
120 \\
50\end{array}$ & $\begin{array}{r}559 \\
102 \\
457 \\
64 \\
237 \\
86 \\
70\end{array}$ & $\begin{array}{l}3 \\
2 \\
16 \\
10\end{array}$ & & $\begin{array}{r}9,515 \\
1,161 \\
8,354 \\
3,292 \\
3,402 \\
954 \\
708\end{array}$ & $\begin{array}{r}10,439 \\
1,353 \\
9,086 \\
3,357 \\
3,807 \\
1,146 \\
776\end{array}$ \\
\hline & \multicolumn{9}{|c|}{ Stocks } \\
\hline \multirow[t]{4}{*}{ 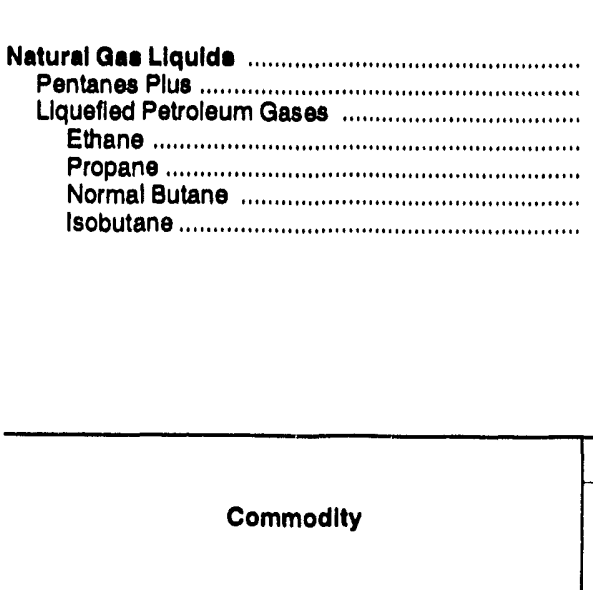 } & $\begin{array}{r}91 \\
14 \\
77 \\
0 \\
28 \\
48 \\
1\end{array}$ & \multicolumn{2}{|c|}{$\begin{array}{r}31 \\
3 \\
28 \\
0 \\
18 \\
5 \\
5\end{array}$} & $\begin{array}{r}122 \\
17 \\
105 \\
0 \\
46 \\
53 \\
6\end{array}$ & $\begin{array}{r}101 \\
15 \\
86 \\
11 \\
43 \\
18 \\
14\end{array}$ & \multicolumn{3}{|c|}{$\begin{array}{r}39 \\
11 \\
28 \\
0 \\
20 \\
8 \\
0\end{array}$} & $\begin{array}{r}2,151 \\
182 \\
1,969 \\
696 \\
769 \\
385 \\
119\end{array}$ \\
\hline & \multicolumn{6}{|c|}{ PAD Dlatrlet III } & \multirow{2}{*}{$\begin{array}{c}\text { PAD Dist. } \\
\text { IV }\end{array}$} & \multirow{2}{*}{$\begin{array}{c}\text { PAD Dist. } \\
\mathrm{V}\end{array}$} & \multirow[b]{3}{*}{$\begin{array}{l}\text { U.S. } \\
\text { Total }\end{array}$} \\
\hline & \multirow{2}{*}{$\begin{array}{l}\text { Texas } \\
\text { Inland }\end{array}$} & \multirow{2}{*}{$\begin{array}{c}\text { Toxas } \\
\text { Gulf } \\
\text { Coavt }\end{array}$} & \multirow{2}{*}{$\begin{array}{l}\text { La. } \\
\text { Cuif } \\
\text { Coait }\end{array}$} & \multirow[b]{2}{*}{$\begin{array}{c}\text { N. La., } \\
\text { Ark. }\end{array}$} & \multirow{2}{*}{$\begin{array}{c}\text { New } \\
\text { Mexlco }\end{array}$} & \multirow[b]{2}{*}{ Total } & & & \\
\hline & & & & & & & Rocky Mt. & West Coast & \\
\hline & & & & & Net Productlo & & & & \\
\hline \multirow[t]{2}{*}{ 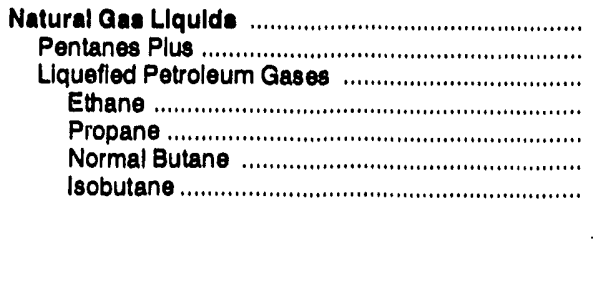 } & $\begin{array}{r}19,305 \\
3,303 \\
16,002 \\
6,716 \\
5,856 \\
2,431 \\
999\end{array}$ & $\begin{array}{r}3,704 \\
671 \\
3,033 \\
1,646 \\
869 \\
-1,736 \\
2,254\end{array}$ & $\begin{array}{r}7,922 \\
1,301 \\
6,621 \\
3,147 \\
2,143 \\
514 \\
817\end{array}$ & $\begin{array}{r}641 \\
191 \\
450 \\
100 \\
182 \\
123 \\
45\end{array}$ & $\begin{array}{r}4,931 \\
600 \\
4,331 \\
2,080 \\
1,439 \\
525 \\
287\end{array}$ & $\begin{array}{r}36,503 \\
6,066 \\
30,437 \\
13,689 \\
10,489 \\
1,857 \\
4,402\end{array}$ & $\begin{array}{r}4,628 \\
1,011 \\
3,617 \\
1,405 \\
1,388 \\
497 \\
327\end{array}$ & $\begin{array}{r}3,280 \\
1,678 \\
1,602 \\
2 \\
316 \\
811 \\
473\end{array}$ & $\begin{array}{r}\mathbf{5 5 , 7 7 1} \\
10,219 \\
45,552 \\
18,701 \\
16,392 \\
4,431 \\
6,028\end{array}$ \\
\hline & \multicolumn{9}{|c|}{ Stocks } \\
\hline 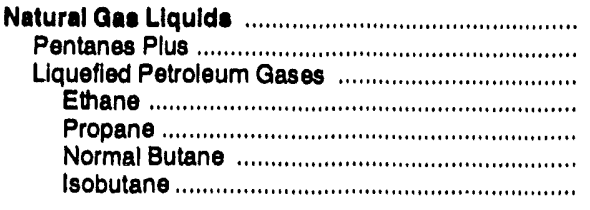 & $\begin{array}{r}409 \\
151 \\
258 \\
46 \\
150 \\
53 \\
9\end{array}$ & $\begin{array}{r}1,914 \\
316 \\
1,598 \\
580 \\
508 \\
334 \\
176\end{array}$ & $\begin{array}{r}882 \\
238 \\
644 \\
9 \\
299 \\
213 \\
123\end{array}$ & $\begin{array}{r}134 \\
17 \\
117 \\
81 \\
15 \\
15 \\
6\end{array}$ & $\begin{array}{r}103 \\
20 \\
83 \\
24 \\
39 \\
14 \\
6\end{array}$ & $\begin{array}{r}3,442 \\
742 \\
2,700 \\
740 \\
1,011 \\
629 \\
320\end{array}$ & $\begin{array}{r}238 \\
66 \\
172 \\
6 \\
92 \\
59 \\
15\end{array}$ & $\begin{array}{r}76 \\
16 \\
60 \\
0 \\
32 \\
11 \\
17\end{array}$ & $\begin{array}{r}6,029 \\
1,023 \\
5,006 \\
1,442 \\
1,950 \\
1,137 \\
477\end{array}$ \\
\hline
\end{tabular}

Note: Refer to Appendix A for Refining District descriptions.

Source: Energy Information Administration (EIA) Form ElA-816, "Monthly Natural Gas Llquids Report." 
Table 28. Refinery Input of Crude Oll and Petroleum Products by PAD and Refining Districts, March 1993

(Thousand Barrels, Except Where Noted)

\begin{tabular}{|c|c|c|c|c|c|c|c|}
\hline \multirow[b]{2}{*}{ Commodity } & \multicolumn{3}{|c|}{ PAD Diatrict I } & \multicolumn{4}{|c|}{ PAD District II } \\
\hline & $\begin{array}{l}\text { East } \\
\text { Const }\end{array}$ & $\begin{array}{c}\text { Appalachian } \\
\text { No. } 1\end{array}$ & Total & Ind., III., Ky. & N. Minn., Wis.s. & $\begin{array}{c}\text { Okla., Kan8., } \\
\text { Mo. }\end{array}$ & Total \\
\hline 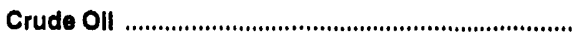 & 31,893 & 3,037 & 34,930 & 62,891 & 10,720 & 20,991 & 94,602 \\
\hline 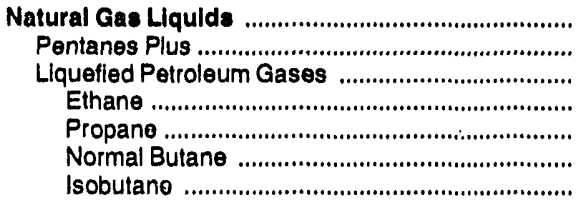 & $\begin{array}{r}264 \\
0 \\
264 \\
0 \\
0 \\
129 \\
135\end{array}$ & $\begin{array}{l}0 \\
0 \\
0 \\
0 \\
0 \\
0 \\
0\end{array}$ & $\begin{array}{r}264 \\
0 \\
264 \\
0 \\
0 \\
129 \\
135\end{array}$ & $\begin{array}{r}1,308 \\
92 \\
1,216 \\
0 \\
0 \\
306 \\
910\end{array}$ & $\begin{array}{r}438 \\
16 \\
422 \\
0 \\
0 \\
314 \\
108\end{array}$ & $\begin{array}{r}1,003 \\
678 \\
325 \\
0 \\
0 \\
10 \\
315\end{array}$ & $\begin{array}{r}2,749 \\
786 \\
1,963 \\
0 \\
0 \\
630 \\
1,333\end{array}$ \\
\hline 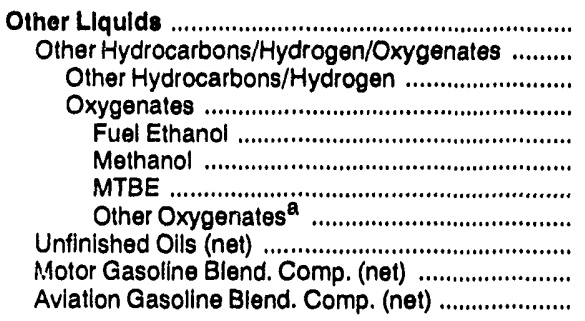 & $\begin{array}{r}6,150 \\
1,029 \\
0 \\
W \\
W \\
W \\
W \\
W \\
3,925 \\
1,196 \\
0\end{array}$ & $\begin{array}{r}94 \\
2 \\
2 \\
W \\
W \\
W \\
W \\
W \\
90 \\
2 \\
0\end{array}$ & $\begin{array}{r}6,244 \\
1,031 \\
2 \\
1,029 \\
W \\
W \\
993 \\
W \\
4,015 \\
1,198 \\
0\end{array}$ & $\begin{array}{r}682 \\
183 \\
45 \\
138 \\
W \\
W \\
W \\
W \\
705 \\
-202 \\
-4\end{array}$ & $\begin{array}{r}149 \\
107 \\
0 \\
107 \\
W \\
W \\
W \\
W \\
9 \\
33 \\
0\end{array}$ & $\begin{array}{r}-1,808 \\
14 \\
0 \\
14 \\
W \\
W \\
W \\
W \\
-1,799 \\
-23 \\
0\end{array}$ & $\begin{array}{r}-977 \\
304 \\
45 \\
259 \\
160 \\
W \\
W \\
W \\
-1,085 \\
-192 \\
-4\end{array}$ \\
\hline Total Input to Refinerles .................................. & 38,307 & 3,131 & 41,438 & 64,881 & 11,307 & 20,186 & 96,374 \\
\hline 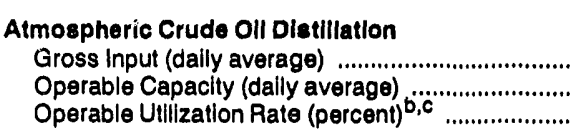 & $\begin{array}{r}1,039 \\
1,442 \\
72.0\end{array}$ & $\begin{array}{r}98 \\
101 \\
97.1\end{array}$ & $\begin{array}{r}1,137 \\
1,543 \\
73.7\end{array}$ & $\begin{array}{r}2,093 \\
2,324 \\
90.1\end{array}$ & $\begin{array}{r}345 \\
358 \\
96.2\end{array}$ & $\begin{array}{r}i \\
688 \\
724 \\
95.1\end{array}$ & $\begin{array}{r}3,126 \\
3,406 \\
91.8\end{array}$ \\
\hline 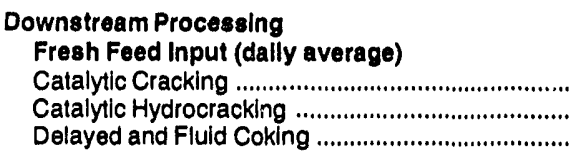 & $\begin{array}{r}557 \\
22 \\
84\end{array}$ & $\begin{array}{r}18 \\
4 \\
0\end{array}$ & $\begin{array}{r}576 \\
26 \\
84\end{array}$ & $\begin{array}{l}712 \\
123 \\
138\end{array}$ & $\begin{array}{r}116 \\
0 \\
54\end{array}$ & $\begin{array}{r}192 \\
3 \\
66\end{array}$ & $\begin{array}{r}1,021 \\
126 \\
258\end{array}$ \\
\hline $\begin{array}{l}\text { Crude Oll Qualities } \\
\text { Sulfur Content, Weighted Average (percent) ......... } \\
\text { API Gravity, Weighted Average (degrees) ............. }\end{array}$ & $\begin{array}{r}0.81 \\
31.46\end{array}$ & $\begin{array}{r}0.80 \\
37.50\end{array}$ & $\begin{array}{r}0.81 \\
31.98\end{array}$ & $\begin{array}{r}1.08 \\
33.83\end{array}$ & $\begin{array}{r}1.71 \\
30.73\end{array}$ & $\begin{array}{r}0.68 \\
36.27\end{array}$ & $\begin{array}{r}1.06 \\
34.01\end{array}$ \\
\hline 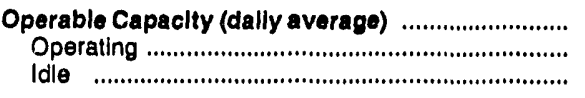 & $\begin{array}{r}1,442 \\
1,372 \\
70\end{array}$ & $\begin{array}{r}101 \\
101 \\
0\end{array}$ & $\begin{array}{r}1,543 \\
1,473 \\
70\end{array}$ & $\begin{array}{r}2,324 \\
2,315 \\
9\end{array}$ & $\begin{array}{r}358 \\
358 \\
0\end{array}$ & $\begin{array}{r}724 \\
693 \\
30\end{array}$ & $\begin{array}{r}3,406 \\
3,367 \\
40\end{array}$ \\
\hline 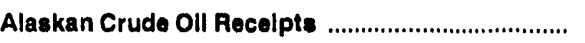 & 0 & 0 & 0 & 745 & 0 & 0 & 746 \\
\hline
\end{tabular}

See footnotes at end of table. 
Table 28. Refinery Input of Crude Oll and Petroleum Products by PAD and Refining Districts, March 1993 (Contlnued)

(Thousand Barrels, Except Where Noted)

\begin{tabular}{|c|c|c|c|c|c|c|c|c|c|}
\hline \multirow{3}{*}{ Commodity } & \multicolumn{6}{|c|}{ PAD Dletilct III } & \multirow{3}{*}{\begin{tabular}{|c|}
$\begin{array}{c}\text { PAD Dlat. } \\
\text { IV }\end{array}$ \\
Rocky Mt. \\
\end{tabular}} & \multirow{3}{*}{\begin{tabular}{|c|}
$\begin{array}{c}\text { PAD Dist. } \\
V\end{array}$ \\
West Coast
\end{tabular}} & \multirow{3}{*}{ U.S. } \\
\hline & \multirow{2}{*}{$\begin{array}{l}\text { Texas } \\
\text { Inland }\end{array}$} & \multirow{2}{*}{$\begin{array}{l}\text { Toxas } \\
\text { Gulf } \\
\text { Coast }\end{array}$} & \multirow{2}{*}{$\begin{array}{l}\text { La. } \\
\text { Couif } \\
\text { Coast }\end{array}$} & \multirow{2}{*}{$\begin{array}{l}\text { N. La., } \\
\text { Ark. }\end{array}$} & \multirow{2}{*}{$\begin{array}{c}\text { New } \\
\text { Mexlco }\end{array}$} & \multirow[b]{2}{*}{ Total } & & & \\
\hline & & & & & & & & & \\
\hline Crude Oll & 14,565 & 92,144 & 74,511 & $\mathbf{5 , 5 8 4}$ & 2,476 & 189,280 & 13,619 & 78,290 & 410,721 \\
\hline 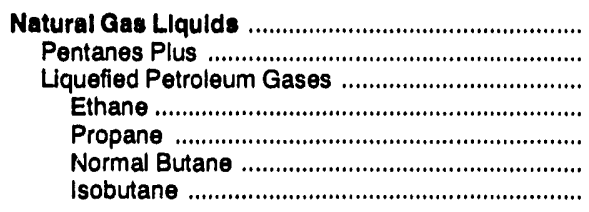 & $\begin{array}{r}1,134 \\
564 \\
570 \\
0 \\
0 \\
432 \\
138\end{array}$ & $\begin{array}{r}2,818 \\
1,138 \\
1,780 \\
0 \\
1 \\
276 \\
1,503\end{array}$ & $\begin{array}{r}2,023 \\
390 \\
1,633 \\
0 \\
0 \\
636 \\
997\end{array}$ & $\begin{array}{r}141 \\
121 \\
20 \\
0 \\
0 \\
1 \\
19\end{array}$ & $\begin{array}{r}251 \\
137 \\
114 \\
0 \\
0 \\
12 \\
102\end{array}$ & $\begin{array}{r}6,467 \\
2,350 \\
4,117 \\
0 \\
1 \\
1,357 \\
2,759\end{array}$ & $\begin{array}{r}508 \\
191 \\
317 \\
0 \\
0 \\
204 \\
113\end{array}$ & $\begin{array}{r}2,779 \\
1,278 \\
1,501 \\
0 \\
0 \\
882 \\
619\end{array}$ & $\begin{array}{r}12,767 \\
4,605 \\
8,162 \\
0 \\
1 \\
3,202 \\
4,959\end{array}$ \\
\hline 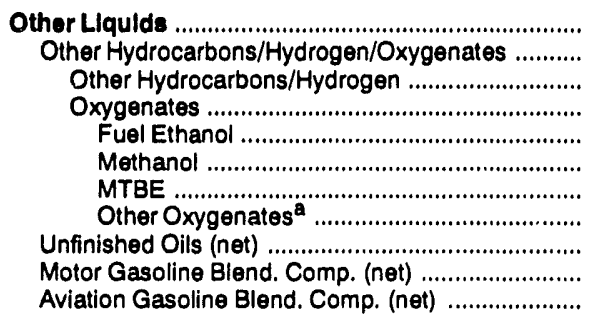 & $\begin{array}{r}205 \\
148 \\
124 \\
24 \\
W \\
W \\
W \\
W \\
-36 \\
93 \\
0\end{array}$ & $\begin{array}{r}9,329 \\
1,259 \\
174 \\
1,085 \\
W \\
W \\
985 \\
W \\
7,914 \\
157 \\
-1\end{array}$ & $\begin{array}{r}3,278 \\
928 \\
481 \\
447 \\
W \\
W \\
W \\
1 \\
2,899 \\
-546 \\
-3\end{array}$ & $\begin{array}{r}-236 \\
8 \\
0 \\
W \\
W \\
W \\
W \\
W \\
-270 \\
26 \\
0\end{array}$ & $\begin{array}{r}136 \\
17 \\
0 \\
W \\
W \\
W \\
W \\
W \\
-46 \\
165 \\
0\end{array}$ & $\begin{array}{r}12,712 \\
2,360 \\
779 \\
1,581 \\
W \\
W \\
1,342 \\
W \\
10,461 \\
-105 \\
.4\end{array}$ & $\begin{array}{r}-461 \\
65 \\
0 \\
65 \\
W \\
W \\
W \\
W \\
.416 \\
-110 \\
0\end{array}$ & $\begin{array}{r}1,027 \\
683 \\
260 \\
423 \\
W \\
W \\
346 \\
W \\
68 \\
267 \\
9\end{array}$ & $\begin{array}{r}18,545 \\
4,443 \\
1,086 \\
3,357 \\
259 \\
240 \\
2,780 \\
78 \\
13,043 \\
1,058 \\
1\end{array}$ \\
\hline 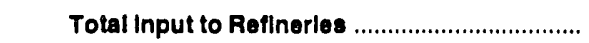 & 15,904 & 104,391 & 79,812 & 5,489 & เ. 2,863 & 208,459 & 13,666 & 82,096 & 442,033 \\
\hline Atmospherlc Crude Oll Distillation & & & & & : & & & & \\
\hline 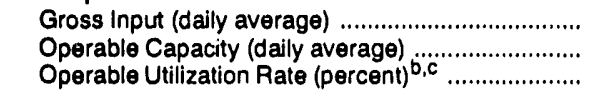 & $\begin{array}{r}474 \\
525 \\
90.3\end{array}$ & $\begin{array}{r}3,002 \\
3,233 \\
92.9\end{array}$ & $\begin{array}{r}2,452 \\
2,663 \\
92.1\end{array}$ & $\begin{array}{r}176 \\
249 \\
71.0\end{array}$ & $\begin{array}{r}80 \\
98 \\
81.8\end{array}$ & $\begin{array}{r}6,185 \\
6,767 \\
91.4\end{array}$ & $\begin{array}{r}446 \\
519 \\
85.8\end{array}$ & $\begin{array}{r}2,642 \\
2,912 \\
90.7\end{array}$ & $\begin{array}{r}13,535 \\
15,148 \\
89.4\end{array}$ \\
\hline $\begin{array}{l}\text { Downstream Processing } \\
\text { Fresh Food Input (dally average) }\end{array}$ & & & & & & & & & \\
\hline 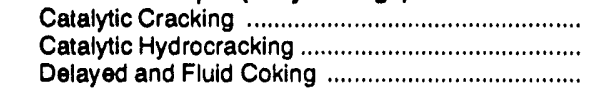 & $\begin{array}{r}166 \\
22 \\
5\end{array}$ & $\begin{array}{r}1,275 \\
189 \\
277\end{array}$ & $\begin{array}{l}852 \\
215 \\
371\end{array}$ & $\begin{array}{r}7 \\
0 \\
11\end{array}$ & $\begin{array}{r}23 \\
0 \\
0\end{array}$ & $\begin{array}{r}2,324 \\
426 \\
664\end{array}$ & $\begin{array}{r}144 \\
11 \\
29\end{array}$ & $\begin{array}{l}719 \\
434 \\
469\end{array}$ & $\begin{array}{l}4,783 \\
1,022 \\
1,503\end{array}$ \\
\hline Crude Oll Quallties & & & & & & & & & \\
\hline $\begin{array}{l}\text { Sulfur Content, Weighted Average (percent) ......... } \\
\text { API Gravity, Weighted Average (degrees) ............. }\end{array}$ & $\begin{array}{r}0.98 \\
39.31\end{array}$ & $\begin{array}{r}1.11 \\
32.25\end{array}$ & $\begin{array}{r}1.25 \\
31.45\end{array}$ & $\begin{array}{r}1.42 \\
32.70\end{array}$ & $\begin{array}{r}1.20 \\
37.02\end{array}$ & $\begin{array}{r}1.17 \\
32.56\end{array}$ & $\begin{array}{r}1.18 \\
34.93\end{array}$ & $\begin{array}{r}1.12 \\
24.86\end{array}$ & $\begin{array}{r}1.10 \\
31.40\end{array}$ \\
\hline $\begin{array}{l}\text { Operable Capaclty (dally average) } \\
\text { Operating } \\
\text { Idle }\end{array}$ & $\begin{array}{r}\mathbf{5 2 5} \\
\mathbf{5 2 5} \\
0\end{array}$ & $\begin{array}{r}3,233 \\
3,206 \\
27\end{array}$ & $\begin{array}{r}2,663 \\
2,663 \\
0\end{array}$ & $\begin{array}{r}249 \\
249 \\
0\end{array}$ & $\begin{array}{r}98 \\
98 \\
0\end{array}$ & $\begin{array}{r}6,767 \\
6,740 \\
27\end{array}$ & $\begin{array}{r}519 \\
509 \\
10\end{array}$ & $\begin{array}{r}2,912 \\
2,819 \\
93\end{array}$ & $\begin{array}{r}15,148 \\
14,908 \\
239\end{array}$ \\
\hline 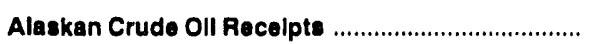 & 86 & 1,123 & 0 & 8 & 0 & 1,227 & 0 & 45,426 & 47,398 \\
\hline
\end{tabular}

a Includes ethyl tertiary butyl ether (ETBE), tertiary amyl methyl ether (TAME), tertiary butyl alcohol (TBA), and other aliphatic alcohols and ethers intended for motor gasoline blending (e.g., isopropyl ether (IPE) or n-propanol).

b Represents gross input divided by operable capacity.

c See Table $\mathrm{H} 2$ in the Highlights Section for additional information concerning utilization rates.

$W=$ Withheld to avoid disciosure of individual company data.

Note: - Totals may not equal sum of components due to independent rounding. - Refer to Appendix A for Refining District descriptions.

Source: Energy Information Administration (EIA) Fom ElA-810, "Monthly Refinery Report." 
Table 29. Refinery Net Production of Finlshed Petroleum Products by PAD and Refining Districts, March 1993

(Thousand Barrels)

\begin{tabular}{|c|c|c|c|c|c|c|c|}
\hline \multirow[b]{2}{*}{ Commodity } & \multicolumn{3}{|c|}{ PAD DIstrlct I } & \multicolumn{4}{|c|}{ PAD District II } \\
\hline & $\begin{array}{l}\text { East } \\
\text { Const }\end{array}$ & $\begin{array}{c}\text { Appalachlan } \\
\text { No. } 1\end{array}$ & Total & Ind., III., Ky. & $\begin{array}{c}\text { MInn., Wis., } \\
\text { N. Dak., } \\
\text { S. Dak. }\end{array}$ & $\begin{array}{c}\text { Okla. Kans., } \\
\text { Mo. }\end{array}$ & Total \\
\hline 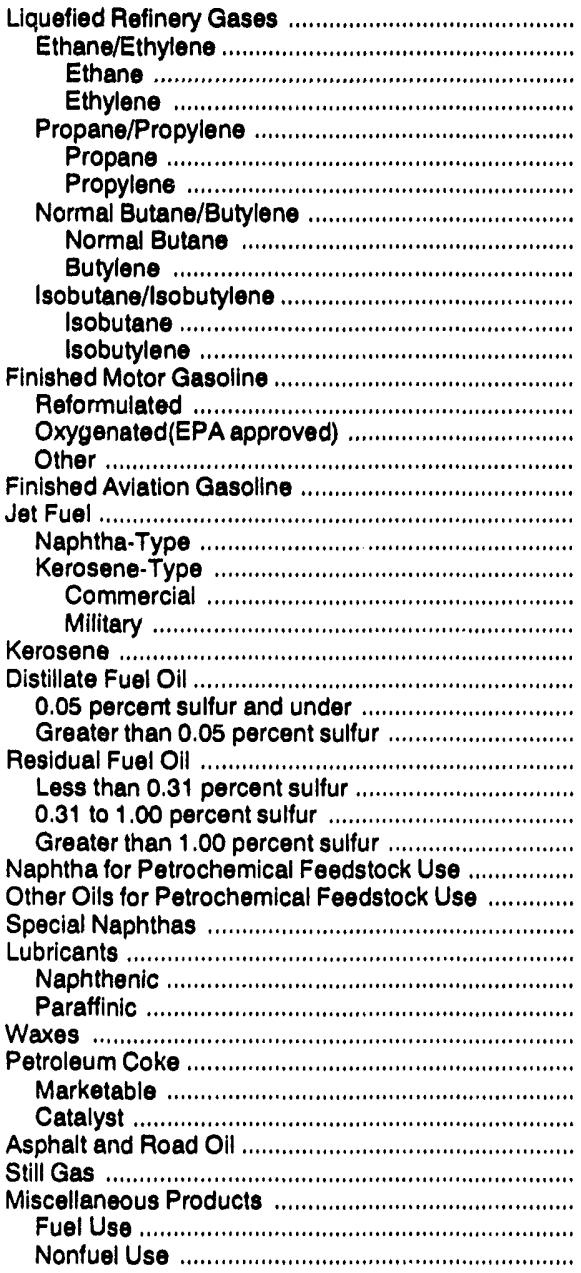 & $\begin{array}{r}1,333 \\
0 \\
W \\
W \\
1,232 \\
W \\
W \\
194 \\
W \\
W \\
.93 \\
W \\
W \\
19,785 \\
0 \\
5,168 \\
14,617 \\
1 \\
2,251 \\
182 \\
2,069 \\
1,905 \\
164 \\
71 \\
9,515 \\
825 \\
8,690 \\
2,696 \\
403 \\
2,156 \\
137 \\
420 \\
10 \\
0 \\
102 \\
0 \\
102 \\
0 \\
1,567 \\
648 \\
919 \\
1,316 \\
1,456 \\
16 \\
0 \\
16\end{array}$ & $\begin{array}{r}-3 \\
0 \\
W \\
W \\
32 \\
W \\
W \\
-32 \\
W \\
W \\
-3 \\
W \\
W \\
1,237 \\
0 \\
0 \\
1,237 \\
0 \\
0 \\
0 \\
0 \\
0 \\
0 \\
93 \\
860 \\
277 \\
583 \\
84 \\
27 \\
57 \\
0 \\
0 \\
0 \\
14 \\
264 \\
0 \\
264 \\
130 \\
23 \\
0 \\
23 \\
263 \\
136 \\
38 \\
0 \\
38\end{array}$ & $\begin{array}{r}1,330 \\
0 \\
W \\
W \\
1,264 \\
W \\
W \\
162 \\
W \\
W \\
-96 \\
W \\
W \\
21,022 \\
0 \\
5,168 \\
15,854 \\
1 \\
2,251 \\
182 \\
2,069 \\
1,905 \\
164 \\
164 \\
10,375 \\
1,102 \\
9,273 \\
2,780 \\
430 \\
2,213 \\
137 \\
420 \\
10 \\
14 \\
366 \\
0 \\
366 \\
130 \\
1,590 \\
648 \\
942 \\
1,579 \\
1,592 \\
54 \\
0 \\
54\end{array}$ & $\begin{array}{r}2,895 \\
0 \\
W \\
W \\
2,265 \\
W \\
W \\
464 \\
W \\
W \\
166 \\
W \\
W \\
32,866 \\
0 \\
860 \\
32,006 \\
39 \\
3,959 \\
161 \\
3,798 \\
3,720 \\
78 \\
714 \\
14,362 \\
401 \\
13,961 \\
1,949 \\
0 \\
352 \\
1,597 \\
637 \\
724 \\
352 \\
618 \\
0 \\
618 \\
35 \\
2,361 \\
1,347 \\
1,014 \\
3,465 \\
2,643 \\
281 \\
0 \\
281\end{array}$ & $\begin{array}{r}299 \\
0 \\
W \\
W \\
257 \\
W \\
W \\
20 \\
W \\
W \\
22 \\
W \\
W \\
5,945 \\
0 \\
9 \\
5,936 \\
40 \\
826 \\
23 \\
803 \\
803 \\
0 \\
66 \\
2,753 \\
0 \\
2,753 \\
290 \\
0 \\
0 \\
290 \\
0 \\
0 \\
0 \\
0 \\
0 \\
0 \\
0 \\
566 \\
405 \\
161 \\
712 \\
362 \\
46 \\
0 \\
46\end{array}$ & $\begin{array}{r}613 \\
0 \\
W \\
W \\
548 \\
W \\
W \\
127 \\
W \\
W \\
-62 \\
W \\
W \\
10,095 \\
0 \\
1,668 \\
8,427 \\
0 \\
1,728 \\
299 \\
1,429 \\
1,429 \\
0 \\
69 \\
5,322 \\
24 \\
5,298 \\
157 \\
32 \\
8 \\
117 \\
8 \\
33 \\
116 \\
233 \\
0 \\
233 \\
35 \\
844 \\
614 \\
230 \\
705 \\
990 \\
62 \\
0 \\
62\end{array}$ & $\begin{array}{r}3,807 \\
0 \\
W \\
W \\
3,070 \\
W \\
W \\
611 \\
W \\
W \\
126 \\
W \\
W \\
48,906 \\
0 \\
2,537 \\
46,369 \\
79 \\
6,513 \\
483 \\
6,030 \\
5,952 \\
78 \\
849 \\
22,437 \\
425 \\
22,012 \\
2,396 \\
32 \\
360 \\
2,004 \\
645 \\
757 \\
468 \\
851 \\
0 \\
851 \\
70 \\
3,771 \\
2,366 \\
1,405 \\
4,882 \\
3,995 \\
389 \\
0 \\
389\end{array}$ \\
\hline Total & 40,539 & 3,139 & 43,678 & 67,900 & 11,908 & 21,010 & 100,815 \\
\hline 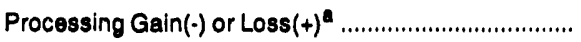 & $-2,232$ & -8 & $-2,240$ & $-3,019$ & -598 & -824 & $-4,441$ \\
\hline
\end{tabular}

See footnotes at end of table. 
Table 29. Refinery Net Production of Finished Petroleum Products by PAD and Refining Districts, March 1993 (Continued)

(Thousand Barrels)

\begin{tabular}{|c|c|c|c|c|c|c|c|c|c|}
\hline \multirow[b]{2}{*}{ Commodity } & \multicolumn{6}{|c|}{ PAD Distrlct III } & \multirow{2}{*}{\begin{tabular}{|c|}
$\begin{array}{c}\text { PAD Dlst. } \\
\text { IV }\end{array}$ \\
Rocky Mt.
\end{tabular}} & \multirow{2}{*}{\begin{tabular}{|c|}
$\begin{array}{c}\text { PAD Dlst. } \\
V\end{array}$ \\
West Coast \\
\end{tabular}} & \multirow[b]{2}{*}{ U.S. } \\
\hline & $\begin{array}{l}\text { Toxas } \\
\text { Inland }\end{array}$ & $\begin{array}{c}\text { Toxas } \\
\text { Qulf } \\
\text { Coast }\end{array}$ & $\begin{array}{c}\text { La. } \\
\text { Gulf } \\
\text { Coast }\end{array}$ & $\begin{array}{l}\text { N. La., } \\
\text { Ark. }\end{array}$ & $\begin{array}{c}\text { New } \\
\text { Mexlco }\end{array}$ & Total & & & \\
\hline 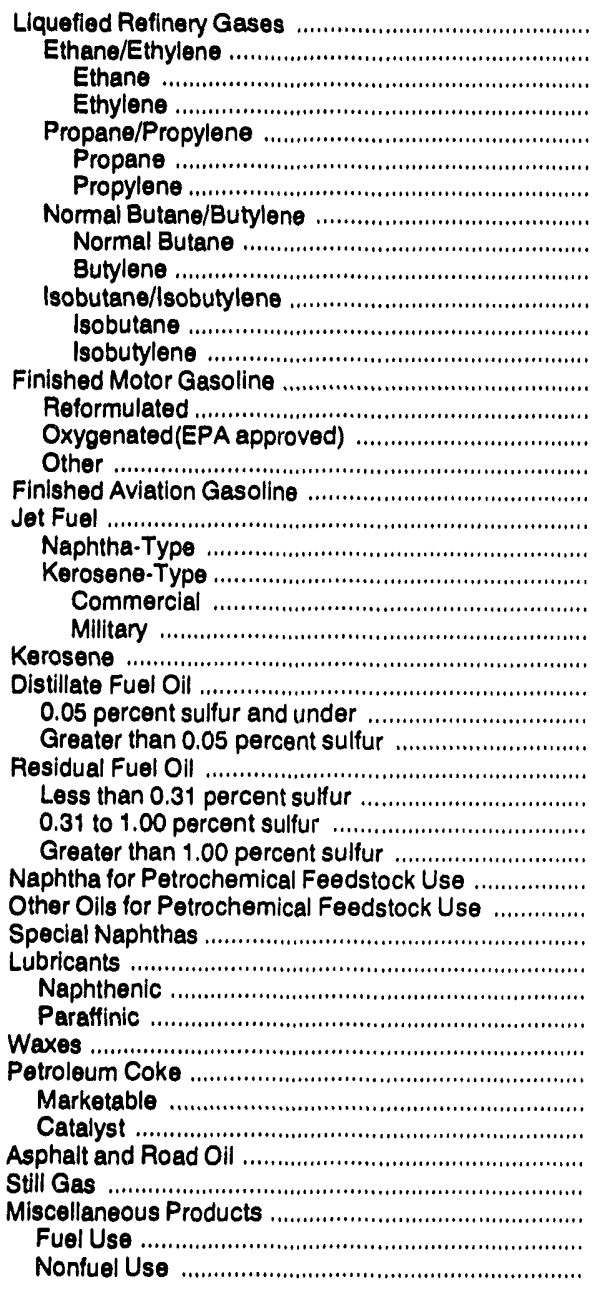 & $\begin{array}{r}709 \\
2 \\
W \\
W \\
588 \\
W \\
W \\
61 \\
W \\
W \\
58 \\
W \\
W \\
8,682 \\
0 \\
37 \\
8,645 \\
192 \\
1,485 \\
326 \\
1,159 \\
1,017 \\
142 \\
4 \\
3,363 \\
8 \\
3,355 \\
386 \\
107 \\
161 \\
118 \\
89 \\
123 \\
70 \\
40 \\
40 \\
0 \\
9 \\
322 \\
35 \\
287 \\
242 \\
623 \\
56 \\
12 \\
44\end{array}$ & $\begin{array}{r}7,309 \\
541 \\
W \\
W \\
4,577 \\
W \\
W \\
2,102 \\
W \\
W \\
89 \\
W \\
W \\
49,071 \\
0 \\
3,052 \\
46,019 \\
152 \\
10,802 \\
714 \\
10,088 \\
9,152 \\
936 \\
131 \\
18,054 \\
696 \\
17,358 \\
5,498 \\
4 \\
923 \\
4,571 \\
2,653 \\
5,234 \\
762 \\
1,780 \\
336 \\
1,444 \\
146 \\
3,930 \\
2,075 \\
1,855 \\
775 \\
4,907 \\
329 \\
0 \\
329\end{array}$ & $\begin{array}{r}3,255 \\
291 \\
W \\
W \\
2,564 \\
W \\
W \\
351 \\
W \\
W \\
49 \\
W \\
W \\
36,085 \\
0 \\
1,403 \\
34,682 \\
78 \\
9,456 \\
693 \\
8,763 \\
8,552 \\
211 \\
248 \\
17,277 \\
2,033 \\
15,244 \\
4,248 \\
560 \\
557 \\
3,131 \\
317 \\
3,322 \\
222 \\
804 \\
0 \\
804 \\
73 \\
4,359 \\
3,162 \\
1,197 \\
1,061 \\
3,063 \\
266 \\
9 \\
257\end{array}$ & $\begin{array}{r}49 \\
-2 \\
W \\
W \\
25 \\
W \\
W \\
18 \\
W \\
W \\
8 \\
W \\
W \\
1,287 \\
0 \\
6 \\
1,281 \\
0 \\
371 \\
194 \\
177 \\
177 \\
0 \\
13 \\
1,404 \\
80 \\
1,324 \\
275 \\
63 \\
202 \\
10 \\
0 \\
0 \\
152 \\
520 \\
397 \\
123 \\
72 \\
116 \\
99 \\
17 \\
1,035 \\
178 \\
0 \\
0 \\
0\end{array}$ & $\begin{array}{r}70 \\
0 \\
W \\
W \\
52 \\
W \\
W \\
18 \\
W \\
W \\
0 \\
W \\
W \\
1,603 \\
0 \\
156 \\
1,447 \\
0 \\
310 \\
291 \\
19 \\
19 \\
0 \\
-4 \\
669 \\
0 \\
669 \\
9 \\
7 \\
2 \\
0 \\
5 \\
0 \\
0 \\
0 \\
0 \\
0 \\
0 \\
15 \\
0 \\
15 \\
53 \\
0 \\
0 \\
0\end{array}$ & $\begin{array}{r}11,392 \\
832 \\
W \\
W \\
7,806 \\
W \\
W \\
2,550 \\
W \\
W \\
204 \\
W \\
W \\
96,728 \\
0 \\
4,654 \\
92,074 \\
422 \\
22,424 \\
2,218 \\
20,206 \\
18,917 \\
1,289 \\
392 \\
40,767 \\
2,817 \\
37,950 \\
10,416 \\
741 \\
1,845 \\
7,830 \\
3,064 \\
8,679 \\
1,206 \\
3,144 \\
773 \\
2,371 \\
300 \\
8,742 \\
5,371 \\
3,371 \\
3,266 \\
8,830 \\
651 \\
21 \\
630\end{array}$ & $\begin{array}{r}237 \\
0 \\
W \\
W \\
215 \\
W \\
W \\
3 \\
W \\
W \\
19 \\
W \\
W \\
6,705 \\
0 \\
111 \\
6,594 \\
18 \\
1,205 \\
378 \\
827 \\
827 \\
0 \\
42 \\
3,493 \\
334 \\
3,159 \\
485 \\
163 \\
152 \\
170 \\
0 \\
23 \\
0 \\
0 \\
0 \\
0 \\
46 \\
401 \\
224 \\
177 \\
763 \\
612 \\
70 \\
0 \\
70\end{array}$ & $\begin{array}{r}2,975 \\
0 \\
W \\
W \\
1,369 \\
W \\
W \\
1,562 \\
W \\
W \\
44 \\
W \\
W \\
36,961 \\
0 \\
-585 \\
37,546 \\
173 \\
12,961 \\
805 \\
12,156 \\
11,350 \\
806 \\
78 \\
13,400 \\
3,191 \\
10,209 \\
9,302 \\
1,207 \\
1,034 \\
7,061 \\
-95 \\
256 \\
69 \\
748 \\
283 \\
465 \\
77 \\
4,625 \\
3,507 \\
1,118 \\
1,069 \\
4,275 \\
227 \\
0 \\
227\end{array}$ & $\begin{array}{r}19,741 \\
832 \\
609 \\
223 \\
13,724 \\
9,634 \\
4,090 \\
4,888 \\
4,845 \\
43 \\
297 \\
297 \\
0 \\
210,322 \\
0 \\
11,885 \\
198,437 \\
693 \\
45,354 \\
4,066 \\
41,288 \\
38,951 \\
2,337 \\
1,525 \\
90,472 \\
7,869 \\
82,603 \\
25,379 \\
2,573 \\
5,604 \\
17,202 \\
4,034 \\
9,725 \\
1,757 \\
5,109 \\
1,056 \\
4,053 \\
623 \\
19,129 \\
12,116 \\
7,013 \\
11,559 \\
19,304 \\
1,391 \\
21 \\
1,370\end{array}$ \\
\hline 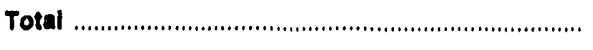 & 16,395 & 111,533 & 84,134 & 5,472 & 2,889 & 220,423 & 14,100 & 87,101 & 466,117 \\
\hline 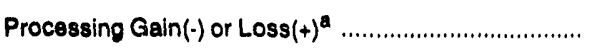 & .491 & $-7,142$ & $-4,322$ & 17 & .26 & $-11,964$ & .434 & $-5,005$ & $.24,084$ \\
\hline
\end{tabular}

- Represents the arithmetic difference between input and production.

$W=$ Withheld to avoid disclosure of individual company data

Note: Refer to Appendix A for refining District descriptions.

Source: Energy Information Administration (EIA) Form EIA-810, "Monthly Refinery Report." 
Table 30. Refinery Stocks of Crude Oll and Petroleum Products by PAD and Refining Districts, March 1993

(Thousand Barrels)

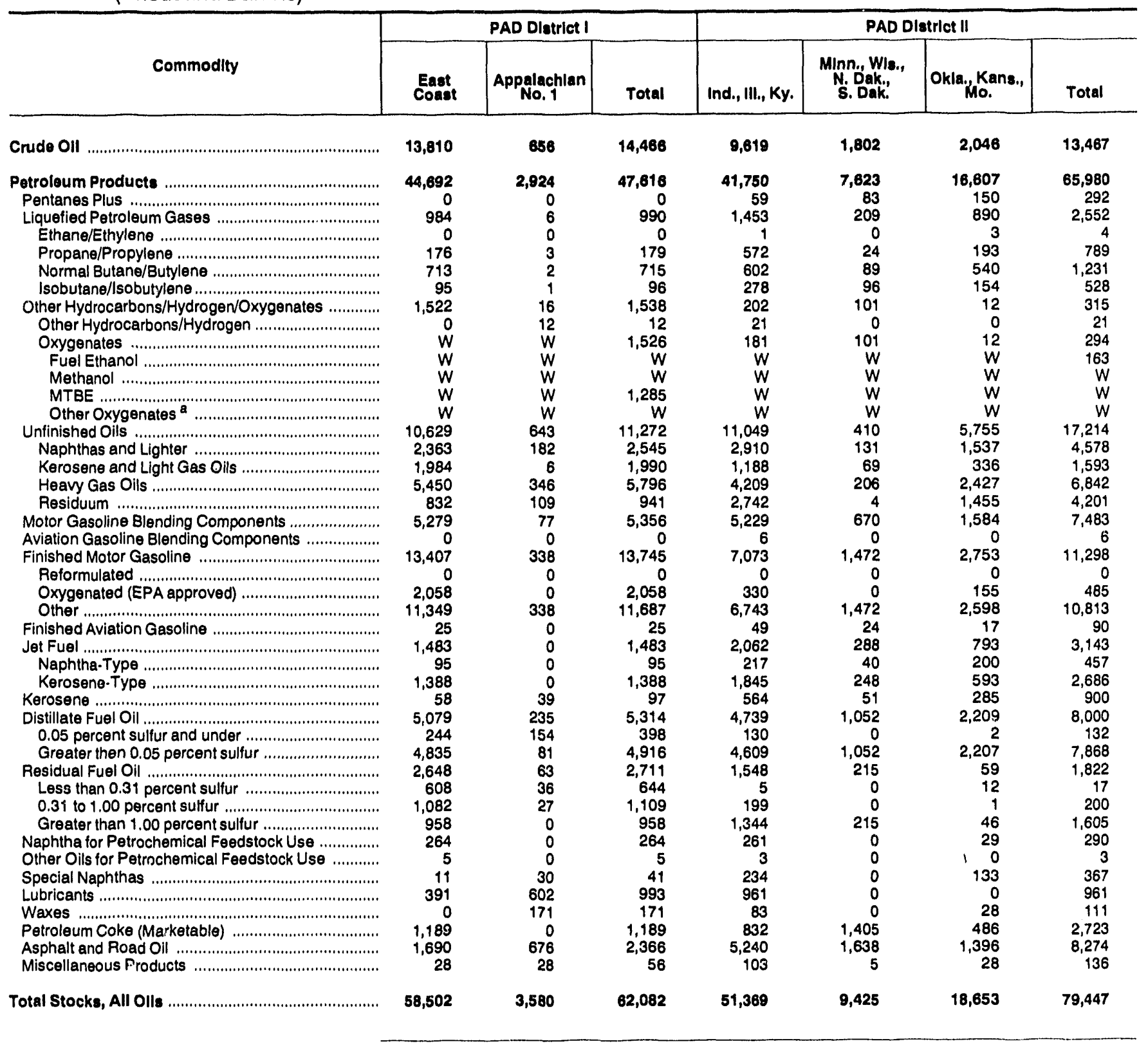

Sge footnotes at end of table. 
Table 30. Refinery Stocks of Crude OII and Petroleum Products by PAD and Refining Districts, March 1993 (Continued) (Thousand Barrels)

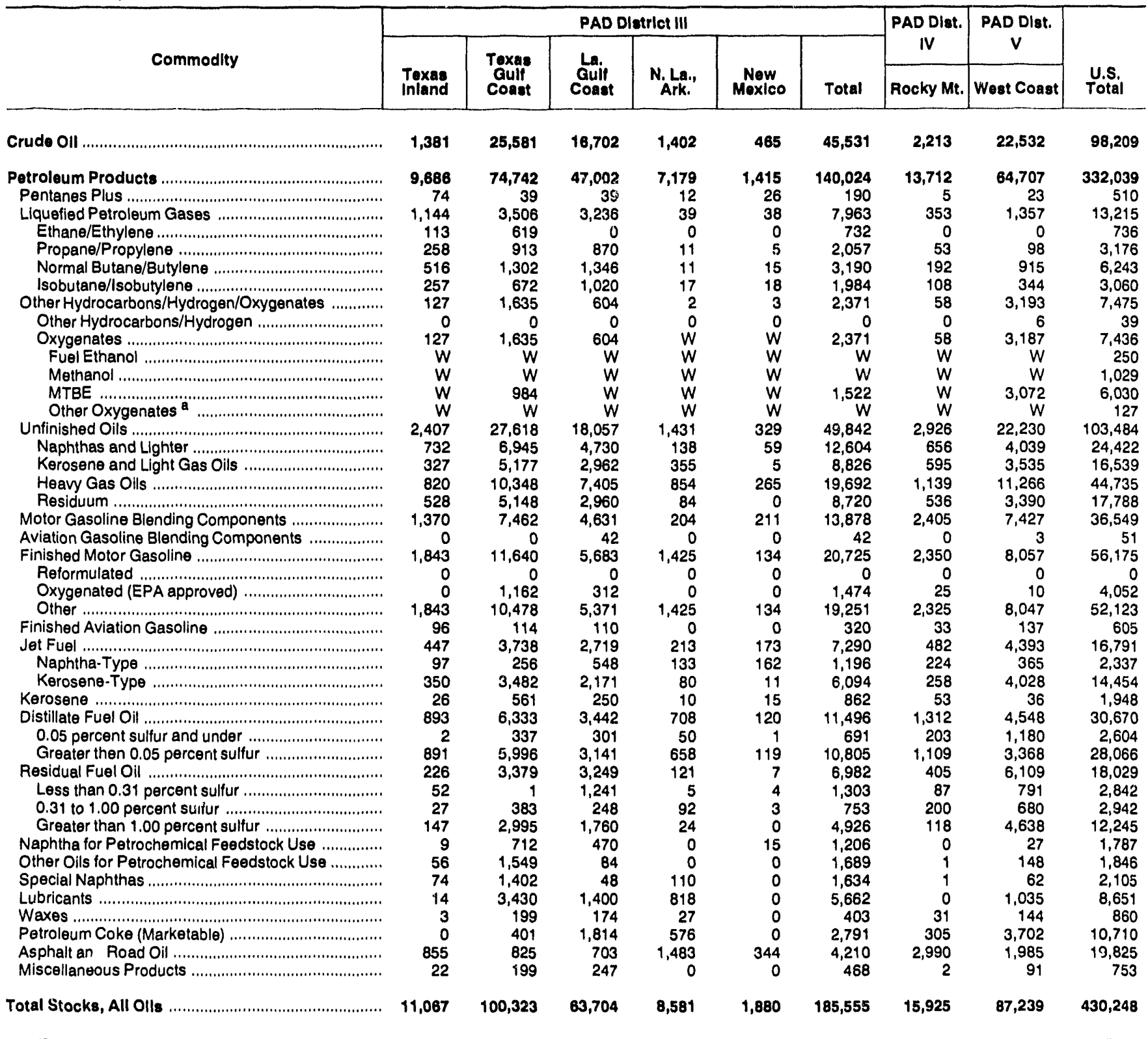

a Includes ethyl tertiary butyl ether (ETBE), tertiary amyl methyl ether (TAME), tertiary butyl alcohol (TBA), and other aliphatic alcohols and ethers intended for motor gasoline blending (e.g., isopropyl ether (IPE) or n-propanol).

$W=$ Withheld to avoid disclosure of individual company data.

Notes: - Stocks are reported as of the last day of the month. - Refer to Appendix A for Refining District descriptions.

Source: Energy Information Administration (EIA) Form EIA-810, "Monthly Refinery Report." 
Table 31. Percent Refinery Yleld of Petroleum Products by PAD ard Refining Districts, ${ }^{\text {a }}$ March 1993

\begin{tabular}{|c|c|c|c|c|c|c|c|}
\hline \multirow[b]{2}{*}{ Commodity } & \multicolumn{3}{|c|}{ PAD Dlatrlct I } & \multicolumn{4}{|c|}{ PAD Dlatrlct II } \\
\hline & $\begin{array}{c}\text { East } \\
\text { Coast }\end{array}$ & $\begin{array}{c}\text { Appalachlan } \\
\text { No. } 1\end{array}$ & Total & Ind., Ill., Ky. & $\begin{array}{l}\text { MInn., Wle., } \\
\text { N. D.ak., } \\
\text { S. Dak. }\end{array}$ & $\begin{array}{c}\text { Okla. Kans., } \\
\text { Mo. }\end{array}$ & Total \\
\hline 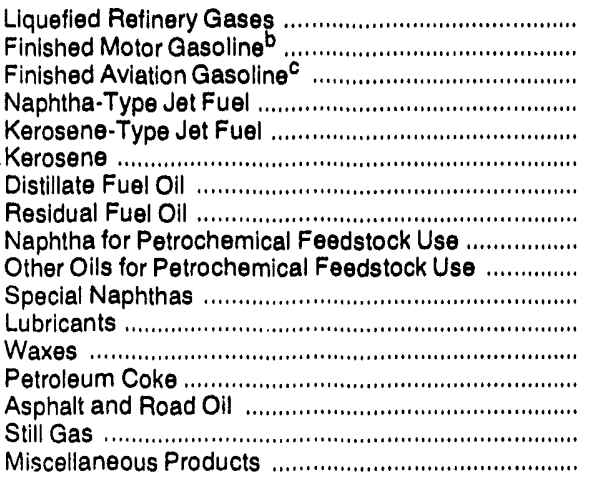 & $\begin{array}{r}3.7 \\
48.3 \\
0.0 \\
0.5 \\
5.8 \\
0.2 \\
26.6 \\
7.5 \\
1.2 \\
0.0 \\
0.0 \\
0.3 \\
0.0 \\
4.4 \\
3.7 \\
4.1 \\
0.0\end{array}$ & $\begin{array}{r}-0.1 \\
39.4 \\
0.0 \\
0.0 \\
0.0 \\
3.0 \\
27.5 \\
2.7 \\
0.0 \\
0.0 \\
0.5 \\
8.4 \\
4.2 \\
0.7 \\
8.4 \\
4.4 \\
1.2\end{array}$ & $\begin{array}{r}3.4 \\
47.6 \\
0.0 \\
0.5 \\
5.3 \\
0.4 \\
26.6 \\
7.1 \\
1.1 \\
0.0 \\
0.0 \\
0.9 \\
0.3 \\
4.1 \\
4.1 \\
4.1 \\
0.1\end{array}$ & $\begin{array}{r}4.6 \\
49.7 \\
0.1 \\
0.3 \\
6.0 \\
1.1 \\
22.6 \\
3.1 \\
1.0 \\
1.1 \\
0.6 \\
1.0 \\
0.1 \\
3.7 \\
5.5 \\
4.2 \\
0.4\end{array}$ & $\begin{array}{r}2.8 \\
50.0 \\
0.4 \\
0.2 \\
7.5 \\
0.6 \\
25.7 \\
2.7 \\
0.0 \\
0.0 \\
0.0 \\
0.0 \\
0.0 \\
5.3 \\
6.6 \\
3.4 \\
0.4\end{array}$ & $\begin{array}{r}3.2 \\
47.4 \\
0.0 \\
1.6 \\
7.5 \\
0.4 \\
27.7 \\
0.8 \\
0.0 \\
0.2 \\
0.6 \\
1.2 \\
0.2 \\
4.4 \\
3.7 \\
5.2 \\
0.3\end{array}$ & $\begin{array}{r}4.1 \\
49.2 \\
0.1 \\
0.5 \\
6.5 \\
0.9 \\
24.0 \\
2.6 \\
0.7 \\
0.8 \\
0.5 \\
0.9 \\
0.1 \\
4.0 \\
5.2 \\
4.3 \\
0.4\end{array}$ \\
\hline 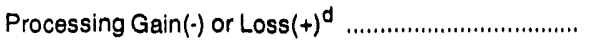 & -6.2 & .0 .3 & .5 .8 & .4 .8 & -5.6 & .4 .3 & -4.8 \\
\hline
\end{tabular}

\begin{tabular}{|c|c|c|c|c|c|c|c|c|c|}
\hline \multirow{3}{*}{ Commodity } & \multicolumn{6}{|c|}{ PAD Distrlct III } & \multirow{3}{*}{\begin{tabular}{|c|}
$\begin{array}{c}\text { PAD Dlst. } \\
\text { IV }\end{array}$ \\
Rocky Mt.
\end{tabular}} & \multirow{3}{*}{\begin{tabular}{|c|}
$\begin{array}{c}\text { PAD Dlst. } \\
\mathrm{V}\end{array}$ \\
Weat Coast
\end{tabular}} & \multirow{3}{*}{$\begin{array}{l}\text { U.S. } \\
\text { Total }\end{array}$} \\
\hline & \multirow[b]{2}{*}{$\begin{array}{l}\text { Texas } \\
\text { Inland }\end{array}$} & \multirow{2}{*}{$\begin{array}{l}\text { Texas } \\
\text { Gulf } \\
\text { Coast }\end{array}$} & \multirow{2}{*}{$\begin{array}{c}\text { La. } \\
\text { Gulf } \\
\text { Coast }\end{array}$} & \multirow{2}{*}{$\begin{array}{c}\text { N. La., } \\
\text { Ark. }\end{array}$} & \multirow{2}{*}{$\begin{array}{l}\text { Now } \\
\text { Mexlco }\end{array}$} & \multirow[b]{2}{*}{ Total } & & & \\
\hline & & & & & & & & & \\
\hline 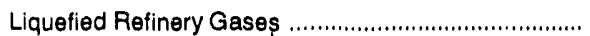 & 4.9 & 7.3 & 4.2 & 0.9 & 2.9 & 5.7 & 1.8 & 3.8 & 4.7 \\
\hline Finished Motor Gasoling & 50.3 & 44.7 & 43.5 & 20.9 & 48.2 & 44.1 & 47.3 & 42.4 & 45.3 \\
\hline Finished Aviation Gasoline ${ }^{c}$ & 1.3 & 0.2 & 0.1 & 0.0 & 0.0 & 0.2 & 0.1 & 0.2 & 0.2 \\
\hline Naphtha-Type Jet Fuel .................................................. & 2.2 & 0.7 & 0.9 & 3.7 & 12.0 & 1.1 & 2.9 & 1.0 & 1.0 \\
\hline 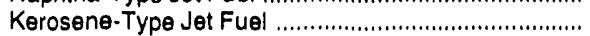 & 8.0 & 10.1 & 11.3 & 3.3 & 0.8 & 10.1 & 6.3 & 15.5 & 9.7 \\
\hline Kerosene & 0.0 . & 0.1 & 0.3 & 0.2 & .0 .2 & 0.2 & 0.3 & 0.1 & 0.4 \\
\hline 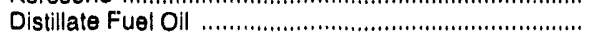 & 23.2 & 18.0 & 22.3 & 26.4 & 27.5 & 20.4 & 26.5 & 17.1 & 21.4 \\
\hline 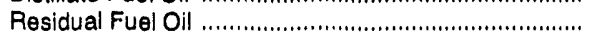 & 2.7 & 5.5 & 5.5 & 5.2 & 0.4 & 5.2 & 3.7 & 11.9 & 6.0 \\
\hline Naphtha for Petrochemical Feedstock Use ................. & 0.6 & 2.7 & 0.4 & 0.0 & 0.2 & 1.5 & 0.0 & .0 .1 & 1.0 \\
\hline Other Oils for Petrochemical Feedstock Use .............. & 0.9 & 5.2 & 4.3 & 0.0 & 0.0 & 4.4 & 0.2 & 0.3 & 2.3 \\
\hline Special Naphthas & 0.5 & 0.8 & 0.3 & 2.9 & 0.0 & 0.6 & 0.0 & 0.1 & 0.4 \\
\hline 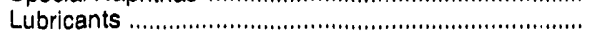 & 0.3 & 1.8 & 1.0 & 9.8 & 0.0 & 1.6 & 0.0 & 1.0 & 1.2 \\
\hline 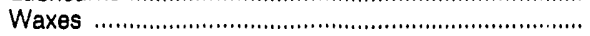 & 0.1 & 0.2 & 0.1 & 1.4 & 0.0 & 0.2 & 0.4 & 0.1 & 0.2 \\
\hline Petroleum Coke & 2.2 & 3.9 & 5.6 & 2.2 & 0.6 & 4.4 & 3.0 & 5.9 & 4.5 \\
\hline 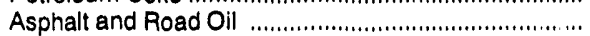 & 1.7 & 0.8 & 1.4 & 19.5 & 6.3 & 1.6 & 5.8 & 1.4 & 2.7 \\
\hline 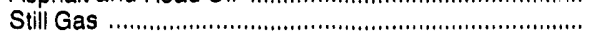 & 4.3 & 4.9 & 4.0 & 3.4 & 2.4 & 4.4 & 4.6 & 5.5 & 4.6 \\
\hline 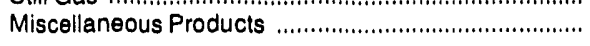 & 0.4 & 0.3 & 0.3 & 0.0 & 0.0 & 0.3 & 0.5 & 0.3 & 0.3 \\
\hline Processing Gain $(-)$ or Loss $(+)^{d}$ & -3.4 & -7.1 & -5.6 & 0.3 & .1 .1 & .6 .0 & $\cdot 3.3$ & $\cdot 6.4$ & -5.7 \\
\hline
\end{tabular}

Based on crude oil input and net reruns of unfinished oils.

b Based on total finished motor gasoline output minus net input of motor gasoline blending components, minus input of natural gas plant liquids, other hydrocarbons and oxygenates.

c Based on finished aviation gasoline output minus net input of aviation gasoline blending components.

d Represents the difference between input and production.

Notes: - Totals may not equal sum of components due to independent rounding. - Refer to Appendix A for Refining District descriptions

Sources: Calculated from data on Tables 28 and 29. 
Table 32. Imports of Resldual Fuel Oll by Sulfur Content and by PAD DIstrict and State of Entry, March 1993 (Thousand Barrels)

\begin{tabular}{|c|c|c|c|c|}
\hline \multirow[b]{2}{*}{ PAD Dlatrlot and State of Entry } & \multicolumn{4}{|c|}{ Reoldual Fual Oll } \\
\hline & $\begin{array}{l}\text { Loe than } \\
\text { 0.31\% } \\
\text { Sulfur }\end{array}$ & $\begin{array}{l}0.31 \text { to } \\
1.00 \% \\
\text { Sulfur }\end{array}$ & $\begin{array}{l}\text { Greater } \\
\text { than } 1.00 \% \\
\text { Sulfur }\end{array}$ & Total \\
\hline 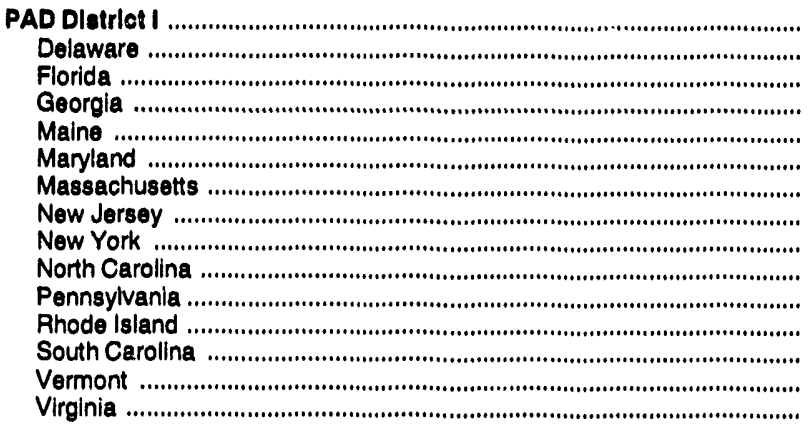 & $\begin{array}{r}498 \\
0 \\
0 \\
0 \\
57 \\
180 \\
0 \\
0 \\
194 \\
0 \\
67 \\
0 \\
0 \\
0 \\
0\end{array}$ & $\begin{array}{r}2,065 \\
0 \\
222 \\
0 \\
225 \\
156 \\
292 \\
173 \\
897 \\
0 \\
100 \\
0 \\
0 \\
0 \\
0\end{array}$ & $\begin{array}{r}7,080 \\
123 \\
1,014 \\
101 \\
410 \\
918 \\
527 \\
1,748 \\
738 \\
444 \\
77 \\
172 \\
195 \\
4 \\
609\end{array}$ & $\begin{array}{r}9,643 \\
123 \\
1,236 \\
101 \\
692 \\
1,254 \\
819 \\
1,921 \\
1,829 \\
444 \\
244 \\
172 \\
195 \\
4 \\
609\end{array}$ \\
\hline $\begin{array}{l}\text { PAD Dlatrict II } \\
\text { North Dakota }\end{array}$ & $\begin{array}{l}0 \\
0\end{array}$ & $\begin{array}{l}2 \\
2\end{array}$ & $\begin{array}{l}0 \\
0\end{array}$ & $\begin{array}{l}2 \\
2\end{array}$ \\
\hline 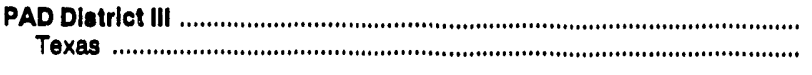 & $\begin{array}{l}0 \\
0\end{array}$ & $\begin{array}{l}0 \\
0\end{array}$ & $\begin{array}{l}609 \\
609\end{array}$ & $\begin{array}{l}609 \\
609\end{array}$ \\
\hline 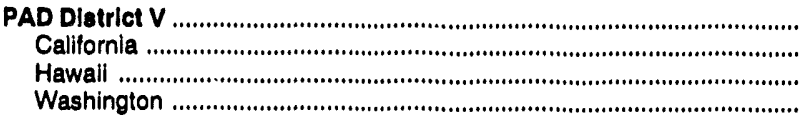 & $\begin{array}{r}100 \\
0 \\
100 \\
0\end{array}$ & $\begin{array}{l}0 \\
0 \\
0 \\
0\end{array}$ & $\begin{array}{r}565 \\
498 \\
0 \\
67\end{array}$ & $\begin{array}{r}665 \\
498 \\
100 \\
67\end{array}$ \\
\hline 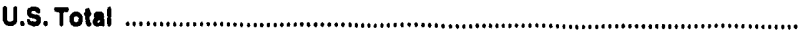 & 598 & 2,067 & 8,254 & 10,919 \\
\hline
\end{tabular}

Source: Energy Information Administration (EIA) Form EIA-814, "Monthly Imports Report." 
Table 33. Imports of Crude Oll and Petroleum Products by PAD District, March 1993 (Thousand Barrels)

\begin{tabular}{|c|c|c|c|c|c|c|c|}
\hline \multirow[b]{2}{*}{ Commodily } & \multicolumn{6}{|c|}{ Petroleum Adminlatration for Defense Distrlcte } & \multirow[b]{2}{*}{$\begin{array}{c}\text { Dally } \\
\text { Average }\end{array}$} \\
\hline & $\mathbf{I}$ & $\|$ & III & N & $\mathbf{V}$ & $\begin{array}{l}\text { U.S. } \\
\text { Total }\end{array}$ & \\
\hline 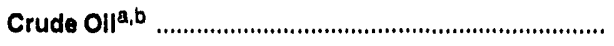 & 38,993 & 42,566 & 111,569 & 2,649 & 6,139 & 201,916 & 6,513 \\
\hline 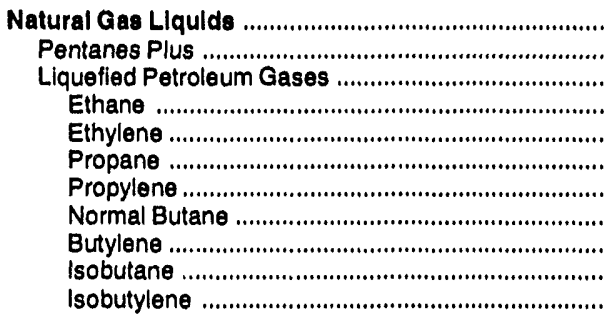 & $\begin{array}{r}720 \\
0 \\
720 \\
0 \\
0 \\
511 \\
0 \\
177 \\
0 \\
32 \\
0\end{array}$ & $\begin{array}{r}2,081 \\
42 \\
2,039 \\
372 \\
0 \\
1,318 \\
129 \\
131 \\
0 \\
89 \\
0\end{array}$ & $\begin{array}{r}1,624 \\
775 \\
849 \\
0 \\
0 \\
576 \\
0 \\
172 \\
0 \\
101 \\
0\end{array}$ & $\begin{array}{r}261 \\
78 \\
183 \\
0 \\
0 \\
100 \\
0 \\
75 \\
0 \\
8 \\
0\end{array}$ & $\begin{array}{r}16 \\
0 \\
16 \\
0 \\
0 \\
4 \\
0 \\
0 \\
0 \\
12 \\
0\end{array}$ & $\begin{array}{r}4,702 \\
895 \\
3,807 \\
372 \\
0 \\
2,509 \\
129 \\
555 \\
0 \\
242 \\
0\end{array}$ & $\begin{array}{r}152 \\
29 \\
123 \\
12 \\
0 \\
81 \\
4 \\
18 \\
0 \\
8 \\
0\end{array}$ \\
\hline 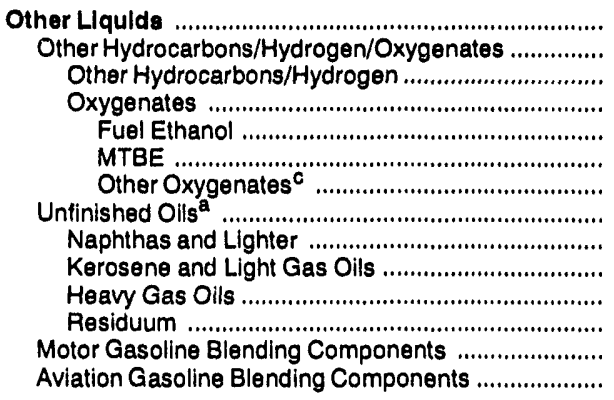 & $\begin{array}{r}5,173 \\
0 \\
0 \\
0 \\
0 \\
0 \\
0 \\
4,448 \\
0 \\
0 \\
2,892 \\
1,556 \\
725 \\
0\end{array}$ & $\begin{array}{r}104 \\
0 \\
0 \\
0 \\
0 \\
0 \\
0 \\
102 \\
102 \\
0 \\
0 \\
0 \\
2 \\
0\end{array}$ & $\begin{array}{r}10,636 \\
0 \\
0 \\
0 \\
0 \\
0 \\
0 \\
9,943 \\
3,011 \\
0 \\
775 \\
6,157 \\
693 \\
0\end{array}$ & $\begin{array}{l}0 \\
0 \\
0 \\
0 \\
0 \\
0 \\
0 \\
0 \\
0 \\
0 \\
0 \\
0 \\
0 \\
0\end{array}$ & $\begin{array}{r}1,280 \\
364 \\
0 \\
364 \\
0 \\
364 \\
0 \\
880 \\
0 \\
0 \\
0 \\
880 \\
36 \\
0\end{array}$ & $\begin{array}{r}17,193 \\
364 \\
0 \\
364 \\
0 \\
364 \\
0 \\
15,373 \\
3,113 \\
0 \\
3,667 \\
8,593 \\
1,456 \\
0\end{array}$ & $\begin{array}{r}555 \\
12 \\
0 \\
12 \\
0 \\
12 \\
0 \\
496 \\
100 \\
0 \\
118 \\
277 \\
47 \\
0\end{array}$ \\
\hline 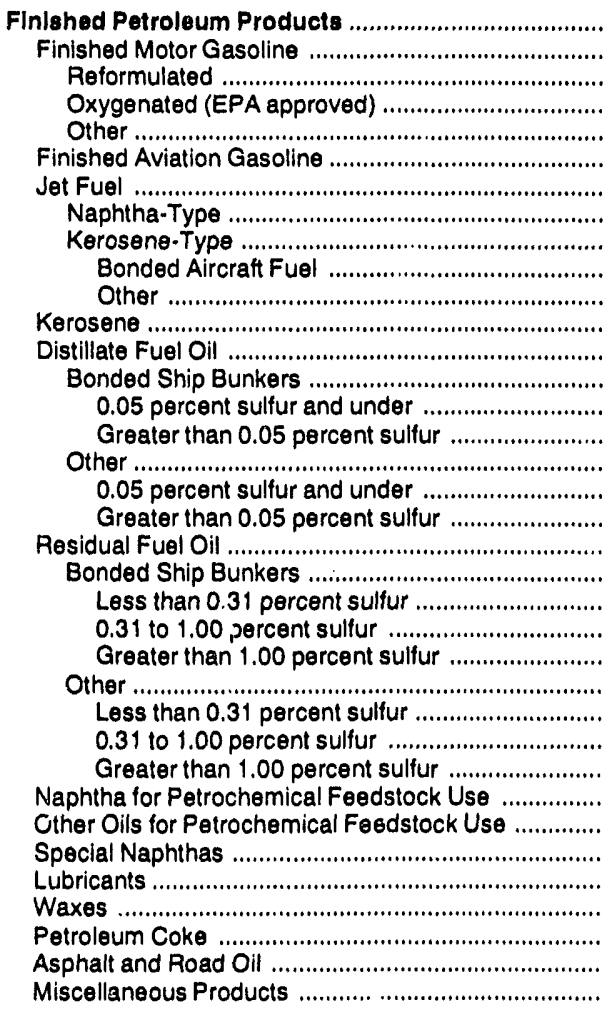 & $\begin{array}{r}26,670 \\
5,844 \\
0 \\
0 \\
5,844 \\
0 \\
2,716 \\
0 \\
2,716 \\
1,890 \\
826 \\
39 \\
7,047 \\
0 \\
0 \\
0 \\
7,047 \\
1,916 \\
5,131 \\
9,643 \\
0 \\
0 \\
0 \\
0 \\
9,643 \\
498 \\
2,065 \\
7,080 \\
66 \\
0 \\
9 \\
410 \\
77 \\
0 \\
816 \\
3\end{array}$ & $\begin{array}{r}346 \\
59 \\
0 \\
0 \\
59 \\
1 \\
71 \\
71 \\
0 \\
0 \\
0 \\
0 \\
110 \\
0 \\
0 \\
0 \\
110 \\
38 \\
72 \\
2 \\
0 \\
0 \\
0 \\
0 \\
2 \\
0 \\
2 \\
0 \\
32 \\
0 \\
53 \\
6 \\
7 \\
0 \\
0 \\
5\end{array}$ & $\begin{array}{r}6,701 \\
0 \\
0 \\
0 \\
0 \\
0 \\
383 \\
141 \\
242 \\
0 \\
242 \\
0 \\
0 \\
0 \\
0 \\
0 \\
0 \\
0 \\
0 \\
609 \\
0 \\
0 \\
0 \\
0 \\
609 \\
0 \\
0 \\
609 \\
880 \\
4,476 \\
131 \\
0 \\
6 \\
181 \\
35 \\
0\end{array}$ & $\begin{array}{r}67 \\
11 \\
0 \\
0 \\
11 \\
0 \\
0 \\
0 \\
0 \\
0 \\
0 \\
0 \\
52 \\
2 \\
2 \\
0 \\
50 \\
28 \\
22 \\
0 \\
0 \\
0 \\
0 \\
0 \\
0 \\
0 \\
0 \\
0 \\
0 \\
0 \\
0 \\
0 \\
4 \\
0 \\
0 \\
0\end{array}$ & $\begin{array}{r}1,020 \\
226 \\
0 \\
0 \\
226 \\
3 \\
4 \\
0 \\
4 \\
3 \\
1 \\
0 \\
77 \\
61 \\
0 \\
61 \\
16 \\
0 \\
16 \\
665 \\
0 \\
0 \\
0 \\
0 \\
665 \\
100 \\
0 \\
565 \\
0 \\
0 \\
8 \\
0 \\
2 \\
22 \\
13 \\
0\end{array}$ & $\begin{array}{r}34,804 \\
6,140 \\
0 \\
0 \\
6,140 \\
4 \\
3,174 \\
212 \\
2,962 \\
1,893 \\
1,069 \\
39 \\
7,286 \\
63 \\
2 \\
61 \\
7,223 \\
1,982 \\
5,241 \\
10,919 \\
0 \\
0 \\
0 \\
0 \\
10,919 \\
598 \\
2,067 \\
8,254 \\
978 \\
4,476 \\
201 \\
416 \\
96 \\
203 \\
864 \\
8\end{array}$ & $\begin{array}{r}1,123 \\
198 \\
0 \\
0 \\
198 \\
(s) \\
102 \\
7 \\
96 \\
61 \\
34 \\
1 \\
235 \\
2 \\
(s) \\
2 \\
233 \\
64 \\
169 \\
352 \\
0 \\
0 \\
0 \\
0 \\
352 \\
19 \\
67 \\
266 \\
32 \\
144 \\
6 \\
13 \\
3 \\
7 \\
28 \\
(s)\end{array}$ \\
\hline Total & 71,556 & 45,097 & 130,530 & 2,977 & 8,455 & 258,615 & 8,342 \\
\hline
\end{tabular}

a Crude oil and unfinished oils are reported by the PAD District in which they are to be processed; all other products are reported by the PAD District of entry. b Includes crude oil imported for storage in the Strategic Petroleum Reserve.

- Includes ethyl tertiary butyl ether (ETBE), tertiary amyl methyl ether (TAME), tertiary butyl alcohol (TBA), and other aliphatic alcohols and ethers intended for motor gasoline biending (e.g., isopropyl ether (IP

(s) = Less than 500 barrels or less than 500 barrels per day.

Note: Totals may not equal sum of components due to independent rounding.

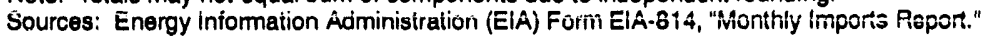


Table 34. Year-to-Date Imports of Crude Oll and Petroleum Products by PAD Distrlct, January-March 1993

(Thousand Barrels)

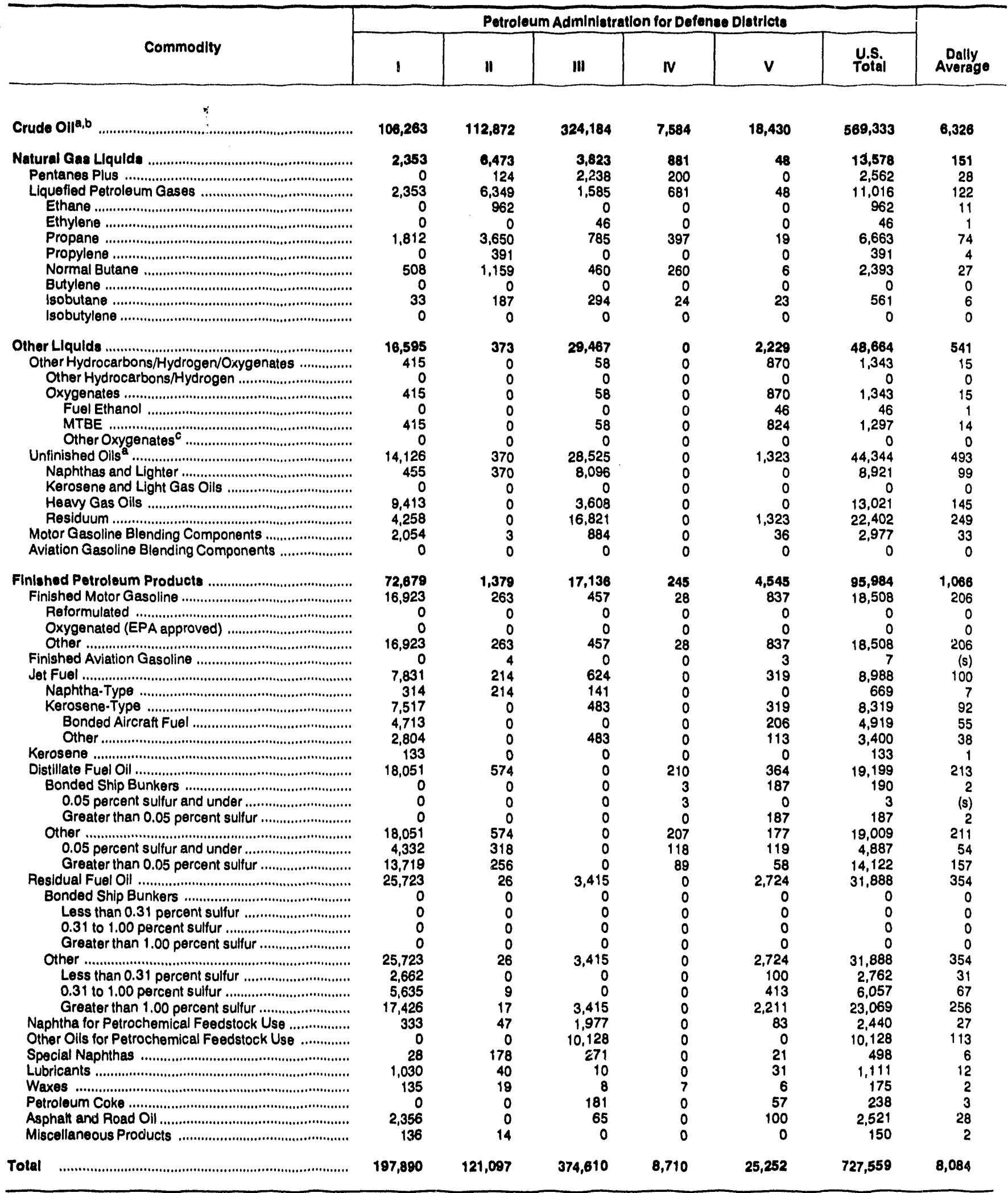

Crude oil and unfinished oils are reported by the PAD District in which they are to be processed; all other products are reported by the PAD District of entry.

Includes crude oil imported for storage in the Strategic Petroleum Reserve.

C Includes ethyl tertiary butyl ether (ETBE), tertiary amyl methyl etl er (TAME), tertiary butyl alcohoi (TBA), and other aliphatic alcohols and ethers intendrd for motor gasolina blending (e.g., isopropyl ether (IPE) or n-propanol).

$(s)=$ Less than 500 barrels or less than 500 barrels per day.

Note: Totals may not equal sum of componenis due to independent rounding.

Source: Energy Information Administration (EIA) Form EIA-814, "Monthly Imports Report." 
Table 35. Imports of Crude Oll and Petroleum Products Into the Unlted States by Country of Origin, March 1993 (Thousand Barrels)

\begin{tabular}{|c|c|c|c|c|c|c|c|c|c|c|}
\hline Country of Origin & $\begin{array}{c}\text { Crude } \\
\text { Oli }\end{array}$ & $\begin{array}{c}\text { Uquefled } \\
\text { Petroleum } \\
\text { Casee }\end{array}$ & $\begin{array}{c}\text { Unflniehed } \\
\text { Olle }\end{array}$ & $\begin{array}{c}\text { Blending } \\
\text { Compo- } \\
\text { nente } \\
\end{array}$ & $\begin{array}{l}\text { Finiahed } \\
\text { Motor } \\
\text { Gacollne }\end{array}$ & Jet Fuel & $\begin{array}{l}\text { Diotlliate } \\
\text { Fuel Oll }\end{array}$ & $\begin{array}{l}\text { Roaldual } \\
\text { Fuel Oll }\end{array}$ & Kerosene & $\begin{array}{c}\text { Speclal } \\
\text { Naphthas }\end{array}$ \\
\hline 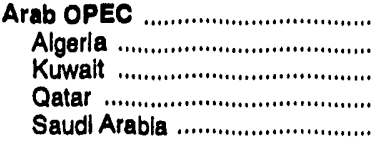 & $\begin{array}{r}51,319 \\
205 \\
9,309 \\
0 \\
41,805\end{array}$ & $\begin{array}{r}658 \\
654 \\
0 \\
0 \\
0\end{array}$ & $\begin{array}{r}3,918 \\
361 \\
0 \\
197 \\
3,360\end{array}$ & $\begin{array}{l}0 \\
0 \\
0 \\
0 \\
0\end{array}$ & $\begin{array}{r}693 \\
0 \\
0 \\
0 \\
693\end{array}$ & $\begin{array}{l}1 \\
0 \\
0 \\
0 \\
1\end{array}$ & $\begin{array}{l}0 \\
0 \\
0 \\
0 \\
0\end{array}$ & $\begin{array}{r}498 \\
0 \\
498 \\
0 \\
0\end{array}$ & $\begin{array}{l}0 \\
0 \\
0 \\
0 \\
0\end{array}$ & $\begin{array}{l}0 \\
0 \\
0 \\
0 \\
0\end{array}$ \\
\hline 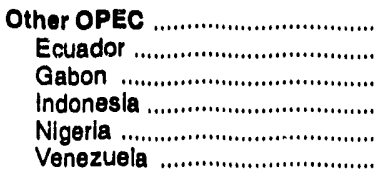 & $\begin{array}{r}60,611 \\
1,814 \\
3,817 \\
1,986 \\
27,667 \\
25,327\end{array}$ & $\begin{array}{r}171 \\
0 \\
0 \\
0 \\
0 \\
171\end{array}$ & $\begin{array}{r}1,559 \\
0 \\
0 \\
0 \\
180 \\
1,379\end{array}$ & $\begin{array}{r}561 \\
0 \\
0 \\
0 \\
0 \\
561\end{array}$ & $\begin{array}{r}1,897 \\
0 \\
0 \\
0 \\
0 \\
1,897\end{array}$ & $\begin{array}{r}1,890 \\
0 \\
0 \\
0 \\
0 \\
1,890\end{array}$ & $\begin{array}{r}3,586 \\
0 \\
0 \\
0 \\
241 \\
3,345\end{array}$ & $\begin{array}{r}3,172 \\
0 \\
104 \\
100 \\
681 \\
2,287\end{array}$ & $\begin{array}{l}0 \\
0 \\
0 \\
0 \\
0 \\
0\end{array}$ & $\begin{array}{l}0 \\
0 \\
0 \\
0 \\
0 \\
0\end{array}$ \\
\hline 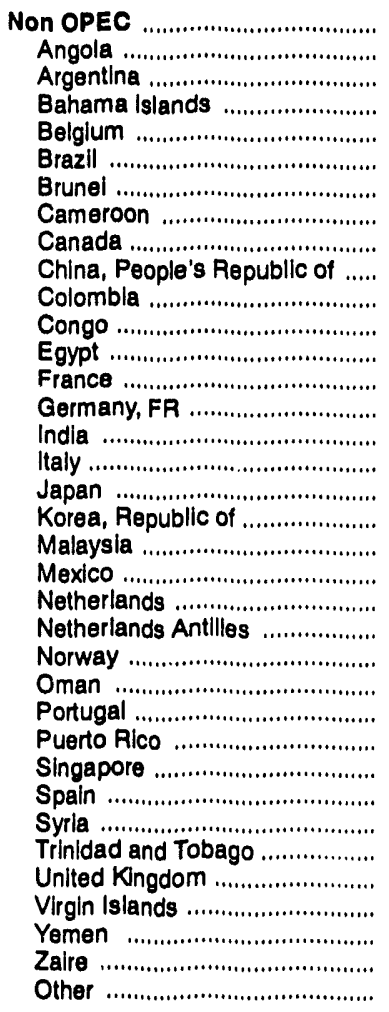 & $\begin{array}{r}89,986 \\
12,633 \\
816 \\
0 \\
0 \\
0 \\
155 \\
0 \\
25,229 \\
2,277 \\
4,008 \\
2,175 \\
1,430 \\
0 \\
0 \\
0 \\
0 \\
0 \\
0 \\
324 \\
25,262 \\
0 \\
0 \\
3,578 \\
0 \\
0 \\
0 \\
0 \\
0 \\
600 \\
2,213 \\
8,704 \\
0 \\
0 \\
348 \\
0\end{array}$ & $\begin{array}{r}2,982 \\
0 \\
0 \\
0 \\
0 \\
0 \\
0 \\
0 \\
2,752 \\
0 \\
0 \\
0 \\
0 \\
20 \\
0 \\
0 \\
0 \\
0 \\
0 \\
4 \\
0 \\
0 \\
0 \\
0 \\
0 \\
0 \\
0 \\
0 \\
0 \\
0 \\
0 \\
206 \\
0 \\
0 \\
0 \\
0\end{array}$ & $\begin{array}{r}8,886 \\
0 \\
0 \\
0 \\
0 \\
0 \\
0 \\
0 \\
375 \\
0 \\
0 \\
0 \\
141 \\
187 \\
678 \\
256 \\
1,086 \\
0 \\
1,084 \\
0 \\
44 \\
306 \\
629 \\
195 \\
702 \\
0 \\
0 \\
1,116 \\
449 \\
0 \\
0 \\
836 \\
1,764 \\
48 \\
0 \\
0\end{array}$ & $\begin{array}{r}895 \\
0 \\
0 \\
0 \\
0 \\
0 \\
0 \\
0 \\
2 \\
0 \\
0 \\
0 \\
0 \\
0 \\
0 \\
0 \\
0 \\
0 \\
36 \\
0 \\
693 \\
0 \\
0 \\
0 \\
0 \\
0 \\
0 \\
0 \\
0 \\
0 \\
0 \\
0 \\
164 \\
0 \\
0 \\
0\end{array}$ & $\begin{array}{r}3,550 \\
0 \\
0 \\
0 \\
0 \\
627 \\
0 \\
0 \\
1,530 \\
162 \\
0 \\
0 \\
0 \\
0 \\
0 \\
0 \\
237 \\
0 \\
0 \\
0 \\
190 \\
0 \\
0 \\
0 \\
0 \\
0 \\
0 \\
0 \\
0 \\
0 \\
0 \\
0 \\
804 \\
0 \\
0 \\
0\end{array}$ & $\begin{array}{r}1,283 \\
0 \\
0 \\
0 \\
0 \\
0 \\
0 \\
0 \\
78 \\
0 \\
0 \\
0 \\
141 \\
0 \\
0 \\
0 \\
0 \\
0 \\
0 \\
0 \\
11 \\
0 \\
0 \\
0 \\
0 \\
0 \\
0 \\
0 \\
0 \\
0 \\
0 \\
0 \\
1,053 \\
0 \\
0 \\
0\end{array}$ & $\begin{array}{r}3,700 \\
0 \\
0 \\
0 \\
0 \\
0 \\
0 \\
0 \\
1,843 \\
0 \\
0 \\
0 \\
0 \\
0 \\
0 \\
0 \\
0 \\
0 \\
0 \\
0 \\
187 \\
0 \\
0 \\
0 \\
0 \\
0 \\
0 \\
0 \\
0 \\
0 \\
0 \\
0 \\
1,670 \\
0 \\
0 \\
0\end{array}$ & $\begin{array}{r}7,249 \\
0 \\
0 \\
935 \\
0 \\
221 \\
0 \\
321 \\
291 \\
0 \\
978 \\
0 \\
0 \\
0 \\
373 \\
0 \\
0 \\
0 \\
0 \\
0 \\
0 \\
0 \\
1,100 \\
0 \\
0 \\
256 \\
0 \\
0 \\
0 \\
0 \\
651 \\
0 \\
2,123 \\
0 \\
0 \\
0\end{array}$ & $\begin{array}{r}39 \\
0 \\
0 \\
0 \\
0 \\
0 \\
0 \\
0\end{array}$ & $\begin{array}{r}201 \\
0 \\
0 \\
0 \\
0 \\
0 \\
0 \\
0 \\
190 \\
0 \\
0 \\
0 \\
0 \\
0 \\
0 \\
0 \\
11 \\
0 \\
0 \\
0 \\
0 \\
0 \\
0 \\
0 \\
0 \\
0 \\
0 \\
0 \\
0 \\
0 \\
0 \\
0 \\
0 \\
0 \\
0 \\
0\end{array}$ \\
\hline 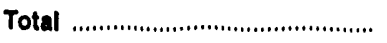 & 201,916 & 3,807 & 15,373 & 1,456 & 6,140 & 3,174 & 7,286 & 10,919 & 39 & 201 \\
\hline 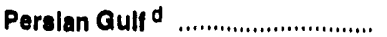 & 51,114 & 0 & 3,557 & 0 & 693 & 1 & 0 & 498 & 0 & 0 \\
\hline
\end{tabular}

See footnotes at end of table. 
Table 35. Imports of Crude Oll and Petroleum Products Into the United States by Country of OrigIn," March 1993 (Continued)

(Thousand Barrels)

\begin{tabular}{|c|c|c|c|c|c|c|c|c|c|c|}
\hline \multirow[b]{2}{*}{ Country of Origln } & \multirow[b]{2}{*}{$\begin{array}{c}\text { Naphtha for } \\
\text { Petrochemloal } \\
\text { Feedetock } \\
\text { Uce }\end{array}$} & \multirow[b]{2}{*}{$\begin{array}{c}\text { Other Olle for } \\
\text { Potrochemical } \\
\text { Feedotock } \\
\text { Une }\end{array}$} & \multirow[b]{2}{*}{ Lubricante } & \multirow[b]{2}{*}{$\begin{array}{c}\text { Asphatt and } \\
\text { Road Oll }\end{array}$} & \multirow[b]{2}{*}{$\begin{array}{c}\text { Other } \\
\text { Productac }\end{array}$} & \multirow[b]{2}{*}{$\begin{array}{c}\text { Total } \\
\text { Producte }\end{array}$} & \multirow[b]{2}{*}{$\begin{array}{c}\text { Total } \\
\text { Crude Oll } \\
\text { and } \\
\text { Producte }\end{array}$} & \multicolumn{3}{|c|}{ Dally Average } \\
\hline & & & & & & & & $\begin{array}{c}\text { Crude } \\
\text { Oll } \\
\end{array}$ & Producte & Total \\
\hline 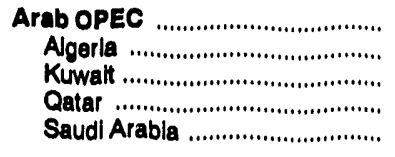 & $\begin{array}{l}0 \\
0 \\
0 \\
0 \\
0\end{array}$ & $\begin{array}{r}4,027 \\
4,027 \\
0 \\
0 \\
0\end{array}$ & $\begin{array}{l}0 \\
0 \\
0 \\
0 \\
0\end{array}$ & $\begin{array}{l}0 \\
0 \\
0 \\
0 \\
0\end{array}$ & $\begin{array}{r}483 \\
483 \\
0 \\
0 \\
0\end{array}$ & $\begin{array}{r}10,274 \\
5,525 \\
498 \\
197 \\
4,054\end{array}$ & $\begin{array}{r}61,693 \\
5,730 \\
9,807 \\
197 \\
45,859\end{array}$ & $\begin{array}{r}1,655 \\
7 \\
300 \\
0 \\
1,349\end{array}$ & $\begin{array}{r}331 \\
178 \\
16 \\
6 \\
131\end{array}$ & $\begin{array}{r}1,987 \\
185 \\
3 \cdot 3 \\
6 \\
1,479\end{array}$ \\
\hline 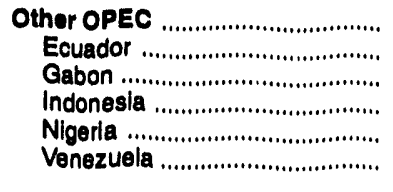 & $\begin{array}{l}0 \\
0 \\
0 \\
0 \\
0 \\
0\end{array}$ & $\begin{array}{l}0 \\
0 \\
0 \\
0 \\
0 \\
0\end{array}$ & $\begin{array}{l}0 \\
0 \\
0 \\
0 \\
0 \\
0\end{array}$ & $\begin{array}{r}469 \\
0 \\
0 \\
0 \\
0 \\
469\end{array}$ & $\begin{array}{r}120 \\
0 \\
0 \\
0 \\
0 \\
120\end{array}$ & $\begin{array}{r}13,425 \\
0 \\
104 \\
100 \\
1,102 \\
12,119\end{array}$ & $\begin{array}{r}74,038 \\
1,814 \\
3,921 \\
2,086 \\
28,769 \\
37,446\end{array}$ & $\begin{array}{r}1,955 \\
59 \\
123 \\
64 \\
892 \\
817\end{array}$ & $\begin{array}{r}433 \\
0 \\
3 \\
3 \\
36 \\
391\end{array}$ & $\begin{array}{r}2,388 \\
59 \\
126 \\
67 \\
928 \\
1,208\end{array}$ \\
\hline 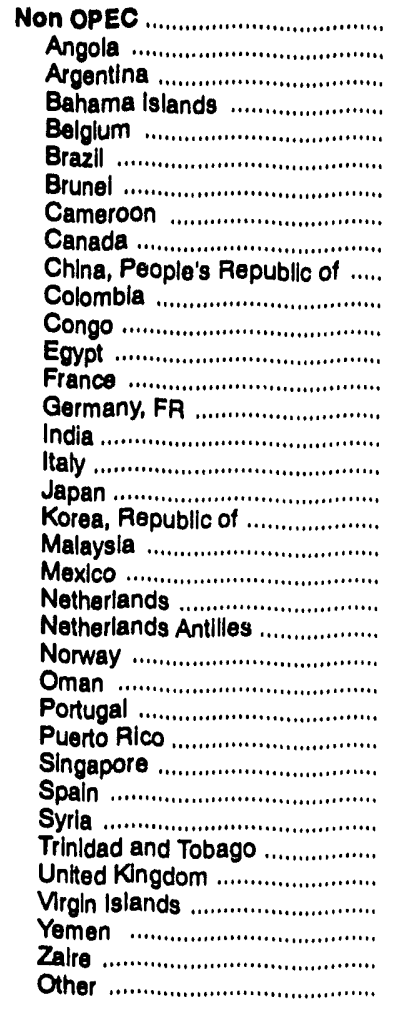 & $\begin{array}{r}978 \\
0 \\
27 \\
0 \\
22 \\
0 \\
0 \\
0 \\
76 \\
0 \\
0 \\
0 \\
0 \\
40 \\
0 \\
0 \\
12 \\
0 \\
55 \\
0 \\
0 \\
7 \\
68 \\
0 \\
0 \\
0 \\
458 \\
0 \\
0 \\
0 \\
0 \\
22 \\
0 \\
191 \\
0 \\
0\end{array}$ & $\begin{array}{r}449 \\
0 \\
180 \\
0 \\
0 \\
0 \\
0 \\
0 \\
0 \\
0 \\
0 \\
0 \\
0 \\
0 \\
0 \\
0 \\
0 \\
0 \\
0 \\
0 \\
0 \\
0 \\
0 \\
0 \\
0 \\
0 \\
0 \\
0 \\
0 \\
0 \\
0 \\
0 \\
0 \\
0 \\
0 \\
269\end{array}$ & $\begin{array}{r}416 \\
0 \\
0 \\
0 \\
0 \\
0 \\
0 \\
0 \\
47 \\
0 \\
0 \\
0 \\
0 \\
0 \\
0 \\
0 \\
0 \\
0 \\
0 \\
0 \\
0 \\
0 \\
0 \\
0 \\
0 \\
0 \\
355 \\
0 \\
14 \\
0 \\
0 \\
0 \\
0 \\
0 \\
0 \\
0\end{array}$ & $\begin{array}{r}395 \\
0 \\
0 \\
0 \\
0 \\
0 \\
0 \\
0 \\
131 \\
0 \\
0 \\
0 \\
0 \\
0 \\
0 \\
0 \\
0 \\
0 \\
0 \\
0 \\
0 \\
0 \\
84 \\
0 \\
0 \\
0 \\
0 \\
0 \\
180 \\
0 \\
0 \\
0 \\
0 \\
0 \\
0 \\
0\end{array}$ & $\begin{array}{r}987 \\
0 \\
181 \\
0 \\
0 \\
1 \\
0 \\
0 \\
427 \\
15 \\
0 \\
0 \\
2 \\
0 \\
3 \\
0 \\
0 \\
1 \\
0 \\
0 \\
294 \\
40 \\
0 \\
0 \\
0 \\
0 \\
0 \\
0 \\
0 \\
0 \\
0 \\
0 \\
0 \\
0 \\
0 \\
3\end{array}$ & $\begin{array}{r}33,000 \\
0 \\
388 \\
935 \\
22 \\
849 \\
0 \\
321 \\
7,781 \\
177 \\
978 \\
0 \\
284 \\
247 \\
1,054 \\
256 \\
1,346 \\
1 \\
1,175 \\
4 \\
1,419 \\
353 \\
1,881 \\
195 \\
702 \\
256 \\
813 \\
1,116 \\
643 \\
0 \\
651 \\
1,064 \\
7,578 \\
239 \\
0 \\
272\end{array}$ & $\begin{array}{r}122,986 \\
12,633 \\
1,204 \\
935 \\
22 \\
849 \\
155 \\
321 \\
33,010 \\
2,454 \\
4,986 \\
2,175 \\
1,714 \\
247 \\
1,054 \\
256 \\
1,346 \\
1 \\
1,175 \\
328 \\
26,681 \\
353 \\
1,881 \\
3,773 \\
702 \\
256 \\
813 \\
1,116 \\
643 \\
600 \\
2,864 \\
9,768 \\
7,578 \\
239 \\
348 \\
272\end{array}$ & $\begin{array}{r}2,903 \\
408 \\
26 \\
0 \\
0 \\
0 \\
5 \\
0 \\
814 \\
73 \\
129 \\
70 \\
46 \\
0 \\
0 \\
0 \\
0 \\
0 \\
0 \\
10 \\
815 \\
0 \\
0 \\
115 \\
0 \\
0 \\
0 \\
0 \\
0 \\
19 \\
71 \\
281 \\
0 \\
0 \\
11 \\
0\end{array}$ & $\begin{array}{r}1,065 \\
0 \\
13 \\
30 \\
1 \\
27 \\
0 \\
10 \\
251 \\
6 \\
32 \\
0 \\
9 \\
8 \\
34 \\
8 \\
43 \\
(8) \\
38 \\
(8) \\
46 \\
11 \\
61 \\
6 \\
23 \\
8 \\
26 \\
36 \\
21 \\
0 \\
21 \\
34 \\
244 \\
8 \\
0 \\
9\end{array}$ & $\begin{array}{r}3,967 \\
408 \\
39 \\
30 \\
1 \\
27 \\
5 \\
10 \\
1,065 \\
79 \\
161 \\
70 \\
55 \\
8 \\
34 \\
8 \\
43 \\
(s) \\
38 \\
11 \\
861 \\
11 \\
61 \\
122 \\
23 \\
8 \\
26 \\
36 \\
21 \\
19 \\
92 \\
315 \\
244 \\
8 \\
11 \\
9\end{array}$ \\
\hline 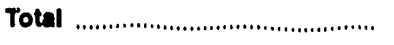 & 978 & 4,476 & 416 & 864 & 1,570 & 56,689 & 258,615 & 6,513 & 1,829 & 8,342 \\
\hline Peraian Gulf d & 0 & 269 & 0 & 0 & 0 & 5,018 & 56,132 & 1,649 & 162 & 1,811 \\
\hline
\end{tabular}

Crude oll and unfinished oils are reported by the PAD District in which they are $t$, ve processed; all other products are reported by the PAD District of entry.

b Includes crude oll imported for storage in the Strategic Petroleum Reserve.

c Includes aviation gasoline, aviation gasoline blending components, miscellaneous proc cts, other hydrocarbons and oxygenates, pentanes plus, petroleum coke, and waxeg.

'Includes Bahrain, Iran, Iraq, Kuwalt, Qatar, Saudl Arabla, and United Arab Emirates.

$(s)=$ Less than 500 barrels or less than 500 barrels per day.

Note: Totals may not equal sum ( components due to independent rounding.

Source: Energy Information Administration (EIA) Form EIA-814, "Monthly Imports Report." 
Table 36. PAD District I-Imports of Crude OII and Potroleum Products by Country of Origin," March 1993

(Thousand Barrels)

\begin{tabular}{|c|c|c|c|c|c|c|c|c|c|c|}
\hline Country of Orlgln & $\begin{array}{c}\text { Crudo } \\
\text { Oll }\end{array}$ & $\begin{array}{c}\text { Liquofled } \\
\text { Potroloum } \\
\text { anees }\end{array}$ & $\begin{array}{c}\text { Unilinishod } \\
\text { Olle }\end{array}$ & $\begin{array}{c}\text { Gasoline } \\
\text { Blending } \\
\text { Compo- } \\
\text { nente }\end{array}$ & $\begin{array}{l}\text { Finished } \\
\text { Motor } \\
\text { Oesolline } \\
\end{array}$ & Jet Fuel & $\begin{array}{l}\text { Dlatiliato } \\
\text { Fuel Oll }\end{array}$ & $\begin{array}{l}\text { Roeldual } \\
\text { Fuel Oll }\end{array}$ & Kerosene & $\begin{array}{c}\text { Special } \\
\text { Naphthas }\end{array}$ \\
\hline $\begin{array}{l}\text { Arab OPEC } \\
\text { Saudi Arabla }\end{array}$ & $\begin{array}{l}3,823 \\
3,823\end{array}$ & $\begin{array}{l}0 \\
0\end{array}$ & $\begin{array}{l}0 \\
0\end{array}$ & $\begin{array}{l}0 \\
0\end{array}$ & $\begin{array}{l}693 \\
693\end{array}$ & $\begin{array}{l}0 \\
0\end{array}$ & $\begin{array}{l}0 \\
0\end{array}$ & $\begin{array}{l}0 \\
0\end{array}$ & $\begin{array}{l}0 \\
0\end{array}$ & $\begin{array}{l}0 \\
0\end{array}$ \\
\hline 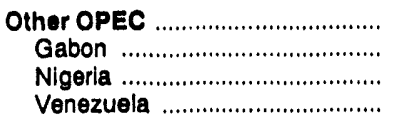 & $\begin{array}{r}20,453 \\
928 \\
14,945 \\
4,680\end{array}$ & $\begin{array}{l}0 \\
0 \\
0 \\
0\end{array}$ & $\begin{array}{r}180 \\
0 \\
180 \\
0\end{array}$ & $\begin{array}{r}561 \\
0 \\
0 \\
561\end{array}$ & $\begin{array}{r}1,897 \\
0 \\
0 \\
1,897\end{array}$ & $\begin{array}{r}1,890 \\
0 \\
0 \\
1,890\end{array}$ & $\begin{array}{r}3,886 \\
0 \\
241 \\
3,345\end{array}$ & $\begin{array}{r}2,836 \\
104 \\
681 \\
2,051\end{array}$ & $\begin{array}{l}0 \\
0 \\
0 \\
0\end{array}$ & $\begin{array}{l}0 \\
0 \\
0 \\
0\end{array}$ \\
\hline 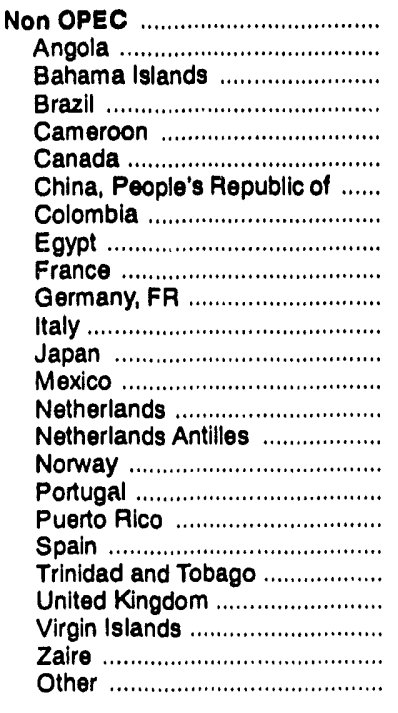 & $\begin{array}{r}14,717 \\
5,485 \\
0 \\
0 \\
0 \\
1,999 \\
682 \\
0 \\
728 \\
0 \\
0 \\
0 \\
0 \\
1,878 \\
0 \\
0 \\
1,651 \\
0 \\
0 \\
0 \\
0 \\
2,046 \\
0 \\
348 \\
0\end{array}$ & $\begin{array}{r}720 \\
0 \\
0 \\
0 \\
0 \\
514 \\
0 \\
0 \\
0 \\
0 \\
0 \\
0 \\
0 \\
0 \\
0 \\
0 \\
0 \\
0 \\
0 \\
0 \\
0 \\
206 \\
0 \\
0 \\
0\end{array}$ & $\begin{array}{r}4,268 \\
0 \\
0 \\
0 \\
0 \\
0 \\
0 \\
0 \\
0 \\
75 \\
353 \\
569 \\
0 \\
0 \\
306 \\
0 \\
0 \\
0 \\
0 \\
449 \\
0 \\
752 \\
1,764 \\
0 \\
0\end{array}$ & $\begin{array}{r}164 \\
0 \\
0 \\
0 \\
0 \\
0 \\
0 \\
0 \\
0 \\
0 \\
0 \\
0 \\
0 \\
0 \\
0 \\
0 \\
0 \\
0 \\
0 \\
0 \\
0 \\
0 \\
164 \\
0 \\
0\end{array}$ & $\begin{array}{r}3,254 \\
0 \\
0 \\
627 \\
0 \\
1,396 \\
0 \\
0 \\
0 \\
0 \\
0 \\
237 \\
0 \\
190 \\
0 \\
0 \\
0 \\
0 \\
0 \\
0 \\
0 \\
0 \\
804 \\
0 \\
0\end{array}$ & $\begin{array}{r}826 \\
0 \\
0 \\
0 \\
0 \\
4 \\
0 \\
0 \\
0 \\
0 \\
0 \\
0 \\
0 \\
0 \\
0 \\
0 \\
0 \\
0 \\
0 \\
0 \\
0 \\
0 \\
822 \\
0 \\
0\end{array}$ & $\begin{array}{r}3,461 \\
0 \\
0 \\
0 \\
0 \\
1,604 \\
0 \\
0 \\
0 \\
0 \\
0 \\
0 \\
0 \\
187 \\
0 \\
0 \\
0 \\
0 \\
0 \\
0 \\
0 \\
0 \\
1,670 \\
0 \\
0\end{array}$ & $\begin{array}{r}6,807 \\
0 \\
935 \\
221 \\
321 \\
222 \\
0 \\
978 \\
0 \\
0 \\
0 \\
0 \\
0 \\
0 \\
0 \\
1,100 \\
0 \\
256 \\
0 \\
0 \\
651 \\
0 \\
2,123 \\
0 \\
0\end{array}$ & $\begin{array}{r}39 \\
0 \\
0 \\
0 \\
0 \\
39 \\
0 \\
0 \\
0 \\
0 \\
0 \\
0 \\
0 \\
0 \\
0 \\
0 \\
0 \\
0 \\
0 \\
0 \\
0 \\
0 \\
0 \\
0 \\
0\end{array}$ & $\begin{array}{l}9 \\
0 \\
0 \\
0 \\
0 \\
9 \\
0 \\
0 \\
0 \\
0 \\
0 \\
0 \\
0 \\
0 \\
0 \\
0 \\
0 \\
0 \\
0 \\
0 \\
0 \\
0 \\
0 \\
0 \\
0\end{array}$ \\
\hline Total & 38,993 & 720 & 4,448 & 725 & 5,844 & 2,716 & 7,047 & 9,643 & 39 & 9 \\
\hline Persian Gulf ${ }^{d}$ & 3,823 & 0 & 0 & $\mathbf{0}$ & 693 & 0 & 0 & 0 & 0 & 0 \\
\hline
\end{tabular}


Table 36. PAD District —Imports of Crude Oll and Petroleum Products by Country of Origin, March 1993 (Continued) (Thousand Barrels)

\begin{tabular}{|c|c|c|c|c|c|c|c|c|c|c|}
\hline \multirow[b]{2}{*}{ Country of Orlgin } & \multirow[b]{2}{*}{$\begin{array}{c}\text { Nephthe for } \\
\text { Potroohemical } \\
\text { Feedotock } \\
\text { Uee }\end{array}$} & \multirow[b]{2}{*}{$\begin{array}{c}\text { Other Olle for } \\
\text { Petroohemloal } \\
\text { Feodstock } \\
\text { Uee }\end{array}$} & \multirow[b]{2}{*}{ Lubricents } & \multirow[b]{2}{*}{$\begin{array}{c}\text { Asphalt and } \\
\text { Rogd oll }\end{array}$} & \multirow[b]{2}{*}{$\begin{array}{c}\text { Othor } \\
\text { Prodycte }\end{array}$} & \multirow[b]{2}{*}{$\begin{array}{c}\text { Total } \\
\text { Producte }\end{array}$} & \multirow[b]{2}{*}{$\begin{array}{c}\text { Total } \\
\text { Crude Oll } \\
\text { and } \\
\text { Products }\end{array}$} & \multicolumn{3}{|c|}{ Dally Average } \\
\hline & & & & & & & & $\begin{array}{c}\text { Crude } \\
\text { Oll }\end{array}$ & Products & Total \\
\hline $\begin{array}{l}\text { Arab OPEC } \\
\text { Saudi Arabla } \ldots \ldots \ldots \ldots \ldots \ldots \ldots \ldots \ldots \ldots \ldots \ldots \ldots \ldots \ldots \ldots \ldots \ldots \ldots \ldots \ldots\end{array}$ & $\begin{array}{l}0 \\
0\end{array}$ & $\begin{array}{l}0 \\
0\end{array}$ & $\begin{array}{l}0 \\
0\end{array}$ & $\begin{array}{l}0 \\
0\end{array}$ & $\begin{array}{l}0 \\
0\end{array}$ & $\begin{array}{l}893 \\
693\end{array}$ & $\begin{array}{l}4,516 \\
4,516\end{array}$ & $\begin{array}{l}123 \\
123\end{array}$ & $\begin{array}{l}22 \\
22\end{array}$ & $\begin{array}{l}146 \\
146\end{array}$ \\
\hline 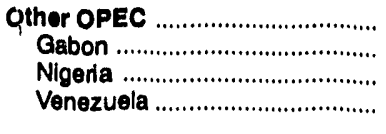 & $\begin{array}{l}0 \\
0 \\
0 \\
0\end{array}$ & $\begin{array}{l}0 \\
0 \\
0 \\
0\end{array}$ & $\begin{array}{l}0 \\
0 \\
0 \\
0\end{array}$ & $\begin{array}{r}434 \\
0 \\
0 \\
434\end{array}$ & $\begin{array}{l}0 \\
0 \\
0 \\
0\end{array}$ & $\begin{array}{r}11,384 \\
104 \\
1,102 \\
10,178\end{array}$ & $\begin{array}{r}31,837 \\
1,032 \\
16,047 \\
14,758\end{array}$ & $\begin{array}{r}660 \\
30 \\
482 \\
148\end{array}$ & $\begin{array}{r}367 \\
3 \\
36 \\
328\end{array}$ & $\begin{array}{r}1,027 \\
33 \\
518 \\
476\end{array}$ \\
\hline 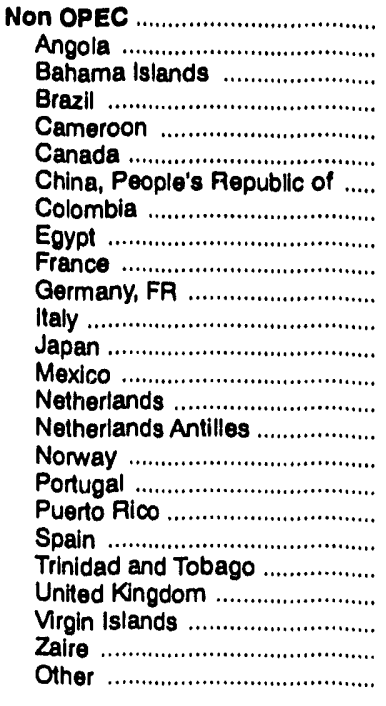 & $\begin{array}{r}68 \\
0 \\
0 \\
0 \\
0 \\
6 \\
0 \\
0 \\
0 \\
0 \\
0 \\
0 \\
0 \\
0 \\
0 \\
0 \\
0 \\
0 \\
60 \\
0 \\
0 \\
0 \\
0 \\
0 \\
0\end{array}$ & $\begin{array}{l}0 \\
0 \\
0 \\
0 \\
0 \\
0 \\
0 \\
0 \\
0 \\
0 \\
0 \\
0 \\
0 \\
0 \\
0 \\
0 \\
0 \\
0 \\
0 \\
0 \\
0 \\
0 \\
0 \\
0 \\
0\end{array}$ & $\begin{array}{r}410 \\
0 \\
0 \\
0 \\
0 \\
41 \\
0 \\
0 \\
0 \\
0 \\
0 \\
0 \\
0 \\
0 \\
0 \\
0 \\
0 \\
0 \\
355 \\
14 \\
0 \\
0 \\
0 \\
0 \\
0\end{array}$ & $\begin{array}{r}382 \\
0 \\
0 \\
0 \\
0 \\
118 \\
0 \\
0 \\
0 \\
0 \\
0 \\
0 \\
0 \\
0 \\
0 \\
84 \\
0 \\
0 \\
0 \\
180 \\
0 \\
0 \\
0 \\
0 \\
0\end{array}$ & $\begin{array}{r}80 \\
0 \\
0 \\
1 \\
0 \\
21 \\
15 \\
0 \\
0 \\
0 \\
1 \\
0 \\
1 \\
0 \\
40 \\
0 \\
0 \\
0 \\
0 \\
0 \\
0 \\
0 \\
0 \\
0 \\
1\end{array}$ & $\begin{array}{r}20,486 \\
0 \\
935 \\
849 \\
321 \\
3,974 \\
15 \\
978 \\
0 \\
75 \\
354 \\
806 \\
1 \\
377 \\
346 \\
1,184 \\
0 \\
256 \\
415 \\
643 \\
651 \\
958 \\
7,347 \\
0 \\
1\end{array}$ & $\begin{array}{r}35,203 \\
5,485 \\
935 \\
849 \\
321 \\
5,973 \\
697 \\
978 \\
728 \\
75 \\
354 \\
806 \\
1 \\
2,255 \\
346 \\
1,184 \\
1,551 \\
256 \\
415 \\
643 \\
651 \\
3,004 \\
7,347 \\
348 \\
1\end{array}$ & $\begin{array}{r}475 \\
177 \\
0 \\
0 \\
0 \\
64 \\
22 \\
0 \\
23 \\
0 \\
0 \\
0 \\
0 \\
61 \\
0 \\
0 \\
50 \\
0 \\
0 \\
0 \\
0 \\
66 \\
0 \\
11 \\
0\end{array}$ & $\begin{array}{r}661 \\
0 \\
30 \\
27 \\
10 \\
128 \\
(8) \\
32 \\
0 \\
2 \\
11 \\
26 \\
(s) \\
12 \\
11 \\
38 \\
0 \\
8 \\
13 \\
21 \\
21 \\
31 \\
237 \\
0 \\
(s)\end{array}$ & $\begin{array}{r}1,136 \\
177 \\
30 \\
27 \\
10 \\
193 \\
22 \\
32 \\
23 \\
2 \\
11 \\
26 \\
(s) \\
73 \\
11 \\
38 \\
50 \\
8 \\
13 \\
21 \\
21 \\
97 \\
237 \\
11 \\
(s)\end{array}$ \\
\hline 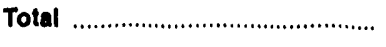 & 66 & 0 & 410 & 816 & 80 & 32,563 & 71,556 & 1,258 & 1,050 & 2,308 \\
\hline Peralan Gulf ${ }^{d}$................................ & 0 & 0 & $\mathbf{0}$ & $\mathbf{0}$ & 0 & 693 & 4,516 & 123 & 22 & 146 \\
\hline
\end{tabular}

a Crude oil and unfinished olls are reported by the PAD District in which they are to be processed; all other products are reported by the PAD District of entry.

b Includes crude oll imported for storage in the Strategic Petroleum Reserve. waxes.

Includes Bahrain, Iran, Iraq, Kuwait, Qatar, Saudl Arabia, and United Arsb Emirates

(s) = Less than 500 barrels or less than 500 barrels p6r day.

Note: Totals may not equal sum of components due to independent rounding

Source: Energy Information Administration (EIA) Form ElA-814, "Monthly Imports Repu.t." 
Table 38. PAD District III-Imports of Crude OII and Petroleum Products by Country of Origin, March 1993

(Thousand Barrels)

\begin{tabular}{|c|c|c|c|c|c|c|c|c|c|c|}
\hline Country of Origin & $\begin{array}{c}\text { Cruda } \\
\text { Oll }\end{array}$ & $\begin{array}{c}\text { Liquefled } \\
\text { Petroleum } \\
\text { Gaese }\end{array}$ & $\begin{array}{c}\text { Unfinishou } \\
\text { Olle }\end{array}$ & $\begin{array}{l}\text { Gasoline } \\
\text { Elending } \\
\text { Compo- } \\
\text { nente } \\
\end{array}$ & $\begin{array}{l}\text { Finisched } \\
\text { Motor } \\
\text { Gasollne }\end{array}$ & Jot Fuel & $\begin{array}{l}\text { Distllate } \\
\text { Fuel Oll }\end{array}$ & $\begin{array}{c}\text { Residual } \\
\text { Fuel Oll }\end{array}$ & Kerosene & $\begin{array}{c}\text { Special } \\
\text { Naphthas }\end{array}$ \\
\hline $\begin{array}{l}\text { Arab OPEC } \\
\text { Algeria } \\
\text { Kuwait } \\
\text { Qatar } \\
\text { Saudi Arabia }\end{array}$ & $\begin{array}{r}42,451 \\
205 \\
7,078 \\
0 \\
35,168\end{array}$ & $\begin{array}{r}654 \\
654 \\
0 \\
0 \\
0\end{array}$ & $\begin{array}{r}3,918 \\
361 \\
0 \\
197 \\
3,360\end{array}$ & $\begin{array}{l}0 \\
0 \\
0 \\
0 \\
0\end{array}$ & $\begin{array}{l}0 \\
0 \\
0 \\
0 \\
0\end{array}$ & $\begin{array}{l}0 \\
0 \\
0 \\
0 \\
0\end{array}$ & $\begin{array}{l}0 \\
0 \\
0 \\
0 \\
0\end{array}$ & $\begin{array}{l}0 \\
0 \\
0 \\
0 \\
0\end{array}$ & $\begin{array}{l}0 \\
0 \\
0 \\
0 \\
0\end{array}$ & $\begin{array}{l}0 \\
0 \\
0 \\
0 \\
0\end{array}$ \\
\hline 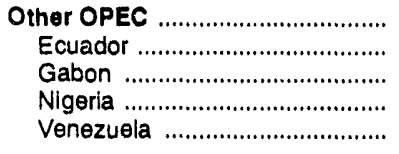 & $\begin{array}{r}28,919 \\
359 \\
2,452 \\
8,936 \\
17,172\end{array}$ & $\begin{array}{r}171 \\
0 \\
0 \\
0 \\
171\end{array}$ & $\begin{array}{r}1,379 \\
0 \\
0 \\
0 \\
1,379\end{array}$ & $\begin{array}{l}0 \\
0 \\
0 \\
0 \\
0\end{array}$ & $\begin{array}{l}0 \\
0 \\
0 \\
0 \\
0\end{array}$ & $\begin{array}{l}0 \\
0 \\
0 \\
0 \\
0\end{array}$ & $\begin{array}{l}0 \\
0 \\
0 \\
0 \\
0\end{array}$ & $\begin{array}{r}238 \\
0 \\
0 \\
0 \\
236\end{array}$ & $\begin{array}{l}0 \\
0 \\
0 \\
0 \\
0\end{array}$ & $\begin{array}{l}0 \\
0 \\
0 \\
0 \\
0\end{array}$ \\
\hline 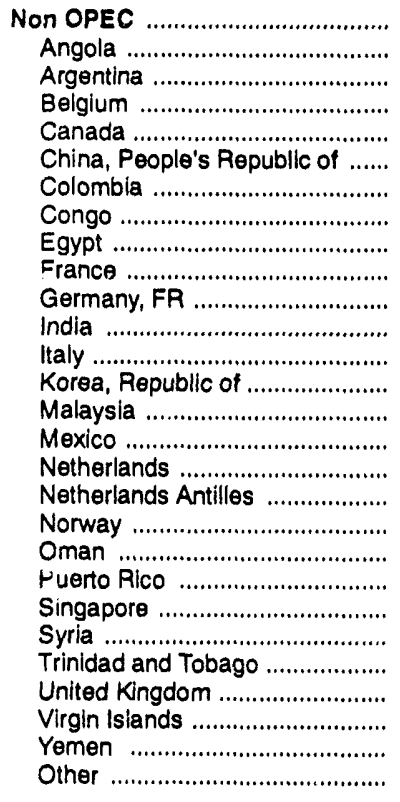 & $\begin{array}{r}40,199 \\
6,275 \\
816 \\
0 \\
0 \\
659 \\
3,502 \\
2,175 \\
702 \\
0 \\
0 \\
0 \\
0 \\
0 \\
324 \\
18,614 \\
0 \\
0 \\
502 \\
0 \\
0 \\
0 \\
600 \\
2,213 \\
3,583 \\
0 \\
0 \\
0\end{array}$ & $\begin{array}{r}24 \\
0 \\
0 \\
0 \\
0 \\
0 \\
0 \\
0 \\
0 \\
20 \\
0 \\
0 \\
0 \\
0 \\
4 \\
0 \\
0 \\
0 \\
0 \\
0 \\
0 \\
0 \\
0 \\
0 \\
0 \\
0 \\
0 \\
0\end{array}$ & $\begin{array}{r}4,646 \\
0 \\
0 \\
0 \\
273 \\
0 \\
0 \\
0 \\
141 \\
112 \\
325 \\
256 \\
517 \\
1,084 \\
0 \\
44 \\
0 \\
629 \\
195 \\
702 \\
0 \\
236 \\
0 \\
0 \\
84 \\
0 \\
48 \\
0\end{array}$ & $\begin{array}{r}893 \\
0 \\
0 \\
0 \\
0 \\
0 \\
0 \\
0 \\
0 \\
0 \\
0 \\
0 \\
0 \\
0 \\
0 \\
693 \\
0 \\
0 \\
0 \\
0 \\
0 \\
0 \\
0 \\
0 \\
0 \\
0 \\
0 \\
0\end{array}$ & $\begin{array}{l}0 \\
0 \\
0 \\
0 \\
0 \\
0 \\
0 \\
0 \\
0 \\
0 \\
0 \\
0 \\
0 \\
0 \\
0 \\
0 \\
0 \\
0 \\
0 \\
0 \\
0 \\
0 \\
0 \\
0 \\
0 \\
0 \\
0 \\
0\end{array}$ & $\begin{array}{r}383 \\
0 \\
0 \\
0 \\
0 \\
0 \\
0 \\
0 \\
141 \\
0 \\
0 \\
0 \\
0 \\
0 \\
0 \\
11 \\
0 \\
0 \\
0 \\
0 \\
0 \\
0 \\
0 \\
0 \\
0 \\
231 \\
0 \\
0\end{array}$ & $\begin{array}{l}0 \\
0 \\
0 \\
0 \\
0 \\
0 \\
0 \\
0 \\
0 \\
0 \\
0 \\
0 \\
0 \\
0 \\
0 \\
0 \\
0 \\
0 \\
0 \\
0 \\
0 \\
0 \\
0 \\
0 \\
0 \\
0 \\
0 \\
0\end{array}$ & $\begin{array}{r}373 \\
0 \\
0 \\
0 \\
0 \\
0 \\
0 \\
0 \\
0 \\
0 \\
373 \\
0 \\
0 \\
0 \\
0 \\
0 \\
0 \\
0 \\
0 \\
0 \\
0 \\
0 \\
0 \\
0 \\
0 \\
0 \\
0 \\
0\end{array}$ & $\begin{array}{l}0 \\
0 \\
0 \\
0 \\
0 \\
0 \\
0 \\
0 \\
0 \\
0 \\
0 \\
0 \\
0 \\
0 \\
0 \\
0 \\
0 \\
0 \\
0 \\
0 \\
0 \\
0 \\
0 \\
0 \\
0 \\
0 \\
0 \\
0\end{array}$ & $\begin{array}{r}131 \\
0 \\
0 \\
0 \\
120 \\
0 \\
0 \\
0 \\
0 \\
0 \\
0 \\
0 \\
11 \\
0 \\
0 \\
0 \\
0 \\
0 \\
0 \\
0 \\
0 \\
0 \\
0 \\
0 \\
0 \\
0 \\
0 \\
0\end{array}$ \\
\hline Total & 111,569 & 849 & 9,943 & 693 & 0 & 383 & 0 & 609 & 0 & 131 \\
\hline Persian Gulf ${ }^{d}$.............................. & 42,248 & $\mathbf{0}$ & 3,557 & 0 & 0 & 0 & 0 & 0 & 0 & 0 \\
\hline
\end{tabular}

See footnotes at end of table. 
Table 38. PAD District III-Imports of Crude Oil and Petroleum Products by Country of Origin, ${ }^{a}$ March 1993 (Continued)

(Thousand Barrels)

\begin{tabular}{|c|c|c|c|c|c|c|c|c|c|c|}
\hline \multirow[b]{2}{*}{ Country of Origin } & \multirow[b]{2}{*}{$\begin{array}{c}\text { Naphtha for } \\
\text { Potrochemical } \\
\text { Foedstock } \\
\text { Use }\end{array}$} & \multirow[b]{2}{*}{$\begin{array}{c}\text { Other Olls for } \\
\text { Petrochemical } \\
\text { Feedstock } \\
\text { Use }\end{array}$} & \multirow[b]{2}{*}{ Lubricants } & \multirow[b]{2}{*}{$\begin{array}{c}\text { Asphalt and } \\
\text { Rogd OII }\end{array}$} & \multirow[b]{2}{*}{$\begin{array}{c}\text { Othar } \\
\text { Products }\end{array}$} & \multirow[b]{2}{*}{$\begin{array}{c}\text { Total } \\
\text { Products }\end{array}$} & \multirow[b]{2}{*}{$\begin{array}{c}\text { Total } \\
\text { Crude OII } \\
\text { and } \\
\text { Products }\end{array}$} & \multicolumn{3}{|c|}{ Dally Average } \\
\hline & & & & & & & & $\begin{array}{c}\text { Crude } \\
\text { Oll } \\
\end{array}$ & Products & Total \\
\hline 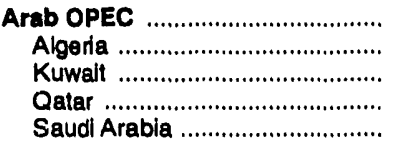 & $\begin{array}{l}0 \\
0 \\
0 \\
0 \\
0\end{array}$ & $\begin{array}{r}4,027 \\
4,027 \\
0 \\
0 \\
0\end{array}$ & $\begin{array}{l}0 \\
0 \\
0 \\
0 \\
0\end{array}$ & $\begin{array}{l}0 \\
0 \\
0 \\
0 \\
0\end{array}$ & $\begin{array}{r}483 \\
483 \\
0 \\
0 \\
0\end{array}$ & $\begin{array}{r}9,082 \\
5,525 \\
0 \\
197 \\
3,360\end{array}$ & $\begin{array}{r}51,533 \\
5,730 \\
7,078 \\
197 \\
38,528\end{array}$ & $\begin{array}{r}1,369 \\
7 \\
228 \\
0 \\
1,134\end{array}$ & $\begin{array}{r}293 \\
178 \\
0 \\
6 \\
108\end{array}$ & $\begin{array}{r}1,662 \\
185 \\
228 \\
6 \\
1,243\end{array}$ \\
\hline 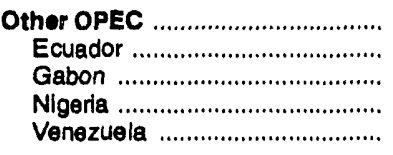 & $\begin{array}{l}0 \\
0 \\
0 \\
0 \\
0\end{array}$ & $\begin{array}{l}0 \\
0 \\
0 \\
0 \\
0\end{array}$ & $\begin{array}{l}0 \\
0 \\
0 \\
0 \\
0\end{array}$ & $\begin{array}{r}35 \\
0 \\
0 \\
0 \\
35\end{array}$ & $\begin{array}{l}0 \\
0 \\
0 \\
0 \\
0\end{array}$ & $\begin{array}{r}1,821 \\
0 \\
0 \\
0 \\
1,821\end{array}$ & $\begin{array}{r}30,740 \\
359 \\
2,452 \\
8,936 \\
18,993\end{array}$ & $\begin{array}{r}933 \\
12 \\
79 \\
288 \\
554\end{array}$ & $\begin{array}{r}59 \\
0 \\
0 \\
0 \\
59\end{array}$ & $\begin{array}{r}992 \\
12 \\
79 \\
288 \\
613\end{array}$ \\
\hline 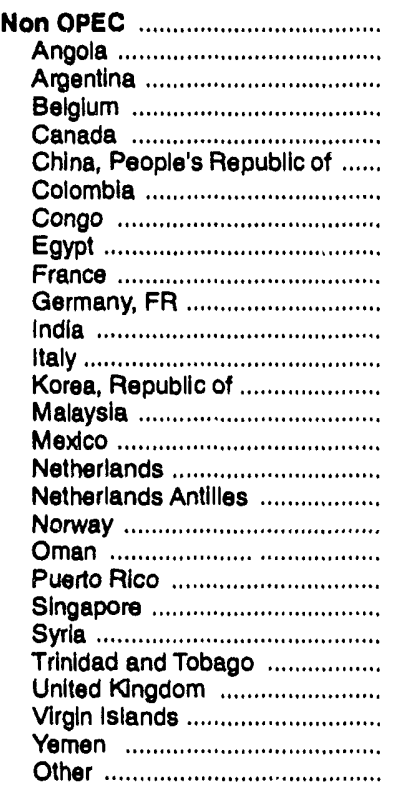 & $\begin{array}{r}880 \\
0 \\
27 \\
22 \\
38 \\
0 \\
0 \\
0 \\
0 \\
40 \\
0 \\
0 \\
12 \\
55 \\
0 \\
0 \\
7 \\
68 \\
0 \\
0 \\
398 \\
0 \\
0 \\
0 \\
22 \\
0 \\
191 \\
0\end{array}$ & $\begin{array}{r}449 \\
0 \\
180 \\
0 \\
0 \\
0 \\
0 \\
0 \\
0 \\
0 \\
0 \\
0 \\
0 \\
0 \\
0 \\
0 \\
0 \\
0 \\
0 \\
0 \\
0 \\
0 \\
0 \\
0 \\
0 \\
0 \\
0 \\
269\end{array}$ & $\begin{array}{l}0 \\
0 \\
0 \\
0 \\
0 \\
0 \\
0 \\
0 \\
0 \\
0 \\
0 \\
0 \\
0 \\
0 \\
0 \\
0 \\
0 \\
0 \\
0 \\
0 \\
0 \\
0 \\
0 \\
0 \\
0 \\
0 \\
0 \\
0\end{array}$ & $\begin{array}{l}0 \\
0 \\
0 \\
0 \\
0 \\
0 \\
0 \\
0 \\
0 \\
0 \\
0 \\
0 \\
0 \\
0 \\
0 \\
0 \\
0 \\
0 \\
0 \\
0 \\
0 \\
0 \\
0 \\
0 \\
0 \\
0 \\
0 \\
0\end{array}$ & $\begin{array}{r}479 \\
0 \\
181 \\
0 \\
0 \\
0 \\
0 \\
0 \\
2 \\
0 \\
2 \\
0 \\
0 \\
0 \\
0 \\
292 \\
0 \\
0 \\
0 \\
0 \\
0 \\
0 \\
0 \\
0 \\
0 \\
0 \\
0 \\
2\end{array}$ & $\begin{array}{r}8,058 \\
0 \\
388 \\
22 \\
431 \\
0 \\
0 \\
0 \\
284 \\
172 \\
700 \\
256 \\
540 \\
1,139 \\
4 \\
1,040 \\
7 \\
697 \\
195 \\
702 \\
398 \\
236 \\
0 \\
0 \\
106 \\
231 \\
239 \\
271\end{array}$ & $\begin{array}{r}48,257 \\
6,275 \\
1,204 \\
22 \\
431 \\
659 \\
3,502 \\
2,175 \\
986 \\
172 \\
700 \\
256 \\
540 \\
1,139 \\
328 \\
19,654 \\
7 \\
697 \\
697 \\
702 \\
398 \\
236 \\
600 \\
2,213 \\
3,689 \\
231 \\
239 \\
271\end{array}$ & $\begin{array}{r}1,297 \\
202 \\
26 \\
0 \\
0 \\
21 \\
113 \\
70 \\
23 \\
0 \\
0 \\
0 \\
0 \\
0 \\
10 \\
600 \\
0 \\
0 \\
16 \\
0 \\
0 \\
0 \\
19 \\
71 \\
116 \\
0 \\
0 \\
0\end{array}$ & $\begin{array}{r}260 \\
0 \\
13 \\
1 \\
14 \\
0 \\
0 \\
0 \\
9 \\
6 \\
23 \\
8 \\
17 \\
37 \\
(5) \\
34 \\
(5) \\
22 \\
6 \\
23 \\
13 \\
8 \\
0 \\
0 \\
3 \\
7 \\
8 \\
9\end{array}$ & $\begin{array}{r}1,557 \\
202 \\
39 \\
1 \\
14 \\
21 \\
113 \\
70 \\
32 \\
6 \\
23 \\
8 \\
17 \\
37 \\
11 \\
634 \\
(s) \\
22 \\
22 \\
23 \\
13 \\
8 \\
19 \\
71 \\
119 \\
7 \\
8 \\
9\end{array}$ \\
\hline Total & 880 & 4,476 & 0 & 35 & 962 & 18,961 & 130,530 & 3,599 & 612 & 4,211 \\
\hline Perslan Gulf ${ }^{d}$ & $\mathbf{0}$ & 269 & 0 & 0 & 0 & 3,826 & 46,072 & 1,363 & 123 & 1,486 \\
\hline
\end{tabular}

a Crude oll and unfinished olls are reported by the PAD District in which they are to be processed; all other products are reported by the PAD District of entry

Includes crude oll imported for storage in the Strategic Petroleum Reserve.

c Includes aviation gasoline, aviation gasoline blending components, miscellaneous products, other hydrocarbons and oxygenates, pentanes plus, petroleum coke, and waxes.

Includes Bahrain, Iran, Iraq, Kuwait, Qatar, Saudi Arable, and United Arab Emirates.

$(s)=$ Less than 500 barrels or less than 500 barrels per day.

Note: Totals may not equal sum of components due to independent rounding.

Source: Energy information Administration (EIA) Form EIA-814, "Monthly Imports Report." 
Table 39. PAD Districts IV and V-Imports of Crude Oll and Petroleum Products by Country of Origin, March 1993

(Thousand Barrels)

\begin{tabular}{|c|c|c|c|c|c|c|c|c|c|c|}
\hline Country of Origln & $\begin{array}{c}\text { Crude } \\
\text { OII }\end{array}$ & $\begin{array}{c}\text { Liquoflod } \\
\text { Potroloum } \\
\text { Geses }\end{array}$ & $\begin{array}{c}\text { Unilnished } \\
\text { Olls }\end{array}$ & $\begin{array}{c}\text { Gasoline } \\
\text { Blending } \\
\text { Compo- } \\
\text { nente }\end{array}$ & $\begin{array}{l}\text { FInlahed } \\
\text { Motor } \\
\text { Gacollne }\end{array}$ & Jet Fuel & $\begin{array}{l}\text { Diatliate } \\
\text { Fuel OII }\end{array}$ & $\begin{array}{l}\text { Rosldual } \\
\text { Fuol Oll }\end{array}$ & Kerosene & $\begin{array}{c}\text { Speclal } \\
\text { Naphthas }\end{array}$ \\
\hline & \multicolumn{10}{|c|}{ PAD Dlotriet IV } \\
\hline $\begin{array}{r}\text { Non OPEC } \\
\text { Canada } \ldots \ldots \ldots \ldots \ldots \ldots \ldots \ldots \ldots \ldots \ldots \ldots \ldots\end{array}$ & $\begin{array}{l}2,649 \\
2,649\end{array}$ & $\begin{array}{l}183 \\
183\end{array}$ & $\begin{array}{l}0 \\
0\end{array}$ & $\begin{array}{l}0 \\
0\end{array}$ & $\begin{array}{l}11 \\
11\end{array}$ & $\begin{array}{l}0 \\
0\end{array}$ & $\begin{array}{l}52 \\
52\end{array}$ & $\begin{array}{l}0 \\
0\end{array}$ & $\begin{array}{l}0 \\
0\end{array}$ & $\begin{array}{l}0 \\
0\end{array}$ \\
\hline 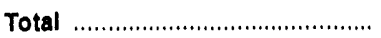 & 2,649 & 183 & 0 & 0 & 11 & 0 & $\mathbf{5 2}$ & 0 & 0 & 0 \\
\hline
\end{tabular}

PAD Dletrlet V

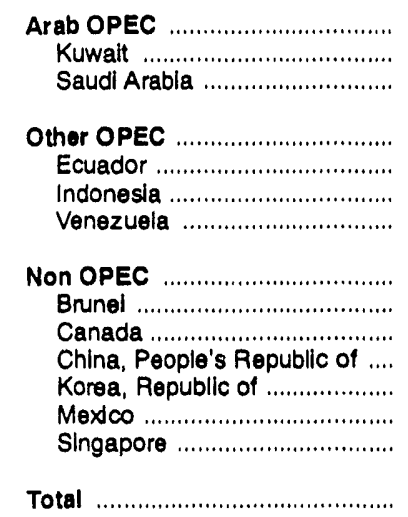

Persian Gulf ${ }^{d}$

PAD DIstrictV

\begin{tabular}{rrrrrrrrrr}
\hline 1,018 & 0 & 0 & 0 & 0 & 1 & 0 & 498 & 0 & 0 \\
1,018 & 0 & 0 & 0 & 0 & 0 & 0 & 498 & 0 & 0 \\
0 & 0 & 0 & 0 & 0 & 1 & 0 & 0 & 0 & 0 \\
3,097 & 0 & 0 & 0 & 0 & 0 & 0 & 100 & 0 & 0 \\
1,111 & 0 & 0 & 0 & 0 & 0 & 0 & 0 & 0 & 0 \\
1,986 & 0 & 0 & 0 & 0 & 0 & 0 & 100 & 0 & 0 \\
0 & 0 & 0 & 0 & 0 & 0 & 0 & 0 & 0 & 0 \\
2,024 & 16 & 880 & 36 & 228 & 3 & 77 & 67 & 0 & 8 \\
155 & 0 & 0 & 0 & 0 & 0 & 0 & 0 & 0 & 0 \\
933 & 16 & 0 & 0 & 64 & 3 & 77 & 67 & 0 & 8 \\
936 & 0 & 0 & 0 & 162 & 0 & 0 & 0 & 0 & 0 \\
0 & 0 & 0 & 36 & 0 & 0 & 0 & 0 & 0 & 0 \\
0 & 0 & 0 & 0 & 0 & 0 & 0 & 0 & 0 & 0 \\
0 & 0 & 880 & 0 & 0 & 0 & 0 & 0 & 0 & 0 \\
6,139 & 16 & 880 & 36 & 228 & 4 & 77 & 665 & 0 & 8 \\
1,018 & 0 & 0 & 0 & 0 & 1 & 0 & 498 & 0 & 0
\end{tabular}

See forthotes at end of table. 
Table 39. PAD Districts IV and V-Imports of Crude Oil and Petroleum Products by Country of Origin, ${ }^{a}$ March 1993 (Continued)

(Thousand Barrels)

\begin{tabular}{|c|c|c|c|c|c|c|c|c|c|c|}
\hline & & & & & & & & & ally Averag & \\
\hline Country of Origln & $\begin{array}{c}\text { Naphtha for } \\
\text { Potrochemlcai } \\
\text { Feedstock } \\
\text { Use }\end{array}$ & $\begin{array}{c}\text { Other Olls for } \\
\text { Petrochemical } \\
\text { Feedstock } \\
\text { Use }\end{array}$ & Lubricants & $\begin{array}{c}\text { Asphalt and } \\
\text { Road OII }\end{array}$ & $\begin{array}{c}\text { Other } \\
\text { Productse }\end{array}$ & $\begin{array}{c}\text { Total } \\
\text { Products }\end{array}$ & $\begin{array}{l}\text { Total } \\
\text { Crude Oll } \\
\text { and } \\
\text { Products }\end{array}$ & $\begin{array}{c}\text { Crude } \\
\text { Oll }\end{array}$ & Products & Total \\
\hline
\end{tabular}

PAD Dlstrlct IV

\begin{tabular}{|c|c|c|c|c|c|c|c|c|c|c|}
\hline 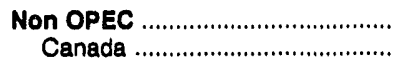 & $\begin{array}{l}0 \\
0\end{array}$ & $\begin{array}{l}0 \\
0\end{array}$ & $\begin{array}{l}0 \\
0\end{array}$ & $\begin{array}{l}0 \\
0\end{array}$ & $\begin{array}{l}82 \\
82\end{array}$ & $\begin{array}{l}328 \\
328\end{array}$ & $\begin{array}{l}2,977 \\
2,977\end{array}$ & $\begin{array}{l}85 \\
85\end{array}$ & $\begin{array}{l}11 \\
11\end{array}$ & $\begin{array}{l}96 \\
96\end{array}$ \\
\hline , & 0 & 0 & 0 & 0 & 82 & 328 & 2,977 & 85 & 11 & 96 \\
\hline
\end{tabular}

PAD Dlatrict V

\begin{tabular}{|c|c|c|c|c|c|c|c|c|c|c|}
\hline 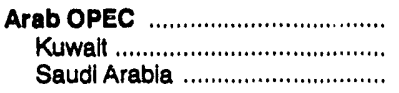 & $\begin{array}{l}0 \\
0 \\
0\end{array}$ & $\begin{array}{l}0 \\
0 \\
0\end{array}$ & $\begin{array}{l}0 \\
0 \\
0\end{array}$ & $\begin{array}{l}0 \\
0 \\
0\end{array}$ & $\begin{array}{l}0 \\
0 \\
0\end{array}$ & $\begin{array}{r}499 \\
498 \\
1\end{array}$ & $\begin{array}{r}1,517 \\
1,516 \\
1\end{array}$ & $\begin{array}{r}33 \\
33 \\
0\end{array}$ & $\begin{array}{l}16 \\
16 \\
(s)\end{array}$ & $\begin{array}{l}49 \\
49 \\
\text { (s) }\end{array}$ \\
\hline 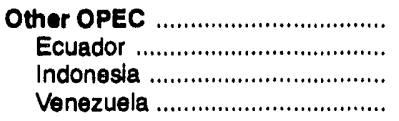 & $\begin{array}{l}0 \\
0 \\
0 \\
0\end{array}$ & $\begin{array}{l}0 \\
0 \\
0 \\
0\end{array}$ & $\begin{array}{l}0 \\
0 \\
0 \\
0\end{array}$ & $\begin{array}{l}0 \\
0 \\
0 \\
0\end{array}$ & $\begin{array}{r}120 \\
0 \\
0 \\
120\end{array}$ & $\begin{array}{r}220 \\
0 \\
100 \\
120\end{array}$ & $\begin{array}{r}3,317 \\
1,111 \\
2,086 \\
120\end{array}$ & $\begin{array}{r}100 \\
36 \\
64 \\
0\end{array}$ & $\begin{array}{l}7 \\
0 \\
3 \\
4\end{array}$ & $\begin{array}{r}107 \\
36 \\
67 \\
4\end{array}$ \\
\hline 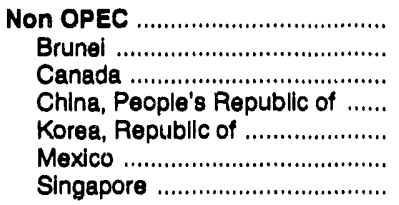 & $\begin{array}{l}0 \\
0 \\
0 \\
0 \\
0 \\
0 \\
0\end{array}$ & $\begin{array}{l}0 \\
0 \\
0 \\
0 \\
0 \\
0 \\
0\end{array}$ & $\begin{array}{l}0 \\
0 \\
0 \\
0 \\
0 \\
0 \\
0\end{array}$ & $\begin{array}{r}13 \\
0 \\
13 \\
0 \\
0 \\
0 \\
0\end{array}$ & $\begin{array}{r}271 \\
0 \\
269 \\
0 \\
0 \\
2 \\
0\end{array}$ & $\begin{array}{r}1,597 \\
0 \\
517 \\
162 \\
36 \\
2 \\
880\end{array}$ & $\begin{array}{r}3,621 \\
155 \\
1,450 \\
1,098 \\
36 \\
2 \\
880\end{array}$ & $\begin{array}{r}65 \\
5 \\
30 \\
30 \\
0 \\
0 \\
0\end{array}$ & $\begin{array}{r}52 \\
0 \\
17 \\
5 \\
1 \\
(s) \\
28\end{array}$ & $\begin{array}{r}117 \\
5 \\
47 \\
35 \\
1 \\
\text { (s) } \\
28\end{array}$ \\
\hline Total & 0 & 0 & 0 & 13 & 391 & 2,316 & 8,455 & 198 & 75 & 273 \\
\hline Porblan Qulf ${ }^{d}$............................. & 0 & 0 & 0 & 0 & 0 & 499 & 1,517 & 33 & 16 & 49 \\
\hline
\end{tabular}

a Crude oll and unflnished oils are reported by the PAD District in which they are to be processed; all other products are reported by the PAD District of entry.

b Includes crude oll imported for storage in the Strategic Petroleum Reserve.

c Includes aviation gasoline, aviation gasoline blending components, miscellaneuus products, other hydrocarbons and oxygenates, pentanes plus, petroleum coke, and waxes.

'Includes Bahrain, Iran, Iraq, Kuwalt, Qatar, Saudi Arabia, and United Arab Emirates.

(s) = Less than 500 barrels or less than 500 barrels per day. '

Note: Totais may not equal sum of components due to independent rounding.

Source: Energy Information Administration (E|A) Form EIA-814, "Monthly Imports Report." 
Table 40. Year-to-Date Imports of Crude Oil and Petroleum Products Into the United States by Country of Origin," January-March 1993 (Thousand Barrels)

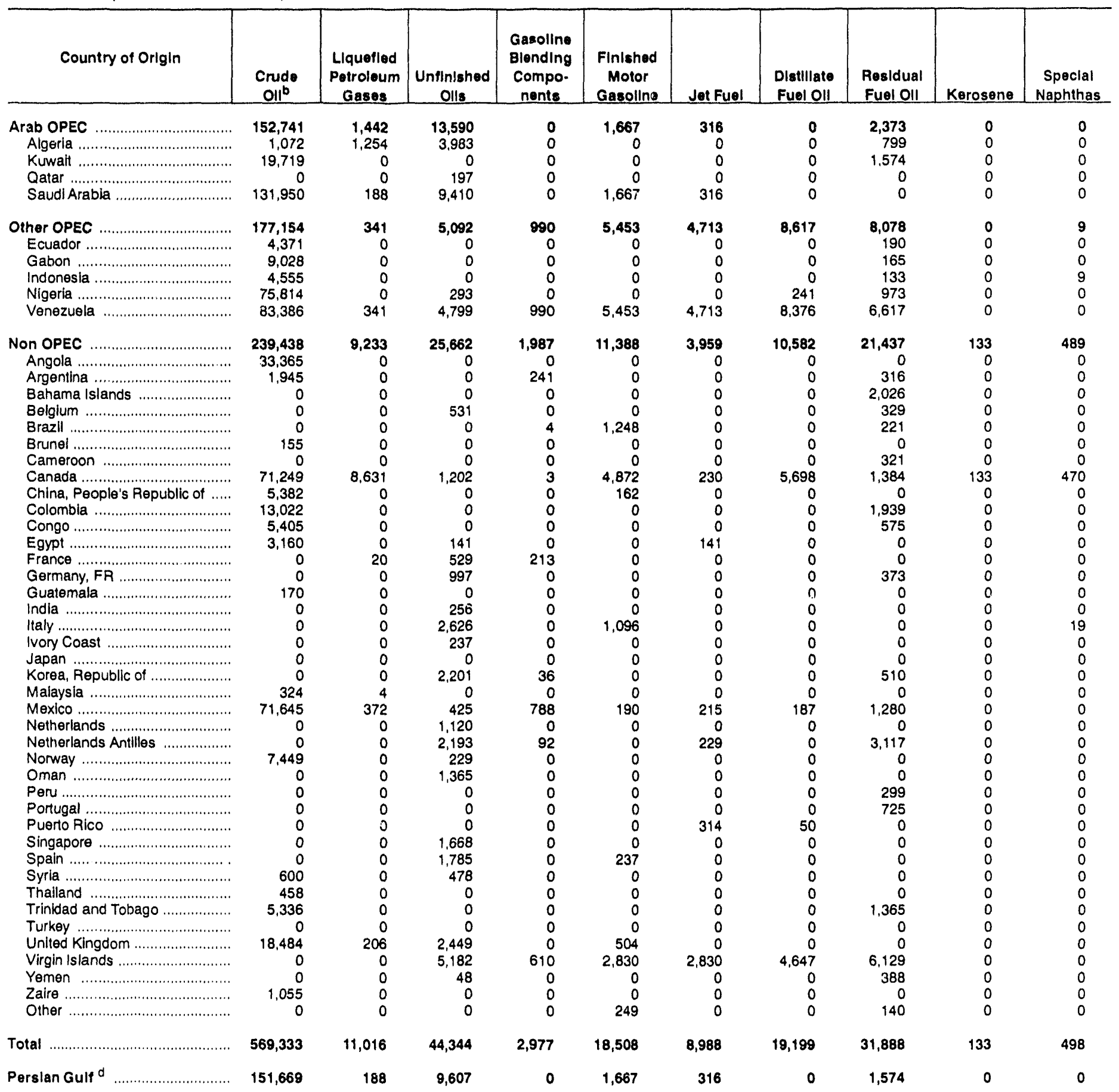


Table 40. Year-to-Date Imports of Crude Oil and Petroleum Products Into the United States by Country of

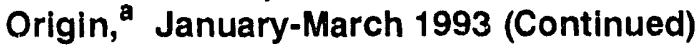
(Thousand Barrels)

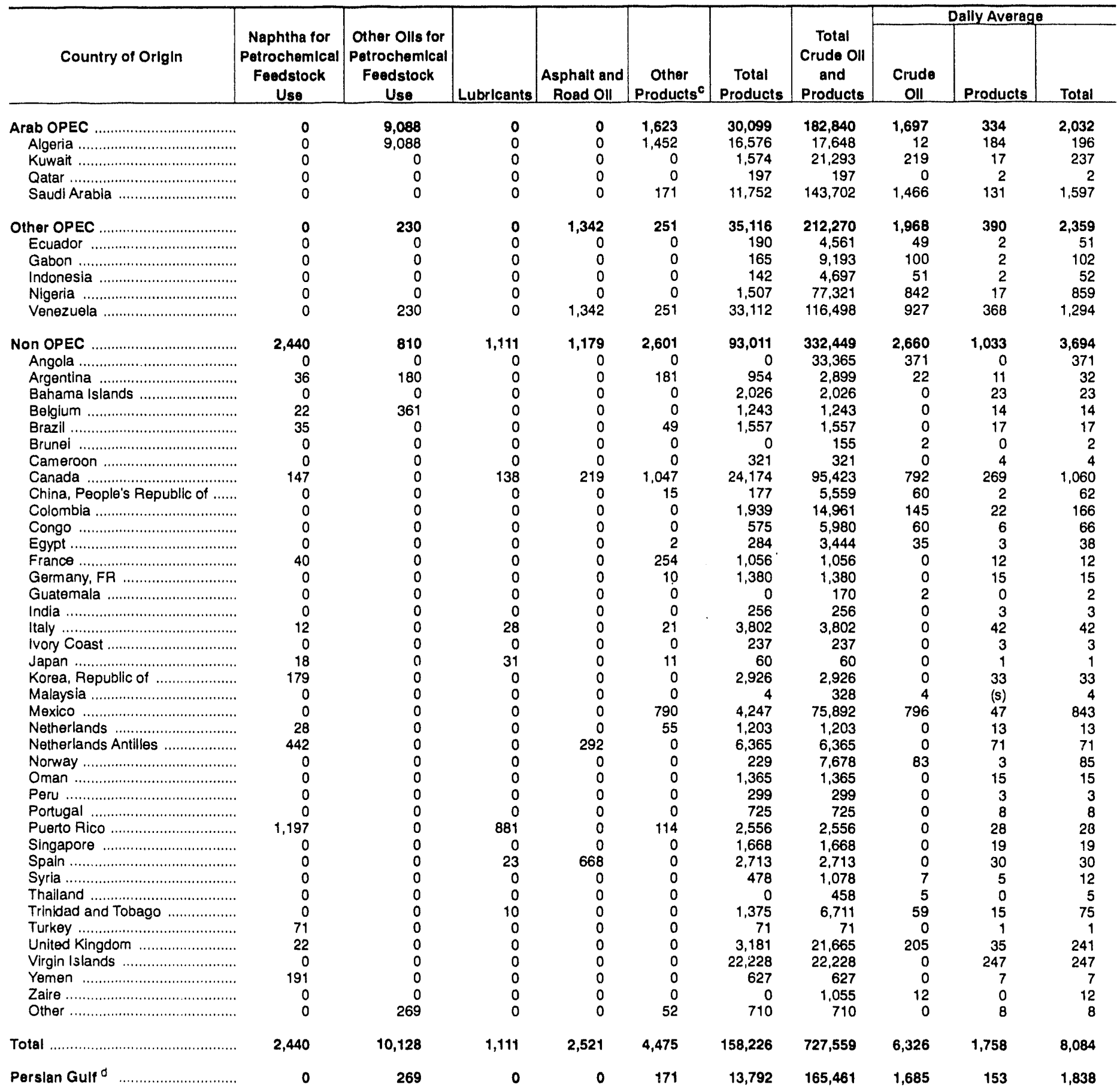

a Crude oil and unfinished oils are reported by the PAD District in which they are to be processed; all other products are reported by the PAD District of entry

b Includes crude oil imported for storage in the Strategic Petroleum Reserve.

c Includes aviation gasoline, aviation gasoline blending components, miscellaneous products, other hydrocarbons and oxygenates, pentanes plus, petroleum coke, and waxes.

Includes Bahrain, Iran, Iraq, Kuwait, Qatar, Saudi Arabia, and United Arab Emirates.

(s) = Less than 500 barrels or less than 500 barrels per day.

Note: Totals may not equal sum of components due to independent rounding.

Source: Energy Information Administration (EIA) Form EIA-814, "Monthly Imports Report." 
Table 41. PAD District I-Year-to-Date Imports of Crude Oll and Petroleum Products by Country of Origin, ${ }^{\text {a }}$ January-March 1993 (Thousand Barrels)

\begin{tabular}{|c|c|c|c|c|c|c|c|c|c|c|}
\hline Country of Orlgin & $\begin{array}{c}\text { Crude } \\
\text { Oll }\end{array}$ & $\begin{array}{c}\text { Llquefled } \\
\text { Potroleum } \\
\text { Gases }\end{array}$ & $\begin{array}{c}\text { Unflnished } \\
\text { Olls }\end{array}$ & $\begin{array}{l}\text { Gasoline } \\
\text { Blending } \\
\text { Compo- } \\
\text { nents }\end{array}$ & $\begin{array}{l}\text { Finiahed } \\
\text { Motor } \\
\text { Gasollne }\end{array}$ & Jet Fuol & $\begin{array}{l}\text { Dlatiliate } \\
\text { Fuol OII }\end{array}$ & $\begin{array}{l}\text { Resldual } \\
\text { Fuel OII }\end{array}$ & Kerosene & $\begin{array}{c}\text { Special } \\
\text { Naphthas }\end{array}$ \\
\hline 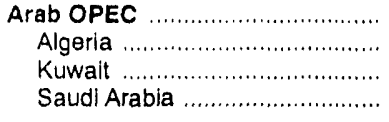 & $\begin{array}{r}12,639 \\
0 \\
602 \\
12,037\end{array}$ & $\begin{array}{r}424 \\
236 \\
0 \\
188\end{array}$ & $\begin{array}{r}1,089 \\
1,089 \\
0 \\
0\end{array}$ & $\begin{array}{l}0 \\
0 \\
0 \\
0\end{array}$ & $\begin{array}{r}1,667 \\
0 \\
0 \\
1,667\end{array}$ & $\begin{array}{r}315 \\
0 \\
0 \\
315\end{array}$ & $\begin{array}{l}0 \\
0 \\
0 \\
0\end{array}$ & $\begin{array}{r}990 \\
799 \\
191 \\
0\end{array}$ & $\begin{array}{l}0 \\
0 \\
0 \\
0\end{array}$ & $\begin{array}{l}0 \\
0 \\
0 \\
0\end{array}$ \\
\hline 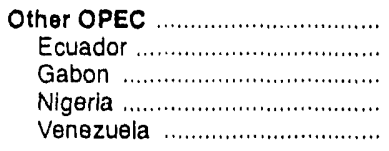 & $\begin{array}{r}54,512 \\
377 \\
1,924 \\
39,911 \\
12,300\end{array}$ & $\begin{array}{r}170 \\
0 \\
0 \\
0 \\
170\end{array}$ & $\begin{array}{r}397 \\
0 \\
0 \\
180 \\
217\end{array}$ & $\begin{array}{r}990 \\
0 \\
0 \\
0 \\
990\end{array}$ & $\begin{array}{r}5,453 \\
0 \\
0 \\
0 \\
5,453\end{array}$ & $\begin{array}{r}4,713 \\
0 \\
0 \\
0 \\
4,713\end{array}$ & $\begin{array}{r}8,617 \\
0 \\
0 \\
241 \\
8,376\end{array}$ & $\begin{array}{r}6,955 \\
190 \\
165 \\
681 \\
5,919\end{array}$ & $\begin{array}{l}0 \\
0 \\
0 \\
0 \\
0\end{array}$ & $\begin{array}{l}0 \\
0 \\
0 \\
0 \\
0\end{array}$ \\
\hline 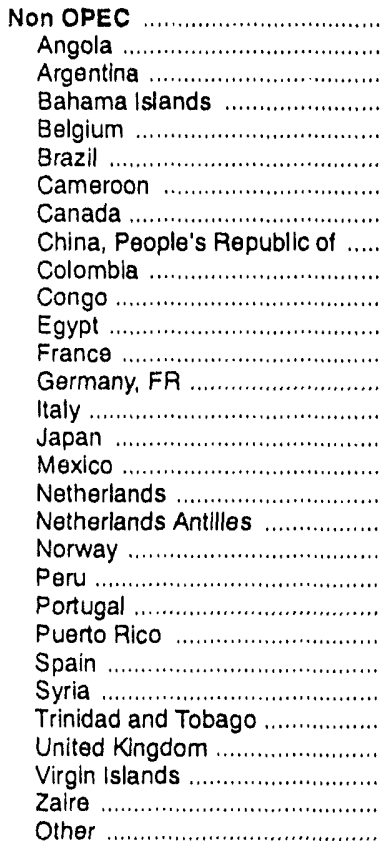 & $\begin{array}{r}39,112 \\
13,481 \\
0 \\
0 \\
0 \\
0 \\
0 \\
5,585 \\
1,331 \\
1,011 \\
0 \\
1,415 \\
0 \\
0 \\
0 \\
0 \\
5,334 \\
0 \\
0 \\
3,760 \\
0 \\
0 \\
0 \\
0 \\
0 \\
546 \\
5,594 \\
0 \\
1,055 \\
0\end{array}$ & $\begin{array}{r}1,759 \\
0 \\
0 \\
0 \\
0 \\
0 \\
0 \\
1,553 \\
0 \\
0 \\
0 \\
0 \\
0 \\
0 \\
0 \\
0 \\
0 \\
0 \\
0 \\
0 \\
0 \\
0 \\
0 \\
0 \\
0 \\
0 \\
206 \\
0 \\
0 \\
0\end{array}$ & $\begin{array}{r}12,640 \\
0 \\
0 \\
0 \\
0 \\
0 \\
0 \\
483 \\
0 \\
0 \\
0 \\
0 \\
417 \\
353 \\
1,131 \\
0 \\
0 \\
306 \\
601 \\
0 \\
0 \\
0 \\
0 \\
1,566 \\
236 \\
0 \\
2,365 \\
5,182 \\
0 \\
0\end{array}$ & $\begin{array}{r}1,064 \\
0 \\
241 \\
0 \\
0 \\
0 \\
0 \\
0 \\
0 \\
0 \\
0 \\
0 \\
213 \\
0 \\
0 \\
0 \\
0 \\
0 \\
0 \\
0 \\
0 \\
0 \\
0 \\
0 \\
0 \\
0 \\
0 \\
610 \\
0 \\
0\end{array}$ & $\begin{array}{r}9,803 \\
0 \\
0 \\
0 \\
0 \\
1,248 \\
0 \\
3,906 \\
0 \\
0 \\
0 \\
0 \\
0 \\
0 \\
1,096 \\
0 \\
190 \\
0 \\
0 \\
0 \\
0 \\
0 \\
0 \\
237 \\
0 \\
0 \\
504 \\
2,373 \\
0 \\
249\end{array}$ & $\begin{array}{r}2,803 \\
0 \\
0 \\
0 \\
0 \\
0 \\
0 \\
4 \\
0 \\
0 \\
0 \\
0 \\
0 \\
0 \\
0 \\
0 \\
0 \\
0 \\
117 \\
0 \\
0 \\
0 \\
314 \\
0 \\
0 \\
0 \\
0 \\
2,368 \\
0 \\
0\end{array}$ & $\begin{array}{r}9,434 \\
0 \\
0 \\
0 \\
0 \\
0 \\
0 \\
4,550 \\
0 \\
0 \\
0 \\
0 \\
0 \\
0 \\
0 \\
0 \\
187 \\
0 \\
0 \\
0 \\
0 \\
0 \\
50 \\
0 \\
0 \\
0 \\
0 \\
4,647 \\
0 \\
0\end{array}$ & $\begin{array}{r}17,778 \\
0 \\
316 \\
2,026 \\
329 \\
221 \\
321 \\
1,186 \\
0 \\
1,939 \\
575 \\
0 \\
0 \\
0 \\
0 \\
0 \\
0 \\
0 \\
3,117 \\
0 \\
299 \\
256 \\
0 \\
0 \\
0 \\
1,365 \\
0 \\
5,828 \\
0 \\
0\end{array}$ & $\begin{array}{r}133 \\
0 \\
0 \\
0 \\
0 \\
0 \\
0 \\
133 \\
0 \\
0 \\
0 \\
0 \\
0 \\
0 \\
0 \\
0 \\
0 \\
0 \\
0 \\
0 \\
0 \\
0 \\
0 \\
0 \\
0 \\
0 \\
0 \\
0 \\
0 \\
0\end{array}$ & $\begin{array}{r}28 \\
0 \\
0 \\
0 \\
0 \\
0 \\
0 \\
24 \\
0 \\
0 \\
0 \\
0 \\
0 \\
0 \\
4 \\
0 \\
0 \\
0 \\
0 \\
0 \\
0 \\
0 \\
0 \\
0 \\
0 \\
0 \\
0 \\
0 \\
0 \\
0\end{array}$ \\
\hline 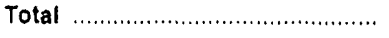 & 106,263 & 2,353 & 14,126 & 2,054 & 16,923 & 7,831 & 18,051 & 25,723 & 133 & 28 \\
\hline 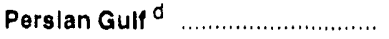 & 12,639 & 188 & 0 & 0 & 1,667 & 315 & 0 & 181 & 0 & 0 \\
\hline
\end{tabular}


Table 41. PAD District —Year-to-Date Imports of Crude Oll and Petroleum Products by Country of Origin, ${ }^{a}$ January-March 1993 (Continued) (Thousand Barrels)

\begin{tabular}{|c|c|c|c|c|c|c|c|c|c|c|}
\hline \multirow[b]{2}{*}{ Country of Origin } & \multirow[b]{2}{*}{$\begin{array}{c}\text { Naphtha for } \\
\text { Petrochemical } \\
\text { Feedstock } \\
\text { Use }\end{array}$} & \multirow[b]{2}{*}{$\begin{array}{c}\text { Other Olts for } \\
\text { Petrochemical } \\
\text { Feedstock } \\
\text { Use }\end{array}$} & \multirow[b]{2}{*}{ Lubricants } & \multirow[b]{2}{*}{$\begin{array}{c}\text { Asphalt and } \\
\text { Road Oll }\end{array}$} & \multirow[b]{2}{*}{$\begin{array}{c}\text { Other } \\
\text { Products }\end{array}$} & \multirow[b]{2}{*}{$\begin{array}{c}\text { Total } \\
\text { Products }\end{array}$} & \multirow[b]{2}{*}{$\begin{array}{l}\text { Total } \\
\text { Crude OlI } \\
\text { and } \\
\text { Products }\end{array}$} & \multicolumn{3}{|c|}{ Dally Average } \\
\hline & & & & & & & & $\begin{array}{c}\text { Crude } \\
\text { OII }\end{array}$ & Products & Total \\
\hline 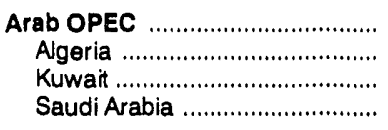 & $\begin{array}{l}0 \\
0 \\
0 \\
0\end{array}$ & $\begin{array}{l}0 \\
0 \\
0 \\
0\end{array}$ & $\begin{array}{l}0 \\
0 \\
0 \\
0\end{array}$ & $\begin{array}{l}0 \\
0 \\
0 \\
0\end{array}$ & $\begin{array}{r}113 \\
0 \\
0 \\
113\end{array}$ & $\begin{array}{r}4,598 \\
2,124 \\
191 \\
2,283\end{array}$ & $\begin{array}{r}17,237 \\
2,124 \\
793 \\
14,320\end{array}$ & $\begin{array}{r}140 \\
0 \\
7 \\
134\end{array}$ & $\begin{array}{r}51 \\
24 \\
2 \\
25\end{array}$ & $\begin{array}{r}192 \\
24 \\
9 \\
159\end{array}$ \\
\hline 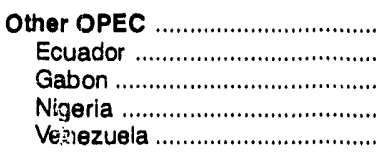 & $\begin{array}{l}0 \\
0 \\
0 \\
0 \\
0\end{array}$ & $\begin{array}{l}0 \\
0 \\
0 \\
0 \\
0\end{array}$ & $\begin{array}{l}0 \\
0 \\
0 \\
0 \\
0\end{array}$ & $\begin{array}{r}1,277 \\
0 \\
0 \\
0 \\
1,277\end{array}$ & $\begin{array}{l}0 \\
0 \\
0 \\
0 \\
0\end{array}$ & $\begin{array}{r}28,572 \\
190 \\
165 \\
1,102 \\
27,115\end{array}$ & $\begin{array}{r}83,084 \\
567 \\
2,089 \\
41,013 \\
39,415\end{array}$ & $\begin{array}{r}606 \\
4 \\
21 \\
443 \\
137\end{array}$ & $\begin{array}{r}317 \\
2 \\
2 \\
12 \\
301\end{array}$ & $\begin{array}{r}923 \\
6 \\
23 \\
456 \\
438\end{array}$ \\
\hline 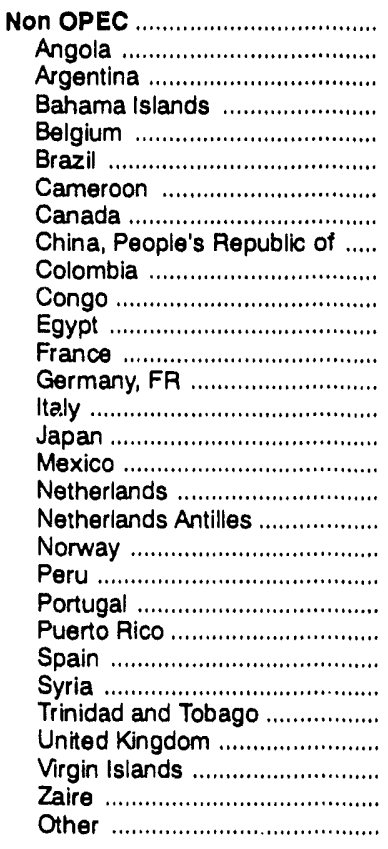 & $\begin{array}{r}333 \\
0 \\
0 \\
0 \\
0 \\
0 \\
0 \\
29 \\
0 \\
0 \\
0 \\
0 \\
0 \\
0 \\
0 \\
11 \\
0 \\
0 \\
0 \\
0 \\
0 \\
0 \\
293 \\
0 \\
0 \\
0 \\
0 \\
0 \\
0 \\
0\end{array}$ & $\begin{array}{l}0 \\
0 \\
0 \\
0 \\
0 \\
0 \\
0 \\
0 \\
0 \\
0 \\
0 \\
0 \\
0 \\
0 \\
0 \\
0 \\
0 \\
0 \\
0 \\
0 \\
0 \\
0 \\
0 \\
0 \\
0 \\
0 \\
0 \\
0 \\
0 \\
0\end{array}$ & $\begin{array}{r}1,030 \\
0 \\
0 \\
0 \\
0 \\
0 \\
0 \\
98 \\
0 \\
0 \\
0 \\
0 \\
0 \\
0 \\
28 \\
0 \\
0 \\
0 \\
0 \\
0 \\
0 \\
0 \\
881 \\
23 \\
0 \\
0 \\
0 \\
0 \\
0 \\
0\end{array}$ & $\begin{array}{r}1,079 \\
0 \\
0 \\
0 \\
0 \\
0 \\
0 \\
189 \\
0 \\
0 \\
0 \\
0 \\
0 \\
0 \\
0 \\
0 \\
0 \\
0 \\
292 \\
0 \\
0 \\
0 \\
0 \\
598 \\
0 \\
0 \\
0 \\
0 \\
0 \\
0\end{array}$ & $\begin{array}{r}573 \\
0 \\
0 \\
0 \\
0 \\
49 \\
0 \\
44 \\
15 \\
0 \\
0 \\
0 \\
254 \\
7 \\
21 \\
11 \\
0 \\
55 \\
0 \\
0 \\
0 \\
0 \\
114 \\
0 \\
0 \\
0 \\
0 \\
0 \\
0 \\
3\end{array}$ & $\begin{array}{r}58,457 \\
0 \\
557 \\
2,026 \\
329 \\
1,518 \\
321 \\
12,199 \\
15 \\
1,939 \\
575 \\
0 \\
884 \\
360 \\
2,280 \\
22 \\
377 \\
361 \\
4,127 \\
0 \\
299 \\
256 \\
1,652 \\
2,424 \\
236 \\
1,365 \\
3,075 \\
21,008 \\
0 \\
252\end{array}$ & $\begin{array}{r}97,569 \\
13,481 \\
557 \\
2,026 \\
329 \\
1,518 \\
321 \\
17,784 \\
1,346 \\
2,950 \\
575 \\
1,415 \\
884 \\
360 \\
2,280 \\
22 \\
5,711 \\
361 \\
4,127 \\
3,760 \\
299 \\
256 \\
1,652 \\
2,424 \\
236 \\
1,911 \\
8,669 \\
21,008 \\
1,055 \\
252\end{array}$ & $\begin{array}{r}435 \\
150 \\
0 \\
0 \\
0 \\
0 \\
0 \\
62 \\
15 \\
11 \\
0 \\
16 \\
0 \\
0 \\
0 \\
0 \\
59 \\
0 \\
0 \\
42 \\
0 \\
0 \\
0 \\
0 \\
0 \\
6 \\
62 \\
0 \\
12 \\
0\end{array}$ & $\begin{array}{r}650 \\
0 \\
6 \\
23 \\
4 \\
17 \\
4 \\
136 \\
(s) \\
22 \\
6 \\
0 \\
10 \\
4 \\
25 \\
(s) \\
4 \\
4 \\
46 \\
0 \\
3 \\
3 \\
18 \\
27 \\
3 \\
15 \\
34 \\
233 \\
0 \\
3\end{array}$ & $\begin{array}{r}1,084 \\
150 \\
6 \\
23 \\
4 \\
17 \\
4 \\
198 \\
15 \\
33 \\
6 \\
16 \\
10 \\
4 \\
25 \\
(5) \\
63 \\
4 \\
46 \\
42 \\
3 \\
3 \\
18 \\
27 \\
3 \\
21 \\
96 \\
233 \\
12 \\
3\end{array}$ \\
\hline 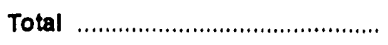 & 333 & 0 & 1,030 & 2,356 & 686 & 91,627 & 197,890 & 1,181 & 1,018 & 2,199 \\
\hline Persian Gulf ${ }^{d}$ & 0 & 0 & 0 & 0 & 113 & 2,474 & 15,113 & 140 & 27 & 168 \\
\hline
\end{tabular}

a Crude oil and unfinished oils are reported by the PAD District in which they are to be processed; all other products are reported by the PAD District of entry.

b Includes crude oil imported for storage in the Strategic Petroleum Reserve.

c Includes aviation gasoline, aviation gasoline blending components, miscellaneous products, other hydrocarbons ano oxygenates, pentanes plus, petroleum coke, and waxes.

Includes Bahrain, Iran, Iraq, Kuwait, Qatar, Saudi Arabia, and United Arab Emirates.

$(s)=$ Less than 500 barrels or less than 500 barrels per day.

Note: Totals may not equal sum of components due to independent rounding.

Source: Energy Information Administration (EIA) Form E|A-814, "Monthly Imports Report." 
Table 42. PAD District II-Year-to-Date Imports of Crude Oll and Petroleum Products by Country of Origin," January-March 1993 (Thousand Barrels)

\begin{tabular}{|c|c|c|c|c|c|c|c|c|c|c|}
\hline Country of Origin & $\begin{array}{c}\text { Crude } \\
\text { Oll }\end{array}$ & $\begin{array}{l}\text { Liquefled } \\
\text { Potroleum } \\
\text { Gases }\end{array}$ & $\begin{array}{c}\text { Unflnlshed } \\
\text { Olls }\end{array}$ & $\begin{array}{l}\text { Gasoline } \\
\text { Blending } \\
\text { Compo- } \\
\text { nente }\end{array}$ & $\begin{array}{l}\text { Finlohed } \\
\text { Motor } \\
\text { Gasoline }\end{array}$ & Jet Fuel & $\begin{array}{l}\text { Distiliate } \\
\text { Fuel OII }\end{array}$ & $\begin{array}{l}\text { Residual } \\
\text { Fuel OII }\end{array}$ & Kerosene & $\begin{array}{c}\text { Special } \\
\text { Naphthas }\end{array}$ \\
\hline 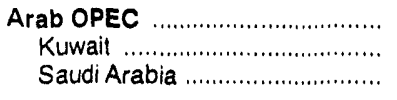 & $\begin{array}{r}10,497 \\
1,213 \\
9,284\end{array}$ & $\begin{array}{l}0 \\
0 \\
0\end{array}$ & $\begin{array}{l}0 \\
0 \\
0\end{array}$ & $\begin{array}{l}0 \\
0 \\
0\end{array}$ & $\begin{array}{l}0 \\
0 \\
0\end{array}$ & $\begin{array}{l}0 \\
0 \\
0\end{array}$ & $\begin{array}{l}0 \\
0 \\
0\end{array}$ & $\begin{array}{l}0 \\
0 \\
0\end{array}$ & $\begin{array}{l}0 \\
0 \\
0\end{array}$ & $\begin{array}{l}0 \\
0 \\
0\end{array}$ \\
\hline 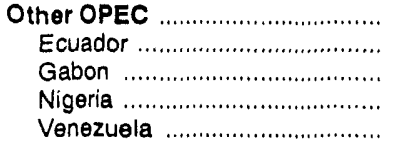 & $\begin{array}{r}23,397 \\
1,821 \\
437 \\
8,352 \\
12,787\end{array}$ & $\begin{array}{l}0 \\
0 \\
0 \\
0 \\
0\end{array}$ & $\begin{array}{l}0 \\
0 \\
0 \\
0 \\
0\end{array}$ & $\begin{array}{l}0 \\
0 \\
0 \\
0 \\
0\end{array}$ & $\begin{array}{l}0 \\
0 \\
0 \\
0 \\
0\end{array}$ & $\begin{array}{l}0 \\
0 \\
0 \\
0 \\
0\end{array}$ & $\begin{array}{l}0 \\
0 \\
0 \\
0 \\
0\end{array}$ & $\begin{array}{l}0 \\
0 \\
0 \\
0 \\
0\end{array}$ & $\begin{array}{l}0 \\
0 \\
0 \\
0 \\
0\end{array}$ & $\begin{array}{l}0 \\
0 \\
0 \\
0 \\
0\end{array}$ \\
\hline 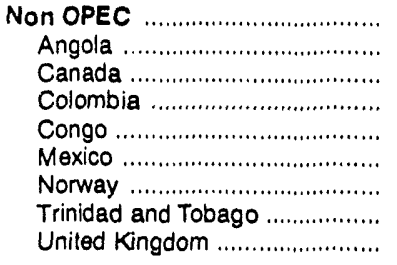 & $\begin{array}{r}78,978 \\
2,122 \\
53,001 \\
994 \\
867 \\
13,153 \\
2,598 \\
1,211 \\
5,032\end{array}$ & $\begin{array}{r}6,349 \\
0 \\
6,349 \\
0 \\
0 \\
0 \\
0 \\
0 \\
0\end{array}$ & $\begin{array}{r}370 \\
0 \\
370 \\
0 \\
0 \\
0 \\
0 \\
0 \\
0\end{array}$ & $\begin{array}{l}3 \\
0 \\
3 \\
0 \\
0 \\
0 \\
0 \\
0 \\
0\end{array}$ & $\begin{array}{r}263 \\
0 \\
263 \\
0 \\
0 \\
0 \\
0 \\
0 \\
0\end{array}$ & $\begin{array}{r}214 \\
0 \\
214 \\
0 \\
0 \\
0 \\
0 \\
0 \\
0\end{array}$ & $\begin{array}{r}574 \\
0 \\
574 \\
0 \\
0 \\
0 \\
0 \\
0 \\
0\end{array}$ & $\begin{array}{r}26 \\
0 \\
26 \\
0 \\
0 \\
0 \\
0 \\
0 \\
0\end{array}$ & $\begin{array}{l}0 \\
0 \\
0 \\
0 \\
0 \\
0 \\
0 \\
0 \\
0\end{array}$ & $\begin{array}{r}178 \\
0 \\
178 \\
0 \\
0 \\
0 \\
0 \\
0 \\
0\end{array}$ \\
\hline Total & 112,872 & 6,349 & 370 & 3 & 263 & 214 & 574 & 26 & 0 & 178 \\
\hline Persian Gulf d & 10,497 & 0 & 0 & 0 & 0 & 0 & 0 & $\mathbf{0}$ & 0 & 0 \\
\hline
\end{tabular}


Table 42. PAD District II-Year-to-Date Imports of Crude Oil and Petroleum Products by Country of Origin, ${ }^{a}$ January-March 1993 (Continued)

(Thousand Barrels)

\begin{tabular}{|c|c|c|c|c|c|c|c|c|c|c|}
\hline \multirow[b]{2}{*}{ Country of Origin } & \multirow[b]{2}{*}{$\begin{array}{c}\text { Naphtha for } \\
\text { Petrochemical } \\
\text { Foedstock } \\
\text { Use }\end{array}$} & \multirow[b]{2}{*}{$\begin{array}{c}\text { Other Olls for } \\
\text { Petrochemlcal } \\
\text { Feedstock } \\
\text { Use }\end{array}$} & \multirow[b]{2}{*}{ Lubricants } & \multirow[b]{2}{*}{$\begin{array}{c}\text { Asphait and } \\
\text { Road Oll }\end{array}$} & \multirow[b]{2}{*}{$\begin{array}{c}\text { Other } \\
\text { Products }\end{array}$} & \multirow[b]{2}{*}{$\begin{array}{c}\text { Total } \\
\text { Products }\end{array}$} & \multirow[b]{2}{*}{$\begin{array}{l}\text { Total } \\
\text { Crude OII } \\
\text { and } \\
\text { Products }\end{array}$} & \multicolumn{3}{|c|}{ Dally Average } \\
\hline & & & & & & & & $\begin{array}{c}\text { Crude } \\
\text { Oll } \\
\end{array}$ & Products & Total \\
\hline 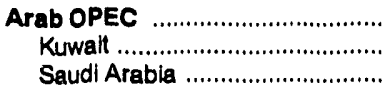 & $\begin{array}{l}0 \\
0 \\
0\end{array}$ & $\begin{array}{l}0 \\
0 \\
0\end{array}$ & $\begin{array}{l}0 \\
0 \\
0\end{array}$ & $\begin{array}{l}0 \\
0 \\
0\end{array}$ & $\begin{array}{l}0 \\
0 \\
0\end{array}$ & $\begin{array}{l}0 \\
0 \\
0\end{array}$ & $\begin{array}{r}10,497 \\
1,213 \\
9,284\end{array}$ & $\begin{array}{r}117 \\
13 \\
103\end{array}$ & $\begin{array}{l}0 \\
0 \\
0\end{array}$ & $\begin{array}{r}117 \\
13 \\
103\end{array}$ \\
\hline 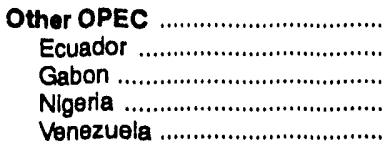 & $\begin{array}{l}0 \\
0 \\
0 \\
0 \\
0\end{array}$ & $\begin{array}{l}0 \\
0 \\
0 \\
0 \\
0\end{array}$ & $\begin{array}{l}0 \\
0 \\
0 \\
0 \\
0\end{array}$ & $\begin{array}{l}0 \\
0 \\
0 \\
0 \\
0\end{array}$ & $\begin{array}{l}0 \\
0 \\
0 \\
0 \\
0\end{array}$ & $\begin{array}{l}0 \\
0 \\
0 \\
0 \\
0\end{array}$ & $\begin{array}{r}23,397 \\
1,821 \\
437 \\
8,352 \\
12,787\end{array}$ & $\begin{array}{r}260 \\
20 \\
5 \\
93 \\
142\end{array}$ & $\begin{array}{l}0 \\
0 \\
0 \\
0 \\
0\end{array}$ & $\begin{array}{r}260 \\
20 \\
5 \\
93 \\
142\end{array}$ \\
\hline 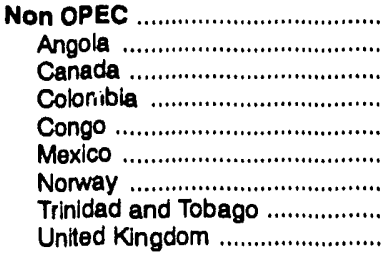 & $\begin{array}{r}47 \\
0 \\
47 \\
0 \\
0 \\
0 \\
0 \\
0 \\
0\end{array}$ & $\begin{array}{l}0 \\
0 \\
0 \\
0 \\
0 \\
0 \\
0 \\
0 \\
0\end{array}$ & $\begin{array}{r}40 \\
0 \\
40 \\
0 \\
0 \\
0 \\
0 \\
0 \\
0\end{array}$ & $\begin{array}{l}0 \\
0 \\
0 \\
0 \\
0 \\
0 \\
0 \\
0 \\
0\end{array}$ & $\begin{array}{r}161 \\
0 \\
161 \\
0 \\
0 \\
0 \\
0 \\
0 \\
0\end{array}$ & $\begin{array}{r}8,225 \\
0 \\
8,225 \\
0 \\
0 \\
0 \\
0 \\
0 \\
0\end{array}$ & $\begin{array}{r}87,203 \\
2,122 \\
61,226 \\
994 \\
867 \\
13,153 \\
2,598 \\
1,211 \\
5,032\end{array}$ & $\begin{array}{r}878 \\
24 \\
589 \\
11 \\
10 \\
146 \\
29 \\
13 \\
56\end{array}$ & $\begin{array}{r}91 \\
0 \\
91 \\
0 \\
0 \\
0 \\
0 \\
0 \\
0\end{array}$ & $\begin{array}{r}969 \\
24 \\
680 \\
11 \\
10 \\
146 \\
29 \\
13 \\
56\end{array}$ \\
\hline Total & 47 & 0 & 40 & 0 & 161 & 8,225 & 121,097 & 1,254 & 91 & 1,346 \\
\hline Persian Qulf ${ }^{d}$............................... & 0 & 0 & 0 & 0 & $\mathbf{0}$ & $\mathbf{0}$ & 10,497 & 117 & 0 & 117 \\
\hline
\end{tabular}

a Crude oil and unfinished olls are reported by the PAD District in which they are to be processed; all other products are reported by the PAD District of entry.

b Includes crude oil imported for storage in the Strategic Petroleum Reserve.

C Includes aviation gasoline, aviation gasoline blending components, miscellaneous products, other hydrocarbons and oxygenates, pentanes plus, petroleum coke, and waxes.

Includes Bahrain, Iran, Iraq, Kuwait, Qatar, Saudi Arabia, and United Arab Emirates.

(8) $=$ Less than 500 barrels or less than 500 barrels per day.

Note: Totals may not equal sum of components due to independent rounding.

Source: Energy Information Administration (EIA) Form EIA-814, "Monthly Imports Report." 
Table 43. PAD District III-Year-to-Date Imports of Crude OIl and Petroleum Products by Country of Origin, ${ }^{a}$ January-March 1993 (Thousand Barrels)

\begin{tabular}{|c|c|c|c|c|c|c|c|c|c|c|}
\hline Country of Origln & $\begin{array}{c}\text { Crude } \\
\text { OlI }^{\mathrm{b}}\end{array}$ & $\begin{array}{c}\text { Liquefled } \\
\text { Potroleum } \\
\text { Gases }\end{array}$ & $\begin{array}{c}\text { Unfinished } \\
\text { Olis }\end{array}$ & $\begin{array}{c}\text { Gasoline } \\
\text { Blending } \\
\text { Compo- } \\
\text { nents }\end{array}$ & $\begin{array}{l}\text { Finished } \\
\text { Motor } \\
\text { Gasollne }\end{array}$ & Jet Fuel & $\begin{array}{l}\text { Distillate } \\
\text { Fuel OII }\end{array}$ & $\begin{array}{l}\text { Residual } \\
\text { Fuel OlI }\end{array}$ & Kerosene & $\begin{array}{c}\text { Spoclal } \\
\text { Naphthas }\end{array}$ \\
\hline 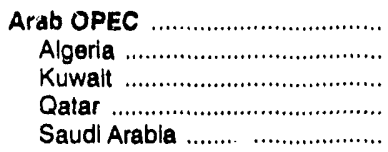 & $\begin{array}{r}127,483 \\
1,072 \\
15,782 \\
0 \\
110,629\end{array}$ & $\begin{array}{r}1,018 \\
1,018 \\
0 \\
0 \\
0\end{array}$ & $\begin{array}{r}12,501 \\
2,894 \\
0 \\
197 \\
9,410\end{array}$ & $\begin{array}{l}0 \\
0 \\
0 \\
0 \\
0\end{array}$ & $\begin{array}{l}0 \\
0 \\
0 \\
0 \\
0\end{array}$ & $\begin{array}{l}0 \\
0 \\
0 \\
0 \\
0\end{array}$ & $\begin{array}{l}0 \\
0 \\
0 \\
0 \\
0\end{array}$ & $\begin{array}{r}885 \\
0 \\
885 \\
0 \\
0\end{array}$ & $\begin{array}{l}0 \\
0 \\
0 \\
0 \\
0\end{array}$ & $\begin{array}{l}0 \\
0 \\
0 \\
0 \\
0\end{array}$ \\
\hline 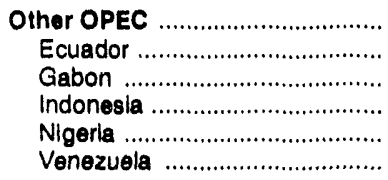 & $\begin{array}{r}92,124 \\
359 \\
6,667 \\
0 \\
27,551 \\
57,547\end{array}$ & $\begin{array}{r}171 \\
0 \\
0 \\
0 \\
0 \\
171\end{array}$ & $\begin{array}{r}4,349 \\
0 \\
0 \\
0 \\
113 \\
4,236\end{array}$ & $\begin{array}{l}0 \\
0 \\
0 \\
0 \\
0 \\
0\end{array}$ & $\begin{array}{l}0 \\
0 \\
0 \\
0 \\
0 \\
0\end{array}$ & $\begin{array}{l}0 \\
0 \\
0 \\
0 \\
0 \\
0\end{array}$ & $\begin{array}{l}0 \\
0 \\
0 \\
0 \\
0 \\
0\end{array}$ & $\begin{array}{r}859 \\
0 \\
0 \\
0 \\
292 \\
567\end{array}$ & $\begin{array}{l}0 \\
0 \\
0 \\
0 \\
0 \\
0\end{array}$ & $\begin{array}{l}9 \\
0 \\
0 \\
9 \\
0 \\
0\end{array}$ \\
\hline 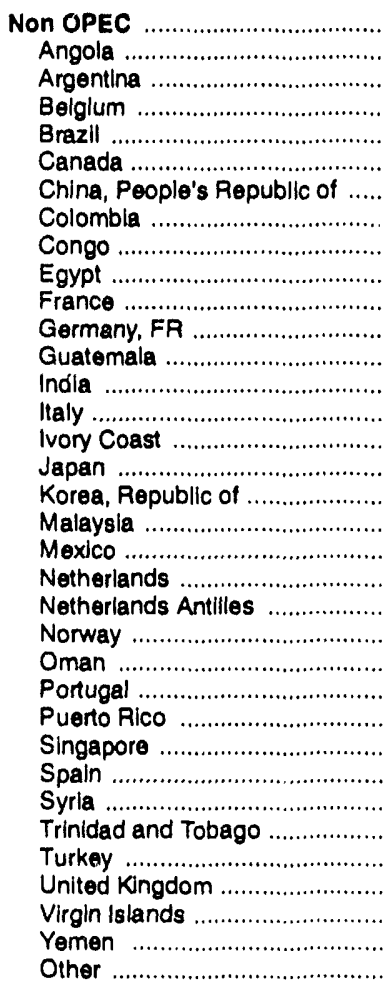 & $\begin{array}{r}104,577 \\
17,762 \\
1,945 \\
0 \\
0 \\
0 \\
1,306 \\
10,267 \\
4,538 \\
1,745 \\
0 \\
0 \\
170 \\
0 \\
0 \\
0 \\
0 \\
0 \\
324 \\
53,158 \\
0 \\
0 \\
1,091 \\
0 \\
0 \\
0 \\
0 \\
0 \\
600 \\
3,579 \\
0 \\
7,858 \\
0 \\
0 \\
0\end{array}$ & $\begin{array}{r}396 \\
0 \\
0 \\
0 \\
0 \\
0 \\
0 \\
0 \\
0 \\
0 \\
20 \\
0 \\
0 \\
0 \\
0 \\
0 \\
0 \\
0 \\
4 \\
372 \\
0 \\
0 \\
0 \\
0 \\
0 \\
0 \\
0 \\
0 \\
0 \\
0 \\
0 \\
0 \\
0 \\
0 \\
0\end{array}$ & $\begin{array}{r}11,675 \\
0 \\
0 \\
531 \\
0 \\
349 \\
0 \\
0 \\
0 \\
141 \\
112 \\
644 \\
0 \\
256 \\
1,495 \\
237 \\
0 \\
2,201 \\
0 \\
425 \\
814 \\
1,592 \\
229 \\
1,365 \\
0 \\
0 \\
691 \\
219 \\
242 \\
0 \\
0 \\
84 \\
0 \\
48 \\
0\end{array}$ & $\begin{array}{r}884 \\
0 \\
0 \\
0 \\
4 \\
0 \\
0 \\
0 \\
0 \\
0 \\
0 \\
0 \\
0 \\
0 \\
0 \\
0 \\
0 \\
0 \\
0 \\
788 \\
0 \\
92 \\
0 \\
0 \\
0 \\
0 \\
0 \\
0 \\
0 \\
0 \\
0 \\
0 \\
0 \\
0 \\
0\end{array}$ & $\begin{array}{r}457 \\
0 \\
0 \\
0 \\
0 \\
0 \\
0 \\
0 \\
0 \\
0 \\
0 \\
0 \\
0 \\
0 \\
0 \\
0 \\
0 \\
0 \\
0 \\
0 \\
0 \\
0 \\
0 \\
0 \\
0 \\
0 \\
0 \\
0 \\
0 \\
0 \\
0 \\
0 \\
457 \\
0 \\
0\end{array}$ & $\begin{array}{r}624 \\
0 \\
0 \\
0 \\
0 \\
0 \\
0 \\
0 \\
0 \\
141 \\
0 \\
0 \\
0 \\
0 \\
0 \\
0 \\
0 \\
0 \\
0 \\
21 \\
0 \\
0 \\
0 \\
0 \\
0 \\
0 \\
0 \\
0 \\
0 \\
0 \\
0 \\
0 \\
462 \\
0 \\
0\end{array}$ & $\begin{array}{l}0 \\
0 \\
0 \\
0 \\
0 \\
0 \\
0 \\
0 \\
0 \\
0 \\
0 \\
0 \\
0 \\
0 \\
0 \\
0 \\
0 \\
0 \\
0 \\
0 \\
0 \\
0 \\
0 \\
0 \\
0 \\
0 \\
0 \\
0 \\
0 \\
0 \\
0 \\
0 \\
0 \\
0 \\
0\end{array}$ & $\begin{array}{r}1,671 \\
0 \\
0 \\
0 \\
0 \\
0 \\
0 \\
0 \\
0 \\
0 \\
0 \\
373 \\
0 \\
0 \\
0 \\
0 \\
0 \\
0 \\
0 \\
0 \\
0 \\
0 \\
0 \\
0 \\
469 \\
0 \\
0 \\
0 \\
0 \\
0 \\
0 \\
0 \\
301 \\
388 \\
140\end{array}$ & $\begin{array}{l}0 \\
0 \\
0 \\
0 \\
0 \\
0 \\
0\end{array}$ & $\begin{array}{r}262 \\
0 \\
0 \\
0 \\
0 \\
247 \\
0 \\
0 \\
0 \\
0 \\
0 \\
0 \\
0 \\
0 \\
15 \\
0 \\
0 \\
0 \\
0 \\
0 \\
0 \\
0 \\
0 \\
0 \\
0 \\
0 \\
0 \\
0 \\
0 \\
0 \\
0 \\
0 \\
0 \\
0 \\
0\end{array}$ \\
\hline 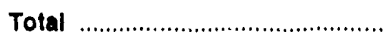 & 324,184 & 1,585 & 28,525 & 884 & 457 & 624 & 0 & 3,415 & 0 & 271 \\
\hline 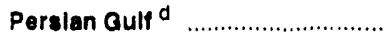 & 126,411 & 0 & 9,607 & 0 & 0 & 0 & 0 & 885 & 0 & 0 \\
\hline
\end{tabular}


Table 43. PAD District II-Year-to-Date Imports of Crude Oll and Petroleum Products by Country of Origin, January-March 1993 (Continued)

(Thousand Barrels)

\begin{tabular}{|c|c|c|c|c|c|c|c|c|c|c|}
\hline \multirow[b]{2}{*}{ Country of Origin } & \multirow[b]{2}{*}{$\begin{array}{c}\text { Naphtha for } \\
\text { Potrochemical } \\
\text { Feedstock } \\
\text { Use }\end{array}$} & \multirow[b]{2}{*}{$\begin{array}{c}\text { Other Olls for } \\
\text { Petrochemicaf } \\
\text { Feedstock } \\
\text { Use }\end{array}$} & \multirow[b]{2}{*}{ Lubricants } & \multirow[b]{2}{*}{$\begin{array}{c}\text { Asphalt and } \\
\text { Road Oll }\end{array}$} & \multirow[b]{2}{*}{$\begin{array}{c}\text { Other } \\
\text { Products }\end{array}$} & \multirow[b]{2}{*}{$\begin{array}{c}\text { Total } \\
\text { Products }\end{array}$} & \multirow[b]{2}{*}{$\begin{array}{c}\text { Total } \\
\text { Crude OII } \\
\text { and } \\
\text { Products }\end{array}$} & \multicolumn{3}{|c|}{ Dally Average } \\
\hline & & & & & & & & $\begin{array}{c}\text { Crude } \\
\text { Oll }\end{array}$ & Products & Total \\
\hline 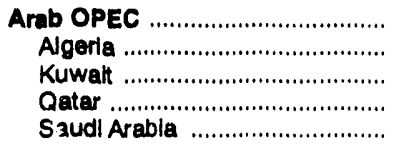 & $\begin{array}{l}0 \\
0 \\
0 \\
0 \\
0\end{array}$ & $\begin{array}{r}9,088 \\
9,088 \\
0 \\
0 \\
0\end{array}$ & $\begin{array}{l}0 \\
0 \\
0 \\
0 \\
0\end{array}$ & $\begin{array}{l}0 \\
0 \\
0 \\
0 \\
0\end{array}$ & $\begin{array}{r}1,510 \\
1,452 \\
0 \\
0 \\
58\end{array}$ & $\begin{array}{r}25,002 \\
14,452 \\
885 \\
197 \\
9,468\end{array}$ & $\begin{array}{r}152,485 \\
15,524 \\
16,667 \\
197 \\
120,097\end{array}$ & $\begin{array}{r}1,416 \\
12 \\
175 \\
0 \\
1,229\end{array}$ & $\begin{array}{r}278 \\
161 \\
10 \\
2 \\
105\end{array}$ & $\begin{array}{r}1,694 \\
172 \\
185 \\
2 \\
1,334\end{array}$ \\
\hline 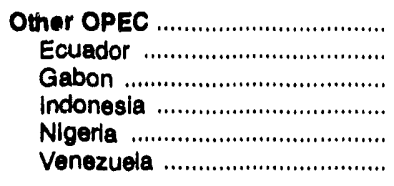 & $\begin{array}{l}0 \\
0 \\
0 \\
0 \\
0 \\
0\end{array}$ & $\begin{array}{r}230 \\
0 \\
0 \\
0 \\
0 \\
230\end{array}$ & $\begin{array}{l}0 \\
0 \\
0 \\
0 \\
0 \\
0\end{array}$ & $\begin{array}{r}65 \\
0 \\
0 \\
0 \\
0 \\
65\end{array}$ & $\begin{array}{l}0 \\
0 \\
0 \\
0 \\
0 \\
0\end{array}$ & $\begin{array}{r}5,683 \\
0 \\
0 \\
9 \\
405 \\
5,269\end{array}$ & $\begin{array}{r}97,807 \\
359 \\
6,667 \\
9 \\
27,956 \\
62,816\end{array}$ & $\begin{array}{r}1,024 \\
4 \\
74 \\
0 \\
306 \\
639\end{array}$ & $\begin{array}{r}63 \\
0 \\
0 \\
(5) \\
5 \\
59\end{array}$ & $\begin{array}{r}1,087 \\
4 \\
74 \\
(8) \\
311 \\
698\end{array}$ \\
\hline 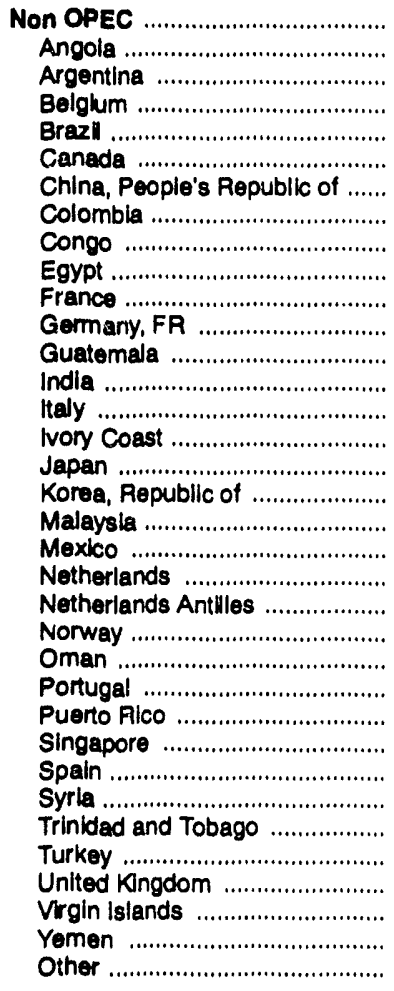 & $\begin{array}{r}1,977 \\
0 \\
36 \\
22 \\
35 \\
71 \\
0 \\
0 \\
0 \\
0 \\
40 \\
0 \\
0 \\
0 \\
12 \\
0 \\
7 \\
96 \\
0 \\
0 \\
28 \\
442 \\
0 \\
0 \\
0 \\
904 \\
0 \\
0 \\
0 \\
0 \\
71 \\
22 \\
0 \\
191 \\
0\end{array}$ & $\begin{array}{r}810 \\
0 \\
180 \\
361 \\
0 \\
0 \\
0 \\
0 \\
0 \\
0 \\
0 \\
0 \\
0 \\
0 \\
0 \\
0 \\
0 \\
0 \\
0 \\
0 \\
0 \\
0 \\
0 \\
0 \\
0 \\
0 \\
0 \\
0 \\
0 \\
0 \\
0 \\
0 \\
0 \\
0 \\
269\end{array}$ & $\begin{array}{r}10 \\
0 \\
0 \\
0 \\
0 \\
0 \\
0 \\
0 \\
0 \\
0 \\
0 \\
0 \\
0 \\
0 \\
0 \\
0 \\
0 \\
0 \\
0 \\
0 \\
0 \\
0 \\
0 \\
0 \\
0 \\
0 \\
0 \\
0 \\
0 \\
10 \\
0 \\
0 \\
0 \\
0 \\
0\end{array}$ & $\begin{array}{l}0 \\
0 \\
0 \\
0 \\
0 \\
0 \\
0 \\
0 \\
0 \\
0 \\
0 \\
0 \\
0 \\
0 \\
0 \\
0 \\
0 \\
0 \\
0 \\
0 \\
0 \\
0 \\
0 \\
0 \\
0 \\
0 \\
0 \\
0 \\
0 \\
0 \\
0 \\
0 \\
0 \\
0 \\
0\end{array}$ & $\begin{array}{r}975 \\
0 \\
181 \\
0 \\
0 \\
0 \\
0 \\
0 \\
0 \\
2 \\
0 \\
3 \\
0 \\
0 \\
0 \\
0 \\
0 \\
0 \\
0 \\
786 \\
0 \\
0 \\
0 \\
0 \\
0 \\
0 \\
0 \\
0 \\
0 \\
0 \\
0 \\
0 \\
0 \\
0 \\
3\end{array}$ & $\begin{array}{r}19,741 \\
0 \\
397 \\
914 \\
39 \\
667 \\
0 \\
0 \\
0 \\
284 \\
172 \\
1,020 \\
0 \\
256 \\
1,522 \\
237 \\
7 \\
2,297 \\
4 \\
2,392 \\
842 \\
2,126 \\
229 \\
1,365 \\
469 \\
904 \\
691 \\
219 \\
242 \\
10 \\
71 \\
106 \\
1,220 \\
627 \\
412\end{array}$ & $\begin{array}{r}124,318 \\
17,762 \\
2,342 \\
914 \\
39 \\
667 \\
1,306 \\
10,267 \\
4,538 \\
2,029 \\
172 \\
1,020 \\
170 \\
256 \\
1,522 \\
237 \\
7 \\
2,297 \\
328 \\
55,550 \\
842 \\
2,126 \\
1,320 \\
1,365 \\
469 \\
904 \\
691 \\
219 \\
842 \\
3,589 \\
71 \\
7,964 \\
1,220 \\
627 \\
412\end{array}$ & $\begin{array}{r}1,162 \\
197 \\
22 \\
0 \\
0 \\
0 \\
15 \\
114 \\
50 \\
19 \\
0 \\
0 \\
2 \\
0 \\
0 \\
0 \\
0 \\
0 \\
4 \\
591 \\
0 \\
0 \\
12 \\
0 \\
0 \\
0 \\
0 \\
0 \\
7 \\
40 \\
0 \\
87 \\
0 \\
0 \\
0\end{array}$ & $\begin{array}{r}219 \\
0 \\
4 \\
10 \\
(s) \\
7 \\
0 \\
0 \\
0 \\
3 \\
2 \\
11 \\
0 \\
3 \\
17 \\
3 \\
(s) \\
26 \\
(s) \\
27 \\
9 \\
24 \\
3 \\
15 \\
5 \\
10 \\
8 \\
2 \\
3 \\
(s) \\
1 \\
1 \\
14 \\
7 \\
5\end{array}$ & $\begin{array}{r}1,381 \\
197 \\
26 \\
10 \\
(\mathrm{~s}) \\
7 \\
15 \\
114 \\
50 \\
23 \\
2 \\
11 \\
2 \\
3 \\
17 \\
3 \\
(\mathrm{~s}) \\
26 \\
4 \\
617 \\
9 \\
24 \\
15 \\
15 \\
5 \\
10 \\
8 \\
2 \\
9 \\
40 \\
1 \\
88 \\
14 \\
7 \\
5\end{array}$ \\
\hline 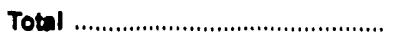 & 1,977 & 10,128 & 10 & 65 & 2,485 & 50,426 & 374,610 & 3,602 & 560 & 4,162 \\
\hline Porslan Gulf ${ }^{d}$ & 0 & 269 & 0 & 0 & 58 & 10,819 & 137,230 & 1,405 & 120 & 1,525 \\
\hline
\end{tabular}

a Crude oll and unfinished olls are reported by the PAD District in which they are to be processed; all other products are reported by the PAD District of entry.

- Includes crude oil imported for storage in the Strategic Petroleum Reserve. waxes.

Includes aviation gasoline, aviation gasollne blending components, miscellaneous products, other hydrocarbons and oxygenates, pentanes plus, petroleum coke, and

Includes Bahrain, Iran, Iraq, Kuwait, Qatar, Saudi Arabla, and United Arab Emirates.

(s) = Less than 500 barrels or less than 500 barrels per day.

Note: Totals may not equal sum of components due to independent rounding

Source: Energy Information Administration (EIA) Form EIA-814, "Monthly Imports Report." 
Table 44. PAD Districts IV and V-Year-to-Date Imports of Crude Oll and Petroleum Products by Country of Origin, January-March 1993 (Thousand Barrels)

\begin{tabular}{|c|c|c|c|c|c|c|c|c|c|c|}
\hline Country of Origin & $\underset{\substack{\text { Cruda } \\
\text { Olb }}}{ }$ & $\begin{array}{l}\text { Llquofied } \\
\text { Potroloum } \\
\text { Coses }\end{array}$ & $\begin{array}{c}\text { Unfinished } \\
\text { Olls }\end{array}$ & $\begin{array}{c}\text { Gasolln: } \\
\text { Blonding } \\
\text { Coripo- } \\
\text { nonte }\end{array}$ & $\begin{array}{l}\text { Finlehed } \\
\text { Motor } \\
\text { Cacollne }\end{array}$ & Jot Fual & $\begin{array}{l}\text { Distllate } \\
\text { Fuel OII }\end{array}$ & $\begin{array}{l}\text { Rooldual } \\
\text { Fuall OII }\end{array}$ & Kerosene & $\begin{array}{c}\text { Special } \\
\text { Naphthae }\end{array}$ \\
\hline
\end{tabular}

PAD District IV

Non OPEC
Canada

Canada ...................................

Total
7,584

7,584

7,584

$\begin{array}{lll}681 & 0 & 0 \\ 681 & 0 & 0 \\ 681 & 0 & 0\end{array}$

28

0
0

210

210

210

0
0
0

0
0

0

28

0

0

0

0

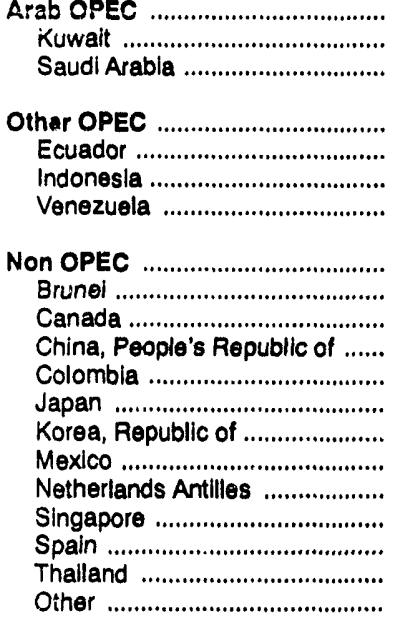

Tota!

Perslan Gule
2,122

2,122

7,121

7,121

1,814

4,555

752

0,187

155

5,079

2,745

750

0

0

0
0
0

458
0

18,430

2,122

(n)

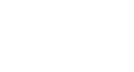

(2)

(28)

210

\section{PAD Dlatrlet $V$}

See footnotes at end of table. 
Table 44. PAD Districts IV and V-Year-to-Date Imports of Crude Oll and Petroleum Products by Country of Origin, January-March 1993 (Continued)

(Thousand Barrels)

\begin{tabular}{|c|c|c|c|c|c|c|c|c|c|c|}
\hline \multirow[b]{2}{*}{ Country of Orlgin } & \multirow[b]{2}{*}{$\begin{array}{c}\text { Naphtha for } \\
\text { Potrochemlcal } \\
\text { Foedstock } \\
\text { Use }\end{array}$} & \multirow[b]{2}{*}{$\begin{array}{c}\text { Other Olls for } \\
\text { Petrochemlcal } \\
\text { Foedstock } \\
\text { Use }\end{array}$} & \multirow[b]{2}{*}{ Lubricants } & \multirow[b]{2}{*}{$\begin{array}{c}\text { Asphalt and } \\
\text { Road OII }\end{array}$} & \multirow[b]{2}{*}{$\begin{array}{c}\text { Other } \\
\text { Products }\end{array}$} & \multirow[b]{2}{*}{$\begin{array}{c}\text { Total } \\
\text { Producte }\end{array}$} & \multirow[b]{2}{*}{$\begin{array}{c}\text { Total } \\
\text { Crude Oll } \\
\text { and } \\
\text { Products }\end{array}$} & \multicolumn{3}{|c|}{ Dally Average } \\
\hline & & & & & & & & $\begin{array}{c}\text { Crude } \\
\text { Oll }\end{array}$ & Products & Total \\
\hline
\end{tabular}

PAD District IV

\begin{tabular}{|c|c|c|c|c|c|c|c|c|c|c|}
\hline $\begin{array}{l}\text { Non OPEC } \\
\text { Canada }\end{array}$ & $\begin{array}{l}0 \\
0\end{array}$ & $\begin{array}{l}0 \\
0\end{array}$ & $\begin{array}{l}0 \\
0\end{array}$ & $\begin{array}{l}0 \\
0\end{array}$ & $\begin{array}{l}207 \\
207\end{array}$ & $\begin{array}{l}1,126 \\
1,126\end{array}$ & $\begin{array}{l}8,710 \\
8,710\end{array}$ & $\begin{array}{l}84 \\
84\end{array}$ & $\begin{array}{l}13 \\
13\end{array}$ & $\begin{array}{l}97 \\
97\end{array}$ \\
\hline Total & 0 & 0 & 0 & 0 & 207 & 1,126 & 8,710 & 84 & 13 & 97 \\
\hline
\end{tabular}

PAD District V

\begin{tabular}{|c|c|c|c|c|c|c|c|c|c|c|}
\hline 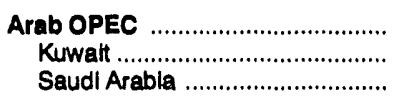 & $\begin{array}{l}0 \\
0 \\
0\end{array}$ & $\begin{array}{l}0 \\
0 \\
0\end{array}$ & $\begin{array}{l}0 \\
0 \\
0\end{array}$ & $\begin{array}{l}0 \\
0 \\
0\end{array}$ & $\begin{array}{l}0 \\
0 \\
0\end{array}$ & $\begin{array}{r}499 \\
498 \\
1\end{array}$ & $\begin{array}{r}2,621 \\
2,620 \\
1\end{array}$ & $\begin{array}{r}24 \\
24 \\
0\end{array}$ & $\begin{array}{r}6 \\
6 \\
(8)\end{array}$ & $\begin{array}{l}29 \\
29 \\
\text { (s) }\end{array}$ \\
\hline 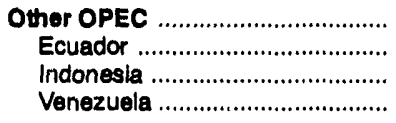 & $\begin{array}{l}0 \\
0 \\
0 \\
0\end{array}$ & $\begin{array}{l}0 \\
0 \\
0 \\
0\end{array}$ & $\begin{array}{l}0 \\
0 \\
0 \\
0\end{array}$ & $\begin{array}{l}0 \\
0 \\
0 \\
0\end{array}$ & $\begin{array}{r}251 \\
0 \\
0 \\
251\end{array}$ & $\begin{array}{r}881 \\
0 \\
133 \\
728\end{array}$ & $\begin{array}{l}7,982 \\
1,814 \\
4,688 \\
1,480\end{array}$ & $\begin{array}{r}79 \\
20 \\
51 \\
8\end{array}$ & $\begin{array}{r}10 \\
0 \\
1 \\
8\end{array}$ & $\begin{array}{l}89 \\
20 \\
52 \\
16\end{array}$ \\
\hline 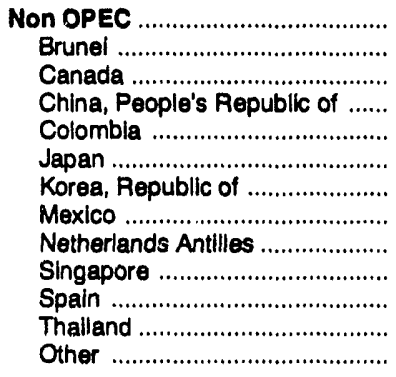 & $\begin{array}{r}83 \\
0 \\
0 \\
0 \\
0 \\
0 \\
83 \\
0 \\
0 \\
0 \\
0 \\
0 \\
0\end{array}$ & $\begin{array}{l}0 \\
0 \\
0 \\
0 \\
0 \\
0 \\
0 \\
0 \\
0 \\
0 \\
0 \\
0 \\
0\end{array}$ & $\begin{array}{r}31 \\
0 \\
0 \\
0 \\
0 \\
31 \\
0 \\
0 \\
0 \\
0 \\
0 \\
0 \\
0\end{array}$ & $\begin{array}{r}100 \\
0 \\
30 \\
0 \\
0 \\
0 \\
0 \\
0 \\
0 \\
0 \\
70 \\
0 \\
0\end{array}$ & $\begin{array}{r}685 \\
0 \\
635 \\
0 \\
0 \\
0 \\
0 \\
4 \\
0 \\
0 \\
0 \\
0 \\
46\end{array}$ & $\begin{array}{r}5,462 \\
0 \\
1,957 \\
162 \\
0 \\
31 \\
629 \\
1,478 \\
112 \\
977 \\
70 \\
0 \\
46\end{array}$ & $\begin{array}{r}14,649 \\
155 \\
7,036 \\
2,907 \\
750 \\
31 \\
629 \\
1,478 \\
112 \\
977 \\
70 \\
458 \\
46\end{array}$ & $\begin{array}{r}102 \\
2 \\
56 \\
31 \\
8 \\
0 \\
0 \\
0 \\
0 \\
0 \\
0 \\
5 \\
0\end{array}$ & $\begin{array}{r}61 \\
0 \\
22 \\
2 \\
0 \\
(s) \\
7 \\
16 \\
1 \\
11 \\
1 \\
0 \\
1\end{array}$ & $\begin{array}{r}163 \\
2 \\
78 \\
32 \\
8 \\
(5) \\
7 \\
16 \\
1 \\
11 \\
1 \\
5 \\
1\end{array}$ \\
\hline 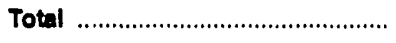 & 83 & 0 & 31 & 100 & 936 & 6,822 & 25,252 & 205 & 76 & 281 \\
\hline Perelan Gulf ${ }^{d}$................................. & 0 & 0 & 0 & 0 & 0 & 490 & 2,621 & 24 & 6 & 29 \\
\hline
\end{tabular}

Crude oll and unfinished olls are reported by the PAD District in which they are to be processed; all other products are reported by the PAD District of entry.

Includes crude oil imporied for storage in the Strategic Petroleum Reserve.

c Includes aviation gasoline, aviation gasoline blending components, miscellaneous products, other hydrocarbons and oxygenates, pentanes plus, petroleum coke, and waxes.

Includes Bahrain, Iran, Iraq, Kuwait, Qatar, Saudl Arabia, and United Arab Emirates.

$(s)=$ Less than 500 barrels or less than 500 barrels per day.

Note: Totals may not equal sum of components due to independent rounding.

Source: Energy Information Administration (EIA) Form ElA-814, "Monthly Imports Report." 
Table 45. Exports of Crude OII and Petroleum Products by PAD District, March 1993

(Thousand Barrels)

\begin{tabular}{|c|c|c|c|c|c|c|c|}
\hline \multirow[b]{2}{*}{ Commodity } & \multicolumn{6}{|c|}{ Petroleum Adminiatration for Defense Districte } & \multirow[b]{2}{*}{$\begin{array}{c}\text { Dally } \\
\text { Average }\end{array}$} \\
\hline & 1 & ॥ & III & IV & V & $\begin{array}{l}\text { U.S. } \\
\text { Total }\end{array}$ & \\
\hline 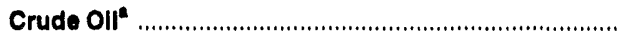 & 0 & 48 & $\mathbf{0}$ & 0 & 4,255 & 4,301 & 139 \\
\hline 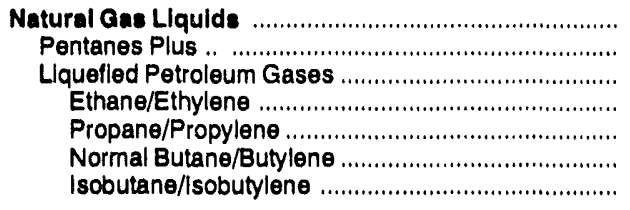 & $\begin{array}{r}16 \\
1 \\
15 \\
0 \\
14 \\
1 \\
0\end{array}$ & $\begin{array}{r}236 \\
27 \\
209 \\
0 \\
83 \\
126 \\
0\end{array}$ & $\begin{array}{r}747 \\
0 \\
747 \\
0 \\
661 \\
86 \\
0\end{array}$ & $\begin{array}{l}1 \\
1 \\
0 \\
0 \\
0 \\
0 \\
0\end{array}$ & $\begin{array}{r}472 \\
0 \\
472 \\
0 \\
233 \\
239 \\
0\end{array}$ & $\begin{array}{r}1,472 \\
29 \\
1,443 \\
0 \\
990 \\
453 \\
0\end{array}$ & $\begin{array}{r}47 \\
1 \\
47 \\
0 \\
32 \\
15 \\
0\end{array}$ \\
\hline 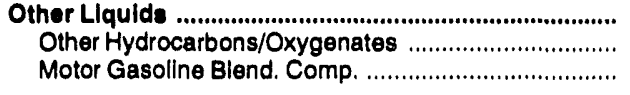 & $\begin{array}{l}1 \\
0 \\
1\end{array}$ & $\begin{array}{l}0 \\
0 \\
0\end{array}$ & $\begin{array}{r}254 \\
0 \\
254\end{array}$ & $\begin{array}{l}0 \\
0 \\
0\end{array}$ & $\begin{array}{r}260 \\
0 \\
260\end{array}$ & $\begin{array}{r}514 \\
0 \\
514\end{array}$ & $\begin{array}{r}17 \\
0 \\
17\end{array}$ \\
\hline 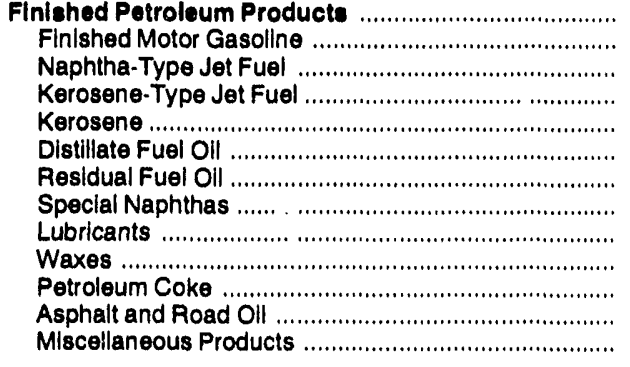 & $\begin{array}{r}485 \\
39 \\
0 \\
0 \\
4 \\
84 \\
220 \\
6 \\
94 \\
15 \\
15 \\
2 \\
7\end{array}$ & $\begin{array}{r}338 \\
16 \\
1 \\
(8) \\
1 \\
(8) \\
1 \\
8 \\
42 \\
3 \\
244 \\
21 \\
(8)\end{array}$ & $\begin{array}{r}10,353 \\
2,849 \\
16 \\
1,482 \\
0 \\
846 \\
2,001 \\
15 \\
306 \\
31 \\
2,799 \\
7 \\
(8)\end{array}$ & $\begin{array}{r}11 \\
(\mathbf{s}) \\
0 \\
0 \\
0 \\
1 \\
0 \\
(8) \\
5 \\
0 \\
4 \\
1 \\
0\end{array}$ & $\begin{array}{r}10,585 \\
460 \\
525 \\
1,092 \\
1 \\
1,845 \\
2,482 \\
4 \\
189 \\
10 \\
3,957 \\
19 \\
1\end{array}$ & $\begin{array}{r}21,773 \\
3,364 \\
542 \\
2,575 \\
6 \\
2,777 \\
4,704 \\
33 \\
635 \\
59 \\
7,018 \\
49 \\
9\end{array}$ & $\begin{array}{r}702 \\
109 \\
17 \\
83 \\
(8) \\
90 \\
152 \\
1 \\
20 \\
2 \\
226 \\
2 \\
(8)\end{array}$ \\
\hline Total & 502 & 621 & 11,354 & 11 & 15,572 & 28,061 & $\mathbf{9 0 5}$ \\
\hline
\end{tabular}

a Crude oll exports are restricted to: (1) crude oll derived from fields under the State waters of Alaska's Cook Inlet; (2) certain domestlcally produced crude oll destined for Canada; and (3) shipments to U.S. territoies, and California crude oil to Pacific Rim countries. On December 6, 1991, the U.S. Department of Commerce approved a license to export 25,000 barrels per day of California heavy crude oil (less than 20 degrees API gravity) to Paclfic Rim countrles for one year. $(s)=$ Less than 500 barrels or less than 500 barrels per day.

Note: Totals may not equal sum of components due to independent rounding.

Sources: Energy Information Administration (EIA) Form EIA-810, "Monthly Refinery Report" and the U.S. Bureau of the Census. 
Table 46. Year-to-Date Exports of Crude Oll and Petroleum Products by PAD District, January-March 1993 (Thousand Barrels)

\begin{tabular}{|c|c|c|c|c|c|c|c|}
\hline \multirow[b]{2}{*}{ Commodity } & \multicolumn{6}{|c|}{ Petroleum Administration for Defense Dlstrlcte } & \multirow[b]{2}{*}{$\begin{array}{c}\text { Dally } \\
\text { Averag }\end{array}$} \\
\hline & 1 & II & III & $\mathbf{N}$ & $\mathbf{V}$ & U.S. & \\
\hline 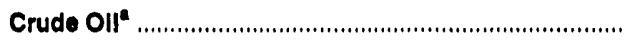 & 0 & 272 & $\mathbf{0}$ & 0 & 12,660 & 12,932 & 144 \\
\hline 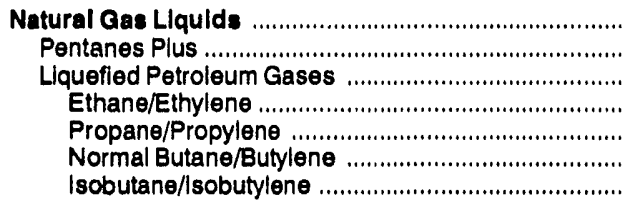 & $\begin{array}{r}60 \\
6 \\
55 \\
0 \\
50 \\
5 \\
0\end{array}$ & $\begin{array}{r}512 \\
81 \\
430 \\
0 \\
222 \\
209 \\
0\end{array}$ & $\begin{array}{r}2,695 \\
1 \\
2,695 \\
0 \\
2,335 \\
360 \\
0\end{array}$ & $\begin{array}{l}2 \\
2 \\
0 \\
0 \\
0 \\
0 \\
0\end{array}$ & $\begin{array}{r}1,000 \\
0 \\
1,000 \\
0 \\
357 \\
643 \\
0\end{array}$ & $\begin{array}{r}4,270 \\
90 \\
4,180 \\
0 \\
2,963 \\
1,217 \\
0\end{array}$ & $\begin{array}{r}47 \\
1 \\
46 \\
0 \\
33 \\
14 \\
0\end{array}$ \\
\hline $\begin{array}{l}\text { Other Liqulds } \\
\text { Other Hydrocarbons/Oxygenates } \\
\text { Motor Gasoline Blend. Comp. }\end{array}$ & $\begin{array}{l}3 \\
0 \\
3\end{array}$ & $\begin{array}{r}(s) \\
0 \\
(s)\end{array}$ & $\begin{array}{r}267 \\
0 \\
267\end{array}$ & $\begin{array}{l}0 \\
0 \\
0\end{array}$ & $\begin{array}{r}548 \\
0 \\
548\end{array}$ & $\begin{array}{r}817 \\
0 \\
817\end{array}$ & $\begin{array}{l}9 \\
0 \\
9\end{array}$ \\
\hline 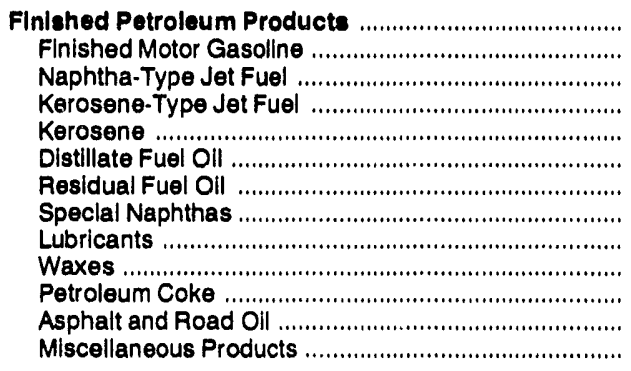 & $\begin{array}{r}2,535 \\
127 \\
1 \\
234 \\
18 \\
127 \\
1,242 \\
16 \\
405 \\
36 \\
300 \\
7 \\
22\end{array}$ & $\begin{array}{r}913 \\
190 \\
29 \\
47 \\
2 \\
4 \\
50 \\
21 \\
102 \\
10 \\
425 \\
31 \\
(8)\end{array}$ & $\begin{array}{r}33,722 \\
7,556 \\
564 \\
3,386 \\
564 \\
3,739 \\
6,420 \\
343 \\
748 \\
95 \\
10,283 \\
18 \\
7\end{array}$ & $\begin{array}{r}22 \\
1 \\
0 \\
0 \\
0 \\
1 \\
0 \\
1 \\
11 \\
0 \\
6 \\
3 \\
0\end{array}$ & $\begin{array}{r}28,279 \\
2,663 \\
1,153 \\
2,335 \\
3 \\
5,550 \\
4,288 \\
95 \\
327 \\
37 \\
9,781 \\
44 \\
2\end{array}$ & $\begin{array}{r}63,471 \\
10,536 \\
1,748 \\
6,002 \\
588 \\
9,421 \\
12,001 \\
475 \\
1,593 \\
178 \\
20,795 \\
103 \\
32\end{array}$ & $\begin{array}{r}705 \\
117 \\
19 \\
67 \\
7 \\
105 \\
133 \\
5 \\
18 \\
2 \\
231 \\
1 \\
(8)\end{array}$ \\
\hline Total & 2,598 & 1,698 & 36,684 & 24 & 40,487 & 81,490 & 905 \\
\hline
\end{tabular}

a Crude oll exports are restricted to: (1) crude oll derlved from flelds under the State waters of Alaska's Cook Inlet; (2) certain domestlcally produced crude oll destined for Canada; and (3) shipments to U.S. territories, and Californla crude oil to Paciflc Rim countries. On December 6, 1991, the U.S. Department of Commerce approved a license to export 25,000 barrels per day of Callfornia heavy crude oll (less than 20 degrees API gravity) to Pacific Rim countries for one year.

(s) = Less than 500 barrels or less than 500 barrels per day.

Note: Totals may not equal sum of components due to independent rounding.

Sources: Energy Information Administration (E|A) Form EIA-810, "Monthly Refinery Report" and the U.S. Bureau of the Census. 
Table 47. Exports of Crude Oll and Petroleum Products by Destination, March 1993 (Thousand Barrels)

\begin{tabular}{|c|c|c|c|c|c|c|c|c|}
\hline Destination & $\begin{array}{c}\text { Crude } \\
\text { Oll }\end{array}$ & $\begin{array}{c}\text { Pentanes } \\
\text { Plue }\end{array}$ & $\begin{array}{c}\text { Liquefled } \\
\text { Potroleum } \\
\text { Ganes } \\
\end{array}$ & $\begin{array}{l}\text { Finilshed } \\
\text { Motor } \\
\text { Gacollne }\end{array}$ & Jet Fual & Kerosene & $\begin{array}{c}\text { Distliate Fuel } \\
\text { OII }\end{array}$ & $\begin{array}{c}\text { Residual } \\
\text { Fuel Oll }\end{array}$ \\
\hline 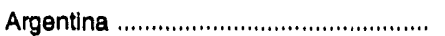 & 0 & 0 & 0 & 0 & 0 & 0 & 0 & 0 \\
\hline 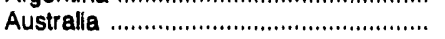 & 0 & 0 & 3 & 0 & 0 & 0 & 0 & 0 \\
\hline 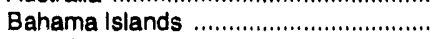 & 0 & 0 & 15 & 41 & 11 & 0 & 76 & 32 \\
\hline 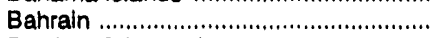 & 0 & 0 & 0 & 0 & 0 & 0 & (s) & 0 \\
\hline Belglum \& Luxembourg ......................... & 0 & 0 & 9 & 1 & 0 & 0 & 0 & 0 \\
\hline 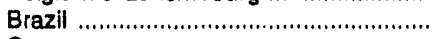 & 0 & 0 & 0 & 0 & 140 & 0 & 226 & 0 \\
\hline 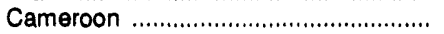 & 0 & 0 & 0 & 0 & 0 & 0 & 0 & 0 \\
\hline 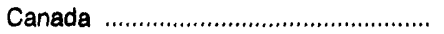 & 46 & 29 & 243 & 218 & 92 & 4 & 3 & 857 \\
\hline 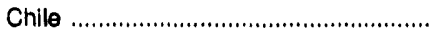 & 0 & 0 & 0 & 0 & 0 & 0 & 0 & 0 \\
\hline China, People's Republic of ................... & 0 & 0 & 0 & 0 & 169 & 0 & 200 & 0 \\
\hline 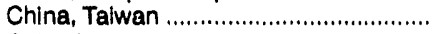 & 0 & 0 & 0 & 252 & 0 & 0 & 600 & 389 \\
\hline 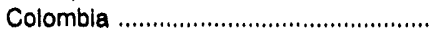 & 0 & 0 & 113 & 0 & 0 & 0 & 0 & 0 \\
\hline 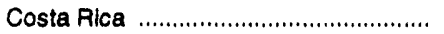 & 0 & 0 & (s) & 0 & 0 & 0 & 0 & 0 \\
\hline 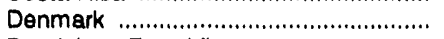 & 0 & 0 & 0 & 0 & 0 & 0 & 0 & 0 \\
\hline Dominican Republic ................................. & 0 & 0 & 0 & 0 & 0 & 0 & 0 & 97 \\
\hline 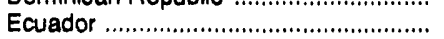 & 0 & 0 & 226 & 0 & 0 & 0 & 0 & 0 \\
\hline Egypt & 0 & 0 & 0 & 0 & 0 & 0 & 0 & 0 \\
\hline 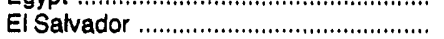 & 0 & 0 & 13 & 0 & 0 & 0 & 0 & 0 \\
\hline Finland & 0 & 0 & 0 & 0 & 0 & 0 & 0 & 0 \\
\hline France & 0 & 0 & 0 & 0 & 0 & 0 & 0 & 0 \\
\hline 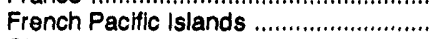 & 0 & 0 & 0 & 0 & 0 & 0 & 0 & 99 \\
\hline 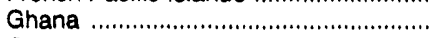 & 0 & 0 & 0 & 0 & 0 & 0 & 0 & 0 \\
\hline Gre日ce & 0 & 0 & 0 & 0 & 0 & (s) & 0 & 0 \\
\hline 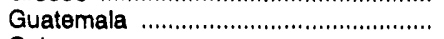 & 0 & 0 & 18 & 179 & 5 & 0 & 0 & 42 \\
\hline Guinea & 0 & 0 & 0 & 0 & 0 & 0 & (s) & 0 \\
\hline 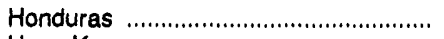 & 0 & 0 & 8 & 65 & 10 & 0 & 0 & 0 \\
\hline 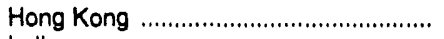 & 0 & 0 & 0 & 0 & 0 & 0 & 0 & (s) \\
\hline India & 0 & 0 & 0 & 0 & 270 & 0 & 0 & 0 \\
\hline 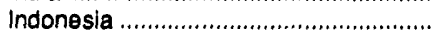 & 0 & 0 & 0 & 0 & 0 & 0 & 0 & 0 \\
\hline |reland & 0 & 0 & 0 & 0 & 0 & 0 & 0 & 0 \\
\hline 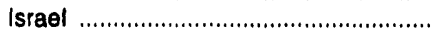 & 0 & 0 & 0 & 0 & 211 & (s) & 0 & 0 \\
\hline 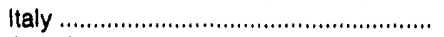 & 0 & 0 & 0 & 0 & 0 & 0 & 0 & 320 \\
\hline 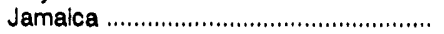 & 0 & 0 & 0 & 0 & 0 & 0 & 23 & 646 \\
\hline 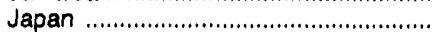 & 0 & 0 & 1 & 194 & 1,254 & 0 & 20 & 382 \\
\hline 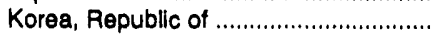 & 0 & 0 & 0 & 0 & 381 & 0 & 899 & 66 \\
\hline Malaysla & 0 & 0 & 0 & 0 & 0 & 0 & 0 & 0 \\
\hline Mexico & 0 & 0 & 593 & 1,696 & 55 & 1 & 33 & 961 \\
\hline 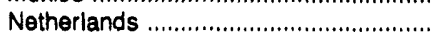 & 0 & 0 & 0 & 0 & 0 & (s) & (s) & 0 \\
\hline Netherlands Antilles ............................... & 0 & 0 & 2 & 302 & 0 & 0 & 150 & 0 \\
\hline 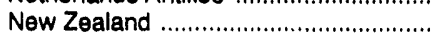 & 0 & 0 & 0 & 0 & 0 & 0 & 0 & 0 \\
\hline 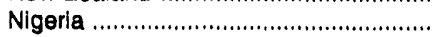 & 0 & 0 & 0 & 0 & 478 & 0 & 0 & 0 \\
\hline Norway & 0 & 0 & 0 & 0 & 0 & 0 & 0 & 0 \\
\hline 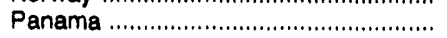 & 0 & 0 & 21 & 0 & 0 & 0 & 184 & 256 \\
\hline 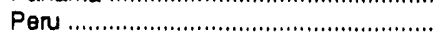 & 0 & 0 & 0 & 10 & 0 & 0 & 1 & 0 \\
\hline 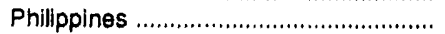 & 0 & 0 & 0 & 0 & 0 & 0 & 0 & 0 \\
\hline Poland & 0 & 0 & 0 & 0 & 0 & 0 & 0 & 0 \\
\hline 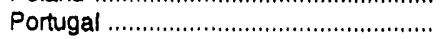 & 0 & 0 & 0 & 0 & 0 & 0 & 0 & 0 \\
\hline 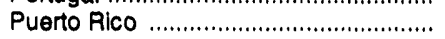 & 0 & 0 & 2 & 366 & 44 & 0 & 89 & $(s)$ \\
\hline Russia & 0 & 0 & 0 & 0 & 0 & 0 & (s) & 0 \\
\hline 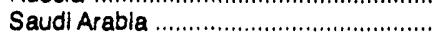 & 0 & 0 & (s) & (s) & 0 & 0 & 1 & 0 \\
\hline 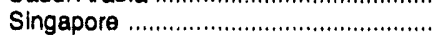 & 0 & 0 & 0 & 0 & 0 & 0 & 146 & 231 \\
\hline South Africa & 0 & 0 & 0 & 0 & 0 & 0 & 0 & 0 \\
\hline 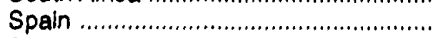 & 0 & 0 & 0 & 0 & 0 & 0 & 0 & 0 \\
\hline 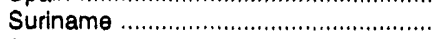 & 0 & 0 & 0 & 0 & 0 & 0 & 0 & 0 \\
\hline 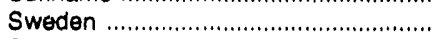 & 0 & 0 & 0 & 0 & 0 & 0 & (s) & 0 \\
\hline 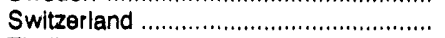 & 0 & 0 & 0 & 0 & 0 & 0 & 0 & 0 \\
\hline 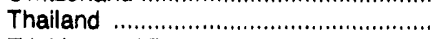 & 0 & 0 & 0 & 0 & 0 & (s) & 0 & 0 \\
\hline 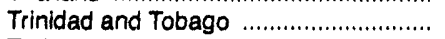 & 0 & 0 & 0 & 0 & 0 & 0 & (s) & 0 \\
\hline 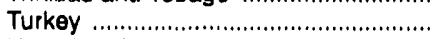 & 0 & 0 & 0 & 0 & 0 & 0 & 0 & 0 \\
\hline United Arab Emirates ............................. & 0 & 0 & 0 & 0 & 0 & 0 & 0 & 326 \\
\hline United Kirgdom .................................... & 0 & 0 & 23 & 0 & 0 & 0 & 1 & 0 \\
\hline 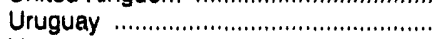 & 0 & 0 & 0 & 0 & 0 & 0 & 0 & 0 \\
\hline 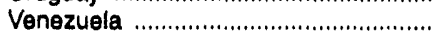 & 0 & 0 & 1 & 0 & 0 & 0 & (s) & 0 \\
\hline 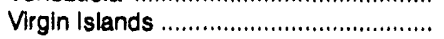 & 4,255 & 0 & 0 & 0 & 0 & 0 & 0 & 0 \\
\hline 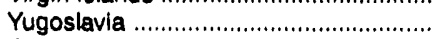 & 0 & 0 & 0 & 0 & 0 & 0 & 1 & 0 \\
\hline 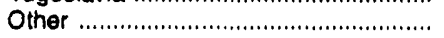 & 0 & 0 & 143 & 40 & 0 & 0 & 125 & 0 \\
\hline 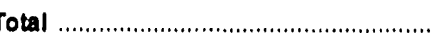 & 4,301 & 29 & 1,443 & 3,364 & 3,117 & 6 & 2,777 & 4,704 \\
\hline
\end{tabular}

See footnotes at end of table. 
Table 47. Exports of Crude Oll and Petroleum Products by Destination, March 1993 (Continued) (Thousand Barrels)

\begin{tabular}{|c|c|c|c|c|c|c|c|c|}
\hline \multirow{2}{*}{ Destination } & \multirow[b]{2}{*}{$\begin{array}{c}\text { Speclal } \\
\text { Naphthas }\end{array}$} & \multirow[b]{2}{*}{ Lubrlcants } & \multirow[b]{2}{*}{ Waxes } & \multirow[b]{2}{*}{$\begin{array}{c}\text { Petroleum } \\
\text { Coke }\end{array}$} & \multirow[b]{2}{*}{$\begin{array}{c}\text { Asphalt } \\
\text { and Road } \\
\text { Oll }\end{array}$} & \multirow[b]{2}{*}{$\begin{array}{c}\text { Other } \\
\text { Products }\end{array}$} & \multicolumn{2}{|c|}{ Crude OII and Products } \\
\hline & & & & & & & Total & $\begin{array}{c}\text { Dally } \\
\text { Average }\end{array}$ \\
\hline 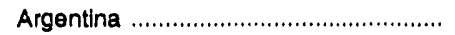 & (s) & 2 & (s) & 0 & (s) & (s) & 3 & (s) \\
\hline 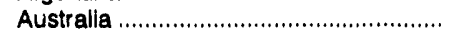 & (s) & 9 & 3 & 262 & (s) & (s) & 278 & 9 \\
\hline 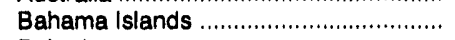 & (s) & 2 & 0 & 0 & (s) & 0 & 176 & 6 \\
\hline 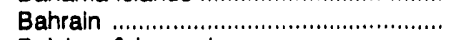 & 0 & 0 & 0 & 0 & (s) & 0 & 1 & (s) \\
\hline Belgium \& Luxembourg ............................. & 4 & 8 & 4 & 806 & (s) & (s) & 833 & 27 \\
\hline 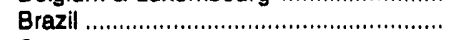 & 0 & 1 & (s) & 0 & 0 & (s) & 367 & 12 \\
\hline 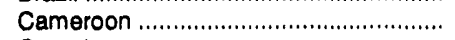 & 0 & (s) & 0 & 37 & 0 & 0 & 37 & 1 \\
\hline 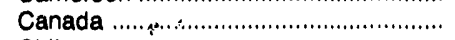 & 10 & 106 & 9 & 350 & 20 & 3 & 1,992 & 64 \\
\hline 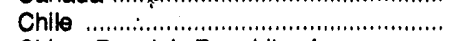 & 0 & 2 & (s) & 0 & (s) & (s) & 3 & (s) \\
\hline China, People's Republic of ................. & 0 & 97 & (s) & 0 & 0 & 0 & 467 & 15 \\
\hline 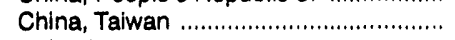 & 1 & 15 & (s) & 0 & 1 & 260 & 1,519 & 49 \\
\hline 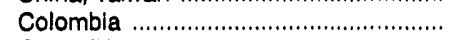 & 0 & 2 & (s) & 1 & (s) & (s) & 117 & 4 \\
\hline 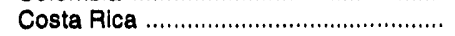 & 2 & 10 & (s) & 0 & 0 & (s) & 13 & (s) \\
\hline 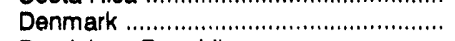 & 0 & (s) & 1 & 0 & 0 & 0 & 1 & (s) \\
\hline Dominican Republic ............................. & 0 & 3 & 0 & 0 & 0 & 0 & 100 & 3 \\
\hline 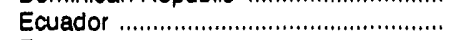 & $(\mathbf{s})$ & (s) & (s) & 0 & 0 & (s) & 227 & 7 \\
\hline 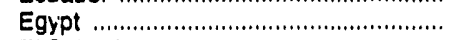 & (s) & (s) & 0 & 0 & 0 & 0 & (s) & (s) \\
\hline 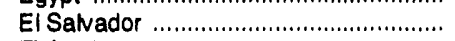 & (s) & 29 & (s) & 0 & 0 & 0 & 43 & 1 \\
\hline Finland & 0 & 1 & (s) & 146 & 0 & (s) & 147 & 5 \\
\hline France & (s) & (s) & 2 & 0 & (s) & 0 & 3 & (s) \\
\hline Fronch Pacific Islands .......................... & 0 & (s) & 0 & 0 & 0 & 0 & 99 & 3 \\
\hline 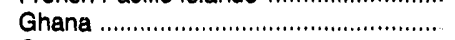 & 0 & (s) & 0 & 0 & 0 & 0 & (s) & (s) \\
\hline Gre日ce & 0 & 2 & (s) & 72 & 0 & 0 & 74 & 2 \\
\hline 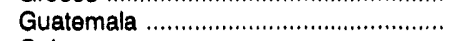 & 2 & 8 & 1 & 0 & 0 & 0 & 254 & 8 \\
\hline Guinea & 0 & 1 & 0 & 0 & 0 & 0 & 1 & (s) \\
\hline 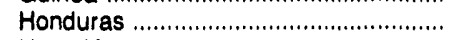 & 1 & 5 & (s) & 0 & 0 & 0 & 90 & 3 \\
\hline 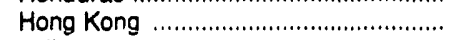 & 0 & 3 & 1 & 0 & 0 & 0 & 5 & (s) \\
\hline India & 0 & (s) & (s) & 0 & 0 & 0 & 270 & 9 \\
\hline 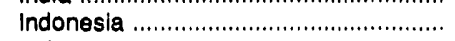 & 0 & 4 & 0 & 0 & (s) & 0 & 4 & (s) \\
\hline Ireland & 0 & (s) & (s) & 0 & 0 & 0 & (s) & (s) \\
\hline 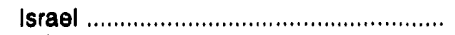 & 0 & 4 & (s) & 0 & 0 & 0 & 215 & 7 \\
\hline Italy & (s) & 1 & 1 & 356 & 0 & (s) & 678 & 22 \\
\hline 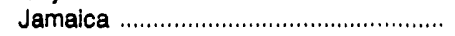 & (s) & 1 & (s) & 0 & 0 & 1 & 670 & 22 \\
\hline 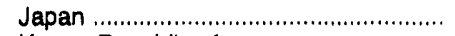 & 1 & 19 & 3 & 2,911 & 1 & 2 & 4,788 & 154 \\
\hline 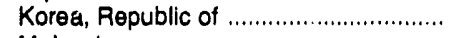 & 0 & 7 & 1 & 238 & (s) & (s) & 1,591 & 51 \\
\hline 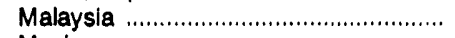 & 0 & 1 & (s) & 0 & 0 & (s) & 1 & (s) \\
\hline Mexico & 4 & 133 & 21 & 85 & 12 & 56 & 3,649 & 118 \\
\hline 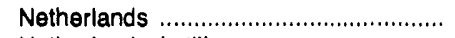 & 2 & 2 & 1 & 435 & 1 & (s) & 441 & 14 \\
\hline 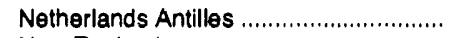 & 0 & 2 & 0 & 0 & 0 & 198 & 654 & 21 \\
\hline 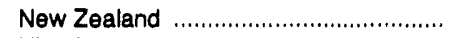 & 0 & 5 & 0 & 0 & 0 & 0 & 5 & (s) \\
\hline 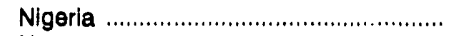 & 0 & (s) & 0 & 0 & 0 & 0 & 478 & 15 \\
\hline 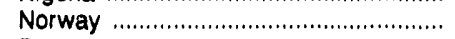 & (s) & (s) & (s) & 0 & 0 & 0 & (s) & (s) \\
\hline 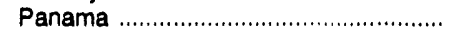 & (s) & 2 & (s) & 72 & 0 & (s) & 534 & 17 \\
\hline 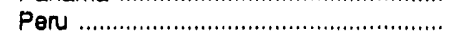 & 0 & 27 & 1 & 1 & 0 & 0 & 39 & 1 \\
\hline 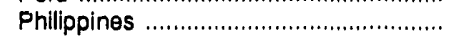 & 0 & 2 & (s) & 0 & 0 & (s) & 2 & (s) \\
\hline 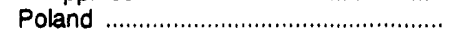 & 0 & $\overline{1}$ & 0 & 0 & 0 & 0 & 1 & (s) \\
\hline 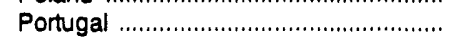 & 0 & (s) & 0 & 0 & 0 & 0 & (s) & (s) \\
\hline 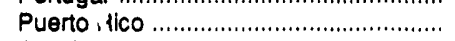 & 1 & 14 & 1 & 0 & 0 & 0 & 516 & 17 \\
\hline Russia & 0 & 2 & (s) & 0 & 0 & 0 & 2 & (s) \\
\hline 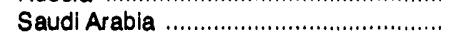 & (s) & $\overline{3}$ & (s) & (s) & 0 & (s) & 4 & (s) \\
\hline Singapore & 0 & 27 & (s) & $\begin{array}{r}101 \\
0\end{array}$ & 1 & (s) & 405 & 13 \\
\hline South Africa & 0 & 1 & (s) & 0 & (s) & 0 & 1 & (s) \\
\hline Spain & 0 & 1 & (s) & 929 & 0 & 0 & 930 & 30 \\
\hline Suriname & 0 & (s) & 0 & 0 & 0 & 0 & (s) & (s) \\
\hline 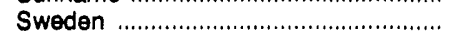 & 0 & 1 & (s) & 138 & 0 & (s) & 139 & 4 \\
\hline 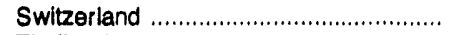 & (s) & (s) & (s) & 0 & (s) & 0 & (s) & (s) \\
\hline 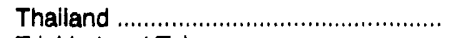 & 0 & 17 & (s) & 0 & (s) & 0 & 17 & 1 \\
\hline Trinidad and Tobago ................................ & 0 & (s) & (s) & 0 & 0 & (s) & 1 & (s) \\
\hline Turkey & 0 & 0 & (s) & 0 & (s) & 0 & (s) & (s) \\
\hline United Arab Emirates ............................ & (s) & (s) & 0 & 58 & 0 & (s) & 384 & 12 \\
\hline United Kingdom .................................... & 1 & 1 & 1 & 2 & 1 & 0 & 29 & 1 \\
\hline 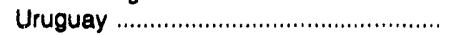 & 0 & 2 & 0 & 0 & 0 & 0 & 2 & (s) \\
\hline 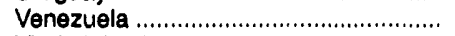 & 4 & 3 & 1 & 109 & 1 & (s) & 119 & 4 \\
\hline 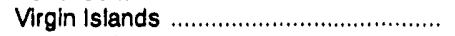 & 0 & 0 & 0 & 0 & 0 & 0 & 4,255 & 137 \\
\hline 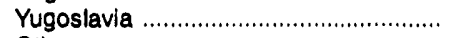 & 0 & (s) & 0 & 0 & 0 & 0 & 1 & (s) \\
\hline 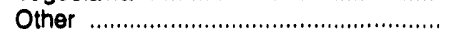 & (s) & 41 & 0 & 0 & 4 & (s) & 353 & 11 \\
\hline 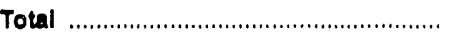 & 33 & 635 & 59 & 7,018 & 49 & 523 & 28,061 & 905 \\
\hline
\end{tabular}

a Crude oil exports are restricted to: (1) crude oll derlved from fields under the State waters of Alaska's Cook Inlet; (2) certain domestically produced crude oil destined for Canada; and (3) shipments to U.S. territories.

includes miscellaneous products, motor gasoline blending components, and other hydrocarbons and oxygenates.

(s) $=$ Less than 500 barrels or less than 500 barrels per day.

Note: Totals may not equal sum of components due to independent rounding.

Sources: Energy Information Administration (EIA) Form EIA-810, "Monthly Refinery Report" and the U.S. Bureau of the Census. 
Table 48. Year-to-Date Exports of Crude Oll and Petroleum Products by Destination, January-March 1993 (Thousand Barrels)

\begin{tabular}{|c|c|c|c|c|c|c|c|c|}
\hline Destination & $\begin{array}{c}\text { Crude } \\
\text { Olla }\end{array}$ & $\begin{array}{c}\text { Pentenos } \\
\text { Pluse }\end{array}$ & $\begin{array}{c}\text { Liquefled } \\
\text { Potroleum } \\
\text { Qases }\end{array}$ & $\begin{array}{l}\text { Finlshed } \\
\text { Motor } \\
\text { Qasollne }\end{array}$ & Jet Fuel & Kerosene & $\begin{array}{c}\text { Dlstlliate Fuel } \\
\text { Oll }\end{array}$ & $\begin{array}{l}\text { Residual } \\
\text { Fuel OII }\end{array}$ \\
\hline 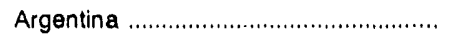 & 0 & 0 & 0 & 0 & 0 & 0 & (s) & 0 \\
\hline Australia & 0 & 0 & 4 & 299 & 0 & 0 & 265 & 0 \\
\hline 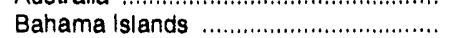 & 0 & 0 & 36 & 120 & 32 & 0 & 104 & 399 \\
\hline 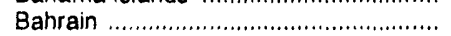 & 0 & 0 & 0 & 0 & 0 & 0 & (s) & 0 \\
\hline 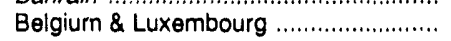 & 0 & 0 & 9 & 1 & 0 & 0 & 10 & 0 \\
\hline 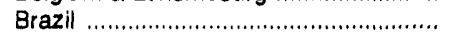 & 0 & 0 & 77 & 0 & 140 & 0 & 226 & 0 \\
\hline Cameroon & 0 & 0 & 0 & 0 & 0 & 0 & 0 & 0 \\
\hline 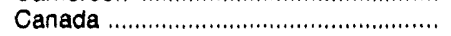 & 272 & 89 & 527 & 869 & 585 & 13 & 40 & 1,940 \\
\hline Chile & 0 & 0 & 0 & 0 & 0 & 0 & 0 & 0 \\
\hline China, People's Republic of .................. & 0 & 0 & 0 & 212 & 308 & 0 & 200 & 0 \\
\hline 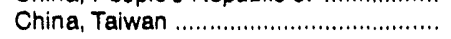 & 0 & 0 & 0 & 1,084 & 0 & (s) & 600 & 389 \\
\hline 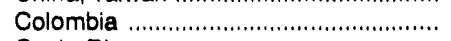 & 0 & 0 & 326 & 0 & 0 & 0 & 4 & 0 \\
\hline 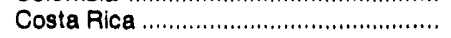 & 0 & 0 & 1 & 0 & 20 & 0 & (s) & 0 \\
\hline 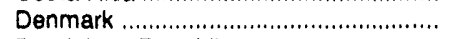 & 0 & 0 & 1 & 0 & 0 & 0 & 0 & 0 \\
\hline Dominican Republic .............................. & 0 & 0 & 135 & 44 & 0 & 0 & (s) & 97 \\
\hline 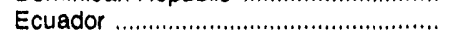 & 0 & 0 & 549 & 0 & 0 & 0 & 0 & 0 \\
\hline 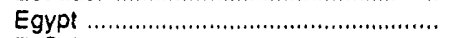 & 0 & 0 & 0 & 0 & 0 & 5 & 9 & 0 \\
\hline 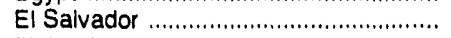 & 0 & 0 & 43 & 122 & 0 & 0 & 0 & 31 \\
\hline 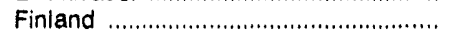 & 0 & 0 & 0 & 0 & 0 & 0 & 0 & 0 \\
\hline France & 0 & 0 & 0 & 0 & 0 & 0 & (s) & 0 \\
\hline French Pacific Islands .......................... & 0 & 0 & 16 & 0 & 0 & 0 & 0 & 99 \\
\hline 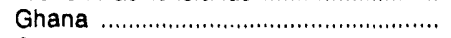 & 0 & 0 & 0 & 0 & 0 & 0 & 0 & 0 \\
\hline 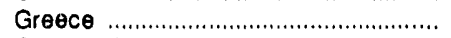 & 0 & 0 & 0 & 0 & 0 & (s) & 0 & 0 \\
\hline 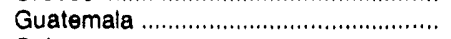 & 0 & 0 & 61 & 414 & 38 & 5 & 0 & 279 \\
\hline 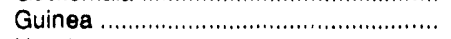 & 0 & 0 & 0 & 0 & (s) & 0 & (s) & 0 \\
\hline 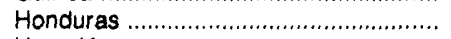 & 0 & 0 & 44 & 223 & 41 & 0 & 0 & 0 \\
\hline 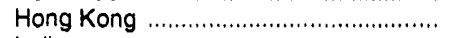 & 0 & 0 & (s) & 0 & 0 & 0 & 0 & 1 \\
\hline 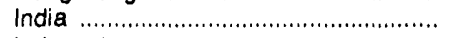 & 0 & 0 & 0 & 0 & 555 & 0 & (s) & 0 \\
\hline 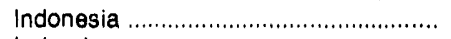 & 0 & 0 & 205 & 0 & 0 & 0 & 799 & 3 \\
\hline 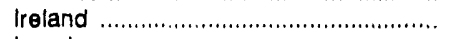 & 0 & 0 & 0 & 0 & 0 & 0 & 0 & 154 \\
\hline 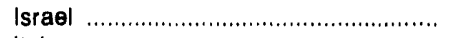 & 0 & (s) & 0 & 0 & 404 & (s) & (s) & 0 \\
\hline 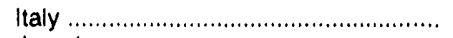 & 0 & 1 & 1 & 0 & 0 & 0 & (s) & 1,806 \\
\hline 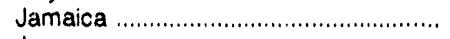 & 0 & 0 & 8 & 0 & 0 & (s) & 23 & 1,726 \\
\hline 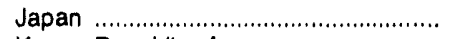 & 0 & 0 & 2 & 194 & 3,301 & 0 & 117 & 531 \\
\hline Korea, Republic of .................................. & 400 & 0 & 0 & 0 & 1,423 & 544 & 3,403 & 917 \\
\hline 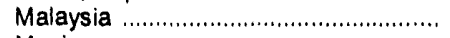 & 0 & 0 & 0 & 0 & 0 & 0 & 0 & 0 \\
\hline 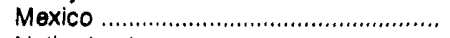 & 0 & 0 & 1,835 & 5,680 & 55 & 2 & 463 & 1,445 \\
\hline 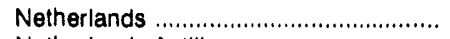 & 0 & 0 & 0 & 0 & 0 & $\overline{1}$ & (s) & 0 \\
\hline Netherlands Antilles ............................. & 0 & 0 & 9 & 407 & 0 & 0 & 735 & 0 \\
\hline New Zealand & 0 & 0 & 0 & 0 & 0 & 0 & 0 & 0 \\
\hline 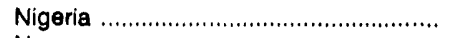 & 0 & 0 & 0 & 0 & 478 & 0 & (s) & 0 \\
\hline 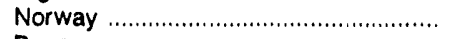 & 0 & 0 & 0 & 0 & 0 & 0 & 0 & 0 \\
\hline 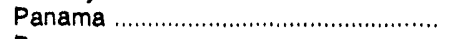 & 0 & 0 & 76 & 0 & 30 & 0 & 374 & 536 \\
\hline 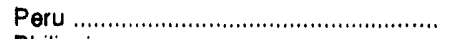 & 0 & 0 & 0 & 10 & 0 & 0 & 331 & 0 \\
\hline 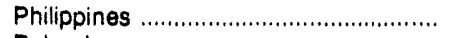 & 0 & 0 & 0 & 0 & 0 & 0 & 0 & 0 \\
\hline 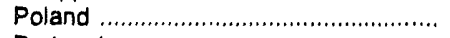 & C & 0 & 0 & 0 & 0 & 0 & 0 & 0 \\
\hline 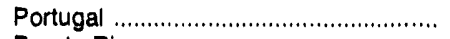 & 0 & 0 & 0 & 0 & 0 & 0 & 127 & 0 \\
\hline 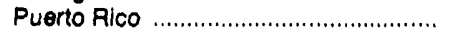 & 0 & 0 & 4 & 733 & 88 & 0 & 184 & 1 \\
\hline 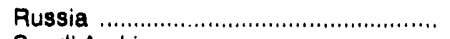 & 0 & 0 & 0 & 0 & 0 & 0 & (s) & (s) \\
\hline 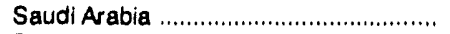 & 0 & 0 & (s) & (8) & 0 & (s) & 1 & 0 \\
\hline 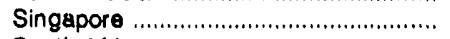 & 0 & 0 & 0 & 0 & 226 & 0 & 817 & 1,077 \\
\hline South Africa & 0 & 0 & 0 & 0 & 0 & 0 & 0 & 0 \\
\hline Spain & 0 & 0 & 0 & 0 & 1 & 0 & 314 & 56 \\
\hline Suriname & 0 & 0 & 11 & 0 & 0 & 0 & 0 & 0 \\
\hline 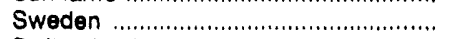 & 0 & 0 & (s) & 0 & 0 & 0 & (s) & 0 \\
\hline Switzerland & 0 & 0 & (s) & 0 & 0 & 0 & 0 & 0 \\
\hline Thailand & 0 & 0 & 0 & 0 & 0 & (s) & 0 & 0 \\
\hline Trinidad and Tobago ............................... & 0 & 0 & (3) & 0 & 0 & 0 & 1 & 0 \\
\hline 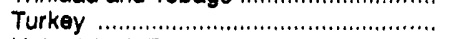 & 0 & 0 & 0 & 0 & 0 & 0 & 0 & 0 \\
\hline United Arab Emirates ............................ & 0 & 0 & 0 & 0 & 0 & 0 & 0 & 998 \\
\hline United Kingdom ...................................... & 0 & 0 & 24 & 2 & 0 & 16 & 1 & 0 \\
\hline Uruguay & 0 & 0 & 0 & 0 & 0 & 0 & 0 & 0 \\
\hline 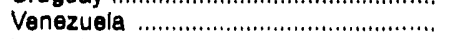 & 0 & 0 & 1 & 0 & 0 & 0 & (s) & 1 \\
\hline 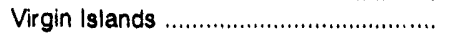 & 12,260 & 0 & 2 & 0 & 0 & 0 & 0 & 0 \\
\hline 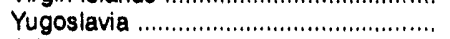 & 0 & 0 & 0 & 0 & 0 & 0 & 1 & 0 \\
\hline 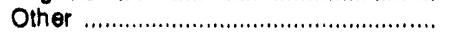 & 0 & 0 & 163 & 124 & 27 & (s) & 269 & 14 \\
\hline 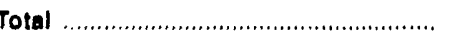 & 12,832 & 80 & 4,180 & 10,536 & 7,749 & 588 & 9,421 & 12,001 \\
\hline
\end{tabular}

See footnotes at end of table. 
Table 48. Year-to-Date Exports of Crude Oll and Petroleum Products by Destination, January-March 1993 (ContInued) (Thousand Barrels)

\begin{tabular}{|c|c|c|c|c|c|c|c|c|}
\hline \multirow{2}{*}{ Destination } & \multirow[b]{2}{*}{$\begin{array}{c}\text { Special } \\
\text { Nephthas }\end{array}$} & \multirow[b]{2}{*}{ Lubricante } & \multirow[b]{2}{*}{ Waxee } & \multirow[b]{2}{*}{$\begin{array}{c}\text { Potroleum } \\
\text { Coke }\end{array}$} & \multirow[b]{2}{*}{$\begin{array}{c}\text { Asphalt } \\
\text { and Road } \\
\text { Oll }\end{array}$} & \multirow[b]{2}{*}{$\begin{array}{l}\text { Other } \\
\text { Products }\end{array}$} & \multicolumn{2}{|c|}{ Crude Oll and Products } \\
\hline & & & & & & & Total & $\begin{array}{c}\text { Dally } \\
\text { Average }\end{array}$ \\
\hline
\end{tabular}

Argentina

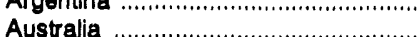

Bahama Islands

Bahrain

Belgium \& Luxembourg

Brazil .....

Cameroon

Canada

Chile

Chine Poople's Republic of

China, Taiwan

Colombia

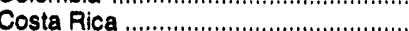

Denmark

Dominican Republic

Ecuador

Egypt ...........

El Salvador

Finland

France

French Pacific Islands

Ghana

Guatemala

Guinea

Honduras

Hong Kong

India.........

indonesia

Ireland

Italy.

Jamaica

Korea, Republic of

Malaysia

Mexico .........

theriands

Netherlands Antilles

New Zealand

Nigeria

Norway

Panama

Peru.....

Philippines

Poland ....

Portugal

Puerto Rico

Russia

Saudi Arabia

Singapore

South Africa

Spain

Suriname

Sweden

Switzerland

Thailand ....

Turkey

Arab Emirates

United Kingdom

Uruguay ......

Venezuela ......

Virgin Islands

Yugoslavia

Other .

Total

\begin{tabular}{|c|c|}
\hline (s) & 19 \\
\hline (s) & 20 \\
\hline (s) & 5 \\
\hline 0 & (s) \\
\hline 5 & 17 \\
\hline 301 & 22 \\
\hline 0 & (s) \\
\hline 18 & 262 \\
\hline (s) & 23 \\
\hline 0 & 101 \\
\hline 10 & 47 \\
\hline (s) & 21 \\
\hline 6 & 28 \\
\hline 0 & 1 \\
\hline 0 & 12 \\
\hline (s) & 2 \\
\hline (s) & (s) \\
\hline 1 & 36 \\
\hline 0 & 2 \\
\hline 7 & 3 \\
\hline 0 & 1 \\
\hline 0 & (s) \\
\hline (s) & 3 \\
\hline 5 & 20 \\
\hline (s) & 4 \\
\hline 4 & 12 \\
\hline 1 & 9 \\
\hline (s) & 11 \\
\hline 0 & 7 \\
\hline 0 & (s) \\
\hline (s) & 5 \\
\hline (s) & 2 \\
\hline (s) & 2 \\
\hline 69 & 51 \\
\hline 11 & 20 \\
\hline (s) & 3 \\
\hline 10 & 352 \\
\hline 13 & 5 \\
\hline 0 & 7 \\
\hline 0 & 7 \\
\hline (s) & 1 \\
\hline (s) & 1 \\
\hline (s) & 5 \\
\hline (s) & 30 \\
\hline (s) & 8 \\
\hline 0 & 1 \\
\hline 0 & (s) \\
\hline 2 & 39 \\
\hline 1 & 9 \\
\hline (s) & 8 \\
\hline 1 & 42 \\
\hline (s) & 18 \\
\hline 0 & 2 \\
\hline 0 & 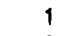 \\
\hline 0 & 2 \\
\hline (s) & 1 \\
\hline (s) & 21 \\
\hline 0 & 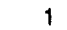 \\
\hline 0 & 12 \\
\hline (s) & 37 \\
\hline 1 & 4 \\
\hline 0 & 3 \\
\hline 4 & 7 \\
\hline (s) & (s) \\
\hline 0 & (s) \\
\hline 1 & 198 \\
\hline
\end{tabular}

475

\section{1}

0

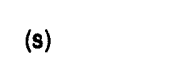

222

(s)

$\begin{array}{ll}5 & 0 \\ & 0\end{array}$

$\begin{array}{rr}712 & 1 \\ 0 & (s) \\ 0 & (s) \\ 2,280 & 3 \\ 0 & (s) \\ 37 & 0 \\ 1,016 & 35 \\ 0 & (s)\end{array}$

(s)
0
0

(s)

1,304
696

1
2,330

766

37
5,705
25

822

2,652

457
56

203

289

552

15

234

360
75

116

$(\mathrm{s})$
367

367
826
5

5
324

13
655

1,015

155

410
3,535

1,761

9,853

6,962
4

10,200

1,350

1,356

119
480

37
1,124

1,124
374

374
10
1

146
1,054

1,054
11

47

2, 164

101
2,367

1

12
141

2
24
2

2,051

$1,151 \quad 13$

790
6

481

12,262

323
883

81,490

849

a Crude oil exports are restricted to: (1) crude oil derived from flelds under the State waters of Alaska's Cook Inlet; (2) certain domestically produced crude oil destined for Canada; and (3) shipments to U.S. territories.

b Includes miscellaneous products, motor gasoline blending components, and other hydrocarbons and oxygenates.

(s) = Less than 500 barrels or less than 500 barrels per day.

Note: Totals may not equal sum of components due to independent rounding.

Sources: Energy Information Administration (EIA) Form EIA-810, "Monthly Refinery Report" and the U.S. Bureau of the Census. 
Table 49. Net Imports of Crude Oll and Petroleum Products Into the United States by Country, March 1993

(Thousand Barrels per Day)

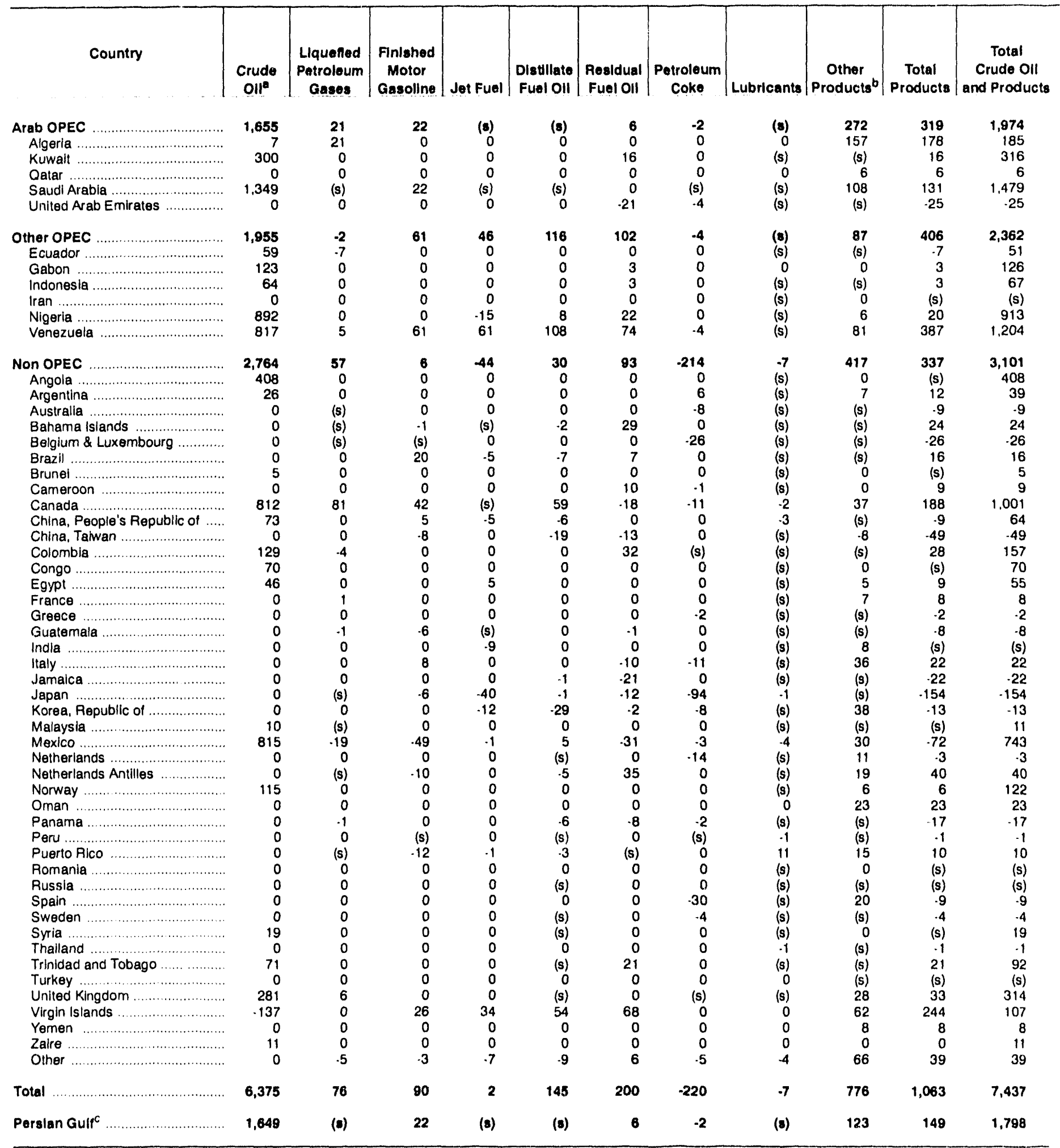

a Includes crude oll imported for storage in the Strategic Petroleum Reserve.

b Includes asphalt and road oll, aviation gasoline, avlation gasoline blending components, kerosene, miscellaneous products, motor gasoline blending components, naphtha for petrochemical feedstock use, other hydrocarbons and oxygenates, other oils for petrochemical feedstock use, pentanes plus, special naphthas. untinished olls, and waxes.

c Includes Bahrain, Iran, Iraq, Kuwait, Qatar. Saudl Arabla, and United Arab Emirates

(s) $=$ Less than 500 barreis per day.

Note: Totals may not equal sum of components due to independent rounding.

Sources: Energy Information Administration (EIA) Forms EIA.810, "Monthly Refinery Report," EIA.814, "Monthly Imports Report" and the U.S. Bureau of the Census 
Table 50. Year-to-Date Net Imports of Crude Oll and Petroleum Products Into the United States by Country, January-March 1993

(Thousand Barrels per Day)

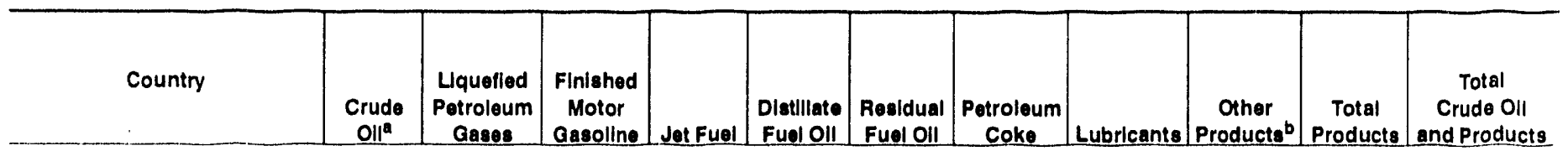

Arab OPEC .

Algeria

Kuvialt

Qatar

Saudl Arabia

United Arab Emirates

Other OPEC

Ecuador

Gabon

Indonesia

Iran ......

Non OPEC

Angola ........

Argentina

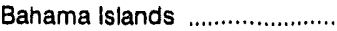

Belglum \& Luxembourg ............

Brazil

Brunel .

Cameroon

(a)

China, People's Republic of .....

China, Taiwan .

Colombia

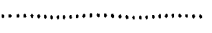

Egypt

France

Greece

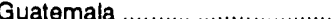

India

Italy ........................

Jamaica

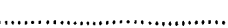

man.

Korea, Republic of .....................

Malaysla .....

Moxico .......

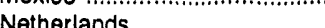

Netherlands Antilles

Norway

Oman .....

(n)

Panama

Peru

Puerto Rico

Romania

(n)

Russia

Spain

Sweden

Syria .........

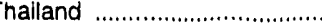

Trinidad and Tobago

Turkey

United Kingdom

Virgin Islands

Yemen

Zaire

.

Other

Total

Perslan Gulf

$\begin{array}{rrr}1,697 & 16 & 19 \\ 12 & 14 & 0 \\ 219 & 0 & 0 \\ 0 & 0 & 0 \\ 1,466 & 2 & 19 \\ 0 & 0 & 0\end{array}$

4
0
0
0
4
0

$\begin{aligned} \text { (o) } & \\ 0 & \\ \text { (s) } & \\ 0 & \\ \text { (s) } & \\ 0 & \end{aligned}$

21
9
17
0
0
.11

-1
0
0
0
$(s)$
-1

$\begin{array}{rr}1,968 & -5 \\ 49 & 6 \\ 100 & 0 \\ 51 & -2 \\ 0 & 0 \\ 842 & 0 \\ 927 & 4\end{array}$

\section{2,517}

371
22

(1)

0

(789

789

789
0
145

145

35

3

0

$+$

4

78

79

8

0

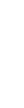

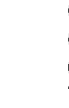$$
5
$$$$
\begin{array}{r}
0 \\
205 \\
.136
\end{array}
$$$$
\begin{array}{r}
.136 \\
0
\end{array}
$$$$
12
$$

8,182

1,685

$\begin{array}{rr}61 & 47 \\ 0 & 0 \\ 0 & 0 \\ 0 & 0 \\ 0 & 0 \\ 0 & -5 \\ 61 & 52\end{array}$

\section{7}

0

90

-9
0

3

93

65

0
0
$(s)$

(s)

(s)

$\begin{array}{ll}-1 & \text { (s) } \\ 0 & 14\end{array}$

$\begin{array}{ll}0 & 0 \\ 0 & 0\end{array}$

$\begin{array}{rr}90 & 44 \\ 0 & -1 \\ 0 & -12\end{array}$

$\begin{array}{rrr}0 & -12 & 0 \\ -4 & 0 & 0\end{array}$

0000

$\begin{array}{rlll}0 & 0 & 2 & (s) \\ (s) & 0 & 0 & \text { (s) }\end{array}$

$\begin{array}{rrr}0 & 0 \\ -1 & -5 & 0 \\ 0 & 0\end{array}$

$\begin{array}{rrrrr}-1 & 0 & & \\ & 12 & 0 & \text { (s) } & \text { (s) }\end{array}$

(s)

(s)
0

$\begin{array}{lll}0 & -2 & 37 \\ 0 & 0 & .16\end{array}$

(s)
.16

$\begin{array}{rr}.16 & 61\end{array}$

(s)

(s)

0

-1
0

0
(s)
0

0

(s)

0

(s)

0

(s)

0

0
.5

76

2

19

Includes crude oil imported for storage in the Strategic Petroleum Reserve.

- Includes asphalt and road oil, aviation gasoline, aviation gasoline blending components, kerosene, miscellaneous products, motor gasoline blending

components, narhtha for petrochemical feedstock use, other hydrocarbons and oxygenates, other oils for petrochemical feedstock use, pentanes plus, special naphthas, unfinished oils, and waxes.

c Includes Bahrain, Iran, Iraq, Kuwait, Qatar, Saudi Arabia, and United Arab Emirates.

(s) = Less than 500 barrels per day.

Note: Totals may not equal sum of components due to independent rounding

Sources: Energy Information Administration (EIA) Forms EIA-810, "Monthly Refinery Report," ElA-814, "Monthly Imports Report" and the U.S. Bureau of the Census. 
Table 51. Stocks of Crude Oll and Petroleum Products by PAD District, March 1993

(Thousand Barrels)

\begin{tabular}{|c|c|c|c|c|c|c|}
\hline \multirow{2}{*}{ Commodity } & \multicolumn{5}{|c|}{ Potroleum AdmInistration for Defense Districts } & \multirow{2}{*}{$\begin{array}{l}\text { U.s. } \\
\text { Total }\end{array}$} \\
\hline & 1 & $\|$ & III & IV & v & \\
\hline 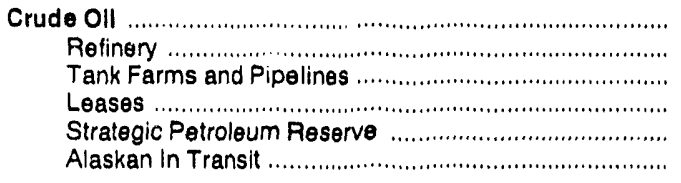 & $\begin{array}{r}15,706 \\
14,466 \\
1,222 \\
18 \\
0 \\
0\end{array}$ & $\begin{array}{r}73,747 \\
13,467 \\
58,983 \\
1,297 \\
0 \\
0\end{array}$ & $\begin{array}{r}740,304 \\
45,531 \\
101,897 \\
15,265 \\
577,611 \\
0\end{array}$ & $\begin{array}{r}12,358 \\
2,213 \\
-9,213 \\
932 \\
0 \\
0\end{array}$ & $\begin{array}{r}72,610 \\
22,532 \\
32,341 \\
1,348 \\
0 \\
16,389\end{array}$ & $\begin{array}{r}914,725 \\
98,209 \\
203,656 \\
18,660 \\
577,611 \\
16,389\end{array}$ \\
\hline 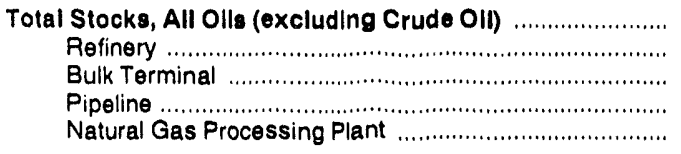 & $\begin{array}{r}148,527 \\
47,616 \\
74,790 \\
25,999 \\
122\end{array}$ & $\begin{array}{r}160,835 \\
65,980 \\
56,585 \\
36,119 \\
2,151\end{array}$ & $\begin{array}{r}244,199 \\
140,024 \\
61,271 \\
39,462 \\
3,442\end{array}$ & $\begin{array}{r}18,963 \\
13,712 \\
2,602 \\
2,411 \\
238\end{array}$ & $\begin{array}{r}96,341 \\
64,707 \\
24,496 \\
7,062 \\
76\end{array}$ & $\begin{array}{r}668,865 \\
332,039 \\
219,744 \\
111,053 \\
6,029\end{array}$ \\
\hline 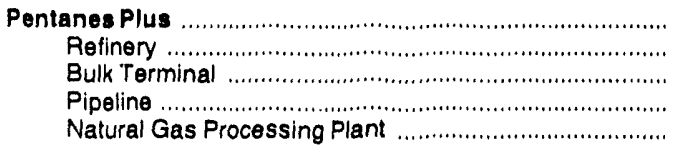 & $\begin{array}{r}55 \\
0 \\
38 \\
0 \\
17\end{array}$ & $\begin{array}{r}3,294 \\
292 \\
2,125 \\
695 \\
182\end{array}$ & $\begin{array}{r}4,767 \\
190 \\
2,904 \\
931 \\
742\end{array}$ & $\begin{array}{r}137 \\
5 \\
0 \\
66 \\
66\end{array}$ & $\begin{array}{r}65 \\
23 \\
26 \\
0 \\
16\end{array}$ & $\begin{array}{r}8,318 \\
510 \\
5,093 \\
1,692 \\
1,023\end{array}$ \\
\hline 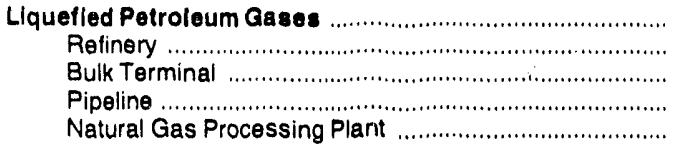 & $\begin{array}{r}2,675 \\
990 \\
692 \\
888 \\
105\end{array}$ & $\begin{array}{r}18,320 \\
2,552 \\
7,132 \\
6,667 \\
1,969\end{array}$ & $\begin{array}{r}42,405 \\
7,963 \\
24,419 \\
7,323 \\
2,700\end{array}$ & $\begin{array}{r}1,061 \\
353 \\
75 \\
461 \\
172\end{array}$ & $\begin{array}{r}2,149 \\
1,357 \\
732 \\
0 \\
60\end{array}$ & $\begin{array}{r}66,610 \\
13,215 \\
33,050 \\
15,339 \\
5,006\end{array}$ \\
\hline 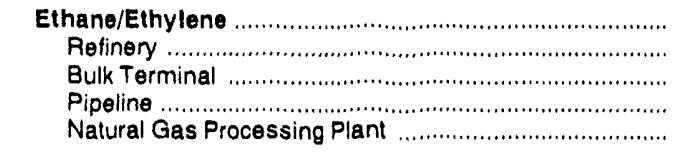 & $\begin{array}{l}0 \\
0 \\
0 \\
0 \\
0\end{array}$ & $\begin{array}{r}4,242 \\
4 \\
1,856 \\
1,686 \\
696\end{array}$ & $\begin{array}{r}13,158 \\
732 \\
8,556 \\
3,130 \\
740\end{array}$ & $\begin{array}{r}196 \\
0 \\
0 \\
190 \\
6\end{array}$ & $\begin{array}{l}0 \\
0 \\
0 \\
0 \\
0\end{array}$ & $\begin{array}{r}17,596 \\
736 \\
10,412 \\
5,006 \\
1,442\end{array}$ \\
\hline 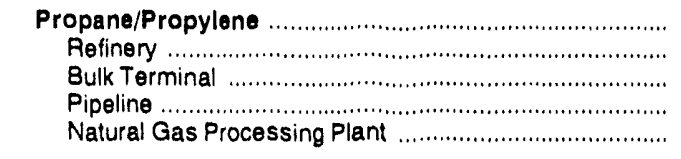 & $\begin{array}{r}1,595 \\
179 \\
519 \\
851 \\
46\end{array}$ & $\begin{array}{r}7,384 \\
789 \\
1,950 \\
3,876 \\
769\end{array}$ & $\begin{array}{r}12,212 \\
2,057 \\
6,263 \\
2,881 \\
1,011\end{array}$ & $\begin{array}{r}374 \\
53 \\
75 \\
154 \\
92\end{array}$ & $\begin{array}{r}269 \\
98 \\
139 \\
0 \\
32\end{array}$ & $\begin{array}{r}21,834 \\
3,176 \\
8,946 \\
7,762 \\
1,950\end{array}$ \\
\hline 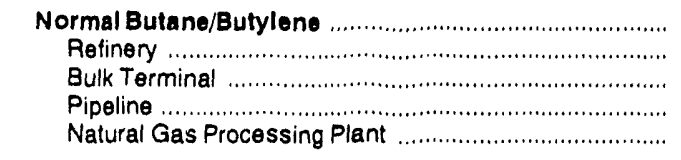 & $\begin{array}{r}940 \\
715 \\
172 \\
0 \\
53\end{array}$ & $\begin{array}{r}3,898 \\
1,231 \\
1,713 \\
569 \\
385\end{array}$ & $\begin{array}{r}9,942 \\
3,190 \\
5,385 \\
738 \\
629\end{array}$ & $\begin{array}{r}300 \\
192 \\
0 \\
49 \\
59\end{array}$ & $\begin{array}{r}1,341 \\
915 \\
415 \\
0 \\
11\end{array}$ & $\begin{array}{r}16,421 \\
6,243 \\
7,685 \\
1,358 \\
1,137\end{array}$ \\
\hline 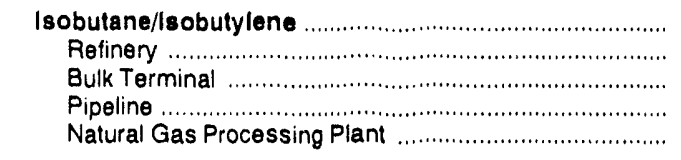 & $\begin{array}{r}140 \\
96 \\
1 \\
37 \\
6\end{array}$ & $\begin{array}{r}2,796 \\
528 \\
1,613 \\
536 \\
119\end{array}$ & $\begin{array}{r}7,093 \\
1,984 \\
4,215 \\
574 \\
320\end{array}$ & $\begin{array}{r}191 \\
108 \\
0 \\
68 \\
15\end{array}$ & $\begin{array}{r}539 \\
344 \\
178 \\
0 \\
17\end{array}$ & $\begin{array}{r}10,759 \\
3,060 \\
6,007 \\
1,215 \\
477\end{array}$ \\
\hline 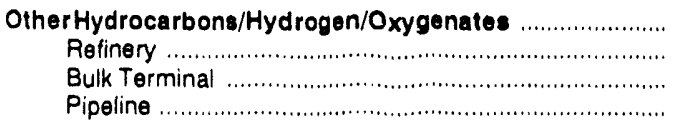 & $\begin{array}{r}2,045 \\
1,538 \\
507 \\
0\end{array}$ & $\begin{array}{r}1,300 \\
315 \\
985 \\
0\end{array}$ & $\begin{array}{r}6,810 \\
2,371 \\
4,197 \\
242\end{array}$ & $\begin{array}{r}146 \\
58 \\
88 \\
0\end{array}$ & $\begin{array}{r}4,227 \\
3,193 \\
736 \\
298\end{array}$ & $\begin{array}{r}14,528 \\
7,475 \\
6,513 \\
540\end{array}$ \\
\hline 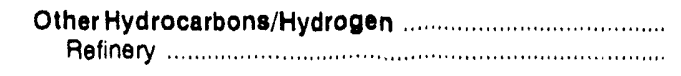 & $\begin{array}{l}12 \\
12\end{array}$ & $\begin{array}{l}21 \\
21\end{array}$ & $\begin{array}{l}0 \\
0\end{array}$ & $\begin{array}{l}0 \\
0\end{array}$ & $\begin{array}{l}6 \\
6\end{array}$ & $\begin{array}{l}39 \\
39\end{array}$ \\
\hline 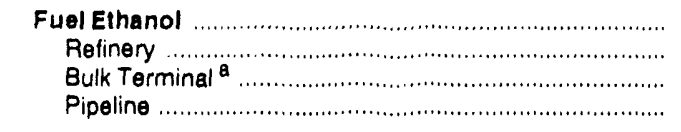 & $\begin{array}{l}62 \\
W \\
W \\
W\end{array}$ & $\begin{array}{r}1,143 \\
163 \\
W \\
W\end{array}$ & $\begin{array}{r}216 \\
W \\
W \\
W\end{array}$ & $\begin{array}{l}45 \\
W \\
W \\
W\end{array}$ & $\begin{array}{r}412 \\
W \\
W \\
W\end{array}$ & $\begin{array}{r}1,878 \\
250 \\
W \\
W\end{array}$ \\
\hline 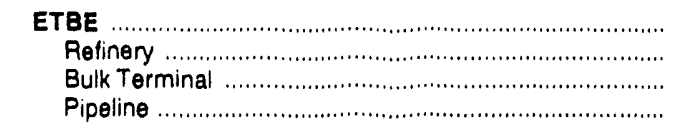 & $\begin{array}{l}W \\
W \\
W \\
W\end{array}$ & $\begin{array}{l}W \\
W \\
W \\
W\end{array}$ & $\begin{array}{l}W \\
W \\
W \\
W\end{array}$ & $\begin{array}{l}W \\
W \\
W \\
W\end{array}$ & $\begin{array}{l}W \\
W \\
W \\
W\end{array}$ & $\begin{array}{l}w \\
w \\
w \\
w\end{array}$ \\
\hline 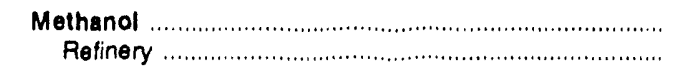 & $\begin{array}{l}W \\
W\end{array}$ & $\begin{array}{l}W \\
W\end{array}$ & $\begin{array}{l}W \\
W\end{array}$ & $\begin{array}{l}W \\
W\end{array}$ & $\begin{array}{l}W \\
W\end{array}$ & $\begin{array}{l}1,029 \\
1,029\end{array}$ \\
\hline
\end{tabular}

See footnotes at end of table. 
Table 51. Stocks of Crude Oll and Petroleum Products by PAD District, March 1993 (Continued)

(Thousand Barrels)

\begin{tabular}{|c|c|c|c|c|c|c|}
\hline \multirow{2}{*}{ Commodity } & \multicolumn{5}{|c|}{ Petroleum AdmInistration for Defense Dlatricts } & \multirow{2}{*}{$\begin{array}{l}\text { U.s. } \\
\text { Total }\end{array}$} \\
\hline & 1 & $\|$ & III & IV & $\mathbf{v}$ & \\
\hline 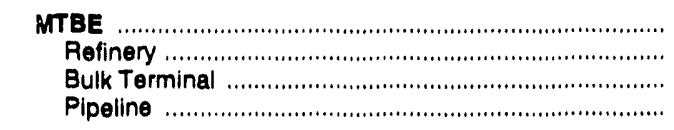 & $\begin{array}{r}1,731 \\
1,285 \\
W \\
W\end{array}$ & $\begin{array}{l}W \\
W \\
W \\
W\end{array}$ & $\begin{array}{r}\mathbf{5 , 5 1 1} \\
1,522 \\
3,747 \\
242\end{array}$ & $\begin{array}{l}W \\
W \\
W \\
W\end{array}$ & $\begin{array}{r}3,742 \\
3,072 \\
373 \\
297\end{array}$ & $\begin{array}{r}11,212 \\
6,030 \\
4,643 \\
539\end{array}$ \\
\hline $\begin{array}{l}\text { Other Oxygenates }{ }^{b} \\
\text { Refinery } \\
\text { Bulk Terminal } \\
\text { Pipeline }\end{array}$ & $\begin{array}{l}W \\
W \\
W \\
W\end{array}$ & $\begin{array}{l}W \\
W \\
W \\
W\end{array}$ & $\begin{array}{l}W \\
W \\
W \\
W\end{array}$ & $\begin{array}{l}W \\
W \\
W \\
W\end{array}$ & $\begin{array}{l}W \\
W \\
W \\
W\end{array}$ & $\begin{array}{l}W \\
W \\
W \\
W\end{array}$ \\
\hline $\begin{array}{l}\text { Unflnlehed Olls } \\
\text { Refinery }\end{array}$ & 11,272 & 17,214 & 49,842 & 2,926 & 22,230 & 103,484 \\
\hline 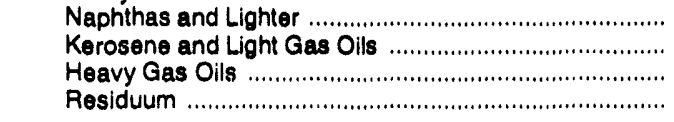 & $\begin{array}{r}2,545 \\
1,990 \\
5,796 \\
941\end{array}$ & $\begin{array}{l}4,578 \\
1,593 \\
6,842 \\
4,201\end{array}$ & $\begin{array}{r}12,604 \\
8,826 \\
19,692 \\
8,720\end{array}$ & $\begin{array}{r}656 \\
595 \\
1,139 \\
536\end{array}$ & $\begin{array}{r}4,039 \\
3,535 \\
11,266 \\
3,390\end{array}$ & $\begin{array}{l}24,422 \\
16,539 \\
44,735 \\
17,788\end{array}$ \\
\hline 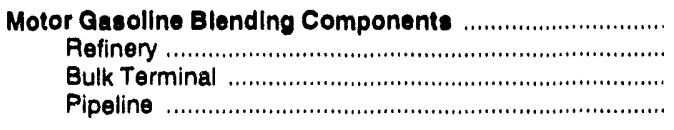 & $\begin{array}{r}5,746 \\
5,356 \\
315 \\
75\end{array}$ & $\begin{array}{r}8,426 \\
7,483 \\
457 \\
486\end{array}$ & $\begin{array}{r}15,488 \\
13,878 \\
1,315 \\
293\end{array}$ & $\begin{array}{r}2,405 \\
2,405 \\
0 \\
0\end{array}$ & $\begin{array}{r}8,307 \\
7,427 \\
100 \\
780\end{array}$ & $\begin{array}{r}40,370 \\
36,549 \\
2,187 \\
1,634\end{array}$ \\
\hline 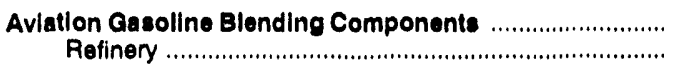 & $\begin{array}{l}0 \\
0\end{array}$ & $\begin{array}{l}6 \\
6\end{array}$ & $\begin{array}{l}42 \\
42\end{array}$ & $\begin{array}{l}0 \\
0\end{array}$ & $\begin{array}{l}3 \\
3\end{array}$ & $\begin{array}{l}51 \\
51\end{array}$ \\
\hline 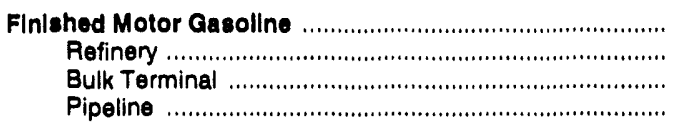 & $\begin{array}{l}58,193 \\
13,745 \\
30,450 \\
13,998\end{array}$ & $\begin{array}{l}50,024 \\
11,298 \\
22,765 \\
16,561\end{array}$ & $\begin{array}{l}50,072 \\
20,725 \\
10,735 \\
18,612\end{array}$ & $\begin{array}{l}4,993 \\
2,350 \\
1,511 \\
1,132\end{array}$ & $\begin{array}{r}23,157 \\
8,057 \\
12,234 \\
2,866\end{array}$ & $\begin{array}{r}187,039 \\
56,175 \\
77,695 \\
53,169\end{array}$ \\
\hline 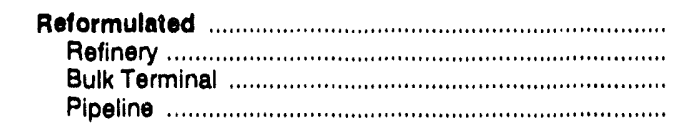 & $\begin{array}{l}0 \\
0 \\
0 \\
0\end{array}$ & $\begin{array}{l}0 \\
0 \\
0 \\
0\end{array}$ & $\begin{array}{l}0 \\
0 \\
0 \\
0\end{array}$ & $\begin{array}{l}0 \\
0 \\
0 \\
0\end{array}$ & $\begin{array}{l}0 \\
0 \\
0 \\
0\end{array}$ & $\begin{array}{l}0 \\
0 \\
0 \\
0\end{array}$ \\
\hline 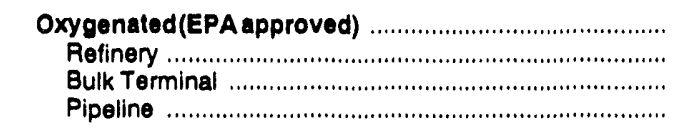 & $\begin{array}{l}8,581 \\
2,058 \\
5,193 \\
1,310\end{array}$ & $\begin{array}{r}1,856 \\
485 \\
1,194 \\
177\end{array}$ & $\begin{array}{r}4,925 \\
1,474 \\
268 \\
3,183\end{array}$ & $\begin{array}{r}300 \\
25 \\
275 \\
0\end{array}$ & $\begin{array}{r}1,819 \\
10 \\
1,079 \\
730\end{array}$ & $\begin{array}{r}17,461 \\
4,052 \\
8,009 \\
5,400\end{array}$ \\
\hline 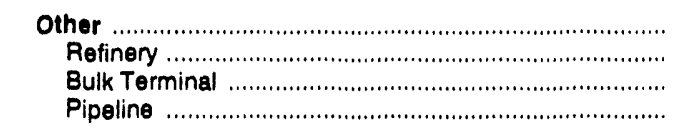 & $\begin{array}{l}49,632 \\
11,687 \\
25,257 \\
12,688\end{array}$ & $\begin{array}{l}48,768 \\
10,813 \\
21,571 \\
16,384\end{array}$ & $\begin{array}{l}\mathbf{4 5 , 1 4 7} \\
19,251 \\
10,467 \\
15,429\end{array}$ & $\begin{array}{l}4,693 \\
2,325 \\
1,236 \\
1,132\end{array}$ & $\begin{array}{r}21,338 \\
8,047 \\
11,155 \\
2,136\end{array}$ & $\begin{array}{r}169,578 \\
52,123 \\
69,686 \\
47,769\end{array}$ \\
\hline 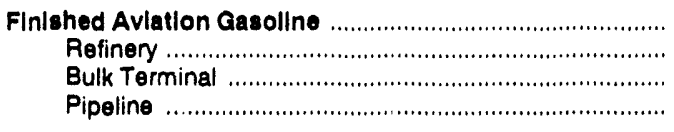 & $\begin{array}{r}476 \\
25 \\
451 \\
0\end{array}$ & $\begin{array}{r}483 \\
90 \\
337 \\
56\end{array}$ & $\begin{array}{r}388 \\
320 \\
68 \\
0\end{array}$ & $\begin{array}{r}45 \\
33 \\
12 \\
0\end{array}$ & $\begin{array}{r}371 \\
137 \\
234 \\
0\end{array}$ & $\begin{array}{r}1,763 \\
605 \\
1,102 \\
56\end{array}$ \\
\hline 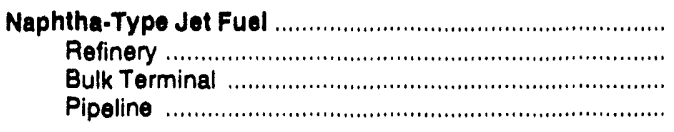 & $\begin{array}{r}374 \\
95 \\
150 \\
129\end{array}$ & $\begin{array}{l}899 \\
457 \\
222 \\
220\end{array}$ & $\begin{array}{r}1,622 \\
1,196 \\
110 \\
316\end{array}$ & $\begin{array}{r}348 \\
224 \\
0 \\
124\end{array}$ & $\begin{array}{r}1,172 \\
365 \\
428 \\
379\end{array}$ & $\begin{array}{r}4,415 \\
2,337 \\
910 \\
1,168\end{array}$ \\
\hline 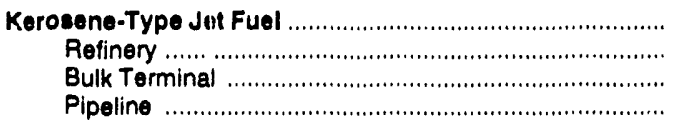 & $\begin{array}{l}9,143 \\
1,388 \\
3,836 \\
3,919\end{array}$ & $\begin{array}{l}7,470 \\
2,686 \\
2,188 \\
2,596\end{array}$ & $\begin{array}{r}12,042 \\
6,094 \\
1,717 \\
4,231\end{array}$ & $\begin{array}{l}583 \\
258 \\
163 \\
162\end{array}$ & $\begin{array}{r}7,776 \\
4,028 \\
2,839 \\
909\end{array}$ & $\begin{array}{l}37,014 \\
14,454 \\
10,743 \\
11,817\end{array}$ \\
\hline
\end{tabular}

See footnotes at end of table. 
Table 51. Stocks of Crude OII and Petroleum Products by PAD Distrlct, March 1993 (Continued)

(Thousand Barrels)

\begin{tabular}{|c|c|c|c|c|c|c|}
\hline \multirow{2}{*}{ Commodity } & \multicolumn{5}{|c|}{ Potroleum Administration for Detense Dlstricts } & \multirow{2}{*}{$\begin{array}{l}\text { U.S. } \\
\text { Total }\end{array}$} \\
\hline & 1 & ॥ & III & IV & v & \\
\hline 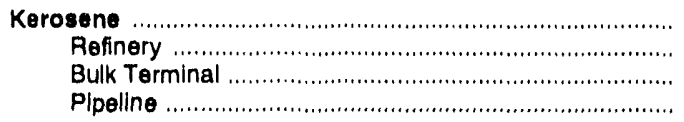 & $\begin{array}{r}1,310 \\
97 \\
1,082 \\
131\end{array}$ & $\begin{array}{r}1,488 \\
900 \\
504 \\
84\end{array}$ & $\begin{array}{r}1,533 \\
862 \\
214 \\
457\end{array}$ & $\begin{array}{r}78 \\
53 \\
9 \\
16\end{array}$ & $\begin{array}{r}46 \\
36 \\
6 \\
4\end{array}$ & $\begin{array}{r}4,455 \\
1,948 \\
1,815 \\
692\end{array}$ \\
\hline 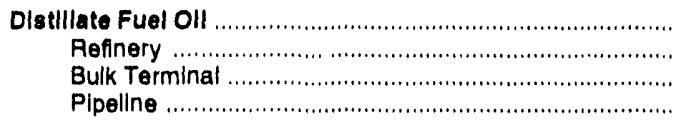 & $\begin{array}{r}33,084 \\
5,314 \\
20,939 \\
6,831\end{array}$ & $\begin{array}{r}28,955 \\
8,000 \\
12,248 \\
8,707\end{array}$ & $\begin{array}{r}23,137 \\
11,496 \\
4,634 \\
7,007\end{array}$ & $\begin{array}{r}2,377 \\
1,312 \\
620 \\
445\end{array}$ & $\begin{array}{l}9,903 \\
4,548 \\
3,730 \\
1,625\end{array}$ & $\begin{array}{l}97,456 \\
30,670 \\
42,171 \\
24,615\end{array}$ \\
\hline 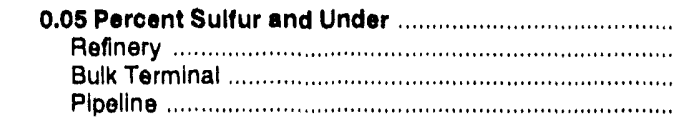 & $\begin{array}{r}4,959 \\
398 \\
4,558 \\
3\end{array}$ & $\begin{array}{r}1,605 \\
132 \\
1,176 \\
297\end{array}$ & $\begin{array}{r}2,828 \\
691 \\
1,376 \\
761\end{array}$ & $\begin{array}{r}458 \\
203 \\
234 \\
21\end{array}$ & $\begin{array}{r}2,544 \\
1,180 \\
1,141 \\
223\end{array}$ & $\begin{array}{r}12,394 \\
2,604 \\
8,485 \\
1,305\end{array}$ \\
\hline 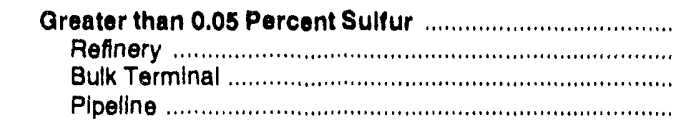 & $\begin{array}{r}28,125 \\
4,916 \\
16,381 \\
6,828\end{array}$ & $\begin{array}{r}27,350 \\
7,868 \\
11,072 \\
8,410\end{array}$ & $\begin{array}{r}20,309 \\
10,805 \\
3,258 \\
6,248\end{array}$ & $\begin{array}{r}1,919 \\
1,109 \\
386 \\
424\end{array}$ & $\begin{array}{l}7,359 \\
3,368 \\
2,589 \\
1,402\end{array}$ & $\begin{array}{l}85,062 \\
28,068 \\
33,688 \\
23,310\end{array}$ \\
\hline 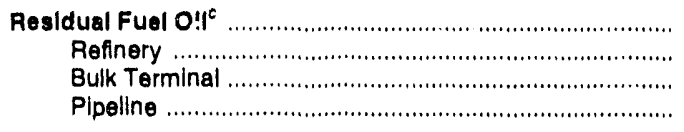 & $\begin{array}{r}13,288 \\
2,711 \\
10,547 \\
28\end{array}$ & $\begin{array}{r}2,756 \\
1,822 \\
934 \\
0\end{array}$ & $\begin{array}{r}15,633 \\
6,982 \\
8,651 \\
0\end{array}$ & $\begin{array}{r}405 \\
405 \\
0 \\
0\end{array}$ & $\begin{array}{r}8,610 \\
6,109 \\
2,300 \\
201\end{array}$ & $\begin{array}{r}40,690 \\
18,029 \\
22,432 \\
229\end{array}$ \\
\hline 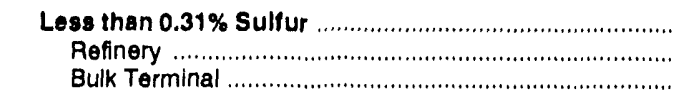 & $\begin{array}{r}2,272 \\
644 \\
1,628\end{array}$ & $\begin{array}{r}17 \\
17 \\
0\end{array}$ & $\begin{array}{r}1,551 \\
1,303 \\
248\end{array}$ & $\begin{array}{r}87 \\
87 \\
0\end{array}$ & $\begin{array}{r}795 \\
791 \\
4\end{array}$ & $\begin{array}{l}4,722 \\
2,842 \\
1,880\end{array}$ \\
\hline 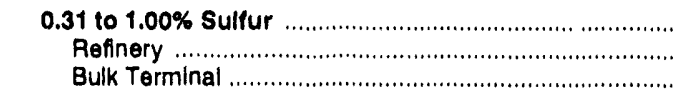 & $\begin{array}{l}4,308 \\
1,109 \\
3,199\end{array}$ & $\begin{array}{l}580 \\
200 \\
380\end{array}$ & $\begin{array}{r}5,018 \\
753 \\
4,265\end{array}$ & $\begin{array}{r}200 \\
200 \\
0\end{array}$ & $\begin{array}{r}1,020 \\
680 \\
340\end{array}$ & $\begin{array}{r}11,126 \\
2,942 \\
8,184\end{array}$ \\
\hline 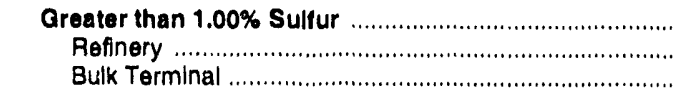 & $\begin{array}{r}6,678 \\
958 \\
5,720\end{array}$ & $\begin{array}{r}2,159 \\
1,605 \\
554\end{array}$ & $\begin{array}{l}9,064 \\
4,926 \\
4,138\end{array}$ & $\begin{array}{r}118 \\
118 \\
0\end{array}$ & $\begin{array}{l}6,594 \\
4,638 \\
1,956\end{array}$ & $\begin{array}{l}24,613 \\
12,245 \\
12,368\end{array}$ \\
\hline 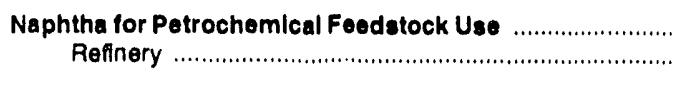 & $\begin{array}{l}264 \\
264\end{array}$ & $\begin{array}{l}280 \\
290\end{array}$ & $\begin{array}{l}1,206 \\
1,206\end{array}$ & $\begin{array}{l}0 \\
0\end{array}$ & $\begin{array}{l}27 \\
27\end{array}$ & $\begin{array}{l}1,787 \\
1,787\end{array}$ \\
\hline 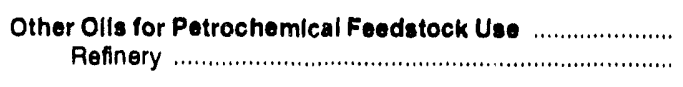 & 5 & $\begin{array}{l}3 \\
3\end{array}$ & $\begin{array}{l}1,689 \\
1,689\end{array}$ & 1 & $\begin{array}{l}148 \\
148\end{array}$ & $\begin{array}{l}1,846 \\
1,846\end{array}$ \\
\hline 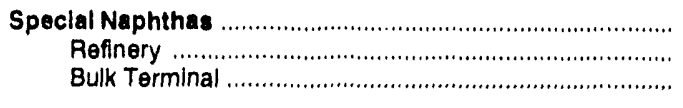 & $\begin{array}{r}134 \\
41 \\
93\end{array}$ & $\begin{array}{l}485 \\
367 \\
118\end{array}$ & $\begin{array}{r}1,707 \\
1,634 \\
73\end{array}$ & $\begin{array}{l}1 \\
1 \\
0\end{array}$ & $\begin{array}{r}62 \\
62 \\
0\end{array}$ & $\begin{array}{r}2,389 \\
2,105 \\
284\end{array}$ \\
\hline 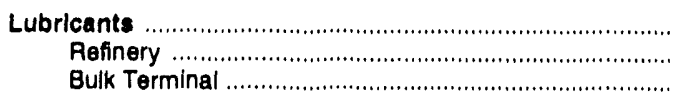 & $\begin{array}{r}3,163 \\
993 \\
2,170\end{array}$ & $\begin{array}{r}2,040 \\
961 \\
1,079\end{array}$ & $\begin{array}{r}6,402 \\
5,662 \\
740\end{array}$ & $\begin{array}{l}0 \\
0 \\
0\end{array}$ & $\begin{array}{r}1,832 \\
1,035 \\
797\end{array}$ & $\begin{array}{r}13,437 \\
8,651 \\
4,788\end{array}$ \\
\hline Refinery & $\begin{array}{l}171 \\
171\end{array}$ & $\begin{array}{l}111 \\
111\end{array}$ & $\begin{array}{l}403 \\
403\end{array}$ & $\begin{array}{l}31 \\
31\end{array}$ & $\begin{array}{l}144 \\
144\end{array}$ & $\begin{array}{l}860 \\
860\end{array}$ \\
\hline 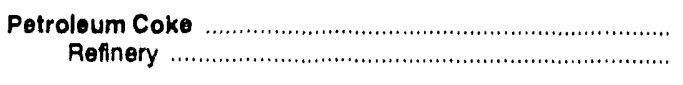 & $\begin{array}{l}1,189 \\
1,189\end{array}$ & $\begin{array}{l}2,723 \\
2,723\end{array}$ & $\begin{array}{l}2,791 \\
2,791\end{array}$ & $\begin{array}{l}305 \\
305\end{array}$ & $\begin{array}{l}3,702 \\
3,702\end{array}$ & $\begin{array}{l}10,710 \\
10,710\end{array}$ \\
\hline $\begin{array}{l}\text { Asphalt and Road Oll } \\
\text { Reffnery } \\
\text { Bulk Terminal }\end{array}$ & $\begin{array}{l}5,208 \\
2,366 \\
2,842\end{array}$ & $\begin{array}{r}13,674 \\
8,274 \\
5,400\end{array}$ & $\begin{array}{r}4,977 \\
4,210 \\
767\end{array}$ & $\begin{array}{r}3,113 \\
2,990 \\
123\end{array}$ & $\begin{array}{r}2,139 \\
1,985 \\
154\end{array}$ & $\begin{array}{r}29,111 \\
19,825 \\
9,286\end{array}$ \\
\hline 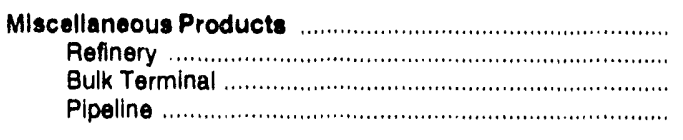 & $\begin{array}{r}734 \\
56 \\
678 \\
0\end{array}$ & $\begin{array}{r}274 \\
136 \\
91 \\
47\end{array}$ & $\begin{array}{r}1,245 \\
468 \\
727 \\
50\end{array}$ & $\begin{array}{l}8 \\
2 \\
1 \\
5\end{array}$ & $\begin{array}{r}271 \\
91 \\
180 \\
0\end{array}$ & $\begin{array}{r}2,532 \\
753 \\
1,677 \\
102\end{array}$ \\
\hline 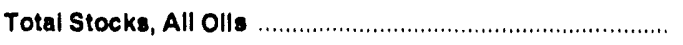 & 164,233 & 234,582 & 984,503 & 31,321 & 168,851 & $1,583,590$ \\
\hline
\end{tabular}

ancludes stocks held by producers.

b Includes tertiary amyl methyl ether (TAME), tertlary butyl alcohol (TBA), and other aliphatic alcohols and ethers Iniended for motor gasoline blending (e.g. isopropyl ether (IPE) or n-propanol

c Sulfur content not avallable for stocks held by pipelines.

$W=$ Withheld to avold disclosure of Individual company data.

Note: Stocks are reported as of the last day of the month.

Sources: Energy Information Administration (ElA) Forms ElÁ.810, "Monthly Refinery Report," ElA-811, "Monthly Bulk Terminal Report," ElA.812, "Monthly Product Pipeline Report," EIA-813, "Monthly Crude Oll Report," and EIA-816, "Monthly Natural Gas Liqulds Report." 
Table 52. Reflnery, Bulk Terminal, and Natural Gas Plant Stocks of Selected Petroleum Products by PAD District and State, March 1993

(Thousand Barrels)

\begin{tabular}{|c|c|c|c|c|c|c|c|c|c|c|}
\hline \multirow[b]{2}{*}{ PAD Dlstrlct and State } & \multicolumn{4}{|c|}{ Motor Gasollne } & \multirow[b]{2}{*}{ Kerosene } & \multicolumn{3}{|c|}{ Dlstillate Fuel Oll } & \multirow[b]{2}{*}{$\begin{array}{c}\text { Resldual } \\
\text { Fuel }\end{array}$} & \multirow[b]{2}{*}{$\begin{array}{l}\text { Propane/ } \\
\text { Propylene }\end{array}$} \\
\hline & Total & Poformulated & $\begin{array}{c}\text { Oxygenated } \\
\text { (EPA } \\
\text { approved) }\end{array}$ & Other & & Total & $\begin{array}{l}0.05 \% \text { Sulfur } \\
\text { and Under }\end{array}$ & $\begin{array}{l}\text { Greater than } \\
0.05 \% \text { Sulfur }\end{array}$ & & \\
\hline 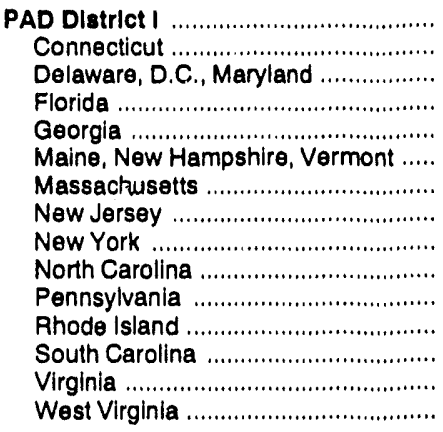 & $\begin{array}{r}44,195 \\
2,130 \\
2,821 \\
4,903 \\
2,016 \\
1,094 \\
2,013 \\
12,087 \\
5,369 \\
2,188 \\
5,214 \\
620 \\
932 \\
2,736 \\
72\end{array}$ & $\begin{array}{l}0 \\
0 \\
0 \\
0 \\
0 \\
0 \\
0 \\
0 \\
0 \\
0 \\
0 \\
0 \\
0 \\
0 \\
0\end{array}$ & $\begin{array}{r}7,251 \\
600 \\
457 \\
291 \\
45 \\
145 \\
305 \\
3,231 \\
1,019 \\
108 \\
866 \\
0 \\
106 \\
78 \\
0\end{array}$ & $\begin{array}{r}36,944 \\
1,530 \\
2,364 \\
4,612 \\
1,971 \\
949 \\
1,708 \\
8,856 \\
4,350 \\
2,080 \\
4,348 \\
620 \\
826 \\
2,658 \\
72\end{array}$ & $\begin{array}{r}1,179 \\
21 \\
52 \\
115 \\
59 \\
73 \\
51 \\
85 \\
245 \\
101 \\
263 \\
W \\
69 \\
30 \\
W\end{array}$ & $\begin{array}{r}26,253 \\
1,844 \\
1,451 \\
1,363 \\
765 \\
1,258 \\
1,896 \\
5,807 \\
4,082 \\
1,006 \\
3,579 \\
657 \\
499 \\
1,999 \\
47\end{array}$ & $\begin{array}{r}4,956 \\
180 \\
565 \\
69 \\
61 \\
140 \\
8 \\
1,303 \\
1,323 \\
93 \\
353 \\
12 \\
38 \\
787 \\
24\end{array}$ & $\begin{array}{r}21,297 \\
1,664 \\
886 \\
1,294 \\
704 \\
1,118 \\
1,688 \\
4, j 04 \\
2,759 \\
913 \\
3,226 \\
645 \\
461 \\
1,212 \\
23\end{array}$ & $\begin{array}{r}13,258 \\
37 \\
2,440 \\
1,233 \\
202 \\
601 \\
628 \\
4,549 \\
1,687 \\
359 \\
746 \\
W \\
W \\
401 \\
W\end{array}$ & $\begin{array}{r}744 \\
W \\
W \\
103 \\
W \\
W \\
W \\
W \\
W \\
W \\
W \\
W \\
W \\
W \\
W\end{array}$ \\
\hline 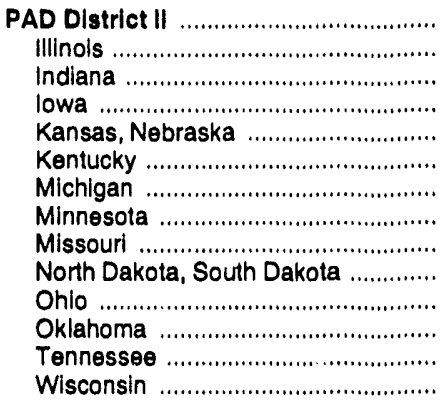 & $\begin{array}{r}34,063 \\
6,647 \\
4,678 \\
1,294 \\
3,495 \\
1,416 \\
2,998 \\
1,813 \\
1,343 \\
930 \\
3,984 \\
1,569 \\
2,140 \\
1,756\end{array}$ & $\begin{array}{l}0 \\
0 \\
0 \\
0 \\
0 \\
0 \\
0 \\
0 \\
0 \\
0 \\
0 \\
0 \\
0 \\
0\end{array}$ & $\begin{array}{r}1,679 \\
332 \\
46 \\
30 \\
44 \\
100 \\
125 \\
0 \\
44 \\
0 \\
466 \\
187 \\
305 \\
0\end{array}$ & $\begin{array}{r}32,384 \\
6,315 \\
4,632 \\
1,264 \\
3,451 \\
1,316 \\
2,873 \\
1,813 \\
1,299 \\
930 \\
3,518 \\
1,382 \\
1,835 \\
1,756\end{array}$ & $\begin{array}{r}1,404 \\
363 \\
63 \\
W \\
9 \\
99 \\
84 \\
W \\
W \\
W \\
385 \\
W \\
42 \\
W\end{array}$ & $\begin{array}{r}20,248 \\
3,812 \\
2,903 \\
1,365 \\
2,233 \\
613 \\
1,652 \\
1,417 \\
672 \\
752 \\
1,469 \\
1,232 \\
856 \\
1,272\end{array}$ & $\begin{array}{r}1,308 \\
197 \\
287 \\
78 \\
165 \\
27 \\
56 \\
26 \\
199 \\
7 \\
130 \\
47 \\
55 \\
34\end{array}$ & $\begin{array}{r}18,940 \\
3,615 \\
2,616 \\
1,287 \\
2,068 \\
586 \\
1,596 \\
1,391 \\
473 \\
745 \\
1,339 \\
1,185 \\
801 \\
1,238\end{array}$ & $\begin{array}{r}2,756 \\
1,038 \\
374 \\
W \\
13 \\
W \\
122 \\
155 \\
W \\
W \\
244 \\
325 \\
138 \\
53\end{array}$ & $\begin{array}{r}3,508 \\
454 \\
W \\
W \\
1,428 \\
W \\
190 \\
W \\
W \\
W \\
W \\
793 \\
W \\
W\end{array}$ \\
\hline 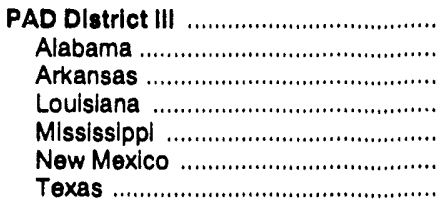 & $\begin{array}{r}31,460 \\
1,213 \\
789 \\
5,610 \\
3,181 \\
334 \\
20,333\end{array}$ & $\begin{array}{l}0 \\
0 \\
0 \\
0 \\
0 \\
0 \\
0\end{array}$ & $\begin{array}{r}1,742 \\
147 \\
0 \\
312 \\
121 \\
0 \\
1,162\end{array}$ & $\begin{array}{r}29,718 \\
1,066 \\
789 \\
5,298 \\
3,060 \\
334 \\
19,171\end{array}$ & $\begin{array}{r}1,076 \\
17 \\
W \\
280 \\
6 \\
W \\
753\end{array}$ & $\begin{array}{r}16,130 \\
573 \\
336 \\
3,657 \\
1,603 \\
191 \\
9,770\end{array}$ & $\begin{array}{r}2,067 \\
51 \\
183 \\
366 \\
70 \\
6 \\
1,391\end{array}$ & $\begin{array}{r}14,063 \\
522 \\
153 \\
3,291 \\
1,533 \\
185 \\
8,379\end{array}$ & $\begin{array}{r}15,633 \\
197 \\
W \\
6,728 \\
W \\
7 \\
8,481\end{array}$ & $\begin{array}{r}9,331 \\
89 \\
W \\
1,354 \\
333 \\
W \\
7,450\end{array}$ \\
\hline 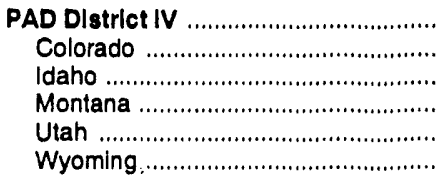 & $\begin{array}{r}3,861 \\
1,218 \\
327 \\
1,117 \\
562 \\
637\end{array}$ & $\begin{array}{l}0 \\
0 \\
0 \\
0 \\
0 \\
0\end{array}$ & $\begin{array}{r}300 \\
252 \\
46 \\
0 \\
2 \\
0\end{array}$ & $\begin{array}{r}3,561 \\
966 \\
281 \\
1,117 \\
560 \\
637\end{array}$ & $\begin{array}{l}62 \\
W \\
W \\
W \\
W \\
W\end{array}$ & $\begin{array}{r}1,932 \\
356 \\
170 \\
535 \\
561 \\
310\end{array}$ & $\begin{array}{r}437 \\
154 \\
69 \\
49 \\
165 \\
0\end{array}$ & $\begin{array}{r}1,495 \\
202 \\
101 \\
496 \\
396 \\
310\end{array}$ & $\begin{array}{r}405 \\
W \\
W \\
68 \\
123 \\
W\end{array}$ & $\begin{array}{r}220 \\
W \\
W \\
33 \\
39 \\
50\end{array}$ \\
\hline 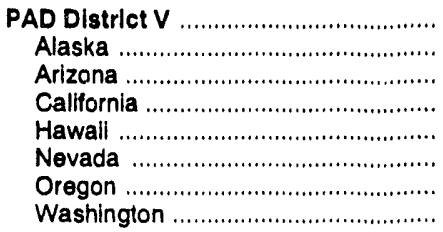 & $\begin{array}{r}20,291 \\
460 \\
687 \\
13,604 \\
670 \\
257 \\
1,254 \\
3,359\end{array}$ & $\begin{array}{l}0 \\
0 \\
0 \\
0 \\
0 \\
0 \\
0 \\
0\end{array}$ & $\begin{array}{r}1,089 \\
1 \\
66 \\
875 \\
0 \\
20 \\
1 \\
126\end{array}$ & $\begin{array}{r}19,202 \\
459 \\
621 \\
12,729 \\
670 \\
237 \\
1,253 \\
3,233\end{array}$ & $\begin{array}{l}42 \\
W \\
W \\
36 \\
W \\
W \\
W \\
W\end{array}$ & $\begin{array}{r}8,278 \\
877 \\
157 \\
4,601 \\
619 \\
70 \\
550 \\
1,404\end{array}$ & $\begin{array}{r}2,321 \\
257 \\
20 \\
1,484 \\
24 \\
4 \\
209 \\
323\end{array}$ & $\begin{array}{r}5,957 \\
620 \\
137 \\
3,117 \\
595 \\
66 \\
341 \\
1,081\end{array}$ & $\begin{array}{r}8,409 \\
W \\
W \\
5,143 \\
W \\
W \\
155 \\
1,567\end{array}$ & $\begin{array}{r}269 \\
W \\
W \\
93 \\
W \\
W \\
W \\
28\end{array}$ \\
\hline 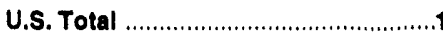 & 133,870 & 0 & 12,061 & 121,809 & 3,763 & 72,841 & 11,089 & 61,752 & 40,461 & 14,072 \\
\hline
\end{tabular}

$W=$ Withheld to avoid disclosure of individual company data.

Notes: - Stocks are reported as of the last day of the month. - Totals may not equal sum of components due to independent rounding.

Sources: Energy Information Administration (EIA) Forms EIA-810, "Monthly Refinery Report," EIA-811, "Monthly Bulk Terminal Report," and EIA-816, "Monthly Natural Gas Liquids Report." 
Table 53. Movements of Crude Oil and Petroleum Products by Pipeline, Tanker, and Barge Between PAD Districts, March 1993

(Thousand Barrels)

\begin{tabular}{|c|c|c|c|c|c|c|c|c|c|}
\hline \multirow[b]{2}{*}{ Commodity } & \multicolumn{3}{|c|}{ From I to } & \multicolumn{4}{|c|}{ From II to } & \multicolumn{2}{|c|}{ From III to } \\
\hline & II & III & V & 1 & III & IV & V & 1 & II \\
\hline 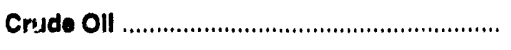 & 78 & 0 & 0 & 147 & 1,552 & 293 & 0 & 0 & 55,482 \\
\hline 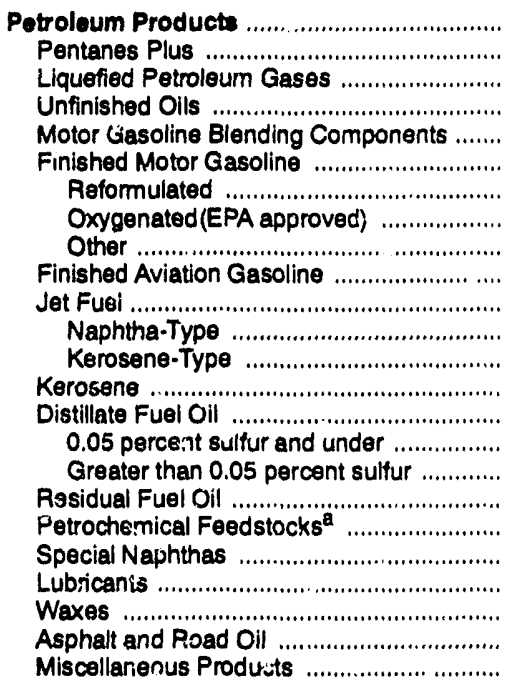 & $\begin{array}{r}7,995 \\
0 \\
0 \\
90 \\
10 \\
5,367 \\
0 \\
0 \\
5,367 \\
0 \\
405 \\
0 \\
405 \\
55 \\
2,118 \\
0 \\
2,018 \\
0 \\
0 \\
0 \\
10 \\
0 \\
0 \\
0\end{array}$ & $\begin{array}{r}68 \\
0 \\
0 \\
0 \\
56 \\
0 \\
0 \\
0 \\
0 \\
0 \\
0 \\
0 \\
0 \\
0 \\
0 \\
0 \\
0 \\
0 \\
0 \\
3 \\
9 \\
0 \\
0 \\
0\end{array}$ & $\begin{array}{l}0 \\
0 \\
0 \\
0 \\
0 \\
0 \\
0 \\
0 \\
0 \\
0 \\
0 \\
0 \\
0 \\
0 \\
0 \\
0 \\
0 \\
0 \\
0 \\
0 \\
0 \\
0 \\
0 \\
0\end{array}$ & $\begin{array}{r}3,747 \\
0 \\
1,323 \\
10 \\
23 \\
1,495 \\
0 \\
0 \\
1,495 \\
0 \\
113 \\
0 \\
113 \\
50 \\
558 \\
63 \\
495 \\
82 \\
0 \\
0 \\
93 \\
0 \\
\therefore . \\
0 \\
0\end{array}$ & $\begin{array}{r}5,506 \\
111 \\
3,432 \\
0 \\
0 \\
895 \\
0 \\
0 \\
895 \\
0 \\
540 \\
101 \\
439 \\
0 \\
242 \\
0 \\
242 \\
265 \\
0 \\
0 \\
10 \\
0 \\
0 \\
11\end{array}$ & $\begin{array}{r}3,131 \\
0 \\
103 \\
0 \\
0 \\
1,650 \\
0 \\
0 \\
1,650 \\
8 \\
797 \\
0 \\
797 \\
0 \\
573 \\
427 \\
146 \\
0 \\
0 \\
0 \\
0 \\
0 \\
0 \\
0\end{array}$ & $\begin{array}{l}0 \\
0 \\
0 \\
0 \\
0 \\
0 \\
0 \\
0 \\
0 \\
0 \\
0 \\
0 \\
0 \\
0 \\
0 \\
0 \\
0 \\
0 \\
0 \\
0 \\
0 \\
0 \\
0 \\
0\end{array}$ & $\begin{array}{r}87,497 \\
0 \\
3,017 \\
146 \\
259 \\
49,377 \\
0 \\
4,811 \\
44,566 \\
144 \\
12,024 \\
288 \\
11,736 \\
610 \\
21,050 \\
87 \\
20,963 \\
204 \\
0 \\
99 \\
309 \\
3 \\
149 \\
106\end{array}$ & $\begin{array}{r}26,592 \\
493 \\
5,178 \\
16 \\
693 \\
12,410 \\
0 \\
0 \\
12,410 \\
99 \\
3,295 \\
73 \\
3,272 \\
55 \\
3,831 \\
38 \\
3,793 \\
0 \\
0 \\
25 \\
338 \\
0 \\
83 \\
76\end{array}$ \\
\hline 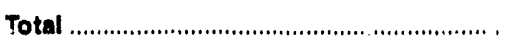 & 8,073 & 68 & 0 & 3,894 & 7,058 & 3,424 & 0 & 87,497 & 82,074 \\
\hline
\end{tabular}

\begin{tabular}{|c|c|c|c|c|c|c|c|c|c|}
\hline \multirow{2}{*}{ Commodity } & \multicolumn{2}{|c|}{ From III to } & \multicolumn{3}{|c|}{ From IV to } & \multicolumn{4}{|c|}{ From $V$ to } \\
\hline & IV & v & $\|$ & III & V & 1 & II & III & IV \\
\hline 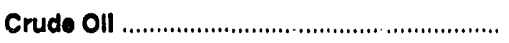 & 0 & 0 & 3,049 & 1,311 & 0 & 0 & 0 & 6,305 & 0 \\
\hline 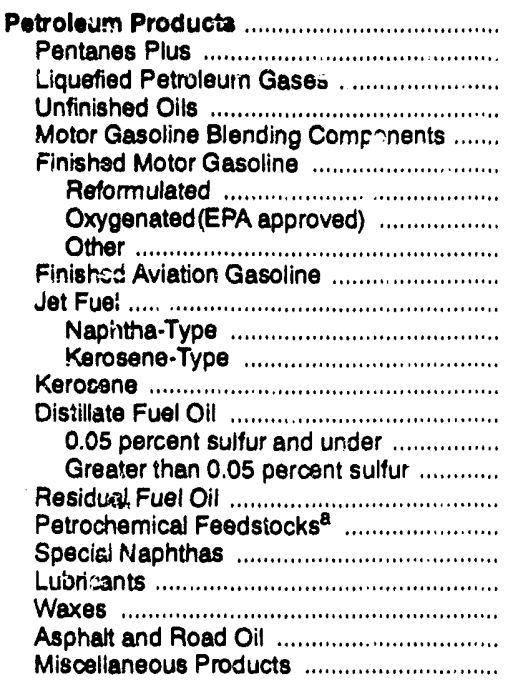 & $\begin{array}{l}0 \\
0 \\
0 \\
0 \\
0 \\
0 \\
0 \\
0 \\
0 \\
0 \\
0 \\
0 \\
0 \\
0 \\
0 \\
0 \\
0 \\
0 \\
0 \\
0 \\
0 \\
0 \\
0 \\
0\end{array}$ & $\begin{array}{r}1,428 \\
0 \\
0 \\
98 \\
51 \\
832 \\
0 \\
0 \\
832 \\
0 \\
191 \\
117 \\
74 \\
0 \\
256 \\
0 \\
256 \\
0 \\
0 \\
0 \\
0 \\
0 \\
0 \\
0\end{array}$ & $\begin{array}{r}1,758 \\
97 \\
710 \\
0 \\
0 \\
470 \\
0 \\
0 \\
470 \\
0 \\
74 \\
71 \\
3 \\
10 \\
397 \\
0 \\
397 \\
0 \\
0 \\
0 \\
0 \\
0 \\
0 \\
0\end{array}$ & $\begin{array}{r}2,033 \\
203 \\
1,830 \\
0 \\
0 \\
0 \\
0 \\
0 \\
0 \\
0 \\
0 \\
0 \\
0 \\
0 \\
0 \\
0 \\
0 \\
0 \\
0 \\
0 \\
0 \\
0 \\
0 \\
0\end{array}$ & $\begin{array}{r}1,095 \\
0 \\
0 \\
0 \\
0 \\
752 \\
0 \\
0 \\
752 \\
0 \\
176 \\
94 \\
82 \\
0 \\
167 \\
0 \\
167 \\
0 \\
0 \\
0 \\
0 \\
0 \\
0 \\
0\end{array}$ & $\begin{array}{l}0 \\
0 \\
0 \\
0 \\
0 \\
0 \\
0 \\
0 \\
0 \\
0 \\
0 \\
0 \\
0 \\
0 \\
0 \\
0 \\
0 \\
0 \\
0 \\
0 \\
0 \\
0 \\
0 \\
0\end{array}$ & $\begin{array}{l}0 \\
0 \\
0 \\
0 \\
0 \\
0 \\
0 \\
0 \\
0 \\
0 \\
0 \\
0 \\
0 \\
0 \\
0 \\
0 \\
0 \\
0 \\
0 \\
0 \\
0 \\
0 \\
0 \\
0\end{array}$ & $\begin{array}{l}0 \\
0 \\
0 \\
0 \\
0 \\
0 \\
0 \\
0 \\
0 \\
0 \\
0 \\
0 \\
0 \\
0 \\
0 \\
0 \\
0 \\
0 \\
0 \\
0 \\
0 \\
0 \\
0 \\
0\end{array}$ & $\begin{array}{l}0 \\
0 \\
0 \\
0 \\
0 \\
0 \\
0 \\
0 \\
0 \\
0 \\
0 \\
0 \\
0 \\
0 \\
0 \\
0 \\
0 \\
0 \\
0 \\
0 \\
0 \\
0 \\
0 \\
0\end{array}$ \\
\hline Total & 0 & 1,428 & 4,807 & 3,344 & 1,095 & 0 & 0 & 6,305 & 0 \\
\hline
\end{tabular}

- Includes naphtha less than $\left.n^{\prime}\right) 1^{\circ} \mathrm{F}$ encipoint and other oils equal to or greater than $401^{\circ} \mathrm{F}$ endpoint.

Scurces: Energy Information Administration (EIA) Forms EIA-E i2, "Monthiy Product Pipeline Peport," ElA-813, "Monthly Crude Oil Report," and EIA-817, "Monthly Tanker and Barge Movement Report." 
Table 54. Movements of Crude Oil and Petroleum Products by Pipeline Between PAD Distrlcts, March 1993

(Thousand Barrels)

\begin{tabular}{|c|c|c|c|c|c|c|c|}
\hline \multirow{2}{*}{ Commodity } & \multicolumn{2}{|c|}{ From I to } & \multicolumn{3}{|c|}{ From II to } & \multicolumn{2}{|c|}{ From III to } \\
\hline & II & III & 1 & III & IV & $\mathbf{I}$ & ॥ \\
\hline 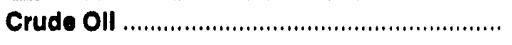 & 78 & 0 & 0 & 1,552 & 293 & $\mathbf{0}$ & 85,402 \\
\hline 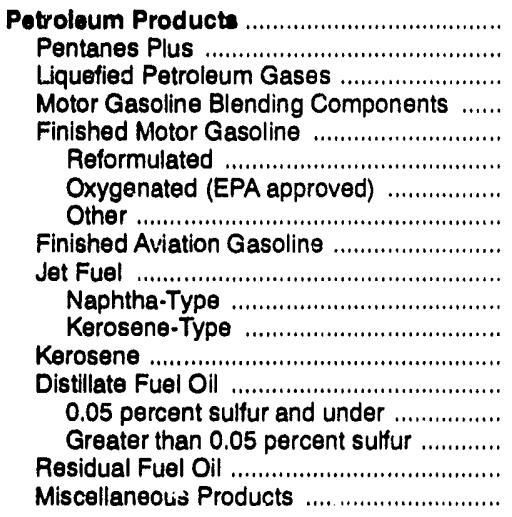 & $\begin{array}{r}7,856 \\
0 \\
0 \\
0 \\
5,338 \\
0 \\
0 \\
5,338 \\
0 \\
405 \\
0 \\
405 \\
95 \\
2,018 \\
0 \\
2,018 \\
0 \\
0\end{array}$ & $\begin{array}{l}0 \\
0 \\
0 \\
0 \\
0 \\
0 \\
0 \\
0 \\
0 \\
0 \\
0 \\
0 \\
0 \\
0 \\
0 \\
0 \\
0 \\
0\end{array}$ & $\begin{array}{r}2,515 \\
0 \\
1,323 \\
23 \\
937 \\
0 \\
0 \\
937 \\
0 \\
64 \\
0 \\
64 \\
21 \\
147 \\
63 \\
84 \\
0 \\
0\end{array}$ & $\begin{array}{r}5,231 \\
111 \\
3,432 \\
0 \\
895 \\
0 \\
0 \\
895 \\
0 \\
540 \\
101 \\
439 \\
0 \\
242 \\
0 \\
242 \\
0 \\
11\end{array}$ & $\begin{array}{r}3,131 \\
0 \\
103 \\
0 \\
1,650 \\
0 \\
0 \\
1,650 \\
8 \\
797 \\
0 \\
797 \\
0 \\
573 \\
427 \\
146 \\
0 \\
0\end{array}$ & $\begin{array}{r}71,781 \\
0 \\
2,747 \\
259 \\
40,147 \\
0 \\
4,027 \\
36,120 \\
0 \\
9,697 \\
114 \\
9,583 \\
610 \\
18,321 \\
0 \\
18,321 \\
0 \\
0\end{array}$ & $\begin{array}{r}23,641 \\
490 \\
5,178 \\
620 \\
10,724 \\
0 \\
0 \\
10,724 \\
90 \\
3,073 \\
73 \\
3,000 \\
0 \\
3,278 \\
8 \\
3,260 \\
0 \\
78\end{array}$ \\
\hline 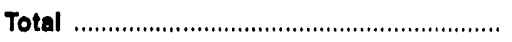 & 7,934 & 0 & 2,515 & 6,783 & 3,424 & 71,781 & 78,043 \\
\hline
\end{tabular}

\begin{tabular}{|c|c|c|c|c|c|c|c|}
\hline \multirow[b]{2}{*}{ Commodity } & \multicolumn{2}{|c|}{ From III to } & \multicolumn{3}{|c|}{ From IV to } & \multicolumn{2}{|c|}{ Fram V to } \\
\hline & IV & $\mathbf{V}$ & II & III & $\mathbf{V}$ & III & N \\
\hline 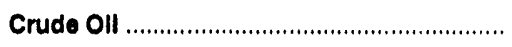 & 0 & 0 & 3,049 & 1,311 & $\mathbf{0}$ & 4,127 & $\mathbf{0}$ \\
\hline 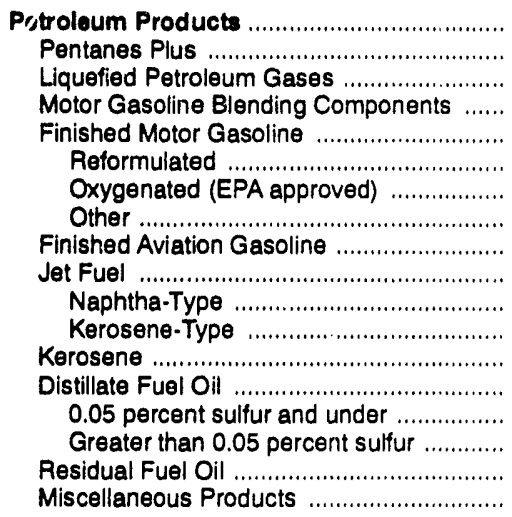 & $\begin{array}{l}0 \\
0 \\
0 \\
0 \\
0 \\
0 \\
0 \\
0 \\
0 \\
0 \\
0 \\
0 \\
0 \\
0 \\
0 \\
0 \\
0 \\
0\end{array}$ & $\begin{array}{r}1,279 \\
0 \\
0 \\
0 \\
832 \\
0 \\
0 \\
832 \\
0 \\
191 \\
117 \\
74 \\
0 \\
256 \\
0 \\
256 \\
0 \\
0\end{array}$ & $\begin{array}{r}1,758 \\
97 \\
710 \\
0 \\
470 \\
0 \\
0 \\
470 \\
0 \\
74 \\
71 \\
3 \\
10 \\
397 \\
0 \\
397 \\
0 \\
0\end{array}$ & $\begin{array}{r}2,033 \\
203 \\
1,830 \\
0 \\
0 \\
0 \\
0 \\
0 \\
0 \\
0 \\
0 \\
0 \\
0 \\
0 \\
0 \\
0 \\
0 \\
0\end{array}$ & $\begin{array}{r}1,095 \\
0 \\
0 \\
0 \\
752 \\
0 \\
0 \\
752 \\
0 \\
176 \\
94 \\
82 \\
0 \\
167 \\
0 \\
167 \\
0 \\
0\end{array}$ & $\begin{array}{l}0 \\
0 \\
0 \\
0 \\
0 \\
0 \\
0 \\
0 \\
0 \\
0 \\
0 \\
0 \\
0 \\
0 \\
0 \\
0 \\
0 \\
0\end{array}$ & $\begin{array}{l}0 \\
0 \\
0 \\
0 \\
0 \\
0 \\
0 \\
0 \\
0 \\
0 \\
0 \\
0 \\
0 \\
0 \\
0 \\
0 \\
0 \\
0\end{array}$ \\
\hline Total & 0 & 1,279 & 4,807 & 3,344 & 1,095 & 4,127 & $\mathbf{0}$ \\
\hline
\end{tabular}

Sources: Energy Information Administration (EIA) Forms EIA-812, "Monthly Froduct Pipeline Report," and EIA-813, Monthly Crude Oil Report." 
Table 55. Movements of Crude OIl and Petroleum Products by Tanker and Barge Between PAD Districts, March 1993

(Thousand Barrels)

\begin{tabular}{|c|c|c|c|c|c|c|c|c|}
\hline \multirow[b]{2}{*}{ Commodity } & \multicolumn{3}{|c|}{ From I to } & \multicolumn{3}{|c|}{ From II to } & \multicolumn{2}{|c|}{ From III to } \\
\hline & II & III & v & 1 & III & V & 1 & $\begin{array}{l}\text { Now } \\
\text { England }\end{array}$ \\
\hline 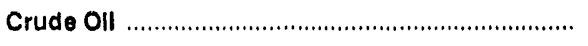 & 0 & 0 & 0 & 147 & 0 & 0 & 0 & 0 \\
\hline 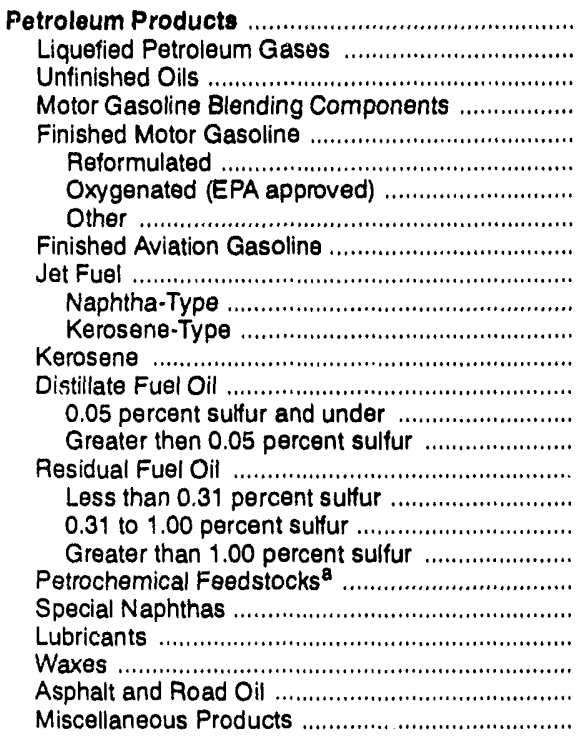 & $\begin{array}{r}139 \\
0 \\
90 \\
10 \\
29 \\
0 \\
0 \\
29 \\
0 \\
0 \\
0 \\
0 \\
0 \\
0 \\
0 \\
0 \\
0 \\
0 \\
0 \\
0 \\
0 \\
0 \\
10 \\
0 \\
0 \\
0\end{array}$ & $\begin{array}{r}68 \\
0 \\
0 \\
56 \\
0 \\
0 \\
0 \\
0 \\
0 \\
0 \\
0 \\
0 \\
0 \\
0 \\
0 \\
0 \\
0 \\
0 \\
0 \\
0 \\
0 \\
3 \\
9 \\
0 \\
0 \\
0\end{array}$ & $\begin{array}{l}0 \\
0 \\
0 \\
0 \\
0 \\
0 \\
0 \\
0 \\
0 \\
0 \\
0 \\
0 \\
0 \\
0 \\
0 \\
0 \\
0 \\
0 \\
0 \\
0 \\
0 \\
0 \\
0 \\
0 \\
0 \\
0\end{array}$ & $\begin{array}{r}1,232 \\
0 \\
10 \\
0 \\
558 \\
0 \\
0 \\
558 \\
0 \\
49 \\
0 \\
49 \\
29 \\
411 \\
0 \\
411 \\
82 \\
0 \\
0 \\
82 \\
0 \\
0 \\
93 \\
0 \\
0 \\
0\end{array}$ & $\begin{array}{r}275 \\
0 \\
0 \\
0 \\
0 \\
0 \\
0 \\
0 \\
0 \\
0 \\
0 \\
0 \\
0 \\
0 \\
0 \\
0 \\
265 \\
0 \\
0 \\
265 \\
0 \\
0 \\
10 \\
0 \\
0 \\
0\end{array}$ & $\begin{array}{l}0 \\
0 \\
0 \\
0 \\
0 \\
0 \\
0 \\
0 \\
0 \\
0 \\
0 \\
0 \\
0 \\
0 \\
0 \\
0 \\
0 \\
0 \\
0 \\
0 \\
0 \\
0 \\
0 \\
0 \\
0 \\
0\end{array}$ & $\begin{array}{r}15,716 \\
270 \\
146 \\
0 \\
9,230 \\
0 \\
784 \\
8,446 \\
144 \\
2,327 \\
174 \\
2,153 \\
0 \\
2,729 \\
87 \\
2,642 \\
204 \\
204 \\
0 \\
0 \\
0 \\
09 \\
309 \\
3 \\
149 \\
106\end{array}$ & $\begin{array}{r}507 \\
0 \\
0 \\
0 \\
0 \\
0 \\
0 \\
0 \\
0 \\
0 \\
0 \\
0 \\
0 \\
507 \\
0 \\
507 \\
0 \\
0 \\
0 \\
0 \\
0 \\
0 \\
0 \\
0 \\
0 \\
0\end{array}$ \\
\hline Total & 139 & 68 & 0 & 1,379 & 275 & 0 & 15,716 & 507 \\
\hline
\end{tabular}

\begin{tabular}{|c|c|c|c|c|c|c|c|}
\hline \multirow[b]{2}{*}{ Commodity } & \multicolumn{4}{|c|}{ From III to } & \multicolumn{3}{|c|}{ From $V$ to } \\
\hline & $\begin{array}{l}\text { Central } \\
\text { Atlantlc }\end{array}$ & $\begin{array}{l}\text { Lower } \\
\text { Atlantlc }\end{array}$ & $\|$ & $\mathbf{v}$ & 1 & $\|$ & III \\
\hline 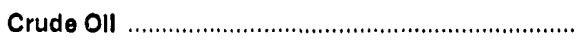 & 0 & 0 & 80 & $\mathbf{0}$ & 0 & 0 & 2,178 \\
\hline 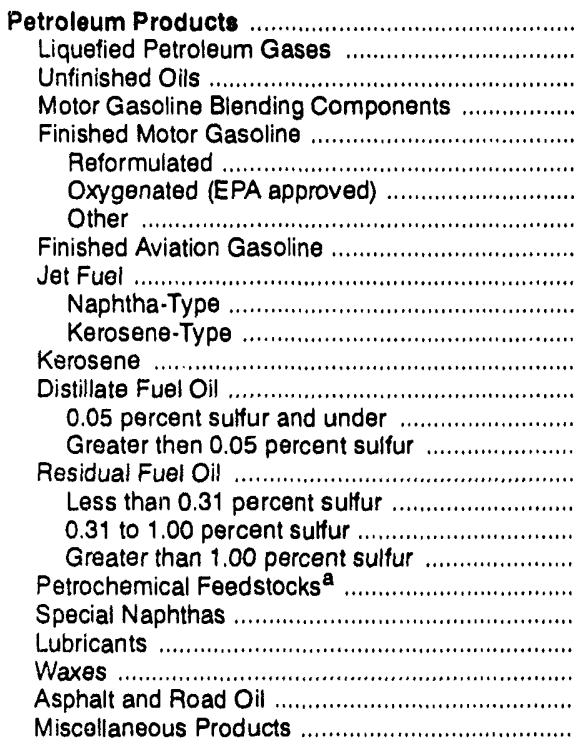 & $\begin{array}{r}1,094 \\
0 \\
36 \\
0 \\
298 \\
0 \\
237 \\
61 \\
44 \\
123 \\
0 \\
123 \\
0 \\
244 \\
0 \\
244 \\
0 \\
0 \\
0 \\
0 \\
0 \\
0 \\
271 \\
3 \\
0 \\
75\end{array}$ & $\begin{array}{r}14,115 \\
270 \\
110 \\
0 \\
8,932 \\
0 \\
547 \\
8,385 \\
100 \\
2,204 \\
174 \\
2,030 \\
0 \\
1,978 \\
87 \\
1,891 \\
204 \\
204 \\
0 \\
0 \\
0 \\
99 \\
38 \\
0 \\
149 \\
31\end{array}$ & $\begin{array}{r}3,051 \\
0 \\
16 \\
73 \\
1,686 \\
0 \\
0 \\
1,686 \\
0 \\
222 \\
0 \\
222 \\
55 \\
553 \\
29 \\
524 \\
0 \\
0 \\
0 \\
0 \\
0 \\
25 \\
338 \\
0 \\
83 \\
0\end{array}$ & $\begin{array}{r}149 \\
0 \\
98 \\
51 \\
0 \\
0 \\
0 \\
0 \\
0 \\
0 \\
0 \\
0 \\
0 \\
0 \\
0 \\
0 \\
0 \\
0 \\
0 \\
0 \\
0 \\
0 \\
0 \\
0 \\
0 \\
0\end{array}$ & $\begin{array}{l}0 \\
0 \\
0 \\
0 \\
0 \\
0 \\
0 \\
0 \\
0 \\
0 \\
0 \\
0 \\
0 \\
0 \\
0 \\
0 \\
0 \\
0 \\
0 \\
0 \\
0 \\
0 \\
0 \\
0 \\
0 \\
0\end{array}$ & $\begin{array}{l}0 \\
0 \\
0 \\
0 \\
0 \\
0 \\
0 \\
0 \\
0 \\
0 \\
0 \\
0 \\
0 \\
0 \\
0 \\
0 \\
0 \\
0 \\
0 \\
0 \\
0 \\
0 \\
0 \\
0 \\
0 \\
0\end{array}$ & $\begin{array}{l}0 \\
0 \\
0 \\
0 \\
0 \\
0 \\
0 \\
0 \\
0 \\
0 \\
0 \\
0 \\
0 \\
0 \\
0 \\
0 \\
0 \\
0 \\
0 \\
0 \\
0 \\
0 \\
0 \\
0 \\
0 \\
0\end{array}$ \\
\hline 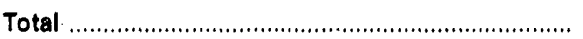 & 1,084 & 14,115 & 3,131 & 149 & 0 & 0 & 2,178 \\
\hline
\end{tabular}

a Includes naphtha less than $401^{\circ} \mathrm{F}$ endpoint and other oils equal to or greater than $401^{\circ} \mathrm{F}$ endpoint.

Sources: Energy Information Administration (EIA) Forms EIA-812, "Monthly Product Pipeline Report" and EIA-817, "Monthly Tanker and Barge Movement Report." 
Table 56. Net Movements of Crude Oll and Petroleum Products by Pipeline, Tanker, and Barge Between PAD Districts, March 1993

(Thousand Barrels)

\begin{tabular}{|c|c|c|c|c|c|c|}
\hline \multirow{2}{*}{ Commodity } & \multicolumn{3}{|c|}{ PAD Dlstrict I } & \multicolumn{3}{|c|}{ PAD District II } \\
\hline & Recelpts & Shipmente & Net Recolpte & Recelpts & Shlpments & Net Recelpts \\
\hline 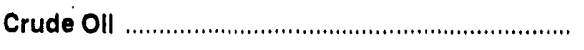 & 147 & 78 & 69 & 58,609 & 1,992 & 56,617 \\
\hline 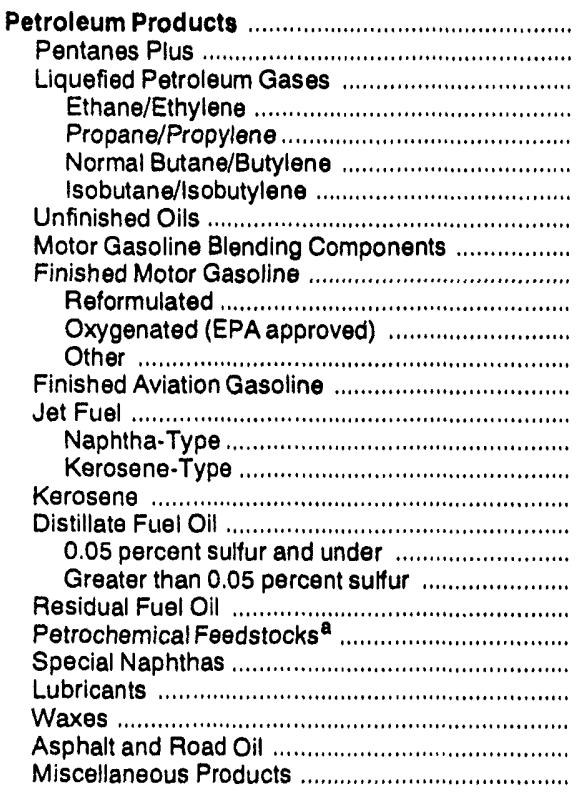 & $\begin{array}{r}91,244 \\
0 \\
4,340 \\
0 \\
4,196 \\
29 \\
115 \\
156 \\
282 \\
50,872 \\
0 \\
4,811 \\
46,061 \\
144 \\
12,137 \\
288 \\
11,849 \\
660 \\
21,608 \\
150 \\
21,458 \\
286 \\
0 \\
99 \\
402 \\
3 \\
149 \\
106\end{array}$ & $\begin{array}{r}8,083 \\
0 \\
0 \\
0 \\
0 \\
0 \\
0 \\
90 \\
66 \\
5,367 \\
0 \\
0 \\
5,367 \\
0 \\
405 \\
0 \\
405 \\
95 \\
2,018 \\
0 \\
2,018 \\
0 \\
0 \\
3 \\
19 \\
0 \\
0 \\
0\end{array}$ & $\begin{array}{r}83,181 \\
0 \\
4,340 \\
0 \\
4,196 \\
29 \\
115 \\
66 \\
216 \\
45,505 \\
0 \\
4,811 \\
40,694 \\
144 \\
11,732 \\
288 \\
11,444 \\
565 \\
19,590 \\
150 \\
19,440 \\
286 \\
0 \\
96 \\
383 \\
3 \\
149 \\
106\end{array}$ & $\begin{array}{r}36,345 \\
590 \\
5,888 \\
670 \\
4,204 \\
382 \\
632 \\
106 \\
703 \\
18,247 \\
0 \\
0 \\
18,247 \\
99 \\
3,774 \\
144 \\
3,630 \\
160 \\
6,246 \\
38 \\
6,208 \\
0 \\
0 \\
25 \\
348 \\
0 \\
83 \\
76\end{array}$ & $\begin{array}{r}12,384 \\
111 \\
4,858 \\
1,836 \\
2,408 \\
446 \\
168 \\
10 \\
23 \\
4,040 \\
0 \\
0 \\
4,040 \\
8 \\
1,450 \\
101 \\
1,349 \\
50 \\
1,373 \\
490 \\
883 \\
347 \\
0 \\
0 \\
103 \\
0 \\
0 \\
11\end{array}$ & $\begin{array}{r}23,961 \\
479 \\
1,030 \\
-1,166 \\
1,796 \\
-64 \\
464 \\
96 \\
680 \\
14,207 \\
0 \\
0 \\
14,207 \\
91 \\
2,324 \\
43 \\
2,281 \\
110 \\
4,873 \\
-452 \\
5,325 \\
-347 \\
0 \\
25 \\
245 \\
0 \\
83 \\
65\end{array}$ \\
\hline 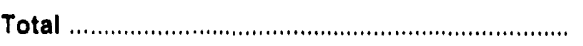 & 91,391 & 8,141 & 83,250 & 94,954 & 14,376 & 80,578 \\
\hline
\end{tabular}

\begin{tabular}{|c|c|c|c|c|c|c|c|c|c|}
\hline \multirow[b]{2}{*}{ Commodity } & \multicolumn{3}{|c|}{ PAD District III } & \multicolumn{3}{|c|}{ PAD Dlatrict IV } & \multicolumn{3}{|c|}{ PAD Distrlat V } \\
\hline & Recelpte & Shipments & $\begin{array}{c}\text { Not } \\
\text { Recelpts }\end{array}$ & Recelpte & Shipments & $\begin{array}{c}\text { Not } \\
\text { Recelpts }\end{array}$ & Recelpts & Shipments & $\begin{array}{c}\text { Net } \\
\text { Rocelpts }\end{array}$ \\
\hline 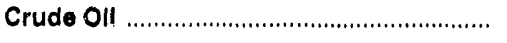 & 8,168 & 55,482 & $.46,314$ & 293 & 4,360 & $-4,067$ & 0 & 6,305 & $\cdot 6,305$ \\
\hline 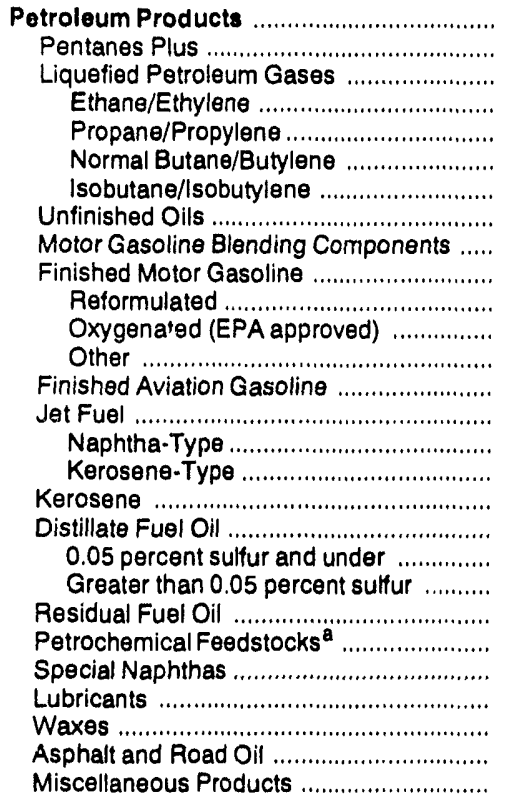 & $\begin{array}{r}7,607 \\
314 \\
5,262 \\
2,873 \\
1,564 \\
620 \\
205 \\
0 \\
56 \\
895 \\
0 \\
0 \\
895 \\
0 \\
540 \\
101 \\
439 \\
0 \\
242 \\
0 \\
242 \\
265 \\
0 \\
3 \\
19 \\
0 \\
0 \\
11\end{array}$ & $\begin{array}{r}115,517 \\
493 \\
8,195 \\
419 \\
6,974 \\
241 \\
561 \\
260 \\
1,003 \\
62,619 \\
0 \\
4,811 \\
57,808 \\
243 \\
15,510 \\
478 \\
15,032 \\
665 \\
25,137 \\
125 \\
25,012 \\
204 \\
0 \\
124 \\
647 \\
3 \\
232 \\
182\end{array}$ & $\begin{array}{r}-107,910 \\
-179 \\
-2,933 \\
2,454 \\
-5,410 \\
379 \\
-356 \\
-260 \\
-947 \\
-61,724 \\
0 \\
-4,811 \\
-56,913 \\
-243 \\
-14,970 \\
-377 \\
-14,593 \\
-665 \\
-24,895 \\
-125 \\
-24,770 \\
61 \\
0 \\
-121 \\
-628 \\
-3 \\
-232 \\
-171\end{array}$ & $\begin{array}{r}3,131 \\
0 \\
103 \\
0 \\
101 \\
2 \\
0 \\
0 \\
0 \\
1,650 \\
0 \\
0 \\
1,650 \\
8 \\
797 \\
0 \\
797 \\
0 \\
573 \\
427 \\
146 \\
0 \\
0 \\
0 \\
0 \\
0 \\
0 \\
0\end{array}$ & $\begin{array}{r}4,886 \\
300 \\
2,540 \\
1,288 \\
683 \\
346 \\
223 \\
0 \\
0 \\
1,222 \\
0 \\
0 \\
1,222 \\
0 \\
250 \\
165 \\
85 \\
10 \\
564 \\
0 \\
564 \\
0 \\
0 \\
0 \\
0 \\
0 \\
0 \\
0\end{array}$ & $\begin{array}{r}-1,755 \\
-300 \\
-2,437 \\
-1,288 \\
-582 \\
-344 \\
-223 \\
0 \\
0 \\
428 \\
0 \\
0 \\
428 \\
8 \\
547 \\
-165 \\
712 \\
-10 \\
9 \\
427 \\
-418 \\
0 \\
0 \\
0 \\
0 \\
0 \\
0 \\
0\end{array}$ & $\begin{array}{r}2,523 \\
0 \\
0 \\
0 \\
0 \\
0 \\
0 \\
98 \\
51 \\
1,584 \\
0 \\
0 \\
1,584 \\
0 \\
367 \\
211 \\
156 \\
0 \\
423 \\
0 \\
423 \\
0 \\
0 \\
0 \\
0 \\
0 \\
0 \\
0\end{array}$ & $\begin{array}{l}0 \\
0 \\
0 \\
0 \\
0 \\
0 \\
0 \\
0 \\
0 \\
0 \\
0 \\
0 \\
0 \\
0 \\
0 \\
0 \\
0 \\
0 \\
0 \\
0 \\
0 \\
0 \\
0 \\
0 \\
0 \\
0 \\
0 \\
0\end{array}$ & $\begin{array}{r}2,523 \\
0 \\
0 \\
0 \\
0 \\
0 \\
0 \\
98 \\
51 \\
1,584 \\
0 \\
0 \\
1,584 \\
0 \\
367 \\
211 \\
156 \\
0 \\
423 \\
0 \\
423 \\
0 \\
0 \\
0 \\
0 \\
0 \\
0 \\
0\end{array}$ \\
\hline Total & 16,775 & 170,999 & $-154,224$ & 3,424 & 9,246 & $\cdot 5,822$ & 2,523 & 6,305 & $-3,782$ \\
\hline
\end{tabular}

Includes naphtha less than $401^{\circ} \mathrm{F}$ endpoint and other oils equal to or greater than $401^{\circ} \mathrm{F}$ endpoint.

Sources: Energy Information Administration (EIA) Forms EIA-812, "Monthly Product Pipeline Report," EIA-813, "Monthly Crude Oil Report," and EIA-817, "Monthly Tanker and Barge Movement Report." 


\section{Appendix A}

\section{District \\ Descriptions and Maps}

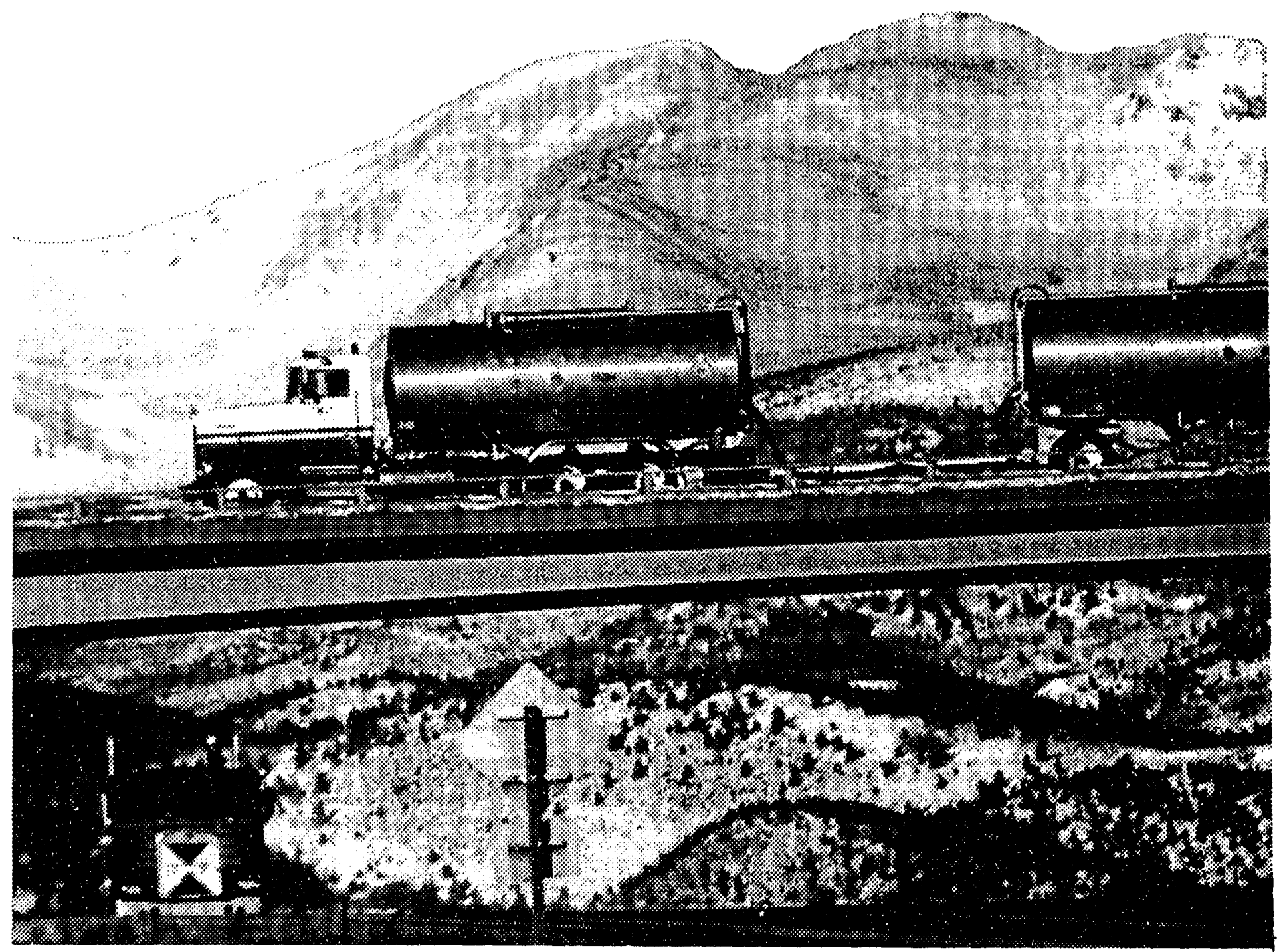

Tank trucks are used to pick up crude oil gathered at leases in remote areas. 


\section{Appendix A}

\section{District Descriptions and Maps}

The following are the Refining Districts which make up the Petroleum Administration for Defense (PAD) Districts.

\section{PAD District I}

East Coast: District of Columbia and the States of Maine, New Hampshire, Vermont, Massachusetts, Rhode Island, Connecticut, New Jersey, Delaware, Maryland, Virginia, North Carolina, South Carolina, Georgia, Florida, and the following counties of the State of New York: Cayuga, Tompkins, Chemung, and all counties east and north thereof. Also the following counties in the State of Pennsylvania: Bradford, Sullivan, Columbia, Montour, Northumberland, Dauphin, York, and all counties east thereof.

Appalachian No. 1: The State of West Virginia and those parts of the States of Pennsylvania and New York not included in the East Coast District.

\section{Sub-PAD District I}

New England: The States of Connecticut, Maine, Massachusetts, New Hampshire, Rhode Island and Vermont.

CentralAtlantic: The District of Columbia and the States of Delaware, Maryland, New Jersey, New York, and Pennsylvania.

Lower Atlantic: The States of Florida, Georgia, North Carolina, South Carolina, Virginia and West Virginia.

\section{PAD District II}

Indiana-Illinois-Kentucky: The States of Indiana, Illinois, Kentucky, Tennessee, Michigan, and Ohio.

Minnesota-Wisconsin-North and South Dakota: The States of Minnesota, Wisconsin, North Dakota, and South Dakota.

Oklahoma-Kansas-Missouri: The States of Oklahoma, Kansas, Missouri, Nebraska, and Iuv'a.

\section{PAD District III}

Texas Inland: The State of Texas except the Texas Gulf Coast District.

Texas Gulf Coast: The following counties of the State of Texas: Newton, Orange, Jefferson, Jasper, Tyler, Hardin, Liberty, Chambers, Polk, San Jacinto, Montgomery, Harris, Galveston, Waller, Fort Bend, Brazoria, Wharton, Matagorda, Jackson, Victoria, Calhoun, Refugio, Aransas, San Patricio, Nueces, Kleberg, Kenedy, Willacy, and Cameron.

Louisiana Gulf Coast: The following parishes of the State of Louisiana: Vernon, Rapides, Avoyelles, Pointe Coupee, West Feliciana, East Feliciana, Saint Helena, Tangipahoa, Washington, and all parishes south thereof. Also the following counties of the State of Mississippi: Pearl River, Stone, George, Hancock, Harrison, and Jackson. Also the following counties of the State of Alabama: Mobile and Baldwin.

North Louisiana-Arkansas: The State of Arkansas and those parts of the States of Louisiana, Mississippi, and Alabama not included in the Louisiana Gulf Coast District.

New Mexico: The State of New Mexico.

\section{PAD District IV}

Rocky Mountain: The States of Montana, Idaho, Wyoming, Utah, and Colorado.

\section{PAD District V}

West Coast: The States of Washington, Oregon, California, Nevada, Arizona, Alaska, and Hawaii. 

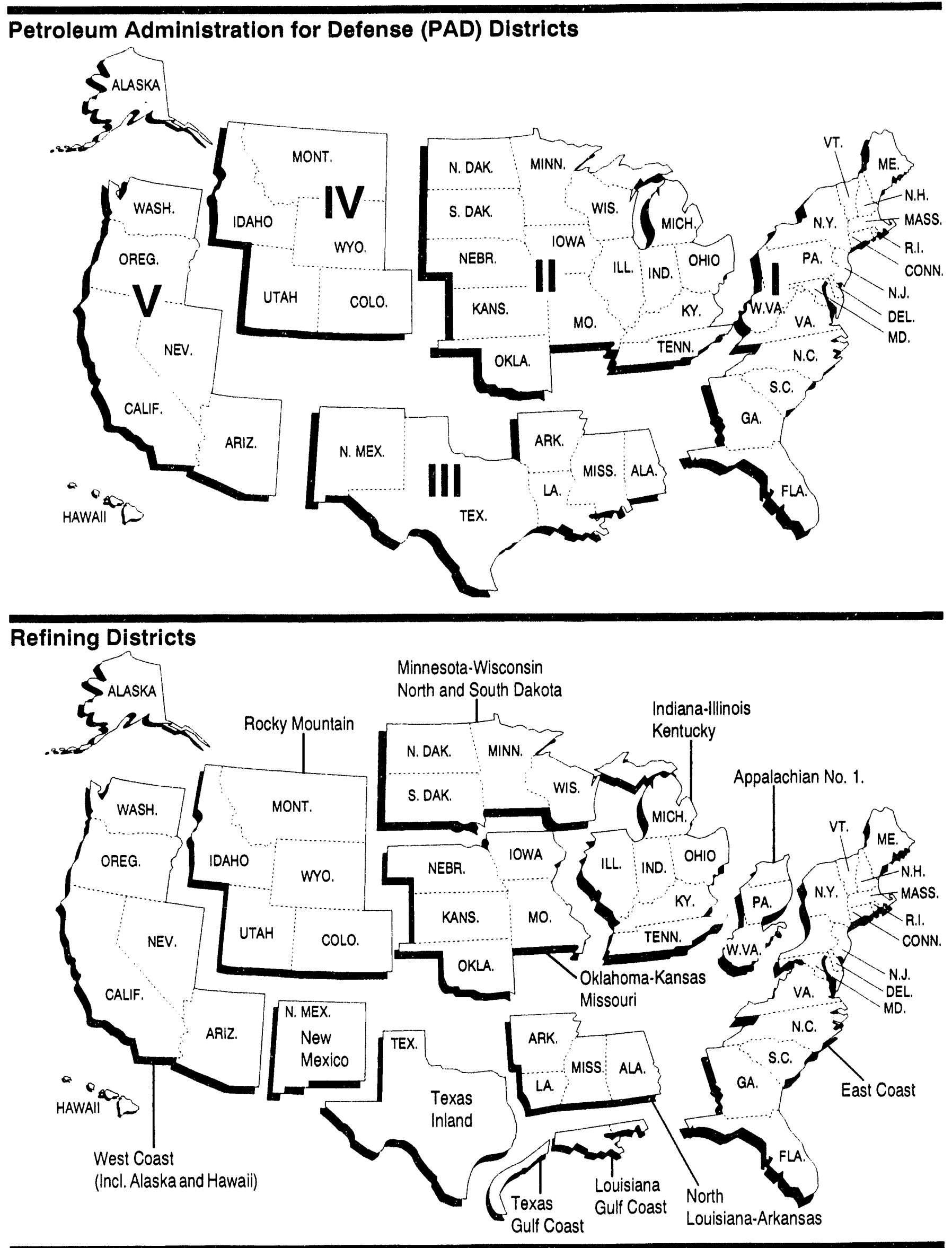


\section{Appendix B}

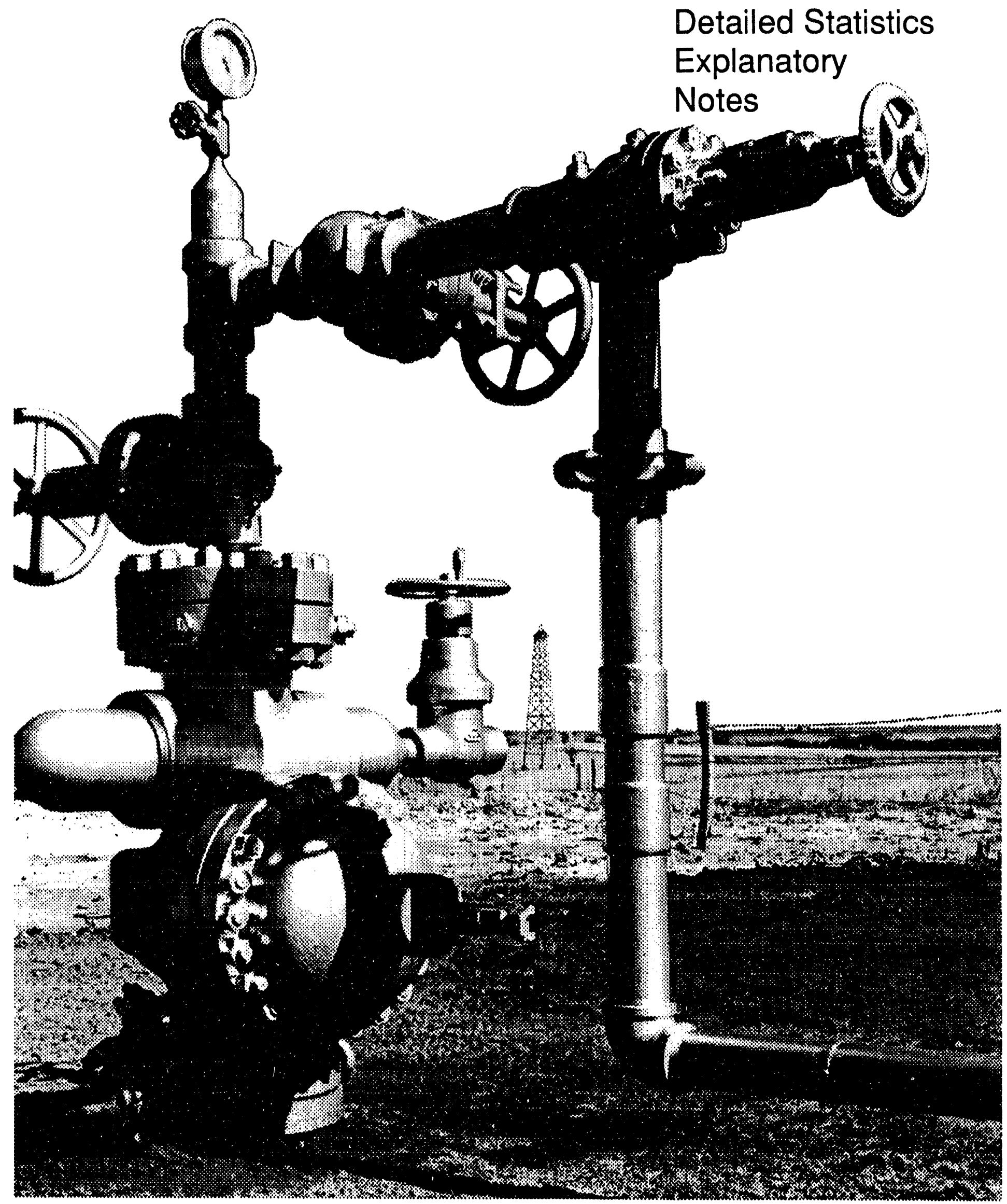

The cluster of pipes and valves that control the flow of oil at the mouth of an oil well is what oilmen call a "Christmas Tree." 


\section{Explanatory Notes}

The following Explanatory Notes are provided to assist in understanding and interpreting the data presented in the Detailed Statistics section in this publication.

- Note 1. Petroleum Supply Reporting System

- Note 2. Monthly Petroleum Supply Reporting System

- Note 3. Technical Notes for Detailed Statistics Tables

- Note 4. Domestic Crude Oil Production

- Note 5. Export Data

- Note 6. Quality Control and Data Revision

- Note 7. Frames Maintenance

- Note 8. 198! Changes in the Petroleum Supply Reporting System

- Noti 9. 1983 Changes in the Petroleum Supply Reporting System

- Note 10. 1984 Changes in the Petroleum Supply Reporting System

- Note 11. 1985 Changes in the Petroleum Supply Reporting System

- Note 12. 1986 Changes in the Petroleum Supply Reporting System

- Note 13. 1987 Changes in the Petroleum Supply Reporting System

- Note 14. 1989 Changes in the Petroleum Supply Reporting System

- Note 15. 1990 Changes in the Petroleum Supply Reporting System

- Note 16. 1991 Changes in the Petroleum Supply Reporting System

- Note 17. 1993 Changes in the Petroleum Supply Reporting System

\section{Note 1. Petroleum Supply Reporting System}

The Petroleum Supply Reporting System (PSRS) represents a family of data collection survey forms, data processing systems, and publication systems that have been consolidated to achieve comparability and consistency throughout. The survey forms that comprise the PSRS are:

\begin{tabular}{|ll|}
\hline Form & \\
Number & Name \\
EIA-800 & "Weekly Refinery Report" \\
EIA-801 & "Weekly Bulk Terminal Report" \\
EIA-802 & "Weekly Product Pipeline Report" \\
EIA-803 & "Weekly Crude Oil Stocks Report" \\
EIA-804 & "Weekly Imports Report" \\
EIA-807 & "Propane Telephone Survey" \\
EIA-810 & "Monthly Refinery Report" \\
EIA-811 & "Monthly Bulk Terminal Report" \\
EIA-812 & "Monthly Product Pipeline Report" \\
EIA-813 & "Monthly Crude Oil Report" \\
EIA-814 & "Monthly Imports Report" \\
EIA-816 & "Monthly Natural Gas Liquids Report" \\
EIA-817 & "Monthly Tanker and Barge Movement \\
& Report" \\
EIA-818 & "Monthly International Energy Agency \\
& Imports/Stocks-At-Sea Report" \\
EIA-819M & "Monthly Oxygenate Telephone Report" \\
EIA-819A & "Annual Oxygenate Report" \\
EIA-820 & "Annual Refinery Report" \\
\hline
\end{tabular}

Forms EIA-800 through 804 comprise the Weekly Petroleum Supply Reporting System (WPSRS). A sample of all petroleum companies report weekly data to the Energy Information Administration (EIA) on crude oil and petroleum product stocks, refinery inputs and production, and crude oil and petroleum product imports. The sample of companies that report weekly is selected from the universe of companies that report on the comparable monthly surveys. Data collected from the WPSRS are used to develop estimates of the most current monthly quantities in the Summary Statistics section of the Petroleum Supply Monthly (PSM) and which appear in the Weekly Petroleum Status Report (WPSR).

The Form EIA-807, "Propane Telephone Survey" is used to collect data on production, stocks, and imports of propane. These data are used to monitor the supply of propane and to report to the Congress and others on supplies when requested. Data are collected from a sample of respondents reporting on the Monthly Petroleum Supply Reporting System (MPSRS) surveys. Data are collected on a weekly basis during the heating season (October through March) and published in the Winter Fuels Report. During the non-heating season (April through September) 
data are collected on end-of-month stocks only. These data are published in the WPSR.

Forms ElA-810 through 814,816 , and 817 comprise the MPSRS. These surveys are used to collect detailed refinery, natural gas plant and oxygenate plant operations data; refinery, bulk terminal, oxygenate plant, natural gas plant, and pipeline stocks data; crude oil and petroleum product imports data; and data on movements of petroleum products and crude oil between Petroleum Administration for Defense (PAD) Districts. A description of the MPSRS forms follows in Explanatory Note 2.

Data from these surveys are published in preliminary form in the PSM. They are published in final form in the Summary Statistics and the Detailed Statistics sections of the Petroleum Supply Annual (PSA), Volumes 1 and 2.

Summary information on the revision error between preliminary and final data is found in the feature article in the PSM entitled, "Timeliness and Accuracy of Petroleum Supply Data." The last article was published in the September 1992 issue and evaluated the accuracy of the data for 1991 compared with previous years.

The Form EIA-818, "Monthly International Energy Agency Imports/Stocks-At-Sea Report" is used to collect data on imports of crude oil and petroleum products and worldwide quantities of petroleum in transit or held as slocks-at-sea by U.S. companies. These data are not published but are used to assist the EIA in fulfilling its datareporting obligation to the International Energy Agency (IEA) and to satisfy requirements of the North Atlantic Treaty Organization Civil Emergency Planning Organization. The data are used by the IEA in ongoing monitoring programs and contribute to petroleum-sharing programs during periods of international petroleum supply disruption.

The Form EIA-819M, "Monthly Oxygenate Telephone Report," is used to collect preliminary data on production, imports, and stocks of oxygenates by PAD District. These data are used to monitor the supply of oxygenates. Data are collected from a sample of respondents reporting on the MPSRS surveys and the EIA-822A/D, "Oxygenate Operations Identification Survey." Data are published in Appendix D of this publication and also in the WPSR.

The Form EIA-819A, "Annual Oxygenate Report" is used to collect data on current and projected name plate production capacity of oxygenates. The results of this survey are published in the Oxygenate Capacity section of the PSA, Volume 1.

The Form ElA-820, "Annual Refinery Report" is used to collect data on refinery fuel use and consumption of steam and electricity, refinery receipts of crude oil by method of transportation, and refinery operable and storage capacity. This survey is the primary source of data in the Refinery Capacity section of the PSA, Volume 1.

\section{Note 2. Monthly Petroleum Supply Reporting System}

The Monthly Petroleum Supply Reporting System (MPSRS) was implemented in January 1983 as the result of an extensive effort by the Energy Information Administration (EIA) to integrate the collection and processing of petroleum supply data that had been collected on other survey forms for many years. The collection of monthly petroleum supply statistics began as early as 1918 when the U.S. Bureau of Mines began collecting data on refinery operations, crude oil stocks and movements. The collection systems were further expanded in 1925 to include natural gas plant liquids production and storage, imports of crude oil and petroleum products and storage and movement of petroleum products in 1959, and tanker and barge movements of crude oil and petroleum products in 1964. Since their inception, each survey has undergone numerous changes, but the MPSRS was the first effort to make them all consistent and comparable. In 1993, the collection system was further expanded to include imports, stocks, and blending of oxygenates.

The forms that comprise the MPSRS are:

\begin{tabular}{|ll|}
\hline Form & \\
Number & Name \\
EIA-810 & "Monthly Refinery Report" \\
EIA-811 & "Monthly Bulk Terminal Report" \\
EIA-812 & "Monthly Product Pipeline Report" \\
EIA-813 & "Monthly Crude Oil Report" \\
EIA-814 & "Monthly Imports Report" \\
EIA-816 & "Monthly Natural Gas Liquids Report" \\
EIA-817 & "Monthly Tanker and Barge Movement \\
& Report" \\
EIA-819M & "Monthly Oxygenate Telephone Report"
\end{tabular}

\section{Respondent Frame}

Form EIA-810, "Monthly Refinery Report" - Operators of all operating and idle petroleum refineries and blending plants located in the 50 States, the District of Columbia, Puerto Rico, the Virgin Islands, Guam and other U.S. possessions. Approximately 240 respondents report on the Form EIA-810.

Form EIA-811, "Monthly Bulk Terminal Report" - Every bulk terminal operating company located in the 50 States, the District of Columbia, Puerto Rico, the Virgin Islands, and other U.S. possessions. A bulk terminal is primarily used for storage and/or marketing of petroleum products and has a total bulk storage capacity of 50,000 barrels or 
more, and/or receives petroleum products by tanker, barge, or pipeline. Bulk terminal facilities associated with a product pipeline are included. In addition, the Form EIA-811 must be completed by merchant oxygenate plants that produce oxygenates. Approximately 350 respondents report on the Form EIA-811.

Form EIA-812, "Monthly Product Pipeline Report" - All product pipeline companies that carry petroleum products (including interstate, intrastate, and intracompany pipelines) in the 50 States and the District of Columbia. Approximately 80 respondents report on the Form EIA812.

Form EIA-813, "Monthly Crude Oil Report" - All companies which carry or store 1,000 barrels or more of crude oil. Included in this survey are gathering and trunk pipeline companies (including interstate, intrastate, and intracompany pipelines), crude oil producers, terminal operators, storers of crude oil (except refineries), and companies transporting Alaskan crude oil by water in the 50 States and the District of Columbia. Approximately 170 respondents report on the Form ElA-813.

Form EIA-814, "Monthly Imports Report" - All companies, including subsidiary or affiliated companies, that import crude oil or petroleum products (1) into the 50 States and the District of Columbia, (2) into Puerto Rico, the Virgin Islands and other U.S. possessions (Guam, Midway Islands, Wake Island, American Samoa, and Northern Mariana Islands), and (3) from Puerto Rico, the Virgin Islands and other U.S. possessions into the 50 States and the District of Columbia. Imports into Foreign Trade Zones located in the 50 States and the District of Columbia are considered imports into the 50 States and the District of Columbia and must be reported. A report is required only if there has been an import during the month unless the importer has been selected as part of a sample to report every month regardless of activity. Approximately 860 respondents report on the Form EIA-814.

Form EIA-816, "Monthly Natural Gas Liquids Report" Operators of all facilities that extract liquid hydrocarbons from a natural gas stream (natural gas processing plant) and/or separate a liquid hydrocarbon stream into its component products (fractionator). Approximately 800 respondents report on the Form EIA-816.

Form EIA-817, "Monthly Tanker and Barge Movement Report" - All comparies that have custody of crude oil or petroleum products transported by tanker or barge between Petroleum Administration for Defense (PAD) Districts or between the Panama Canal and the United States. For purposes of this report, custody is defined as physical possession of crude oil or petroleum products on a company-owned tanker or barge. Also, companies which lease vessels or contract for the movement of crude oil or petroleum products on a tanker or barge between PAD Districts or between the Panama Canal and the United States are considered to have custody. Approximately 35 respondents report on the Form ElA-817.

Form EIA-819M, "Monthly Oxygenate Telephone Report" - The sample of companies that report on the ElA-819M are selected from the universe of companies that report on the MPSRS surveys and the EIA-822A/D, "Oxygenate Operations Identification Survey." The universe consists of (1) operators of facilities that produce (manufacture or distill) oxygenates (including MTBE plants, petrochemical plants, and refineries that produce oxygenates as part of their operations); (2) operators of petroleum refineries; (3) operators of bulk terminals, bulk stations, blending plants, and other nonrefinery facilities that store and/or blend oxygenates; and (4) importers of oxygenates (importer of record) located in or importing oxygenates into the 50 States and the District of Columbia. Approximately 136 respondents report on the Form EIA-819M.

\section{Description of Survey Forms}

The Form EIA-810, "Monthly Refinery Report," is used to collect data on refinery input and capacity, sulfur content and API gravity of crude oil, and data on supply (beginning stocks. receipts, and production) and disposition (inputs, shipments, fuel use and losses, and ending stocks) of crude oil and refined products.

The Form EIA-811, "Monthly Bulk Terminal Report," is used to collect data on end-of-month stock levels of finished petroleum products by State in the custody of the bulk terminal company or merchant oxygenate plant regardless of ownership. Leased tankage at other facilities is excluded. All domestic and foreign stocks held at bulk terminals and in-transit thereto, except those in-transit by pipeline are included. Petroleum products in-transit by pipeline are reported by pipeline operators on Form EIA812, "Monthly Product Pipeline Report."

The Form EIA-812, "Monthly Product Pipeline Report," is used to collect data on end-of-month stock levels and movements of petroleum products transported by pipeline. Intermediate movements for pipeline systems operating in more than two PAD Districts are included.

The Form EIA-813, "Monthly Crude Oil Report," is used to collect data on end-of-month stocks of crude oil held at pipeline and tank farms (associated with the pipelines) and terminals operated by the reporting company. Also, crude oil consumed by pipelines and on leases as pump fuel, boiler fuel, etc., is reported. Data are reported on a PAD District basis. 
Total Alaskan crude oil stocks in-transit by water (including stocks held at transshipment terminals between Alaska and the continental United States) to the 50 States, the District of Columbia, Puerto Rico, and the Virgin Islands are also reported by the transporting company having custody of the stocks.

Inter-PAD District movements of crude oil by pipeline are collected by the shipping and receiving PAD District. Intermediate movements for pipeline systems operating in more than two PAD Districts are not included.

The Form EIA-814, "Monthly Imports Report," is used to collect data on imports of crude oil and petroleum products (1) into the 50 States and the District of Columbia, (2) into Puerto Rico, the Virgin Islands, and other U.S. possessions (Guam, Midway Islands, Wake Island, American Samoa, and Northern Mariana Islands), and (3) from Puerto Rico, the Virgin Islands, and other U.S. possessions into the 50 States and the District of Columbia. Imports into Foreign Trade Zones located in the 50 States and the District of Columbia are considered imports into the 50 States and the District of Columbia.

The type of commodity, port of entry, country of origin, quantity (thousand barrels), sulfur percent by weight, API gravity, and name and location of the processing or storage facility are reported. Sulfur percent by weight is requested for crude oil, crude oil burned as fuel, and residual fuel oil only. API gravity is requested for crude oil only. The name and location of the processing or storage facility is requested for crude oil, unfinished oils, other hydrocarbons/hydrogen/oxygenates and blending components only.

The Form ElA-816, "Monthly Natural Gas Liquids Report," is used to collect data on the operations of natural gas processing plants and fractionators. Beginning and end-of-month stocks, receipts, inputs, production, shipments, and plant fuel use and losses during the month are collected from operators of natural gas processing plants. End-of-month stocks are collected from fractionators.

The Form EIA-817, "Monthly Tanker and Barge Movement Report," is used to collect data on the movements of crude oil and petroleum products between PAD Districts. Data are reported by shipping and receiving PAD District and sub-PAD District. Shipments to and from the Panama Canal are also included if the shipment was delivered to the Canal.

The Form EIA-819M, "Monthly Oxygenate Telephone Report," is used to collect data on production, stocks, and imports of oxygenates. Data on end-of-month stocks are reported on a custody basis regardless of ownership. Data are reported on a PAD District basis.

\section{Collectlon Methods}

Except for the EIA-819M, survey forms for the MPSR can be submitled by mail, facsimile, or electronic trąn mission. Completed forms are required to be postmarked by the 20th calendar day following the end of the report month. Data for the EIA-819M are solicited by telephone or transmitted to the EIA by facsimile. Data for the EIA$819 \mathrm{M}$ survey are collected beginning on the seventh working day of each month. Receipt of the reports are monitored using an automated respondent mailing list. Telephone follow-up calls are made to nonrespondents prior to the publication deadline.

\section{Response Rate}

The response rate is generally 98 to 100 percent. Chronic nonrespondents and late filing respondents are contacted in writing and reminded of their requirement to report. Companies that file late or fail to file are subject to criminal fines, civil penalties, and other sanctions as provided by Section 13(i) of the Federal Energy Administration (FEA) Act.

\section{Data Imputation}

Imputation is performed for companies that fail to file Forms EIA-810 through 813,816 , and $819 \mathrm{M}$. For such companies, previous monthly values are used for current values. The ending stock value of the previous month is used as the value for beginning and ending stocks for the current month.

On the EIA-819M, data are aggregated for each geographic region. Estimation factors, which are derived from 1992 reported data, are then applied to each cell to generate published estimates.

Data for nonrespondents on the Forms EIA-814 and 817 are not imputed because these data series, by respondent, are highly variable.

\section{Confidentlality}

The Office of Legal Counsel of the Department of Justice concluded on March 20,1991, that the Federal Energy Administration Act requires the Energy Information Administration to provide company-specific data to the Department of Justice, or to any Federal agency when requested for official use, which may include enforcement of Federal law. The information contained on this form may also be made available, upon request, to another component of the Department of Energy (DOE), to any Committee of Congress, the General Accounting Office, or other Congressional agencies authorized by law to receive such information. A court of competent jurisdiction may obtain this information in response to an order. 
The information contained on Forms EIA-810 through $813,816,817$, and $819 \mathrm{M}$ are kept confidential and not disclosed to the public to the extent that they satisfy the criteria for exemption under the Freedom of Information Act (FOIA), 5 U.S.C. 552, the Department of Energy (DOE) regulations, 10 C.F.R. 1004.11, implementing the FOIA, and the Trade Secrets Act, 18 U.S.C. 1905. The information contained on Form ElA-814 are not considered confidential and historically has not been treated as such.

Upon receipt of a request for this information under the FOIA, the DOE shall make a final determination whether the information is exempt from disclosure in accordance with the procedures and criteria provided in the regulations. To assist us in this determination, respondents should demonstrate to the DOE that, for example, their information contains trade secrets or commercial or financial information whose release would be likely to cause substantial harm to their company's competitive position. A letter accompanying the submission that explains (on an element-by-element basis) the reasons why the information would be likely to cause the respondent substantial competitive harm if released to the public would aid in this determination. A new justification does not need to be provided each time information is submitted on the form, if the company has previously submitted a justification for that information and the justification has not changed. Company specific data are also provided to other DOE offices for the purpose of examining operations in the context of emergency response planning and actual emergencies.

The data collected on Forms EIA-810 through 814, 816, and 817 appear in EIA publications such as Petroleum Supply Monthly (PSM), Monthly Energy Review, Petroleum Supply Annua' (PSA), and the Annual Energy Review.

Data on the breakdown between liquefied refinery gases and olefins is suppressed on PSM Table 29, "Refinery Net Production of Finished Petroleum Products by PAD and Refining Districts" and the corresponding PSA table to avoid disclosure of company identifiable data.

Data on PSM Table 28, "Refinery Input of Crude Oil and Petroleum Products by PAD and Refining Districts," Table 30, "Refinery Stocks of Crude Oil and Petroleum Products by PAD and Refining Districts," Table 51, "Stocks of Crude Oil and Petroleum Products by PAD District," and Table 52, "Refinery, Bulk Terminal, and Natural Gas Plant Stocks of Selected Petroleum Products, by State" as well as data on the production and stocks of oxygenates published in Appendix D are subject to statistical nondisclosure procedures for oxygenates. Statistics representing data aggregated from less than three com- panies or aggregated data representing 60 percent or more of a single company's data are suppressed.

With the exception of Tables $28,29,30,51$, and 52 in the PSM (and corresponding PSA tables), the tables are not subject to statistical nondisclosure procedures. Thus, there may be some table cells which are based on data from only one or two respondents, or which are dominated by data from one or two large respondents. In these cases, it may be possible for a knowledgeable user of the data to make inferences about the data reported by a specific respondent.

\section{Note 3. Technical Notes for Detailed Statistics Tables}

The detailed statistics tables in the Petroleum Supply Monthly (PSM) provide complete supply and demand information for the current year. The tables are organized to locate National and Petroleum Administration for Defense (PAD) District summary data at the front followed by tables on crude oil and petroleum product production, import/export data, stocks information, and lastly, data on crude oil and petroleum product movements. To assist in the interpretation of these tables, the following technical notes are provided. Column and row headings are defined in the Glossary.

\section{Supply}

Field Production - Field production is the sum of crude oil production, natural gas plant liquids production, other liquids production, and finished petroleum products production.

Crude oil production is an estimate based on data received from State conservation agencies and the Mineral Management Service of the U.S. Department of the Interior. Refer to Explanatory Note 4 for further details.

Field production of natural gas plant liquids is reported on Form EIA-816 and published on a net basis (i.e., production minus inputs) in this column.

Other liquids field production is calculated by forcing the product supplied to be zero; thereby backing into field production.

Field production of finished petroleum products is calculated by (1) adding the amount of fuel ethanol that has been blended into finished motor gasoline, and (2) plus $(+)$ or minus (-) the field production of motor gasoline blending components. Refer to Explanatory Note 17 for a further discussion of this calculation. 
Negative field production of motor gasoline blending components represents an understatement for finished motor gasoline.

Negative field production of other finished motor gasoline represents an overstatement of other finished motor gasoline and an understatement of oxygenated motor gasoline.

Refinery Production - Published production of these products equal refinery production minus refinery input. Refinery production of other hydrocarbons, hydrogen and oxygenates, unfinished oils, and motor and aviation gasoline blending components appear on a net basis under refinery input. Negative refinery production will occur when the amount of a product produced during the month is less than the amount of that same product that is reprocessed (input) or reclassified to become another product during the same month.

Unaccounted for Crude Oil - This column is a balancing item for crude oil. This data element represents the difference between crude oil supply and disposition. Crude oil supply is the sum of field production and imports. Crude oil disposition is the sum of stock change, losses, refinery inputs, exports, and products supplied. A positive result indicates that refiners and exporters reported use of more crude oil than was reported to have been available to them. (This occurs, for example, when imports are undercounted due to late reporting or other problems). A negative result indicates that more crude oil was reported to have been supplied to refiners and exporters than they reported to have used.

\section{Disposition}

Stock Change - This column is calculated as the difference between the Ending Stocks column of this table and the Ending Stocks column of this table in the prior month's publication. A negative number indicates a decrease in stocks and a positive number indicates an increase in stocks.

Crude Losses - The volume of crude oil reported by petroleum refineries as being lost in their operations. These losses are due to spills, contamination, fires, etc., as opposed to refining processing losses or gains.

Refinery Inputs - Refinery inputs of crude oil and intermediate materials (unfinished oils, gasoline blending components, other hydrocarbons and oxygenates, liquefied petroleum gases, and pentanes plus) that are processed at refineries to produce finished petroleum products.

Crude oil inputs represents total crude oil (domestic and foreign) inpit to atmospheric crude oil distillation units and other refinery processing units (i.e., catalytic cracking units, cokers).

Inputs of natural gas liquids are natural gas liquids received from natural gas plants for blending and processing. Published inputs of natural gas liquids are reported on a gross basis.

Inputs of unfinished oils, motor and aviation gasoline blending components, and other hydrocarbons and oxygenates are published on a net basis (i.e., refinery input minus refinery production).

Inputs of finished petroleum products are published on a net basis (i.e., refinery production minus refinery inputs) and displayed under the refinery production column.

Exports - Exports include crude oil shipments from the 50 States to Puerto Rico, and the Virgin Islands.

Products Supplied - Products supplied is equal to field production, plus refinery production, plus imports, plus unaccounted for crude oil, (plus net receipts on a PAD District basis), minus stock change, minus crude losses, minus refinery inputs, minus exports.

Products supplied indicates those quantities of petroleum products supplied for domestic consumption. Occasionally, the result for a product is negative because total disposition of the product exceeds total supply. Negative product supplied may occur for a number of reasons: (1) product reclassification has not been reported; (2) data were misreported or reported late; (3) in the case of calculations on a PAD District basis, the figure for net receipts was inaccurate because the coverage of interdistrict movements was incomplete; and (4) products such as gasoline blending components and unfinished oils have entered the primary supply channels with their production not having been reported, e.g., streams returned to refineries from petrochemical plants.

Product supplied for crude oil is the sum of crude oil burned on leases and by pipelines as fuel. Prior to January 1983, crude oil burned on leases and by pipelines as fuel were reported as either distillate or residual fuel oil and were included in product supplied for these products.

\section{Ylelds}

The refinery yield of finished motor gasoline is calculated by subtracting the inputs of pentanes plus, liquefied petroleum gases, other hydrocarbons/oxygenates and motor gasoline blending components from the production of finished motor gasoline before dividing by the sum of crude oil input and unfinished oils input (net). 
The refinery yield of finished aviation gasoline is calculated by subtracting the inputs of aviation gasoline blending components from the roduction of finished aviation gasoline before dividing by the sum of crude oil in.put and unfinished oils input (net).

Refinery yields for all products (except finished motor gasoline and finished aviation gasoline) are calculated by dividing the production for each product by the sum of crude oil input and unfinished oils input (net) reported in the U.S. total.

\section{Stocks}

Primary stocks of petroleum products do not include either secondary stocks held by dealers and jobbers or tertiary stocks held by consumers.

\section{Movements}

Movements of crude oil b: pipeline between PAD Districts include trunk pipeline companies (interstate, intrastate, and intracompany pipelines). Intermediate movements for crude oil pipeline systems operating in more than two PAD Districts are not included.

Movements of petroleum products by pipeline between PAD Districts include trunk pipeline companies (interstate, intra state and intracompany pipelines). Intermediate movements for prod"ct pipeline systenis operating in more than two PAD Districts are included. For example, a shipment originating in PAD District 3, passing through PAD District 2 to PAD District 1 , is reported as a movement from PAD District 3 to PAD District 2 and also from PAD District 2 to PAD Disirict 1.

Waterborne movements of crude oil and petroleum products between PAD Districts include all shipments of crude oil or petroleum products for which the transporter has custody at the time of shipment. Custody is defined as physical possession of crude oil or petroleum products on a company-owned tanker and barge.

\section{Note 4. Domestic Crude Oil Production}

The Energy Information Administration (EIA) collects monthly crude oil production data on an ongoing basis. Data on crude oil production for States are reported to the EIA by State gavernment agencies. Data on crude oil production for Federal offshore areas are reported to the EIA by the Minerals Management Service of the U.S. Department of the Interior and the Conservation Committee of California Oil Producers.

Currently, all except four crude oil producing States (Michigan, New York, Ohio, and Pennsylvania) report production on a monthly basis. These four States report crude oil production on an annual basis. Estimates of monthly crude oil production for these four States are made by the EIA using data reported on Form EIA-182, "Domestic Crude Oil First Purchase Report." After the end of each calendar year, the monthly crude oil production estimates are updated using annual reports from various State agencies, the Minerals Management Service, and the Conservation Committee of California Oil Producers. The final estimate is published in the Petroleum Supply Annual (PSA).

Table 26 of this publication provides estimates of crude oil production in the latest month for which most State production data are available. There is a time lag of approximately 4 months between the end of the production month and the time when most monthly State crude oil production data become available.

In order to present more timely crude oil production estimates, the EIA prepares a weekly crude oil production estimate, which is used in the Weekly Petroleum Status Report. At the end of the production month, rhese weekly estimates are aggregated into an original estimate of monthly crude oil production. Approximately 45 days later, this original estimate is replaced by State-level interim estimates. The State-level interim estimates are based on: (a) data reported by the States (e.g., production data for Alaska are typically reported to the EI $\rho$ before the interim estimate is made); (b) first purchase data reported on Form EIA-182, "Domestic Crude Oil First Purchase Report;" (c) exponential or hyperbolic curve fitted projections based on recent State data; or (d) constant level projections based on the average production rate during a recent time period.

Table B1 is intended to provide further insight into the EIA's estimates of monthly U.S. crude oil production. It shows: (a) how the aggregate of reported State data evolves over a period of 18 months; (b) the number of prodicing States that have not reported production for a given month within that period; and (c) various EIA estimates of monthly crude oil production within that period:

- The original estimate is a monthly aggregate of the weekly crude oil production estimates published in the Weekly Petroleum Status Report. This original monthly estimate is used in the Petroleum Supply Monthly (PSM) Tables S1 and S2 until replaced by the interim estimate.

- The interim estimate is used in the PSM Tables 1 through 25, and in Tables S1 and S2 until replaced by the final estimate.

- The initial estimate based upon first purchase data collected on the Form EIA-182 is used as an estimation 
Table B1. U.S. Crude Oila Production Estimates and Reported States ${ }^{b}$ Data by Month (Thousand Barrels per Day)

\begin{tabular}{|c|c|c|c|c|c|c|c|c|c|c|c|c|c|c|c|c|c|c|}
\hline \multirow{2}{*}{$\begin{array}{l}\text { Date of Data } \\
\text { Avallability }\end{array}$} & \multicolumn{18}{|c|}{ Mcnth of Production } \\
\hline & $11-91$ & $12-91$ & 1.92 & 2.92 & 3-92 & $4-92$ & 5.92 & 6.92 & 7.92 & 8.92 & 9.92 & $10-92$ & 11.92 & $12-92$ & 1.93 & $2-93$ & 3.93 & $4-93$ \\
\hline \multicolumn{19}{|c|}{ Reported State Data ${ }^{c}$} \\
\hline $1-14-92$ & 1786 & 0 & & & & & & & & & & & & & & & & \\
\hline $2-14-92$ & 2589 & 1788 & 0 & & & & & & & & & & & & & & & \\
\hline $3-14-92$ & 4892 & 2674 & 1854 & 0 & & & & & & & & & & & & & & \\
\hline 4-14-92 & 6898 & 6766 & 2634 & 1875 & 0 & & & & & & & & & & & & & \\
\hline $5-14-92$ & 7331 & 7277 & 4860 & 2434 & 1849 & 0 & & & & & & & & & & & & \\
\hline $6 \cdot 14-92$ & 7368 & 7320 & 6767 & 4898 & 2665 & 1828 & 0 & & & & & & & & & & & \\
\hline $7-14-92$ & 7371 & 7324 & 7226 & 6834 & 4073 & 2566 & 1749 & 0 & & & & & & & & & & \\
\hline $8-14-92$ & 7371 & 7325 & 7231 & 7242 & 6760 & 4046 & 2538 & 1773 & 0 & & & & & & & & & \\
\hline $9-14-92$ & 7370 & 7324 & 7240 & 7260 & 7206 & 6688 & 4738 & 2680 & 1740 & 0 & & & & & & & & \\
\hline $10-14-92$ & 7372 & 7326 & 7243 & 7266 & 7224 & 7152 & 6638 & 4767 & 2572 & 1652 & 0 & & & & & & & \\
\hline $11-14-92$ & 7374 & 7328 & 7244 & 7267 & 7225 & 7166 & 7046 & 6954 & 6520 & 2465 & 1765 & 0 & & & & & & \\
\hline $12-14-92$ & 7375 & 7329 & 7244 & 7271 & 7232 & 7173 & 7054 & 7050 & 7005 & 4959 & 2562 & 1696 & 0 & & & & & \\
\hline $1-14-93$ & 7374 & 7328 & 7244 & 7272 & 7233 & 7174 & 7055 & 7055 & 7006 & 6726 & 6416 & 2514 & 1743 & 0 & & & & \\
\hline $2-14-93$ & 7374 & 7328 & 7245 & 7272 & 7233 & 7174 & 7056 & 7054 & 7009 & 6816 & 6810 & 5644 & 4121 & 1725 & 0 & & & \\
\hline $3-14-93$ & 7374 & 7328 & 7244 & 7272 & 7233 & 7174 & 7057 & 7053 & 7019 & 6817 & 6822 & 6482 & 5636 & 4290 & 1717 & 0 & & \\
\hline $4-14-93$ & 7374 & 7328 & 7254 & 7282 & 7242 & 7185 & 7068 & 7065 & 7031 & 6828 & 6922 & 6917 & 6820 & 6873 & 4048 & 1700 & 0 & \\
\hline $5-14-93$ & 7374 & 7328 & 7256 & 7283 & 7245 & 7188 & 7069 & 7067 & 7032 & 6822 & 6924 & 6997 & 6829 & 6877 & 5549 & 4119 & 1738 & 0 \\
\hline
\end{tabular}

\begin{tabular}{|c|c|c|c|c|c|c|c|c|c|c|c|c|c|c|c|c|c|c|}
\hline \multirow[b]{2}{*}{$5-14-93$} & \multicolumn{18}{|c|}{ Producing States Without Reported Monthly Production } \\
\hline & 0 & 0 & 5 & 5 & 5 & 5 & 5 & 5 & 5 & 5 & 5 & 5 & 6 & 7 & 11 & 19 & 29 & 33 \\
\hline & \multicolumn{18}{|c|}{ Month of Production } \\
\hline & 11.91 & 12.91 & $1-92$ & $2-92$ & $3-92$ & $4-92$ & $5-92$ & $6-92$ & $7-92$ & $8-92$ & 9.92 & $10-92$ & 11.92 & $212-92$ & $1-93$ & $2-93$ & 3.93 & 4.93 \\
\hline
\end{tabular}

\begin{tabular}{|c|c|c|c|c|c|c|c|c|c|c|c|c|c|c|c|c|c|c|}
\hline Original $^{\ominus} \ldots \ldots \ldots$ & 7302 & 7270 & 7344 & 7360 & 7324 & 7279 & 7212 & 7217 & 7114 & 6958 & 6996 & 7031 & 7014 & 7078 & 7023 & 7001 & 6980 & 6904 \\
\hline Interim ${ }^{1}$.............. & 7307 & 7281 & 7363 & 7373 & 7315 & 7291 & 7110 & 7138 & 7096 & 6928 & 7019 & 7065 & 702.7 & 7125 & 7008 & 6957 & 6976 & \\
\hline \multicolumn{18}{|l|}{ Form EIA-182 } & \\
\hline Initial ............. & 7119 & 7357 & 7171 & 7219 & 7168 & 7161 & 6878 & 6981 & 6945 & 6748 & 6836 & 6942 & 6908 & 6925 & 7001 & 6732 & 6962 & \\
\hline Revised........ & $724 \hat{c}$ & 7156 & 7176 & 7231 & 7065 & 7160 & 6918 & 6981 & 6936 & 6753 & 6862 & 6958 & 6887 & 6951 & 6775 & 6812 & & \\
\hline Final ${ }^{9}$. & 7328 & 7299 & 7361 & 7389 & 7348 & 7293 & 7169 & 7167 & 7131 & 6922 & 7030 & 7126 & 7024 & 7103 & & & & \\
\hline
\end{tabular}

\footnotetext{
a Includes lease condensate.

b Includes Federal offshore areas, Gulf of Mexico (PADD III) and Pacific (PADD V), as two separate reporting entities.

c Includes EIA prorated monthly production in 1991 (annual average of 83 thousand barrels per day) for four States (Michigan, New York, Ohio, and Pennsylvania) for which only annual State data are available.

'Michigan, New York, Ohio, and Pennsyivania are counted as having monthly reported data in 1991 after their annual reports were received. These data are first reported as of 4-14-92.

O Original estimates are weighted averages based on the weekly estimates published in the Weekly Petroleum Status Report.

1 Interim estimates were made 44 days after the end of the production month.

9 Published in the Petroleum Supply Annual 1991, DOE/EIA 0340(91)/2.
} 
tool in generating the interim estimate. The initial volume represents the best estimate available 40 days after the end of the production month and includes imputation for nonresponse and possible reporting errors. The revised volume is the best estimate available about 70 days after the production month and includes imputation as needed. A final revision is published concurrent with publication of Form EIA-182 price data in the Petroleum Marketing Annual.

- The final estimate is published in the PSA.

\section{Note 5. Export Data}

Each month the Energy Information Administration (EIA) receives magnetic tapes of aggregated export statistics from the U.S. Bureau of the Census (EM-522 and EM594).

Census export statistics used in the Petroleum Supply Monthly reflect both government and nongovernment exports of domestic and foreign merchandise from the United States (the 50 States and the District of Columbia) to foreign countries and U.S. possessions, without regard to whether or not the exportation involves a commercial transaction. The following types of transactions are excluded from the statistics:

(1) Merchandise shipped in transit through the United States from one foreign country to another, when documented as such with U.S. Customs.

(2) Bunker fuels and other supplies and equipment for use on departing vessels, planes, or other carriers engaged in foreign trade.

\section{Source of Export Information}

The official U.S. export statistics are compiled by the U.S. Bureau of the Census. Exporters are required to file export documents with U.S. Customs officials (Customs Form 7525).

\section{Country and Area of Destination}

The country of destination is defined as the country of ultimate destination or the country where the goods are to be consumed, further processed, or manufactured, as known to the shipper at the time of exportation. If the shipper does not know the country of ultimate destination, the shipment is credited to the last country to which the shipper knows that the merchandise will be shipped in the same form as it was when exported.

\section{Note 6. Quality Control and Data Revision}

\section{Quality Control}

The Energy Information Administration (EIA) monitors the supply and disposition of crude oil, petroleum products, and natural gas liquids in the United States. Through a tracking system, the EIA provides insight into the activities of primary operators and distributors in the petroleum industry. The tracking system, known as the Petroleum Supply Reporting System (PSRS), consists of production, inputs, imports, inventories, mov :ments, and other petroleum-related data collected on weekly, month$l y$, and annual surveys.

Survey forms are periodically reviewed for completeness, meaningfulness, and clarity. Modifications are made, when needed, to maintain efficient measure of the intended data items and to track product movement accurately throughout the industry. Through this process, the EIA can maintain consistency among forms, minimize respondent burden, and ekiminate ambiguity.

In any survey, nonresponse can be a major concern because the effects can cause serious bias in survey results. Nonresponse occurs whenever requested information is not obtained from all units in a survey. The PSRS surveys have a very high response rate. In general, response rates average above 95 percent for the weekly survey and above 98 percent for monthly surveys. Whecever survey responses are not received in time to be included in published statistics, the data are imputed. Although imputing for missing data may not eliminate the total error associated with nonresponse, it can serve to reduce the error. The data reported in the previous month are used as imputed values for missing data for all surveys except the Forms EIA-814, "Monthly Imports Report," and EIA-817, "Monthly Tanker and Barge Movement Report." There is no imputation procedure for these surveys because these data series, by respondent, are highly variable.

Response error is the major factor affecting the accuracy of PSRS data. Response, or reporting error, is the difference between the true value and the value reported on a survey form. Response error can occur for any number of reasons. For example, figures may be entered incorrectly when written on forms by the respondent, or errors may result from the misunderstanding of survey form instructions or definitions. Response error can also occur from the use of preliminary data when final data are not available. This can result in differences between published preliminary and final data. To help detect and minimize probable reporting errors, automated editing procedures are used to check current data for consistency with past data, as well as for internal consistency (e.g., totals equal 
to the sums of the parts), and to flag those data elements that fail edit criteria.

Errors can also be introduced during data processing. For example, while creating computer data files, key errors can occur in transcribing or coding the data; or information can be entered into the wrong cell. Using well designed edit criteria which examine orders of magnitude, cell position, and historical reporting patterns, many of these errors can be identified and corrected.

A principal objective of PSRS surveys is to provide a timely and accurate picture of petroleum industry activities. As part of this objective, a comparison of the data collected on the PSRS with other similar data series from sources outside of the Petroleum Supply Division is performed each year. The results of this data comparison are published in the Petroleum Supply Monthly (PSM) feature article, "Comparison of Independent Statistics on Petroleum Supply" and in subsequent explanatory notes.

The $819 \mathrm{M}$ data, which are based on sample estimates, serve as leading indicators of the PSRS monthly data for oxygenates. To assess the accuracy of the $819 \mathrm{M}$ statistics, data are compared with the monthly aggregate data for the EIA-810, 811, and 812 surveys. Although monthly data are still subject to error, they have been thoroughly reviewed and edited, and are considered to be the most accurate data available.

\section{Resubmlssions}

Resubmissions are any changes to the originally submitted data that were either requested by the EIA or initiated by the respondent. Resubmissions are compared with the original submission and processed at the time of receipt. For Forms EIA- 810 through 813,816 , and 817 the Resubmission Tracking System (RTS) is run after resubmissions have been processed for the month. The RTS enables the user to study major products and data series to see how company resubmissions impact published data on a month by month basis. During the processing year, a summary of the effect of these resubmissions to major series is provided in Appendix C.

For the EIA-819M data, a determination is made on whether to process the resubmissions based on the magnitude of the revision. Cell entries on publication tables are marked with an " $\mathrm{R}$ " for revised.

\section{Late Response}

Respondents who fail to respond within the prescribed time limit (25th day following the end of the report month) become nonrespondents for that particular report period and are contacted by phone to obtain the current month's data. Respondents who are chronically late (i.e.,
3 consecutive months) are notified by EIA either by letter or telephone.

\section{Nonresponse}

Follow-up action is taken when a company fails to respond adequately to data requests from the EIA. Preliminary attempts to gather delinquent reports are made by phone. Noncompliance form letters are sent to those companies that have not submitted reports and have not responded to data requests by phone.

\section{Note 7. Frames Maintenance}

The Petroleum Supply Division (PSD) maintains complete lists of respondents to its monthly surveys. Each survey has a list of companies and facilities required to submit petroleum activity data. This list is known as the survey frame. Frame maintenance procedures are used to monitor the status of petroleum companies and facilities currently contained in each survey frame as well as to identify new members to be added to the frame. As a result, all known petroleum supply organizations falling within the definition of "Who Must Submit" participate in the survey.

The activities for frames maintenance are conducted within three time frames: monthly, annually, and triennially. Monthly frames maintenance procedures focus on examining several frequently published industry periodicals that report changes in status (births, deaths, sales, and acquisitions) of petroleum facilities producing, transporting, importing, and/or storing crude oil and petroleum products. These sources are augmented by articles in newspapers, letters from respondents indicating changes in status, and information received from survey systems operated by other offices. Survey managers review these sources to monitor changes in company operations and to develop lists of potential respondents. These activities assure coverage of the reporting universe and maintain accurate facility information on addresses and ownership.

The sample frame for the Form EIA-819M, "Monthly Oxygenate Telephone Report," is also updated on a monthly basis as needed.

Annual frames maintenance focuses on re-evaluating the "must submit" companies filing the Form EIA-814. In addition, the PSD conducts a comprehensive frame identifier survey of companies that produce, blend, store, or import oxygenates. This information is used in determining which companies are eligible respondents to the Monthly Petroleum Supply Reporting System surveys.

To supplement monthly and annual frames maintenance activities and to provide more thorough coverage, the 
Table B2. New Basis Stocks ${ }^{1}$

\begin{tabular}{|c|c|c|c|}
\hline Commodity & 1980 & 1982 & 1983 \\
\hline \multicolumn{4}{|l|}{ Crude Oil } \\
\hline Total . & 488 & 645 & -. \\
\hline Other Primary & 380 & 351 & -. \\
\hline Crude Oil and & & & \\
\hline $\begin{array}{l}\text { Petroleum Products .. } \\
\text { Motor Gasoline }\end{array}$ & 1,425 & 1,461 & -- \\
\hline Total $\ldots \ldots \ldots \ldots$ & 263 & 244 & -. \\
\hline Finished ... & 214 & 202 & -. \\
\hline Distillate Fuel Oil . . . . . . . & 205 & 186 & -. \\
\hline $\begin{array}{l}\text { Residual Fuel Oil ....... } \\
\text { Jet Fuel }\end{array}$ & 91 & 69 & - \\
\hline Total & 42 & 39 & -- \\
\hline Kerosene-type ....... & 36 & 32 & $\cdots$ \\
\hline Liquefied & & & \\
\hline Petroleum Gases .... & 128 & 102 & 108 \\
\hline $\begin{array}{l}\text { Propane/Propylene ..... } \\
\text { Other Petroleum }\end{array}$ & 69 & 57 & 55 \\
\hline Products .......... & 207 & 219 & 210 \\
\hline
\end{tabular}

${ }^{1}$ Stocks as of December 31.

PSD also conducts a comprehensive triennial frames investigation. These triennial evaluations result in the reassessment and recompilation of the complete frame for each survey.

\section{Changes in Survey Frames}

Beginning in January 1981, the Energy Information Administration (EIA) expanded its universe to include nonrefinery blenders; redefined motor gasoline into two categories (finished leaded and finished unleaded); and separated blending components from finished motor gasoline as a reporting category. Refer to Explanatory Note 8 for future discussion.

In January 1981, 1983, and 1984 numerous respondents were added to bulk terminal and pipeline surveys affect- ing subsequent stocks reported and stock change calculations. Table B2 displays the end-of-year stocks, in million barrels using the expanded coverage (new basis).

Beginning in January 1986, as a result of frames maintenance activities, 39 respondents were added to the monthly survey frames: 2 motor gasoline blenders, 30 bulk terminal operators, 3 pipeline operators, 3 crude oil stock holders, and 1 tanker and barge operator. Table B3 shows the impact of the data reported by the new respondents on published data for production and stocks of major petroleum products.

Also, beginning in January 1986, a major petroleum company consolidated production and stocks reporting for some of its facilities. Data previously reported separately on Form EIA-811, "Monthly Bulk Terminal Report," and on Form EIA-816, "Monthly Natural Gas Liquids Report" for two facilities were combined with data reported for two refineries on Form EIA-810, "Monthly Refinery Report." The primary impact of this reporting change is on Table 24, "Stocks of Crude Oil and Petroleum Products by PAD District," which showed a decrease in natural gas liquids (NGL) stocks at bulk terminals and natural gas processing plants, and an increase in NGL stocks at refineries.

Beginning no later than November 1992, the Clean Air Act Amendments of 1990 require that all gasoline sold in carbon monoxide nonattainment areas have an oxygen content of 2.7 percent (by weight) during winter months. Beginning in 1995 further requirements are that only reformulated gasoline having an average oxygen content of 2.0 percent be sold in the nine worst ozone nonattainment areas.

In 1991, the ElA conducted a frame identifier survey of companies that produce, blend, store, or import

Table B3. Impact of New Respondents to December 1985 PSM Data

\begin{tabular}{|c|c|c|c|c|}
\hline \multirow[b]{2}{*}{ Product } & \multicolumn{2}{|c|}{$\begin{array}{l}\text { Refinery Production } \\
\text { (thousand barrels per day) }\end{array}$} & \multicolumn{2}{|c|}{$\begin{array}{c}\text { Stocks }^{\mathrm{a}} \\
\text { (thousand barrels) }\end{array}$} \\
\hline & $\begin{array}{c}\text { Reported } \\
\text { by New } \\
\text { Respondents }\end{array}$ & $\begin{array}{l}\text { Published } \\
\text { U.S. Total }\end{array}$ & $\begin{array}{c}\text { Reported } \\
\text { by New } \\
\text { Respondents }\end{array}$ & $\begin{array}{l}\text { Published } \\
\text { U.S. Total }\end{array}$ \\
\hline Leaded Gasoline & 1.3 & 2,326 & 224 & 81,379 \\
\hline Unleaded Gasoline & 0.6 & 4,323 & 276 & 108,422 \\
\hline Distillate Fuel Oil & 0 & 3,174 & 1,217 & 143,911 \\
\hline Residual Fuel Oil & 0 & 1,055 & 1,747 & 50,671 \\
\hline NGLs \& LRGs & 0 & 393 & 409 & 80,898 \\
\hline Other Products & 0 & 3,302 & 1,413 & 239,158 \\
\hline Crude Oil (excl. SPR) & - & - & 2,314 & 318,695 \\
\hline
\end{tabular}

Stocks as of December 31, 1985. 
oxygenates. The purpose of this survey was to (1) identify all U.S. producers, blenders, storers, and importers of oxygenates; and (2) collect supply and blending data for 1990 and end of 1990 inventory data on those oxygenates blended into motor gasoline. A summary of the results from the identification survey were published in the Weekly Petroleum Status Report dated February 12, 1992 and in the February 1992 issue of the Petroleum Supply Monthly.

In order to continue to provide relevant information about U.S. and regional gasoline supply, the EIA conducted a second frame identifier survey of these companies during 1992. As a result, a number of respondents were added to the monthly surveys effective in January 1993: 19 blenders, 25 stock holders, and 8 importers. This change affected stocks reported and stock change calculations. However, a new basis stock level was not calculated.

\section{Sampling}

The sampling procedure used for the survey Form EIA$819 \mathrm{M}$ is the cut-off method and is performed using software developed by EIA's Office of Statistical Standards. In the cut-off method, companies are ranked from largest to smallest on the basis of quantities reported (oxygenate production, oxygenate stocks, and oxygenate imports) during 1992. Companies are chosen for the sample beginning with the largest and adding companies until the total sample covers approximately 90 percent of the total for each oxygenate item and supply type by geographic region (PAD Districts I through V) for which data may be published.

\section{Note 8. 1981 Changes in the Petroleum Supply Reporting System}

Petroleum statistics contained in this report for all years through 1980 were developed using definitions, concepts, reporting procedures, and aggregation methods that are consistent with those developed by the U.S. Bureau of Mines. Research conducted by the Energy Information Administration (EIA) in 1979 and 1980 indicated that changes had occurred in the petroleum industry that were not being adequately reflected in EIA's reporting system.

The EIA reporting forms, definitions, and procedures were modified beginning in January 1981 to describe industry operations more accurately. Unfortunately, empirical information is not available to precisely measure the data shortcomings through 1980 . Estimates of the magnitudes of differences in the major data series are described below to form a basis for comparing 1979, 1980 , and 1981 data.

\section{Motor Gasoline}

Prior to 1979, the ElA product-supplied series for motor gasoline was consistently about 2 percent lower than the Federal Highway Administration (FHWA) gasoline sales data series, which is derived from State tax receipts. The difference increased to about 3 percent in 1979 and 1980. There were two primary causes for this growing difference. First, refinery operations, particularly the flows of unfinished oils and the redesignation of some finished products, were not being accurately described on the EIA survey forms. Second, a large amount of gasoline was being produced away from refineries at "downstream blending stations" to take advantage of provisions in regulations governing the amount of lead that could be added. These blending stations were not reporting gasoline production to the EIA until the data system was changed in January 1981.

Quantitative estimates of the magnitude of the difference in EIA's gasoline product supplied data in 1979 and 1980 have been made by the EIA and the American Petroleum Institute (API). Table B4 provides 1979 and 1980 data as published in the Petroleum Statement, Annual, as well as EIA and API estimates of "recast" motor gasoline product supplied.

The EIA recast estimates were based upon preliminary monthly information in the Monthly Petroleum Statement. The ranges displayed in the EIA column reflect uncertainty in the estimates. Also shown are the FHWA motor gasoline sales statistics for those years.

Table B4. Finished Motor Gasoline Product Supplied (Thousand Barrels per Day)

\begin{tabular}{lllll}
\hline & $\begin{array}{c}\text { EIA } \\
\text { Reported }\end{array}$ & $\begin{array}{c}\text { API } \\
\text { Recast }\end{array}$ & $\begin{array}{c}\text { ElA } \\
\text { Recast }\end{array}$ & FHWA $^{\text {a }}$ \\
\hline $1979 \ldots \ldots$ & 7,034 & 7,302 & $7,183-7,347$ & 7,258 \\
$1980 \ldots \ldots$ & 6,579 & 6,882 & $6,806-6,889$ & 6,792 \\
\hline
\end{tabular}

FHWA gasoline statistics based on data from Federal Highway Administration. Estimate of Total Gasoline Use, Table MF-21A published October 1980 and September 1981. Aviation gasoline (Table MF-24) has been subtracted from FHWA product supplied quantities to make data comparable.

\section{Distillate and Residual Fuel Oil}

Distillate and residual fuel oil refinery production statistics through 1980 were adjusted to account for an imbalance between unfinished oil supply and disposition. The reported quantities of refinery inputs of unfinished oils typically exceed the available supply of unfinished oils. It has been assumed that this occurs when distillate 
and residual fuel oils produced by a refinery are shipped to another refinery, where it is treated as unfinished oil. This oil is then reprocessed rather than used or sold as distillate or residual fuel oil.

For many years (including 1980), the difference between unfinished oil disposition and supply was subtracted from distillate and residual fuel oil production to adjust for this discrepancy. Two-thirds of the difference was applied to distillate fuel oil, and one-third to residual fuel oil.

Beginning in January 1981 , this adjustment was discontinued because there was not sufficient empirical evidence to support it. Table B5 presents distillate and residual fucl oil refinery production in 1979 and 1980 as published (adjusted) and on the same basis as 1981 statistics (unadjusted) to permit comparison.

Adjusted distillate and residual fuel oil product supplied volumes differ from the unadjusted volumes by the same amounts as the adjusted and unadjusted production volumes.

Table B5. Distillate and Residual Fuel Oil Production and Product Supplied (Thousand Barrels per Day)

\begin{tabular}{|c|c|c|c|c|}
\hline & $\begin{array}{l}\text { Adjusted } \\
\text { Refinery } \\
\text { Production }\end{array}$ & $\begin{array}{l}\text { Unadjusted } \\
\text { Refinery } \\
\text { Production }\end{array}$ & Difference & $\begin{array}{c}\text { Unadjusted } \\
\text { Product } \\
\text { Supplied }\end{array}$ \\
\hline $\begin{array}{l}\text { Distillate } \\
\text { Fuel Oil } \\
1979 \ldots \ldots \\
1980 \ldots \ldots\end{array}$ & $\begin{array}{l}3,152 \\
2,661\end{array}$ & $\begin{array}{l}3,169 \\
2,764\end{array}$ & $\begin{array}{r}16 \\
103\end{array}$ & $\begin{array}{l}3,327 \\
2,969\end{array}$ \\
\hline $\begin{array}{l}\text { Residual } \\
\text { Fuel Oil } \\
1979 \ldots \ldots \\
1980 \ldots \ldots\end{array}$ & $\begin{array}{l}1,687 \\
1,580\end{array}$ & $\begin{array}{l}1,695 \\
1,634\end{array}$ & $\begin{array}{r}8 \\
54\end{array}$ & $\begin{array}{l}2,834 \\
2,562\end{array}$ \\
\hline
\end{tabular}

\section{Total Petroleum Products}

The imbalance between the supply and disposition of unfinished oils and gasoline blending components is included with other products (line 35 ) in Table 1 . These imbalances are reported as negative product supplied in Table 2. Since these changes only involve redistribution of the volumes of finished motor gasoline, distillate and residual fuel oil, gasoline blending components, and unfinished oils, the total volume of petroleum products supplied remains unaffected by them.

\section{Alaskan In-Transit Stocks}

Stocks of Alaskan crude oil in-transit were included for the first time in January 1981. The major impact of this change is on the reporting of stock change calculations.
Using the expanded coverage (new basis), 1980 end-ofyear crude oil stocks would have been 488 million barrels for Total and 380 million barrels for Other Primary.

\section{Note 9. 1983 Changes in the Petroleum Supply Reporting System}

January 1983 marked the implementation of recent changes in the collection, processing and availability of the Energy Information Administration's (EIA) petroleum supply data. Survey forms and definitions were made consistent; frames for bulk terminals, petroleum product pipelines and crude oil stock holders were updated, and the survey processing system was redesigned and incorporated into the new Petroleum Supply Reporting System (PSRS).

\section{Changes in Data Collection}

Changes in data collection can be grouped into five categories. Some were made to improve consistency, others to classify activity more precisely, and others to combine or eliminate information elements or to reduce the frequency of reporting in recognition of the trade-off between data value and reporting burden. The changes are itemized below.

- Motor gasoline was divided into three standard categories (finished leaded motor gasoline, finished unleaded motor gasoline and motor gasoline blending components).

- Aviation gasoline blending components were added to Form EIA-817.

- Crude oil burned as fuel on leases and by pipelines is reported as a single item on Form EIA-813. Previously it was reported as distillate or residual fuel oil consumption.

- No. 4 Fuel Oil is now included with distillate fuel oil.

- Gasohol was eliminated as a separate category and is now reported as either "finished leaded motor gasoline" or "finished unleaded motor gasoline."

- Waterborne movements of petrochemical feedstocks are now divided into naphtha-less than 400 degrees end-point and other-oils equal to or greater than 400 degrees end-point on Form EIA-817.

- Data aggregation for Petroleum Administration for Defense District (PADD) I was divided into three subdistricts on Forms EIA-812 and 817. 
- Detailed categories of Gross Input to Crude Oil Distillation Units were eliminated, and only Total Gross Inputs is collected on Form EIA-810.

- Waterborne movements of crude oil and petroleum products between PADD's, on Form EIA-817, no longer reflect shipping and receiving States.

- Reporting of production and stocks of No. 4 Fuel Oil by sulfur levels were eliminated from Forms EIA-810, 811,812 , and 817 .

- Crude oil stocks are collected at PADD levels rather than State levels on Form EIA-813.

- Shipments from natural gas processing plants no longer reflect destination by facility type on Form EIA-816.

- The four categories for unfinished oils were reduced to two on Form EIA-810.

- The five categories for sulfur content of residual fuel oil were reduced to three on Forms EIA-810, 811, and 817.

- Normal Butane and Other Butanes were combined into a single category on Forms EIA-810, 811, and 816.

- Three subcategories of lubricating oils (bright stock, neutral, and other) were combined into a single category on the Form EIA-810.

- Three subcategories of waxes (microcrystalline, crystalline-fully refined, and crystalline-other) were combined into a single category on the Form EIA-810.

- Asphalt and Road Oil were combined into a single category on Forms EIA-810 and 811.

- Plant fuel use and Losses were combined on Form EIA-816.

- Natural Gasoline and Isopentane were combined on Form EIA-816.

\section{Change in Crude Oil Lease Stocks}

The end-of-month crude oil stocks held on leases are reported on the Form EIA-813, "Monthly Crude Oil Report." However, only those companies that store 1,000 barrels or more of crude oil are required to submit a report. Previous frames analysis has shown that crude oil stocks held on leases reported to the Energy Information Administration (EIA) are consistently lower than the lease stocks reported to individual states.
Up until 1983, monthly state government data on lease stocks were substituted for EIA data wherever possible in order to rectify the understatement of lease crude oil stocks. State date were available from three states -Texas, New Mexico, and Montana. To calculate the "lease adjustment", a comparison between the EIA reported data and the state government data was made and the difference added to the EIA data for respective states.

In 1983, the EIA modified the Form EIA-813 to eliminate state data on crude oil stocks and began collecting crude oil stock data by PAD District. With this change, the "lease adjustment" could no longer be calculated on a state basis and was changed to a PAD District level.

\section{Note 10. 1984 Changes in the Petroleum Supply Reporting System}

In January 1984, a number of changes in the reporting of natural gas liquids (NGL) were implemented. The modified system reflects supply and disposition of NGL on a component, rather than a product, basis.

From 1979 to 1983, the Energy Information Administration (EIA) collected and reported information on the supp$1 y$ and disposition of nine NGL products. Beginning with January 1984, NGL supply and disposition data were reported for 5 components to be consistent with record keeping practices used by the industry. Table B6 shows the product category under the new and old basis.

Table B6. Product Basis vs. Component Basis Reporting

\begin{tabular}{|c|c|c|c|c|c|}
\hline \multirow[b]{2}{*}{$\begin{array}{l}\text { 1979-1983 } \\
\text { Product Basis }\end{array}$} & \multicolumn{5}{|c|}{$\begin{array}{c}1984 \text { Component } \\
\text { Basis }\end{array}$} \\
\hline & 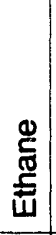 & 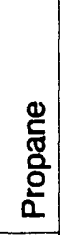 & 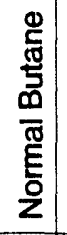 & 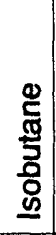 & 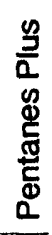 \\
\hline Ethane & - & & & & \\
\hline Ethane-Propane Mixtures & - & - & & & \\
\hline Propane & & - & & & \\
\hline Butane-Propane Mixtures & & - & - & & \\
\hline Butane & & & $\cdot$ & & \\
\hline Isobutane & & & & $\cdot$ & \\
\hline Unfractionated Stream & - & $\bullet$ & $\bullet$ & $\cdot$ & $\cdot$ \\
\hline Natural Gasoline and Isopentane & & & & & $\cdot$ \\
\hline Plant Condensate & & & & & - \\
\hline
\end{tabular}




\begin{tabular}{|c|c|c|c|c|c|}
\hline \multirow{2}{*}{ Product } & \multicolumn{5}{|c|}{ EIA Component Slate } \\
\hline & Ethane & Propane & Normal Butane & Isobutane & Pentanes Plus \\
\hline \multicolumn{6}{|l|}{ Import Product } \\
\hline Natural Gasoline and Isopentane (EIA-814) ... & - & - & - & - & 100 \\
\hline Plant Condensate (EIA-814) $\ldots \ldots \ldots \ldots$ & - & - & - & - & 100 \\
\hline Ethane $(I M-145) \ldots \ldots \ldots \ldots \ldots \ldots \ldots$ & 100 & - & $\ldots$ & - & - \\
\hline Propane $(I M-145) \ldots$ & - & 100 & - & - & - \\
\hline Butane (IM-145) & - & - & 65 & 35 & - \\
\hline Butane-Propane Mixtures (IM-145) $\ldots \ldots$ & - & 40 & 35 & 20 & 5 \\
\hline Ethane-Propane Mixtures (IM-145) $\ldots \ldots \ldots$ & 60 & 40 & - & - & - \\
\hline \multicolumn{6}{|l|}{ Export Product } \\
\hline Ethane (A\|l PAD Districts) & 100 & - & - & - & - \\
\hline Propane (All PAD Districts) $\ldots \ldots \ldots \ldots \ldots$ & - & 100 & - & - & - \\
\hline Butane (All PAD Districts) $\ldots \ldots \ldots \ldots \ldots$ & - & - & 100 & - & - \\
\hline \multicolumn{6}{|l|}{ Mixed Streams } \\
\hline PAD Districts I, IV, V & - & 40 & 60 & - & - \\
\hline PAD District $11 \ldots \ldots \ldots \ldots \ldots \ldots \ldots$ & 30 & 25 & 15 & 15 & 15 \\
\hline PAD District III $\ldots \ldots \ldots \ldots \ldots \ldots \ldots$ & - & 80 & 20 & - & - \\
\hline
\end{tabular}

Four Petroleum Supply Reporting System surveys were modified beginning in January 1984. They were:
EIA-810
ELA-811
EIA-812
ELA-816

\author{
"Monthly Refinery Report" \\ "Monthly Bulk Terminal Report" \\ "Monthly Product Pipeline Report" \\ "Monthly Natural Gas Liquids Report"
}

This change affected stocks reported and stock change calculations. Under the new basis, end-of-year 1983 stocks would have been 108 million barrels for Liquefied Petroleum Gases and 210 million barrels for Other Petroleum Products.

A fifth survey, Form EIA-814, "Monthly Imports Report" (formerly Form ERA-60), was not modified. Therefore, to allocate imports and exports of mixed NGL streams to individual component parts, the EIA developed a statistical algorithm.

\section{Imports}

The imports algorithm was based on information gathered from the larger importers of NGL, who were asked to provide component analysis of the products they imported during the first 6 months of 1983. The percentages shown in Table B7 are derived from the weighted averages of the data provided by the importers.

\section{Exports}

The exports algorithm was based on information gathered from the larger exporters of NGL, who were asked to provide component analysis of the products they exported during 1983. The percentages shown in Table B7 are derived from the weighted averages of the data provided by the exporters. It was necessary to derive percentages by Petroleum Administration for Defense District of exportation, due to the wide variation of components included in the mixed streams.

\section{Note 11. 1985 Changes in the Petroleum Supply Reporting System}

Beginning in January 1985, inter-Petroleum Administration for Defense (PAD) District pipeline movements of crude oil were included in the crude oil supply balance at the PAD District level but did not affect National level statistics. As a result of including these movements, Net Receipts of crude oil and Unaccounted for Crude Oil at the PAD District level changed significantly. Also affected were crude oil imports and unfinished oil imports at the PAD District level which are provided by PAD District of Entry (Tables 6-10) and by PAD District of Processing (Tables 16-19). 
The tables in the Petroleum Supply Monthly that were changed duc to the inclusion of inter-PAD District pipeline movements of crude oil are the following:

- Tables 6 through 10, "PAD Districts I to V, Supply and Disposition of Crude Oil and Petroleum Products."

- Effective January 1985, crude oil imports and unfinished oil imports in Tables 4 through 8 were reported at the PAD District of Entry rather than at the PAD District of Processing. Net Receipts now include movements by pipeline as well as by tanker and barge.

- Table 26, "Movements of Crude Oil and Petroleum Products by Pipeline, Tanker, and Barge Between PAD Districts."

- The crude oil line includes movements by pipeline as well as by tanker and barge.

- Table 27, "Movements of Crude Oil and Petroleum Products by Pipeline Between PAD Districts."

- A line was added to report crude oil movements.

- Table 29, "Net Movements of Crude Oil and Petroleum Products by Pipeline, Tanker, and Barge Between PAD Districts."

- The crude oil line includes movements by pipeline as well as by tanker and barge.

\section{Note 12. 1986 Changes in the Petroleum Supply Reporting System}

Beginning in January 1986, several changes to the Petroleum Supply Reporting System (PSRS) went into effect. These changes affected the frame of operators of petroleum facilities required to complete the monthly surveys in the PSRS and resulted in some changes to the tables presented in the Petroleum Supply Monthly (PSM). Refer to Explanatory Note 7 for detailed description of frames maintenance and updates.

\section{Changes in Data Collection}

- The unit of measure used on Form EIA-814, "Monthly Imports Report," was changed from barrels to thousands of barrels.

- Unfinished oil imports data, previously reported as one product on the Form EIA-814, were separated into the following four classifications.

- Naphthas and lighter
- Kerosene and light gas oils

- Heavy gas oils

- Residuum

- The number of categories for reporting natural gas li. quids and liquefied petroleum gases data on Form EIA. 814 was reduced from 19 to 5 by climinating the requirement to separately identify categories for further processing, petrochemical use, and fuel usc.

- The requirements to report the type of processing facility and the applicable section of the oil import regulations were eliminated for the Form ElA-814.

- The requirement to report data for imports of crude oil, unfinished oils, and finished products on separate schedules of the Form EIA-814 was eliminated.

- The requirement to report two end-use categories, petrochemical use and other use, for still gas and liquefied refinery gases, was eliminated on Form EIA810, "Monthly Refinery Report."

- Form EIA-815, "Monthly Shipments from Puerto Rico to the United States Report," was discontinued. The data previously reported on this form are now reported on Form EIA-814.

\section{Changes in Publication Tables}

Several changes were also made to tables in the PSM either as a direct result of changes in reporting requirements or to improve the usefulness of the publication. These changes were:

- Table 13, "Refinery Input of Crude Oil and Petroleum Products by PAD District."

- Alaskan crude oil receipts were shown separately.

- Table 14, "Refinery Production of Petrolcum Products by PAD District."

- The breakout between "petrochemical feedstock use" and "other use" were no longer shown separately for still gas or for liqueficd refinery gases.

- Tables 16 and 17, "Imports of Crude Oil and Petroleum Products by PAD District."

- Imports of unfinished oils were separated into four categories: naphthas and lighter, kerosene and light gas oils, heavy gas oils, and residuum.

- Tables 18 and 19, "Imports of Crude Oil and Pctroleum Products by Source." 
- Countries formerly included in the categories "Other Western Hemisphere" and "Other Eastern Hemisphere" were shown individually.

- Table 24, "Stocks of Crude Oil and Petroleum Products by PAD District."

- The breakout between "petrochemical feedstock use" and "other use" for each liquefied petroleum gas was eliminated.

\section{Note 13. 1987 Changes in the Petroleum Supply Reporting System}

Several changes to the Petroleum Supply Reporting System (PSRS) went into effect at the beginning of January 1987. These changes were made as part of the Energy Information Administration's (EIA's) continuing effort to provide pertinent, timely, and consistent energy information. These changes were subsequently reflected in the Petroleum Supply Annual.

\section{Changes In Data Collection}

Fresh feed input to catalytic cracking units, hydrocracking units, and cokers were added to the Form EIA-810, "Monthly Refinery Report."

\section{Changes in Publication Tables}

- The "Appalachian No. 2" Refining District was combined with the "Indiana, Illinois, Kentucky," Refining District. This affected Petroleum Supply Monthly (PSM) Tables 12 through 15, 24, 30, and 31 .

- Fresh feed inputs to catalytic cracking units, hydrocracking units, and cokers were added to Table 13, "Refinery Input of Crude Oil and Petroleum Products by PAD District."

\section{Clarification}

In 1986, several refineries and terminals in the United States applied for Foreign Trade Zone (FTZ) status and applications from three refineries were approved. Consequently, during 1986, some refineries with FTZ status were treated as if they were within the United States while the Hawaiian FTZ was considered outside.

Effective with the January 1987 data, all FTZ facilities located within the 50 United States are considered domestic entities and are included in PSM statistics. The principal differences in the PSM data series as a result of adding the Hawaiian FTZ was an approximate 1-percent increase in crude imports and a 3-percent decrease in product imports.

\section{Note 14. 1989 Changes in the Petroleum Supply Reporting System}

Several changes to the Petroleum Supply Reporting System (PSRS) went into effect at the beginning of January 1989. These changes were made to reduce respondent burden, to fulfill user requests for additional data, and to improve accuracy and consistency in reporting. To reflect these changes and to improve the usefulness of the Petroleum Supply Monthly (PSM) publication, the following changes were made in January 1989 and subsequently reflected in the Petroleum Supply Annual (PSA).

\section{Changes in Data Collection}

- Data on inputs and production of naphthenic and paraffinic lubricants were added to the Form EIA-810, "Monthly Refinery Report."

- Separate lines for the collection of inputs and production of olefins (ethylene, propylene, and butylene)were added to Form EIA-810, "Monthly Refinery Report."

- The collection of data on the movement of Liquefied Petroleum Gases (LPGs) and Liquefied Refinery Gases (LRGs) on a component basis were added to the Forms EIA-812, "Monthly Product Pipeline Report," and the EIA-817, "Monthly Tanker and Barge Movement Report."

- Bonded imports of jet fuel and fuel oils and imports of LPGs previously published from data provided by the U.S. Bureau of the Census were discontinued. Data are now published from the data reported on Form EIA814, "Monthly Imports Report."

- Exports of butane/propane and ethane/propane mixtures were split in a ratio of 60 percent for the butane and ethane portions and 40 percent for the propane portion.

- The reporting of products other than Natural Gas Liquids (NGLs) by natural gas processing plants was eliminated on Form EIA-816, "Monthly Natural Gas Liquids Report."

- Fractionators were required to report only end-ofmonth stocks of NGLs on Form EIA-816, "Monthly Natural Gas Liquids Report." 
Beginning with the January 1989 issue of the PSM, adjustments were being made to refinery inputs and product supplied of NGLs and refinery inputs of crude oil to account for refiner misreporting. Substantial volumes of NGLs are produced at natural gas processing plants in Alaska and injected into the crude oil moving in the Trans Alaska Pipeline System (TAPS). Refiners receiving any crude oil commingled with NGLs are instructed to report the NGL portion of that stream separately from the crude oil portion. This has not been done for Alaskan crude oil because refiners are unable to identify these volumes for accounting purposes. As a result, the NGL production in Alaska has been credited directly toward product supplied and also toward product supplied from refinery production when the refiner processes the crude oil-NGL mixture. In addition, the reporting of the commingled stream as crude oil by the refiner has overstated crude oil inputs and resulted in an increase in unaccounted for crude oil equal to the volume of NGL in the crude oil.

To offset this reporting error, an adjustment was made to refinery input in all Petroleum Administration for Defense (PAD) Districts receiving Alaskan crude oil. The adjustment reduces the crude oil inputs and increases the NGL inputs by an equal amount. Each PAD District adjustment is a portion of the known Alaskan NGL production that is proportional to the PAD District's share of Alaskan crude oil received at all refineries in the United States. The greatest impact occurs in PAD District $V$ for butane and pentanes plus.

The reporting problem began in 1987 and has grown as injections of NGLs into the TAPS have increased. Data for 1988 was revised to account for the adjustment in the PSA published in May 1989. Revisions for 1987 data are not planned.

\section{Changes in Publication Tables}

- Year-to-Date tables on Supply and Disposition by PAD District (Tables 7, 9, 11, 13, and 15) were added.

- "Stock Withdrawal" was renamed "Stock Change" and was moved from Supply to Disposition in Tables 2 through 15. A negative number indicates a decrease in stocks and a positive number indicates an increase in stocks.

- A jet fuel total line was added to Tables 2-15, 19, 20, $23,24,43-46$.

- PAD District Supply and Disposition tables (Tables 6 through 15) now display liquefied petroleum gases on a component basis.

- Tables showing net imports by country for the current month and year-to-date (Tables 39 and 40) were added.

- Table numbers were changed as a result of data additions and table reorganization. Table $\mathbf{B} 8$ is provided to show the old to new table numbers for the detailed statistics tables.

- Table 17, "Natural Gas Plant Net Production and Stocks of Petroleum Products by PAD and Refining District."

- Stocks at natural gas processing plants by Refining District previously published in Table 24 was included with net production of petroleum products at natural gas plants.

- The reporting of products other than natural gas liquids by natural gas processing plants was eliminated.

- Table 19, "Net Refinery Production of Finished Petroleum Products by PAD and Refining District."

Table B8. Conversion Table for 1989 PSM

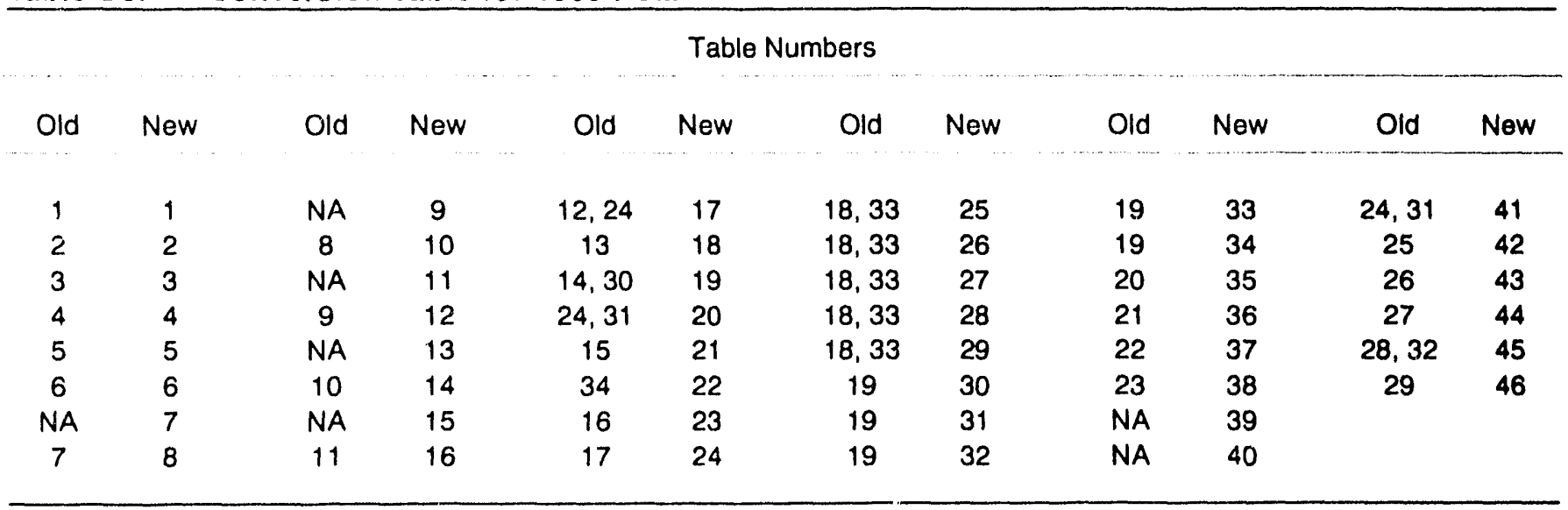

NA $=$ Not Applicable 
- Net production of olefins (ethylene, propylene, and butylene) was added.

- Net production of naphthenic and paraffinic lubricants was added.

- Net production of residual fuel oil by percent sulfur, previously published as Table 30, was added.

- Table 20, "Refinery Stocks of Crude Oil and Petroleum Products by PAD and Refining District."

- Stocks at refineries by Refining District were added from Table 24.

- Stocks of residual fuel oil by percent sulfur content, previously published as Table 31 , were added.

- Tables 25 through 34, "Imports of Crude Oil and Petroleum Products by Country of Origin."

- Data previously included in the "Other Products" category were displayed separately for naphthas for petrochemical feedstock use, other oils for petrochemical feedstock use, lubricants, and asphalt and road oil.

- Sulfur content categories for residual fuel oil, previously published as Table 33, were added.

- Tables 37 and 38, "Exports of Crude Oil and Petroleum Products by Destination."

- Data for exports by destination previously included in the "Other Products" category were displayed separately for pentanes plus, kerosene, naphthas for petrochemical feedstock use, and other oils for petrochemical feedstock use.

- Table 41, "Stocks of Crude Oil and Petroleum Products by PAD District."

- Refining District data were eliminated. Refinery stocks and natural gas processing plant stocks by Refining District were added to Tables 17 and 20, respectively.

- Sulfur content categories for residual fuel oil, previously published as Table 31 , were added.

\section{Note 15. 1990 Changes in the Petroleum Supply Reporting System}

Beginning with the May 1990 issue of the Petroleum Supply Monthly, stocks of propane/propylene were added to Table 42, "Refinery, Bulk Terminal, and Natural Gas Plant Stocks of Selected Petroleum Products by State." This change was subsequently reflected in the Petroleum Supply Annual.

\section{Note 16. 1991 Changes in the Petroleum Supply Reporting System}

Several changes were made to the Petroleum Supply Reporting System effective with the March issue of the Petroleum Supply Monthly (PSM). These changes were made to provide additional data and to improve the usefulness of the publication.

\section{Changes in Publication Tables}

\section{Summary StatisticsTables}

- A new table was added to show jet fuel supply and disposition.

- Table S8, "Other Petroleum Products Supply and Disposition" was redesignated as Table S9. Jet fuel data is no longer included. Historical data were revised to exclude jet fuel.

- Table S3, "Crude Oil and Petroleum Product Imports" was expanded to display all Organization of Petroleum Exporting Countries (OPEC) and additional NonOPEC countries. A separate column for crude oil imports was also been added for each country.

- Time periods were included in table titles.

\section{Figures}

- Annual graphs were eliminated.

- Time periods were included in figure titles.

- Sources were provided for each figure.

- Bar graphs used to display end-of-month stocks were replaced with line graphs.

\section{Sources}

The sources and explanatory notes for this section were updated and relocated to the end of the Summary Statistics section.

\section{Detailed Statistics Tables}

- Table 1, "U.S. Petroleum Balance" 
Table B9. Conversion Table for 1991 PSM

\begin{tabular}{|c|c|c|c|c|c|c|c|c|c|c|c|}
\hline \multirow[b]{2}{*}{ Old } & \multirow[b]{2}{*}{ New } & \multicolumn{5}{|c|}{ Table Numbers } & \multirow[b]{2}{*}{ New } & \multirow[b]{2}{*}{ Old } & \multirow[b]{2}{*}{ New } & \multirow[b]{2}{*}{ Old } & \multirow[b]{2}{*}{ New } \\
\hline & & Old & New & Old & New & Old & & & & & \\
\hline 1 & 1 & $N$ & 11 & 13 & 21 & 21 & 31 & 31 & 41 & 41 & 51 \\
\hline 2 & 2 & $N$ & 12 & 14 & 22 & 22 & 32 & 32 & 42 & 42 & 52 \\
\hline 3 & 3 & 9 & 13 & $N$ & 23 & 23 & 33 & 33 & 43 & 43 & 53 \\
\hline 4 & 4 & 10 & 14 & $N$ & 24 & 24 & 34 & 34 & 44 & 44 & 54 \\
\hline 5 & 5 & $N$ & 15 & 15 & 25 & 25 & 35 & 35 & 45 & 45 & 55 \\
\hline 6 & 6 & $N$ & 16 & 16 & 26 & 26 & 36 & 36 & 46 & 46 & 56 \\
\hline$N$ & 7 & 11 & 17 & 17 & 27 & 27 & 37 & 37 & 47 & & \\
\hline$N$ & 8 & 12 & 18 & 18 & 28 & 28 & 38 & 38 & 48 & & \\
\hline 7 & 9 & $N$ & 19 & 19 & 29 & 29 & 39 & 39 & 49 & & \\
\hline 8 & 10 & $N$ & 20 & 20 & 30 & 30 & 40 & 40 & 50 & & \\
\hline
\end{tabular}

$\mathrm{N}=$ Now Table

- A line was added to display jet fuel as a separate category for Total Products Supplied and Total Stocks (lines 34 and 44, respectively).

- PAD District Supply and Disposition Tables

- A year-to-date table in thousand barrels and a current month table in thousand barrels per day were added for each PAD District.

- Imports of Crude Oil and Petroleum Products by PAD District

- Residual fuel oil sulfur categories were added.

- Imports of Crude Oil and Petroleum Products by Country of Origin

- Residual fuel oil sulfur categories by country of origin were eliminated. These categories are now reported on a PAD District basis.

- Separate daily average columns were added for crude oil and petroleum products.

- Table numbers were changed as a result of table additions. Table B9 is provided to show the old to new table numbers for the detailed statistics tables.

\section{Note 17. 1993 Changes in the Petroleum Supply Reporting System}

In keeping with the Department of Energy's (DOE's) mandated responsibilities, the Energy Information Administration (EIA) made several changes to the Petroleum Supply Reporting System (PSRS) effective in January
1993. These changes were designed to accommodate the revisions to the Clean Air Act of 1990, and to reflect current and upcoming changes in the petroleum industry. These changes will be subsequently reflected in the 1993 Petroleum Supply Annual.

\section{Changes In Data Collection}

- Motor gasoline categories have been revised to reflect the change in the type of fuels produced. The new categories are: reformulated gasoline, oxygenated gasoline (EPA approved), and other finished gasoline. These changes were made to Forms EIA-810, "Monthly Refinery Report," EIA-811, "Monthly Bulk Terminal Report, "EIA-812, "Monthly Product Pipeline Report," EIA-814, "Monthly Imports Report," and EIA-817, "Monthly Tanker and Barge Movement Report."

- Distillate Fuel Oil has been split into two sulfur categories to meet Environmental Protection Agency requirements effective in October 1993. The new categories for inputs, production, end-of-month stocks and movements are: $0.05 \%$ sulfur and under, and greater than $0.05 \%$ sulfur. These changes were made to Forms EIA-810, "Monthly Refinery Report," EIA-811, "Monthly Bulk Terminal Report," EIA-812, "Monthly Product Pipeline Report," EIA-814, "Monthly Imports Report," and EIA-817, "Monthly Tanker and Barge Movement Report."

- Other hydrocarbons, hydrogen, and alcohol (Code 090) has been renamed "Other hydrocarbons, hydrogen, and oxygenates" on Form EIA-810, "Monthly Refinery Report." A new line has also been added to report Other hydrocarbons and hydrogen separately.

- Data on inputs and end-of-month stocks of oxygenates (i.e., fuel ethanol, ethyl tertiary butyl ether (ETBE), 
methanol, methyl tertiary butyl ether (MTBE), tertiary amyl methyl ether (TAME), tertiary bityl alcohol (TBA), and other oxygenates) has been added to Form EIA-810, "Monthly Refinery Report."

- Inputs and production of Isobucylene (Code 634) has been added as sub-categories to Isobutane (Code 615) on Form EIA-810, "Monthly Refinery Report."

- Data on inputs and production of military kerosenetype jet fuel and commercial $k$ _rosene-type jet fuel has been added to Form EIA-810, "Monthly Refinery Report."

- Liquefied Petroleum and hefinery Gases column headings for Ethane, Propane, Normal Butane, and Isobutane have been revised to include olefins (e.g., Ethane/Ethylene etc.) on Form EIA-811, "Monthly Bulk Terminal Report."

- Data on end-of-month stocks of oxygenates, (i.e., fuel ethanol, ethyl tertiary butyl ether (ETBE), methyl tertiary butyl ether (MTBE), tertiary amyl methyl ether (TAME), tertiary buiyl alcohol (TBA), and other oxygensies) have been added to Forms EIA-811, "Monthly Bulk Terminal Report," and EIA-812, "Monthly Product Pipeline Report." Data for methanol are not collected at this time but has been included on the form for future use.

- Imports of oxygenates (i.e., fuel ethanol, ethyl tertiary butyl ether (ETBE), methyl tertiary butyl ether (MTBE), tertiary amyl methyl ether (TAMF), tertiary butyl alcohol (TBA), and other oxygenates) have been added to Form EIA 914, "Monthly Imports Report." Data for methanol are not requested at this time.

- Imports of olefins are collected separately from liquefied petroleum gases (i.e., ethylene, propylene, butylene, ind isobutylene) on Form EIA-814, "Monthly Imports Report."

- Data on blended into motor gasoline has been eliminated on the Form EIA-819M, "Monthly Oxygenate Telephone Report."

- Data on methanol is no longer required on the Form EIA-819M, "Monthly Oxygenate Telephone Report" but remains on the form for future use.

\section{Charges in Summary Statistics Tables}

- Table S1. Crude and Petroleum Products Overview

- History data for 1973 through $₫ 780$ has been dropped. The table title has been changed to reflect the change in time series.
- Table S2. Crude Oil Supply and Disposition

- History data for 1973 through 1980 has been dropped. The table title has been changed to reflect the change in time series.

- The Crude Used Directly column has been eliminated. This coluri $n$ is no longer applicable since the years 1973 through 1980 have been eliminated. The data for 1981 and 1982 are provided in a footnote.

- Table S3. Crude Oil and Petroleum Product Imports

- History data for 1973 through 1980 has been dropped. The table title has been changed to reflect the change in time series.

- The Former USSR has been renamed Russia. The remaining states that comprised the Former USSR have been included in the Other Non-OPEC column.

- Table S4. Finished Motor Gasoline Supply and Disposition

- History data for 1973 through 1980 has been dropped. The table title has been changed to reflect the change in time series.

- Product supplied-unleaded and product supplied-unleaded (percent of Total) columns have been eliminated. A new column has been added to display end-of-month stocks of oxygenates. These stocks are not included in the Total Motor Gasoline end-ofmonth stocks.

- Table S5. Distillate Fuel Oil Supply and Disposition

- History data for 1973 through 1980 has been dropped. The table title has been changed to reflect the change in time series.

- Distillate fuel oil siocks have been separated into two sulfur categories $(0.05 \%$ sulfur and under and greater than $0.05 \%$ sulfur).

- The Crude Used Directly column has been eliminated. This column is no longer applicable since the years 1973 through 1980 have been eliminated. The data for 1981 and 1982 are provided in a footnote.

- Table S6. Residual Fuel Oil Supply and Disposition

- History data for 1973 through 1980 has been dropped. The table title has been changed to reflect the change in time series. 
- The Crude Used Directly column has been eliminated. This column is no longer applicable since the years 1973 through 1980 have been eliminated. The data for 1981 and 1982 are provided in a footnote.

- Table S7. Jet Fuel Supply and Disposition

- History data for 1973 through 1980 has been dropped. The table title has been changed to reflect the change in time series.

- Table S8. Propane/Propylene Supply and Disporition

- A new summary table has been added to display supply and disposition data for propane/propylene. This information will continue to be included in the $\mathrm{Li}$ quefied Petroleum Gases Supply and Disposition table (renumbered as Table S9).

- Table S9. Liquefied Petroleum Gases Supply and Disposition

- Formerly numbered as Table S8.

- History data for 1973 through 1980 has been dropped. The table title has been changed to reflect the change in time series.

- Table S10. Other Petroleum Products Supply and Disposition

- Formerly numbered as Table S9.

- History data for 1973 through 1980 has been dropped. The table title has been changed to reflect the change in time series.

\section{Changes In Detailed Statistics Tables}

\section{- Table 1. U.S. Petroleum Balance}

- Line 14 includes fuel ethanol blended into finished motor gasoline. This quantity is comparable to the sum of field production of finished motor gasoline and natural gas liquids and LRGs on Table 2.

- Line 20 has been modified to read: Other Liquids New Supply (Field Production) to accommodate motor gasoline blending components field production.

- Tables 2 through 25. Supply and Disposition

- Isobutane has been renamed Isobutane/Isobutylene under Liquefied Petroleum Gases for clarification.
- Other Hydrocarbons/Hydrogen/Alcohol has been renamed Other Hydrocarbons/Hydrogen/Oxygenates for clarification.

- Unleaded and leaded motor gasoline categories have been replaced with the new types of gasolines produced: reformulated, oxygenated (EPA approved), and other.

- Distillate fuel oil sulfur categories (0.05\% sulfur and under and greater than $0.05 \%$ sulfur) have been added.

- Table 28. Refinery Input

- Other Hydrocarbons/Hydrogen/Alcohol has been renamed Qther Hydrocarbons/Hydrogen/Oxygenates for clarification. Sub-categories are displayed for Other Hydrocarbons/Hydrogen and for Oxygenates.

- Oxygenates are displayed separately for fuel ethanol, methanol, MTBE, and other oxygenates. Other oxygenates includes ethyl tertiary butyl ether (ETBE), tertiary amyl methyl alcohol (TAME), tertiary butyl alcohol (TBA), and other aliphatic alcohols and ethers intended for motor gasoline blending (e.g., isopropyl ether (IPE) or n-propanol).

- Table 29. Refinery Net Production

- Isobutane has been renamed Isobutane/Isobutylerc under Liquefied Petroleum Gases for clarification. Isobutylene is displayed as a sub-category to be consistent with the other liquefied gases.

- Unleaded and leaded motor gasoline categories have been replaced with the new types of gasolines produced: reformulated, oxygenated (EPA approved), and other.

- Military and commercial kerosene-type jet fuel has been added.

- Distillate fuel oil sulfur categories (0.05\% sulfur and under and greater than $0.05 \%$ sulfur) have been added.

- Table 30. Refinery Stocks

- Isobutane has been renamed Isobutane/Isobutylene under Liquefied Petroleum Gases for clarification.

- Other Hydrocarbons/Hydrogen/Alcohol has been renamed Qther Hydrecarbons/Hydrogen/Oxygenates for clarification. Sub-categories are displayed for Other Hydrocarbons/Hydrogen and for Oxygenates.

- Oxygenates are displayed separately for fiuel ethanol, methanol, MTBE, and other oxygenates. Other 
oxygenates includes ethyl tertiary butyl ether (ETBE), tertiary amyl methyl alcohol (TAME), tertiary butyl alcohol (TBA), and other aliphatic alcohols and ethers intended for motor gasoline blending (e.g., isopropyl ether (IPE) or n-propanol).

- Unleaded and leaded motor gasoline categories have been replaced with the new types of gasolines produced: reformulated, oxygenated (EPA approved), and other.

- Distillate fuel oil sulfur categories $(0.05 \%$ sulfur and under and greater than $0.05 \%$ sulfur) have been added.

\section{- Tables 33 and 34. Imports by PAD District}

- Data on olefins are displayed separately from liquefied petroleum gases.

- Other Hydrocarbons/Hydrogen/Alcohol has been renamed Other Hydrocarbons/Hydrogen/Oxygenates for clarification. Sub-categories are displayed for Other Hydrocarbons/Hydrogen and for Oxygenates.

- Oxygenates are displayed separately for fuel ethanol, MTBE, and other oxygenates. Other oxygenates includes ethyl tertiary butyl ether (ETBE), tertiary amyl methyl alcohol (TAME), tertiary butyl alcohol (TBA), and other aliphatic alcohols and ethers intended for motor gasoline blending (e.g., isopropyl ether (IPE) or n-propanol).

- Unleaded and leaded motor gasoline categories have been replaced with the new types of gasolines produced: reformulated, oxygenated (EPA approved), and other.

- Distillate fuel oil sulfur categories $(0.05 \%$ sulfur and under and greater than $0.05 \%$ sulfur) have been added to both bonded ship bunkers and other.

- Tables 35-44. Imports by Countiy of Origin

- A new line has been added to appear below the Total line to show the sum of the Persian Gulf countries.

- Former USSR has been changed to read Russia. States formerly included in USSR are now included in the Other countries category under Non-OPEC.

- Tables 45 and 46. Exports

- Isobutane has been renamed Isobutane/lsobutylene under Liquefied Petroleum Gases for clarification.
- Other Hydrocarbons/Oxygenates and Motor Gasoline Blending Components have been added as export products under the Other Liquids category.

- Tables 47-48. Exports by Destination

- Miscellaneous products category has been renamed Other Products to accommodate exports of other hydrocarbons/ oxygenates and motor gasoline blending components.

- Tables 49-50. Net Imports

- A new line has been added to appear below the Total line to show the sum of the Persian Gulf countries.

- Former USSR has been changed to read Russia. States formerly included in USSR are now included in the Other countries category under Non-OPEC.

- Table 51. Stocks

- Other Hydrocarbons/Hydrogen/Alcohol has been renamed Qther Hydrocarbons/Hydrogen/Oxygenates for clarification. Sub-categories are displayed for Other hydrocarbons/hydrogen fuel ethanol, ETBE, methanol, MTBE, and other oxygenates.

- Other oxygenates includes tertiary amyl methyl alcohol (TAME), tertiary butyl alcohol (TBA), and other aliphatic alcohols and ethers intended for motor gasoline blending (e.g., isopropyl ether (IPE) or npropanol.

- Unleaded and leaded motor gasoline categories have been replaced with the new types of gasolines produced: reformulated, oxygenated (EPA approved), and other.

- Distillate fuel oil sulfur categories $(0.05 \%$ sulfur and under and greater than $0.05 \%$ sulfur) have been added.

- Table 52. Refinery, Bulk Terminal, and Natural Gas Plant Stocks

- Unleaded and leaded motor gasoline categories have been replaced with the new types of gasolines produced: reformulated, oxygenated (EPA approved), and other.

- Distillate fuel oil sulfur categories $(0.05 \%$ sulfur and under and greater than $0.05 \%$ sulfur) have been added.

- Table 53. Movements by Pipeline, Tanker, and Barge

- Unleaded and leaded motor gasoline categories have been replaced with the new types of gasolines 
produced: reformulated, oxygenated (EPA approved), and other.

- Distillate fuel oil sulfur categories $(0.05 \%$ sulfur and under and greater than $0.05 \%$ sulfur) have been added.

- Table 54. Movements by Pipeline

- Unleaded and leaded motor gasoline categories have been replaced with the new types of gasolines produced: reformulated, oxygenated (EPA approved), and other.

- Distillate fuel oil sulfur categories $(0.05 \%$ sulfur and under and greater than $0.05 \%$ sulfur) have been added.

- Table 55. Movements by Tanker and Barge

- Unieaded and leaded motor gasoline categories have been replaced with the new types of gasolines produced: reformulated, oxygenated (EPA approved), and other.

- Distillate fuel oil sulfur categories $(0.05 \%$ sulfur and under and greater than $0.05 \%$ sulfur) have been added.

- Table 56. Net Movements

- Isobutane has been renamed Isobutane/Isobutylene under Liquefied Petroleum Gases for clarification.

- Unleaded and leaded motor gasoline categories have been replaced with the new types of gasolines produced: reformulated, oxygenated (EPA approved), and other.

- Distillate fuel oil sulfur categories $(0.05 \%$ sulfur and under and greater than $0.05 \%$ sulfur) have been added.

\section{Changes in Appendix C}

- Inputs

- Other hydrocarbons has been renamed Other Hydrocarbons/Oxygenates for clarification.

- Production

- Isobutane has been renamed Isobutane/Isobutylene under Liquefied Petroleum Gases for clarification.

- Unleaded and leaded motor gasoline categories have been replaced with the new types of gasolines produced: reformulated, oxygenated (EPA approved), and other.
- A new line has been added to display field production of motor gasoline blending components.

- Imports

- Isobutane has been renamed Isobutane/Isobutylene under Liquefied Petroleum Gases for clarification.

- Unleaded and leaded motor gasoline categories have been replaced with the new types of gasolines produced: reformulated, oxygenated (EPA approved), and other.

- Stocks

- Other hydrocarbons has been renamed Other Hydrocarbons/Oxygenates for clarification.

- Isobutane has been renamed Isobutane/Isobutylene under Liquefied Petroleum Gases for clarification.

- Unleaded and leaded motor gasoline categories have been replaced with the new types of gasolines produced: reformulated, oxygenated (EPA approved), and other.

- Product Supplied

- Isobutane has been renamed Isobutane/Isobutylene under Liquefied Petroleum Gases for clarification.

- Unleaded and leaded motor gasoline categories have been replaced with the new types of gasolines produced: reformulated, oxygenated (EPA approved), and other.

\section{Changes in Appendix D}

- Table D1. U.S. Summary Table

- Data on blended into motor gasoline has been eliminated. This information is no longer collected on the survey EIA-819M, "Monthly Oxygenate Telephone Report."

- Table D2. Monthly Fuel Ethanol Production and Ending Stocks

- Data for the previous year as well as current year are displayed.

- Data on blended into motor gasoline has been eliminated. This information is no longer collected on the survey EIA-819M, "Monthly Oxygenate Telephone Report." 
- Data for fuel ethanol imports has been dropped due to small volumes reported by respondents.

- Table D3. Monthly MTBE Production and Ending Stocks

- Data for the previous year as well as current year are displayed.
- Data on blended into motor gasoline has been eliminated. This information is no longer collected on the survey EIA-819M, "Monthly Oxygenate Telephone Report."

- Data on MTBE imports has been dropped from the table due to small volumes reported by respondents. 


\section{Appendix C}

This table contains information on revisions to published statistics caused by resubmission of respondent survey forms. The table shows the published value in the Petroleum Supply Monthly (PSM) and the cumulative difference resulting from resubmissions for the major product series. The official published petroleum supply statistics are not changed to reflect revisions until publication of the Petroleum Supply Annual (PSA), except in cases of catastrophic error.

This table is provided as a service to analysts who need to know the latest available statistics. It should be used with caution because resubmissions are received on an irregular basis and the impact on published data can change from month to month. In some cases, the pattern of revision caused by resubmissions during the year is a poor indicator of final statistics that will be published in the PSA.

\section{Impact of Resubmissions on Major Series, 1992}

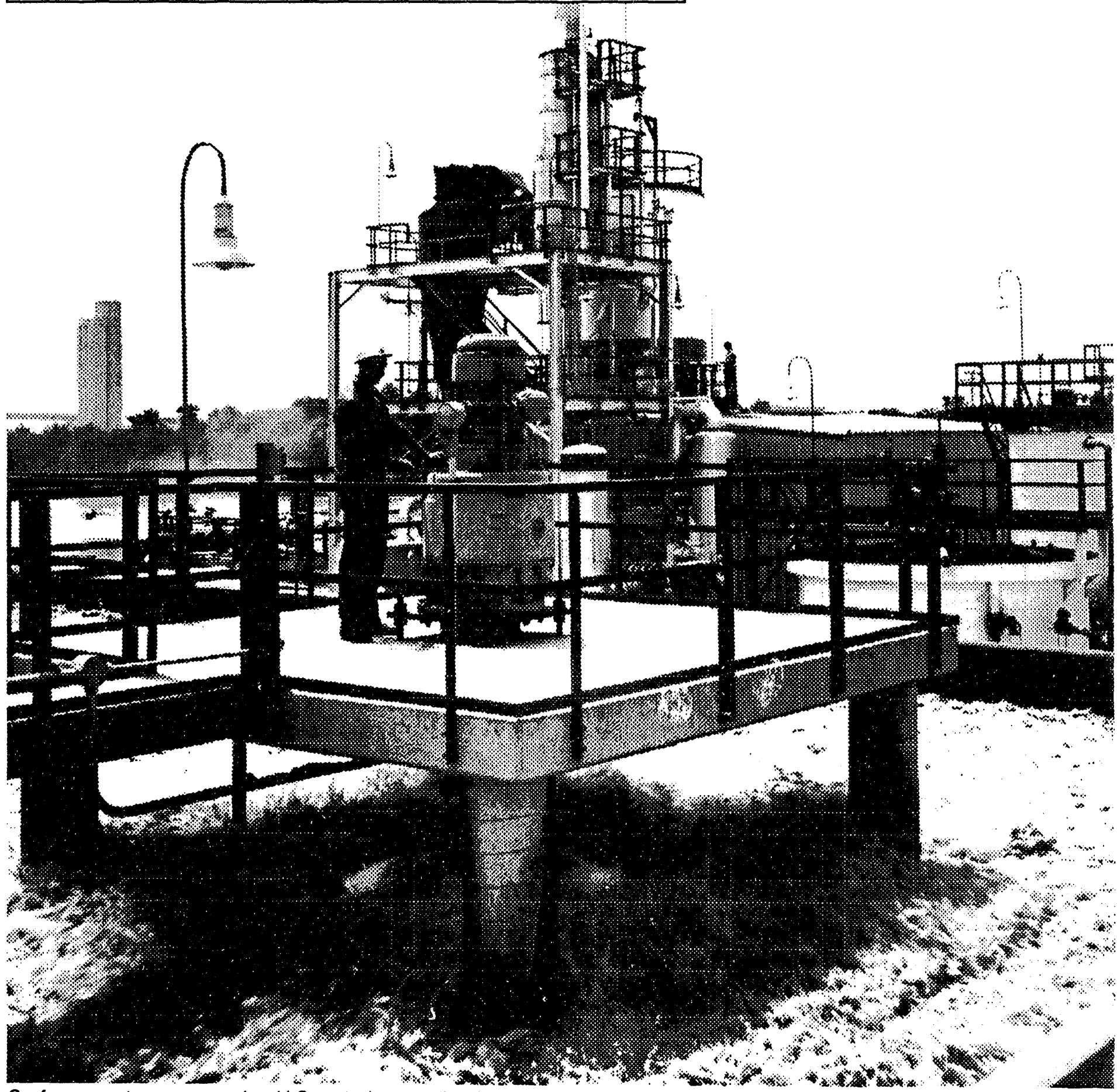


Table C1. Impact of Resubmissions on Major Serles, 1993

(Thousand Barrels per Day, Except Where Noted)

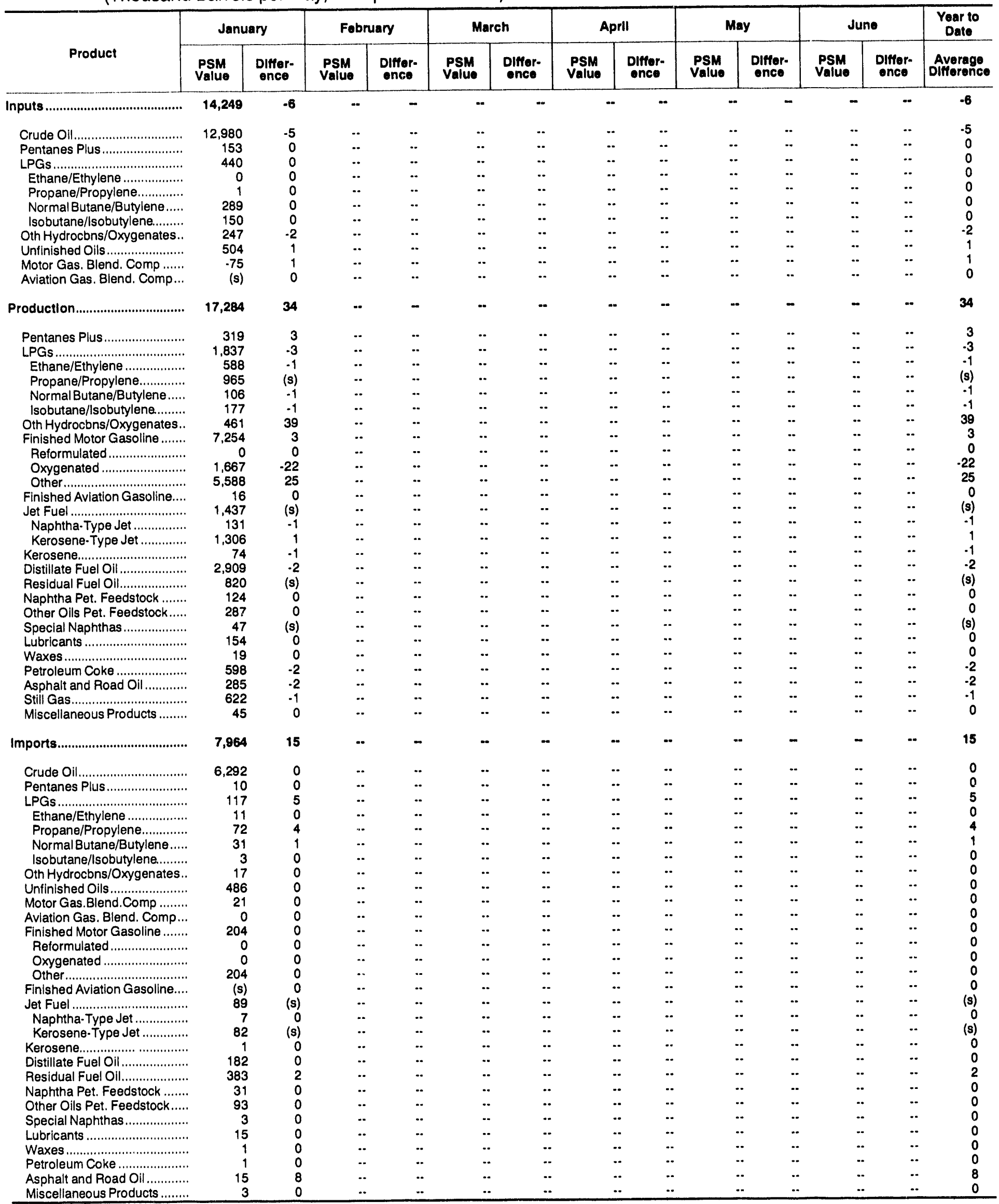

(s) = Less than 500 barrels per day.
Note: - Volumes indlcate cumulative changes resulting from resubmlsslons received for that month as of the date of this publication. - Totals may not equal sum of components due to independent rounding. 
Table C1. Impact of Resubmissions on Major Serles, 1993 (Thousand Barrels per Day, Except Where Noted)

\begin{tabular}{|c|c|c|c|c|c|c|c|c|c|c|c|c|c|c|}
\hline \multirow{2}{*}{ Product } & \multicolumn{2}{|c|}{ January } & \multicolumn{2}{|c|}{ Fobruary } & \multicolumn{2}{|c|}{ March } & \multicolumn{3}{|c|}{ Aprll } & \multicolumn{2}{|c|}{ May } & \multicolumn{2}{|c|}{ June } & \multirow{2}{*}{$\begin{array}{c}\begin{array}{c}\text { Year to } \\
\text { Date }\end{array} \\
\begin{array}{c}\text { Average } \\
\text { Difference }\end{array} \\
\end{array}$} \\
\hline & $\begin{array}{c}\text { PSM } \\
\text { Value }\end{array}$ & $\begin{array}{c}\text { Dlffer- } \\
\text { ence }\end{array}$ & $\begin{array}{c}\text { PSM } \\
\text { Value }\end{array}$ & $\begin{array}{l}\text { Differ- } \\
\text { ence }\end{array}$ & $\begin{array}{c}\text { PSM } \\
\text { Value }\end{array}$ & $\begin{array}{l}\text { Differ- } \\
\text { ence }\end{array}$ & $\begin{array}{l}\text { PSM } \\
\text { Value }\end{array}$ & & $\begin{array}{l}\text { Differ- } \\
\text { once }\end{array}$ & $\begin{array}{c}\text { PSM } \\
\text { Value }\end{array}$ & $\begin{array}{l}\text { Differ- } \\
\text { once }\end{array}$ & $\begin{array}{c}\text { PSM } \\
\text { Value }\end{array}$ & $\begin{array}{l}\text { Differ- } \\
\text { once }\end{array}$ & \\
\hline Stocks (Thousand Barrels) .... 1 & $1,611,388$ & 1,523 & - & -- & - & -- & & - & - & - & -- & - & - & 1,523 \\
\hline Crude Oil (excl. SPR) ............ & 325,640 & 1,332 &.- & .. & -. & -. & & -. & $\cdots$ & - & .- & -. & -. & 1,332 \\
\hline Pentanes Plus ............................ & 8,260 & 150 & .. & .. & -. &.. & & -- & -. & $\cdots$ & 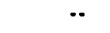 & -. & .. & 150 \\
\hline 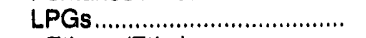 & 74,993 & $-1,804$ &.. & .. & .. & .. & & .. & $-\cdot$ & .. & .. & .. & -. & $-1,804$ \\
\hline Ethane/Ethylene .................... & 16,496 & .92 & -. & 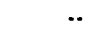 & -. &.$\cdot$ & & -- & $-\cdot$ & .. & $\cdot \cdot$ & -. & -. & .92 \\
\hline Propane/Propylene............. & 33,489 & $-1,119$ & .. & -. & .. &.. & & -• & .• & -. & .. & .. & .. & $-1,119$ \\
\hline Normal Butane/Butylene..... & 14,383 & -481 & 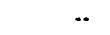 & -. & .. & .* & & .. & -. & .. & .. & ". & .. & -481 \\
\hline Isobutane/Isobutylene........ & 10,625 & .112 & .. & -. & .. &.. & & .. & .. & -. & -. & .. & $\cdots$ & -112 \\
\hline Oth Hydrocbns/Oxygenates.. & 14,016 & 1,278 & -. & 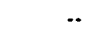 & .. & -. & & .. & -. & -. & -. & -. & -. & 1,278 \\
\hline Unfinished Oils....................... & 99,259 & 163 & .. & $\cdots$ & -. & .. & & -- & .. & -- & .. & -. & .. & 163 \\
\hline Motor Gas. Blend. Comp ...... & 41,309 & -6 & $\cdot-$ & $\cdot$. & -. & .. & & -- & -. & -• & .. & $\cdot \cdot$ & $\cdots$ & $\cdot 6$ \\
\hline Aviation Gas. Blend. Comp... & 61 & 0 & -. & 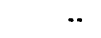 & .. & •• & & -. & 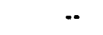 & -. & -. & .. & -. & 0 \\
\hline Finished Motor Gasoline....... & 195,291 & 268 & .. & .. & -. & -. & & -. & -. & -. & .. & -. & -. & 268 \\
\hline Reformulated........................ & 0 & 0 & - & .. & .- & .• & & -. & $\cdot \cdot$ & -. & $-\cdot$ & .. & -. & 0 \\
\hline 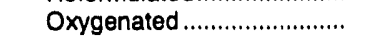 & 32,349 & .175 & -. & -. & .. & $\ddot{*}$ & & -. & $\cdot-$ & 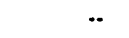 & -. & .. & .. & -175 \\
\hline 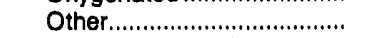 & 162,942 & 443 &.. & .. & $\cdot \cdot$ & .• & & .. & 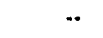 & .. & .. & .. & .- & 443 \\
\hline Finished Aviation Gasoline ... & 1,717 & 0 &. & -. & $\cdots$ & -. & & .• & $\cdot \cdot$ & $\cdot-$ & .. & $\cdots$ & .. & 0 \\
\hline 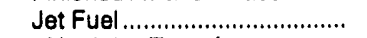 & 41,014 & 7 &.- & -. & $\cdot$. & .. & &.. & -. & .. & .. & .. &.- & 7 \\
\hline Naphtha-Type Jet ................. & 4,763 & 0 & .. & .- & -. & .. & &.$\cdot$ & $\cdots$ & $\cdot \cdot$ & -. & .. & .. & 0 \\
\hline Kerosene-Type Jet .............. & 36,251 & 7 & .. & .. & 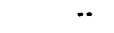 & -. & & -. & .. & .. & .. & .. & .. & 7 \\
\hline 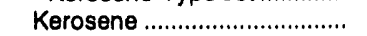 & 5,289 & .4 &.. &.. & $\cdot-$ & .. & & .. & -. & $\cdots$ & .. & .. &.- & .4 \\
\hline Distillate Fuel Oll.................... & 130,222 & .78 & -. & .. & -. & .. & & .. & $-\cdot$ & .. & .. &.$\cdot$ &.. & .78 \\
\hline Residual Fuel Oll..................... & 44,221 & .1 &.- & -. & 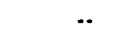 & $\ddot{*}$ & & -. & -. & $\because$ & $*$ &.. & -. & -1 \\
\hline Naphtha Pet. Feedstock ....... & 1,616 & 0 & .. &.. & $\cdot-$ & -. & & .. & -. & -. & .. & .. &.. & 0 \\
\hline Other Olis Pet. Feedstock..... & 1,680 & 0 & $-\cdot$ & $\cdots$ & $\cdots$ & -. & & .. & $\cdots$ & $\cdots$ & -. & .. & .. & 0 \\
\hline Speclal Naphthas.................. & 2,216 & -3 & .. & .. & $\cdots$ & -- & & .. & $*$ & -. & .. &.. &.. & -3 \\
\hline 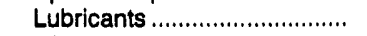 & 13,514 & 0 & $-\cdot$ & -. & $-\cdot$ & $\cdot$. & & .. & -. & .. & -. & -. & .. & 0 \\
\hline 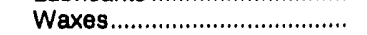 & 919 & 0 &.. & .- & .. & -. & & -. & $\cdot-$ & -. & .. & -. & .. & 0 \\
\hline Petroleum Coke ...................... & 10,494 & 0 & -. & -. & -. & .. & &.. & .. &.. & .. & .. & .. & 0 \\
\hline Asphalt and Road Oil............ & 22,115 & 221 &.. & .. & .. & .. & & .. & .. & 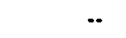 & -. & $\cdots$ & .. & 221 \\
\hline Miscellaneous Products ......... & 2,231 & 0 &.- & 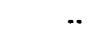 & -. & -. & & -. & 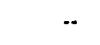 & 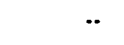 & $\cdots$ & $\cdots$ & -. & 0 \\
\hline Product Supplied......................... & 16,502 & 43 & - & -- & $\cdots$ & - & & - & - & - & $\cdots$ & $\cdots$ & - & 43 \\
\hline Crude Oil.................................. & 10 & 0 &.. &.. & -. & -. & & .. & -. & $\cdots$ & -. & $\cdot \cdot$ & .. & 0 \\
\hline Pentanes Plus........................ & 196 & -2 & .. & .. & $\cdots$ & .. & & -- &.. & -. & * & $\cdot \cdot$ & .. & .2 \\
\hline 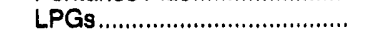 & 1,917 & 61 & -. &.. & $\cdots$ & -. & & -- & $-\cdot$ & -. & $\cdots$ & -. & .. & 61 \\
\hline Ethane/Ethylene.................. & 672 & 2 & .. & .. & $\because$ & $\cdots$ & & .. & $\cdots$ & -. & $\cdots$ & -. & .. & 2 \\
\hline Propane/Propylene.............. & 1,179 & 40 & -. & -. & .. & -- & &.$\cdot$ & -. & -. & 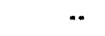 & 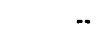 & -- & $4 \overline{0}$ \\
\hline Normal Butane/Butylene..... & 31 & 15 & .. & .. & $\cdots$ & 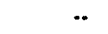 & & .. &.- & $\cdots$ & -. & $\cdots$ & -• & 15 \\
\hline Isobutane/Isobutylene......... & 35 & 3 &.. & .. & -. & 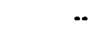 & & -. &.. & -* & $\because$ & $\because$ & .. & 3 \\
\hline Oth Hydrocbns/Oxygenates.. & 0 & 0 & .. &.. & $\cdots$ & -- & & .- & $\cdot$. & $\because$ & $\cdots$ & -. & .. & 0 \\
\hline Unfinished Oils....................... & .144 & .6 &.. & .. & -. & .. & & .. &.- & $\cdots$ & -. & -. & .. & .6 \\
\hline Motor Gas. Blend. Comp ...... & 0 & 0 & -. & .. & 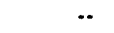 & $\cdots$ & & $\cdot \cdot$ & -. & $\cdots$ & $\cdots$ & $\cdots$ & -. & 0 \\
\hline Aviation Gas. Biend. Cump... & (s) & 0 & .. & .. & -. & $\cdot$. & &.- &.. & -. & $-\cdot$ & $\cdot-$ & .. & 0 \\
\hline Finished Motor Gasoline....... & 6,746 & -6 &.- & .. & $-\cdot$ & $\cdot \cdot$ & & $-\infty$ & -. & $-\cdot$ & $\cdots$ & $\cdots$ & -. & .6 \\
\hline Reformulated........................ & NA & NA &.. & .. & -. &.- & & -- & $-\cdot$ & -. & $\cdots$ & .. & $\cdots$ & NA \\
\hline 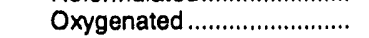 & NA & NA & .. & .. & 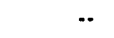 & $\cdot$. & & .. & $\cdots$ & .. & 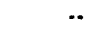 & -. & .. & NA \\
\hline 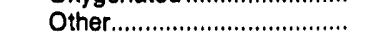 & NA & NA &.. &.. & $\cdots$ & -. & &.$\cdot$ &.$\cdot$ & .. & 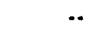 & -- & -. & NA \\
\hline Finished Aviation Gasollne ... & 10 & 0 & -. & .. & .. & $\cdots$ & &.. & .• & .. & $\cdots$ & $\cdots$ & -. & 0 \\
\hline 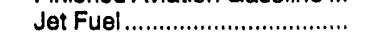 & 1,464 & -1 &.. & .. & .. & $\cdots$ & & -. & -. & .. & 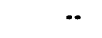 & 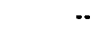 & -• & -1 \\
\hline Naphtha-Type Jet ................ & 93 & -1 & .. & -. & $\cdot \cdot$ & -. & & .. & -. & -. & $\cdots$ & $\cdot \cdot$ & .. & -1 \\
\hline Kerosene-Type Jet .............. & 1,371 & 1 & -. & -. &.- & .. & & .. & .. & .. & .. & .. & .. & 1 \\
\hline 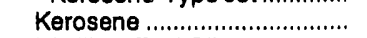 & 63 & .1 & .. & .. & .. & .. & & .. & .. & .. & .. & 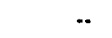 & -. & .1 \\
\hline Distillate Fuel Oil ..................... & 3,322 & 1 & .. &.. & $\cdot$. & *. & &.. & $\cdot \cdot$ & .. &.. & .. & .. & 1 \\
\hline Residual Fuel Oil..................... & 1,020 & 2 & .. &.. & $\cdot$. & .. & &.- & $\cdot$. & -. & -. & .. & -. & 2 \\
\hline Naphtha Pet. Feedstock ....... & 158 & 0 &.. & .. & -. & .. & & .. &. & -. & .- &.- & -. & 0 \\
\hline Other Olls Pet. Feedstock..... & 388 & 0 & .. & .. & $-\cdot$ &.. & & .. & 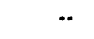 & 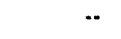 & 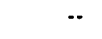 &.- & $\cdot \cdot$ & 0 \\
\hline Special Naphthas.................. & 40 & (s) &.. & .. & .. &.- & & -- & -. & $\cdots$ & $\cdot \cdot$ &.. & .. & (s) \\
\hline 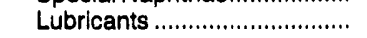 & 149 & 0 & .. & .. & $\cdots$ &.. & & -. & 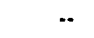 & -. & .. & -- & .. & 0 \\
\hline 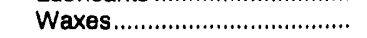 & 18 & 0 & .. & .. & -. & -. & & .. & 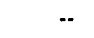 & .. &.. & .. & -. & 0 \\
\hline Petroleum Coke ..................... & 339 & -2 & -. & -. & $\ddot{*}$ & .• & & $\cdots$ & $\cdot$. & $-\cdot$ & .. & $\ddot{*}$ & .. & -2 \\
\hline Asphalt and Road Oil............. & 145 & -1 & -. & -. & $\cdots$ & .. & & 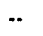 & .. & $\cdots$ & .. & .. & .. & -1 \\
\hline 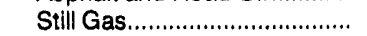 & 622 & -1 & -. & .. & $\cdots$ & - & & $\cdots$ & 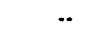 & 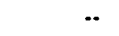 & .. & -. & -. & -1 \\
\hline Miscellaneous Products ........ & 39 & 0 & .. & .. & .. & .. & & -. & .. & 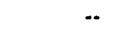 & $\cdot \cdot$ &.$\cdot$ & 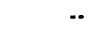 & 0 \\
\hline
\end{tabular}

(s) = Less than 500 barrels per day.

Note: - Volumes indicate cumulative changes resulting from resubmissions received for that month as of the date of this publication. - Totals may not equal sum of components due to independent rounding. 


\section{Appendix D}

\section{EIA 819M Monthly Oxygenate Telephone Report}

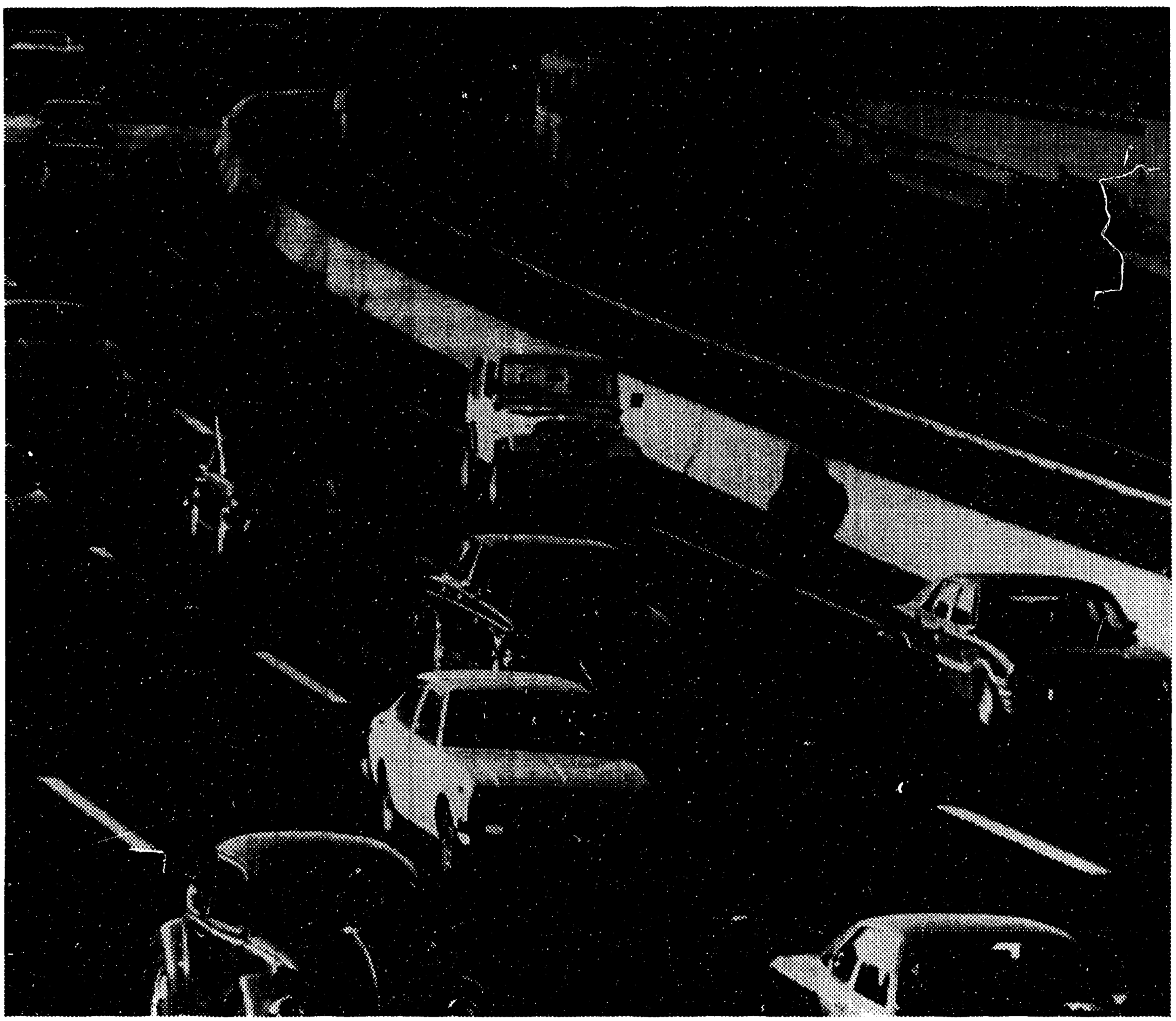

The Clean Air Act Amendments of 1990 include provisions intended to reduce toxic vehicle emissions. 


\section{EIA-819M \\ Monthly Oxygenate Telephone Report}

The EIA-819M, "Monthly Oxygenate Telephone Report," provides production data and preliminary stock data for fuel ethanol and methyl tertiary butyl ether (MTBE) in the United States and major U.S. geographic regions. These data have been published in the Weekly Petroleum Status Report (WPSR) and the Petroleum Supply Monthly since March 1992.

Data are collected from a sample of respondents reporting on the Monthly Petroleum Supply Reporting System surveys. Final data on production and stocks of fuel ethanol and MTBE are presented in the Detailed Statistics section beginning with the March 1993 issue. The quantity of oxygenates blended into motor gasoline previously published in this appendix is now presented in the Highlights section.

Table D1. U.S. Summary Table, Aprll 1993

\begin{tabular}{|c|c|c|c|c|c|c|}
\hline \multirow[b]{2}{*}{ Products } & \multicolumn{2}{|c|}{ Aprll 1993} & \multicolumn{2}{|c|}{ March 1993} & \multicolumn{2}{|c|}{ Year-to-Date } \\
\hline & $\begin{array}{c}\text { Thousand } \\
\text { Barrels }\end{array}$ & \begin{tabular}{|c|} 
Thousand \\
Barrels per Day
\end{tabular} & $\begin{array}{c}\text { Thousand } \\
\text { Barrels }\end{array}$ & \begin{tabular}{|c|} 
Thousand \\
Barrels per Day
\end{tabular} & $\begin{array}{c}\text { Thousand } \\
\text { Barrels }\end{array}$ & $\begin{array}{c}\text { Thousand } \\
\text { Barrels per Day }\end{array}$ \\
\hline \multicolumn{7}{|l|}{ Fuel Ethanol } \\
\hline $\begin{array}{l}\text { Production } \\
\text { Stocks }\end{array}$ & $\begin{array}{l}2,274 \\
2,069\end{array}$ & $\begin{array}{l}76 \\
--\end{array}$ & $\begin{array}{l}2,373 \\
1,878\end{array}$ & $\begin{array}{r}77 \\
--\end{array}$ & $\begin{array}{l}9,059 \\
2,069\end{array}$ & $\begin{array}{l}75 \\
--\end{array}$ \\
\hline \multicolumn{7}{|l|}{ MTBE } \\
\hline Production & 4,125 & 138 & 3,472 & 112 & 14,333 & 119 \\
\hline Stocks & 11,953 & -- & 10,550 & -- & 11,953 & -- \\
\hline
\end{tabular}

Source: Energy Information Administration (EIA) Form EIA-819M, "Monthly Oxygenate Telephone Report." 
Table D2. Monthly Fuel Ethanol Production and Stocks by Potroleum AdmInlstration for Defense Districts (PADD)

(Thousand Barrels per Day, Except Where Noted)

\begin{tabular}{|c|c|c|c|c|c|c|c|c|c|c|c|c|}
\hline DlstrlceYear & Jan & Fob & Mar & Apr & May & Jun & Jul & Aug & Sop & Oct & Nov & Dec \\
\hline \multicolumn{13}{|l|}{$\begin{array}{l}\text { Total U.S. } \\
\text { Production }\end{array}$} \\
\hline 1993 & 76 & 73 & 77 & 76 & & & & & & & & \\
\hline \multicolumn{13}{|c|}{ Stocks (thous. bbls.) } \\
\hline 1992 & 1,076 & 1,287 & 1,462 & 1,457 & 1,858 & 1,941 & 2,362 & 2,530 & 2,973 & 2,980 & 2,547 & 1,791 \\
\hline
\end{tabular}

\begin{tabular}{|c|c|c|c|c|c|c|c|c|c|c|c|c|}
\hline \multicolumn{13}{|c|}{ East Coast (PADD I) } \\
\hline \multicolumn{13}{|c|}{ Production } \\
\hline 1992 & $w$ & $W$ & $w$ & W & $w$ & $w$ & $w$ & $W$ & $w$ & $w$ & W & W \\
\hline 1993 & $w$ & $W$ & $w$ & W & & & & & & & & \\
\hline 1992 & 85 & 93 & 100 & 82 & 88 & 67 & 200 & 207 & 177 & 163 & 139 & 99 \\
\hline 1993 & 117 & 64 & 62 & 41 & & & & & & & & \\
\hline
\end{tabular}

\begin{tabular}{|c|c|c|c|c|c|c|c|c|c|c|c|c|}
\hline \multicolumn{13}{|c|}{ MIdwest (PADD II) } \\
\hline \multicolumn{13}{|c|}{ Production } \\
\hline 1992 & 73 & 66 & 63 & 64 & 64 & 61 & 61 & 66 & 66 & 72 & 72 & 73 \\
\hline 1993 & 74 & 71 & 75 & 74 & & & & & & & & \\
\hline 1992 & 532 & 662 & 791 & 794 & 1,010 & 1,143 & 1,344 & 1,361 & 1,339 & 1,553 & 1,279 & 889 \\
\hline 1993 & 1,094 & 1,124 & 1,143 & 1,310 & & & & & & & & \\
\hline
\end{tabular}

\begin{tabular}{|c|c|c|c|c|c|c|c|c|c|c|c|c|}
\hline \multicolumn{13}{|c|}{ Gulf Coast (PADD III) } \\
\hline \multicolumn{13}{|c|}{ Production } \\
\hline 1992 & W & W & $w$ & $w$ & W & $W$ & W & $w$ & W & W & W & W \\
\hline 1993 & $w$ & $w$ & $w$ & $w$ & & & & & & & & \\
\hline 1992 & 248 & 344 & 394 & 452 & 530 & 464 & 562 & 612 & 405 & 477 & 465 & 254 \\
\hline 1993 & 203 & 244 & 216 & 294 & & & & & & & & \\
\hline
\end{tabular}

\begin{tabular}{|c|c|c|c|c|c|c|c|c|c|c|c|c|}
\hline \multicolumn{13}{|c|}{ Rocky Mountaln (PADD IV) } \\
\hline \multicolumn{13}{|c|}{ Production } \\
\hline 1992 & W & $W$ & $w$ & W & W & $w$ & $w$ & $W$ & W & W & W & W \\
\hline 1993 & w & W & w & W & & & & & & & & \\
\hline 1992 & 27 & 11 & 20 & 14 & 15 & 12 & 17 & 20 & 21 & 44 & 60 & 70 \\
\hline 1993 & 61 & 44 & 45 & 41 & & & & & & & & \\
\hline
\end{tabular}

\begin{tabular}{|c|c|c|c|c|c|c|c|c|c|c|c|c|}
\hline West Coast (PADD V) & & & & & & & & & & & & . \\
\hline \multicolumn{13}{|l|}{ Production } \\
\hline 1992 & W & W & $w$ & w & $W$ & $w$ & W & $w$ & $w$ & $w$ & $w$ & W \\
\hline 1993 & W & w & $w$ & w & & & & & & & & \\
\hline 1992 & 184 & 177 & 156 & 114 & 214 & 254 & 240 & 330 & 732 & 743 & 604 & 479 \\
\hline 1993 & 561 & 453 & 412 & 383 & & & & & & & & \\
\hline
\end{tabular}

$W=$ Withheld to avoid disclosure of individual company data.

Note: - Geographic coverage is the 50 States and the Distriat of Columbia. - Totals may not equal sum of components due to independent rounding.

Source: Energy Information Administration (EIA) Form EIA-819M, "Monthly Oxygenate Telephone Report." 
Table D3. Monthly Methyl Tertlary Butyl Ether (MTBE) Productlon and Stocks by Petroleum Adminlstration for Defense Dlstrlcts (PADD)

(Thousand Barrels per Day, Except Where Noted)

\begin{tabular}{|c|c|c|c|c|c|c|c|c|c|c|c|c|}
\hline Districe/Month & Jan & Fob & Mar & Apr & May & Jun & Jul & Aug & Sop & Oct & Nov & Dec \\
\hline \multicolumn{13}{|l|}{$\begin{array}{l}\text { Total U.S. } \\
\text { Production }\end{array}$} \\
\hline 1992 & 98 & 94 & 89 & 79 & 90 & 90 & 101 & 91 & 104 & 118 & 128 & 125 \\
\hline 1993 & 115 & 114 & 112 & 138 & & & & & & & & \\
\hline \multicolumn{13}{|c|}{ Stocks (thous. bble.) } \\
\hline 1992 & 11,999 & 12,681 & 13,966 & 14,962 & 15,961 & 18,887 & 20,436 & 23,131 & 22,853 & 19,208 & 16,342 & 13,818 \\
\hline 1993 & 10,648 & 10,148 & 10,550 & 11,953 & & & & & & & & \\
\hline \multicolumn{13}{|c|}{ East Coast (PADD I) } \\
\hline \multicolumn{13}{|l|}{ Production } \\
\hline 1992 & $w$ & W & W & W & $W$ & $w$ & $w$ & $w$ & $w$ & $w$ & $w$ & W \\
\hline 1993 & W & $w$ & W & W & & & & & & & & \\
\hline \multicolumn{13}{|c|}{ Stocks (thous. bble.) } \\
\hline 1992 & 3,086 & 2,944 & 3,551 & 3,929 & 4,453 & 4,663 & 4,824 & 5,046 & 4,875 & 3,839 & 3,098 & 2,613 \\
\hline 1993 & 1,881 & 1,833 & 1,492 & 1,598 & & & & & & & & \\
\hline \multicolumn{13}{|c|}{ MIdweot (PADD II) } \\
\hline \multicolumn{13}{|l|}{ Production } \\
\hline 1992 & $w$ & w & $w$ & W & $W$ & $W$ & $w$ & $w$ & W & W & $w$ & $w$ \\
\hline 1993 & W & w & $W$ & $w$ & & & & & & & & \\
\hline \multicolumn{13}{|c|}{ Stocks (thous. bble.) } \\
\hline 1992 & $w$ & $w$ & $w$ & $w$ & $w$ & $W$ & $W$ & $w$ & $w$ & $w$ & $w$ & W \\
\hline 1993 & $w$ & w & $w$ & $w$ & & & & & & & & \\
\hline \multicolumn{13}{|c|}{ Gult Coast (PADD III) } \\
\hline \multicolumn{13}{|l|}{ Production } \\
\hline 1992 & 88 & 82 & 77 & 69 & 77 & 77 & 88 & 78 & 93 & 108 & 118 & 114 \\
\hline 1993 & 102 & 101 & 99 & 124 & & & & & & & & \\
\hline \multicolumn{13}{|c|}{ Stocks (thous. bbls.) } \\
\hline 1992 & 5,104 & 5,711 & 6,058 & 6,728 & 6,870 & 8,549 & 8,928 & 9,847 & 9,192 & 8,309 & 7,380 & 6,159 \\
\hline 1993 & 4,987 & 4,707 & 5,304 & 6,152 & & & & & & & & \\
\hline
\end{tabular}

\begin{tabular}{|c|c|c|c|c|c|c|c|c|c|c|c|c|}
\hline \multicolumn{13}{|c|}{ Rocky Mountaln (PADD IV) } \\
\hline \multicolumn{13}{|c|}{ Production } \\
\hline 1992 & $w$ & $w$ & $w$ & $w$ & W & w & $w$ & W & $w$ & W & w & $w$ \\
\hline 1993 & W & $w$ & $w$ & $w$ & & & & & & & & \\
\hline \multicolumn{13}{|c|}{ Stocks (thous. bble.) } \\
\hline 1992 & $w$ & W & $w$ & $W$ & W & $w$ & w & W & $w$ & W & W & W \\
\hline 1993 & $w$ & $w$ & $w$ & $w$ & & & & & & & & \\
\hline \multicolumn{13}{|c|}{ West Coast (PADD V) } \\
\hline \multicolumn{13}{|c|}{ Production } \\
\hline 1992 & W & w & $w$ & $W$ & $w$ & $w$ & $w$ & W & $w$ & $w$ & $w$ & W \\
\hline 1993 & W & $w$ & $w$ & $w$ & & & & & & & & \\
\hline \multicolumn{13}{|c|}{ Stocks (thous. bble.) } \\
\hline 1992 & 3,418 & 3,673 & 4,011 & 4,064 & 4,309 & 5,385 & 6,419 & 7,936 & 8,466 & 6,723 & 5,543 & 4,768 \\
\hline 1993 & 3,536 & 3,333 & 3,516 & 3,921 & & & & & & & & \\
\hline
\end{tabular}

$W=$ Withheld to avold disclosure of individual company data.

Note: - Geographic coverage is the 50 States and the District of Columbia. - Totals may not equal sum of componente due to independent rounding.

Source: Energy Information Administration (EIA) Form EIA-819M, "Monthly Oxygenate Telephone Report." 


\section{Glossary}

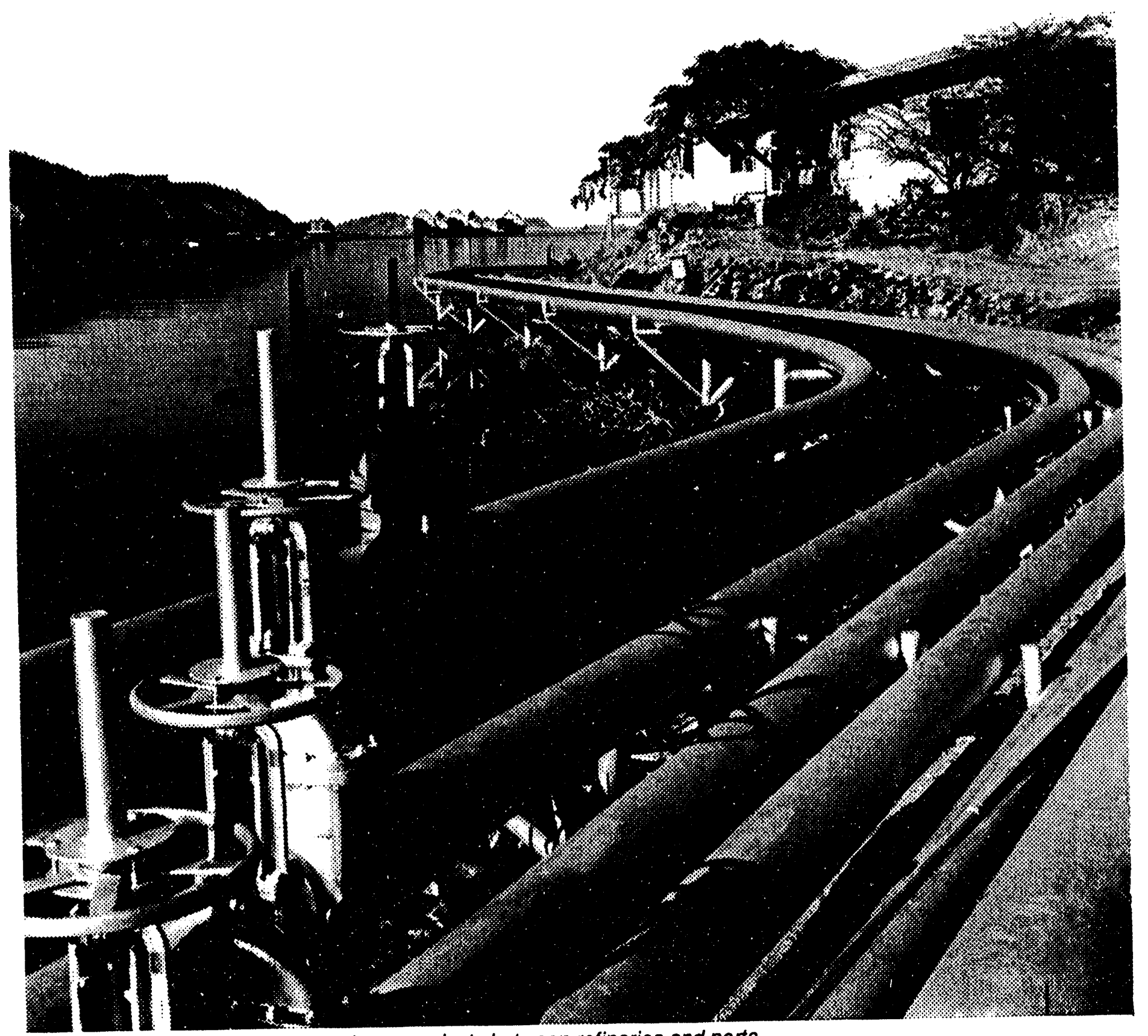

Pipelines carry crude oil and petroleum products between refineries and ports. 


\section{Definitions of Petroleum Products and Other Terms}

Alcohol. The family name of a group of organic chemical compounds composed of carbon, hydrogen, and oxygen. The series of molecules vary in chain length and are composed of a hydrocarbon plus a hydroxyl group; $\mathrm{CH}_{3}-\left(\mathrm{CH}_{2}\right) \mathrm{n}-\mathrm{OH}$ (e.g., methanol, ethanol, and tertiary butyl alcohol).

Alkylate. The product of an alkylation reaction. It usually refers to the high octane product from alkylation units. This alkylate is used in blending high octane gasoline.

Alkylation. A refining process for chemically combining isobutane with olefin hydrocarbons (e.g., propylene, butylene) through the control of temperature and pressure in the presence of an acid catalyst, usually sulfuric acid or hydrofluoric acid. The product, alkylate, an isoparaffin, has high octane value and is blended with motor and aviation gasoline to improve the antiknock value of the fuel.

API Gravity. An arbitrary scale expressing the gravity or density of liquid petroleum products. The measuring scale is calibrated in terms of degrees API; it is calculated as follows:

$$
\text { Degrees API }=\frac{141.5}{s p .8 r .60^{\circ} \mathrm{F} / 60^{\circ} \mathrm{F}} \cdot 131.5
$$

Aromatics. Hydrocarbons characterized by unsaturated ring structures of carbon atoms. Commercial petroleum aromatics are benzene, toluene, and xylene (BTX).

Asphalt. A dark-brown-to-black cement-like material containing bitumens as the predominant constituent obtained by petroleum processing. The definition includes crude asphalt as well as the following finished products: cements, fluxes, the asphalt content of emulsions (exclusive of water), and petroleum distillates blended with asphalt to make cutback asphalts. The conversion factor for asphalt is 5.5 barrels per short ton.

ASTM. The acronym for the American Society for Testing and Materials.

Atmospheric Crude Oil Distillation. The refining process of separating crude oil components at atmospheric pressure by heating to temperatures of about $600^{\circ}$ to $750^{\circ} \mathrm{F}$ (depending on the nature of the crude oil and desired products) and subsequent condensing of the fractions by cooling.
Aviation Gasoline (Finished). All special grades of gasoline for use in aviation reciprocating engines, as given in ASTM Specification D910 and Military Specification MIL-G-5572. Excludes blending components which will be used in blending or compounding into finished aviation gasoline.

Aviation Gasoline Blending Components. Naphthas which will be used for blending or compounding into finished aviation gasoline (e.g., straight-run gasoline, alkylate, reformate, benzene, toluene, and xylene). Excludes oxygenates (alcohols, ethers), butane, and pentanes plus. Oxygenates are reported as other hydrocarbons, hydrogen, and oxygenates.

Barrel. A volumetric unit of measure for crude oil and petroleum products equivalent to 42 U.S. gallons. This measure is used in most statistical reports. Factors for converting petroleum coke, asphalt, still gas and wax to barrels are given in the definitions of these products.

Barrels Per Calendar Day. The maximum number of barrels of input that can be processed during a 24-hour period after making allowances for the following limitations:

the capability of downstream facilities to absorb the output of crude oil processing facilitics of a given refinery. No reduction is made when a planned distribution of intermediate streams through other than downstream facilities is part of a refinery's normal operation;

the types and grades of inputs to be processed;

the types and grades of products expected to be manufactured;

the environmental constraints associated with refinery operations;

the reduction of capacity for scheduled downtime such as routine inspection, mechanical problems, maintenance, repairs, and turnaround; and

the reduction of capacity for unscheduled downtime such as mechanical prcblems, repairs, and slowdowns.

Barrels Per Stream Day. The amount a unit can process running at full capacity under optimal crude oil and product slate conditions. 
Benzene $\left(\mathrm{C}_{6} \mathrm{H}_{6}\right)$. An aromatic hydrocarbon present in small proportion in some crude oils and made commercially from petroleum by the catalytic reforming of naphthenes in petroleum naphtha. Also made from coal in the manufacture of cole. Used as a solvent, in manufacturing detergents, synthe.1c fibers, and petrochemicals and as a component of high-octane gasoline.

Blending Components. See Motor or Aviation Gasoline Blending Components.

Blending Plant. A facility which has no refining capability but is either capable of producing finished motor gasoline through mechanical blending or blends oxygenates with motor gasoline.

Bonded Petroleum Imports. Petroleum imporied and entered into Customs bonded storage. These imports are not included in the import statistics until they are: (1) withdrawn from storage free of duty for use as fuel for vessels and aircraft engaged in international trade; or (2) withdrawn from storage with duty paid for domestic use.

BTX. The acronym for the commercial petroleum aromatics benzene, toluene, and xylene. See individual categories for definitions.

Bulk Station. A facility used primarily for the storage and/or marketing of petroleum products which has a total bulk storage capacity of less than 50,000 barreis and receives its petroleum products by tank car or truck.

Bulk Terminal. A facility used primarily for the storage and/or marketing of petroleum products which has a total bulk storage capacity of 50,000 barrels or more and/or receives petroleum products by tanker, barge, or pipeline.

Butane $\left(\mathrm{C}_{4} \mathrm{H}_{10}\right)$. A normally gaseous straight-chain or branch-chain hydrocarbon extracted from natural gas or refinery gas streams. It includes isobutane and normal butane and is designated in ASTM Specification D1835 and Gas Processors Association Specifications for commercial butane.

Isobutane ( $\mathrm{C}_{4} \mathrm{H}_{10}$ ). A normally gaseous branch-chain hydrocarbon. It is a colorless paraffinic gas that boils at a temperature of $10.9^{\circ} \mathrm{F}$. It is extracted from natural gas or refinery gas streams.

Normal Butane $\left(\mathrm{C}_{4} \mathrm{H}_{10}\right)$. A normally gaseous straight-chain hydrocarbon. It is a colorless paraffinic gas that boils at a ter. perature of $31.1^{\circ} \mathrm{F}$. It is extracted from natural gas or refinery gas streams.

Butylene $\left(C_{4} h_{8}\right)$. An olsfinic hydrocarbon recovered Irom refinery processes.
Captive Refinery Oxygenate Plants. Oxygenate production facilities located within or adjacent to a .efinery complex.

Catalytic Cracking. The refining process of breaking down the larger, heav ier, and more complex hydrocarbon molecules into simpler and lighter molecules. Catalytic cracking is accomplished by the use of a catalytic agent and is an effective process for increasing the yield of gasoline from crude oil. Catalytic cracking processes fresh feeds and recycled feeds.

Fresh Feeds. Crude oil or petroleum distillates which are being fed to processing units for the first time.

Recycled Feeds. Feeds that are continuously fed back for additional processing.

Catalytic Hydrocracking. A refining process that uses hydrogen and catalysts with relatively low temperatures and high pressures for converting middle boiling or residual material to high-octane gasoline, reformer charge stock, je: fuel, and/or high grade fuel oil. The process uses one or more catalysts, depending upon product output, and can handle high sulfur feedstocks without prior desulfurization.

Catalytic Hydrotreating. A refining process for treating petroleum fractions from atmospheric or vacuum distillation units (e.g., naphthas, middle distillates, reformer feeds, residual fuel oil, and heavy gas oil) and other petroleum (e.g., cat cracked naphtha, coker naphtha, gas oil, etc.) in the presence of catalysts and substantial quantities of hydrogen. H'drotreating includes desulfurization, removal of substances (e.g., nitrogen compounds) that deactivate catalysts, conversion of olefins to paraffins to reduce gum formation in gasoline, and other processes to upgrade the quality of the fractions.

Catalytic Reforming. A refining process using controlled heat and pressure with catalysts to rearrange certain hydrocarbon molecules, thereby converting paraffinic and raphthenic type hydrocarbons (e.g., low-octane gasoline boiling range fractions) into petrochemical feedstocks and higher octane stocks suitable for blending into finished gasoline. Catalytic reforming is reported in two categories. They are:

Low Pressure. A processing unit operating at less than 225 pounds per square inch gauge (PSIG) measured at the outlet separator.

High Pressure. A processing unit operating at either equal to or greater than 225 pounds per square inch gauge (PSIG) measured at the outlet separator. 
Charge Capacity. The input (feed) capacity of the refinery processing facilities.

Coal. A black or brownish-blaci solid combustible substance formed by the partial decomposition of vegetable matter without access to air. The rank of coal, which includes anthracite, bituminous coal, subbituminous coal, and lignite, is based on fixed carbon, volatile matter, and heating value. Coal rank indicates the progressive alteration, or coalification, from lignite to anthracite. Lignite contains approximately 9 to 17 million BTU per ton. The heat contents of subbituminous and bituminous coal range from 16 to 24 million BTU per ton, and from 19 to 30 million BTU per ton, respectively. Anthracite contains approximately 22 to 28 million BTU per ton.

\section{Commercial Kerosene-Type Jet Fuel. See} Kerosene-Type Jet Fuel.

Crude Oil (Including Lease Condensate). A mixture of hydrocarbons that exists in liquid phase in underground reservoirs and remains liquid at atmospheric pressure after passing through surface-separating facilities. Included are lease condensate and liquid hydrocarbons produced from tar sands, gilsonite, and oil shale. Drip gases are also included, but topped crude oil (residual oil) and other unfinished oils are excluded. Liquic.: produced at natural gas processing plants and mixed with crude oil are likewise excluded where identifiable. Crude oil is considered as either domestic or foreign, according to the following:

Domestic. Crude oil produced in the United States or from its "outer continental shelf" as defined in 43 USC 1331.

Foreign. Crude oil produced outside the United States. Imported Athabasca hydrocarbons are included.

Crude Oil, Refinery Receipts. Receipts of domestic and foreign crude oil at a refinery. Includes all crude oil in transit except crude oil in transit by pipeline. Foreign crude oil is reported as a receipt only after entry through customs. Crude oil of foreign origin held in bonded storage is excluded.

Crude Oil Losses. Represents the volume of crude oil reported by petroleum refineries as being lost in their operations. These losses are due to spills, contamination, fires, etc. as opposed to refinery processing losses.

Crude Oil Production. The volume of crude oil produced from oil reservoirs during given periods of time. The amount of such production for a given period is measured as volumes delivered from lease storage tanks (i.e., the point of custody transfer) to pipelines, trucks, or other media for transport to refineries or terminals with adjustments for (1) net differences between opening and closing lease inventories, and (2) basic sediment and water (BS\&W).

Crude Oil Qualities. Refers to two properties of crude oil, the sulfur content and A.PI gravity, which affect processing complexity and product characteristics.

Delayed Coking. A process by which heavicr crude oil fractions can be thermally decomposed under conditions of elevated temperatures and pressure to produce a mixture of lighter oils and petroleum coke. The light oils can be processed further in other refinery units to meet product specifications. The coke can be used either as a fuel or in other applications such as the manufacturing of steel or aluminum.

Disposition. The components of petroleum disposition are stock change, crude oil losses, refinery inputs, exports, and products supplied for domestic consumption.

Distillate Fuel Oil. A general classification for one of the petroleum fractions produced in conventional distillation operations. It is used primarily for space heating, on-and-off-highway diesel engine fuel (including railroad engine fuel and fuel for agricultural machinery), and electric power generation. Included are products known as No. 1, No. 2, and No. 4 fuel oils; No. 1, No. 2, and No. 4 diesel fucls. Distillate fuel oil is reported in the following sulfur categories: $0.05 \%$ sulfur and under and Greater than $0.05 \%$ sulfur.

No. 1 Distillate. A petroleum distillate which meets the specifications for No. 1 heating or fuel oil as defined in ASTM D 396 and/or the specifications for No. 1 diesel fuel as defined in ASTM Specification D 975 with distillation temperatures of $420^{\circ} \mathrm{F}$ at the 10 -percent recovery point and $550^{\circ} \mathrm{F}$ at the 90-percent recovery point, and kinematic viscosities between 1.4 and 2.2 centistokes at $100^{\circ} \mathrm{F}$.

No. 2 Distillate. A petroleum distillate which meets the specifications for No. 2 heating or fuel oil as defined in ASTM D 396 and/or the specifications for No. 2 diesel fuel as defined in ASTM Specification D 975 with distillation temperatures of $540^{\circ}$ and $640^{\circ} \mathrm{F}$ at the 90 -percent recovery point, and kinematic viscosities between 2.0 and 4.3 centistokes at $100^{\circ} \mathrm{F}$.

No. 4 Fuel Oil. A fuel oil for commercial burner installations not equipped with preheating facilities. It is used extensively in industrial plants. This grade is a blend of distillate fuel oil and residual fuel oil stocks that conforms to ASTM Specification D396 or Federal Specification VV-F-815C; with minimum and maximum kinematic viscosities between 5.8 and 26.4 centistokes at 
$100^{\circ} \mathrm{F}$. Also included is No, 4-D, a fuel oil for low and medium-speed diesel engines that conforms to ASTM Specification D975.

Electricity (Purchased). Electricity purchased for refinery operations that is not produced within the refinery complex.

Ending Stocks. Primary stocks of crude oil and petroleum products held in storage as of 12 midnight on the last day of the month. Primary stocks include crude oil or petroleum products held in storage at (or in) leases, refineries, natural gas processing plants, pipelines, tank farms, and bulk terminals that can store at least 50,000 barrels of petroleum products or that can receive petroleum products by tanker, barge, or pipeline. Crude oil that is in-transit by water from Alaska, or that is stored on Federal leases or in the Strategic Petroleum Reserve is included. Primary Stocks exclude stocks of foreign origin that are held in bonded warehouse storage.

ETBE (Ethyl tertiary butyl ether) $\left(\mathrm{CH}_{3}\right)_{3} \mathrm{COC}_{2} \mathrm{H}_{5}$. An oxygenate blend stock formed by the catalytic etherification of isobutylene with ethanol.

Ethane $\left(\mathrm{C}_{2} \mathrm{H}_{6}\right)$. A normally gaseous straight-chain hydrocarbon. It is a colorless paraffinic gas that boils at a temperature of $-127.48^{\circ} \mathrm{F}$. It is extracted from natural gas and refinery gas streams.

Ether. A generic term applied to a group of organic chemical compounds composed of carbon, hydrogen, and oxygen, characterized by an oxygen atom attached to two carbon atoms (e.g., methyl tertiary butyl ether).

Ethylene $\left(\mathrm{C}_{2} \mathrm{H}_{4}\right)$. An olefinic hydrocarbon recovered from refinery processes or petrochemical processes.

Exports. Shipments of goods from the 50 States and the District of Columbia to foreign countries, Puerto Rico, the Virgin Islands, and other U.S. possessions and territories.

Field Production. Represents crude oil production on leases, natural gas liquids production at natural gas processing plants, new supply of other hydrocarbons/ oxygenates and motor gasoline blending components, and fuel ethanol blended into finished motor gasoline.

Flexicoking. A thermal cracking process which converts heavy hydrocarbons such as crude oil, tar sands bitumen, and distillation residues into light hydrocarbons. Feedstocks can be any pumpable hydrocarbons including those containing high concentrations of sulfur and metals.

Fluid Coking. A thermal cracking process utilizing the fluidized-solids technique to remove carbon (coke) for continuous conversion of heavy, low-grade oils into lighter products.

Fresh Feed Input. Represents input of material (crude oil, unfinished oils, natural gas liquids, other hydrocarbons and oxygenates or finished products) to processing units at a refinery that is being processed (input) into a particular unit for the first time.

\section{Examples:}

(1) Unfinished oils coming out of a crude oil distillation unit which are input into a catalytic cracking unit are considered fresh feed to the catalytic cracking unit.

(2) Unfinished oils coming out of a catalytic cracking unit being looped back into the same catalytic cracking unit to be reprocessed are not considered fresh feed.

Fuel Ethanol $\left(\mathrm{C}_{2} \mathrm{H}_{5} \mathrm{OH}\right)$. An anhydrous denatured aliphatic alcohol intended for gasoline blending as described in Oxygenates definition.

Fuels Solvent Deasphalting. A refining process for removing asphalt compounds from petroleum fractions, such as reduced crude oil. The recovered stream from this process is used to produce fuel products.

Gas Oil. A liquid petroleum distillate having a viscosity intermediate between that of kerosene and lubricating oil. It derives its name from having originally been used in the manufacture of illuminating gas. It is now used to produce distillate fuel oils and gasoline.

Gasoline Blending Components. Naphthas which will be used for blending or compounding into finished aviation or motor gasoline (e.g., straight-run gasoline, alkylate, reformate, benzene, toluene, and xylene). Excludes oxygenates (alcohols, ethers), butane, and pentanes plus.

Gross Input to Atmospheric Crude Oil Distillation Units. Total input to atmospheric crude oil distillation units. Includes all crude oil, lease condensate, natural gas plant liquids, unfinished oils, liquefied refinery gases, slop oils, and other liquid hydrocarbons produced from tar sands, gilsonite, and oil shale.

Heavy Gas Oil. Petroleum distillates with an approximate boiling range from $651^{\circ}$ to $1000^{\circ} \mathrm{F}$.

Hydrogen. The lightest of all gases, occurring chiefly in combination with oxygen in water; exists also in acids, bases, alcohols, petroleum, and other hydrocarbons. 
Idle Capacity. The component of operable capacity that is not in operation and not under active repair, but capable of being placed in operation within 30 days; and capacity not in operation but under active repair that can be completed within 90 days.

Imported Crude Oil Burned As Fuel. The amount of foreign crude oil burned as a fuel oil, usually as residual fuel oil, without being processed as such. Imported crude oil burned as fuel includes lease condensate and liquid hydrocarbons produced from tar sands, gilsonite, and oil shale.

Imports. Receipts of goods into the 50 States and the District of Columbia from foreign countries, Puerto Rico, the Virgin Islands, and other U.S. possessions and territories.

Isobutane. See Butane.

Isobutylene $\left(\mathrm{C}_{4} \mathrm{H}_{8}\right)$. An olefinic hydrocarbon recovered from refinery processes or petrochemical processes.

Isohexane $\left(\mathrm{C}_{6} \mathrm{H}_{14}\right)$. A saturated branch-chain hydrocarbon. It is a colorless liquid that boils at a temperature of $156.2^{\circ} \mathrm{F}$.

Isomerization. A refining process which alters the fundamental arrangement of atoms in the molecule without adding or removing anything from the original material. Used to convert normal butane into isobutane $\left(\mathrm{C}_{4}\right)$, an alkylation process feedstock, and normal pentane and hexane into isopentane $\left(\mathrm{C}_{5}\right)$ and isohexane $\left(\mathrm{C}_{6}\right)$, high-octane gasoline components.

\section{Isopentane. See Natural Gasoline and Isopentane.}

Kerosene. A petroleum distillate that has a maximum distillation temperature of $401^{\circ} \mathrm{F}$ at the 10-percent recovery point, a final boiling point of $572^{\circ} \mathrm{F}$, and a minimum flash point of $100^{\circ} \mathrm{F}$. Included are the two grades designated in ASTM D3699: No. 1-K and No. $2-\mathrm{K}$, and all grades of kerosene called range or stove oil. Kerosene is used in space heaters, cook stoves, and water heaters and is suitable for use as an illuminant when burned in wick lamps.

Kerosene-Type Jet Fuel. A quality kerosene product with a maximum distillation temperature of $400^{\circ} \mathrm{F}$ at the 10-percent recovery point and a final maximum boiling point of $572^{\circ} \mathrm{F}$. The fuel is designated in ASTM Specification D1655 and Military Specification MIL-T-5624L (Grades JP-5 and JP-8). A relatively low-freezing point distillate of the kerosene type used primarily for turbojet and turboprop aircraft engines.
Commercial. Kerosene-type jet fuel intended for commercial use.

Military. Kerosene-type jet fuel intended for military use.

Lease Condensate. A natural gas liquid recovered from gas well gas (associated and non-associated) in lease separators or natural gas field facilities. Lease condensate consists primarily of pentanes and heavier hydrocarbons.

Light Gas Oils. Liquid petroleum distillates heavier than naphtha, with an approximate boiling range from $401^{\circ} \mathrm{F}$ to $650^{\circ} \mathrm{F}$.

Liquefied Petroleum Gases (LPG). Ethane, ethylene, propane, propylene, normal butane, butylene, isobutane, and isobutylene produced at refineries or natural gas processing plants, including plants that fractionate raw natural gas plant liquids.

Liquefied Refinery Gases (LRG). Liquefied petroleum gases fractionated from refinery or still gases. Through compression and/or refrigeration, they are retained in the liquid state. The reported categories are ethane/ethylene, propane/propylene, normal butane/butylene, and isobutane/isobutylene. Excludes still gas.

Lubricants. A substance used to reduce friction between bearing surfaces or as process materials either incorporated into other materials used as processing aids in the manufacturing of other products, or as carriers of other materials. Petroleum lubricants may be proruced either from distillates or residues. Other substances may be added to impart or improve certain required properties. Do not include byproducts of lubricating oil refining such as aromatic extracts derived from solvent extraction or tars derived from deasphalting. "Lubricants" includes all grades of lubricating oils from spindle oil to cylinder oil and those used in greases. Reporting categories include:

Paraffinic. Includes all grades of bright stock and neutrals with a Viscosity Index $>75$.

Naphthenic. Includes all lubricating oil base stocks with a Viscosity Index $<75$.

Note: The criterion for categorizing the lubricants is based solely on the Viscosity Index of the stocks and is independent of crude sources and type of processing used to produce the oils.

Exceptions: Lubricating oil base stocks that have been historically classified as naphthenic or paraffinic by a refiner may continue to be so categorized irrespective of the Viscosity Index criterion. 
Example:

(1) Unextracted paraffinic oils that would not meet the Viscosity Index test.

Merchant Oxygenate Plants. Oxygenate production facilities that are not associated with a petroleum refinery. Production from these facilities is sold under contract or on the spot market to refiners or other gasoline blenders.

Methanol $\left(\mathrm{CH}_{3} \mathrm{OH}\right)$. A light, volatile alcohol eligible for gasoline blending as described in Oxygenate definition.

Middle Distillates. A general classification of refined petroleum products that includes distillate fuel oil and kerosene.

Military Kerosene-Type Jet Fuel. See Kerosene-Type Jet Fuel.

Miscellaneous Products. Includes all finished products not classified elsewhere (e.g., petrolatum, lube refining byproducts (aromatic extracts and tars), absorption oils, ram-jet fuel, petroleum rocket fuels, synthe tic natural gas feedstocks, and specialty oils).

Motor Gasoline (Finished). A complex mixture of relatively volatile hydrocarbons, with or without small quantities of additives, that has been blended to form a fuel suitable for use in spark-ignition engines. Motor gasoline, as given in ASTM Specification D439 or Federal Specification VV-G-1690B, includes a range in distillation temperatures from $122^{\circ}$ to $158^{\circ} \mathrm{F}$ at the 10 -percent recovery point and from $365^{\circ}$ to $374^{\circ} \mathrm{F}$ at the 90 -percent recovery point. "Motor gasoline" includes reformulated gasoline, oxygenated gasoline (EPA approved), and other finished gasoline. Blendstock is excluded until blending has been completed.

Reformulated Gasoline. Gasoline formulated for use in motor vehicles, the composition and properties of which are certified as "reformulated gasoline" by the U.S. Environmental Protection Agency.

Oxygenated Gasoline (EPA approved). Gasoline formulated for use in motor vehicles, that is intended for use in the EPA CO nonattainment program. Exclude reformulated gasoline.

Other Finished. Motor gasoline not included in the oxygenated or reformulated gasoline categories.

Motor Gasoline Blending. Mechanical mixing of motor gasoline blending components and oxygenates to produce finished motor gasoline (see definition of Motor Gasoline).
Motor Gasoline Blending Components. Naphthas which will be used for blending or compounding into finished motor gasoline (e.g. straight-run gasoline, alkylate, reformate, benzene, toluene, and xylene). Excludes oxygenates (alcohols, ethers), butane, and pentanes plus. Oxygenates are reported as individual components and included in the total for other hydrocarbons, hydrogens, and oxygenates.

MTBE (Methyl tertiary butyl ether) ( $\left.\mathrm{CH}_{3}\right)_{3} \mathrm{COCH}_{3}$. An ether intended for gasoline blending as described in Oxygenate definition.

Nameplate Capacity. The maximum design production capacity specified by the manufacturer of a processing unit or the maximum amount of a product that can be produced running the manufacturing unit at full capacity.

Naphtha. A generic term applied to a petroleum fraction with an approximate boiling range between $122^{\circ}$ and $400^{\circ}$ F.

Naphtha Less Than $401^{\circ}$ F. See Petrochemical Feedstocks.

Naphtha-Type Jet Fuel. A fuel in the heavy naphtha boiling range. ASTM Specification D1655 specifies for this fuel maximum distillation temperatures of $290^{\circ} \mathrm{F}$ at the 20-percent recovery point and $470^{\circ} \mathrm{F}$ at the 90 -percent point, meeting Military Specification MIL-T-5624L (Grade JP-4). JP-4 is used for turbojet and turboprop aircraft engines, primarily by the military. Excludes ram-jet and petroleum rocket fuels.

Natural Gas. A mixture of hydrocarbons and small quantities of various nonhydrocarbons existing in the gaseous phase or in solution with crude oil in underground reservoirs.

Natural Gas Field Facility. A field facility designed to process natural gas produced from more than one lease for the purpose of recovering condensate from a stream of natural gas; however, some field facilities are designed to recover propane, normal butane, pentanes plus, etc., and to control the quality of natural gas to be marketed.

Natural Gas Plant Liquids. Natural gas liquids recovered from natural gas in gas processing plants, and in some situations, from natural gas field facilities. Natural gas liquids extracted by fractionators are also included. These liquids are defined according to the published specifications of the Gas Processors Association and the American Society for Testing and Materials and are classified as follows: ethane, propane, normal butane, isobutane, and pentanes plus. 
Natural Gas Processing Plant. A facility designed (1) to achieve the recovery of natural gas liquids from the stream of natural gas which may or may not have been processed through lease separators and field facilities, and (2) to control the quality of the natural gas to be marketed. Cycling plants are classified as gas processing plants.

Natural Gasoline and Isopentane. A mixture of hydrocarbons, mostly pentanes and heavier, extracted from natural gas, that meets vapor pressure, end-point, and other specifications for natural gasoline set by the Gas Processors Association. Includes isopentane which is a saturated branch-chain hydrocarbon, $\left(\mathrm{C}_{5} \mathrm{H}_{12}\right)$, obtained by fractionation of natural gasoline or isomerization of normal pentane.

Net Receipts. The difference between total movements into and total movements out of each PAD District by pipeline, tanker, and barge.

Normal Butane. See Butane.

OPEC. The acronym for the Organization of Petroleum Exporting Countries, that have organized for the purpose of negotiating with oil companies on matters of oil production, prices and future concession rights. Current members are Algeria, Gabon, Indonesia, Iran, Iraq, Kuwait, Libya, Nigeria, Qatar, Saudi Arabia, United Arab Emirates, and Venezuela. The Neutral Zone between Kuwait and Saudi Arabia is considered part of OPEC.

Operable Capacity. The amount of capacity that, at the beginning of the period, is in operation; not in operation and not under active repair, but capable of being placed in operation within 30 days; or not in operation but under active repair that can be completed within 90 days. Operable capacity is the sum of the operating and idle capacity and is measured in barrels per calendar day or barrels per stream day.

Operating Capacity. The component of operable capacity that is in operation at the beginning of the period.

Operable Utilization Rate. Represents the utilization of the atmospheric crude oil distillation units. The rate is calculated by dividing the gross input to these units by the operable refining capacity of the units.

Operating Utilization Rate. Represents the utilization of the atmospheric crude oil distillation units. The rate is calculated by dividing the gross input to these units by the operating refining capacity of the units.

\section{Other Finished. See Motor Gasoline (Finished).}

Other Hydrocarbons. Materials received by a refinery and consumed as a raw material. Includes hydrogen, coal tar derivatives, gilsonite, and natural gas received by the refinery for reforming into hydrogen. Natural gas to be used as fuel is excluded.

Other Oils Equal To or Greater Than $401^{\circ} \mathrm{F}$. See Petrochemical Feedstocks.

Other Oxygenates. Other aliphatic alcohols and aliphatic ethers intended for motor gasoline blending (e.g., isopropyl ether (IPE) or n-propanol).

Oxygenated Gasoline. See Motor Gasoline (Finished).

Oxygenates. Any substance which, when added to gasoline, increases the amount of oxygen in that gasoline blend. Through a series of waivers and interpretive rules, the Environmental Protection Agency (EPA) has determined the allowable limits for oxygenates in unleaded gasoline. The "Substantially Similar" Interpretive Rules (56 FR (February 11, 1991)) allows blends of aliphatic alcohols other than methanol and aliphatic ethers, provided the oxygen content does not exceed 2.7 percent by weight. The "Substantially Similar" Interpretive Rules also provides for blends of methanol up to 0.3 percent by volume exclusive of other oxygenates, and butanol or alcohols of a higher molecular weight up to 2.75 percent by weight. Individual waivers pertaining to the use of oxygenates in unleaded gasoline have been issued by the EPA. They include:

Fuel Ethanol. Blends of up to 10 percent by volume anhydrous ethanol (200 proof) (commonly referred to as the "gasohol waiver").

Methanol. Blends of methanol and gasoline-grade tertiary butyl alcohol (GTBA) such that the total oxygen content does not exceed 3.5 percent by weight and the ratio of methanol to GTBA is less than or equal to 1 . It is also specified that this blended fuel must meet ASTM volatility specifications (commonly referred to as the "ARCO" waiver).

Blends of up to 5.0 percent by volume methanol with a minimum of 2.5 percent by volume cosolvent alcohols having a carbon number of 4 or less (i.e., ethanol, propanol, butanol, and/or GTBA). The total oxygen must not exceed 3.7 percent by weight, and the blend must meet ASTM volatility specifications as well as phase separation and alcohol purity specifications (commonly referred to as the "DuPont" waiver).

MTBE (Methyl tertiary butyl ether). Blends up to 15.0 percent by volume MTBE which must meet the ASTM D4814 specifications. Blenders must take precautions that the blends are not used as base gasolines for other oxygenated blends (commonly referred to as the "Sun" waiver). 
Pentanes Plus. A mixture of hydrocarbons, mostly pentanes and heavier, extracted from natural gas. Includes isopentane, natural gasoline, and plant condensate.

Persian Gulf. The countries that comprise the Persian Gulf are: Bahrain, Iran, Iraq, Kuwait, Qatar, Saudi Arabia, and the United Arab Emirates.

Petrochemical Feedstocks. Chemical feedstocks derived from petroleum principally for the manufacture of chemicals, synthetic rubber, and a variety of plastics. The categories reported are "Naphtha Less Than $401^{\circ} \mathrm{F}$ " and "Other Oils Equal To or Greater Than $401^{\circ}$ F."

Naphtha Less Than $401^{\circ} \mathrm{F}$. A naphtha with a boiling range of less than $401^{\circ} \mathrm{F}$ that is intended for use as a petrochemical feedstock.

Other Oils Equal To or Greater Than $401^{\circ} \mathrm{F}$. Oils with a boiling range equal to or greater than $401^{\circ} \mathrm{F}$ that are intended for use as a petrochemical feedstock.

Petroleum Administration for Defense (PAD) Districts. Geographic aggregations of the 50 States and the District of Columbia into five districts by the Petroleum Administration for Defense in 1950. These districts were originally instituted for economic and geographic reasons as Petroleum Administration for War (PAW) Districts, which was established in 1942.

Petroleum Coke. A residue, the final product of the condensation process in cracking. This product is reported as marketable coke or catalyst coke. The conversion factor is 5 barrels per short ton.

Marketable Coke. Those grades of coke produced in delayed or fluid cokers which may be recovered as relatively pure curbon. This "green" coke may be sold as is or further puritied by calcining.

Catalyst Coke. In many catalytic operations (c.g., catalytic cracking) carbon is deposited on the catalyst, thus deactivating the catalyst. The catalyst is reactivated by burning off the carbon, which is used as a fuel in the refining process. This carbon or coke is not recoverable in a concentrated form.

Petroleum Products. Petroleum products are obtained from the processing of crude oi! (including lease condensate), natural gas, and other hydrocarbon compounds. Petroleum products include unfinished oils, liquefied petroleum gases, pentanes plus, aviation gasoline, motor gasoline, naphtha-type jet fuel, kerosene-type jet fuel, kerosene, distillate fuel oil, residual fuel oil, petrochemical feedstocks, special naphthas, lubricants, waxes, petroleum coke, asphalt, road oil, still gas, and misccllancous products.

Pipeline (Petroleum). Crude oil and product pipelines used to transport crude oil and petroleum products respectively, (including interstate, intrastate, and intracompany pipelines) within the 50 States and the District of Columbia.

Plant Condensate. Onc of the natural gas liquids, mostly pentanes and heavicr hydrocarbons, recovered and separated as liquids at gas inlet separators or scrubbers in processing plants.

Processing Gain. The volumetric amount by which total output is greater than input for a given period of time. This difference is due to the processing of crude oil into products which, in total, have a lower specific gravity than the crude oil processed.

Processing Loss. The volumetric amount by which total refinery output is less than input for a given period of time. This difference is due to the processing of crude oil into products which, in total, have a higher specific gravity than the crude oil processed.

Product Supplied, Crude Oil. Crude oil burned on leases and by pipelines as fucl.

Production Capacity. The amount of product that can be produced from processing facilitics.

Products Supplied. Approximately represents consumption of petroleum products because it measures the disappearance of these products from primary sources, i.c., refincrics, natural gas processing plants, blending plants, pipclines, and bulk terminals. In general, product supplied of each product in any given period is computed as follows: ficld production, plus refinery production, plus imports, plus unaccounted for crude oil, (plus net receipts when calculated on a PAD District basis), minus stock change, minus crude oil losses, minus refinery inputs, minus exports.

Propane $\left(\mathrm{C}_{3} \mathrm{H}_{8}\right)$. A normally gascous straight-chain hydrocarbon. It is a colorless paraffinic gas that boils at a temperature of $-43.67^{\circ} \mathrm{F}$. It is extracted from natural gas or refinery gas streams. It includes all products designated in ASTM Specification D1835 and Gas Processors Association Specifications for commercial propane and HD-5 propane.

Propylene $\left(\mathrm{C}_{3} \mathrm{H}_{6}\right)$. An olefinic hydrocarbon recovered from refincry processes or petrochemical processes. 
Refinery. An installation that manufactures finished petroleum products from crude oil, unfinished oils, natural gas liquids, other hydrocarbons, and oxygenates.

Refinery Input, Crude Oil. Total crude oil (domestic plus foreign) input to crude oil distillation units and other refinery processing units (cokers, etc.).

Refinery Input, Total. The raw materials and intermediate materials processed at refineries to produce finished petroleum products. They include crude oil, products of natural gas processing plants, unfinished oils, other hydrocarbons and oxygenates, motor gasoline and aviation gasoline blending components and finished petroleum products.

Refinery Production. Petroleum products produced at a refinery or blending plant. Published production of these products equals refinery production minus refinery input. Negative production will occur when the amount of a product produced during the month is less than the amount of that same product that is reprocessed (input) or reclassified to become another product during the same month. Refinery production of unfinished oils, and motor and aviation gasoline blending components appear on a net basis under refinery input.

Refinery Yield. Refinery yield '(expressed as a percentage) represents the percent of finished product produced from input of crude oil and net input of unfinished oils. It is calculated by dividing the sum of crude oil and net unfinished input into the individual net production of finished products. Before calculating the yield for finished motor gasoline, the input of natural gas liquids, other hydrocarbons and oxygenates, and net input of motor gasoline blending components must be subtracted from the net production of finished motor gasoline. Before calculating the yield for finished aviation gasoline, input of aviation gasoline blending components must be subtracted from the net production of finished aviation gasoline.

\section{Reformulated Gasoline. See Motor Gasoline (Finished).}

Residual Fuel Oil. The heavier oils that remain after the distillate fuel oils and lighter hydrocarbons are distilled away in refinery operations and that conform to ASTM Specifications D396 and 975. Included are No. 5, a residual fuel oil of medium viscosity; Navy Special, for use in steam-powered vessels in government scrvice and in shore power plants; No. 6, which includes Bunker $\mathrm{C}$ fuel oil, and is used for commercial and industrial heating, electricity generation and to power ships. Imports of residual fuel oil include imported crude oil burned as fuel.
Residuum. Residue from crude oil after distilling off all but the heaviest components, with a boiling range greater than $1000^{\circ} \mathrm{F}$

Road Oil. Any heavy petroleum oil, including residual asphaltic oil used as a dust pallative and surface treatment on roads and highways. It is generally produced in six grades from 0 , the most liquid, to 5 , the most viscous.

Shell Storage Capacity. The design capacity of a petroleum storage tank which is always greater than or equal to working storage capacity.

Special Naphthas. All finished products within the naphtha boiling range that are used as paint thinners, cleaners, or solvents. These products are refined to a specified flash point. Special naphthas include all commercial hexane and cleaning solvents conforming to ASTM Specification D1836 and D484, respectively. Naphthas to be blended or marketed as motor gasoline or aviation gasoline, or that are to be used as petrochemical and synthetic natural gas (SNG) feedstocks are excluded.

Steam (Purchased). Steam, purchased for usc by a refinery, that was not generated from within the refinery complex.

Still Gas (Refinery Gas). Any form or mixture of gases produced in refincries by distillation, cracking, reforming, and other processes. The principal constituents are methane, ethane, ethylene, normal butane, butylenc, propane, propylene, etc. Still gas is used as a refinery fucl and a petrochemical feedstock. The conversion factor is 6 million BTU's per fucl oil equivalent barrel.

Stock Change. The difference between stocks at the beginning of the month and stocks at the end of the month. A negative number indicates a decrease in stocks and a positive number indicates an increase in stocks.

Strategic Petroleum Reserve (SPR). Petrolcum stocks maintained by the Federal Government for use during periods of major supply interruption.

Sulfur. A nonmetallic clement of lemon-ycllow color, sometimes known as "brimstonc".

Supply. The components of petrolcum supply are field production, refinery production, imports, and net receipts when calculated on a PAD District basis.

TAME (Tertiary amyl methyl ether) $\left(\mathrm{CH}_{3}\right)_{2}\left(\mathrm{C}_{2} \mathrm{H}_{5}\right) \mathrm{COCH}_{3}$. An oxygenate blend stock formed by the catalytic etherification of isoamylene with methanol. 
Tank Farm. An installation used by gathering and trunk pipcline companies, crude oil producers, and terminal operators (except refineries) to store crude oil.

Tanker and Barge. Vessels that transport crude oil or petroleum products. Data are reported for movements between PAD Districts; from a PAD District to the Panama Canal; or from the Panama Canal to a PAD District.

TBA (Tertiary butyl alcohol) ( $\left.\mathrm{CH}_{3}\right)_{3} \mathrm{COH}$. An alcohol primarily used as a chemical feeds'ock, a solvent or feedstock for isobutylene production for MTBE; produced as a co-product of propylene oxide production or by direct hydration of isobutylene.

Thermal Cracking. A refining process in which heat and pressure are used to break down, rearrange, or combine hydrocarbon molecules. Thermal cracking includes gas oil, visbreaking, fluid coking, delayed coking, and other thermal cracking processes (c.g., flexicoking). See individual categories for definition.

Toluene $\left(\mathrm{C}_{6} \mathrm{H}_{5} \mathrm{CH}_{3}\right)$. Colorless liquid of the aromatic group of petroleum hydrocarbons, made by the catalytic reforming of petroleum naphthas containing methyl cyclohexane. A high-octane gasoline-blending agent, solvent, and chemical intermediate, base for TNT.

Unaccounted for Crude Oil. Represents the arithmetic difference between the calculated supply and the calculated disposition of crude oil. The calculated supply is the sum of crude oil production plus imports minus changes in crude oil stocks. The calculated disposition of crude oil is the sum of crude oil input to refineries, crude oil exports, crude oil burned as fuel, and crude oil losses.

Unfinished Oils. Includes all oils requiring further processing, except those requiring only mechanical blending. Includes naphthas and lighter oils, kerosene and light gas oils, heavy gas oils, and residuum. See individual catcgorics for definition.

Unfractionated Streams. Mixtures of unsegregated natural gas liquid components excluding those in plant condensate. This product is extracted from natural gas.

United States. The United States is defined as the 50 States and the District of Columbia.

Vacuum Distillation. Distillation under reduced pressure (less the atmospheric) which lowers the boiling temperature of the liquid being distilled. This technique with its relatively low temperisures prevents cracking or decomposition of the charge stock.

Visbreaking. A thermal cracking process in which heavy atmospheric or vacuum-still bottoms are cracked at moderate temperatures to increase production of distillate products and reduce viscosity of the distillation residues.

Wax. A solid or semi-solid material derived from petroleum distillates or residues by such treatments as chilling, precipitating with a solvent, or de-oiling. It is light-colored, morc-or-less translucent crystalline mass, slightly greasy to the touch, consisting of a mixture of solid hydrocarbons in which the paraffin series predominates. Includes all marketable wax whether crude scale or fully refined. The three grades included are microcrystalline, crystalline-fully refined, and crystalline-other. The conversion factor is 280 pounds per 42 U.S. gallons per barrel.

Microcrystalline Wax. Wax extracted from certain petroleum residues having a finer and less apparent crystalline structure than paraffin wax and having the following physical characteristics: penetration at $77^{\circ} \mathrm{F}$ (D1321)-60 maximum; viscosity at $210^{\circ} \mathrm{F}$ in Saybolt Universal Seconds (SUS); (D88)-60 SUS (10.22 centistokes) minimum to 150 SUS (31.8 centistokes) maximum; oil content (D721)-5 percent minimum.

Crystalline-Fully Refined Wax. A light-colored paraffin wax having the following characteristics: viscosity at $210^{\circ} \mathrm{F}$ (D88)-59.9 SUS (10.18 centistokes) maximum; oil content (D721)-0.5 percent maximum; other +20 color, Saybolt minimum.

Crystalline-Other Wax. A paraffin wax having the following characteristics: viscosity at $210^{\circ} \mathrm{F}$ (D88)-59.9 SUS (10.18 centistokes) maximum; oil content (D721)-0.51 percent minimum to 15 percent maximum.

Working Storage Capacity. The difference in volume between the maximum safe fill capacity and the quantity below which pump suction is ineffective (bottoms).

Xylene $\left(\mathrm{C}_{6} \mathrm{H}_{4}\left(\mathrm{CH}_{3}\right)_{2}\right)$. Colorless liquid of the aromatic group of hydrocarbons made the catalytic reforming of certain naphthenic petroleum fractions. Used as high-octane motor and aviation gasoline blending agents, solvents, chemical intermediates. Isomers are metaxylene, orthoxylene, paraxylene. 

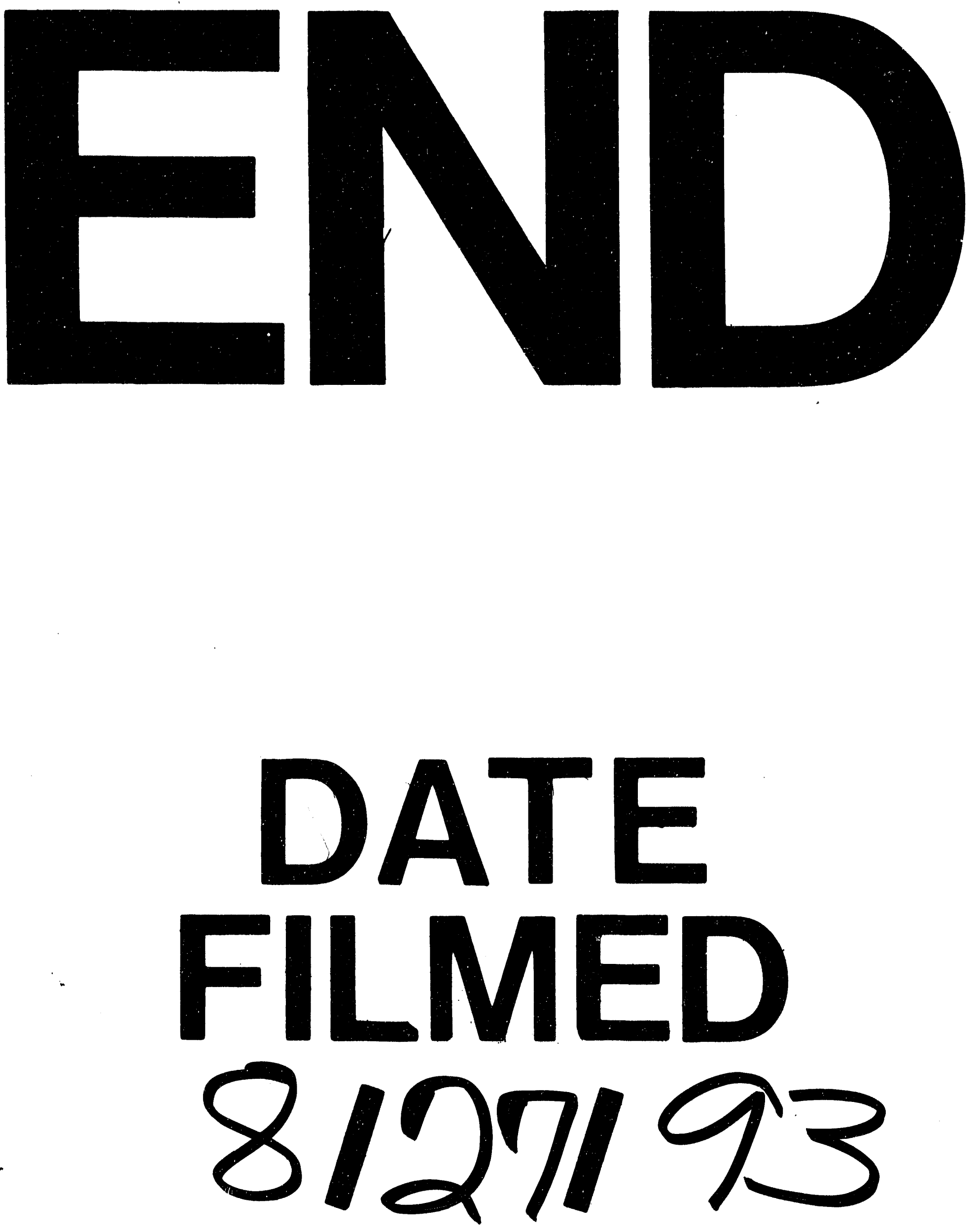


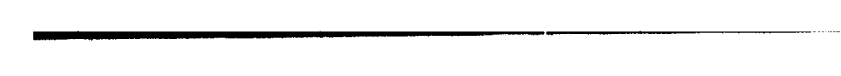

